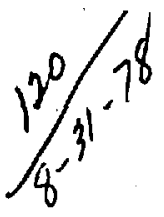

\title{
REFERENCE DESIGN FOR THE STANDARD MIRROR HYBRID REACTOR
}

\section{A Joint Report by Lawrence Livermore Laboratory and General Atomic Company}

Lewrence Livermore Laboratory

D. J. Bender, Editor

R. S. Devoto, J. H. Fink, T. R. Galloway,

W. E. Kastenberg, J. D. Lee, W. S. Neef, Jr.

\section{MASTER}

\section{General Atomic Company}

K. R. Schultz, D. W. Culver,

B. Bao, S. R. Rao

May 22, 1978
Work performed under the auspices of the U.S. Department af Energy by the UCLLL under contract number W-7405-ENG-48 and by General Atomic Co. under contracts number E-04-3-167. Project Agreement 38, and number EY-76-C-03-0167. Project Agreement 38.

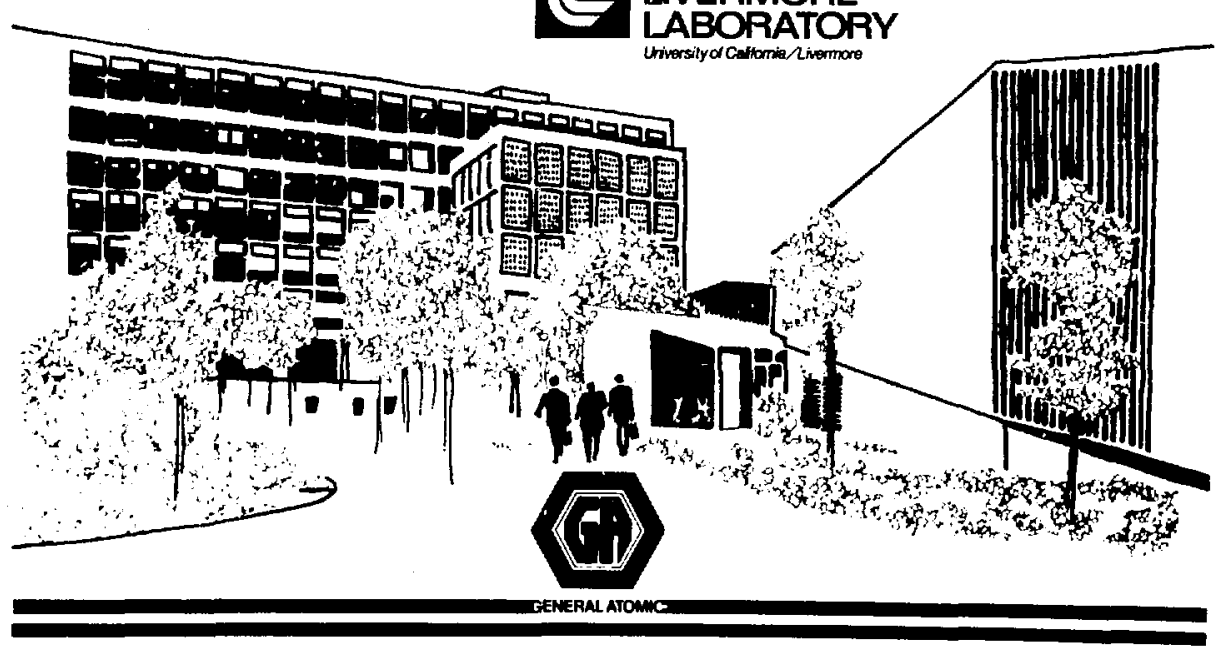




\section{近}

\section{LAWRENCE LIVERMORE LABORATORY}

University of Calfornia Livermore,Catifornia 94550

\section{UCRL-52478 \\ GA-A14796 \\ 'REFERENCE DESIGN FOR THE STANDARD MIRROR HYBRID REACTOR

\author{
A Joint Report by \\ Lawrence Livermore Laboratory \\ and \\ General Atomic Company
}

\section{Lawrence Livermore Laboratory \\ D. J. Bender, Editor}

J. H. Fink, T. R. Galloway, W. E. Kastenberg", J. D. Lee,

R. S. Devoto, W. S. Neef, Jr.

General Atomic Company

K. R. Schultz, D. W. Culver, S. B. Rao, S. R. Rao

This repen wa pepared it an woosmt of work tpon wored by the Unised Stutes Govemment. Neither the Und led Sutes ner the United States Deputment of Exetgy, not any of thett emptornes, not any of their

comtractors, sibcontrietion, of thet employer, mathes

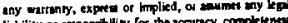

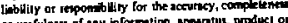

or unfulnes of any in farmation, appantun, producl o

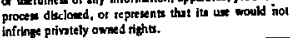

University of California at Los Angeles

MS. date: May 22, 1978

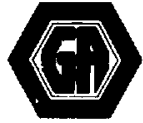




\section{FOREWORD}

This study was sponsored by the Energy Research and Development Administration and the Department of Energy, and was performed as a cooperative investigation by Lawrence Livermore Iaboratory (under Contract No. W-7405-EMG-48) and General Atonic Company (under Contracts Nos. E-04-3-167, Project Agreenent 38, and EY-76-C-03-0167, Project Agreement 38). 


\section{CONTENTS}

Abstract

1. Introduction

References

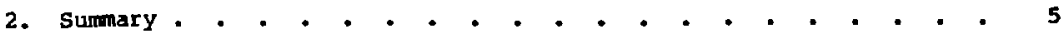

3. Reactor Description - . - . . . - . . . . . . • . . 28

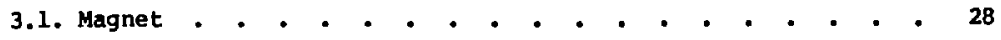

3.2. Injectors . . . . . . . . . . . . . . . . . 30

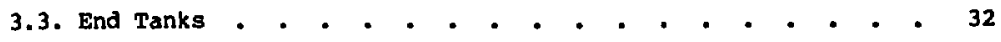

3.4. Vacuur System . . . . . . . . . . . . . . . . 34

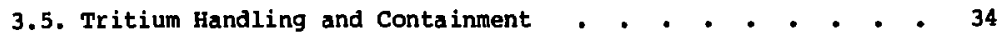

3.6. Cryogenic system. . . . . . . . . . . . . . . 38

3.7. Prestressed Concrete Reactor vessel . . . . . . . . 38

3.8. Blanket . . . . . . . . . . . . . . . . . . 40

3.9. Power Conversion System . . . . . . . . . . . . 48

4. System Model and Economic Analysis (D. J. Bender) . . . . . . 49

4.1. Introduction . . . . . . . . . . . . . . . . 49

4.2. Description . . . . . . . . . . . . . . . . 49

4.3. Reference Design . . . . . . . . . . . . . . . 53

4.4. Parametric Analysis . . . . . . . . . . . . . . 71

References . . • . . . . . . . . . . • . . . . 87

5. Plasma Physics (R. S. Devoto) . . . . . . . . . . . . 88

5.1. Introduction . . . . . . . . . . . . . . . . 88

5.2. Magnetohydrodynamic Stability . • . • . • . . . . 92

5.3. Drift Surfaces . . . . . . . • . . . . . . 96

5.4. Equilibrium Magnetic Fields . - . . • • • . . . . 97

5.5. Fokker-Planck Computations . . . . . . . . . . 110

5.6. Radial Density Profiles . . . . . . . . . . . . 119

5.7. Discussion . . . . . . . . . . . . . . . . . 135

References . . . . . . . . . . . . . . . . . . 138

6. Fusion Components . . . . . . . . . . . . . . . . 140

6.1. Magnet Design (D. J. Bender) . . . . . . . . . . . 140

6.2. Neutral Bean Injectors (J. H. Bink) - • • • • . . . 171

6.3. Beam Line Layout (D. J. Bender) . . . . . . . . . . 203

6.4. End Tanks (D. J. Bender) . . . . . . . . . . . . 210

6.5. Vacuum System (D. J. Bender) . . . . . . . . . . . 228 
6.6. Tritium Containment and Handling (T. R. Galloway) . . . . 236

6.7. Cryogenic Systems (D. J. Bender) . . . . . . . . . 298

References . . . . . . . . . . . . . . . . . . 304

7. Nuclear Analysis (J. D. Lee) . . . . . . • . • . . . . 310

7.1. Blanket Nuclear Analysis . . . . . . . . . . . 310

7.2. Shielding . . . . . . . . . . . . . . . . . 341

References . • • • • • • • • • . • . • • • 346

8. Mechanical Design . . . . . . . . . . . . . . • 348

8.1. Prestressed Concrete Reactor Vessel

(D. W. Culver, W. S. Neef, Jr.) . . . . . . . . . . 348

8.2. Blanket Moâlu Design (D. W. Culver, K. R. Schultz) • . 361

References . . . . . . . . . . . . . . . . . . 381

9. Heat Transport Systems . . • . . . . • • • • • • • 382

9.1. Fuel Design (S. B. Rao, K. R. Schultz) . . . . . . . 382

9.2. Power Conversion System (S. R. Rao) . . . . . . . . 404

References . . . . . . . . . . . . . . . . 443

10. Plant Layout (W. S. Neef, Jr.) • . . . . . • . • • . . 144

10.1. General Considerations . . . . . . . . . . . . . 444

10.2. Magnet Considerations . . . . . . . . . . . . . 445

10.3. PCRV Considerations . . . . . . . . . . . . . . 448

10.4. End Tank Considerations . . . . . . . . . . . . 448

10.5. Containment Considerations . . . . . . . . . . . 450

11. Power Conversion Loop Control System (5. R. Rao) . . . . . . 451

11.1. Introduction . . . . . . . . . . . . . . . 451

11.2. Plant Control System + . . . . . . . . . . . 452

11.3. Plant Protection System . . . . . . . . . . . . 462

12. Safety . . . . . . . . . . . . . . . . . . . . 466

12.1. Safety Design (S. R. RaO) . . . . . . . . . . . . 466

12.2. Safety and Environmental Effects (w. B. Kastenberg) . . . 00

References - . . . . . . . . . . . . . . . . . . 511

Appendix A: Alternate Mechanical Design (W. S. Neef, Jr.) . • • 514

Appendix B: Evaluation of Hybrid Cost Accounts (D. J. Bender,

$K$. R. Schultz, and D. W. Culver) . . . . . . . . . 524

B.l. Capital Costs . . . . . . . . . . . . . . . . 524

B.2. Fuel Cycle Costs . . . . . . . . . . . . . . . 535

B.3. Blanket and NSSS Cost E itimates . . . . . . . . . . 537

References . . . . . . . . . . . . . . . . . . . 542 


\section{ABSTRACT}

This report describes the results of a two-year study by Lawrence Livermore Laboratory and General Atomic Co. to develop a conceptual design for the standard (minimum-B) mirror hybrid reactor. The reactor parameters have been chosen to minimize the cost of producing nuclear fuel $\left({ }^{239}\right.$ Pu) for consumption in fission power reastors (light water reactors).

The deuterium-tritium plasma produces r $400 \mathrm{~kW}$ of fusion power with a plasma $Q$ of 0.64 . The fast-fission blanket, which is fueled with depleted uranium and lithium, generates sufficient tritium to run the reactor, has a blanket energy multiplication of $M=10.4$, and has a net fissile breeding ratio of $\mathrm{Pu} / \mathrm{n}=1.51$. The reactor has a net electrical output of $600 \mathrm{MHe}$, a fissile production of $2000 \mathrm{~kg}$ of plutoniur. per year (at a capacity factor of $0.74)$, and a net plant efficiency of 0.18 .

The plasma-containment field is generated by a Yin-Yang magnet using NbTi superconductor, and the neutral beam system uses positive-ion acceleration with beam direct conversion. The spherical blanket is based on gas-cooled fast reactor technology. The fusion components, blanket, and primary heattransfer loop components are all contained within a prestressed-concrete reactor vessel, which provide magnet restraint and supports the primary heattransfer loop and the blanket.

The degign is based on existing technology where possible and a minimum extrapolation of technology otherwise. The projections for the plasma physics are conservative, in that they are well-fcunded on the experiments in $2 x I I B$ and the interpretation of these experiments. The resulting reactor may therefore be viewed as a comparatively near-term goal of the magnetic fusion program. 


\section{INTRODUCTION}

Lawrence Livermore Laboratory has been investigating the hybrid reactor concept since 1970. The present report represents a milestone in this research, in that it culminates two years of work by a joint Lawrence Livermore Laboratory/General Atomic Co. design team to develop an optimized conceptual design of a commercial hybrid reactor based on standard, or minimum-B, mirror plasma confinement. Here, the term "optimized" means that the hybrid parameters are selected to minimize the cost of producing electrical power from a fusion-fiseion power system. This power system consists of the hybrid reactor plus the light-water fission reactors which are supplied with nuclear fuel produced by the hybrid $\left({ }^{239} \mathrm{Pu}\right.$ in this case).

In 1972, J. D. Lee of LLL published the first report of the nuclear performance of fast-fission blankets. ${ }^{l}$ In the next two years at LLI, hybrid blanket neutronics continued, ${ }^{2,3}$ preliminary system studies ${ }^{4}$ and blanket designs ${ }^{5}$ were undertaken, and a mirror hybrid conceptual design study with Battelle Pacific Northwest Laboratories was completed. ${ }^{6}$ That hybrid design employed a thermal-fission blanket with high energy multiplication but comparatively low fissile-fuel production. At this point in their evolution, the mirror hybrid studies were reviewed by werner et $a 1.7$ and Leonard et $a 1^{8}$

In 1975, LUI undertook the first point-design study of a mirror hybrid with a fast-fission blanket, ${ }^{9}$ with the objectives of determining a marner in which all of the necessary components could be integrated into the reactor, assessing the technological problems, and obtaining a rough cost estimate. It was concluded that the mirror hykrid with a fast-spectrum uranium blanket was an attractive concept, and that the point design would benefit from refinement and optimization. Also as a result of this study, we concluded that the economically most advantageous configuration for the hybrid is as a nuclear fuel producer. This rationale was discussed by Moir 10,11 and has been subsequently quantified by Bender. ${ }^{12}$

In 1976, the mirror hybrid studies emphasized reactor optinization, including the neutronic analysis of metallic fuels and development of a system analysis model. 13 The system model allowed identification of key ecommic parameters, such as the importance of optimum first-wall loading $\left(1-2 \mathrm{~m} / \mathrm{m}^{2}\right)$ and a large fertile-material volume fraction in the blanket. 
Also in 1976, General Atonic Company joined with ILL in a cooperative study, to apply their expertise in gas-cooling technology to the mirror hybrid and to evaluate the 1975 design $^{14}$ from the standpoint of a reactor vendor with considerable experience in the-design of fission reactors. The results of the $1976 \mathrm{LLL} / \mathrm{GA}$ studies were reported at the 1976 US-OSSR Fusion-Pission Symposium. ${ }^{15}$

The present conceptual design study is founded upon this experience in pcint design, component optinization, and system analysis. The design employs the earliest available fusion technology (classical mirror plasma confinenent, positive-ion neutral beam systems, NbTi superconducting magnets) and state-of-the-art fission technology (primarily, gas-cooled fast reactor technology). It is thus intended to represent the characteristics of an early comercial reactor.

The report is organized as follows: Chapter 2 is a sumary of the reactor design; Chapter 3 contains brief descriptions of the component designs; and Chapter 4 presents the final reactor parameters and the economics of the design and discusses the results of the parametric optimization. Chapters 5-10 present the detalled design information. Chapter 11 discusses plant control, and Chapter 12, safety. 


\section{References}

1. J. D. Lee, "Neutronics of Sub-Critical Fast Fission Blankets for D-T Fusion Reactors," Proc. 7th Intersociety Energy Conversion Engineering Conf., 1972, San Diego (American Chemical Society, 1972), p. 1294; also, Lawrence Livermore Laboratory, Rept. UCRL-73952 (1972).

2. J. D. Lee, "Neutronic Analysis of a 2500 Mat Fast Fission Natural Uranium Blanket for a DT Fusion Reactor," Proc. 1st Topical Meeting on the Technology of Controlled Nuclear Fusion, San Diego (USAEC, 1974), CONF-740402-P1, p. 223; also, Lawrence Livermore Laboratory, Rept. UCRL-75304 (1974).

3. R. C. Haight and J. D. Lee, "Calculations of a Fast Fission Blanket for DT Fusion Reactors with Two Evaluated Nuclear Data Libraries," Proc. lst Topical Meeting on the Technology of Controlled Nuclear Fusion, San Diego (USAEC, 1974), CONF-740402-P1, D. 271; also, Lawrence Livermore Laboratory, Rept. UCRL-75627 (1974).

4. R. W. Moir, "Conceptual Design Consideration for D-T Mirror Reactors With and Without a Fission Blanket," Proc. lst Topical Meeting on the Technology of Controlled Nuclear Fusion, San Diego (USAEC, 1974), CONF-740402-P2, p. 373; also, Lawrence Livermore Laboratory, Rept. UCRL-75596 (1974).

5. L. D. Hansborough and R. W. Werner, "A Modular Fusion-Fission Hybrid Blanket," Proc. Ist Topical Meeting on the Technology of Controlled Nuclear Fusion, San Diego (USAEC, 1974), CONF-740402-P1, p. 211; also, Lawrence Livermore Laboratory, Rept. UCRL-51610 (1974).

6. W. C. Wolkenhauer, B. R. Leonard, Jr., A. M. Sutey, and R. W. Moir, "Conceptual Design of a Fusion-Fission Hybrid Reactor with a Subcritical Gas-Cooled Fission Blanket," Proc. lst Topical Meeting on the Technology of Controlled Nuclear Fusion, San Diegc (USAEC, 1974), CONE-740402-P1, P. 238 =

7. R. W. Werner, J. D. Lee, R. W. Moir, G. A. Carlson, M. A. Peterson, W. C. Wolkenhauer, and B. R. Leorard, Jr., "The Interesting Possibilities of Fusion-Fission," Proc. 5th Conf. on Plasma Physics and Controlled Nuclear Fusior, Research, Tokyo (IAEA, 1975), Vol. 3, p. 641; also, Lawrence Livermore Laboratory, Rept. UCRL-75527 (1974). 
8. B. R. Leonard, Jr, and W. C. Wolkenhauer, "Progress Toward the Development of a Mirror Hybrid (Fusion-Fission) Reactor," Proc. 5th Conf. on Plasma Physics and Controlled Nuclear Fugion Regearah, Tokyo (IAEA, 1975), Vol. 3, p. 649.

9. R. W. Moir, J. D. Lee, R. J. Burleigh, W. L. Barr, J. H. Fink, G. W. Hamilton, D. J. Bender, G. A. Carlson, W. L. Dexter, J. Holdren, C. L. Folkers, M. A. Peterson, M. E. Rensink, H. W. Patterson, R. L. Nelson, and C. E. Taylor, Progreas on the Conceptuaj Design of a Mirror Hybrid Fusion-Fission Reactor, Lawrence Livermore Laboratory, Rept. UCRL-51797 (1975).

10. R. W. Moir, "Mirror Fusion-Fission Reactor Designs," Proc. US-USSR Symposium on Fusion-Fission Reactors, Livermore (1976), CONF-760733, p. 15; 2.1so, Lawrence Livermore Laboratory, Rept. UCRL-78629 (1976).

11. Mirror Hybrid Reactor Studies, July 2975 through September 1976, J. D. Lee, ed., UCRL-50043-1, Lawrence Livermore Laboratory (1976).

12. D. J. Bender, Performance Parameters for Fusion-Fission Power Sygtems, Lawrence Iivermore Laboratory, Rept. UCRL-80589 Rev. 1 (1978) (submitted to Nuclear Technology).

13. D. J. Bender and G. A. Carlson, System Model for Analygis of the Mirror Fusion-Fission Reactor, Lawrence Livermore Laboratory, Rept. UCRL-52293 (1977).

14. K. R. Schultz, C. B. Baxi, R. Rao, A. R. Veca, G. A. Backus, J. B. Dee, E. A. Estrine, M. T. Sinnad, C. C. Baker, F. L. Openshaw, and H. J. Snyder, Jr., Conceptual Design of the Blanket and Power Conversion System for a Mirror Hybrid Fusion-Fission Reactor, General Atomic Co., Rept. GA-A14021 (1976).

15. Proc. US-USSR Symposium on Funion-Fission Reactors, Iivernore (1976), CONF-760733. 


\section{SUMMARY}

\subsection{Overall Design}

The major design cholces that were made for the Mirror Hybrid Reactor are:

- Minimum-B mirror confinement of deutarium-tritium plasma

- Yin-Yang coil design, NbTi superconductor

- Positive-ion injectors with direct recovery

- Fast-spectrum blanket neutronics

- single-stage plasma direct convertor

- Cryocondensation vacuun pumping

- Blanket

$\mathrm{U}_{3} \mathrm{Si}$ fuel (depleted uranium)

IiD tritium breeder (natural lithium)

Inconel 718 structural material

- Helium primary heat-transfor loop (PHTL)

- Prestressed-concrete reactor vessel (PCRV)

Magnet restraint

PHTL restraint

Blanket support and restraint

- stean thermal conversion system

A cutaway view of the reactor is shown in Fig. 2-1. The containnent building houses the prestressed-concrete reactor vessel (PCRV), which in turn contains the fusion components and che primary heat-transfer loop (PHiL). The service crane (500-tonne capacity) is mounted atop the PCRV, with vertical access to the components within the concrete structure. Since the magnet is mounted with its axis vertical, the end tanks for plasma leakage are above and below the magnet. The magnet is designed so that only 10 of the exhaust plasma leaks into the top end tank, in order that the top end tank can be small. The small size of the top eni tank peraits access to the blanket and confinement region from the top of the PCRV by removal of the top shielding blocks and entry through the top of the end tank.

The blanket is the spherical shell inside the Yin-Yang magnet. Two neutral beam injector tanks are located within the PCRV and penetrate the blanket just above the lobes, or turnarounds, of the lower magnetic coil. 


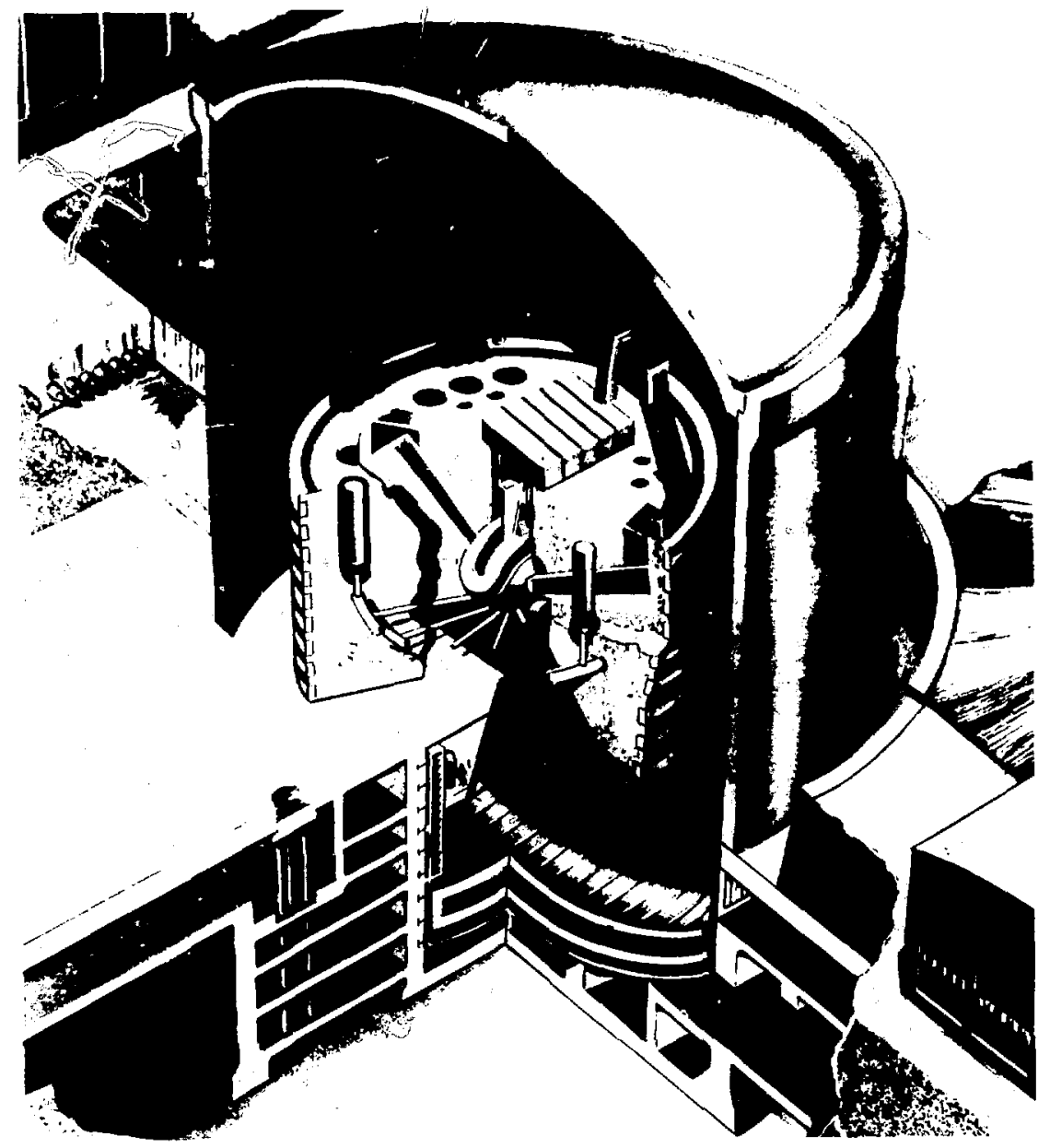

Fig. 2-1. Cutaway view of the reactor. 
The steam generators and helium circulators are arrayed around the periphery of the PCRV. A:l helium duciing is integra? to the PCRV; two large ring manifolds (supply and return) are located beneath the steam generators and circulators, and coolant is exchanged with the sianket via the radial pipe runs shown in the figure.

Reactor parameters are stimmarized in Table 2-1, and Fig, 2-2 is a power flow diagram for the reactor. The D-T plasma has a fusion power output of $\sim 400 \mathrm{MW}$ (neutrons plus alphas) and the reactor produces $\sim 600 \mathrm{MH}$ of net electrical outyut and $-2000 \mathrm{~kg}$ of piutonium per year. The plasma $Q$ of 0.64 was found to be an optimum within the constraints of the design. Higher $Q$ was possible, but it resulted in a lower first-wall loading, and this configuration was found to yield poorer economics than the reference design parameters. Higher $Q(\sim 1)$ with high first-wall loading could be achieved only if the magnetic fieid at the conductor were raised to 12-14 $\mathrm{T}$, and to do that would require using $\mathrm{Nb}_{3} \mathrm{Sn}$ as the superconductor.

\subsection{Plasma Physics}

Mirror plasma physics experiments have progressed rapidly in the last few years. The next-generation mirror experiment, MFTF, will represent the physics prototype for a reactor-grade plasma. This proposition is illustrated in Table 2-2, where we list key physics parameters for 2 XIIB, the present mirror experiment; for MFTF; and for the commercial hybrid reactor being discussed here. 2 XIIB has 20-keV neutral-beam injectors, some of which are being upgraded to $40 \mathrm{keV}$; MFTF will have $20-\mathrm{keV}$ injectors for start-up and $80-\mathrm{keV}$ injectors for sustained $(0.5 \mathrm{~s})$ operation. The major differences between the MFTF plasma and that required for a commercial reactor are in size and duty factor; however, MFTF is versatile enough so that it should establish scaling laws that will allow us to extrapolate to the larger plasma with a high degree of confidence. Also. $n \tau$ in the comercial facility is a factor of 10 larger than in MFTF, as a result of the higher plasma energy and the use of D-T instead of deuterium. Thus, achieving the anticipated plasma performance from MFTF will provide an adequate physics base from which development of a mirror hybrid could proceed. 
Table 2-1. Sumary of reactor characterlstics.

Magnet

Central field, $\mathbf{T}$

2.52

Mirror ratio

2.25

Max field at conductor, T

8.5

\section{Injectors}

Type

Positive-ion acceleration

Extraction voltage, $\mathrm{kV}$

Deuteriun

125.0

Tritium

187.5

Efficiency

0.58

Injected current, A

Deuterium

Tritium

\section{Plagma}

Av injection energy, kev

121

plasma mirror catio

4.15

Beta

Central plasma density, $\mathrm{cm}^{-3}$

0.71

$n T, s / \mathrm{cm}^{3}$

$9 \times 10^{13}$

$\mathbf{Q}$

$2 \times 10^{13}$

0.64

Dimensions

Mirror-to-mirror length, m

13.0

Radius at midplane, m

2.5

\section{Blanket}

Fractional blanket coverage

0.86

Av blanket multiplication (864 coverage)

10.4

First-wall load, MN/m $\mathrm{m}^{2}$

1.7

Peak fuel power density, $w / \mathrm{cm}^{3}$

280

coolant

Pressure, ats

60

Inlet/outlet temperatures, ${ }^{\circ} \mathrm{C}$ $280 / 540$

End-of-life exposure, $w-y / m^{2}$

6.6

Av fissile breeding ratio ( $86 *$ coverage)

1.5 
Table 2-1. (Continued).

End tankB

$\begin{array}{ll}\text { Weak mirror (large tank) } & \\ \text { Input power, wN } & 607 \\ \text { ifect converter efficiency } & 0.10 \\ \text { iectical output, w } & 243 \\ \text {.ong mirror (small tank) } & 103 \\ \text { Input power, ww } & \\ \text { No direct conversion }\end{array}$

Thermal conversion cycle

$\begin{array}{lc}\text { Type } & \text { Steam } \\ \text { Turbine inlet conditions } & \\ \text { Temperature, }{ }^{\circ} \mathrm{C} & 440 \\ \text { Pressure, MPa } & 0.42 \\ \text { Reheat conditions } & \\ \text { Temperature, }{ }^{\circ} \mathrm{C} & 504 \\ \text { Pressure, MPa } & 5.26 \\ \text { Efficiency } & 0.355\end{array}$

\section{Overall}

$\begin{array}{ll}\text { Net electrical output, MN } & 603 \\ \text { Plant capacity factor } & 0.74 \\ \text { Annual plutonium production, kg } & 2016 \\ \text { Recirculating power fraction } & 0.65 \\ \text { Net plant efficiency } & 0.18\end{array}$




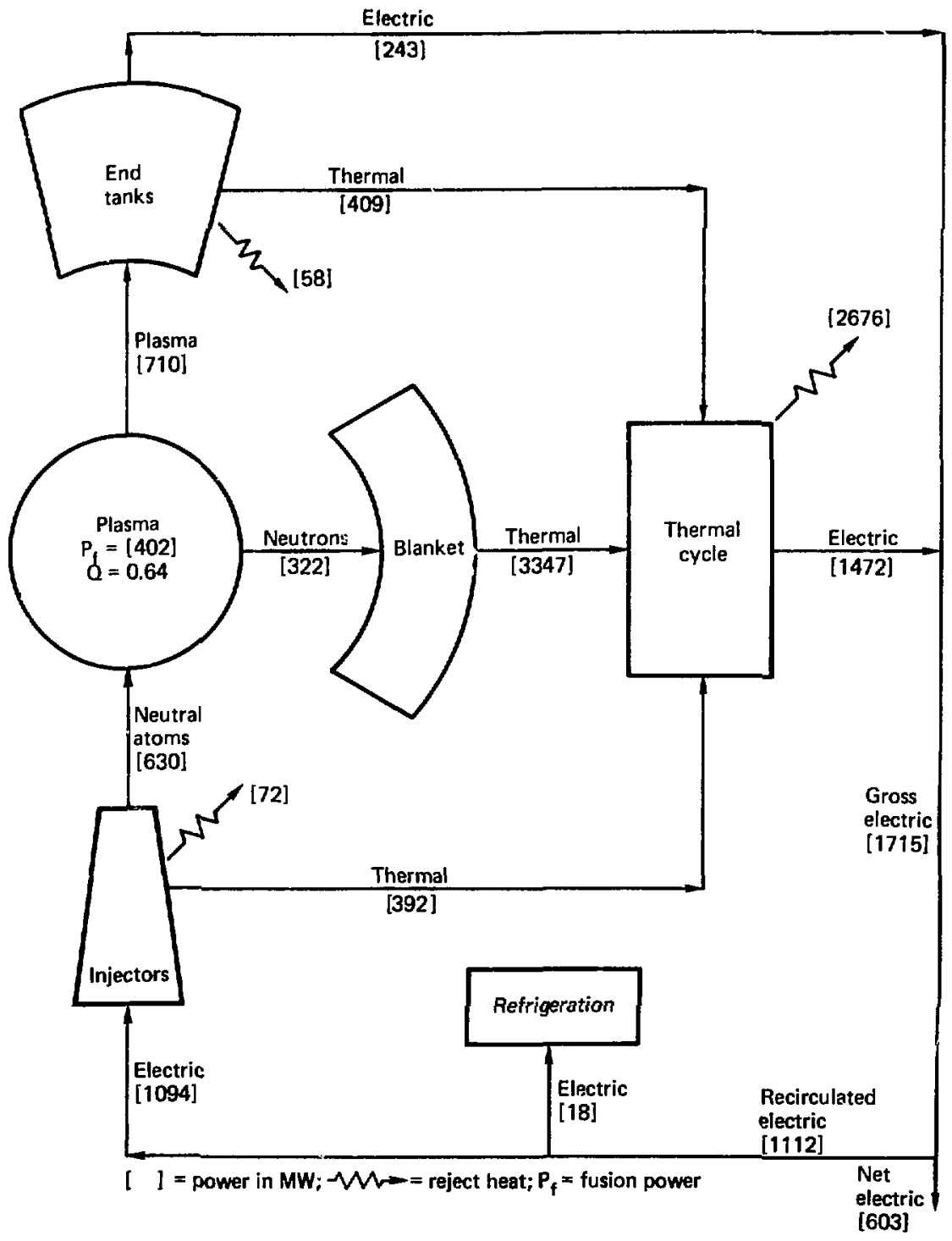

Fig. 2-2. Reactor power flow. 
Table 2-2. Comparison of plasma parameters in 2XIIB, MFTF, and a comercial hybrio reactor.

\begin{tabular}{llll}
\hline & 2XIIB & MrTF & Comercial hybrid \\
\hline Plasma length, m & 1.6 & 3.4 & 13 \\
Conductor field, T & - & 7 & 8.5 \\
$\mathrm{~B}$ & $0.5-1$ & 0.5 & 0.7 \\
Injector extraction energy, keV & $20^{\mathrm{a}}$ & $80^{\mathrm{b}}$ & $125^{\mathrm{C}}$ \\
Average plasma energy, keV & 13 & 50 & 135 \\
$\mathrm{nt}, \mathrm{s} / \mathrm{cm}^{3}$ & $7 \times 10^{10}$ & $2 \times 10^{12}$ & $2 \times 10^{13}$ \\
$\mathrm{Q}$ & - & $0.15^{\mathrm{d}}$ & 0.64 \\
$\mathrm{~L} / \mathrm{a}_{\mathrm{i}}$ (see note e) & 35 & $100-300$ & 225 \\
$\mathrm{R}_{\mathrm{p}} / \mathrm{a}_{\mathrm{i}}$ (see note e) & 3 & $10-50$ & 45 \\
\hline
\end{tabular}

${ }^{a}$ some of the $2 X I I B$ neutral-beam injectors are being upgraded to $10 \mathrm{keV}$. $b_{80} \mathrm{keV}$ for susti.ined operation; start-up injectors will be $20 \mathrm{kev}$. c Deuterium component.

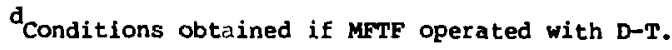

$e_{t}$ is the plasma length, $R_{p}$ the plasma radius at the midplane, and $a_{i}$ the gyroradius.

The plasma modeling for the hybrid used the full range of analytical tools that have been developed to support mirror physics investigations in $2 \mathrm{XIIB}$ and MFTF.

- The MCFUS equilibrium code was used to calculate three-dimensional, finite- $\beta$ plasma equilibrium, and to check for stability against the interchange mode.

- A two-dimensional (in velocity space) Fokker-Planck code was used to evaluate $n \tau$ under the condition of classical mirror confinement, i.e., charged-particle scattering into the loss-cone in velocity space, and also to compute the maximum $B$ for the mirror mode.

- Current theories were used to evaluate stability against the two dominant loss-cone microinstabilities, the drift-cyclotron loss-cone (DCLC) mode and the convective loss-cone mode.

- A radial transport code was used to calculate plasma buildup and sustaining of steady state, with allowance for stabilization of the plasma surface boundary layer (region of large density gradients) againat the DCrC node. 
In some instances, we had to modify these codes in order to generalize them from experimental conditions to those appropriate to a reactor-grade plasma.

\subsection{Fusion Components}

Figure 2-3 illustrates the basic plasma-coil geometry. A minimum-B magnetic well is established with the Yin-Yang conductor geometry, and the plasma confinement volume is the spherical region inside the conductor bundles. Plasma leaks through the slots, or mouths, in the two coils; in Fig. 2-3, the large "fans" outside the coils represent unconfined plasma streaming from the confinement volume. Figure 2-4 shows the variation of the basic Yin-Yang magnet that has been developed for reactor applications. This design uses a large main coil with a small mirror coil inside and has the virtue of locating the mirror point very near the inner radius of the conductor: since a plasma leakage fan is narrowest at the mirror point, this location for the mirror point implies a minimum-size opening in the blanket for the leakage fans. The magnet has an outside diameter of about $22 \mathrm{~m}$, and is designed with a maximum field at the conductor of $8.5 \mathrm{~T}$, dictated by the use of NbTi superconductor.

Energetic neutral atoms of deuterium and tritium are injected into the plasma by two injector arrays, each made up of a large number of individual sources (see Fig. 2-5). The neutral injection system is based on Lawrence Berkeley Laboratory's positive-ion-injection technology with beam direct conversion, and also incorporates features appropriate to the steady-state environment of a reactor - including features that stem from considerations of efficiency, punping, lifetime, and reliability.

Each array of injectors delivers to the plasma a 2600-A bearn of neutral atoms, of which two-thirds $(1733 \mathrm{~A})$ are $\mathrm{D}^{0}$ and one-third $(867 \mathrm{~A})$ are $T^{0}$. The energy of the tritium atoms is designed to be 1.5 times the energy of the deuterium atoms, so that both species are at the same velocity, and thus have equal mean free paths for penetration and capture in the plasma. The extraction voltages of the deuterium and tritium injectors are 125 and $187.5 \mathrm{kV}$, respectively. In the neutral beam, 70 of the atoms are at full energy, 17 are at one-half energy, and 13 t are at one-third energy; this mix of particle energies results in bean energies for the deuterium and tritium of 103.5 and $155.3 \mathrm{kev}$, respectively. Thus the 


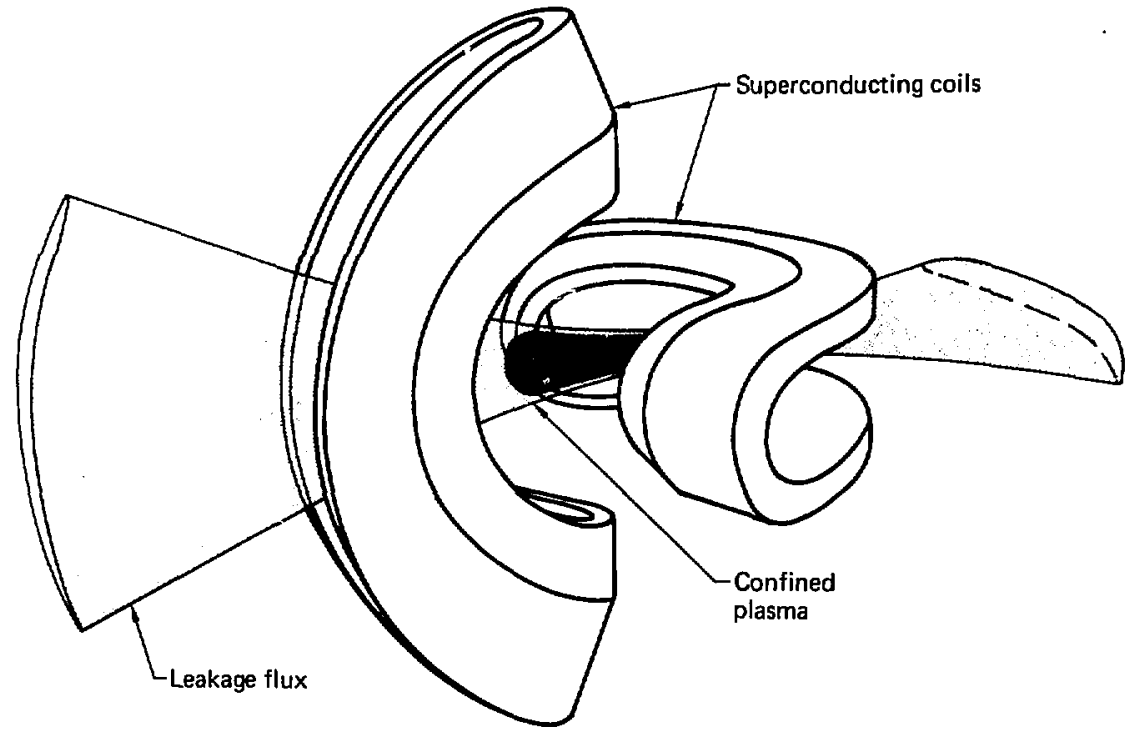

Fig. 2-3. Plasma and coil geometry. 


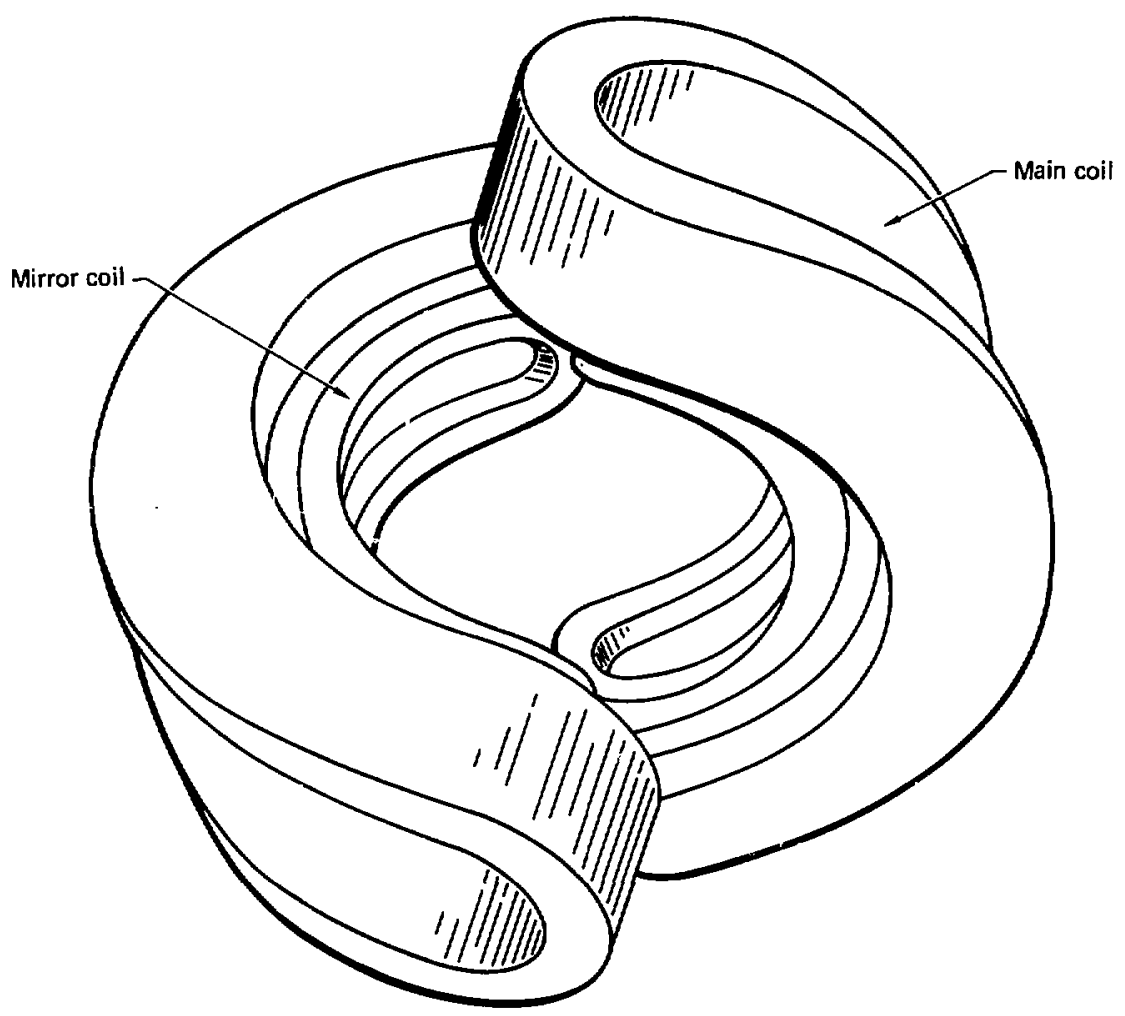

Fig. 2-4. Magnet conductor geometry. 

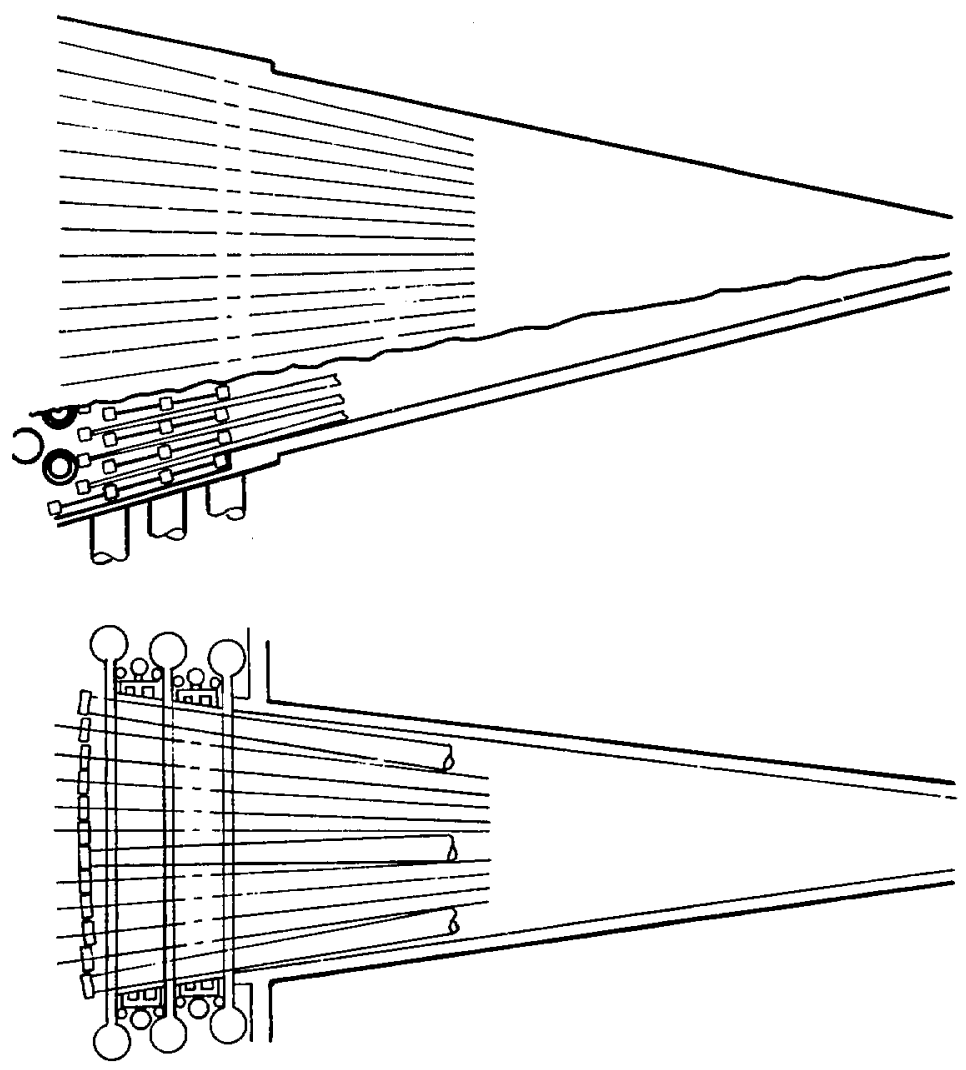

Fig. 2-5. Neutral-beam injector array. 
average energy of the beam - two-thirds deuterium and one-thira tritiun is $120.8 \mathrm{kev}$. Our analysis predicts an efficiency for the injectors of about 58 excluding recovery of the waste heat.

Outboard of the coils, end tanks receive the plasma leakage (see Fig. 2-1). Some of the kinetic energy of the leakage ion flow is converted directly into electricity; the remainder is deposited as thermal energy in the direct-converter electrodes and is removed by active cooling. Since the plasma is neutralized upon striking the direct-converter electrodes, the end tanks contain vacuum pumping equipment to remove the resulting gas.

To provide access to the blanket from outside the machine, it is convenient to make one of the end tanks as small as possible. We do this by designing the magnet so that one of the mirror fields is 58 stronger than the other. This moderate field perturbation has a dramatic effect on the distribution of the plasma leakage: 908 escapes through the weak mirror and only 10 s escapes through the strong mirror. Since the size of the end tank is proportional to the amount of plasma flow, we can use a small end tank behind the strong mirror.

To keep the small tank as simple as possible, we do no direct conversion there; the plasma energy is deposited as thermal energy only. The large end tank, which receives 908 of the end leakage flow, is designed with a simple, single-stage direct converter having an efficiency of 408. Such a converter design is appropriate since the amount of energy flowing into the end tank is small compared with the blanket energy, and thus the reactor power balance is not very sensitive to the efficiency of the direct converter. Both end tanks have provisions for active $2 \infty$ ling and vacuum pumping.

The primary gas loads in the vacuum system result from 1) gas feed to the nentral bean system that is not converted to energetic neutral atoms, and 2) plasma leakage from the confinement volume $\left(\mathrm{D}^{+}, \mathrm{T}^{+}\right.$, and $\mathrm{He}{ }^{-{ }^{-+}}$) that is neutralized in the end tanks. In the injector arrays, the required vacuum conditions are maintained by a combination of mercury ejector pups near the high-pressure sources and helium cryopanels downstream from the bean direct converter. In the end tanks, $D_{2}$ and $r_{2}$ gas is pumped by helium cryopanels on the tank sidewalls, where they are out of the path of direct neutron streaming from the plasma. (Provisions for puming of the fusion ash, helium, were not included in the design for reasons discussed in Secs. 6.4 and 6.5.) 
Significani quantities of cryogens are required for operation of the reactor; the magnet, cryopanels, and isotopic separation equipment are cooled with liquid helium (ifHe), and the chevrons and liners on the cryopanels are cooled with liquid nitrogen (LN). The largest heat load on the cryogenic cooling system is due to neutron heating in the magnet. All cryogenic cooling requirements are supplied with closed-cyele refrigeration systems, requiring a total electrical input of $18 \mathrm{~mW}$.

The reactor hall has a low-humidity air atmisph-te which is continuousiy reprocessed. The air processor is designed to handle leakage and permeaibility losses from the nuclear island at $180 \mathrm{Ci} / \mathrm{day}$, while maintaining lerels of tritium below the maximum permissible concentration (MPC). The processor is also able to handle severe accidental releases of tritium at the kilogram level so as to permit reentry, by workers wearing ventilated suits, in a matter of several weeks.

\subsection{Blanket Nuclear Design}

Table 2-3 lists the beginning-of-life and end-of-life neutronic parameters for the blanket. Economic optimization of the fuel cycle for this reactor dictates a total exposure of the fuel to $6.6 \mathrm{MW}-\mathrm{y} / \mathrm{m}^{2}$ of 14-MeV-neutron energy through the first wall. These parameters assume that tritium is continuously extracted from the blanket (by a purge flow of helium supplied to the breeding pins).

As the fissile breeding fuel we are advocating the use of $U_{3} S i$, a fuel being developed in the Canadian nuclear power program for the CANDU reactor. In the past, we have primarily examined uranium carbide, uraniun-molybdenum alloy, and thorium for use as the fertile fuel in the blanket. However, we chose $\mathrm{U}_{3} \mathrm{Si}$ in the present study because 1 ) being a metallic alloy, it has a high uranium density; 2) it is easy to fabricate; and 3) for a metallic fuel it has a comparatively high burnup capability on the order of 2-3*. In this design, the $\mathrm{U}_{3} \mathrm{Si}$ is encapsulated in Inconel 718 fuel rods.

The tritiun-breeding region is located immediately behind the uranium region in the blanket. The breeding material is LiD + Li, encapsulated in pins of aluminum-beryllium alloy (a good $\mathrm{T}_{2}$ diffusion barrier at $\sim 300^{\circ} \mathrm{C}$, the collant inlet tengerature). The average of the tritium breeding ratios in Table 2-3 is greater than 1, compensating for loss of 14-MeV neutrons 
Table 2-3. Local blanket neutronic parameters.

\begin{tabular}{lcc}
\hline & $\begin{array}{c}\text { Beginning of } \\
\text { life }\end{array}$ & $\begin{array}{c}\text { End of } \\
\text { life }\end{array}$ \\
\hline Exposure, w-y/m ${ }^{2}$ & 0 & 6.6 \\
Energy multiplication & 9.1 & 16.2 \\
Net fissile breeding ratio & 1.85 & 1.65 \\
Burnup & 0 & 0.01 \\
Tritium breeding ratio & 0.99 & 1.35 \\
\hline
\end{tabular}

through holes in the blanket and for decay of the tritium inventory outside the blanket.

In this reactor study, we examined the approach of holding up all of the bred tritilin and recovering it by processing the tritium-breeding pins outside the reactor, in much the same way as the bred fissile material is recovered. It was felt that its inherent simplicity (which implies good safety characteristics) made this design option worthy of consideration. The blanket and tritium-containment designs described in secs. B.2 and 6.6 of this report were developed on this basis.

However, when the final reactor optimization was performed, it was found that the economic penalties associated with the large initial tritium inventory and decay of this inventory were unacceptable: 1) Because the tritium would be ieft in the blanket for the full blanket life, tritium would initially bave to be purchased, until the first blanket was removed. 2) In addition, by that time a significant fraction of the bred tritium would have decayed. Thus the final blanket parameters assume a purge recovery systen, which supplies a small flow of helium to the tritium-breeding pins, continuousiy extracting the tritium from the blanket.

\subsection{Mechanical Design}

One of our primary concerns in the mechanical design of the reactor was to provide highly reliable sugport and containment of the blanket and the components of the primary heat-transfer loop. The basis of our concern was the conclusion that the primary safety consideration for the reactor was a loss-of-flow accident, and the design therefore had to be one in which the 
maintenance of forced cooling to the blanket could be assured to a very high level of confidence.

The design approach we have selected is to mount the magnet, blanket, and primary heat-transfer loop all within a prestressed-concrete reactor vessel (PCRV) of the type developed for gas-cooled fission reactors. This is shown in Fig. 2-1. In the cercer of the PCRV is the magnet and blanket, and the steam generators and helium circulators are located around the periphery. The plan view of the PCRY (Fig. 2-6) indicates the locations, within the concrete structure, of the major fusion components and primary heat-transfer loop components, which include 8 circulators and 12 steam generators. In this way, the blanket and its cooling system are locked together so that no relative motion between them can occur, thus precluding the rupturing of any of the coolant ducts.

The PCRV also serves a second function: it provides the main restraining forces for the magnet. Since the PCRV operates at room temperature, while the nagnet operates at $4 \mathrm{~K}$, ways had to be found to transmit forces from the magnet to the concrete and to limit heat flow from the concrete to the magnet. Our design solution has been to use a high-compressive-strength thermal insulation for both purposes. The material, a silicate refractory, is capable of sustaining about 5000 psi in compression, and at a thickness of about $50 \mathrm{~cm}$ is adequate to limit the heat leak from the concrete to the magnet to an acceptable value.

Changing the blanket does not require any major disassembly of the reactor; instead, the design relies on remote operations to assemble and disassemble the blanket inside the PCRV. The blanket is made up of small cylindrical modules approximately $50 \mathrm{cn}$ in diameter, and the blanket structure is suspended directly from the inside wall of the PCRV, as shown in Fig. 2-7. Blanket modules are removed and replaced with the refueling rachine shown in Fig. 2-8, which consists of a post which is inserted down through the center of the reactor and has a pivoting arm to operate on the modules. The intenance operation involves a series of manipulations of each of the several hundred modules.

The module, as shown in Fig. 2-9, is a cylindrical pressure vessel with a hexagonal base. One of the more challenging aspects of the module design has been to devise a fast, reliable method of making up the seal that isolates the high-pressure helium coolant from the vacuu region that contains the plasma. He discarded the idea of a melded joint, since remote 


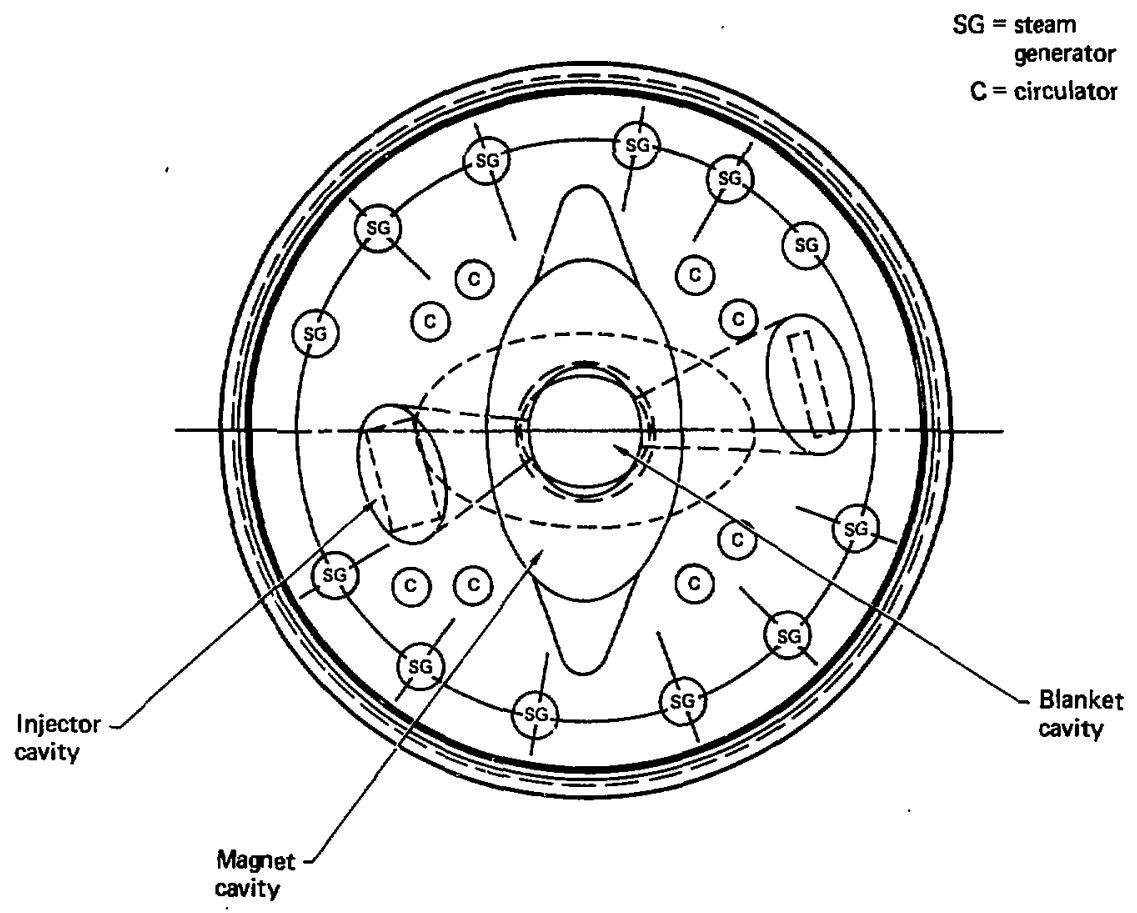

Fig. 2-6. Plan view of the PCRV. 


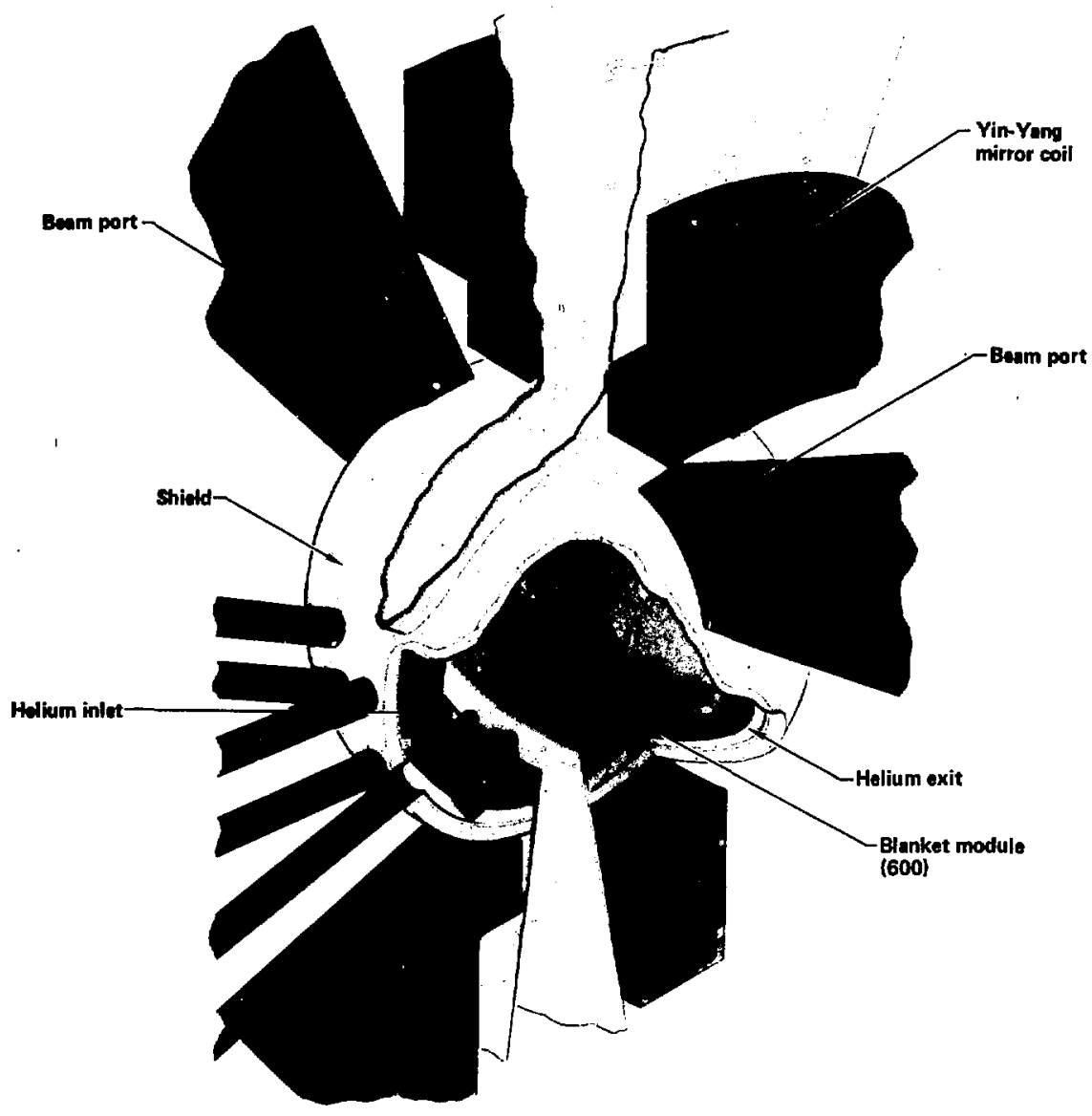

Fig. 2-7. Cutaway view of the blanket and shield. 


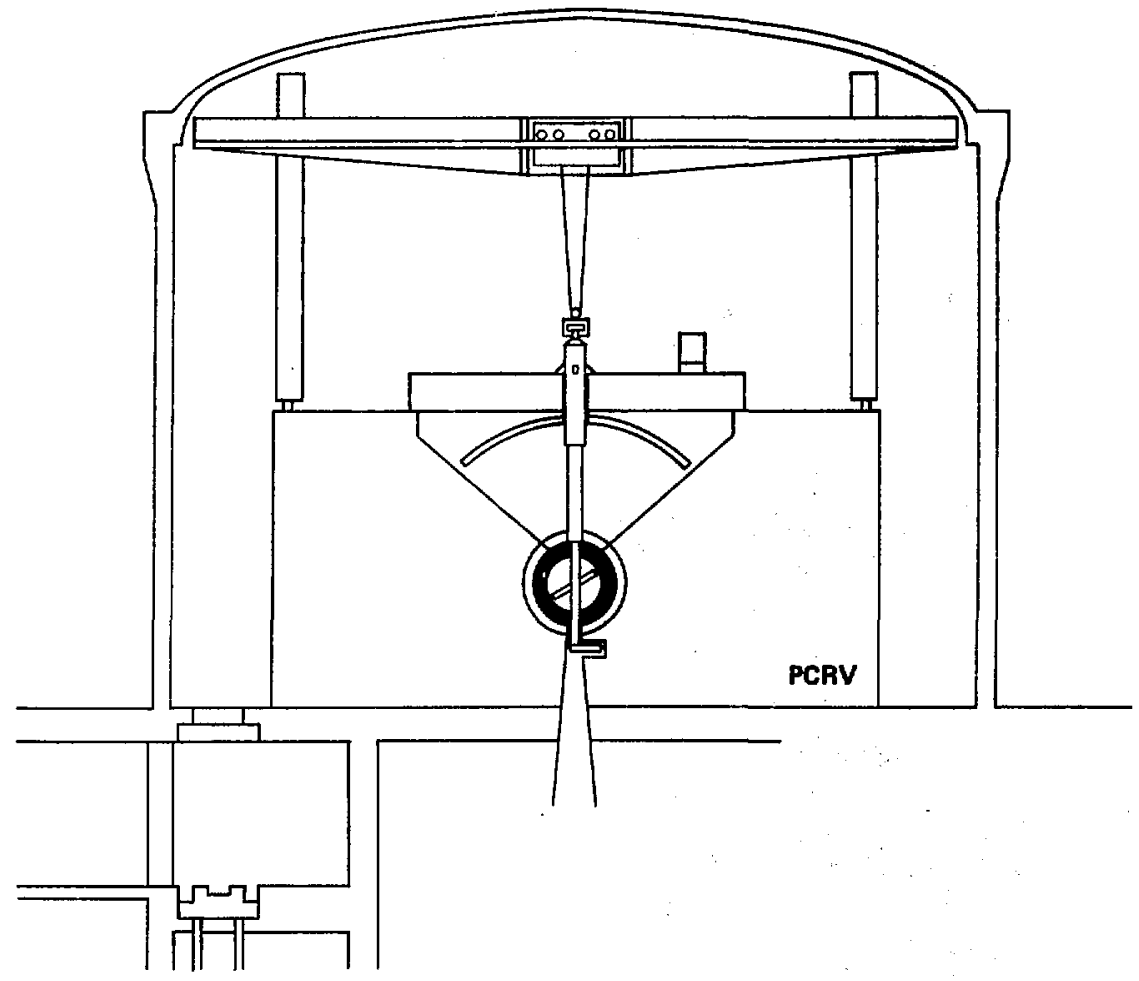

Fig. 2-8. Refueling machine. 


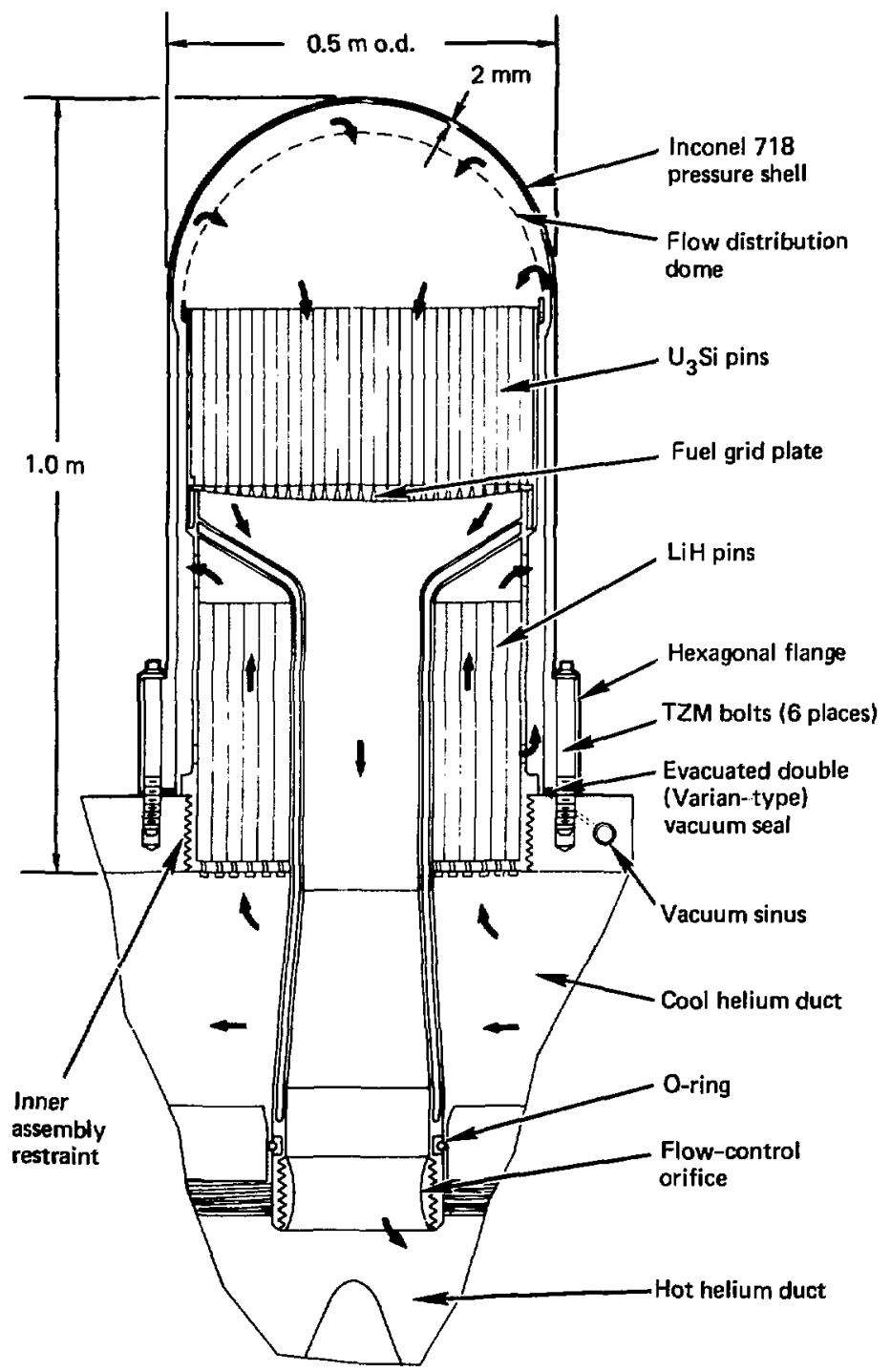

Fig. 2-9. Blanket module. 
grinding and welding are time-consuming operations and we have serious doubts about being able to make remote vacuum-tight welds consistently. Instead, we adopted a bolted joint using a double knife-edge (Varian-type) seal with differential pumping between the two knife-edges. The pressure vessel is bolted in place with six bolts, one at each corner of the hexagonal base. The internals of the module are fabricated as a single unit containing the $\mathrm{U}_{3} \mathrm{Si}$ pins, the tritium breeding pins, and the internal flow ducting, which threads into a socket in the permanent structure. Thus, to ranew a module, the pressure vessel is unscrewed, the pin assembly is removed, a new pin assembly is inserted, and a new pressure vessel is bolted in place. The coolant flow is re-entrant; the tritium pins are cooled by the inlet flow, and the coolant then proceeds down to the first wall, turns, and cools the uranium pins on its exit path out through the module.

\subsection{Power Conversion Loop}

The primary heat-transfer loop is designed to operate with helium as the working flutd. The coolant pressure is $60 \mathrm{~atm}$; inlet temperature to the blanket is $280^{\circ} \mathrm{C}$, and outlet temperature is $540^{\circ} \mathrm{C}$. The flow path is designed to maintain the relative pressure drop, $\Delta \mathrm{p} / \mathrm{p}$, at about 2.58 through the entire loop (blanket, ducting, and steam generator). This combination of conditions permits the use of conventional gas-cooled reactor technology for the design of the helium circulators and steam generators.

As shown in Table 2-3 the local blanket multiplication, and therefore local blanket power density, increases by about a factor of 2 over the life of the fuel. By devising an appropriate fuel management scheme for the blanket, we are able to linit the peak-to-average variation in the total blanket thermal power to about 78 (3350 average, $3600 \mathrm{~m}$ peak), and the capacity of the primary heat-transfer power corvergion loop is designed to accomodate this power variation. The blanket modules are grouped into quadrants; at tine intervals of one quarter of the blanket life, the reactor is shut down and one quadrant of the blanket is refurbished with new fuel assemblies. In this way we are able to establish an equilibrium fuel cycle whereby the four quadrants are each at a different exposure.

The thermal-hydraulic design for the fuel, on the other hand, must provide adequate cooling of the fuel pins during the lifetime power density variation of $16.2 / 9.1=1.8$. The fuel design was based on peak fuel power 
densities (i.e., at the first wall) of about $240 \mathrm{w} / \mathrm{cm}^{3}$ at beginning of life and $500 \mathrm{w} / \mathrm{cm}^{3}$ at end of life. However, the values actually achieved in the final reactor design were 160 and $280 \mathrm{w} / \mathrm{cm}^{3}$. The fuel pins are $0.7 \mathrm{~cm}$ in diameter with $0.15-\mathrm{mm}-t h i c k$ Inconel $718 \mathrm{clad}$ on a pitch-todiameter ratio of 1.05 . The maximum mid-wall clad temperature (hot channel) is limited to $700^{\circ} \mathrm{C}$ with a coolant inlet temperature to the module of $280^{\circ} \mathrm{C}$ and outlet temperature of $540^{\circ} \mathrm{C}$.

The secondary heat-transfer loop uses a conventional (steam) regenerative Rankine cycle. The high-pressure turbine inlet conditions are $140^{\circ} \mathrm{C}\left(820^{\circ} \mathrm{F}\right.$ ) and $8.42 \mathrm{MPa}(1240 \mathrm{psia})$. Main steam flow is used to drive the helium circulators by means of circulator turbines, a procedure developed for application in gas-cooled fission reactors. The thermal efficiency of the cycle is projected to be about $36 \%$.

\subsection{Economics}

Economic evaluation of a conceptual design study is subject to considerable uncertainty due to the preliminary nature of the component design and to lack of fabrication and construction experience. The cost estimates presented in Appendix $B$ and summarized in Table 2-4 are best estimates based on extrapolations of cost data from fusion physics experiments and fission reactor design experiments.

Our estimate of the total plant capital cost is $\$ 1.55$ billion, as sumarized in Table 2-4. This total results in unit capital costs of $\$ 2577 / \mathrm{kWe}$ (net), $\$ 906 / \mathrm{kWe}$ (gross) and $\$ 375 / \mathrm{kWt}$.

For the economic analysis, we required that the hybrid sell its net electrical output at the same unit price as the light-water reactors (LwR's) to which it supplies make-up fuel requirements. By considering all capital costs (hybrid plus LWR's), and all fuel-cycle and operation and maintenance (OsM) costs, the unit price for electricity was evaluated to be 29.7 mills/kwh from the fusion-fission power system.

Based on this selling price for electrical power, the unit cost to the hybrid for producing the fissile fuel was calculated to be $\$ 109 / 9$ (Table 2-5). In turn, the power generation cost in the LWR for fissile fuel at $\$ 109 / 9$ is $7.7 \mathrm{mills} / \mathrm{kWh}$. 
Table 2-4. Plant capital cost sumary.

Costs in $\$$ million (1977)

Structures and facilitles 124.5

Reactor plant 480.4

Reactor equipment

Primary heat-transfer loop

Auxiliary cooling

Radioactive waste facilitles

Fuel handling and storage

Instrumentation and control
284.0

77.3

74.9

10.9

26.9

6.4

Turbine plant equipment

140.6

Electrical plant equipment

59.0

Miscellaneous

8.0

Total direct cost

812.5

Indirect field cost

Total field cost

162.5

975.0

Engineering

146.3

1121.3

Contingency

Total construction cost

112.1

other owner cost

1233.4

61.7

1295.1

Interest during construction

259.0

Total capital cost

1554.1 
Table 2-5. Fissile-fuel production cost.

Costs in
dollars per gram of plutonium

Expenditures

Capital

134.2

Fuel cycle

27.2

OCM

10.1

Total

171.5

Revenues

Electricity at $29.7 \mathrm{mills} / \mathrm{kwh}$

$-62.2$

Net cost

109.3 


\section{REACTOR DESCRIPTION}

In this section, we present a brief description of each of the major components in the reactor.

\subsection{Magnet}

The required minimum-B field configuration is generated with the Yin-Yang conductor geometry. The magnet is superconducting, using NbTi superconductor with copper stabilizer, ano is designed to a maximum field in the conductor of 8.5 T. The central field is $\ddot{z} .63 \mathrm{~T}$ and the mirror field $5.95 \mathrm{~T}$, resulting in a vacuum mirror ratio of 2.26 and a coil efficiency (ratio of mirror field to maximum conductor field) of 0.70 . The reactor design specified that the field at one mirror be 5 greater than the field at the other mirror, to establish plasma selective leakage. This minor perturbation of the symmetric coil was not included in the magnet design.

The overall dimensions of the coil are approximately $22 \mathrm{~m}$ in diameter and the distance between mirror points is $13 \mathrm{~m}$ (Fig. 3-1). The conductor bundle is split into an inner or "mirror" coil and an outer or "main" coil. The major arc of the coils sweeps through a $75^{\circ}$ half-angle and the mirror coil has been spread in the turnaround (minor arc) to ameliorate field concentration in this region.

The magnet conductor consists of KDTi filanentary superconductor embedded in stabilizing copper with a square $(1.2 \times 1.2 \mathrm{~cm})$ cross section. The maxinun current density in the bundle cross section is approximately $1 \mathrm{kA} / \mathrm{cm}^{2}$ and in the superconducting filaments is $100 \mathrm{~A} / \mathrm{mm}^{2}$; the conductor dimensions and currents are sized to insure operation in the cryostatically stable regime.

Each half of the magnet winding is encased in a stainless steel coil case. The coil case is designed to react the "C-opening" forces and the torques. The normal forces on the coil, and the attractive forces between the two halves of the coil are reacted by the PCRV through load-bearing thermal insulation. Each coil half, including conductor and coil case, is estimated to weigh 3000 tonnes. 


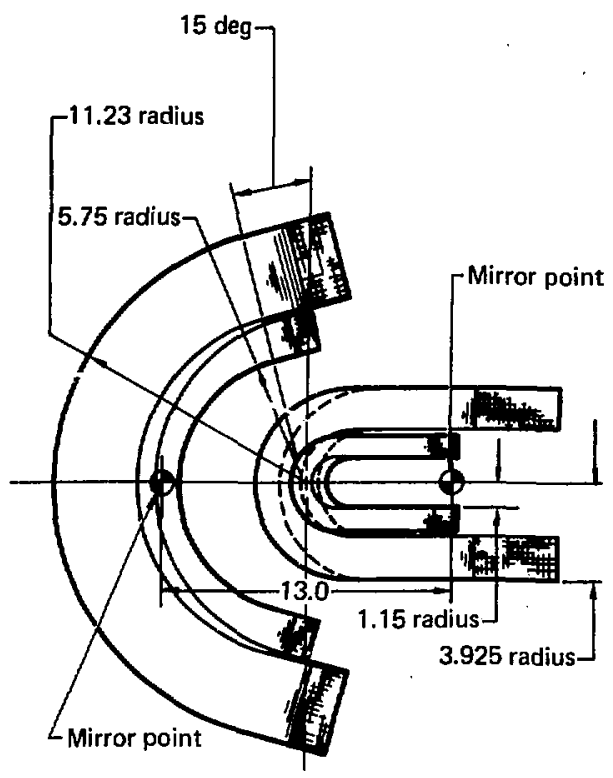

Fig. 3-1. Magnet conductor dimensions. 


\subsection{Injectors}

The neutral beam injector system is based on Lawrence Berkeley Laboratory (LBL) positive-ion injector technology, but differs from the LBL source in several respects. The proposed system would have the following new features:

- A hollow cathode ion source with tungsten impregnated emitters for long life.

- High-voltage insulators and power supplies, all of which are shielded from the neutron and gamma flux.

- A cesium vapor curtain to reduce the flow of gas along the beam line.

- Stationary cryopanels that are periodically outgassed.

- Injector system built of many ion sources to establish system reliability and redundancy.

Each injector array consists of 216 ion sources, arranged in 18 columns of 12 units, spread over an area of $6 \times 9 \mathrm{~m}$. To confine the injected beam within a maximum half angle of $15^{\circ}$, the distance from the sources to the beam entrance aperture in the blanket is $17 \mathrm{~m}$.

Only 180 sources need operate at any one time to deliver the required 2600 A of neutral current per array. Thus, three colunns of sources are available as standbys to be activated if needed. Each column of 12 sources is supported by large alumina insulators above and below the beam line. Surrounding the sources are perforated electrostatic shields at ground potential. In the event of a high-voltage breakdown, an entire column of sources may have to be turned off. However, should an arc occur, we assume that the safety circuits (crowbars, fast interrupts, and arc snubbers) can prevent damage to the electrodes. As a result, a faulted column, having been turned off, will be available for subsequent reactivation.

Figure 3-2 shows a schematic of the components along a deuterium beam line, along with the corresponding variations in the potential and pressure profiles. The ions extracted from the source by the acceleration grids are neutralized in a gas cell that is terminated by a cesium-vapor curtain. Mercury ejector pumps maintain the pressure around the ion sources and in the gas cell at $2 \times 10^{-3}$ Torr. The low-temperature gas escaping from the ion sources fills the gas cell. Not being capable of penetrating the cesium-vapor cell (which acts as a curtain), the gas is removed at relatively high pressure and good efficiency via large pump ducts behind the array of sources. 


\section{Component profile}

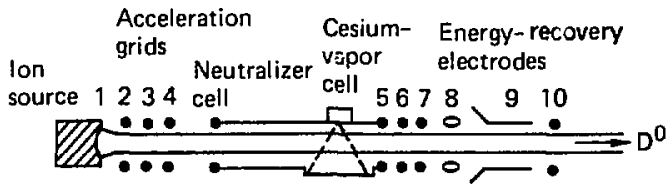

\section{Potential profile}

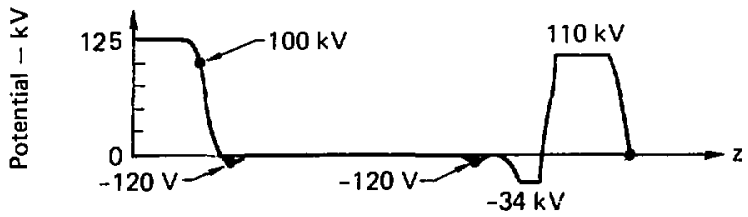

Pressure profile

Cesium

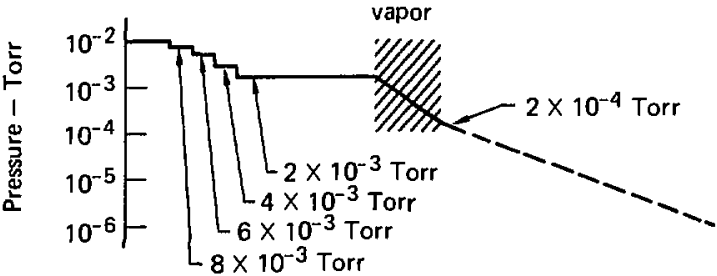

Pig. 3-2. Components, potential, and pressure profiles along a $D^{0}$ bean line. 
Thereby, the gas load is reduced on the cryopanel punps further down the beam line. Beyond the cesium cell, the high-energy ions, which are traveling with the beam, are removed by the energy-recovery electrodes.

\subsection{End Tanks}

In this reactor, the magnet has one mirror field 58 stronger than the other, which causes a selective leakage situation such that 90 of the confined ions $\left(D^{+}\right.$and $T^{+}$) flow out of the weak mirror and the remaining $10 *$ out of the strong mirror. The alrma particles, because they do not behave adiabatically, are not magnetically confined. Thus the alphas are not subject to selective leakage and equal currents of these ions flow out of the two mirrors.

The motivation for using selective leakage is that the size of the end tank is proportional to the amount of plasma flow into it. Thus the tank receiving the exhaust from the strong mirror is much smaller than the weakmirror end tank, and provides a convenient access path from outside the reactor into the plasma confinement chamber for blanket maintenance operations. The strong mirror is located on top of the reactor and the remote handling equipment is lowered into the reactor from the top of the PCRV through the small end tank.

The major features of an end tank are shown in Fig. 3-3. The plasma exhaust expands along the field lines of the external, or fringe, field of the magnet. The tank sidewalls enclose the fan and the end wall is oriented perpendicular to the field lines. The plasma flow is terminated on the plasma collector, which in the weak-mirror tank is a single-stage direct converter and in the strong mirror tank is a plasma thermal dump, e.g., a grounded, convectively-cooled wall. The vacuum pumping equipment, cryopanels, are located on the sidewalls where they are not subject to direct bombardment by nutrons from the plasma. The plasma collectors are cooled by high-tenperature helium, making this thermal energy available for thernal conversion. The tank sidewalls are covered by water-cooled chevrons (to provide thermal protection for the cryogenic pumping equipnent), and this thermal energy is thus renoveo as low grade heat.

The input plasma power to the large end tank is $607 \mathrm{~m}$ and the grounded grid of the single stage direct converter is located $\sim 30 \mathrm{~m}$ from the center of 

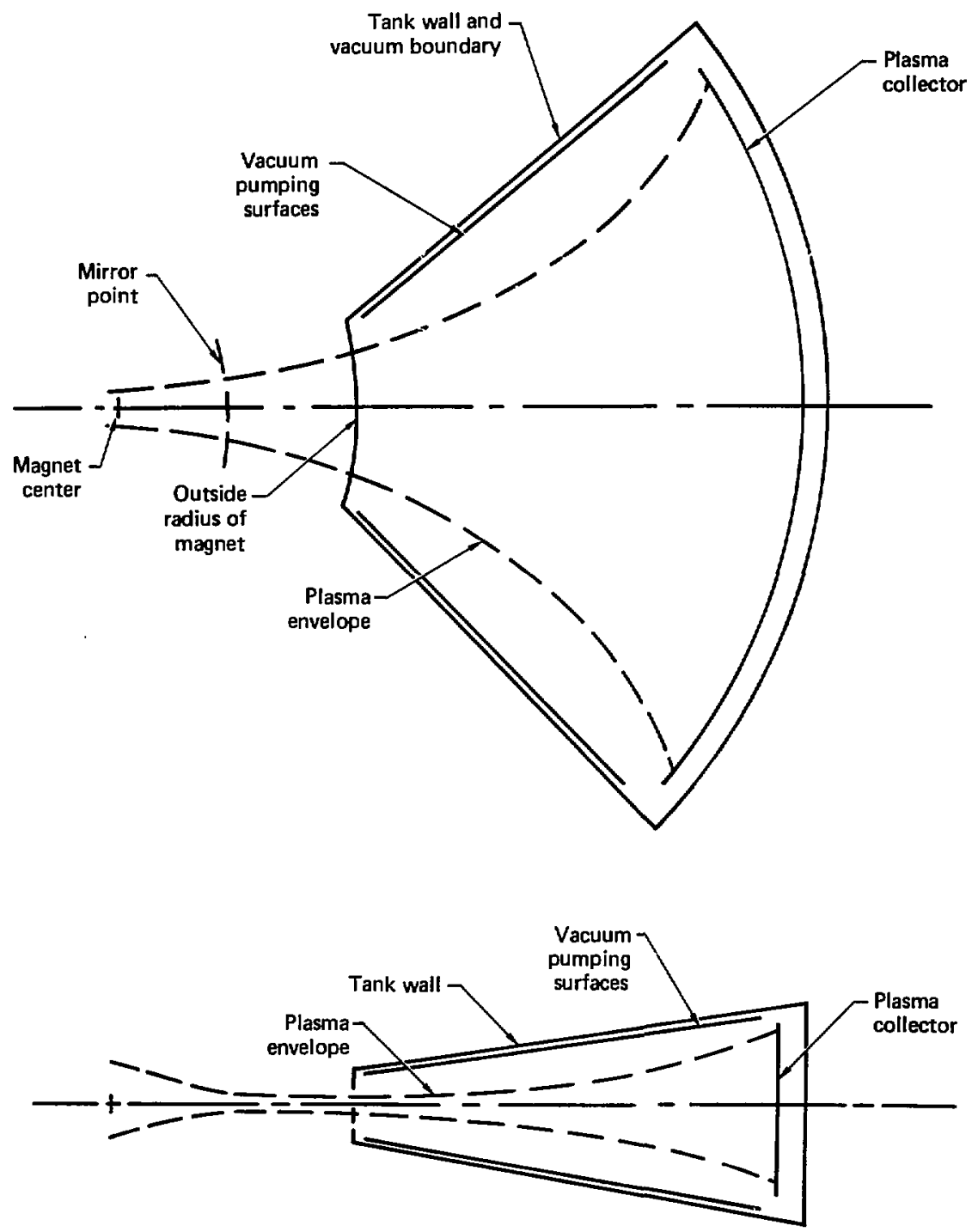

Fig. 3-3. End tank features. 
the magnet. These conditions result in a plasma power flux at the converter grids of $175 \mathrm{w} / \mathrm{cm}^{2}$. The grounded grid and collector are convectively cooled, and the negative grid is radiatively cooled. The direct converter parameters were selected to minimize the $\$ / \mathrm{kw}$ of the reactor, and resulted in a direct converter efficiency of $40 \%$. Of the remaining 60 of the input power, $52 \%$ is recovered as high-grade thermal energy and $8:$ as low-grade (waste) heat.

The input power to the small end tank is 103 and the incident power flux on the plasma collector is $250 \mathrm{w} / \mathrm{cm}^{2}$, thus locating the collector $15 \mathrm{~m}$ from the center of the magnet. Of the total input power, 908 is recovered as high-grade thermal energy and 10 as waste heat.

\subsection{Vacuum System}

The major source of gas in the reactor vacuum system is the gas feed to the neutral beam injectors. This gas is pumped in the injector tanks and in the end tanks. In addition, a gas flow is used to provide stabilization to the surface of the plasma and helium is generated in the plasma due to fusion reactions.

The vacuum system gas flow is shown in Fig. 3-4. The injector tanks use a combination pumping system composed of positive displacement (Roots) blowers (chamber 1), mercury ejector pumps (chamber 2) and LHe cryopanels (chamber 3 ). The $D_{2}$ and $T_{2}$ gas in the end tanks, as well as the $D_{2}$ in the stream tanks, are pumped with LHe cryopanels. He pumping capacity in the end tanks was not addressed because of the absence, at present, of a viable the pumping technology for reactor applications.

\subsection{Tritium Handling and Containment}

Tritiun control in the reactor is possible with emissions to the environment below $10 \mathrm{cl} / \mathrm{d}$, while still maintaining worker safety and cost effectiveness. A number of new methods have been employed to achieve such low levels in a near-term machine with today's technology. Figure 3-5 summarizes the various tritiun leak source terms and the associated processing loops.

The tritium breeding pins are designed as tritium containment vessels filled with lithium deuteride and are constructed of a special lon perneability, high strength aluminum alloy. In the hybrid reactor these tritium 


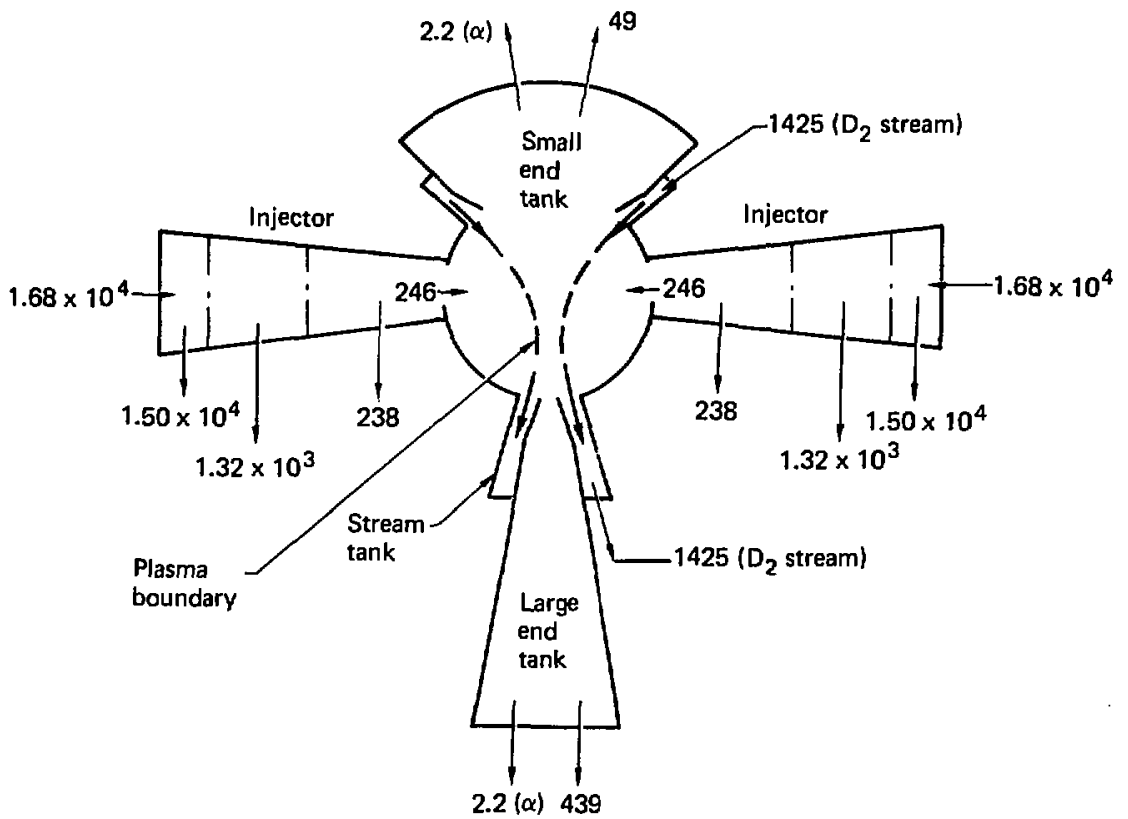

Fig. 3-4. Vacuum system gas flows (flows in Torr-litre/s). 


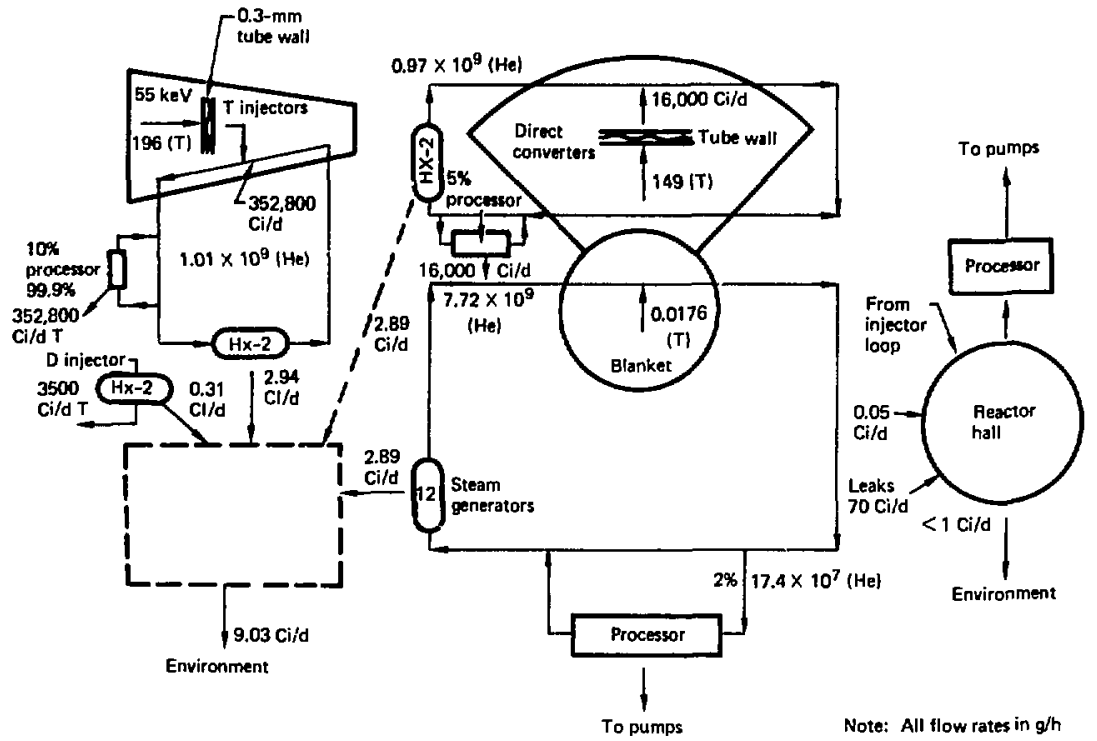

Fig. 3-5. Tritium leak source terms and processing loops. 
breeding pins are "harvested" together with the uranium pins. The tritiun is extracted from these tubes in-plant and the lithium deuteride-filled tubes are remanufactured and reinstalled for the next cycle. A breeding pin failure rate of $10^{-3}$ is assumed, thus releasing $448 \mathrm{ci} / \mathrm{d}$ into the helium coolant, which is then scavenged by a slip stream processor hanaling 2 of the helium flow. The tritium-contaminated helium will permeate about $3 \mathrm{ci} / \mathrm{d}$ into the steam generators, which is in turn lost to the cooling towers and then to the environment.

The next major source of tritium leakage is from the tritium neutral beam injector, where $55 \mathrm{keV}$ energetic tritons implant tritium into the surface of the electrode tubing. Once within the tube metal, tritium will diffuse producing a flux of tritium into the electrode helium coolant flow at about $70.560 \mathrm{Ci} / \mathrm{d}$. A $10 \mathrm{~s}$ fraction of the coolant flow is then processed to remove 996 of the tritium and the resulting residual tritium leaks into the steam generators at about $3 \mathrm{Ci} / \mathrm{d}$, where it eventualiy passes into the cooling water and then to the enviromment. The deuterium injector uses deuterium with a maximum 18 tritium impurity level and it creates a leak to the steam of 0.3 $\mathrm{Ci} / \mathrm{d}$.

A similar tritium loss phenomenon occurs in the hybrid's end tanks where $55 \mathrm{keV}$ tritons produce a loss of $8,000 \mathrm{Ci} / \mathrm{d}$ into the helium flow. Again a 99.91 processor is used, handling 58 of the flow to reduce this source of tritium in the main helium coolant to less than $3 \mathrm{Ci} / \mathrm{d}$.

All of the remaining miscellaneous leaks are taken to be from the process equipment and piping or ducting directly into the reactor hall - totaling a maximum of $180 \mathrm{ci} / \mathrm{d}$. The reactor hall atmosphere is processed and kept at low humidity to maintain tritium gas levels of below $40 \mu \mathrm{Ci} / \mathrm{m}^{3}$, and tritium water of below $5 \mu \mathrm{Ci} / \mathrm{m}^{3}$. This reactor hall processing design is expected to result in routine as well as credible accident losies through the reactor hall containment of less than $1 \mathrm{ci} / \mathrm{d}$.

In this way, the tritium loss to the environment is designed to be less than $3 \mathrm{Ci} / \mathrm{d}$ from breeding pin losses, $3 \mathrm{Ci} / \mathrm{d}$ from injectors, $3 \mathrm{Ci} / \mathrm{d}$ from the end tanks, and less than $1 \mathrm{Ci} / \mathrm{d}$ from reactor hall containment permeation. This is some 40 times lower than is required by ARC's regulations required of all nuclear fuel cycle operations, but about the level suggested by the ALARA cost/benefit analysis amounting to an expenditure cutoff at the $\$ 66,000$ level. It was found no further reductions in enissions could be accomplished by an expenditure of $\$ 56,000$. 


\subsection{Cryogenic System}

The LHe and IN required for cooling in the magnet, cryopanels, and isotopic separation equipment, are supplied by closed cycle refrigeration Bystems as shown in Fig. 3-6. The working fluid is first isothermally compressed, and then transfers heat to the returning flow via the heat exchangers in the cold box. The cold gas is then expanded through a Joule-Thompson valve into the two-phase region. The liquid produced flows to the heat load where it is boiled. The resulting saturated vapor is combined with the saturated vapor from the separator, used to cool the inlet flow and returned to the compressor.

The heat load on the LHe system is $14 \mathrm{~kW}$, which is dominated by $12 \mathrm{~kW}$ due to neutron heating in the magnet. The required electrical input power for this refrigerator is $4 \mathrm{MW}$. The heat load of $\sim 1000 \mathrm{~kW}$ on the LN system originates in the chevrons and liners used for (thermal) protection of the cryopanels; the input power to this refrigerator is $\sim 10$

\subsection{Prestressed-Concrete Reactor Vessel}

Two problen areas identified in previous work on the mirror hybrid were the support of the blanket modules and the containment of the primary loop components. Both require containment of hot, high pressure helium and support of large components against pressure, gravity and seismic forces. Similar problems are encountered at cryogenic temperatures in the support and restraint of the large superconducting Yin-Yang magnet. These three problems can be solved by use of a prestressed-concrete reactor vessel (PCRV) similar to that used by the BTGR.

The PCRV is $64 \mathrm{~m}$ in diameter and $31 \mathrm{~m} \mathrm{high.} \mathrm{It} \mathrm{performs} \mathrm{five} \mathrm{major}$ functions:

- Restraint of main coil forces.

- Pressure containment and positive constraint of helium coolant ducts.

- Positioning and restraint of prinary heat transfer loop components, i.e.. steam generators, helium circulators.

- Support of the spherical modular blanket and shield.

- Biological shielding.

The PCRV will operate at room temperature; the cryogenic magnet is interfaced to the concrete through 0.5-m-thick load-bearing silica insulating pads 


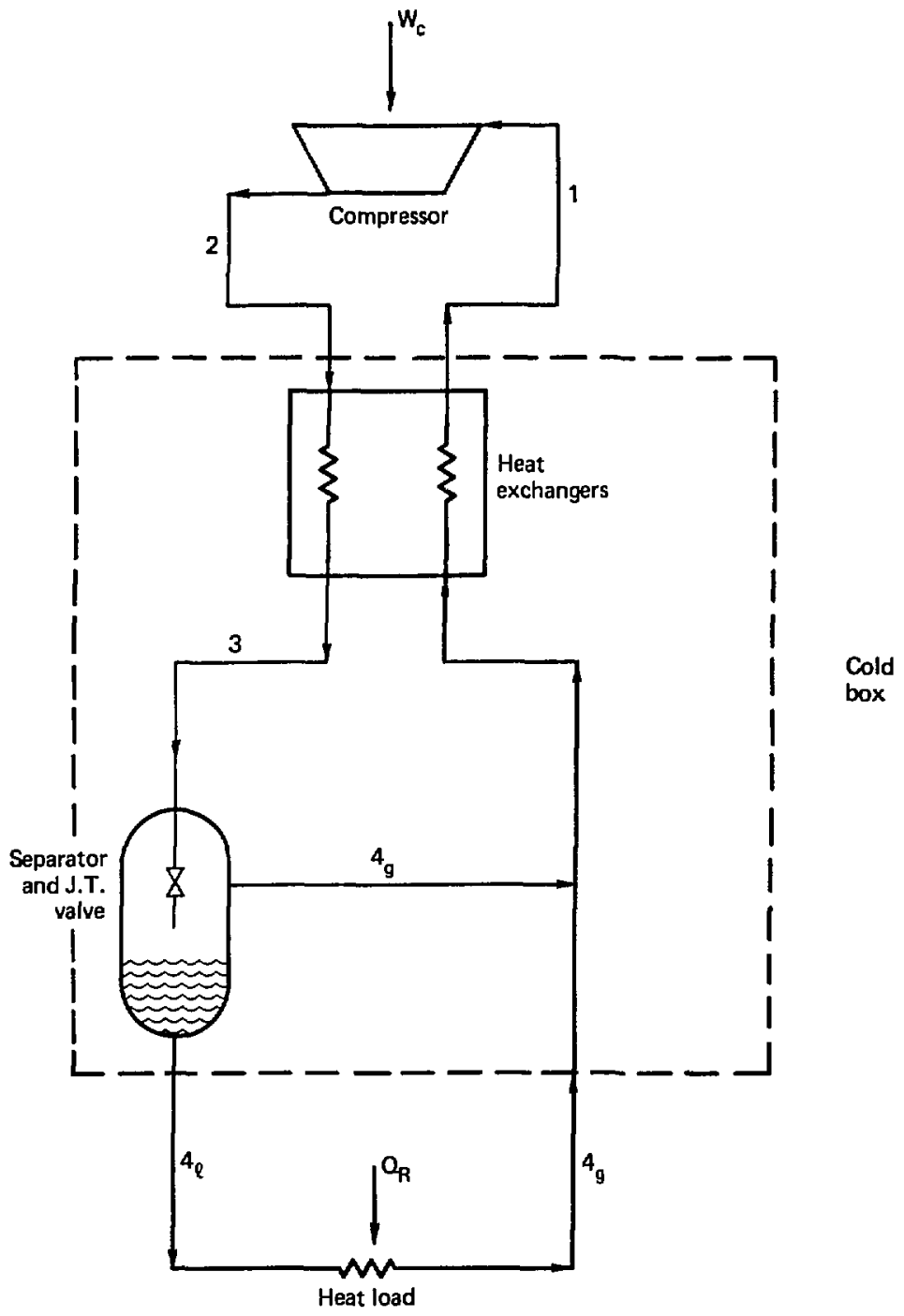

Fig. 3-6. Refrigeration equipment. 
with $35 \mathrm{MPa}$ (5200 psi) compressive strength. The concept of cryogenic loadbearing insulation is important in that it allows integration of the magnet restraint structure and the PCRV into one large concrete monolith. In addition to economic advantages, the PCRV concept offers safety advantages because of the inherent redundancy of the primary coolant pressure boundary. The PCRV design is shown in Figs, 3-7,8.

The reactor blanket and shield structure are mounted in a spherical cavity inside the PCRV. The blanket structure consists of removable inner portions (modules) and a permanent outer one. The outer structure, containing the coolant manifolding and neutron shielding, is embedded into the PCRV concrete where possible. All blanket cooling lines are interfaced through the embedments and no primary coolant flows outside the blanket or PCRV. Coolant flows from the outer blanket manifold to toroidal manifolds that completely encircle the reactor and act as coolant plena to equalize the helium flow to the 12 stean generators. The manifolding is show in Fig. 3-9. The details of the hot and cold helium ducts in the permanent blanket structure are shown in Fig. 3-10.

\subsection{Blanket}

Helium was chosen for the blanket coolant because it does not degrade the neutron spectrum thus allowing a high blanket breeding ratio to be attained. Helium is chemically stable and noncorrosive, which is important for reasons of safety and operability. Further, helium is not electrically conductive, which is important in magnetically confined fusion systems.

On the basis of reutronic and economic analysis the uranium alloy uraniun silicide $\left(\mathrm{U}_{3} \mathrm{Si}\right)$ was chosen as the fuel material. Uranium silicide has been developed for the CNDU reactor program and has been irradiated up to $2.5 \%$ burnup with very low swelling. It has a high uranium density, low parasitic absorption, and good irradiation stability at temperatures up to $900^{\circ} \mathrm{C}$.

To optimize the hybrid blanket neutronic performance, the amount of structural material in the blanket and the thickness of the first wall must be minimized. This requires a structural material that exhibits high strength at elevated temperature and also has good irradiation life. Inconel 718 was chosen because it has these characteristics. The design strength is in excess of $400 \mathrm{MPa}(50,000 \mathrm{psi})$ to above $600^{\circ} \mathrm{C}$. Radiation induced swelling is expected 


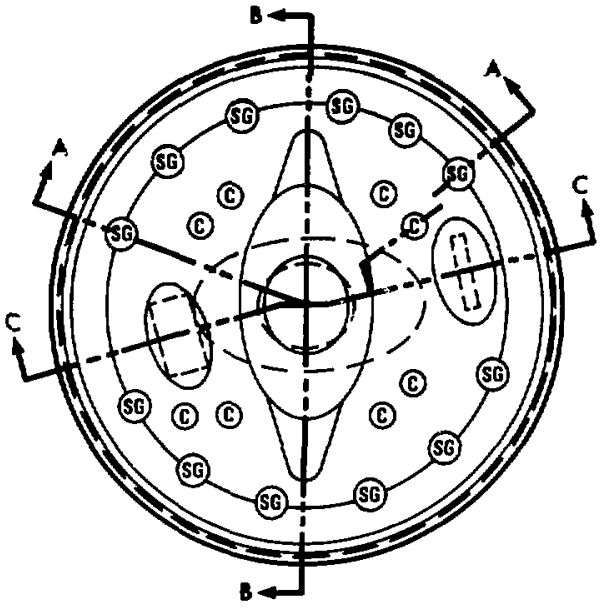

Fig. 3-7. Prestressed concrete reactor vessel, top view. 
Section A - A through steam generator and helium circulator

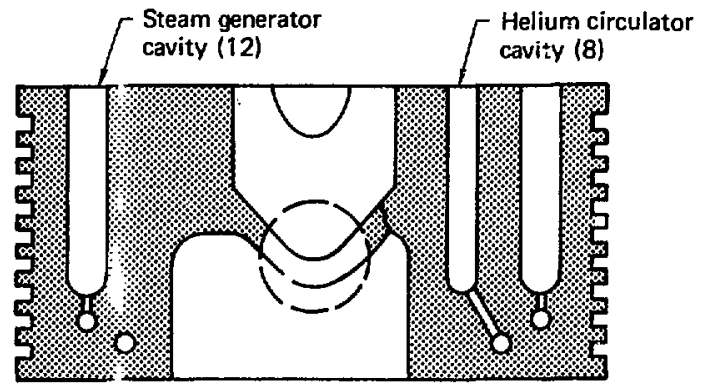

Section B - B

Grooves for post-tensionir.g cables (9)

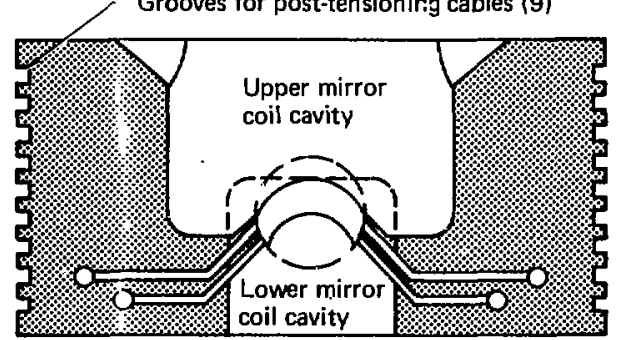

Section $\mathrm{C}-\mathrm{C}$ through beam injectors

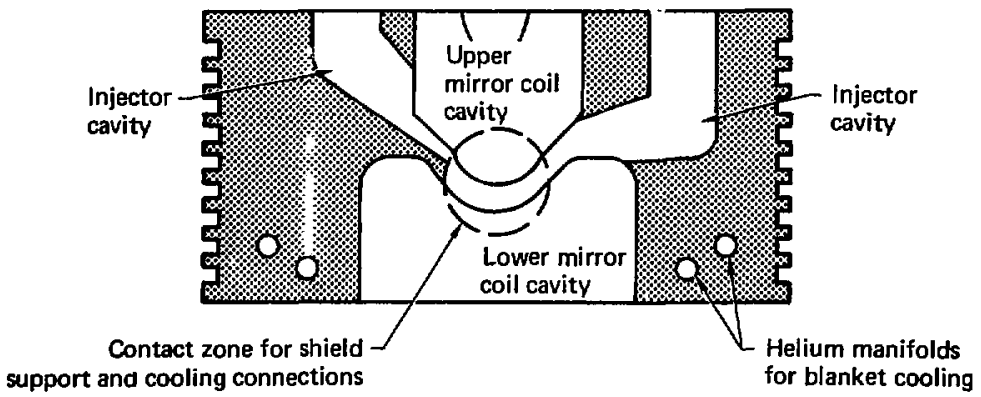

Fig. 3-8. Prestressed concrete reactor vessel, sections. 


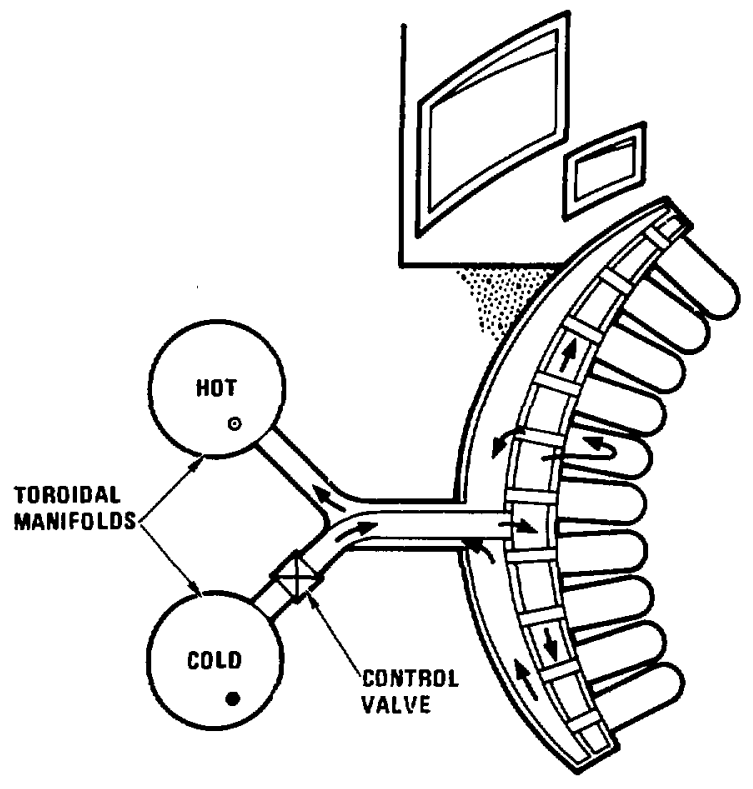

Fig. 3-9. Coolant manifolding. 


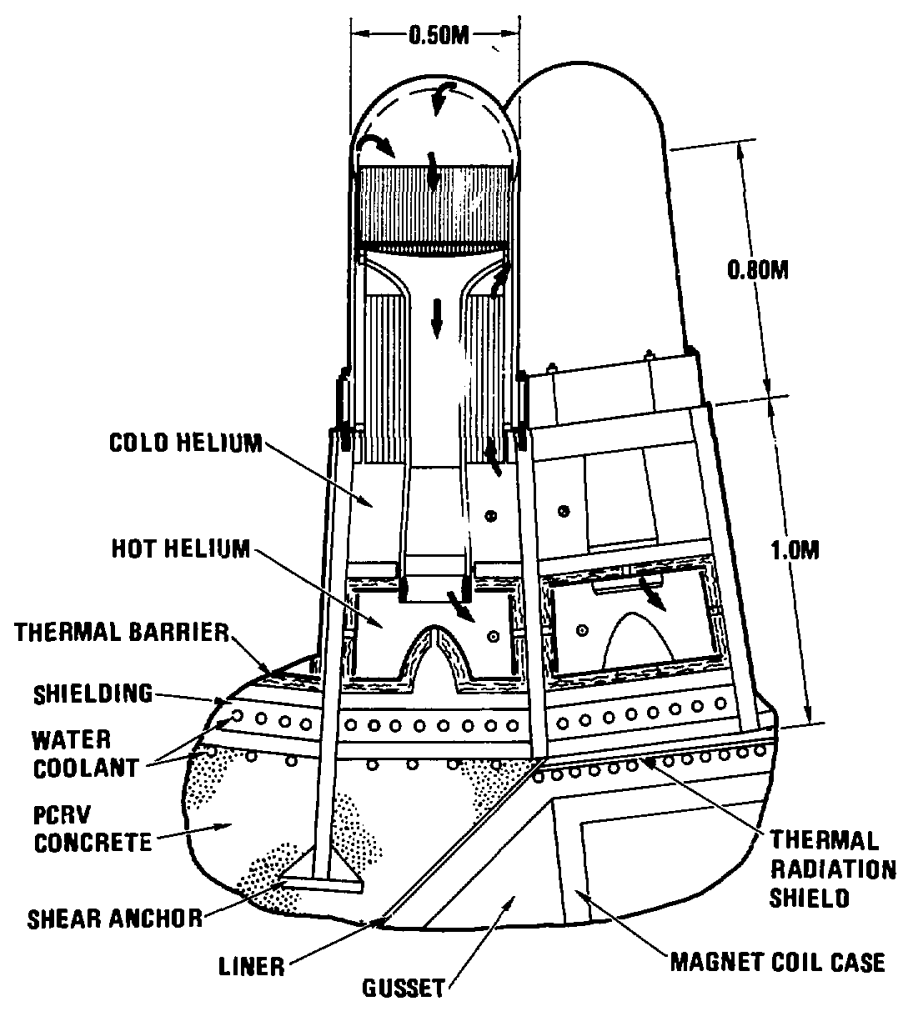

Fig. 3-10. Blanket manifold structure. 
to be quite low on the basis of experimental data from irradiations of Inconel 718 and the similar alloy PE-16 in HFIR and EBR-II. For irradiation temperatures in the range $320^{\circ}$ to $700^{\circ} \mathrm{C}$, radiation embrittlement of Inconel 718 is not expected to be a problem based on HFIR irradiation data for the similar PE-16 alloy which exhibits at least 18 residual ductility after the radiation equivalent to about $6 \mathrm{mw}-\mathrm{y} / \mathrm{m}^{2}$.

Lithium delteride (LiD) was chosen as the tritium breeding compound. This material has a high lithium density plus deuterium to moderate the neutrons. It also appears to have excellent irradiation stability as radiation damage anneals out at temperatures above $300^{\circ} \mathrm{C}$. A potential problem with LiD is its high deuterium vapor pressure. In a system designed to release tine bred tritium, dehydriding of the LiD would be a serious concern. We have opted to contain the tritium in the fuel rods by using hydrogen retentive aluminum alloy cladding and low lithium zone temperatures. The aluminum-beryllium alloy Lockalloy 43 was used.

The blanket module is shown in Fig. 3-11. It is composed of only two pieces, the fuel assembly and the Inconel 718 pressure shell. The pressure shell is in the shape of a cylinder capped with a hemisphere and has a first wall thickness of only $2.4 \mathrm{~mm}$. The fuel assembly threads into a socket in the permanent module support structure. The pressure shell is bolted to the support structure with six large bolts in a hexagonal flange at the base of the cylinder. The vacuum seal is a double mechanical varian-type knife edge seal with secondary vacuum pumping between the two knife edges.

Inconing $280^{\circ} \mathrm{C}$ helium flows first through and cools the LiD zone. It emerges, at only $300^{\circ} \mathrm{C}$ due to the low power density in the LiD, and flows past the first wall, cooling it. The coolant flow turns and flows radially outward through the $\mathrm{U}_{3} \mathrm{Si}$ fuel zone, emerging at $540^{\circ} \mathrm{C}$.

The radial outward coolant flow path is important because of the very steep radial outward power density gradient characteristic of a hybrid reactor blanket. Figure 3-12 shows th: steep radial power density gradient and the hot spot temperature characteristics that are obtained by using radial outward coolant flow. As can be seen, the limiting temperatures, the hot spot fuel centerline temperature and the hot spot cladding temperature, are almost constant along the length of the fuel rod.

The fuel configuration chosen for both the $\mathrm{U}_{3} \mathrm{Si}$ zone and the LiD zone is radially oriented fuel rods. The rods are wire-wrapped to maintain spacing 


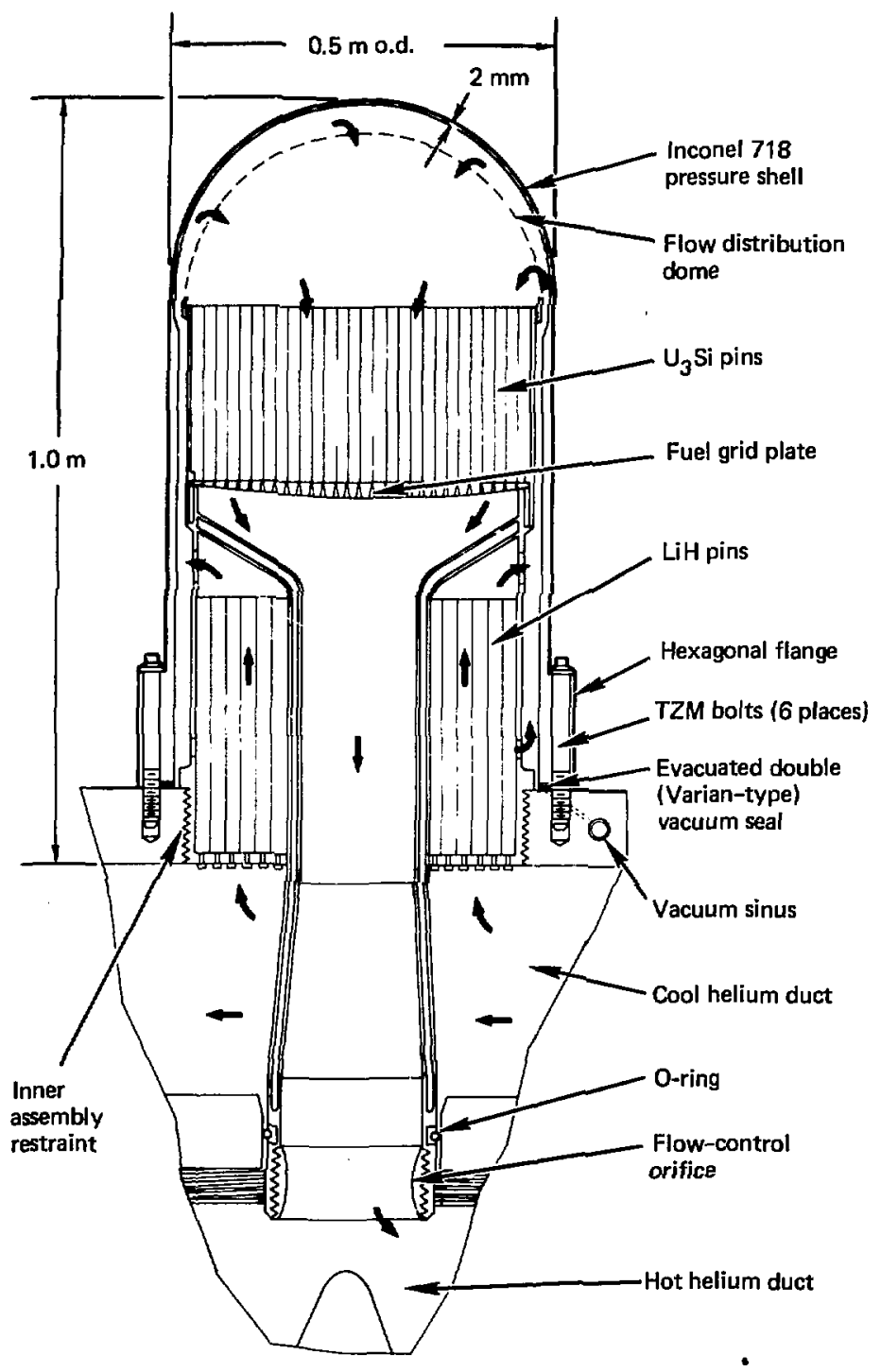

Fig. 3-11. Blanket module. 


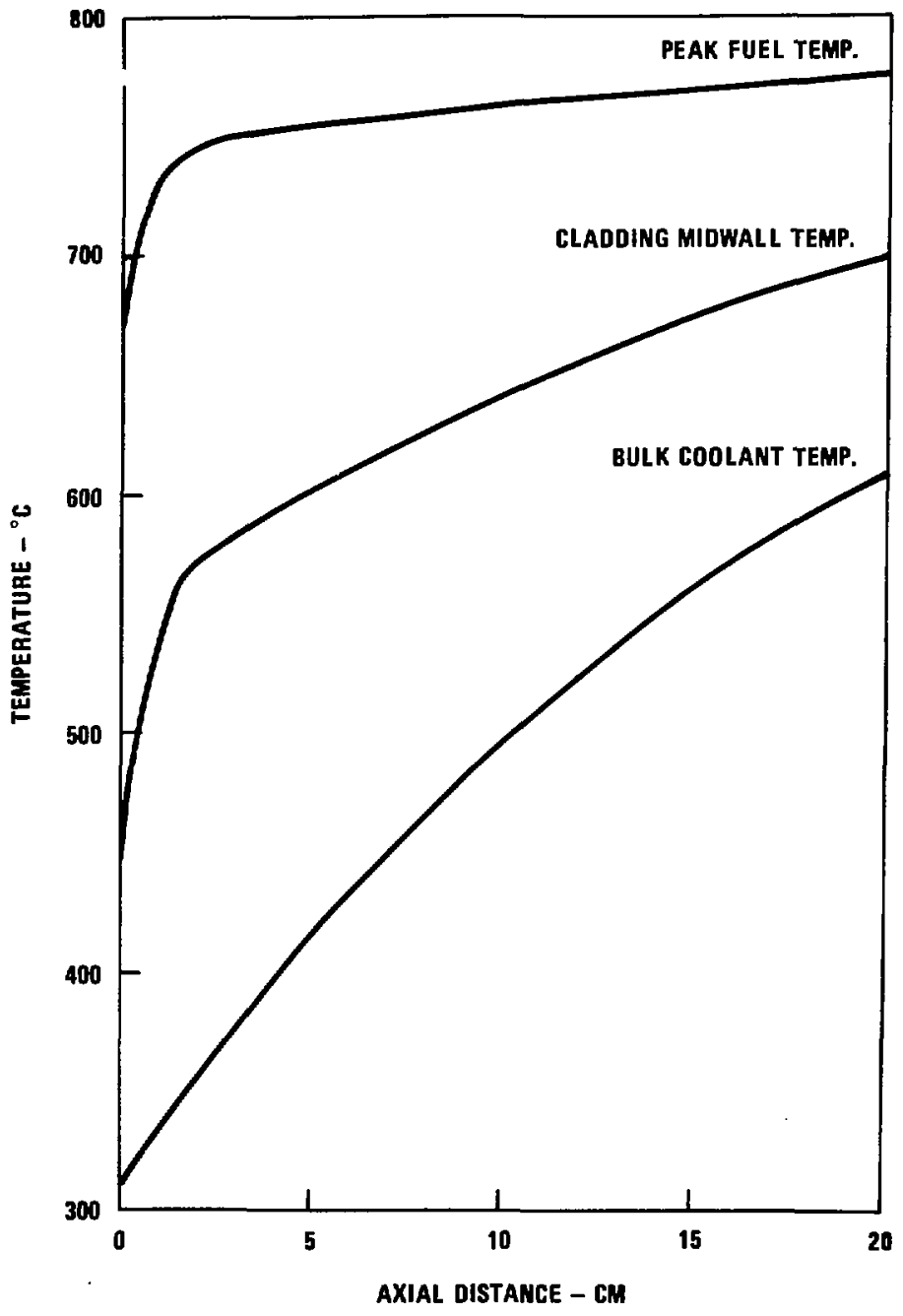

Fig. 3-12. Blanket power distribution and hot-spot terperatures. 
for coolant flow. No fission gas plenum or tritium plenum are necessary due to the highly retentive nature of the $U_{3} \mathrm{si}$ and $\mathrm{LiD}$ fuel materials and the modest burnup experienced by both $\mathrm{U}_{3} \mathrm{Si}$ and LiD before being reprocessed. The rods are not pressurized and the cladding is allowed to creep dow upon the fuel pellet. The high design peak power density in the fission zone 1500 $\mathrm{w} / \mathrm{cm}^{3}$ ) leads to a small diameter fuel $\operatorname{rod}(7 \mathrm{~mm})$ while the lower, $30 \mathrm{w} / \mathrm{cm}^{3}$ peak heating rate in the LiD allows larger rods $(30-m-d i a m)$ in the lithium zone.

A new blanket module starts life with depleted uranium fuel and has an energy multiplication $(M)$ of 9.1 , a net fissile breeding ratio $(f / n)$ of 1.85 and a tritium breeding ratio $(T / n)$ of 0.99 . By the end of its life at an exposure of $6.6 \mathrm{MN}-y / \mathrm{m}^{2}$, it has attained a burnup of 18 , and the nuclear performance parameters are then $M=16.2, f / n=1.65$ and $T / n=1.65$. The change in nuclear performance with exposure is due to the buildup of ${ }^{239} \mathrm{Pu}$, a fraction of which is fissioned in-situ, thus decreasing $f / n$ and increasing $M$ and $T / n$.

\subsection{Power Conversion System}

The power conversion system used in the mirror hybrid is derived from the conventional steam cycle systems developed for the HTGR and GCFR. Hot helium from the blanket modules flows to the toroidal hot coolant duct. Equal flows from this duct are directed to twelve helically-wound steam generators. The cool helium leaving the steam generators flows to the eight steam turbinedriven helium circulators which pump it back to the toroidal cool helium duct. The helium returns to the blanket modules through flow control valves which regulate the flow to each blanket segment to maintain the power helium temperature conditions of $280^{\circ} \mathrm{C}$ inlet and $540^{\circ} \mathrm{C}$ outlet at $6 \mathrm{MPa}(60 \mathrm{~atm})$.

The steam cycle power conversion system is conventional, using $440^{\circ} \mathrm{C} / 8.42 \mathrm{MPa}$ superheated steam conditions with a single reheat of $504^{\circ} \mathrm{C} / 5.26 \mathrm{MPa}$. An unusual feature is the use of $389 \mathrm{MWt}$ of waste heat from the neutral bean injectors for feedwater heating. 


\section{SYSTEM MODEL AND ECONOMIC ANALYSIS}

\subsection{Introduction}

At the beginning of this study, the existing version of the mirror hybrid reactor model, described in Ref. 1, was used to obtain an optimized set of reactor parameters for the conceptual design study. The component designs, described in the following sections of this report, were based on these parameters. The design parameters were chosen to minimize the cost of producing electricity from a fusion-fission power system, which consists of a mirror hybrid reactor and a group of light water reactors that are supplied with makeup fissile fuel $\left({ }^{239} \mathrm{Pu}\right)$ requirements by the hybrid.

The size of the hybrid was selected to be as small as possible without incurring any significant size-related penalty. A plasma length, mirrorto-mirror, of $13 \mathrm{~m}$ was found to be approximately the minimum size plasma that would permit acceptable blanket coverage and adequate space for shielding in the mouth of the Yin-Yang coil. Also, the injection energy and magnetic field intensity were constrained to permit the use of positive-ion injector technology and NbTi superconductor, respectively.

As the component design information evolved during the course of the study, the system model was updated to reflect the design changes. Because there was not enough time available to iterate on the reactor design, the component descriptions presented in the following sections are not entirely consistent as to power levels, dimensions, etc. These descriptions should therefore be regarded as representative of the reactor component designs. However, the final system model does contain a consistent description of the reactor, and here we present the set of reactor parameters obtained with the finished version of the model.

\subsection{Model Description}

The systen model contains the following features:

- A plasma physics nodel.

- A hybrid reactor model to size reactor components and evaluate power flows, blanket geometry, and first-wall neutron loading.

- A capital cost model for the hybrid. 
- A fuel-management model to evaluate the thermal power and fissile breeding of the blanket as a function of blanket exposure.

- An economic analysis to evaluate the cost of producing electricity from the hybrid plus i.ts associated burner reactors.

Thıis model has been described in detail in Ref. 1 . Here, the model is briefly reviewed, emphasizing changes that have been made since the publication of the report.

\subsubsection{Plasma Physics and Reactor Model}

The plasma physics model used in this study is described in ch. 5. We treat primarily plasma energetics and confinement. The plasma is treated with a two-region model. The inner or "core" region is free from radial density gradients, and ion confinement is determined by ion-ion scattering collisions into the (electrostatic-magnetic) loss cone. We term this condition classical mirror confinement. The outer or "boundary-layer" region, assumed to be 3 gyroradii in thickness, is the region of the plasma where the density drops from its central value to zero. Thus, this region contains significant density gradients and it is assumed to require stream stabilization with warm plasma. Ion confinement in the boundary layer is electron-drag dominated (with a low electron temperature as compared to the core), which results in a lower confinement time than in the core. The overall $Q$ for the plasma is therefore a result of an area and density-weighted average of confinement in the core and boundary layer.

The reactor model contains scaling laws for the Yin-Yang magnet design, magnet neutron shield, blanket geometry, blanket neutronics, direct converter, vacuum pumping requirements, injector efficiency, cryogenic equipment and a reactor power balance. The efficiency of the thermal conversion equipment is specified as input. The major new features here are the direct converter model (described in Ref. 2), the analytic blanket neutronics model (described in Sec. 7.1.6), and the blanket design.

The procedure in the blanket management scheme is to load fertile material into the reactor, expose the fuel to a specified maximum burnup, remove the fuel from the reactor, and then reprocess it to obtain the fissile fuel that has been bred. As the fissile isotopes are created in the blanket, some of this material is fissioned in situ, the result being an increased blanket energy multiplication and therefore, an increased thermal output. The blanket management scheme is used to decrease the power swing that resuits from the 
enrichment and to provide an output of fissile material (e.g., product) from the blanket at intervals shorter than the blanket life.

The individual blanket modules are grouped into a number of fuel management regions, denoted as $N_{B}$. If $\tau_{B L}$ is the blanket life (e.g., the length of time the fuel stays in the reactor), then at time intervals of $\tau_{B L} / N_{B}$ the reactor is shut down and the modules in one fuel management region $\left(1 / \mathrm{N}_{B}\right.$ of the total blanket) are removed and replaced with new, unexposed modules. By appropriate choice of a startup procedure, each blanket region is at a different exposure and the swing in the overall blanket multiplication can be maintained below that occurring in an individual module.

At the beginning of the reactor life all $\mathrm{N}_{B}$ blanket fuel management regions are loaded with unexposed fuel, and at time intervals of $\tau_{B I N}=\tau_{B L} / N_{B}$ the reactor is shut down and one region of the blanket is removed and refurbished with fresh fuel. Thus, if $\Phi_{m x}$ is the (specified) maximum blanket exposure, the region removed at the end of the first interval has exposure $\Phi_{m X} / N_{B}$, the region removed at the end of the the second interval has exposure $2 \Phi_{m x} / N_{B}$, and so on. After $\left(N_{B}-1\right)$ periods, we reach an equilibrium fuel cycle where, at the beginning of the fuel management interval ( $\tau_{\text {BIN }}$ ) the exposures for the various regions are

$$
\Phi_{i}=(i-1) \Phi_{m x} / N_{B^{\prime}} i=1,2, \ldots, N_{B^{\prime}}
$$

and at the end of the interval, the exposures are

$$
\Phi_{i}=i \Phi_{m x} / N_{B^{\prime}} i=1,2, \ldots, \mathrm{N}_{B^{*}}
$$

The subscript $i$ is the fuel management region index. Note that to reach the equilibrium cycle we pay a penalty; the first $\left(N_{B}-1\right)$ regions removed do not reach maximum exposure.

As described in Ref. 1, we also calculate a capacity factor for the reactor, $\delta_{c}$, that reflects the time and frequency required to shut down the reactor and perform blanket change operations. The capacity factor is taken to be

$$
\delta_{C}=\frac{1}{1.25+\frac{\mathrm{N}_{B} \tau_{B C} \Gamma}{\Phi_{\operatorname{mx}}}}
$$

where $\tau_{B C}$ is the time required to change a blanket region, $\Gamma$ is the first 
wall neutron loading $\left(\mathrm{wN} / \mathrm{m}^{2}\right)$ and $\Phi_{\mathrm{mx}}$ is the maximum blanket exposure $\left(\mathrm{k} / \mathrm{y} / \mathrm{m}^{2}\right)$. This quantity is defined to yield $\delta_{\mathrm{c}}=0.8$ if no blanket change were required.

\subsubsection{Costs}

The hybrid reactor costs, which determine the cost of producing the two hybrid products (fissile fuel and electrical power), are plant capital costs, fuel cycle costs, and operating and maintenance (OGM) costs.

The reactor capital costs are broken down into individual accounts as recommended in Ref. 3. A description of these accounts, for both direct and indirect costs, is given in Appendix B, along with the method of evaluating each account.

The fuel cycle costs are also described by individual accounts for fabrication, reprocessing, etc. We specify unit costs $(\$ / \mathrm{kg})$ for each fuel cycle account, and then, based on blanket mass flows evaluated in the fuel management analysis, the cost of each account is determined. The individual fuel cycle accounts and the unit costs used are also described in Appendix $B$.

The OsM costs are scaled from present fission reactor experience at $10^{4} \$ / Y$ per $k W t$. This cost is approximately equivalent to an electricity cost in an LWR of $1.5 \mathrm{mills} / \mathrm{kW}-\mathrm{h}$.

\subsubsection{Economics}

In contrast to the economic analysis of fission and pure fusion reactors, the corresponding analysis for the hybrid is more complex as a result of the following considerations:

- A primary product of the hybrid, fissile material, does not produce revenues on a continuous basis but rather only when the blanket is removed from the reactor and the fuel is reprocessed. Thus, the economic model must reflect propeily the "inventory charge" for producing a product, which is not sold until the end of the period. In contrast, for example, the electricity production from a fission reactor is sold as it is produced and revenues are realized (almost) simultaneously. - The blanket thermal output, and thus the plant net electrical output, varies with time.

- The hybrid reactor produces two products, fissile material and electricity. To fix the unit price of each product, the economic analysis must be constrained in some way. 
The economic analysis is constrained by requiring that the hybrid electricity cost be equal to the electricity cost from the fission reactors that are supported by the hybrid fissile fuel production. To determine this common electricity cost, we consider a single economic unit, denoted as a fusion-fission power system, which consists of the hybrid and its associated thermal reactors. Fissile fuel is thus a quantity internal to the system and does not enter the economics; the single product is electrical power. Given the capital costs, fuel cycle costs (without fissile material) and OsM costs, the electricity production cost can be determined.

Having thus established the selling price for the hybrid electricity, we may now focus on the hybrid as an economic unit. Given its capital cost, fuel cycle costs, O\&M costs and electrical power selling price, we may determine a production cost for the fissile fuel. To properly account for the time dependent effects in the hybrid economics, we use a present worth cash-flow analysis.

A detailed derivation of the equations describing the economics of the fusion-fission power system and the hybrid is presented in Ref. 1.

\subsection{Reference Design}

Here we present descriptions of the optimized hybrid reactor (parameters, power flow, economics) and the fusion-fission power system (fuel cycle, fission burner reactor, economics). In Sec. 4.4, results of a parametric study of the hybrid are described which demonstrates that the reactor is optimized. In addition, in Sec. 4.4 the improvements in reactor economics are discussed which would result from removing the constraints on magnet and injector technologies.

\subsubsection{Hybrid Reactor}

Parameters of the reference reactor are listed in Table 4-1. The reactor has a fusion power level of $402 \mathrm{~mm}$, a blanket thermal power of $2350 \mathrm{mw}$, and produces $\sim 600 \mathrm{kN}$ of net electrical power and $\sim 2000 \mathrm{~kg} / \mathrm{Y}$ of plutonium-239. The plant has a 65 recirculating power fraction with a plasma $Q=0.64$. The blanket energy multiplication is $M=10.4$, the net fissile breeding ratio is $\mathrm{Pu} / \mathrm{n}=1.51$, and the first wall loading is $\sim 1.7 \mathrm{kw} / \mathrm{m}^{2}$. The blanket performance parameters, $M$ and $P u / n$, are time-averaged over the equilibrium fuel cycle, and account for the 86\% fractional blanket coverage while breeding sufficient tritium to make the reactor self-sustaining in this isotope. 
Magnet

Geometry

Conductor

Naximum conductor field, T

Maximum mirror field, $T$

Minimum mircor field, T

Central field, T

Mirror ratio based on min mirror field

Overall magnet diameter, m

Mirror-to-mirror length, m
Yin-Yang

Nbri/Cu

$$
\text { 8.5 }
$$

5.95

5.66

2.52

2.25

22.5

13.0

Injectors

Type

Source species mix

$$
x^{+}, x_{2}^{+}, x_{3}^{+}
$$

Extraction voltage, $k v$

D

$\mathbf{T}$

Average neutral beam energy, keV

D

$\mathbf{T}$

$2 / 3 \mathrm{D}+1 / 3 \mathrm{~T}$

Efficiency ${ }^{a}$

Injected current, $A$

D

$\mathbf{T}$

Neutral bean power, $N$

Input electrical power, $\$$
Positive ion acceleration

$908,7 \%, 38$

187.5

103.7

155.7

121.0

0.576

5200

3467

1733

630

1094 
Plasma

Average injection energy, kev

121.0

Injection angle

$65^{\circ}$

Plasma mirror ratio

4.15

$\mathbf{Q}$

0.64

Burn fraction

0.98

plasma radius, m

2.5

Plasma length, m

13.0

Fusion Power, NA

402

Core region

Average ion energy, kev

134.6

Average electron energy, kev

15.0

Ambipolar potential, kV

$$
\begin{aligned}
& \text { center to mirror } \\
& \text { center to end wall }
\end{aligned}
$$

63.9

Beta

0.71

Central magnetic field, T

1.37

Central plasma density, $\mathrm{cm}^{-3}$

$8.9 \times 10^{13}$

$\mathrm{nt}, \mathrm{s} / \mathrm{cm}^{3}$

$2.3 \times 10^{13}$

(ov) ${ }_{\mathrm{DT}}, \mathrm{cm}^{3} / \mathrm{s}$

$8.2 \times 10^{-16}$

$\mathbf{Q}$

0.69

Boundary layer region

Average ion energy, kev

. 26.6

Electron temperature, kev

0.42

Average plasma density, $\mathrm{cm}^{-3}$

$4.5 \times 10^{13}$

$\mathrm{m}, \mathrm{s} / \mathrm{cm}^{3}$

$1.6 \times 10^{12}$

Thickness, cro

4.0

Stream current, A

$15 \times 10^{3}$

Energetic current, A 
Blanket

Geometry

$$
\begin{array}{lc}
\text { First wall radius, } \mathrm{m}^{\mathrm{b}} & 3.9 \\
\text { Fractional blanket coverage } & 0.86 \\
\text { First wall area, } \mathrm{m}^{2} & 166
\end{array}
$$

Fuels

Fissile breeding material

$$
\mathrm{U}_{3} \mathrm{~s}_{1}
$$

$\mathrm{U}_{3} \mathrm{~s}_{i}$ pin length, cm

30.0

Uranium inventory, $\mathrm{kg}$

$4.3 \times 10^{5}$

Tritium breeding material

LiD

$\mathrm{Li}$ inventory, $\mathrm{kg}$

$3.7 \times 10^{4}$

Energetics

Average blanket multiplication ${ }^{c}$

10.4

Peak blanket multiplication ${ }^{c}$

11.2

First wall load

$$
\begin{aligned}
& \text { 14- Hev neutron energy flux, } \mathrm{mH} / \mathrm{m}^{2} \mathrm{~b} \\
& \text { 14-HeV neutron current, } \mathrm{n} / \mathrm{cm}^{2} \cdot \mathrm{B}
\end{aligned}
$$

Average thermal power, $N$

Average fuel power density, $\omega / \mathrm{cm}^{3} \mathrm{~d}$

Peak fuel power density, $w / \mathrm{cm}^{3} \mathrm{e}$

115

280

Fuel managenent

Capacity factor

0.74

Number of fuel managenent regions

4

Blanket life, $y$

5.3

Fuel managenent interval, $y$

Time required for blanket change, $y$

0.077

End-of-life exposure, $M-y / \pi^{2}$

6.6

Yearly Pu production, kg 
End tanks

\begin{tabular}{|c|c|}
\hline \multicolumn{2}{|l|}{ Direct converter } \\
\hline Collector Fotential, kV & 63.1 \\
\hline Radius to collector, m & 30.3 \\
\hline Background pressure, Torr & $5.8 \times 10^{-6}$ \\
\hline Efficiency & 0.40 \\
\hline \multicolumn{2}{|l|}{ Output power, w } \\
\hline Electrical & 243 \\
\hline Hi-grade thermal & 315 \\
\hline Low-grade thermal & 49 \\
\hline \multicolumn{2}{|l|}{ Strong mirror (thermal dump) } \\
\hline Input power, $\mathrm{N}$ & 103 \\
\hline Radius to collector, m & 15.4 \\
\hline Background pressure, Torr & $2.1 \times 10^{-5}$ \\
\hline \multicolumn{2}{|l|}{ Output power, w } \\
\hline Hi-grade thermal & 93 \\
\hline Low-grade thermal & 10 \\
\hline
\end{tabular}

Thersal conversion system

Conversion efficiencies

Blanket heat

0.355

End tank heat

0.355

Injector heat $\mathbf{f}$

0.30

Input thernal power, w

BIanket

End tanks

Injectors

output electrical power, w

Blanket

End tanks

Injectors 
Electrical output, $N$ W

Gross

1715

Net

Recirculating power fraction

0.65

$a_{\text {This efficiency describes the conversion of electrical energy into }}$ energetic: neutral energy. Recovery and conversion of the thermal energy (resulting from the injector inefficiency) is treated separately. See "thermal conversion system" in Table $1-1$.

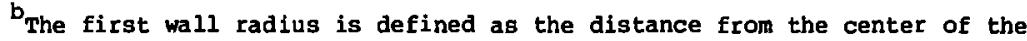
magnet to the front of the $\mathrm{U}_{3} \mathrm{Si}$ fuel pins.

CIn the equilibrium fuel cycle, accounting for the $86 \%$ fractional blanket coverage.

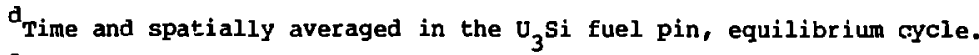

At the front of the $\mathrm{U}_{3} \mathrm{Si}$ fuel pin at end of life.

$f_{A} 308$ efficiency for conversion of injector heat to electricity (as compared to 35.58 for blanket heat) was used, reflecting our present estimate that not all of the injector heat can be recovered as high-grade thermal energy.

Figure 4-1 is a power flow diagram for the reactor with the average equilibrium blanket multiplication $(M=10.4)$. All component efficiencies are listed in Table 4-1, which in turn are described in detail in the appropriate sections of this report.

The heavy metal mass flow for the hybrid is diagramed in Fig. 4-2. The indicated flow is on a per fuel management interval, which is a time of 1.32 $\mathrm{y}$. We have neglected losses in the fabrication/reprocessing operations. Note that the uranium, coming from the blanket reprocessing operation, is recycled back into the blanket fabrication facility. Thus the recycle uranium, after a few passes through the reactor, will be essentially completely devoid of ${ }^{235} \mathrm{U}$, as compared to the original $0.258{ }^{235} \mathrm{U}$ in the (depleted) uranium purchased for the initial blanket and makeup requirements. In the neutronics calculations we have neglected the degradation of ${ }^{235} \mathrm{U}$ content in successive recycles. However, we have verified that this small fissile uranium component contributes very little to blanket neutron and energy multiplication. 


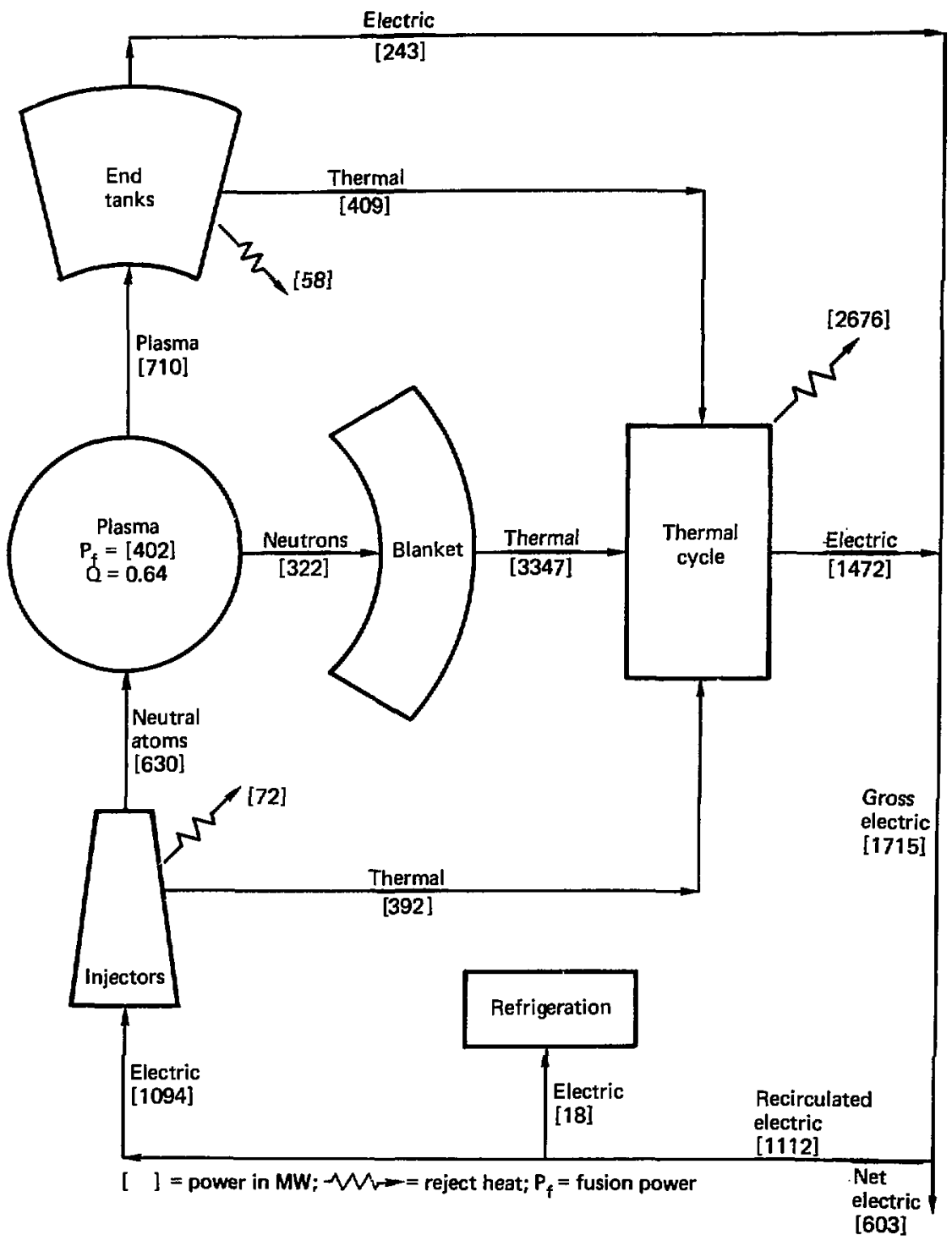

Fig. 1-1. Reactor power flow. 


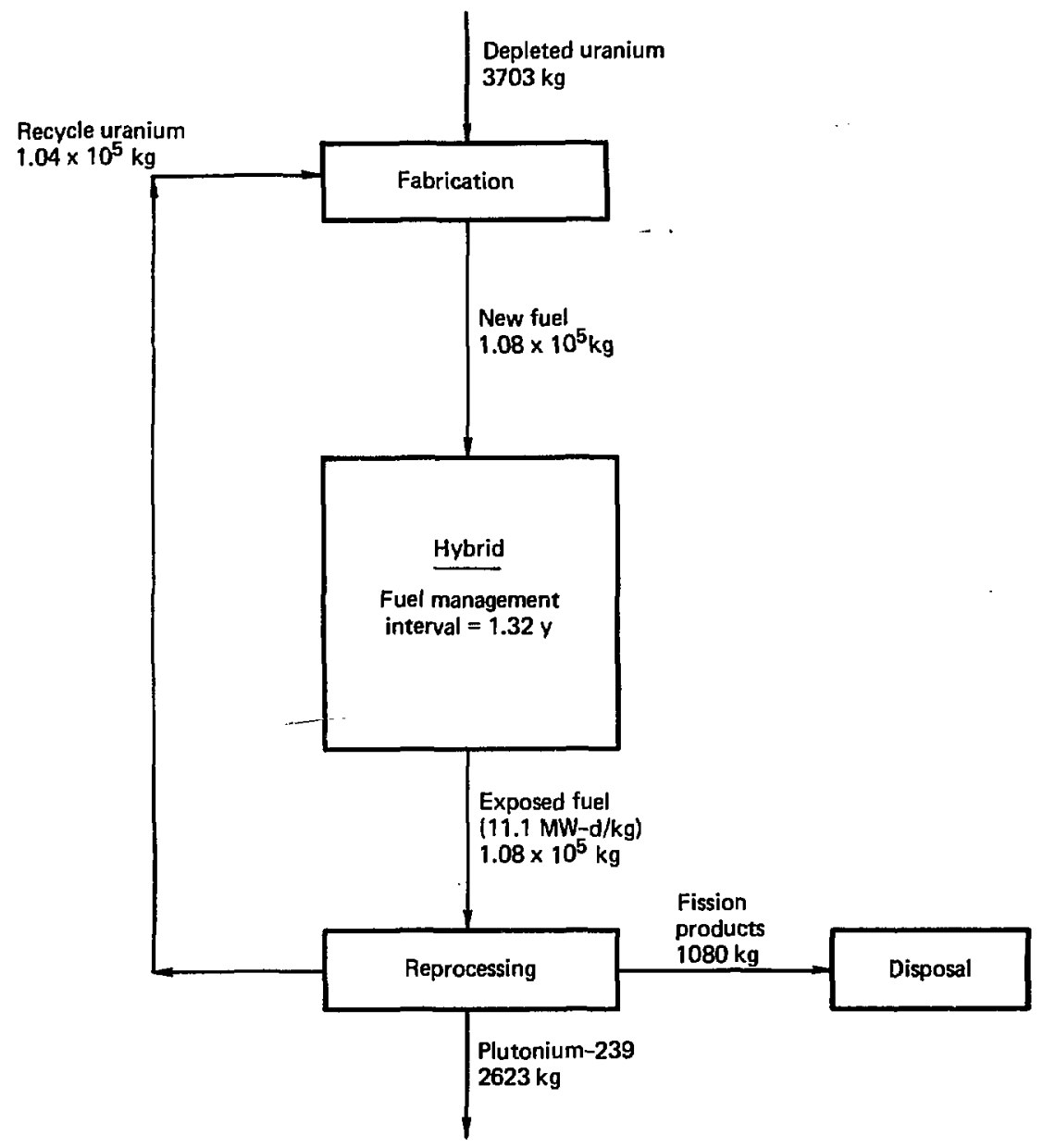

Fig. 4-i. Hybrid heavy metal mass flow per fuel management interval (equilibrium cycle). 
The "local" blanket neutronics parameters, e.g., the performance of an individual fuel bundle, are listed in Table 4-2. These parameters are for a purge tritium recovery scheme (see Sec. 7.1.5). The blanket and tritiun handling/containment designs presented in other sections of this report, were based on the batch tritium recovery scheme. However, when the reactor with a batch tritium recovery scheme was subsequently analyzed with the system code, it was found that the costs associated with the resulting large tritium inventory were unacceptable. Thus the reactor modeling presented in this section assumes the $T_{2}$ purge blanket. Unfortunately, time was not available to make the appropriate modifications in the blanket and tritium handling/ containment designs. However, we have concluded that the batch method of tritium recovery, with its associated large $\mathrm{T}_{2}$ inventory, is unattractive both from the standpoint of cost and $T_{z}$ availability.

With the purge system, we only have to compensate the tritium breeding for the fractional blanket coverage and decay in the external inventory (cryopanels, isotopic separators) of $\sim 3.2 \mathrm{~kg}$. The local (equilibrium) $\mathrm{T} / \mathrm{n}=1.17$ (average) reflects these requirements. The initial blanket performance, before equilibrium is reached, has a lower $\mathrm{T} / \mathrm{n}$ than in the equilibrium fuel cycle. This condition plus the external inventory requires purchase of $\sim 10 \mathrm{~kg}$ $\mathbf{T}_{2}$ during the first three fuel management periods. At the end of the reactor life the $\sim 3 \mathrm{~kg}$ of external inventory are recovered. Alternately, it would be possible to increase the blanket $T / n$ slightly to completely recover

Table 4-2. Local blanket neutronics (purge $T_{2}$ recovery scheme).

\begin{tabular}{|c|c|c|c|}
\hline & $\begin{array}{l}\text { Beginning } \\
\text { of life }\end{array}$ & $\begin{array}{c}\text { Time } \\
\text { Average }\end{array}$ & $\begin{array}{l}\text { End of } \\
\text { life }\end{array}$ \\
\hline Exposure, $M-y / m^{2}$ & 0 & 3.29 & 6.58 \\
\hline Energy multiplication, $M$ & 9.11 & 11.72 & 16.19 \\
\hline Net fissile breeding ratio $(\mathrm{Pu} / \mathrm{n})$ & 1.85 & 1.75 & 1.65 \\
\hline $\begin{array}{l}\text { Pu enrichment } \\
\text { (atoms Pu/atom of initial heavy metal) }\end{array}$ & 0 & & 0.024 \\
\hline $\begin{array}{l}\text { Burnup (fraction of initial heavy } \\
\text { metal atoms fissioned) }\end{array}$ & 0 & & 0.010 \\
\hline Tritiun breeding ratio & 0.99 & 1.17 & 1.35 \\
\hline
\end{tabular}


the initial $\mathrm{T}_{2}$ purchase requirements by the end of the reactor life. In equilibrium, the blanket produces $\sim 17 \mathrm{~kg}$ of $\mathrm{T}_{2}$ per year.

The local energy multiplication increases by a factor of $16.2 / 9.1 \simeq 1.8$ during the fuel bundle life. Note from Table 4-1 that with the fuel management scheme chosen we are able to ameliorate the overall power swing in the blanket consider: ' $Y$, with a peak-to-average power variation of $11.2 / 10.4=1.08$. The blanket modules are required to achieve a maximur exposure of $\sim 6.6 \mathrm{~m}-y / \mathrm{m}^{2}$, resulting in a 18 burnup $(\sim 11 \mathrm{~N}-\mathrm{d} / \mathrm{kg})$ and the enrichment at this point is 0.024 atoms Pu/atom of initlal heavy metal.

A breakdown of the reactor capital costs are presented in Table 4-3 (evaluation is described in Appendix B). The total direct cost of the reactor is estimated to be $\$ 812.5$ million, of which approximately 35 is attributable to the "fusion island" (e.g., reactor equipment, Account 221). The total capital cost, with indirects, is $\$ 1554$ million. This figure includes contingency but no escalation from 1977 dollars. The total cost, on a per unit of output basis, is $\$ 2577$ per kWe (net), $\$ 906$ per kWe (gross), and $\$ 375$ per kWt.

The hybrid reactor economics, expressed as the unit cost of producing plutonium, are presented in Table 4-4. The analysis was performed with the present worth cash-flow model described in Kef. 1. The economic parameters used for the evaluation are listed in Table 4-5. The total expenditures are dominated by capital cost, fuel cycle costs being responsible for only 168 of the total. Electricity sales account for 368 of the plant revenues, fissile material sales for the remaining 648. The electricity selling cost of $29.7 \mathrm{mills} / \mathrm{kW} \cdot \mathrm{h}$ was determined by the analysis described in the following section (Sec. 4.3.2).

A fixed charge rate on capital of 0.15 per year was specified, and the discount factor of 0.063 was determined from the relation (see Eq. 2.99, Ref. 1)

$$
k_{c}=c_{R}\left[1+\frac{k_{t}}{1-k_{t}}\left(1-\frac{1}{c_{R}{ }^{n}}\right)\right]
$$

where $k_{c}$ is the fixed charge rate, $k_{t}$ the effective tax rate, $N$ the number of years in the reactor life, and $C_{R}$ the capital recovery factor, 
Table 4-3. Reactor capital cost (\$ million).

21 Structures + facilities

211 Site improvement

6.03

212 Reactor bldg.

69.41

213 Turbine bldg.

20.09

215 Auxiliary bldg.

16.07

218A Control room bldg.

6.03

218B Diesel gen. bldg.

1.61

218C Administration bldg.

2.41

218D Service bldg.

2.81

22 Reactor plant

480.38

221 Reactor equipment

284.88

.l Magnet

54.16

.11 windings

27.85

.12 Coil case

24.32

.13 Insulation

1.99

.2 Injectors

120.86

.21 Sources

18.00

.22 Power supplies

96.26

.23 Accessories

6.60

.3 Direct converter

16.05

.31 Vacuum tanks

3.25

.32 Grids and collectors

1.29

.33 Elec equipment

10.93

.34 Cooling (side-wall)

0.58

.4 Shielding

2.00

.41 coil

2.00

.5 Vacuum system

20.47

.51 Cryopanels

16.45

.52 External vac. equ.

4.02

.6 Blanket structure

1.47

.7 Reactor vessel

64.95

.71 PCRV

36.63

.72 Liners and insul.

28.32 
222 Main ht. tran. 10op

77.31

.1 Steam generators

64.29

.2 Helium circulators

10.25

.3 Helium purification

2.77

223 Auxiliary cooling

74.85

.1 Refrigeration

46.27

.11 Heliun

18.05

.12 Nitrogen

28.02

.2 Blanket aux. cooling

16.96

.3 Injector cooling

11.61

.4 Coil shield cooling

0.02

224 Rad waste facilities

10.05

225 Fuel handling and storage

26.9

.1 Refueling equip.

18.51

$.2 \mathrm{~T}_{2}$ Reproc. and storage

4.79

. $3 \mathrm{~T}_{2}$ Clean-up

3.60

227 Instr. and control

6.39

23 Turbine plant equipment

140.63

24 Electrical plant equipnent

59.00

25 Miscellaneous

8.04

251 Transport and lifting

2.01

252 Air, water, stm. service

6.03

Total direct cost

812.50

91 Indirect field cost $(0.20)$

162.50

Total field cost

975.00

92 Engineering cost (0.15)

146.25

1121.25

Contingency (0.10)

112.13

1233.37

Total construction cost

61.67

93 Other owner costs $(0.05)$

94 Interest during construction (0.20) 
Expenditures

Capital

134.2

Fuel cycle

27.2

OCM

10.1

Total

171.4

Revenues

Electricity at $29.7 \mathrm{mills} / \mathrm{kW} \cdot \mathrm{h}$

$-62.2$

Net cost

109.3

Table 4-5. Parameters for the economic analysis.

Accounting interval, $y$

0.25

- Reactor life, $y$

30.0

Capital fixed charge rate, $y^{-1}$

0.15

Discount factor, $y^{-1}$

0.063

Effective tax rate

0.64

Fuel cooling tine, $y^{a}$

0.5

plant capacity factor

0.74

at was assumed that exposed fuel bundles were allowed to $c \infty 01$ for 6 months before reprocessing and associated fissile revenue generation.

b Includes federal and state income tax, property tax and revenue (sales) tax. 


$$
C_{R}=\frac{x}{1-(1+x)^{-N}} \text {, }
$$

where $\mathrm{x}$ is the discount factor. This discount factor was then used to evaluate the present worth of the fuel cycle and ock cash flow over the reactor life.

The individual fuel cycle costs are listed in Table 1-6. The major contributors are initial tritium purchase and uranium fabrication/reprocessing costs. Negative indirect costs for reprocessing and spent fuel shipping occur

Table 4-6. Hybrid Fuel Cycle costs (in $\$ / g$ of fissile material).

\begin{tabular}{|c|c|c|c|c|}
\hline $\begin{array}{l}\text { Component } \\
\text { description }\end{array}$ & $\begin{array}{l}\text { Direct } \\
\text { cost }\end{array}$ & $\begin{array}{l}\text { Indirect } \\
\text { cost }\end{array}$ & $\begin{array}{c}\text { Subcomponent } \\
\text { totals }\end{array}$ & $\begin{array}{l}\text { Componen } \\
\text { totals }\end{array}$ \\
\hline Initial fertile materials & & & & 0.705 \\
\hline Uranium & 0.084 & 0.412 & 0.496 & \\
\hline LiD & 0.031 & 0.179 & 0.209 & \\
\hline Recovered fertile materials & & & & -0.088 \\
\hline Uranium & -0.068 & 0.007 & -0.061 & \\
\hline LiD & -0.030 & 0.003 & -0.027 & \\
\hline Initial fusile materials & & & & 8.999 \\
\hline $\mathrm{D}_{2}$ & 0.000 & 0.001 & 0.001 & \\
\hline & 1.383 & 7.615 & 8.998 & \\
\hline Recovered fusile materials & & & & -0.407 \\
\hline $\mathrm{D}_{2}$ & 0.000 & 0.000 & 0.000 & \\
\hline $\mathbf{T}_{2}$ & -0.453 & 0.047 & -0.407 & \\
\hline Fabrication & & & & 8.838 \\
\hline Oranium & 4.889 & 3.800 & 8.609 & \\
\hline LiD & 0.029 & 0.120 & 0.149 & \\
\hline Reprocessing & & & & 8.520 \\
\hline Uranium & 9.911 & -1.391 & 8.520 & \\
\hline Spent fuel shipping & & & & 0.600 \\
\hline Uranium & 0.698 & -0.098 & 0.600 & \\
\hline \multirow[t]{3}{*}{ Blanket structure } & & & & 1.587 \\
\hline & $\underline{0.893}$ & 0.694 & 1.587 & \\
\hline & 16.474 & 10.694 & & 27.167 \\
\hline
\end{tabular}


because these expenses arise after fissile production (and thus after revenue generation). Unit costs for the fuel cycle services are given in Appendix B.

\subsubsection{Fusion-Fission Power System}

In this study, a light water reactor (LWR) was chosen as the fission burner reactor which consumes the hybrid-produced plutonium fuel.

The fuel cycle for the LWR is shown in Fig. 4-3, which was adapted from Pigford and Ang. 4 In Ref. 4, the fuel cycle considered was an "allpi.utonium" LWR, which recycles the plutonfum from its spent fuel (about 56 t fissile, e.g., plutonium-239 and plutonium-241) and is supplied with makeup plutonium generated in a uranium once-through LWR fuel cycle (about 70 s fissile). The plutoniun feed specified was $0.478 \mathrm{~kg} / \mathrm{y} \cdot \mathrm{kHe}$, of which $0.333 \mathrm{~kg} / \mathrm{Y}^{*}$ MWe is fissile. The uranium in the reprocessing stream is not recycled, but rather the reactor is fed with fresh natural uranium, which contains a significant fissile component in the form of uranium-235.

In tne present study, the fissile content $(0.333 \mathrm{~kg} /$ MWe' $\mathrm{y})$ of the makeup plutonium was simply replaced with hybrid-generated plutonium, which is about 98 fissile (see $\mathrm{Ch}$. 7.1.4). A one-for-one replacement is not exactly correct, as the hybrid plutonium does not introduce into the reactor the nonfissile isotopes plutonium-240 and plutonium-242, which exist j.n the LWRproduced plutonium and degrade the reactor performance somewhat. Thus we anticipate improved performance of the LWR on hybrid-produced plutonium, but did not have the tools to evaluate this difference. The effective conversion ratio of the fuel cycle, which describes the makeup fissile plutonium requirements, is $\mathrm{CR} \simeq 1-0.333 / 1.013=0.67$.

The unit costs used to evaluate the LWR economics are listed in Table 4-7. Now, combining the economic descriptions of the hybrid and the

Table 4-7. INR unit costs.

Capital cost, \$/kHe

Fuel cycle ${ }^{a}$

Natural uranium, $\$ / \mathrm{kg}$ HM

Mixed-oxide fabrication, $\$ / \mathrm{kg}$ EM

Reprocessing and disposal, \$/kg HM

spent Fuel Shipping, \$/kg HM

OCH, $\$ / Y-$ wat

$10^{4}$

Capacity factor

0.8

arom Ref. 5. 
Hybrid

plutonium-239

(0.333)

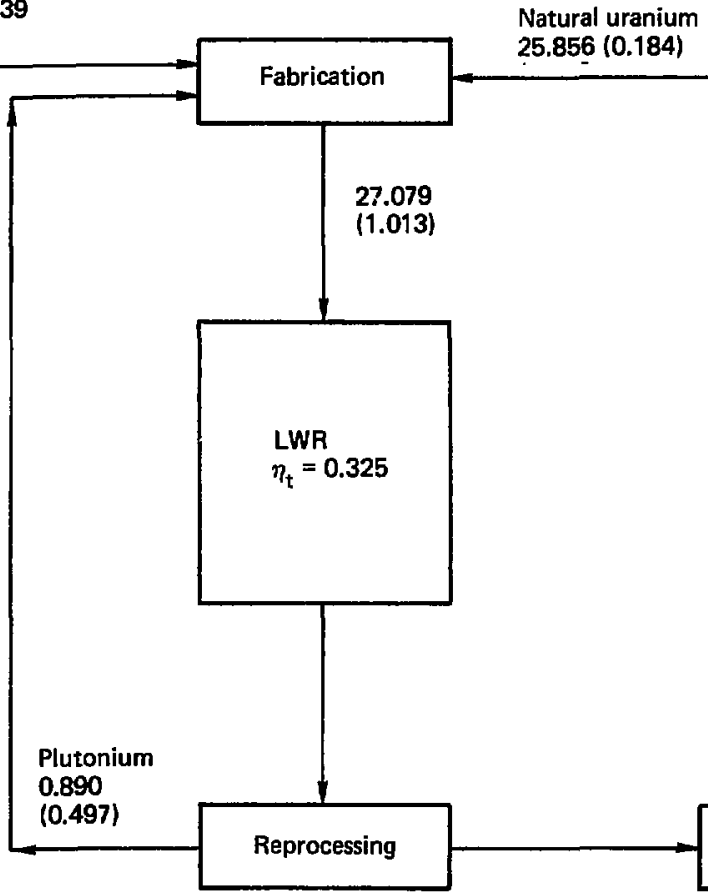

Disposal

Uranium 25.245 (0.081)

Fission products 0.950

Actinides 0.136

Plutonium 0.009

Fig. 4-3. Fuel cycle mass flow for 1000 wive LWR at 0.8 capacity factor and $32 \mathrm{kw}-d / \mathrm{kg}$ burnup. Mass flow in $t / y$; fissile mass in brackets;

$n_{t}=$ thermal efficiency. 
LWR's, which are coupled via their respective fissile production/consumption rates, the results of the cost of electrical power generation from this nuclear complex are presented in Table 4-8. Here, the parameters listed in Table 4-5 were used again. The reference hybrid supports approximately 6,000 we of LWR's, and the capital cost of the system is a weighted average of the LWR's (\$750/kWe) and the hybrid $(\$ 2577 / \mathrm{kWe})$. The electrical power cost of $29.7 \mathrm{mills} / \mathrm{kW} \cdot \mathrm{h}$ obtanned here is the value used in Sec. 4.3.1 to evaluate the hybrid economics.

Having established the plutonium production cost we may also examine the LWR as a single economic unit, purchasing make-up plutonium at $\$ 109.3 / 9$ (Table 4-4), to evaluate the impact of this cost on the LFR economics. This calculation is presented in Table 4-9. Here, the fuel cycle charges are about $12 \mathrm{mills} / \mathrm{kW} \cdot \mathrm{h}$ as compared to about $5 \mathrm{mills} / \mathrm{kW} \cdot \mathrm{h}$ using the once-through uraniun cycle with $\mathrm{U}_{3} \mathrm{O}_{8}$ at about $\$ 75 / \mathrm{kg}^{5}$ Thus we estimate that producing plutonium fuel for an LWR with this hybrid will increase present power generation costs by about 308 , from 23 to $30 \mathrm{mills} / \mathrm{kw} \cdot \mathrm{h}$.

A list of performance parameters ${ }^{6}$ for the fusion-fission power system is evaluated in Table 4-10. The overall fusion energy multiplication is

$$
M^{*}=\frac{\text { (fission reactors }+ \text { hybrid reactor) nuclear power }}{\text { fusion power }} \text {, }
$$

where the hybrid nuclear power is the blanket power plus the power carried by the DT-produced alphas. The thermal power ratio is

$$
\mathbf{R}_{t}=\frac{\text { fission reactor thermal power }}{\text { hybrid thermal power }} \text {, }
$$

where the numerator is the thermal power in all of the fission reactors supported by the hybrid, and the hybrid thermal power is the thermal power produced in the blanket and and tanks. Since the thermal power level is the basic measure of plant size, the hybrid supports about 5 LKR's lof comparable thermal rating). The electrical energy multiplication is

$$
Q_{\mathrm{E}}=\frac{\text { gross electrical output from (fission }+ \text { hybrid) reactors }}{\text { injector electrical input }}
$$




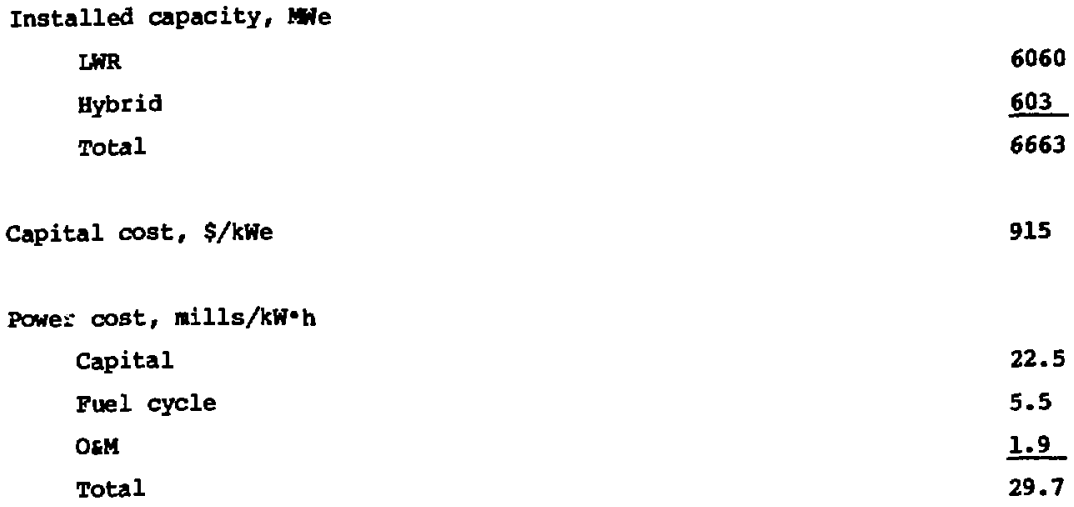

Hybrid

Total

Powe: cost, mills/kw*h

Capital

Fuel cycle

Total

Table 4-9. IwR power generation cost with hybrid fissile fuel.

mills/kWh

Capital cost

Fuel cycle

12.1

All Components except nake-up plutonium 4.4

Make-up plutoniun e $109 \$ / 9$

7.7

OEY 1.5

Total 29.7

Table 4-10. Performance paraneters for the fusion-fission power systen.

Overall fusion energy multiplication, $M$ *

54.9

Thermal power ratio, $R_{t}$ 4.97

Blectrical energy nultiplication, $Q_{E}$

7.1

Hybrid capital cost ratio, $K$

Systen capital cost ratio, $R_{c}$ 
and represents the overall multiplication of the electrical power needed to drive the hybrid plasma. The hybrid capital cost ratio is

$$
\kappa=\frac{\$ / k W t \text { for the hybrid }}{\$ / k \text { Wt for the LWR }} \text {, }
$$

and represents a comparison of the unit costs of the two types of plants that constitute the power system. The system capital cost ratio is

$$
R_{c}=\frac{\$ / k W e \text { for the system }}{\$ / k W e \text { for an LWR }} \text {, }
$$

where $\left(R_{c}-1\right)$ is proportional to the incremental cost of making the LWR's self-sufficient in fuel.

\subsection{Parametric Analysis}

\subsubsection{Fuel Manngement}

The primary fuel management parameters, used to specify the fuel management scheme, are (1) the maximum fuel burnup, (2) the number of fuel management regions in the blanket, $N_{B}$, and (3) the time required for a blanket change operation, $\tau_{\mathrm{BC}}$.

In the present study, we did not perform a detailed analysis of the blanket change operation to obtain an estimate for $\tau_{\mathrm{BC}} \cdot$ Rather, we selected $\tau_{B C}=4$ wk $(0.077 \mathrm{y})$ as a vaiue representative of the analogous operation in a LWR. We also examined the limiting case of $\tau_{B C}=0$ to illustrate the sensitivity to this parameter.

The fissile fuel production cost is shown in Fig. 4-4 as a function of burnup and $\mathrm{N}_{B}$, for the nominal case of $\tau_{B C}=0.077 \mathrm{y}$. Considering first the effect of burnup variation, for each value of $N_{B}$ there is a value of burnup which minimizes costs. The tradeoff here is that at very low burnup, high fabrication/reprocessing costs are incurred and a low capacity factor results from the need to frequently shut down the reactor and perform the blanket change operation. At high burnup, there are penalties for plutoniun inventory charges and large temporal variations in the blanket thermal output. Another underlying effect is that for low burnup, fuel production is maximized and thermal power production is minimized; as burnup is increased, energy 


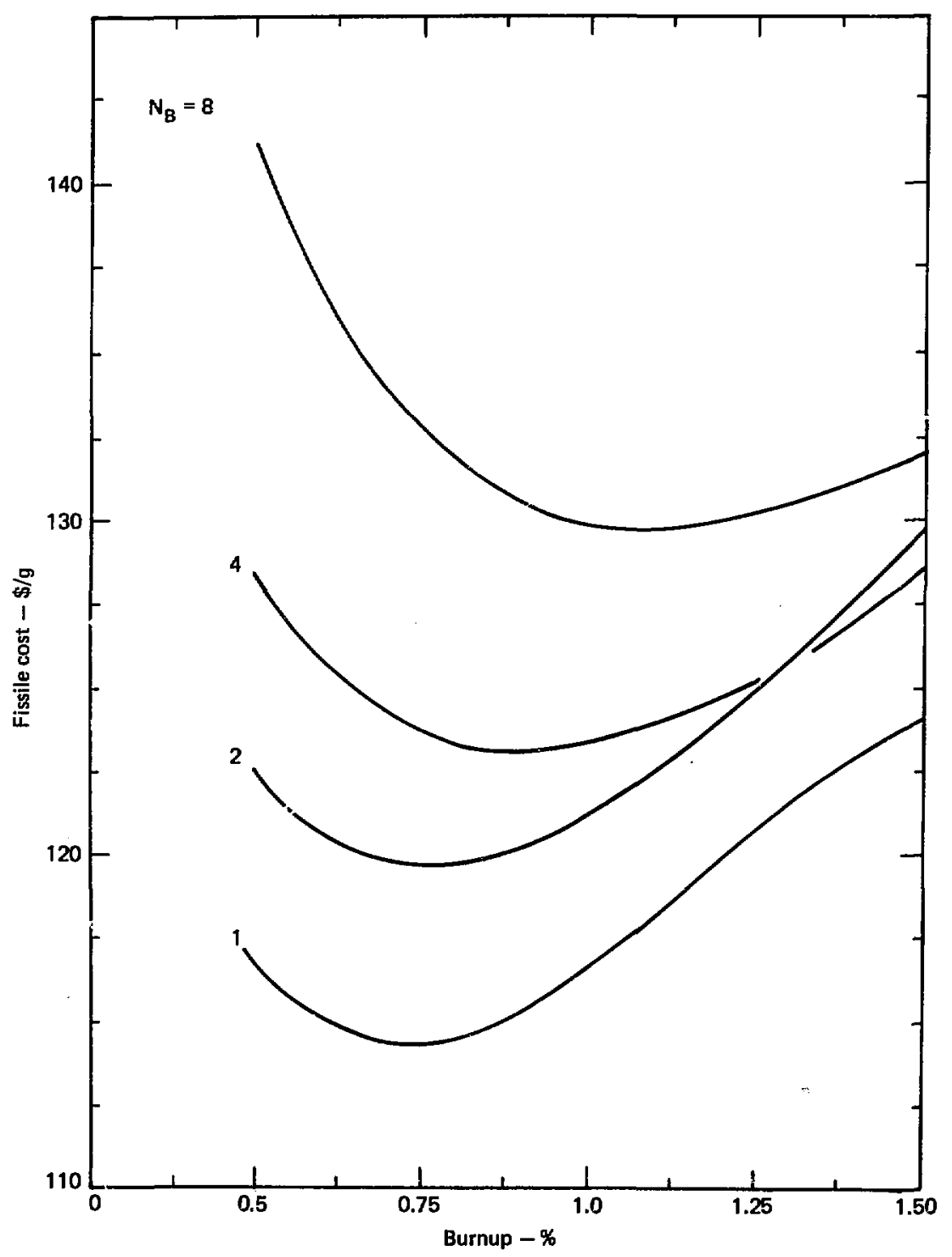

Fig. 4-4. Variation of costs with mximum fuel burnup and number of fuel managenent regions $\left(u_{B}\right)$ for $\tau_{B C}=4 w k$. 
production increases at the expense of fuel production. In general, the burnup optimizes at a low value (compared to fission reactors), where the fuel production rate is within ab?ut 108 of its initial (beginning-of-life) value.

In Fig. 4-4 $\left(\tau_{B C}=4 \mathrm{wk}\right)$, it can be seen that fuel production costs increase as $N_{B}$ increases, and in Fig. 4-5 $\left(\tau_{B C}=0\right)$, the reverse is true. The tradeoff here is that at $\operatorname{low}_{B}$ (and the nominal $\tau_{B C}$ ), there are large temporal swings in the blanket power and high plutonium inventory charges, but these effects are compensated by a bish capacity factor. However, when the capacity factor is constant $\left(\tau_{\mathrm{BC}}=0\right.$, Fig. 4-5), the fuel management scheme optimizes at high $N_{B}$, where power swings and plutonium inventory charges are small. We conclude that for a given $\tau_{B C}$, there is an optimum value of $N_{B}$. However, because of the absence of information at this time on representative values of $\tau_{B C}$ ' we follow fission reactor experience and choose $\tau_{B C}=4$ wk, and select a value of $N_{B}=4$. This value of $N_{B}$ restricts the temporal variation in blanket power to about $16 \%$ during a fuel management interval, and produces fuel from the blanket on approximately a yearly basis. This configuration represents, in our opinion, a reasonable engineering design and economic choice. Then, from Fig. 4-4, the maximum burnup is set at 18 .

\subsubsection{Mirror Ratio and Injection Ei.ergy}

We next examine the effect of varying the injection energy and the vacuum mirror ratio of the magnetic coil. The trade-off here is, to first order, one between power density and "engineering $Q^{\prime \prime}$ of the reactor. The engineering $Q$ is defined as the product of the injector efficiency, $\eta_{i}$ and the plasma $Q$,

$$
Q_{e}=\eta_{i} \mathbf{Q}
$$

For a zositive ion injector, the injector efficiency decreases as extraction energy increases in the energy range greater than about $75 \mathrm{kev}$. The dependence of the plasma $Q$ on the parameters under discussion is 


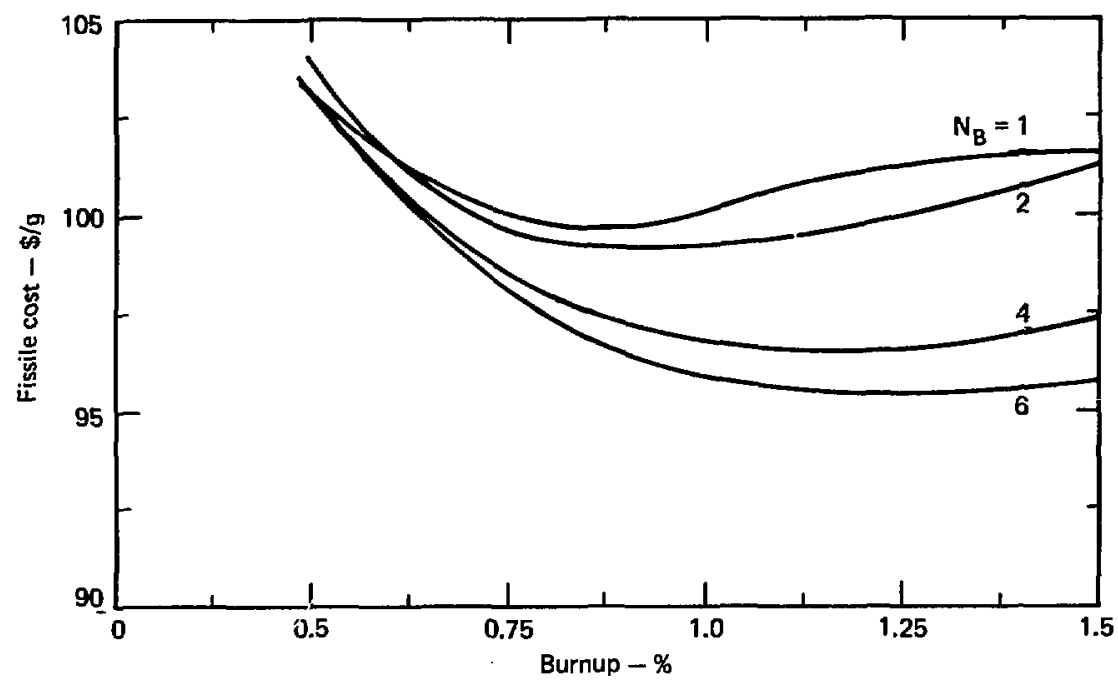

Pig. 4-5. Variation of costs with maximum fuel burnup and number of fuel management regions $\left(N_{B}\right)$ for $\tau_{B C}=0$. 


$$
Q \sim\left(\bar{E}_{i}\right)^{3 / 2} \text { In } R_{\text {VAC }} \text {. }
$$

where $\bar{E}_{i}$ is the average injection energy and $R_{v A C}$ is the vacuum mirror ratio.

Values for $Q_{e}$ as a function of mirror ratio ( $R_{v_{A}}$ ) and the deuterium extraction energy $\left(H_{D}\right)$ are shown in Fig. 4-6. The tritium extraction energy $\left(H_{T}\right)$ is 1.5 times that for deuterium (equal velocity injection), and the injected current is two-thirds deuterium and one-third tritium. The average beam energy, $\vec{E}_{i}$, was evaluated from consideration of the ion species $\mathrm{mix}$ $\left(x^{+}, x_{2}^{+}, x_{3}^{+}\right)$extracted from the source and the reaction rates for neutralization. In general, we see that the decreasing values of $\eta_{i}$ with $\bar{E}_{i}$ and the increasing values of $Q$ with $\bar{E}_{i}$ maximizes the product $Q_{e}$ in the vicinity of $100 \mathrm{keV}$. At any given energy, $Q_{e}$ increases with $R_{\text {ac }}$ ' resulting in about a 40 variation with $R_{V A C}$ in the range of 2 to 3.

The variation of first-wall loading with $W_{D}$ and $R_{V A C}$ is shown in Fig. 4-7. The first-wall loading is proportional to fusion power density, $P_{F}$, which in turn is proportional to the square of the central ion density and the fusion reaction rate,

$$
P_{F} \sim n_{0}^{2}[\sigma v]_{D T}
$$

But $\mathrm{n}_{0} \sim \mathrm{p} / \overline{\mathrm{E}}_{\mathrm{i}}$, where $\mathrm{p}$ is plasma pressure, and $\mathrm{p} \sim \mathrm{B}_{0}^{2}$ where $\mathrm{B}_{0}$ is the central magnetic field. In the present reactor design, where we have constrained the maximum field at the conductor $\left(B_{c}\right)$, we have the relation $B_{0} \sim B_{C} / R_{\text {VAC. }}$ Thus folding all these relations together, the dependence of power density on $\bar{E}_{i}$ and $R_{\text {VAC }}$ is 


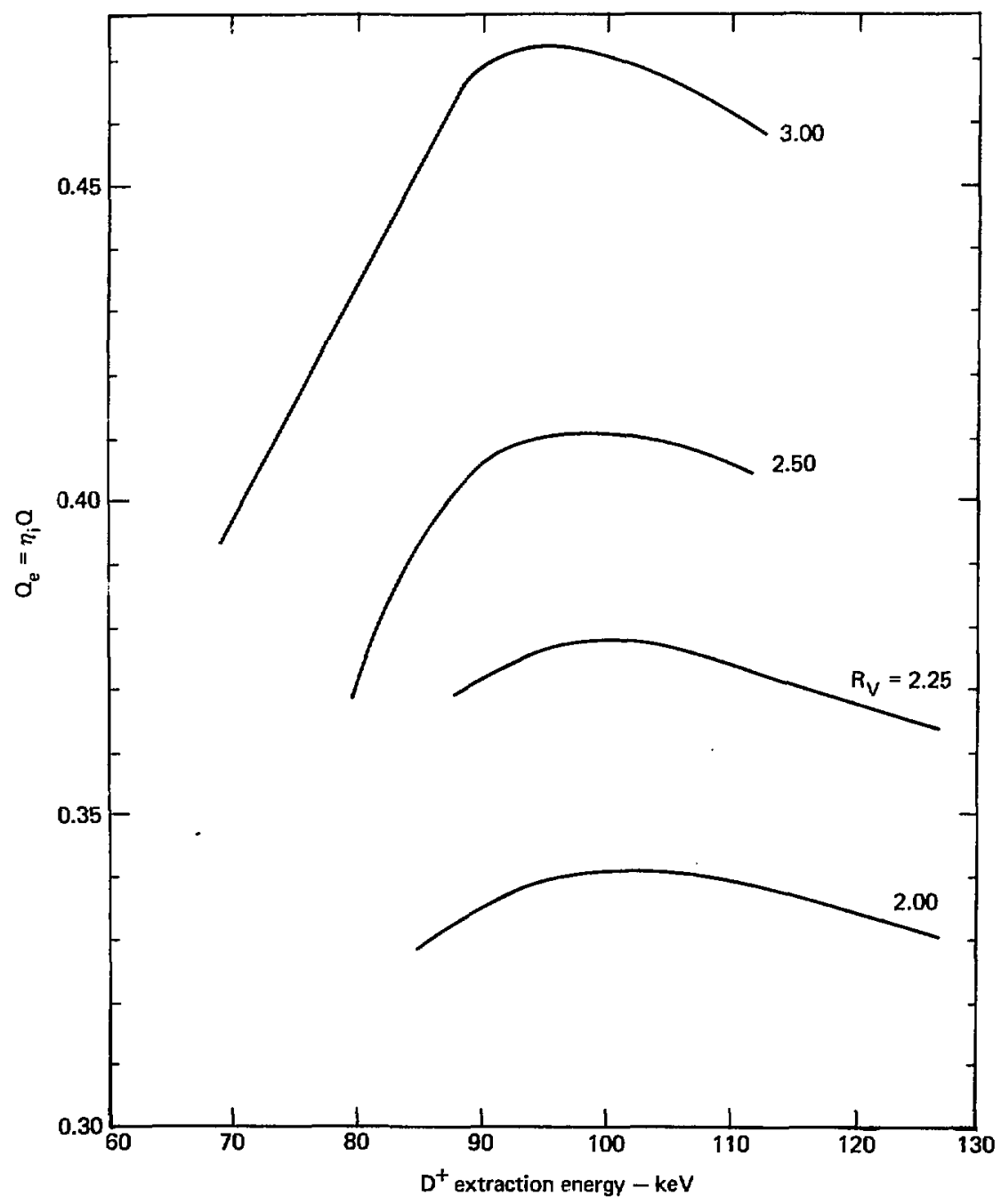

Fig. 4-6. Variation of engineering $Q$ with injector energy and mirror ratio. 


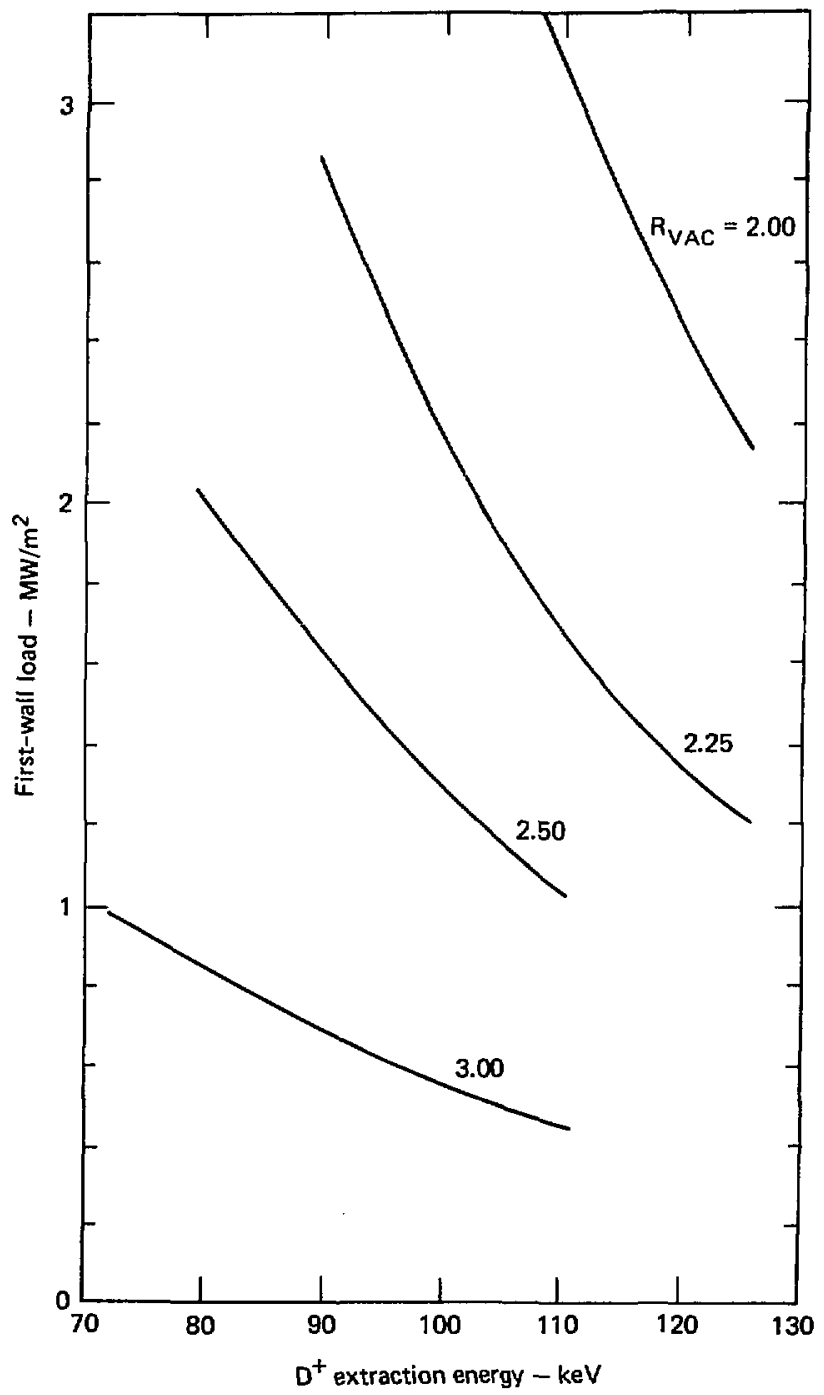

Fig. 4-7. Variation of first-wall loading with injector energy and mirror ratio. 


$$
P_{F} \sim \frac{[\sigma v]_{D T}}{R_{V A C}^{4} \bar{E}_{i}^{2}}
$$

The fusion reaction rate is only weakly dependent on $\bar{E}_{i}$ in the energy range of interest $\left(H_{D}=75-125 \mathrm{keV}\right)$. This dependence on $\bar{E}_{i}$ and $R_{V A C}$ is quantified in Fig. 4-7 where the first-wall loading can be seen to increase both with decreasing energy and decreasing mirror ratio. Note, in particular, the strong variation with mirror ratio.

The variation in fissile production cost as a function of energy and mirror ratio is shown in Fig. 4-8. At $R_{V A C}=3$, the first-wall loading is too low to be economically attractive. This situation occurs because there are some plant components which are independent of fusion power level (e.g., buildings, magnet, PCRV) and low fuel and energy production rates (proportional to first-wall loading) force these plant costs to be comparatively high. Also, at very high wall loading $\left(R_{\text {VAC }}=2.0\right)$, the reactor economics are nonoptimum. Here, the problem is that the high first wall loading requires a comparatively frequent blanket change operation, resulting in low plant capacity factor. The optimum thus occurs in the range of $R_{\text {VAC }}=2.25-2.50$, where the first-wall load is in the range $1.5-2.5 \mathrm{MH} / \mathrm{m}^{2}$.

In general, each curve for a fixed $R_{V A C}$ minimizes at the energy where $Q_{e}$, the engineering $Q$, is a maximum (for that value of $R_{V A C}$ ). Compare the minima on the curves in Fig. 4-8 with the maxima on the curves in Fig. 4-6. We thus conclude that the optimum reactor configuration maximizes $Q_{e}$ (with respect to energy) while maintaining a first-wall load of 1.5 to $2.5 \mathrm{MW} / \mathrm{m}^{2}$. It is interesting to note that the plasma $Q$ for the optimized configuration is $Q \sim 0.6-0.7$, and higher values are possible at higher mirror ratios (Fig. 4-9). However, because the higher $Q$ reduces fuel and energy production rates, it is nonoptimum. Thus, in the trade-off between power dersity and power balance, power density is the more important quantity. 


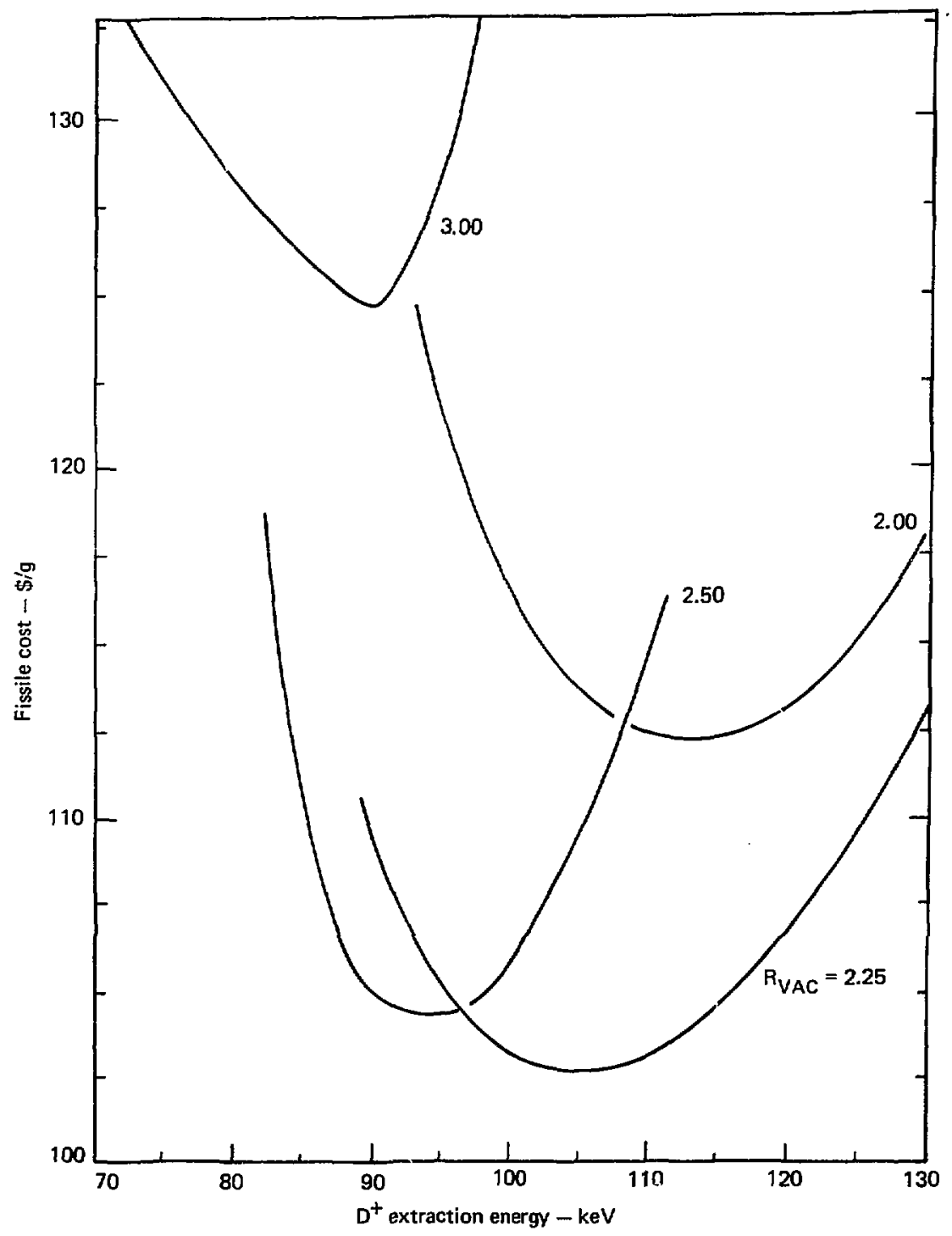

Fig. 4-8. Fissile costs as a function of injector energy and mirror ratio. 


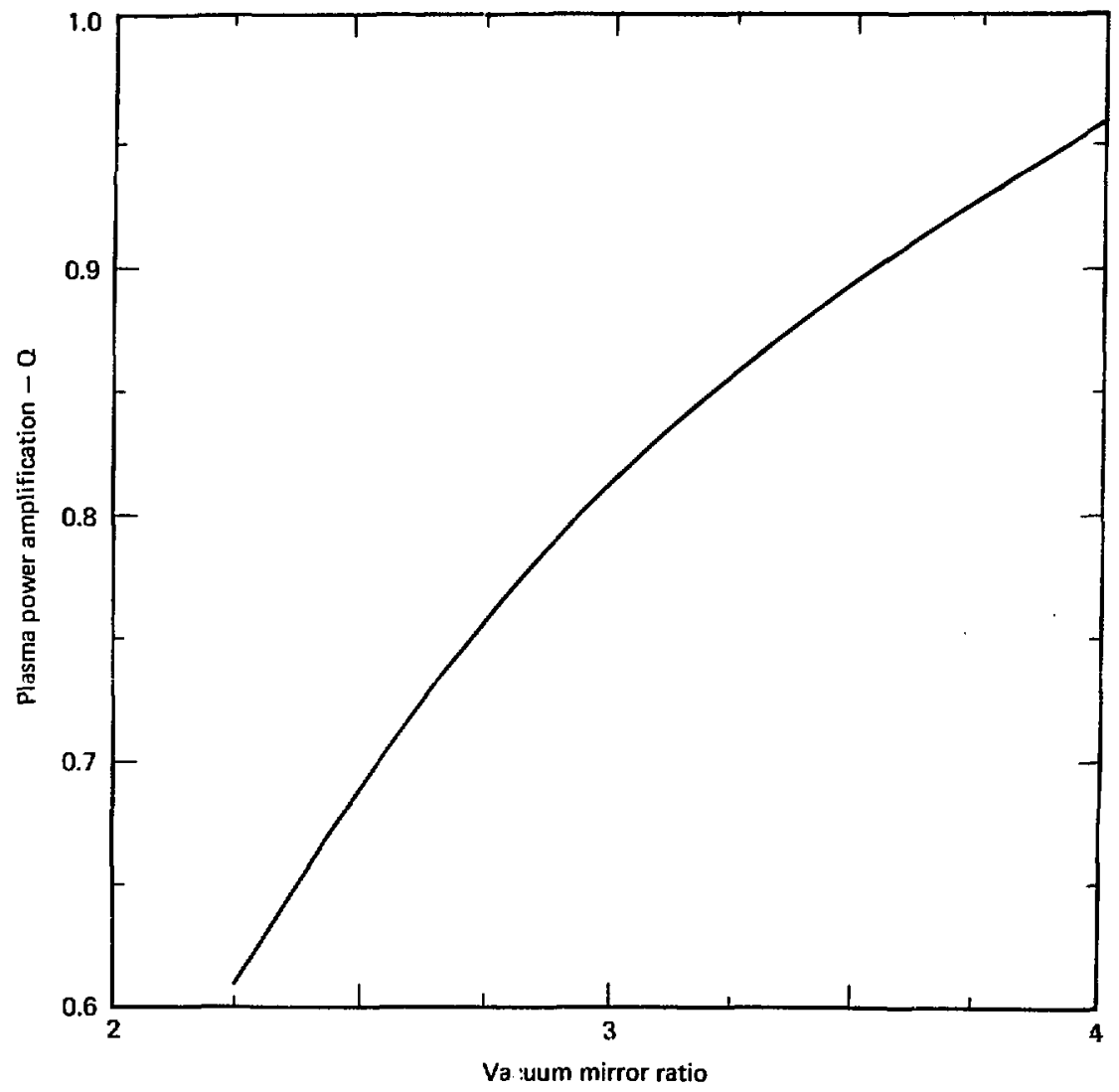

Fig. 4-9. Plasma $Q$ as a function of mirror ratio at a $D^{+}$extraction energy of $100 \mathrm{kev}$. 
We note, in Fig. 4-8, that the reference design choices of $R_{\text {VAC }}=2.25$ and $w_{D}=125 \mathrm{keV}$ are somewhat off optimum in the variable $w_{D^{*}}$ Our initial design choice of $W_{D}=125 \mathrm{keV}$ is about 68 above the minimum fissile cost at $W_{D}=105 \mathrm{keV}$. The optimization on $R_{V_{A C}}$ and $W_{D}$ is sumarized in Fig. 4-10.

\subsubsection{Plasma $Q$}

We also performed the exercise, with the system model, of arbitrarily increasing the plasma $Q$ to determine the economic incentives for higher $Q$ in a hybrid reactor. The result of this parameter variation is shown in 4 ig. 4-11, where we plot both fissile cost from the hybrid and power cost from the fusion-fission system. The effect of increasing $Q$ is (1), to reduce the recirculating power fraction (Fig. 4-12), thus increasing plant revenues from electricity sales and reducing the cost of producing plutonium, and (2), to realize some savings in plant capital costs because of a reduced injection current (smaller injector system and vacuum pumping capacity). Expressed another way, we reduce the $\$ / k W e$ (net) of the plant. We see that at high $Q$ $(Q>5)$ the fissile production costs are reduced from $\$ 110 / 9$ (reference design) to $\sim \$ 30 / g$. However, above $Q \sim 2$, where the recirculating power fraction has been reduced to $\sim 0.25$, there is little incentive for further improvements in plasma performance. Our target condition appears to be $Q_{\mathrm{e}}=\eta_{\mathrm{i}} \mathrm{Q} \simeq 1$.

Note that increasing $Q$ to a value of 2 reduces the electricity cost by only about 208 , from $\sim 30 \mathrm{mills} / \mathrm{kW} \cdot \mathrm{h}$ to $\sim 25 \mathrm{mills} / \mathrm{kH} \cdot \mathrm{h}$, and for $Q>2$, there is an almost negligible impact on power cost. Also, even with a very high $Q$ hybrid $(Q>5)$, fissile production costs cannot be reduced to zero. This is because, based on our model of the hybrid, it is a more expensive plant than the LWR.

\subsubsection{High Technology Hybrid}

As was mentioned previously in this section, the design study restricted the technology to the use of NbTi superconductor and a positive ion injection system. The result was a rather low injector efficiency $\left(\eta_{i} \simeq 58 \%\right.$ ) and a 


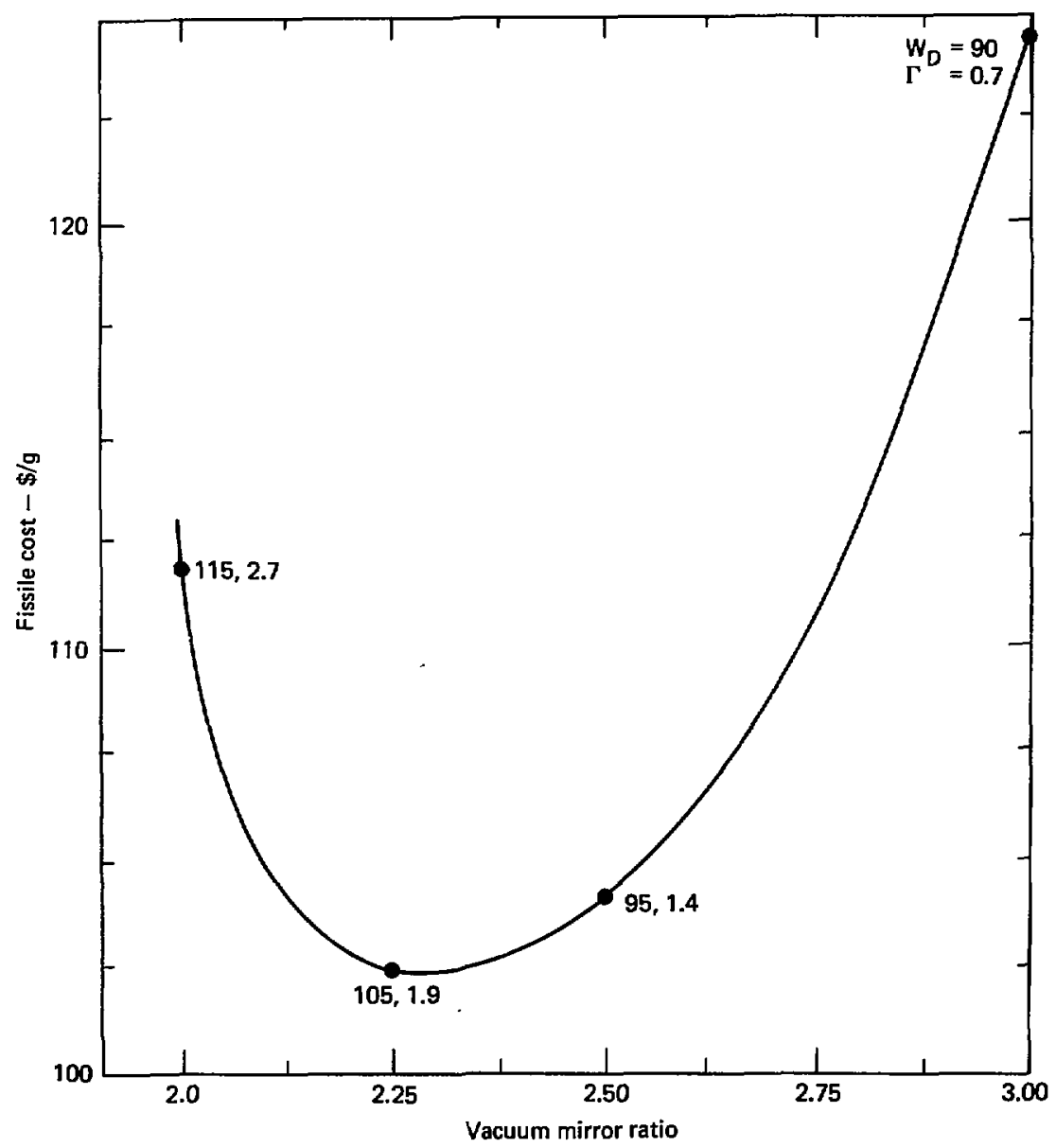

Fig. 4-10. Variation of fissile cost with mirror ratio at the optimum $\mathrm{D}^{+}$ extraction energy $W_{D}(\mathrm{keV}) . \Gamma$ is the first-wall load in w/ $/ \mathrm{m}^{2}$. 


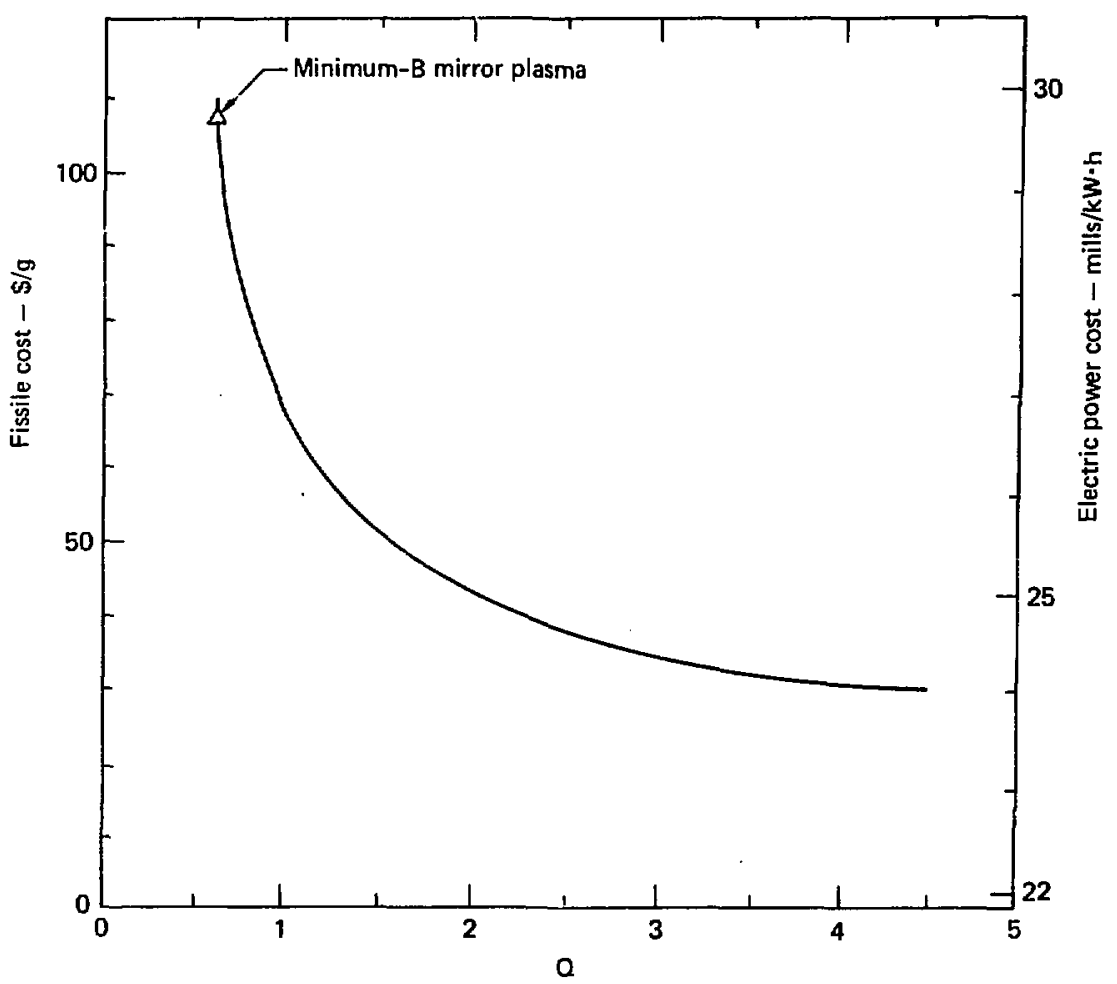

Fig. 4-11. Variation of fissile cost with an (arbitrary) increase in plasma $Q . W_{D}=100 \mathrm{kev}, R_{V_{A C}}=2.25$. 


$$
1
$$


comparatively $l 0 w Q(Q=0.64)$. Here, we examine the effect on plant economics of lifting the two technology restrictions.

Considering first the magnet, we indicated previously that with the $N \cap T_{i}$ superconductor we were able to achieve an optimum first wall loading, which depends on the central field $B_{0}$, but higher plasma $Q$ was attainable only if we could increase $R_{\text {vac }}$ Thus, in our higher technology mirror hybrid, we hold $B_{0}$ and $W_{D}$ constant at $2.52 \mathrm{~T}$ and $125 \mathrm{keV}$ (respectively), resulting in a constant first wall load of $1.7 \mathrm{MN} / \mathrm{m}^{2}$, and increase the mirror ratio by increasing the maximum conductor field, ${ }^{B}{ }_{c}$. The effect on fissile production cost is shown in the upper curve in Fig. 4-13. At a conductor field of 12T, the cost minimizes. At higher field, the increasing gains in $Q$ are offset by rapidly increasing magnet costs. At $B_{c}=12 T$ (RAc $\simeq 3.2$ ), we achieve $Q \simeq 0.85$. This result is most encouraging, in that the present goal of the $\mathrm{Nb}_{3} \mathrm{Sn}$ magnet development program is $\mathrm{B}_{\mathrm{c}}=12 \mathrm{~T}$.

In Fig. 4-13, we also show the effect of increasing both $B_{C}$ (in the manner described above) ar 7 increasing the injector efficiency to $n_{i}=0.75$, which may possibly be achieved with a negative-ion injector system.? In this case, we are able to reduce the fissile production cost to $\sim \$ 75 / 9$, about a 30 reduction from the reference design. 


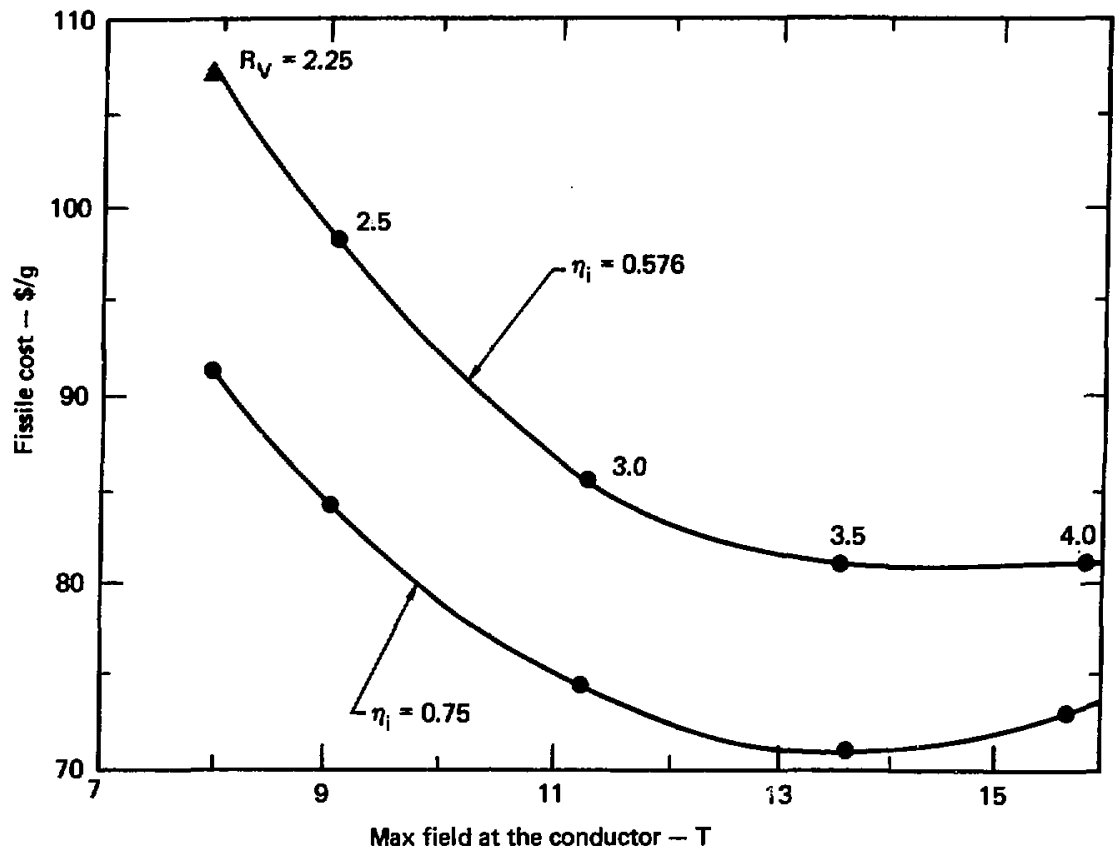

Fig. 4-13. Reduction of fissile cost with a high technology mirror hybrid. $\omega_{D}=100 \mathrm{keV} ; \Gamma=1.7 \mathrm{M} / \mathrm{m}^{2}$ (first-wall loading). 


\section{References}

1. D. J. Bender and G. A. Carlson, System Model for Analysis of the Mirror Fusion-Fission Reactor, Lawrence Livernore Laboratory, Rept. UCRL-52293 (1977).

2. M. A. Hoffman, Electrostatic Direct Energy Converter Perfornance and Scaling Laws, Lawrence Livermore Laboratory, Rept. UCI?-17460 (1977).

3. Guide for Economic Evaluation of Nuclear Reactor P: nt Designs, Nus Corp.., Rockville, Md., Rept. Nus-531 (1969).

4. T. H. Pigford and K. P. Ang, "The Plutoniun Fuel Cyc] es," Health Physics 29. 451 (1975).

5. T. H. Pigford and J. Choi, "Econorics of Fuel Cycle Options in a PrR," TRNSSAO 27, 463 (1977).

6. D. J. Bender, Performance Paraneters for Fusion-Fission Power Systems, Lawrence Livermore Laboratory, Rept. UCRL-80589 (1978).

7. J. H. Fink, W. L. Barr and G. W. Hamilton, "Efficient Bisq-Power, BighEnergy Neutral Beans for the Reference Mirror Reactor," subuitted to Nuclear Fusion; also, Lawronce Livermore Laboratory, Rept. UCRE-79205 (1977). 


\section{PLASMA PHYSICS}

\subsection{Introduction}

In the last several years, studies have been underway at Lawrence Iivermore Laboratory of possible fusion and fusion-fission reactors based on the minimum-B mirror. The fusion studies have recently been concluded with a report covering the details of the reactor. ${ }^{1}$ In this paper we describe the methods used to compute the parameters for the plasma in the hybrid reactor. Included are plasma stability criteria, computation of $Q$, maximum $\beta$ and bulld-up of plasma density profiles, and discussion of several effects, as yet only partially studied, which may change the accessible range of plasma size and $\beta$.

Before proceeding to these topics, it is useful to introduce some basic relations. The total fusion power is given by

$$
P_{F}=\frac{E_{F}}{4} \int n^{2}\langle\sigma v\rangle_{F} d V,
$$

where $E_{F}$ is the total energy released by the fusion reaction conly D-T is considered here so $\left.E_{F}=17.6 \mathrm{MeV}\right), \mathrm{n} / 2$ is the number density of deuterium and tritium, assumed equal throughout the volume, and $\langle\sigma v\rangle_{F}$ is the fusion reaction rate, dependent chiefly on the mean energies of the ions. For regions of the plasma free from if fluctuations and stabilizing warm plasma, the ion energy will be constant and nearly equal to the neutral-beam energy. $\langle\sigma v\rangle_{F}$ can then be removed from the j.ntegral. As a further approximation, typically well satisfied, we assume that the density can be witten as the product of two factors, ${ }^{2}$

$$
n(\underline{x})=n_{0}\left[1-\left(r_{0} / r_{p}\right)^{j}\right] f(p)
$$

The first factor is the density profile at the center of the reactor, assured to be cylindrically symetric there and dependent on the parameter $j$. $f(\rho)$ is a factor giving the distribution of density along the flux burface passing through radius $r_{0}$ at the midplane, and is taken as depending only on $\rho$ $B / B_{0}$, the ratio of local $|B|$ to the minimum value on the flux surface. 
In general, the minimum $B_{0}$ does not occur exactly at the midplane, following instead the seam of a baseball, intersecting the midplane only at certain symetry planes. This small difference in location will be neglected.

The volume element can be related to the midplane area $\mathrm{dA}_{0}$ by conservation of $f l u x d V=d x_{0} d s / \rho$, where $s$ is the distance along a field line. The total fusion power is then found to be

$$
P_{F}=\pi r_{p}^{2} L n_{0}^{2}\langle O V\rangle_{F} E_{F} \frac{j^{2}}{2(j+1)(j+2)} c^{(2)}(R)
$$

where $L$ is the distance from midplane to mirror, $R$ is the mirror ratio, $B_{m} / B_{0}$, and

$$
C^{(2)}(R)=\int_{0}^{1} \rho^{-1} f^{2}(\rho) d(s / L)
$$

$c^{(2)}$ is a weak function of mirror ratio; its value is computed with the aid of a Fokker-Planck code described later. For the large reactors considered in this work, computations with a radial code discussed in a later section show $j$ to be about 20 .

The beam power input to the plasma can be computed from the loss rate of ions since $I_{I N}=I_{\text {OUT' }}$ and $P_{I N}=E_{I N} I_{I N}$. This is the only power input for the reactor designs considered. The loss rate, expressed as a current, is

$$
I_{\text {ours }}=e \int \frac{n}{\tau} d v=e \int \frac{n^{2}}{n \tau} d v,
$$

where $\tau$ is the mean confinement time for ions. The product $n \tau$ depends most strongly on the mean ion energy $\bar{E}_{i}$, the angle $\theta$ of neutral beam injection and effective mirror ratio. These are nearly constant across the plasma and we obtain

$$
P_{I N}=\frac{2 \pi r_{p}^{2} L n_{0}^{2} E_{I N} j^{2} c^{2}(R)}{\langle n \tau\rangle(j+1)(j+2)}
$$


The analysis leading to $\mathrm{Eq}$. (6) has neglected differences in 〈nT> and $\mathrm{E}_{\mathrm{IN}}$ for deuteriun and tritium. We can define averages of these quantities and obtain the same Eq. (6)

$$
\begin{aligned}
& E_{I N}=\frac{I_{D} E_{D}+I_{T} E_{T}}{I_{D}+I_{T}}=\frac{E_{D}\langle n \tau\rangle_{T}+E_{T}\langle n \tau\rangle_{D}}{\langle n \tau\rangle_{D}+\langle n \tau\rangle_{T}} \\
& \frac{4}{\langle n \tau\rangle}=\frac{1}{\langle n \tau\rangle_{D}}+\frac{1}{\langle n \tau\rangle_{T}} .
\end{aligned}
$$

Note that the density in the expression for component <nt>'s is the actual species density $(=n / 2)$.

A more convenient quantity is the power amplification factor, defined as,

$$
Q \equiv \frac{P_{\text {OUT }}}{P_{\text {IN }}}=\frac{\langle n \tau\rangle}{4 E_{I N}} \frac{\langle\sigma v\rangle_{F} E_{F}}{E_{\text {IN }}} .
$$

If the principal loss mechanism is charged-partıcle collisions which diffuse particles into the "loss-cone," then a falrly accurate scaling law is ${ }^{3}$

$$
\left\langle n t>=c_{1} \sqrt{M_{i}} \bar{E}_{i}^{3 / 2} \log _{10} R\right.
$$

Results from codes which solve the Fokker-planck equations for alectrons and ions give values of $C_{1}$ of about $2 \times 10^{10}$ for $M_{1}$ in $A y, \bar{B}_{1}$ in $k e v$, and <nt> in $\mathrm{cm}^{-3}-8$. To relate the nirror ratio $\mathrm{R}$ to the vacuum mirror ratio $R_{V} \equiv B_{m} / B_{0 V}$, we use pressure balance perpendicular to the field lines

$$
\nabla_{\perp}\left(p_{\perp}+\frac{B^{2}}{2 \mu_{0}}\right)=-\underset{\sim}{K} \frac{B^{2}}{\mu_{0}}+p_{\perp}-p_{\eta} .
$$

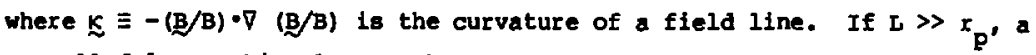
so-called long, thin plasma, the curvature may be neglected and we obtain,

$$
p_{\perp}=\frac{B_{v}^{2}\left(r_{p}\right)-B^{2}}{2 \mu_{0}} \quad \text { (LTA). }
$$


We define now a plasma $\beta$ by

$$
B_{1} \equiv \frac{p_{1}}{B_{v}^{2} / 2 \mu_{0}} \text {. }
$$

With the aid of Eg. (12) and neglecting the small ( $\approx 58)$ radial variation of $B_{v}$ we find the following useful relations (long-thin approximation only) :

$$
\begin{array}{ll}
B=\left(1-\beta_{\perp}\right)^{1 / 2} B_{v} & \text { (ITA) } \\
R=R_{V} /\left(1-\beta_{\perp}\right)^{1 / 2} & \text { (ITA) }
\end{array}
$$

Combining Eqs. (9), (10), and (15) and noting that $\overline{\mathrm{E}}_{i} \approx \mathrm{E}_{I N}$, we obtain

$$
Q \propto\langle\sigma v\rangle_{F} E_{I N}^{1 / 2}\left[\log _{10} R_{v}-\frac{1}{2} \log _{10}\left(1-\beta_{\perp}\right)\right] \text {. }
$$

$\langle\sigma v\rangle_{F}$ has a broad maximum near $\overline{\mathrm{E}}_{\mathrm{i}} \approx 80 \mathrm{kev}$, beyond which $\langle\sigma v\rangle_{\mathrm{F}} \approx \overline{\mathrm{E}}_{i}{ }^{-1 / 2}$. Thus beyond this energy, $Q$ is chiefly governed by $R_{v}$ and $\beta_{\perp}$. The total power can be seen from Eq. (3) to follow the scaling

$$
P_{F} \propto \frac{\langle\sigma v\rangle_{F} \beta_{1}^{2} B_{0 V}^{4}}{E_{I N}^{2}}
$$

From Eqs. (16) and (17), we see that for a fusion reactor, where the cost of the power is very sensitive to $Q$ for $Q$ near unity, ${ }^{1}$ we need a bean energy of at least $100 \mathrm{kev}$ and large $R_{v}$ and $\beta_{\perp}$. But the field at the superconductor is limited, thus restricting $B_{m}$ and we dare not make $R_{v}$ too high, thereby reducing $B_{0 V}$, because of its drastic effect on $P_{F}$. For neutral-beam injection at $90^{\circ}$ to the field lines, $\beta_{1, \max } \approx 0.4$, as determined by stability considerations. For smaller angles $\beta_{\perp \text {, max }}$ may be increased to $\approx 0.8$, however at the expense of a small reduction in $\langle n \tau\rangle$. 4

For a hybrid reactor the economics is sot so sensitive to $Q$ because of the high blanket multiplication. We can thus tolerate somewhat lower $R_{\mathbf{V}}$. Requirements on the magnet design, e.g. choice of superconductor, are therefore not so severe. 
In the following sections, a more detailed discussion will be given of the methods used to predict or choose the parameters $E_{I N^{\prime}} I_{I N}, r_{p^{\prime}} I^{\prime} \beta_{L^{\prime}}$ $R_{v^{\prime}}$ and $B_{0 v}$. In the first section we describe restrictions on the maximum plasma pressure and on the vacuum magnetic field imposed by requirements for magnetohydrodynamic stability. Sanples of ion drift surfaces in the vacuum magnetic field are given in the second section. Characteristics of the equilibrium magnetic field for model $p_{\|}$(B) and radial density profile are given in next section. The fourth section includes methods by which the 2 value and axial density profiles are deduced from solutions to the Fokker-Planck equation, while radial density profiles are covered in the following section. This part concludes with a discussion of some effects, as yet only partly understood, which could require changes in the possible parameters of a mirror reactor.

\subsection{Magnetohydrodynamic Stability}

The conditions for MHD stability of a mirror plasma have been obtained by Hall. 5,6 Equilibriun-destroying instabilities have been classified as due to the firehose mode, the mirror mode, interchanges, ballooning, and kink modes. Simple but general criteria for avoiding these instabilities have been worked out only for the first two cases

$$
\begin{aligned}
& \text { B }-\mu_{0} \frac{\partial p_{1}}{\partial B}>0 \\
& B+\mu_{0} \frac{\partial p_{1}}{\partial B}>0 .
\end{aligned}
$$

In these equations, $B \equiv|\mathrm{B}|$ and $\mathrm{p}_{\|}$and $\mathrm{p}_{\perp}$ are the local pressures parallel and perpendicular to the magnetic-field. Neither of these conditions iequire krowledge of the spatial dependence of $\underset{\sim}{\mathrm{P}}$ or $\underset{\sim}{\mathrm{B}}$ and are, in effect, the conditions for the thresholds of these instabilities in an infinite, homogeneous, anisotropic plasma. They are also the conditions that the equilibrium problen for the plasma and magnetic field, treated as a fluid, be well posed. ${ }^{7}$ It is probable that equilibrium does not even exist if these conditions are not satisfied. 
The functions $p_{\perp}$ (B), $p_{\|}$(B) needed to evaluate conditions in Eqs. (18) and (19) can be found from the solutions to the Fokker-Planck equation, described later. It is found that condition (18) is generally always satisfied in a mirror-confined plasma. Condition (19) in effect limits the $\beta_{\perp}$ value which can be obtained in the plasma.

Several different conditions sufficient for stability against interchanges can be obtained depending on the functional dependence of $p_{\|}$ and $p_{\perp}$. One model for the pressures, applicable to mirror devices, takes the form

$$
p_{H}=\omega(\psi) \hat{p}_{\|}(B, \psi),
$$

where $\psi$ is a magnetic flux coordinate.

For pressure distributions of the form of $\mathrm{Eq} .(20)$, a condition sufficient for interchange stability is ${ }^{6}$

$$
\frac{\partial \bar{\Gamma}}{\partial \omega}+\frac{\partial \bar{\Gamma}}{\partial B} \frac{\partial B}{\partial \omega} \geq 0
$$

where

$$
\bar{\Gamma}=\oint_{\alpha, \beta} \hat{p}_{\|}(B, \psi) d \ell / \varepsilon
$$

is evaluated along field lines passing through the midplane, as indicated here by the $\alpha, \beta$ coordinates of a field line. 6

To evaluate the interchange criterion [inequality Eq. (21)], the equilibrium magnetic field must be known. Two codes for computation of three-dimensional mirror equilibria have recently been developed: MC GUS, by Boyd, Hall, and McNamara ${ }^{8}$ and VEPEC, by Anderson, Breazeal and Sharp. 9 We use the former code for the present problem. Results for a particular magnet set are described in a later section. In connection with interchange stability we can refer to Fig. 5-1 which shows contours of $\bar{\Gamma}$ in the midplane. While $\omega$ decreases with radius, $\vec{\Gamma}$ is monotonically increasing out to a radius of about $3 \mathrm{~m}$. We thus expect that the plasma will be interchange-stable if we do not attempt to fill the magnetic well beyond this radius. The filling is easily controlled with the bean injection current. For a given volume loss rate, which aepends chiefly on the injection energy, a higher current will in general cause the plasa to expand to a larger radius. Thus, for the vacuum field considered here, the 


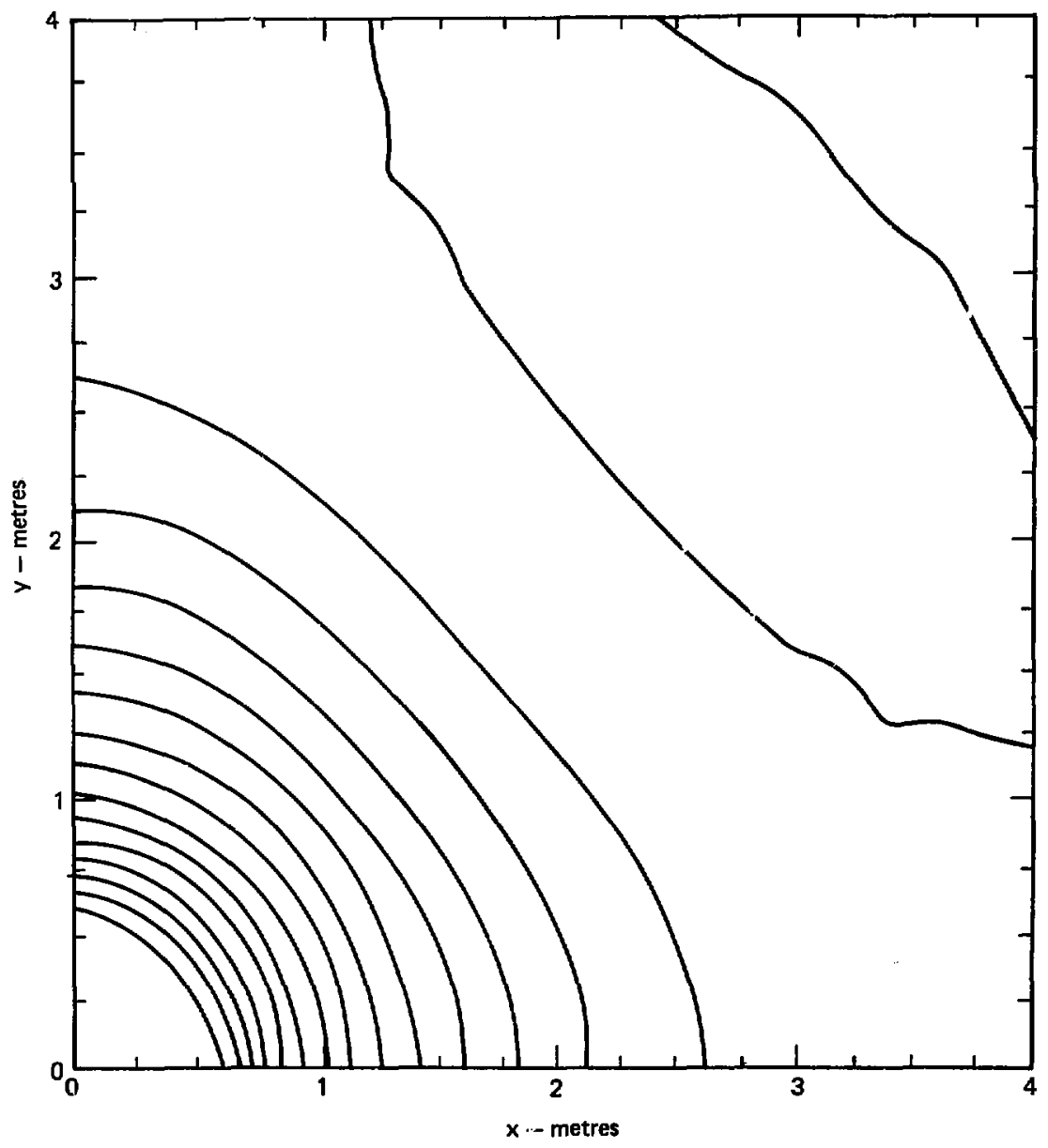

Pig. 5-1. Contours of $\bar{\Gamma}$ in the midplane for the equilibriun field, 
interchange stability limits the plasma radius rather than the plasma $\beta$. For other vacuum fields in which the curvature of the vacuum field lines is not pronounced, there may be sufficient reversal of total curvature by the plasma field to limit the plasma $\beta_{\perp}$ to a value less than that found from the mirror-mode criterion. Sich a result indicates that insufficient curvature was designed into the vacuum field, particularly in the region of high $p_{\|}$

Equilibrium fields are time consuming to compute and, in present codes, allow only a restricted set of $\hat{p}_{\|}(B), \hat{b}_{\perp}(B)$ and $\omega(\psi)$ functions. It is desirable to have a rough criterion for interchange stability, involving only the vacuum field, and which can be used by a field designer to judge the suitability of the field coils. For this purpose we use the low $B$, isotropic pressure criterion of Rosenbluth and Longmire, 10

$$
\frac{d}{d r_{0}} \oint \frac{a l}{B} \leq 0 \text {. }
$$

This inequality is trivially satisfied if all field lines on which the plasma is located curve away from the plasma. While it is possible to design magnets for such fields, it is at the expense of access for neutralbeam liras, etc., and the weaker condition of inequality Eq. (21) is preferred. Comparison of the two criteria indicates that inequality Eq. (23) should be conservative if regions of "bad" field-line curvature are located where plasma density is low. For the present magnet design, the two criteria agree that the plama should be interchange-stable.

The other possible instabilities, ballooning and kinks, have only recently come under close scrutiny in mirror devices. ${ }^{1,12}$ Ballooning is thought to be the most serious problem at high $\beta$ if the plasma is otherwise interchange-stable. However, B limitations due to ballooning can usually be overcome in mirror devices by adjustment of the vacuun fields to improve the curvature where the ballooning is predicted to occur. As with interchanges, sufficiency criterla for ballooning stability require knowledge of the equilibrium field. To date, the ballooning criteria have not been coupled to the equilibrium codes, so we have not been able to examine this problem for the reactor plasmas and coils. 


\subsection{Drift Surfaces}

It is evident that the magnetic field must be designed so as to allow closed drift surfaces within the plasma. An additional, more stringent requirement, is that particles on a given line of force drift on surfaces that are not widely separated. Should they be widely separated, a collision can cause the ion to shift outward (or imward) to a new drift surface causing a net diffusion. In the limiting case, in which all such particles drift on the same surface, the surfaces have beun termed omnigenous. 6,8 It is evident in this latter case that the drift surfaces in the midplane may be denoted by a single flux surface coordinate $\psi$. Hall and McNamara have shown ${ }^{6}$ that the pressures and flux surface dependence are separable [see Eq. (20)] in the case of omnigenous drift gurfaces.

Near the central axis of mirror-quadrupole fields, the quadrupole effects are weak, so the drift surfaces in the midplane are circular. As one approaches the plasma radius, quadrupole fields become more lisportant and the midplane drift surfaces flatten and become more rectangular. At large enough radil, the drift surfaces are open and particles found on these surfaces are probably lost from the plasma.

To study the drift surfaces for the present coils, we have used a code which solves for the trajectories of the guiding centers. ${ }^{13}$ A more accurate particle code is not necessary because the magnetic moment

$$
\mu \equiv \frac{\pi v_{1}^{2}}{2 B}
$$

is well-conserved away from the origin in this field. Conservation of the longitudinal adiabatic invariant

$$
J \equiv \oint_{\alpha, \beta} v_{\|} d s
$$

is not assuned in this code. We study the trajectories in the vacuum field only; the drift surfaces preserve their general shape in the equilibriun field. 
Since the field is four-fold symmetric, we need only follow ions through total azimuthal angle of $90^{\circ}$. It is convenient to start ions in a plane midway between the (real or equivalent) Ioffe bars, because the drift surface is locally circular there. Deuterons of total energy $\mathrm{E}=100 \mathrm{keV}$ were started at the minimum $|\mathrm{B}|$ on field lines that pass through a given radius at $z=0$. It is easily seen that ions with starting parallel energy $\mathbf{E}_{\|}$will turn at a bilrror ratio

$$
R_{t}=\frac{E}{E-E_{\Downarrow}}
$$

Since the total vacuum mirror ratio is $\approx 2.25$, thus containing ions with up to $\mathrm{E}_{\|} \approx 55.6 \mathrm{kev}$, ions of starting energy $\mathrm{E}_{\|}=5$ to $50 \mathrm{keV}$ were studied. Lower $E_{\|}$, corresponding to $J \approx 0$, results in drift surfaces which intersect the midplane only over a limited azimuthạl angle (the minimum-|B| line corresponds to a baseball seam).

Results of the calculations for ions started at $r=1$ and $2.5 \mathrm{~m}$ are shown in l'ig. 5-2. We note the near-circular drift surfaces (at $z=0$ ) of all ions for the smaller radius. At the larger radius, the effect of the quadrupole field is quite pronounced. The drift surface fur ions with $E_{\|}=$ $50 \mathrm{keV}$ deviate by about $9 \mathrm{a}_{i}$ from a circular path at $45^{\circ}$. The ions started with $\mathrm{E}_{\|}=5 \mathrm{keV}$ follow drift surfaces which dip further by $15 \mathrm{a}_{i}$ towards the axis. At even larger radii scme of the drift surfaces (not shown do not enclose the axis.

These results confirm that the drift surfaces are closed out to the expected plasma radius. The deviation of drift surfaces for different classes of ions by $\approx 6 a_{i}$ is undesirable, but should not cause additional losses since the plasma density drops to zero near this radius. It appears satisfactory to replace those drift surfaces by an average drift surface as is necessary in solving for the equilibrium fields.

\subsection{Equilibrium Magnetic Fieks}

In the section on wH stability we have seen that it is necessary to know the equilibrium magnetiz field to evaluate stability against interchanges. We have obtained the equilibrium field for the Yin-Yang coil set for the hybrid reactor with the mc Gus code dereloped by Boyd, Hall, and WcNanara. Details of this code have not yet been published, although 


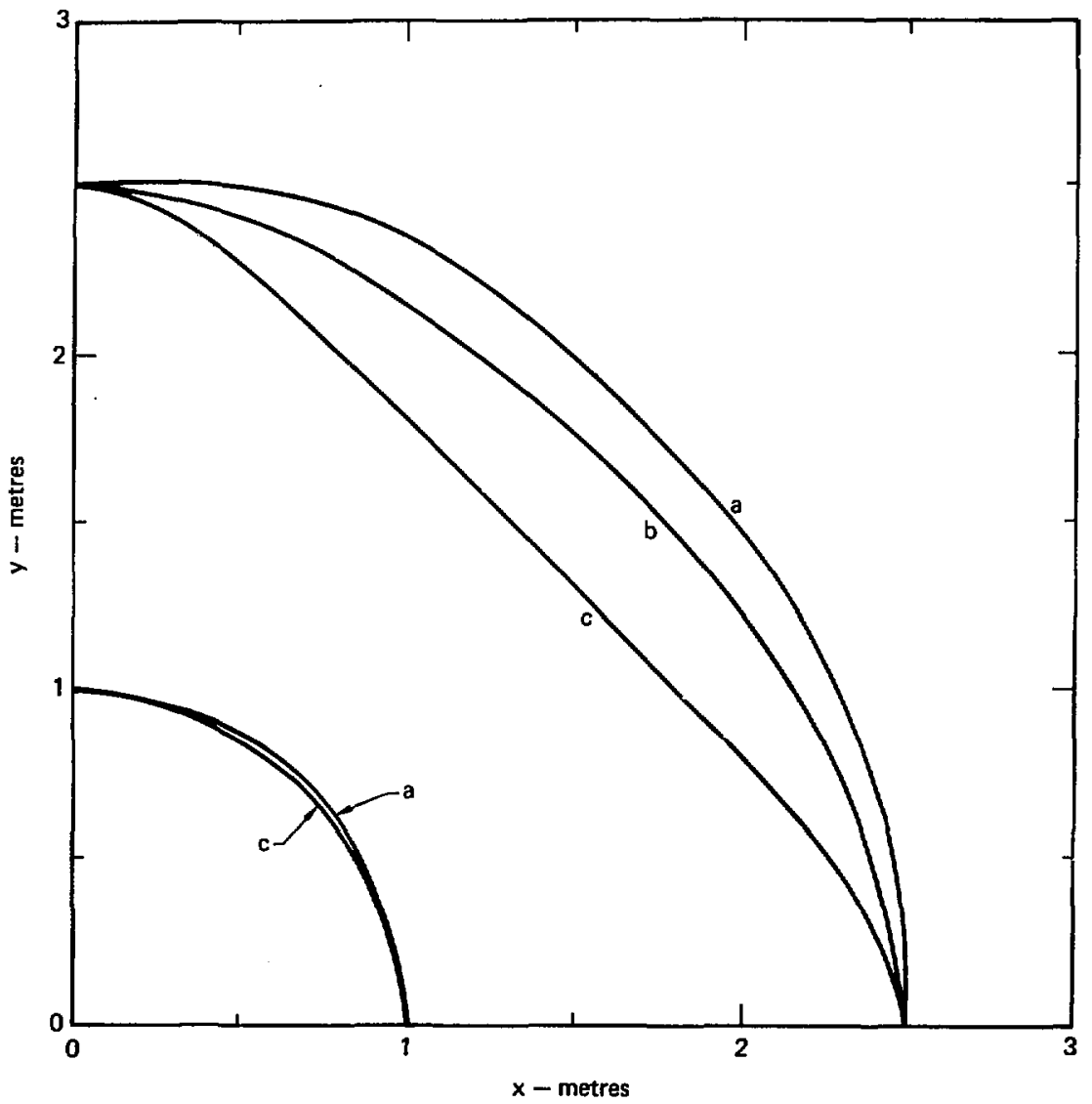

3ig. 5-2. Drift surfaces in the vacuun field of the hybrid Yin-Yang magnet compared to cylindrical surface (a); b: parallel energy, $\mathrm{B}_{\|}=50 \mathrm{keV}$; $c: E_{\|}=5 \mathrm{keV}$. 
certain aspects are discussed by McNamara. 8 The basic equations governing mirror equilibria are the guiding-center fluid equations

$$
\begin{aligned}
& \nabla \cdot \underline{Z}=0, \\
& \nabla \times B=\mu_{0} j^{\prime} \\
& \nabla \cdot \underline{D}=j \times \underset{\sim}{\mathrm{B}} .
\end{aligned}
$$

with

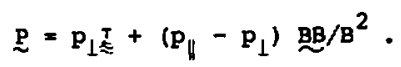

$p_{1}$ and $p_{\|}$are found from monents of the distribution function [see Eq. (12)]. Pressure balance parallel to the field lines imposes the requirement

$$
p_{\perp}--B^{2} \frac{\partial}{\partial B}\left(\frac{p_{b}}{B}\right) \text {. }
$$

The MC GUS code actually solves a different set of equations, 6,8 fully equivalent to the above.

Because of the symmetries of the quadrupole field produced by Ioffe bars, Yin-Yang and Baseball coils, it is only necessary to solve for the field in a single octant. The code can use up to $32^{3}$ grid points in that octant, but, because of the fanning of the magnetic field lines in the mirror region, only about 15 radial grid points are actually within the plasma at the mid-plane. The final plasma pressure is not known a priori, but must be obtained as part of the solution. Thus, solutions are obtained by increasing the plasma pressure in fixed increments of $\widetilde{B}=2 \mu_{0}\left(2 p_{1}+p_{\|}\right) /$ $B_{m}^{2}$. At some value of $\widetilde{\beta}$, the code will fail to meet the specified error criterion. This failure can be associated with violation of the nirror mode or interchange criteria, substantial local reversal of originally favorable curvature or large gradients within the plasma which cannot be acequately represented on the grid. It is possible to continue the solution to higher plasma pressure by relaxing the error criterion, but the solution becomes obviously nonphysical.

Under the assurption of omigeneity, discussed in the last chapter pressure may be written in the form already given,

$$
p_{\|}=\omega(\psi) \hat{p}_{\|}(B, \psi) \text {, }
$$


which is just the form needed for application of the criterion of Eq. (22) for interchange stability. The dependence of $\hat{p}_{\|}$on $\psi$ is unimportant and neglected in the present case. The functions $\omega$ and $\hat{p}_{k}$ should come from computations of the radial density profiles and of the distribution function. Unfortunately, it has not yet been possible to incorporate numerically computed $\omega$ and $\hat{p}_{\|}$functions into the $\mathrm{MC}$ GUS code; we have therefore used model functions for these computations. The $\hat{p}_{\|}$function is the "optimal" profile of Hall, Hooper, and Newcomb ${ }^{14}$ [their Eq. (3.14)] and is too lengthy to reproduce here. This functional form has the property of always satisfying the mirror mode criterion. The model $p_{\|}$(B) and $p_{\perp}$ (B) is compared with results from the distribution function in the next section. The $w$ function is taken as

$$
w=\left(1-x^{2} / a^{2}\right)^{2} \text {, }
$$

since the $x-z$ plane is a symmetry plane of the field.

Results for the equilibrium field for the $75^{\circ}$ sweep Yin-Yang coil described previously are collested in Figs. 5-1 and 5-3 through 5-12. Computations were carried out for plasma pressures up to $\widetilde{\beta}=0.13$ or $\beta_{\perp}$ $=0.77$. The equilibrium mirror ratio $R$ was found to be 3.7 compared to vacuum mirror ratio $R_{v}$ of 2.25 . The long-thin approximation of Eq. (15) yields

$$
B_{\perp, \text { LTX }}=1-\left(\frac{R_{v}}{R}\right)^{2}=0.63 .
$$

Thus, because of curvature effects the magnetic field is able to confine a higher plasma pressure than would be expected from the long-thin approximation. $A$ plot of $\beta_{\perp}$ and $\beta_{1, L T h}$ as found for increasing plasma pressure, or $R$, is given in Fig. 5-3.

Figures 5-4 and 5-5 show the contours of $B_{v}$ in the midplane $(2=0)$ and in the transverse $y=0$ plane, while the final $B\left(\beta_{\perp}=0.77\right.$ ) is show in the same views in Figs. 5-6 and 5-7. Comparison of the figures (the same contour values are used on each) show the marked depression of the magnetic well because of the plasma diamagnetiam. Note particularly the appearance of additional contours inside contour labeled 1 in Figs. 5-6 and 5-7. The field depression shows up more clearly in plots of $B_{v}$ and $B$ vs $x$ in

[text conilinues on p. 110] 


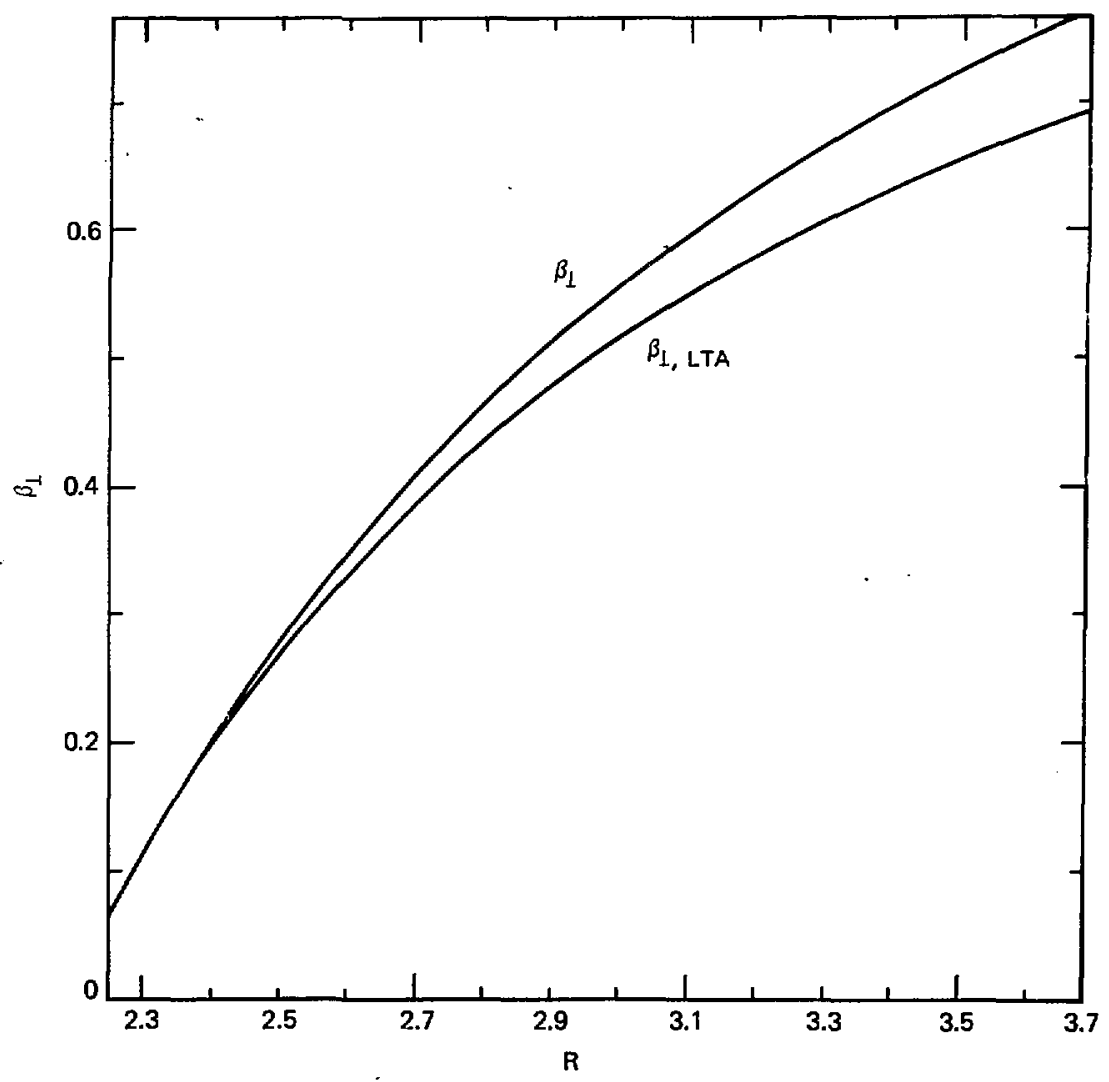

Fig. 5-3. $\beta_{1}$ and $\beta_{L^{\prime} L T A}=1-R_{V}^{2} / R^{2}$ plotted vs $R$ for
equilibriu field. 


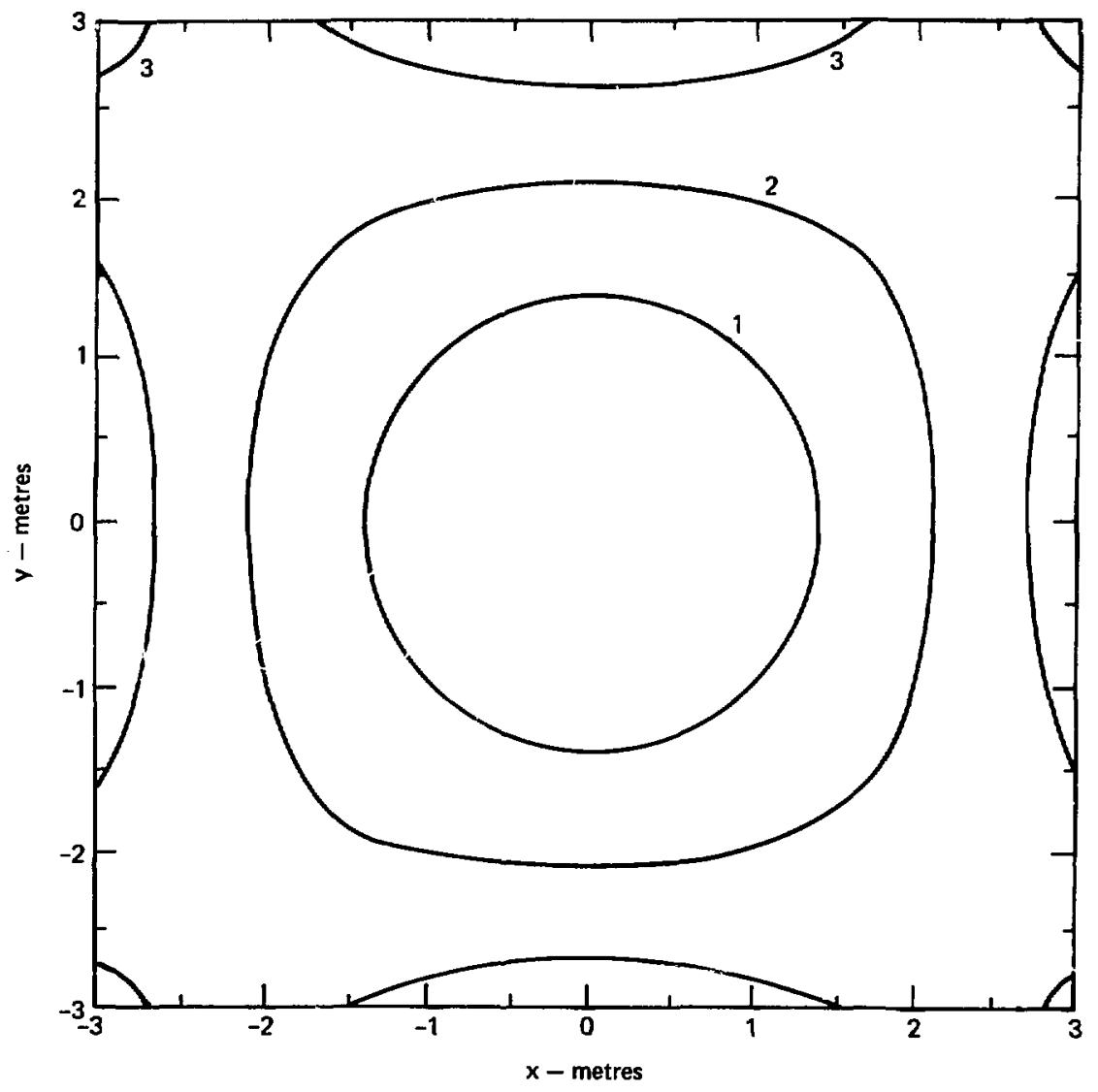

Fig. 5-4. Contours of $B\left(\beta_{\perp}=0\right)$ in $x-y$ plane at $z=0$. Contours labeled 1,2 and 3 have values of $2.52,2.66$ and $2.79 \mathrm{~T}$, respectively. 


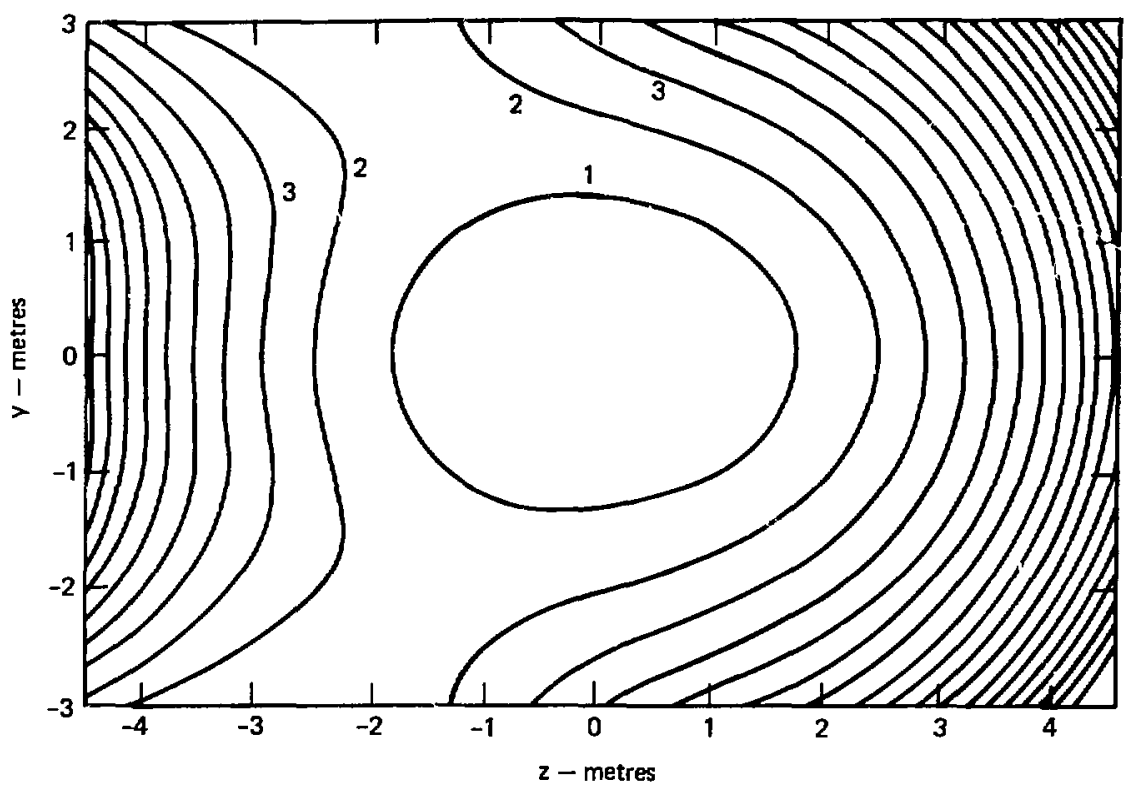

Fig. 5-5. Contours of $B\left(\beta_{1}=0\right)$ in $y-z$ plane $(x=0)$. Contours labeled 1,2 , and 3 have values of $2.52,2.66$, and $2.79 \mathrm{~T}$. 


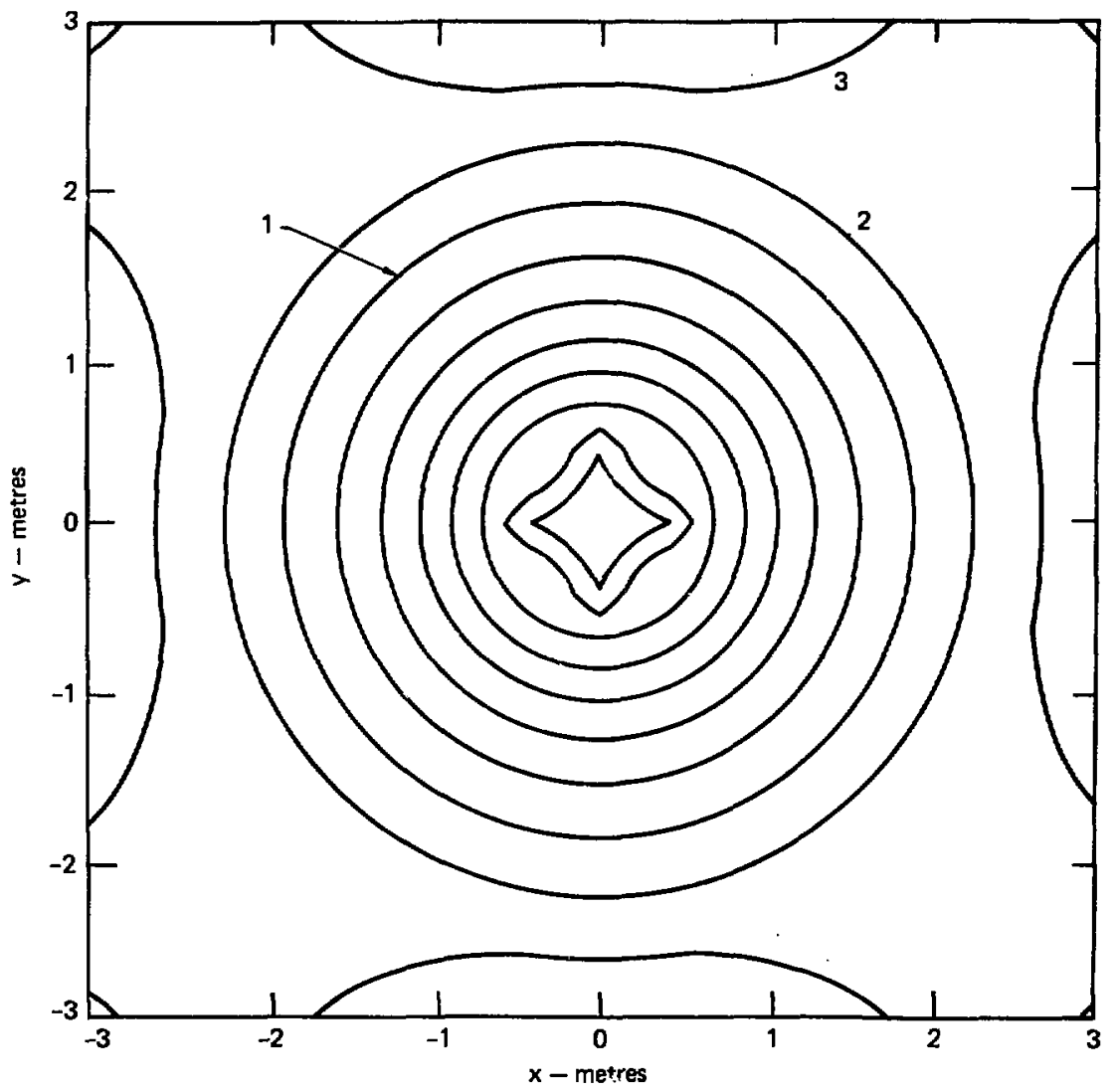

Fig. 5-6. Sane as Fig. 5-3, but $\beta_{\perp}=0.77$. 


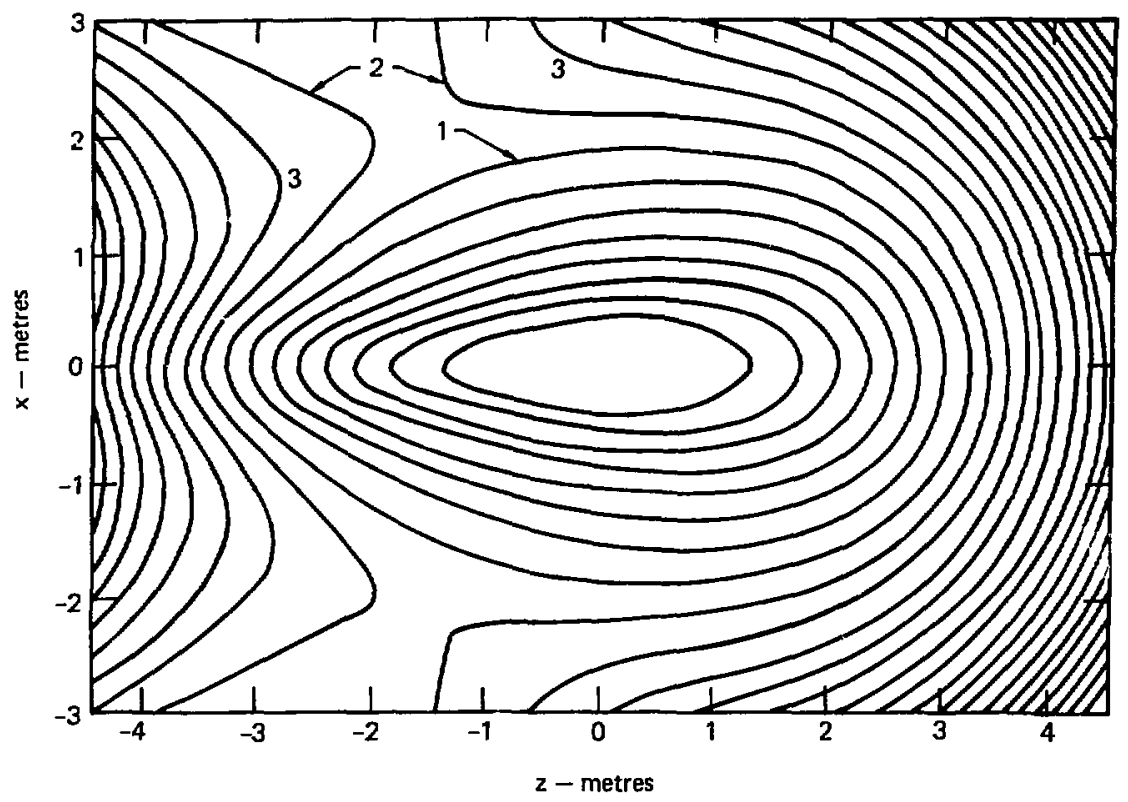

Fig. 5-7. Same as Fig. 5-4, but $\beta_{1}=0.77$. 


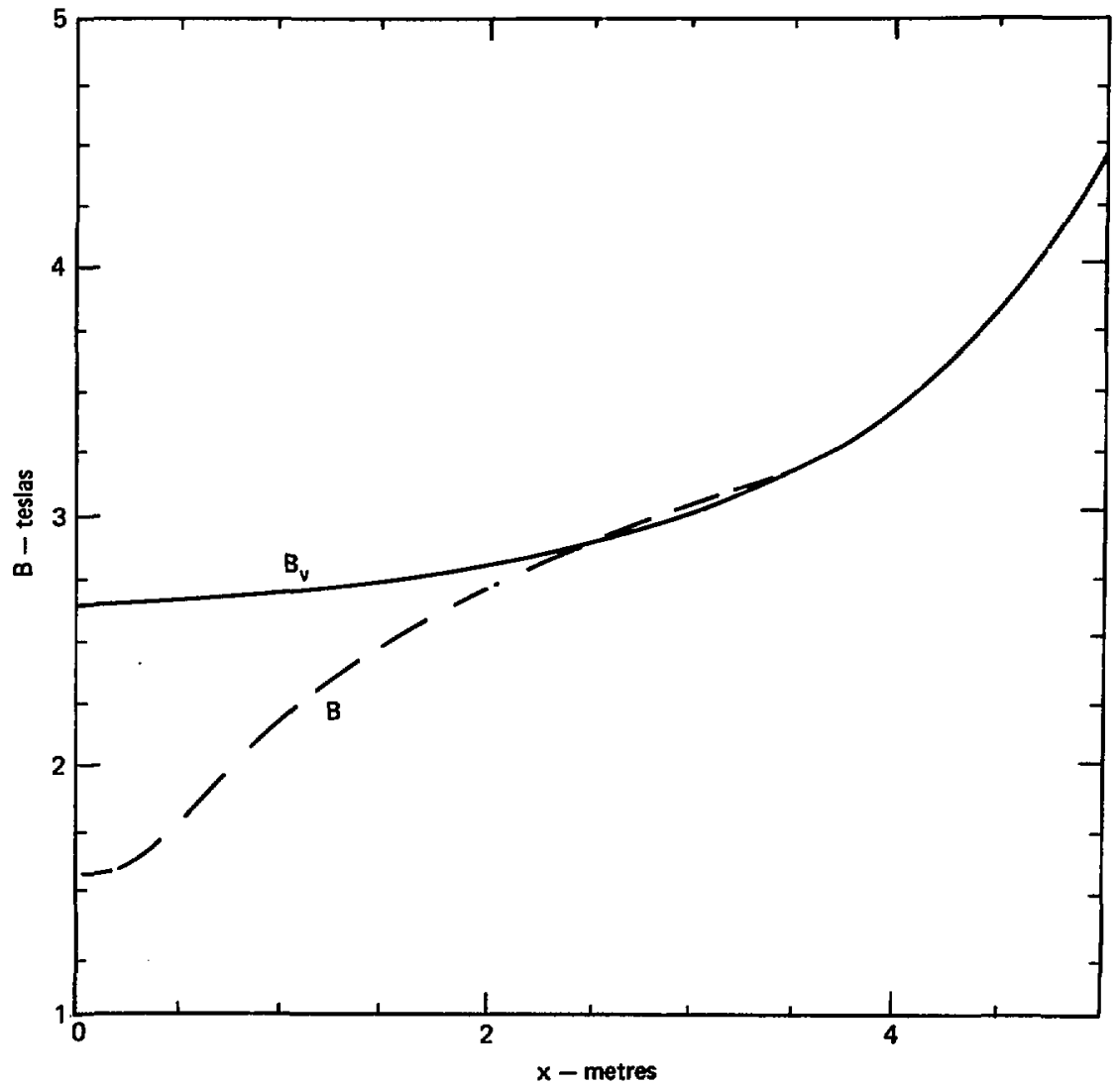

Fig. 5-8, $B_{y}$ and $B\left(B_{1}=0.77\right)$ vo $x$.

106 


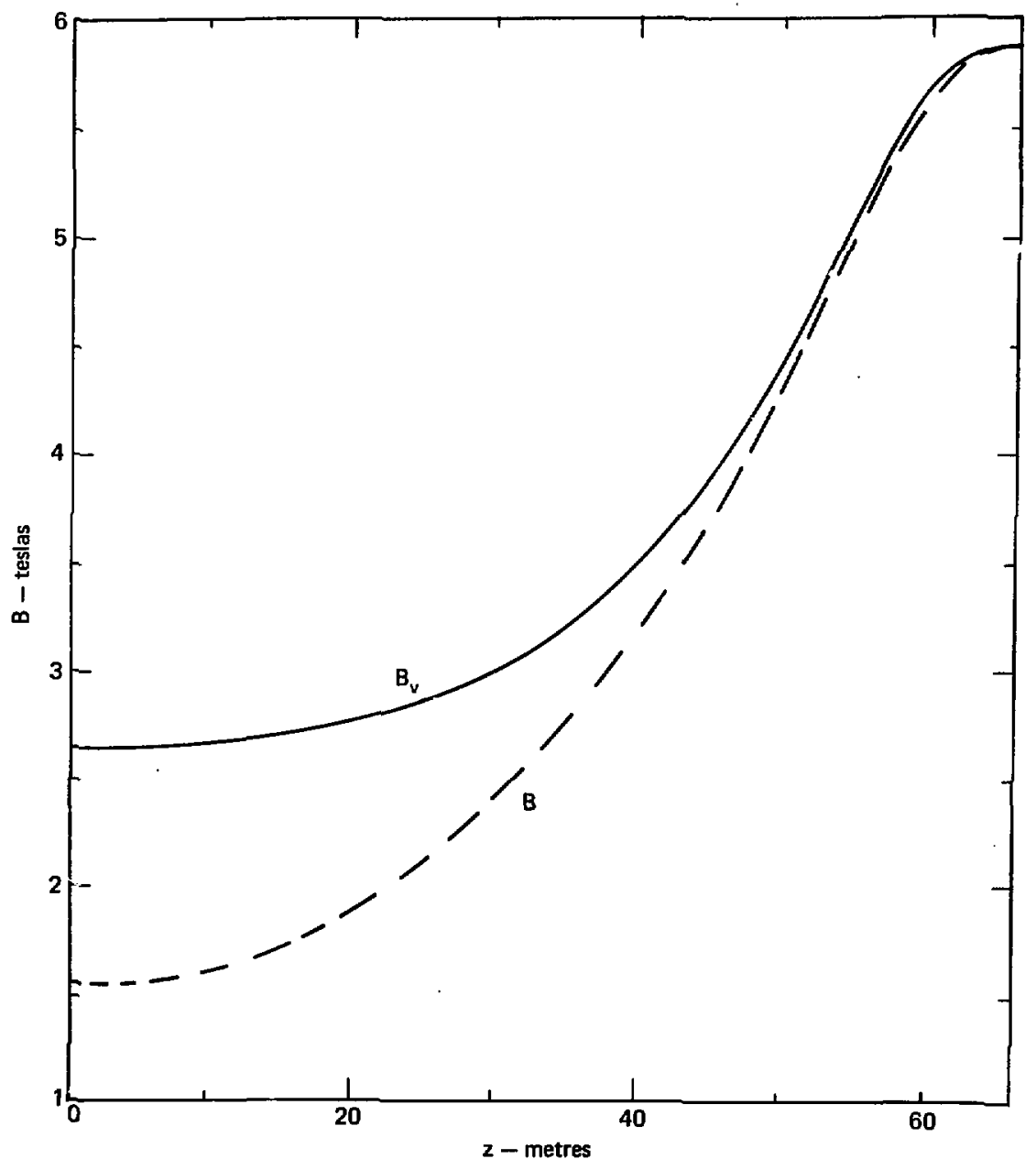

Fig. 5-9. $B_{v}$ and $B\left(\beta_{1}=0.77\right)$ v8 $z$. 


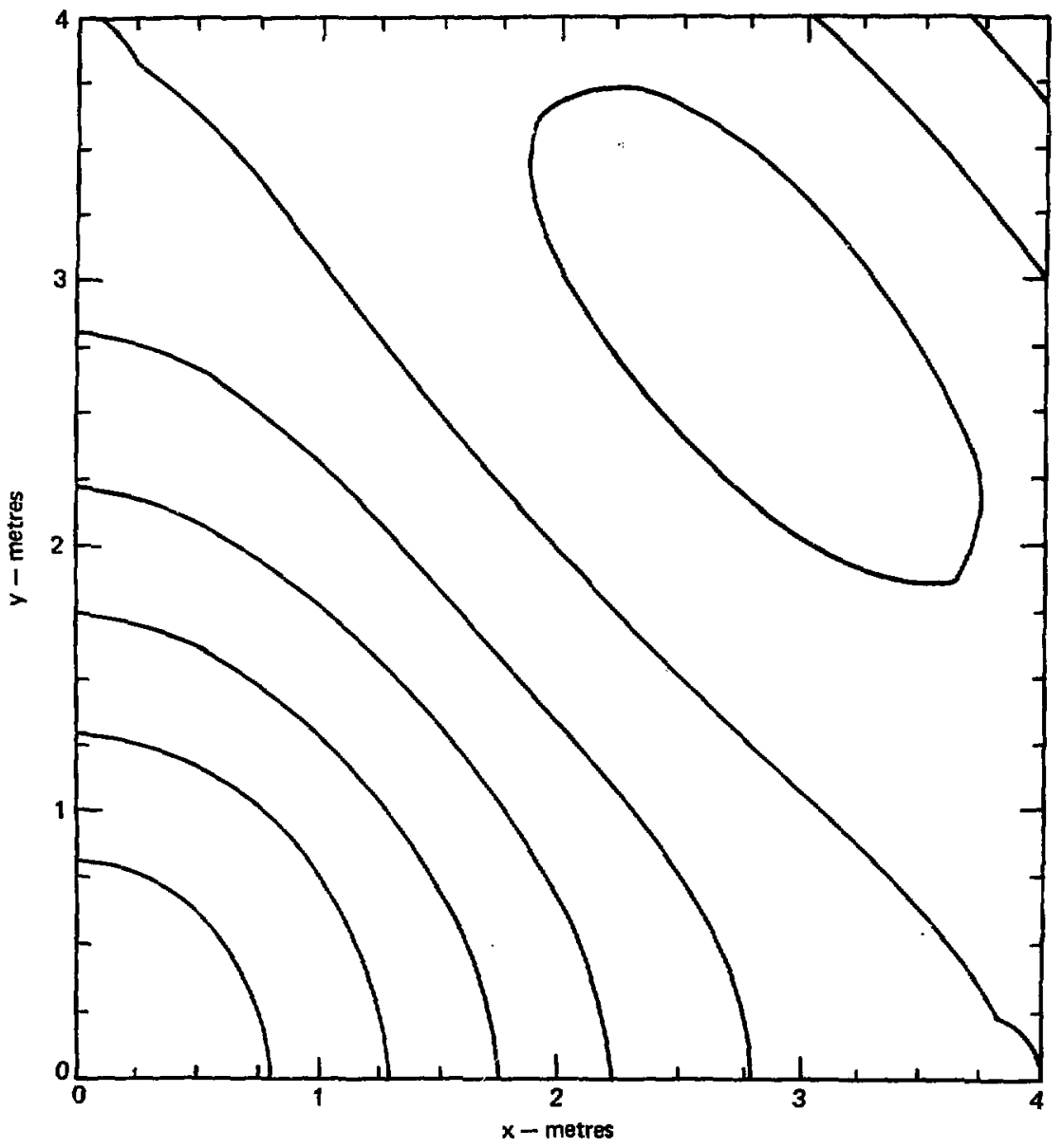

Fig. 5-10. Contours of $\bar{T}$ in the midplane for the vacuun field. 


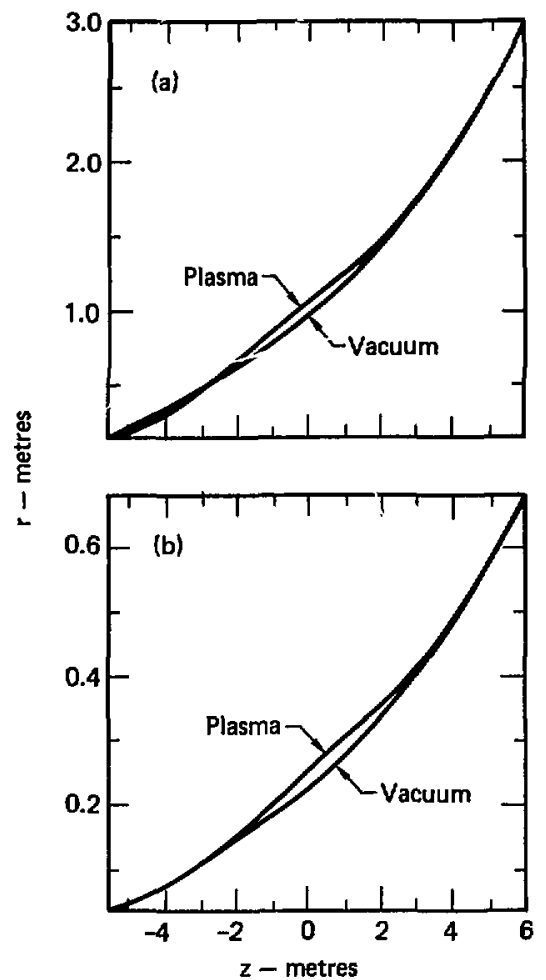

Fig. 5-11 (a,b). Trajectories of two representative magnetic field lines in the vacuum and plasma fields.

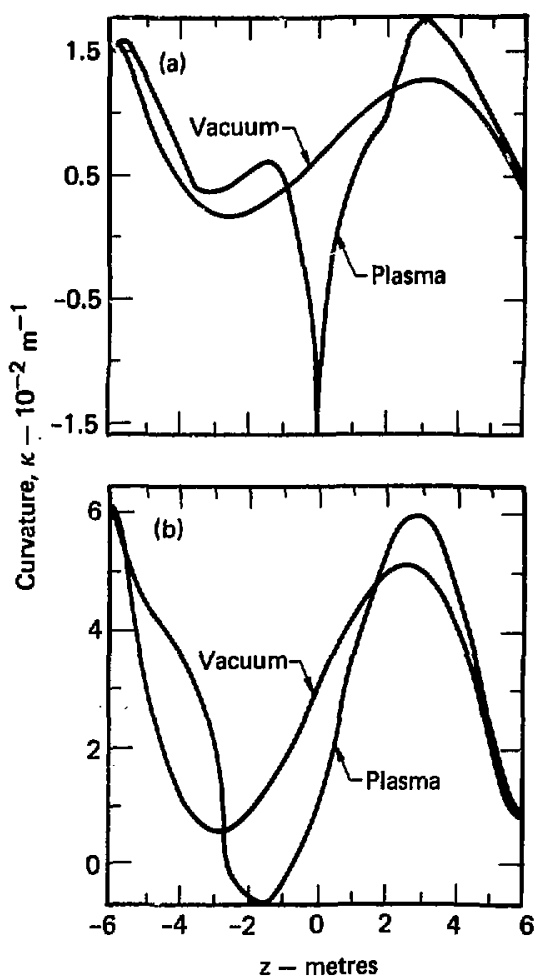

Fig. 5-12 $(a, b)$. Curvature $k$ of the field lines of Fig. 5-11 as a function of axial position, $K>0$ denotes lines convex as seen by plasma. 
Fig. 5-8 and vs $z$ in Fig. 5-9. Barely discernible in Fig. 5-B is a slight increase in $B$ over $B_{y}$ at $x \approx 3.0 \mathrm{~m}$. Conservation of magnetic fiux requires the slight increase in response to the field depression in the plasma center.

Shown in Figs. 5-10 and 5-11 are contours of $\bar{\Gamma}$ without and with plasma present. Noting from Eq. (32) that $d w / d x<0$, we see from Fig. 5-11 that the interchange criterion $D \vec{\Gamma} / D W \leq 0$ is satisicied out to a plasma radius of about $3 \mathrm{~m}$. Thus we expect interchange stability for this plasma.

It is instructive to examine the effect of the plasma field on the curvature of the field lines. The trajectory of two field lines in the $x-z$ plane (i.e. midway between the equivalent roffe bars) is given in Fig. 5-11, both with and without plasma present. These field lines pass through the midplane at $r=24$ and $101 \mathrm{~cm}$ in the vacuum field, but are displaced outward to 27 and $107 \mathrm{~cm}$ by the plasma. The curvatuis for these two field lines is shown in Fig. 5-12. We note that the "good" curvature for botin lines in the vacuum is actually improved by the plasma in certain regions, but reverses to give bad curvature near the midplane. One could anticipate that this region of bad curvature would lead to ballooning instabilities. 6

\subsection{Fokker-Planck Computations}

To compute the losses from the plasma due to collisions, it is necessary to solve for the distribution function of the electrons and the ion species $\left(f_{j}, j=e, D, T, \alpha\right)$. The kinetic equations to be solved can be written in the form

$$
\frac{\partial f_{j}}{\partial t}=\left(\frac{\partial f_{j}}{\partial t}\right)_{c}+s_{j}-L_{j} \text {, }
$$

where $f_{j}=f_{j}(E, \mu, J, t)$ depends only on time and the constants of the particle motion: $\mathrm{E}=$ energy $\mu \equiv \mathrm{m} v_{1}^{2} / 2 \mathrm{~B}=$ magnetic nonent and $J \equiv \oint_{\alpha, \beta} v_{l} d l=$ longitudinal Invariant. The collision term $(\partial f / \partial t)_{c}$ represents the effects of coulomb collisions and is taken to be of the Fokker-planck form. The source term $s_{j}$ models the effects of 
energetic neutral beam injection and $\alpha$-particle generation by fusion reactions. The loss term $\mathrm{L}_{j}$ accounts for the escape of particles which are scattered into the loss cone in velocity space. In the calculations for reactors we are only concerned with steady-state plasmas so

$$
\frac{\partial f_{j}}{\partial t}=0 \text {. }
$$

The distribution functions adjust themselves to balance the source, 10ss, and crilisinn terms in each element of velunity space.

In general, our kinetic equations should be areraged over the orbital motion of the particles within the plasma. However, this would be a prohibitively complex calculational problem for realistic plasma and magnetic field geometries, so we simplify by using a magnetic-square-well model. In this approximation the plasma and magnetic field are uniform over some finite region of space and the kinetic equations reduce to relatively simple velocity-space diffusion equations. Sophisticated coitputer codes have been developed to solve velocity-space Fokker-Planck equations and have been used extensively for studying mirror-confined plasmas. Our calculations here are based on the latest version of these rodes, HYBRID-II, described by Killeen, Mirin, and Rensink. 15 some features of the model particularly relevant for our reactor stuc:as are described in the following paragraphs. A more refined code which averages over the bounce motion of the ions has recently been developed. 16 Results for <nt> and $\beta_{1 \text { max }}$ are in generally good agreement with the simpler square-well calculations. 17 Agreement is best for injection angles $\theta \approx 50-60^{\circ}$, near the angle chosen for the hybrid and fusion reactors.

An important feature included in our Fokker-Planck model is the electrostatic ambipolar potential which arises due to the tendency for electrons to escape faster than ions. The potential is plotted in Fig. 5-13 for the hybrid reactor. In the absence of an ambipolar potential the "loss cone* angle is given by

$$
\left(\frac{v_{1}}{v_{0}}\right)_{L C}=\sin ^{2} \theta_{L C}=\frac{1}{R} .
$$

and $z l l$ Ions with velocity ratio $v_{1} / v_{0}<\left(v_{1} / v_{0}\right)_{\text {LC }}$ are unconfined. This "loss cone" is modified in the presence of an ambipolar potential to 


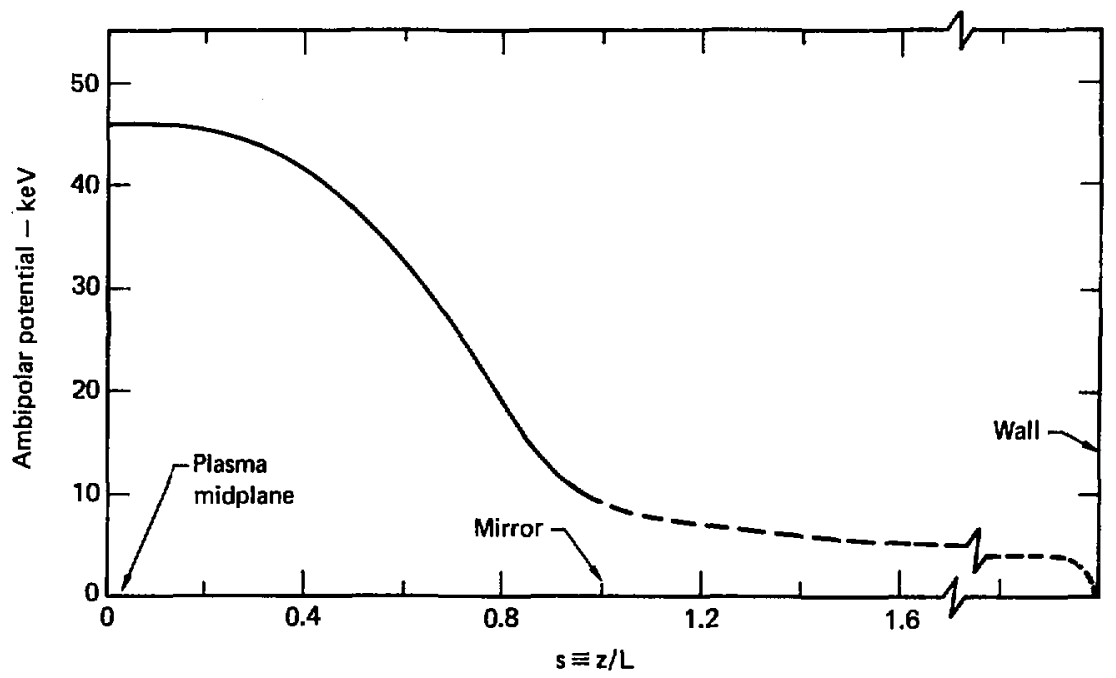

Fig. 5-13. Ambipolar potential vo distance along a field line. The exact shape of the potential from the mirror to the wall (or direct converter) is unknown; the ratio $\phi_{W} / \phi_{m}$ has been chosen as $5 / 4$ (see text). 


$$
\sin ^{2} \theta_{L C}=\left[1+2 z e\left(\phi_{0}-\phi_{m}\right) / m v_{0}^{2}\right] / R \text {. }
$$

$\phi_{0}$ and $\phi_{m}$ are the electrostatic potentials at the midplane and at the mirror. Clearly, all ions with

$$
v_{0}<v_{m i}=\left[\frac{22 e\left(\phi_{0}-\phi_{m}\right)}{m_{i}(R-1)}\right]^{1 / 2}
$$

are unconfined, being swept out by the slectrostatic potential. The loss boundaries in phase space given by Eqs. (34) through (36) are sketched in Fig. 5-14. Mirror ratio $R=2$ is used in this figure for clarity. The situation for electrons is much different. The low-energy electrons will te confined by the mirrors and the electrostatic potential drop from midplane to mirrors. Higher energy particles with sufficient $v$ can overcome the mirror potential, but will be reflected by the potential barrier at the end wall or direct converter $\left(=\phi_{0}-\phi_{w}\right)$. Still higher energy electrons can overcome this barrier and are lost. Thus, all electrons whose midplane speed satisfies.

$$
v_{0}<v_{m e} \equiv\left[\frac{2 e\left(\phi_{0}-\phi_{m}\right)}{m_{e}}\right]^{1 / 2}
$$

are confined to the region between the mirrors by the electrostatic potential. Those electrons with midplane speed satisfying

$$
v_{0}<v_{w e} \equiv\left[\frac{2 e\left(\phi_{0}-\phi_{w}\right)}{m_{e}}\right]^{1 / 2}
$$

are confined to the region between the end walls. The full electron lass boundaries in phase space are sketched in Fig. 5-15 for $R=2$ and $\left(\phi_{0}-\phi_{w}\right)$ $\left(\phi_{0}-\phi_{m}\right)=5 / 4$.

The magnitude of $\phi_{m}-\phi_{w}$ is difficult to compute, ${ }^{18}$ but must be of order $T_{e^{*}}$ We model this effect in the Fokker-Planck code by specifying the potential ratio $\left(\phi_{0}-\phi_{w}\right) /\left(\phi_{0}-\phi_{m}\right)$ as input. The magnitude of $\phi_{0}-\phi_{w}$ is determined self consistently so as to equalize the loss rates of electrons and ions. Typically, we find $\phi_{0}-\phi_{w} \approx 5 T_{e}$ and 


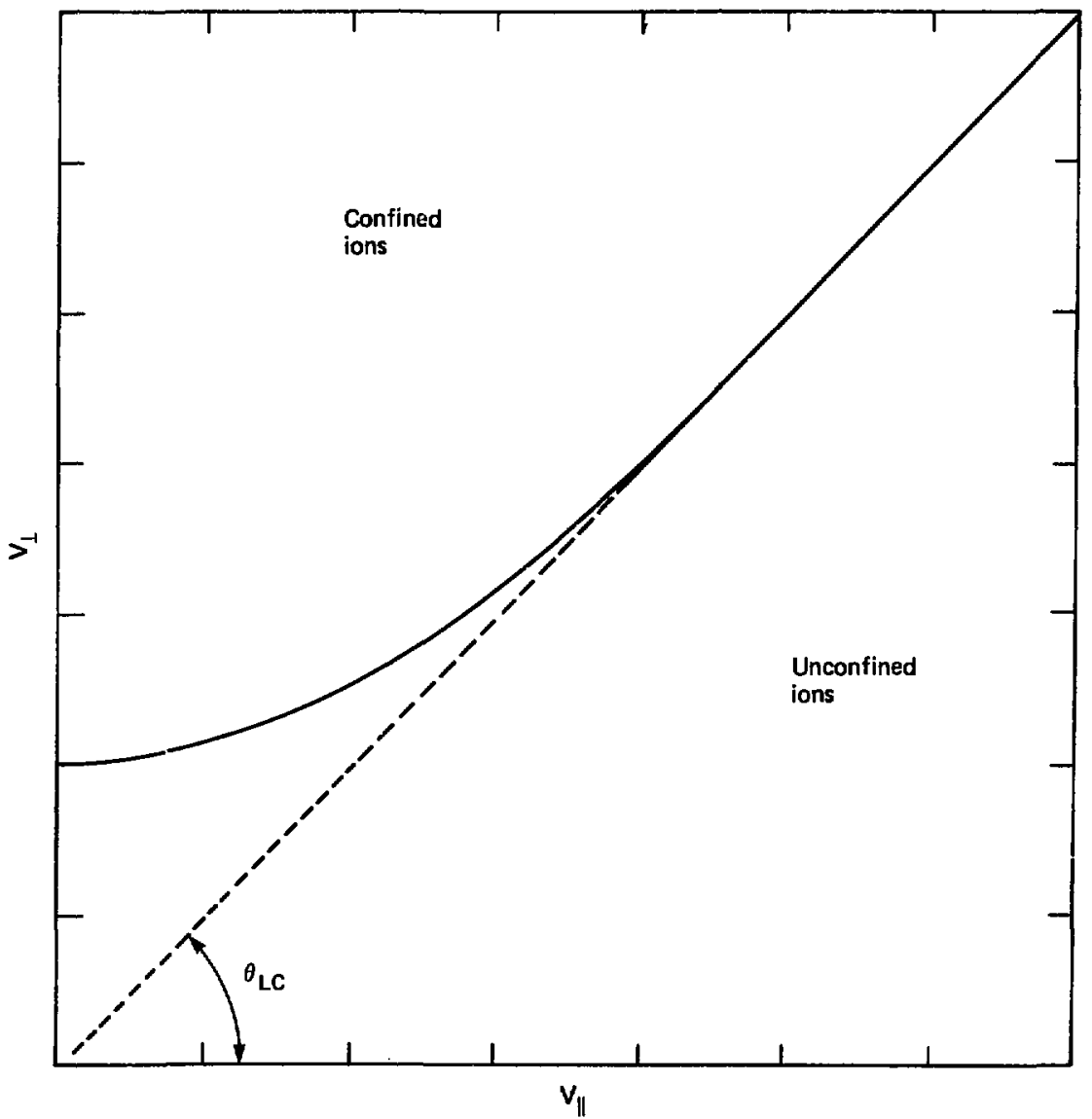

Fig. 5-14. Ion 1088 boundaries in phase space for $\mathrm{R}=2$. (---) Eq. (34); (一) Eq. (35). 


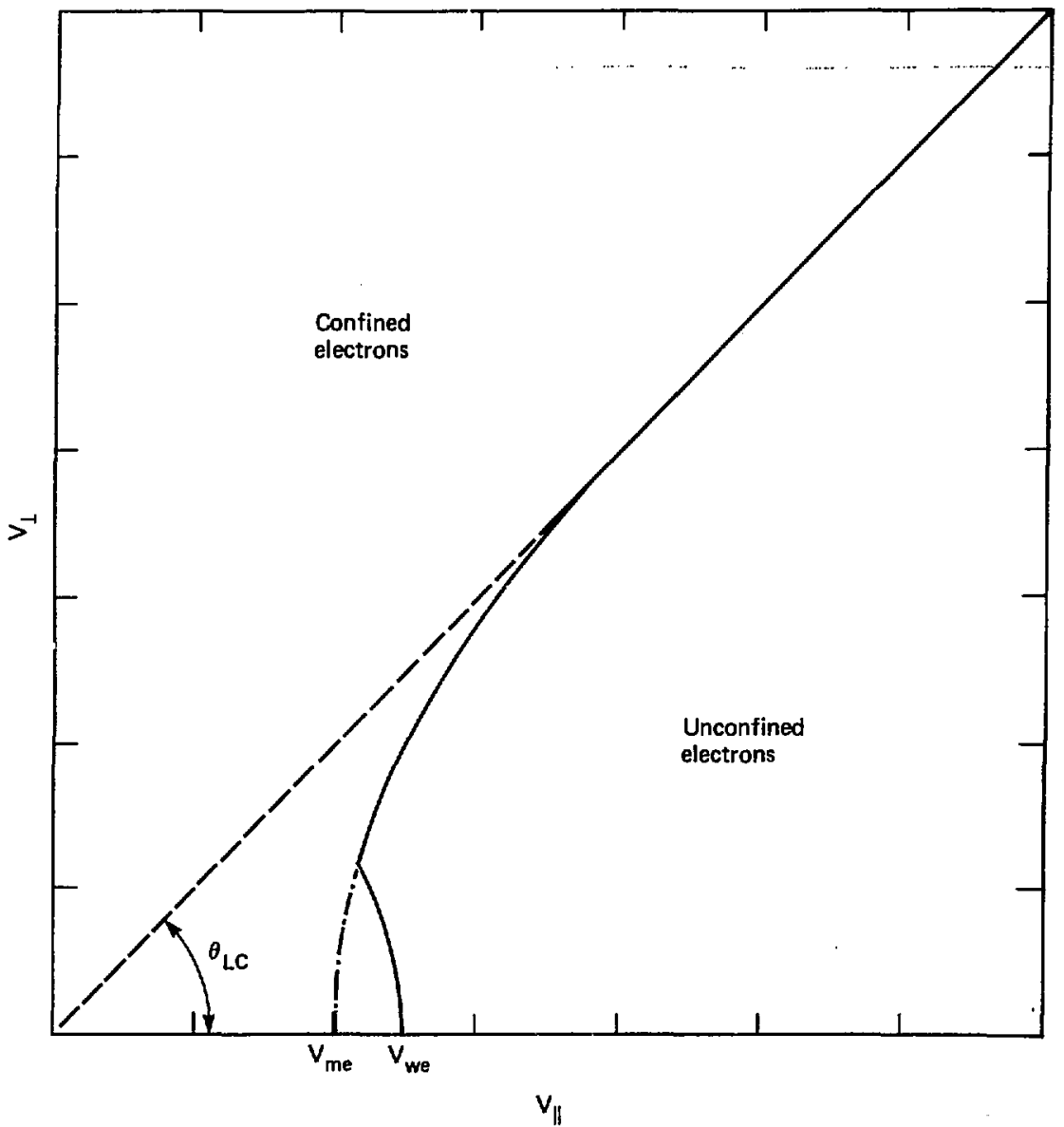

Fig. 5-15. Rlectron $108 s$ boundaries in phase space for $R=2$. (---) Eq. (34); (-- -) Eq. (37); and $(\longrightarrow$ Bq. (38). 
take $\left(\phi_{0}-\phi_{w}\right) /\left(\phi_{0}-\phi_{m}\right)=5 / 4$. This value has the effect of raising <nt> by 5-10s relative to the case where the potential drop beyond the mirrors is neglected.

The velocity-space source terms in our Fokkex-planck model are schenatically of the form

$$
\begin{aligned}
& s_{e}(v, \theta)=J_{e} \delta\left(E-E_{e}\right), \\
& s_{D}(v, \theta)=J_{D} \delta\left(E-E_{D}\right) \delta\left(\theta-\theta_{D}\right), \\
& S_{T}(v, \theta)=J_{T} \delta\left(E-E_{T}\right) \delta\left(\theta-\theta_{T}\right), \\
& s_{\alpha}(v, \theta)=J_{\alpha} \delta\left(E-E_{\alpha}\right),
\end{aligned}
$$

where the subscripts $(e, D, T, \alpha$ ) refer to electrons, deuterons, tritons and alpha particles. The speed variable, $v$, for apecies " $a$ " is $v=$ $\left(2 \mathrm{E}_{\mathrm{a}} / \mathrm{m}_{\mathrm{a}}\right)^{1 / 2}$, and $\theta$ denotes the pitch angle of the particle velocity vector relative to the $B$ field at the mid-plane of the systen. For deuterons (and similarly for tritons) we specify as input the curreit per unit volume, $J_{D^{\prime}}$, the neutral beam injection energy, $E_{D^{\prime}}$ and the effective midplane injection angle, $\theta_{D^{*}}$. In most cases we assune that the injection speeds and angles for deuterium and tritiun are the same, i.e., $\mathrm{B}_{\mathrm{T}}=3 \mathrm{E}_{\mathrm{D}} / 2$ and $\theta_{\mathrm{D}}=\theta_{T^{*}}$. In the present case $\alpha$-particles are not adiabatically confined. We therefore set $J_{\alpha}=0$.

To obtain the maximum $Q$, one needs equal densities for deuterium and tritium in the steady-state plasma. However, deuteriun ions tend to escape faster than tritium ions because of their lower mass, so to obtain equal steady-state densities one must inject more deuterium current. Generally, we find that $J_{D} \approx 2 J_{T}$ is required. The actual magnitudes of $J_{D}$ and $J_{T}$ in our Fokker-Planck calculations may be chosen rather arbitrarily since the value of $Q$ in the square-well model depends only weakly on the actual steady-state plasma density through a logarithmic term in the coulcanb scattering term $(\partial \mathrm{f} / \partial \mathrm{t}) \mathrm{q}^{*}$. We find that $J_{T} \approx 10^{15}$ particles/ $\mathrm{cm}^{3} / \mathrm{sec}$ yields $n_{T} \approx 10^{19}$ particles/cm ${ }^{3}$ for most reactor-like cases.

For the electron source term the current density is just the sun of the ion current densities, $J_{e}=J_{D}+J_{F}$. The electron source energy due to impact ionization of the injected neutral beans is negligible compared to the energy input from the neutral beans and alpha particles, so we simply choose some very low value for $\mathrm{E}_{e^{\prime}} \mathrm{e}_{. \mathrm{g}}, 0.01 \mathrm{kev}$. 
Particle losses in our Fokker-Elanck model are represented by simple finite-transit-time escape rates in the unconfined region of velocity space, i.e., for species " $a$ " we have

$$
L_{a}(v, \theta)=\left\{\begin{array}{cc}
\frac{f_{a}(v, \theta)}{\tau_{k}(v, \theta)}, \text { unconfined }(v, \theta) \\
0, \text { confined }(v, \theta)
\end{array},\right.
$$

where $\tau_{\|}=I_{\pi} / v_{\|}$is the trancit time for a particle with parallel velocity $v_{\|}=v \cos \theta$ in a system where the distance from the mid-plane to the mirrors is $L_{m}$. Since the ion transit time is several orders of magnitude less than the ion-ion collision time, we effectively find that the velocityspace distribution function is zero in the loss-cone region.

Several quantities of interest are computed from the results for the distribution functions. These include the fusion reaction rate parameter defined by

$$
\langle\sigma v\rangle_{F}=\int d y \int d y^{\prime} f_{D}(y) f_{T}\left(\underline{w}^{\prime}\right)\left|y-\underline{v}^{\prime}\right| \sigma_{F}\left(\left|\underline{q}-\underset{\sim}{v^{\prime}}\right|\right)
$$

and the number density and parallel and perpendicular pressures

$$
\begin{aligned}
\left\{\begin{array}{l}
n(B) \\
p_{\|}(B) \\
p_{\perp}(B)
\end{array}\right\}=\pi & \sum_{j}\left(\frac{2}{m_{j}}\right)^{3 / 2} \int_{0}^{\infty} d E \int_{0}^{E / B} \frac{B d \mu}{[E-\mu B]^{1 / 2}} \\
\times f_{j}(E, \mu, B) & \left\{\begin{array}{c}
1 \\
2(E-\mu B) \\
\mu B
\end{array}\right\} .
\end{aligned}
$$

Note that the distribution functions effectively vanish in the "loss cone," a fact not explicitly indicated in the limits of integration. The method by which the midplane distribution function in the Fokker-Planck code is converted to a function of the local B field is given in Ref. 5-15. At steady-state, we can compute the energy anplification factor from

$$
Q=\frac{n_{D} n_{T}\langle\sigma v\rangle_{F} E_{F}}{\left(J_{D} E_{D}+J_{T} E_{T}\right)}
$$


The mirfor and firehose-rode stability criteria [Egs. (18) and (19)] can also be checked from the solution with the aid of Eq. (42) for $P_{\mid}$(B) and $p_{1}(B)$.

To make it possible to find the optimum combination of parameters, we need to calculate $Q$ and $\beta_{1, \max }$ as function of injection energy, injection angle, and vacuum mirror ratio. We have simplified this task by using a single-species version of the code to find $\langle n \tau\rangle$ and $\beta_{1, \max }$ as $a$ function of mircor ratio and injection angle at a single energy. 4,15 These results, together with the scaling law of $\mathrm{Eq}$. (16) enable the choice of an optimum combination of physics input parameters. The multispecies code is then used to obtain the final $Q$ and $B_{\perp, \max }$ and other quantities of interest. The values so computed for the hy,rid reactor are collected in Table 5-1.

The positive Ion injectors used in the hybrid reactor accelerate $\mathrm{D}^{+}$ ions to $125 \mathrm{keV}$ and $\mathrm{T}^{+}$ions to $187.5 \mathrm{keV}$. The energies of the three components and their percentage in the beams as well as the mean energies are listed in Table 5-1. The Fokker-Planck calculations are for the actual mirror ratio, taken as 4.15; the plasma $\beta_{1}$ is specifled (as $\beta_{1,0}$ ) to enable the computation of the vacuum mirror ratio $R_{v}$ from the long-thin approximation [Eq. (15)] . The actual $\beta_{1, \max }$ from the mirror-mode limit is somewhat higher. It is necessary to maintain $\beta_{\perp}$ somewhat below the mirror-node limit to ensure adiabaticity of the ion orbits. 19

The computed mean species energies are somewhat different from the injection energies due to the energy exchange between species and because low energy particles vanish into the "loss cone." The effect of the latter is to raise the mean ion energy to $126 \mathrm{keV}$ from its injected value of $110 \mathrm{keV}$. The computed midplane densities for deuterium and tritium are nearly equal; the $Q$ value for exactly equal species densities would be slightly higher. The small correction can be computed from

$$
Q=\frac{Q_{E P}}{4\left[n_{D}\left(B_{0}\right) / n\left(B_{0}\right)\right]\left[1-n_{D}\left(B_{0}\right) / n\left(B_{0}\right)\right]} \text {, }
$$

where the $\left(B_{0}\right)$ refers to midplane values from the code. The number densities listed in Table 5-1 are those computed for the specified source strengths and not those actually expected in the machine. They must be 
scaled to the actual magnetic field. The true midplane densities can be found from the code results with the expression

$$
n=\frac{B_{10} B_{m}^{2} n\left(B_{0}\right)}{2 \mu_{0} R_{v}^{2} p_{1}\left(B_{0}\right)}
$$

Also listed in Table 5-1 are the mean energies of the escaping particles at the wall and the axial factors $c^{(l)}$ defined by Eq. (1). In computing the latter, the long-thin approximation was used with the model vacuum field given by

$$
\frac{B_{v}(s)}{B_{m}}=\frac{1}{R_{v}}+\left(1-\frac{1}{R_{v}}\right)\left\{\exp \left[-\alpha\left(s^{\prime}-1\right)^{2}\right]+\exp \left[-\alpha\left(s^{\prime}-1\right)^{2}\right]\right\}
$$

where $s^{\prime} \equiv s / L$, and $\alpha$ is a parameter determined by fitting to the actual field along the center line. A value of 10 for $\alpha$ was found appropriate for the Yin-Yang coil set used in the hybrid reactor.

The $p_{\|}(B)$ and $p_{\perp}(B)$ found from the distribution functions with the aid of Eq. (42) are shown in Figs. 5-16. Also plotted are the "optimal" pressure functions used for the equilibriun magnetic-field computations. We note the excellent agreement between the curves. The ratio $p_{1} / p_{\|}$at the midplane is found to be 2.5, considerably above the value of 1 it would have if the distributions were Maxwellian.

The vacuum field, as computed from Eq. (46), and the equilibriun field, obtained from the Fokker-Planck results with the aid of the long-thin approximation, are shown in Fig. 5-17. Also shown is the vaculum field computed with the aid of the MAFCO program. The agreement between the two vacuum fields is surprisingly good. The number density of ions is similarly plotted in Fig. 5-18. The distribution of electrostatic potential has already been given in Fig. 5-13.

\subsection{Radial Density Profiles}

As shown in the introduction, the fusion power output is strongly dependent on the radial density profile of the plasma. In a mirror device, the radial profile is determined by a balance between source terms, due to 
Table 5-1. Plasma parameter for the fusion-fission hybrid reactor as computed from the Hybrid II Fokker-Planck code.

\section{INPUT}

Injection angle, $\theta_{\text {in }}$

Injection energies, deuterium $\left(\overline{\mathrm{E}}_{\mathrm{D}, \mathrm{In}}\right)$

$65^{\circ}$

Injection energies, tritium $\left(\bar{E}_{\mathrm{T}, i n}\right)$

$125,62.5,41.7(94 \mathrm{keV})$

Deuterium source strength, $J_{D}$

$187.5,93.75,62.5$ (141 keV)

Tritium source strength, $J_{T}$

$2 \times 10^{15} \mathrm{~cm}^{-3}-\mathrm{s}^{-1}$

$1 \times 10^{15} \mathrm{~cm}^{-3}-\mathrm{s}^{-1}$

Percentage full, 1/2, 1/3 energy in beams,

$57,23,20$

wall potential/mirror potential,

$$
\left(\phi_{0}-\phi_{w}\right) /\left(\phi_{0}-\phi_{m}\right)
$$

1.25

specified plasma $\beta_{1}, \beta_{1,0}$

0.706

Actual mirror ratio

4.15 .

\section{OUTPUT}

Hean species energies:

Midplane number densities ${ }^{a}$

Maximum $B, \beta_{\perp}, \max$

$\langle$ nT $>$

$\langle 0 v\rangle_{F}$

$Q$

Mirror potential, $\phi_{0}-\phi_{\mathrm{m}}$

wall potential, $\phi_{0}-\phi_{W}$

sean energy of escaping particles at wall:

Axial factors

$$
\begin{aligned}
& \overline{\mathrm{E}}_{\mathrm{D}} \\
& \overline{\mathrm{E}}_{\mathrm{T}} \\
& \overline{\mathrm{E}}_{\mathrm{e}} \\
& \mathrm{n}_{\mathrm{D}} \\
& \mathrm{n}_{\mathrm{T}}
\end{aligned}
$$

$113 \mathrm{keV}$

$139 \mathrm{keV}$

$13.6 \mathrm{keV}$

$1.15 \times 10^{14} \mathrm{~cm}^{-3}$

$1.29 \times 10^{14} \mathrm{~cm}^{-3}$

$$
\begin{gathered}
2 \times 10^{13} \mathrm{~cm}^{-3}-\mathrm{s} \\
8.44 \times 10^{-16} \mathrm{~cm}^{3}-\mathrm{s}^{-1} \\
0.673
\end{gathered}
$$

$37.1 \mathrm{keV}$

$46.3 \mathrm{kev}$

$\bar{E}_{D, w}$
$\bar{E}_{T, w}$
$\bar{E}_{e, w}$

$98.7 \mathrm{kev}$

$112.0 \mathrm{kev}$

$8.5 \mathrm{keV}$

$c^{(2)}$

0.609

0.398

0.341 

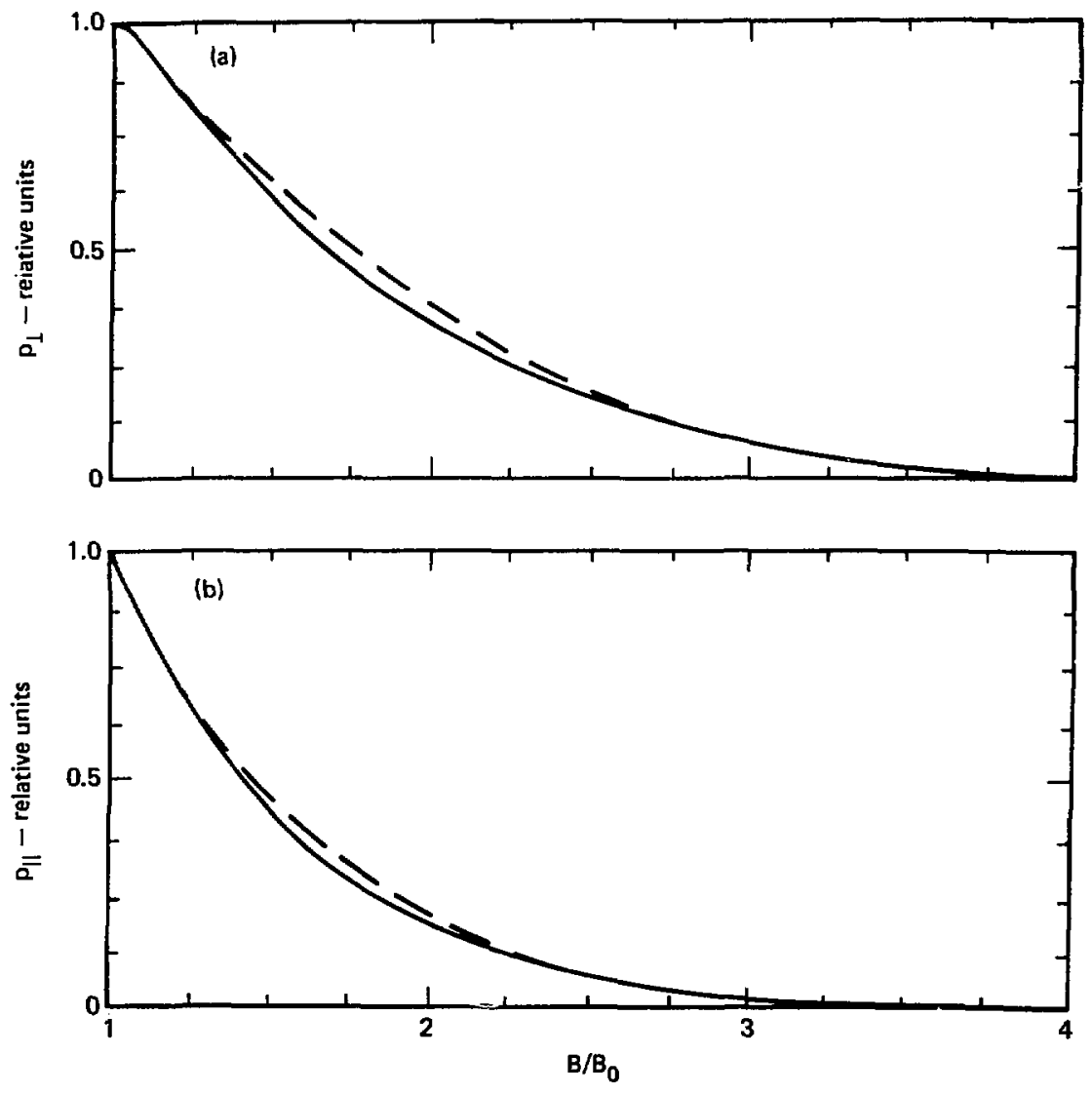

Fig. 5-16(a,b). $P_{1}$ and $P_{1}$ plotted vB $B / B_{0}:(\longrightarrow$ from Fokker-Planck computation; (-- - ) used in equilibrium code. 


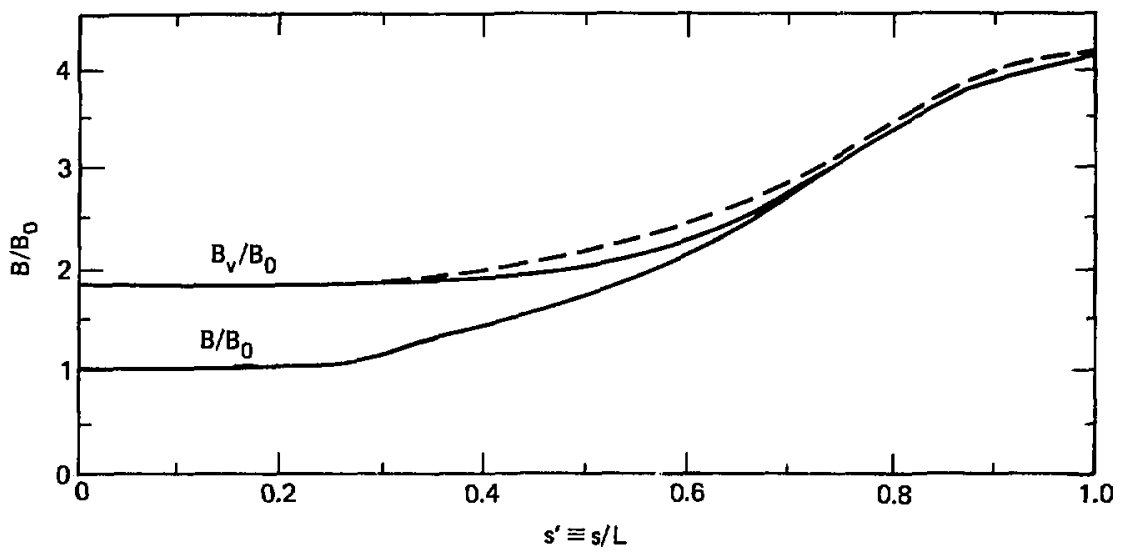

Fig. 5-17. $\mathrm{B}_{\mathrm{v}} / \mathrm{B}_{0}$ from MAFCO computer program $(-)$ and from Eq. (46) with $\alpha=10^{v}(---)$ vs $\mathrm{s}^{\prime}=\mathrm{s} / \mathrm{L} ; \mathrm{B} / \mathrm{B}_{0}$ from Hybrid-II code with $\mathrm{Eq}$. (46) and long-thin approximation [Eg. (12)].

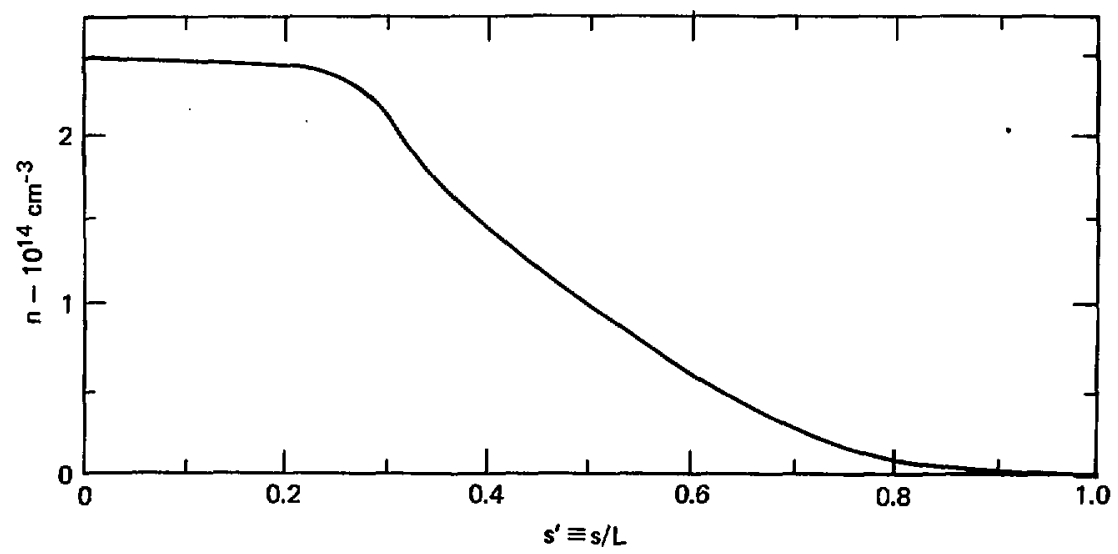

Fig. 5-18. $n\left(=n_{D}+n_{T}\right)$ vs $a^{\prime} \equiv g / L$ from Hybrid-II code with vacuum B-field from $\mathrm{Bq}$. $(46 \mathrm{f}$. 
absorption of neutral beams, and loss terms, predominantly Coulcab collisions and losses due to rf fluctations, and, near the surface, charge exchange off cold neutral gas refluxing from the wall. In contrast to closed systems, radial diffusion of particles and energy has been found to play a minor role in shaping the radial profile.

Enhanced losses due to rf Eluctuations from the drift-cyclotron-losscone (DCLC) instability ${ }^{20}$ have plagued mirror experiments for many years. This instability can be surpressed by weak enough radial density gradients coupled with finite plasma $\beta$ or by the introduction of warm streaming plasma to partlally fill the hole in the distribution function due to the loss cone. This latter technique has been used to dramatically improve confinement times in 2XIIB. 21,22 However, confinement times are still far from the results of the Fokker-Planck code because the streaming plasma depresses the electron temperature resulting in very large electron drag on the ions.

The short lifetimes cannot be tolerated in a reactor and it is necessary to suppress the DCLC mode by the other method, reduction of radial density gradients. It turns out that proper aiming of the neutral beams can cause the density profile to be nearly flat over the bulk of the plasma, thus eliminating the DCLC mode in this reglon. Near the edge of the plasna, where the density must drop to zero, the gradients will be large, and it is necessary to supply warm plasma to stabilize this mode. This boundary layer will lower the effective $Q$ for the plasma since it is of lower energy and with reduced $\langle n \tau\rangle$, so it is desirable to keep it as thin as possible. However, it is not possible to reduce it below about 3 gyroradii since the finite gyro-orbits coupled with charge-exchange at the surface cause effective radial diffusion to maintain this minimum thickness.

Our model of the radial density variation includes a central region of uniform density $n_{0}$ and radius $r_{p}$ with $\langle n T\rangle_{0}$ computed from the FokkerPlanck equation. Surrounding this is the boundary layer of thickness $\delta r$ with $\langle n \tau\rangle_{B L}$ governed by the stream-stabilized DCLC considerations. 22 we will verify with a radial buildup code in subsequent paragraphs that this profile can be attained; for the moment let us focus on the boundary layer and its effects on $Q$. 
To stabilize DCL instabllities, the minimum warm (or unconfined) density $n_{u}$ is given by ${ }^{22}$

$$
n_{u}=\ln \frac{e\left(\phi_{0}-\phi_{m}\right)}{(R-1) E_{i}}
$$

where $\mathrm{n}$ is the local density, $\left(\phi_{0}-\phi_{m}\right)$ the ambipolar potential, $\overrightarrow{\mathrm{E}}_{i}$ the mean ion energy, $R$ the mirror ratio, all at the midplane, and $\lambda$ a nunerical factor about $1 / 2$. We can relate the ambipolar potential to the electron temperature through the equation

$$
e\left(\phi_{0}-\phi_{m}\right)=(n-1) r_{e} .
$$

wher $n \approx 4$. The current dengity of the warm streaming plasma is found from

$$
j_{\text {st }}=e I_{p} n_{u} v_{t} \text {, }
$$

where $L_{p}$ is the plasma length, and $v_{t}^{-1}$ is the transit time for the unconfined stream ions and given by

$$
v_{t}=\frac{a}{L_{p}}\left[\frac{e\left(\phi_{0}-\phi_{m}\right)}{m_{1}}\right]^{1 / 2} \text {. }
$$

Alpha is a factor equal to about 1/2. From Egs. (47) through (50) we find

$$
n_{u}=n \frac{\lambda(n-1) T_{e}}{(R-1) E_{i}}
$$

and

$$
j_{s t}=4.959 \times 10^{-12} \frac{\left(\lambda(n-1)^{3 / 2}\right.}{(R-1) M_{i}^{1 / 2}} \frac{n_{e}^{3 / 2}}{E_{1}} \mathrm{~A} / \mathrm{cm}^{2}
$$

for $T_{e}$ and $\bar{E}_{i}$ in $\mathrm{keV}$ and $\mathrm{n}$ in $\mathrm{cm}^{-3}$. With the suggested values for $\alpha, \lambda$, and $n$ and measurements of $n$ (peak), $T_{e}$ and $\bar{E}_{1}$, this expression yields a value of $j_{s t}$ slightly higher than inferred from endloss measurements on 2XIIB. The discrepancy is probably due to a somewtat longer transit time than predicted by Eq. (50) with $\alpha=1 / 2$.

To obtain the electron temperature, we apply the electron energy equation to the steady state, noting that $T_{e} \ll \overline{\mathbf{s}}_{1}$ and the current of warm plasma is much greater than the current of lost hot lons, 


$$
\frac{n^{2} \bar{E}_{i}}{n \tau_{E}}=\eta T_{e} \frac{j_{g t}}{e L_{p}},
$$

with the ion-electron energy transfer term given by ${ }^{23}$

$$
n \tau_{E}=\frac{9.9 \times 10^{12} M_{i} T_{e}^{3 / 2}}{\ln \Lambda} \text {. }
$$

To apply these relations to the boundary layer, we take constart average values for $\bar{E}_{i}$ and $\pi$ and let $n$ in the midplane vary linearly from the core value $n_{0}$ at $r=r_{p}$ to 0 at $r_{p}+\delta r$. Integrating $\mathrm{Eq}$. (52) across the boundary layer, we obtain

$$
I_{S T}=4.96 \times 10^{-12} \frac{\alpha \lambda(\eta-1)^{3 / 2}}{(R-1) M_{i}^{1 / 2}} \frac{T_{e}^{3 / 2}}{\bar{E}_{i}} \frac{n_{0}}{2}\left(2 \pi r_{p} \delta r\right) .
$$

Note that this is the minimum current for stabilizing the midplane plasma; at axial locations other than the midplane, the minimum current is less.

We must also average Eq. (53) over radius and over the length of the flux tube. With the aid of Eq. (52) we find an expression for $T_{e^{\prime}}$

$$
T_{e}=6.8310^{-6}\left[\frac{C_{B L}^{(2)}(R-1) \ln \Lambda}{n \alpha \lambda(n-1)^{3 / 2} M_{i}^{1 / 2}}\right]^{1 / 4}\left(L_{p} n_{0} \bar{E}_{i}\right)^{1 / 4} .
$$

$\mathrm{C}_{\mathrm{BL}}^{(2)}$ as defined by Eq. (4), is the value for the boundary layer, and is less than the value in the core because of the narrower, drag-dominated distribution function in DCLC-dominated regions.

Equations (55) and (56) give $I_{S T}$ and $T_{e}$ in terms of known quantities and the mean ion energy in the boundary region. In $2 \times I I B$, where the mean bean energy is about $16 \mathrm{kev}$, charge-exchange plays a dominant role in determining the mean plasma energy which turns out to be 10 to $14 \mathrm{keV}$. At the much higher injection energy for reactors, charge-exchange is much less ingortant and it is to be expected that electron drag will be dominant. We can therefore use the ion energy equation in the form

$$
\frac{n^{2} E_{i}}{n \tau_{E}} \frac{n^{2}\left(E_{I N}-E_{O U T}\right)}{\langle n \tau\rangle} \text {, }
$$


where $\langle n \tau\rangle$ is the confinement tine, and the energy at which ions are lost is $E_{\text {OUT }} \approx \mathrm{e} \phi /(\mathrm{R}-1) \ll E_{T N}$. Thus

$$
\overline{\mathbf{E}}_{\mathrm{i}} \approx \frac{\mathrm{nT} \mathrm{E}}{\langle\mathrm{n \tau}\rangle} \mathrm{E}_{\mathrm{IN}}
$$

An individual ion injected at energy $\mathrm{E}_{\mathrm{TN}}$ must drag down to $\mathrm{E}_{\text {OUT }}$ before it is lost. Assuming this process linear in $E_{1}$ we find

$$
\frac{E_{\text {OUT }}}{E_{\text {IN }}}=e^{-\frac{\langle n T\rangle}{n \tau_{E}}}
$$

and

$$
\frac{\langle n T\rangle}{n T_{E}}=\ln \left[\frac{(R-1) E_{I N}}{(n-1) T_{e}}\right] .
$$

Thus we find

$$
\frac{E_{I N}}{E_{i}}=\ln \left[\frac{(R-1) E_{I N}}{(\eta-1) T_{e}}\right] .
$$

The reduced confinement time in the boundary layer causes a reduction in the effective $Q$ for the plasma. We find

$$
Q=\frac{Q_{0}}{1+\frac{2\langle n \tau\rangle_{0} \delta r c_{B L}^{(2)}}{3\langle n \tau\rangle_{B L} r_{P} c_{0}^{(2)}}}
$$

Equations (58) through (62) suffice to determine quantities of interest for the boundary layer. We take the following values for the parameters:

$$
\begin{aligned}
& \alpha=\lambda=1 / 2, \quad \eta=4, \quad R=2, \\
& \ell n A=15, \quad C_{B L}^{(2)}=0.25, \quad c_{0}^{(2)}=0.34, \\
& I_{P}=1300 \mathrm{~cm}, \quad r_{P}=250, \quad n_{0}=9 \times 10^{13} \mathrm{~cm}^{-3}, \\
& E_{I M}=125 \mathrm{keV}, \quad M_{1}=2.5, \quad\langle n \tau\rangle_{0}=2 \times 10^{13} \mathrm{~cm}^{-3}-\mathrm{s}
\end{aligned}
$$


and $\delta r=3 a_{i}=0.9 \sqrt{E_{i}}$ (based on $\left.B_{v}\right):$

and find

$$
\begin{aligned}
T_{e} & \approx 600 \mathrm{eV}, \\
E_{i} & \approx 29.5 \mathrm{keV}, \\
\delta r & \approx 4.9 \mathrm{~cm}, \\
I_{s t} & \approx 18 \mathrm{kA} \\
n \tau_{E} & =7.7 \times 10^{11} \mathrm{~cm}^{-3}-3, \\
<n \tau>_{B L} & =3.2 \times 10^{12} \mathrm{~cm}^{-3}-3, \\
Q & =0.94 \Omega_{0} .
\end{aligned}
$$

The streaming plasma could be supplied via axial stream guns, as in 2 XIIB. For an acceleration voltage of $1 \mathrm{keV}$, this requires an additional power of 18 which must be supplied to the plasea, thus cscreasing the effective $Q$ somewhat wore. An alternative technique is to furnish at least part of $n_{u}$ by bleeding in cold molecules. When these are incident on the surface, they will be ionized or will charge-exchange with the hot plasma and create a warm plasina which streams out the ends of the reactor on the surface. If the hot ion energy at the surface is high enough, the majority of the collisions will be ionizing, ant no great loss of confined plasma will occur. To complete the study of the radial profiles, we need to show that the neutral beams can be aimed so as to obtain a constant density in the central region of the plasma. In addition, we must specify the total beam currents and progranming of current with time to obtain a plasma buildup. The chief tool for this study is a code ${ }^{24}$ which computes radial variation of the guiding center density of the plasma as a function of time. The equation for the guiding center density $w(r, t)$ can be written as

$$
\frac{\partial y}{\partial t}=s_{b}-L_{c}-L_{s},
$$

where $S_{b}, L_{c^{\prime}}$ and $L_{S}$ are, respectively, the rates of increase of guiding center density due to beam absorption, loss due to scattering into the loss cone via Couloab collisions and rf fluctuations and loss due to charge-exchange with neutral atoms and molecules refluxing from the walls. The number density $n(r, t)$ is related to $N(r, t)$ through the equation 


$$
n(r, t)=\frac{2}{\pi} \int \frac{\left[\theta\left(r^{\prime}-\left|r-a_{i}\right|\right)-\theta\left(r^{\prime}-r-a_{i}\right] N\left(r^{\prime}, t\right) r^{\prime} d r^{\prime}\right.}{\left\{\left[r^{2}-\left(r^{\prime}-a_{i}\right)^{2}\right]\left[\left(r^{\prime}+a_{i}\right)^{2}-r^{2}\right]\right\}^{1 / 2}},
$$

where $\theta\left(x-x_{0}\right)$ is the Heaviside function. Equations (63) and (64) are solved under the assumption of constant ion and electron energies. In accord with the boundary layer model, the particle confinement times are different in the core and in the outer region of the plasma. The boundary layer region is assigned a thickness of $\delta r=3 a_{i}$, its inner edge at $r_{p}$ being obtained as part of the solution for $N(r, t)$.

A certain amount of control over the radial profile is available through proper aining of the beams. This can be illustrated by reference to Fig. 5-19. A beam atom can be ionized or charge-exchanged at $T$. Depending on the direction of the magnetic field, the guiding center is deposited outward or inward from T. Figure 5-19 illustrates an outward displacement. If the bean atom undergoes charge-exchange at $T$, then the net effect is an outward displacement of the guiding center from $A$ to B. This displacement effect is properly accounted for in the code.

To compute the term $s_{b}(r, t)$ of $\mathrm{Eq}$. (63), it is necessary to compute the attenuation of the incident neutral beam as well as the contributions of charge-exchanged ions through retrapping. The neutral beam attenuates exponentially along its path in the plasma through ionization and chargeexchange. The plasma ions which charge-exchange with beam atoms are approximated as charge-exchange beams traveling either in the same or opposite direction as the injected beams. Reionization or charge-exchange of these beams is included.

Solution of Eq. (63) as a function of time allows the entire start-up process to be followed. Initially, there must be a plasma target for the neutral beans. As in the experiments in 2XIIB, this is assumed to be generated by plasa guns ained along the axis of the reactor $s o$ as to form a circular plasna of radius $2.5 \mathrm{~m}$ at the midplane. The stream density is taken as $3 \times 10^{13} \mathrm{~cm}^{-3}$ and the duration as $1 \mathrm{~s}$. The aiming of the neutral beans, and their equivalent currents as a function of time nust meet two requirements: the density profile should be nearly flat out to $r=2.5$ - at steady state with $n \approx 9 \times 10^{13} \mathrm{~cm}^{-3}$, and the uniabsorbed bean incident on the wall should not deposit wore than about $100 \mathrm{w} / \mathrm{cm}^{2}$, the 


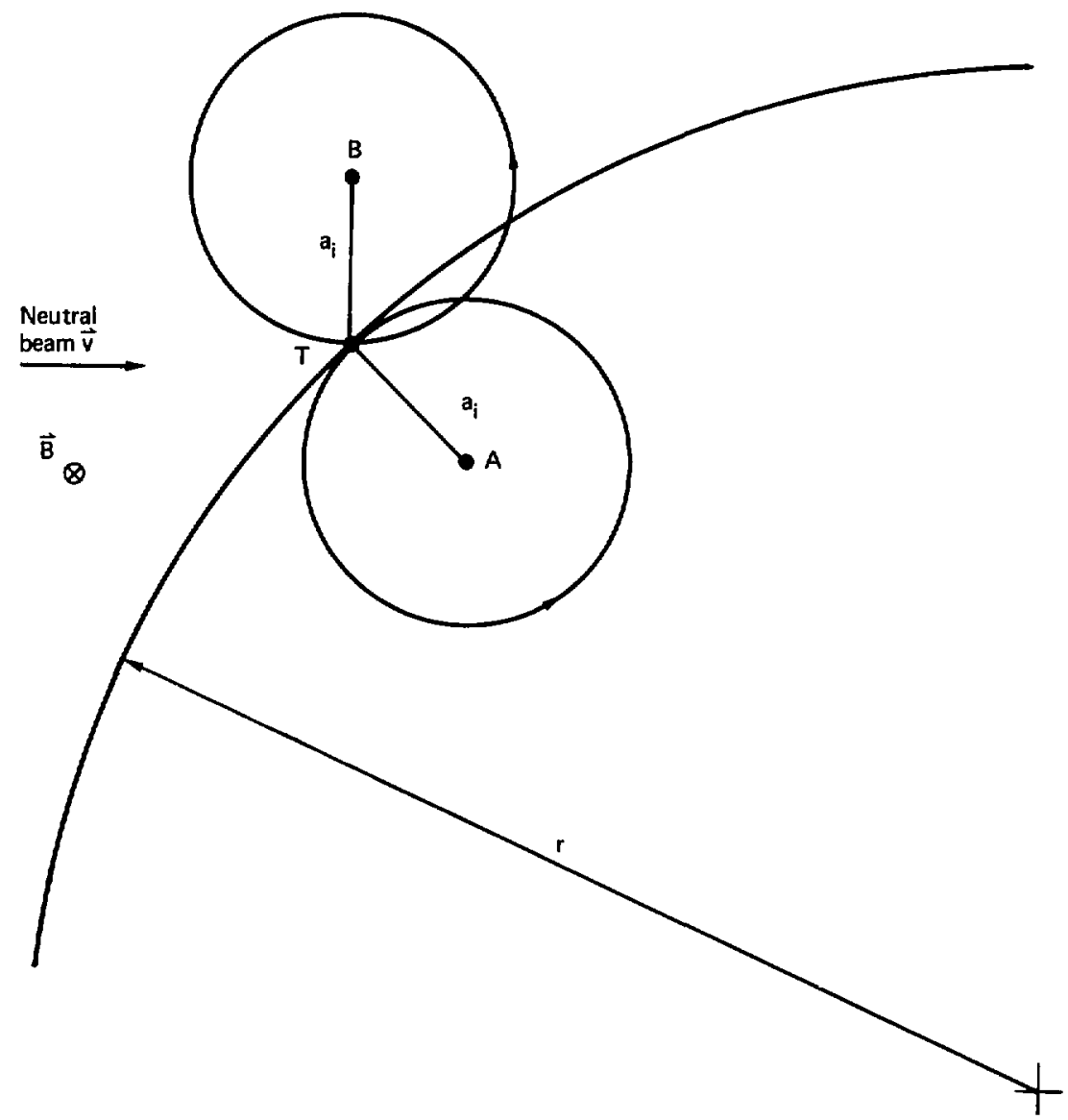

Fig. 5-19. Schematic of guiding-center displacement due to beam trapping T: location of ionization or charge exchange event. Ionization deposits guiding center at $B$. Charge-exchange moves guiding center from $A$ to $B$. 
linit with reasonable wall cooling. It is therefore necessary to estinate the proper beam positioning and currents as a function of tive, carry out the computations of $\mathrm{N}(\mathrm{r}, \mathrm{t})$ to the steady state, and iterate on the position and currents until the desired results are obtained. We have performed several such iterations, but have by no means arrived at a final optimum choice.

The aining of the neutral beams has been discussed earlier. We recall that the placement of some beams in the fan region is necessary to allow bean absorption to occur primarily near the axis of the plasma. The beam currents are shown as a function of time in Fig. 5-20. Bean 1 is placed in the fan and aimed to cover a 1-m-wide area at the center of the plasma. Beans 2 and 3 are placed in the center and aimed to cover radii from 0.5 to $1.5 \mathrm{~m}$. They are aimed to accomplish an outward displacement of guiding centers as illustrated in Fig. 5-19. Reference to Fig. 5-20 shows that the beams are brought to full current at varying rates to linit the rate of energy deposition in the wall. It should be noted that no beans are directly aimed to pass tangentially through the outer portion, $I=1.5$ to $2.5 \mathrm{~m}$, of the plasma. Absorption of the beans ained at the central regions in these outer zones proved to be adequate to support the losses.

Only during the initial stages of startup is there appreciable penetrating beam. The penetrating beam is shown vs time for the fir: $0.5 \mathrm{~s}$ in Fig. 5-21. The peak current of $50 \mathrm{~A}$ (equivalent) in the fan, cor sisponds to a peak wall-heat load of approximately $100 \mathrm{w} / \mathrm{cm}^{2}$. just at the tolerable limit. This load drops to half the peak value by $0.1 \mathrm{~s}$. The load at the center is only about $30 \mathrm{w} / \mathrm{cm}^{2}$.

The evolution of the radial density profiles over the first $0.5 \mathrm{~B}$ is shown at $0.1 \mathrm{~s}$ intervals in Fig. 5-22. We note that the profile is quite flat, even at $0.1 \mathrm{~s}$, with some tendency to peak near the boundary, in spite of the fact that no beams are specifically aimed in the region $r=1.5$ to 2.5 of the plasna. The plasma profile after startup is shown in Fig. 5-23 and shows the large central region of $n \approx 10^{14} \mathrm{~cm}^{-3}$. The deviations from uniformity in the central region are due to the representation of a large number of beam modules by these beams. Reaking near the edge of the plasia is because all beass nust pass through this region. The peaking could be reduced by positioning nore of the beams in the fan region. 


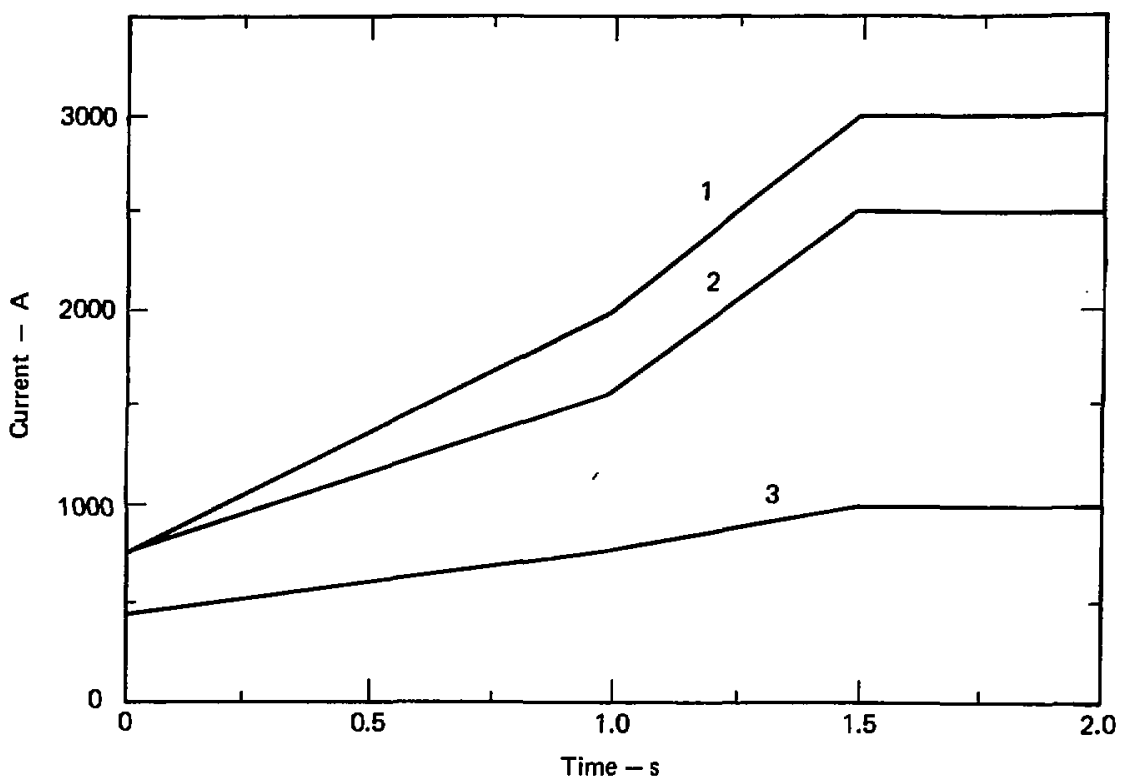

Fig. 5-20. Injected beam (equivalent) currents as a function of time during startup. $1: I=0-0.5 \mathrm{~m}$ in fan; $2: r=0.5-1.0$ in midplane; $3: r=$ 1.0 - 1.5 in midplane. 


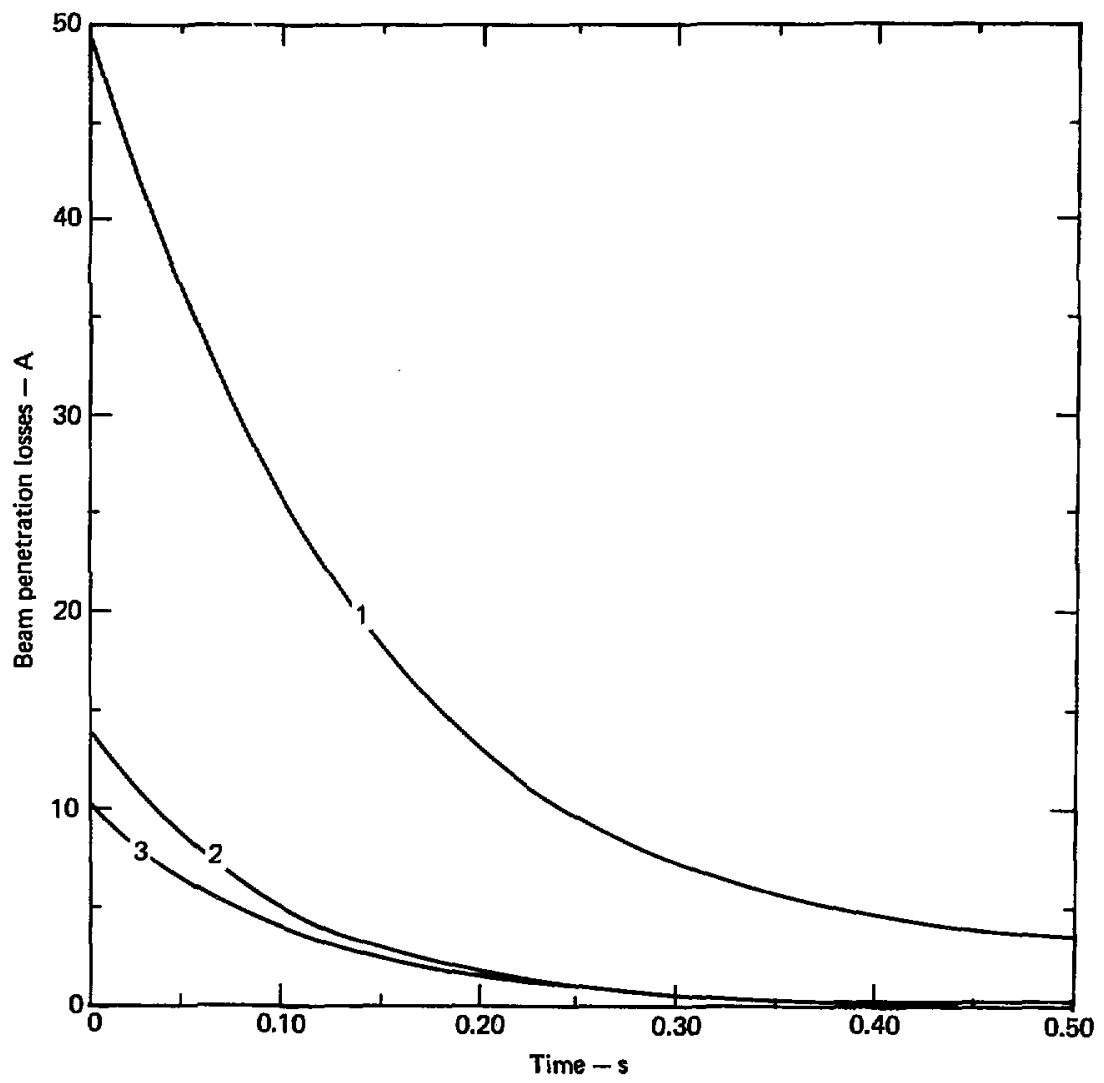

Fig. 5-21. Unabsorbed beam (equivalent) currents as a function of time. Same notation as Fig. 5-20. 


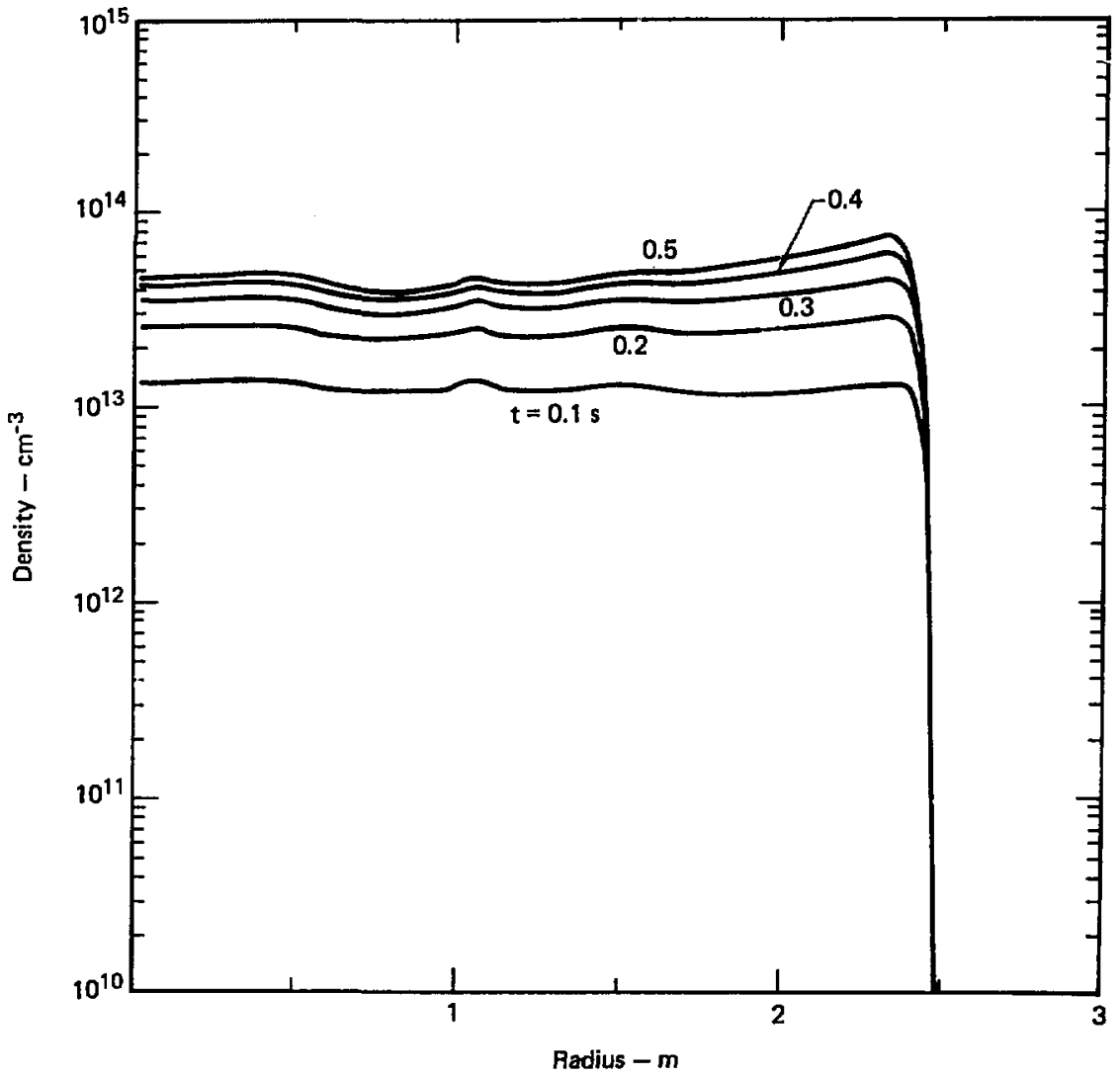

Fig. 5-22. Radial density profiles at $0.1,0.2,0.3,0.4$, and $0.5 \mathrm{~s}$. 


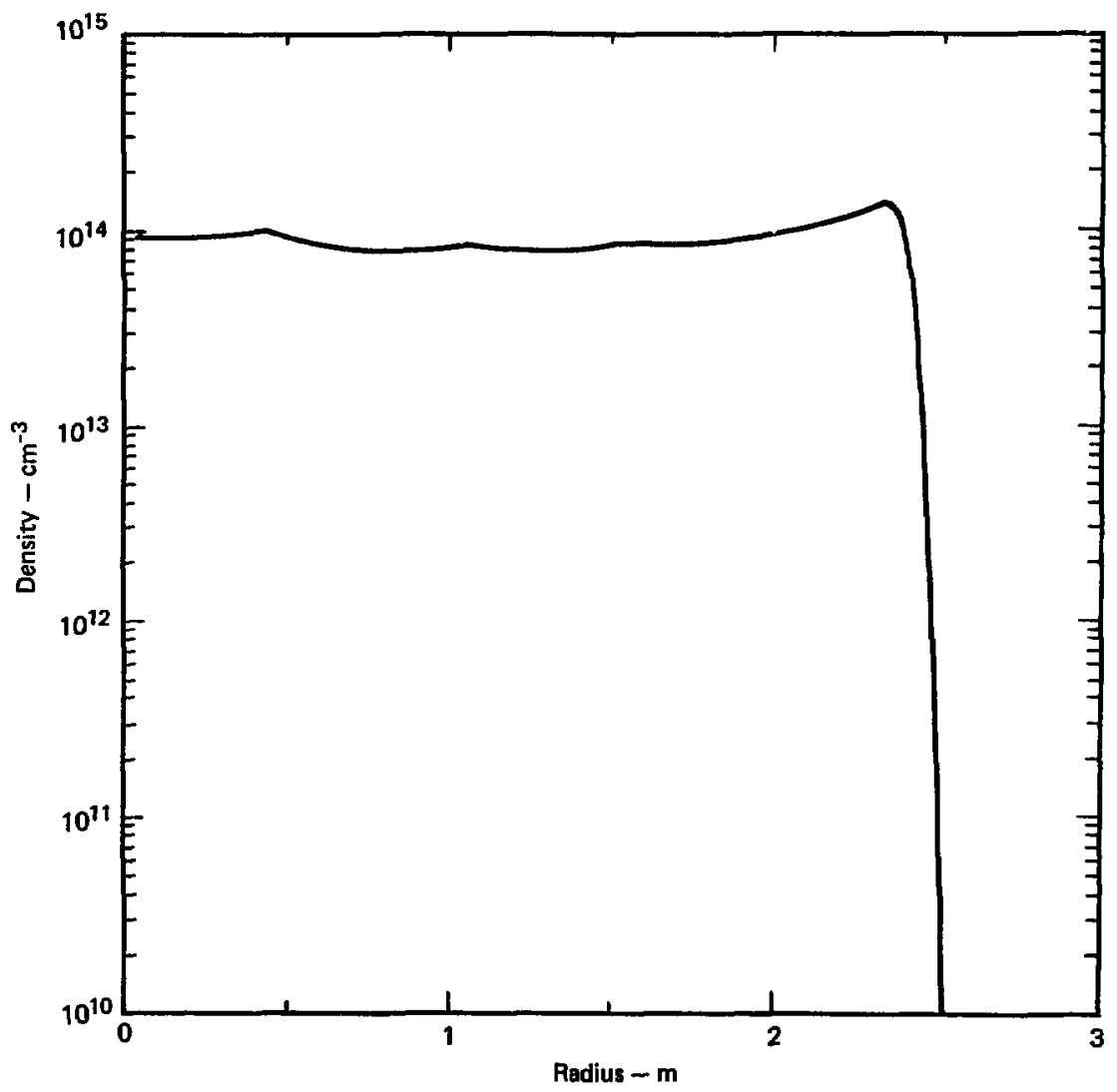

Fig. 5-23. Radial density profile at the end of start-up phase (t $>1.5 \mathrm{~s}$ ). 
Another quantity of interest in connection with startup is the charge-exchange off the incident beams. This is show in Fig. 5-24 (a,b) . prominent in Fig. 5-24(b) is the jump in losses when the target streaning plasma is terminated. The target plasma density of $3 \times 10^{13} \mathrm{~cm}^{-3}$ contributes appreciably to the attenuation of both the incident and charge-exchange beams.

The results of this chapter show that the density profiles can be made flat by proper aiming of the neutral beams, thus confining the DCLC instability to a thin boundary layer.

\subsection{Discussion}

In the previous chapters we have described how several tools are used to predict the behavior of a mirror reactor. Most of the parameters of the reactor have been collected in Table 5-1. We discuss here two additional effects that could be of importance and that will cone under study in the next large mirror experiments in MFTF.

An instability that might limit the axial length of a mirror machine is the convective loss-cone mode of Rosenbluth and Post. ${ }^{20}$ The presence of this mode could lead to enhanced velocity-space diffusion of ions. Estimates $^{25}$ indicate this mode could limit the length of a mirror reactor to $\approx 100 a_{i}$ to prevent excess wave amplification. However, the convective mode has never been fully identified in mirror machines. If the mode is to be a problem in mirror reactors, it should be apparent in MFTF.

Another instability, the Alfvén Ion Cyclotron (AIC) mode, has recently come under close scrutiny. 26 It is an electromagnetic mode that arises as a result of anisotropy in the distribution function or pressures, in contrast to the loss-cone modes that are electrostatic and arise because of the absence of low energy ions in the distribution function. Thus, the AIC instability cannot be suppressed by a stabilization stream, but could be eliminated, if it were a probler, by reducing the anisotropy of the pressures. Such a reduction is equivalent to reducing the permitted $\beta_{\perp}$. thus reducing the fusion power for a given magnetic field. 


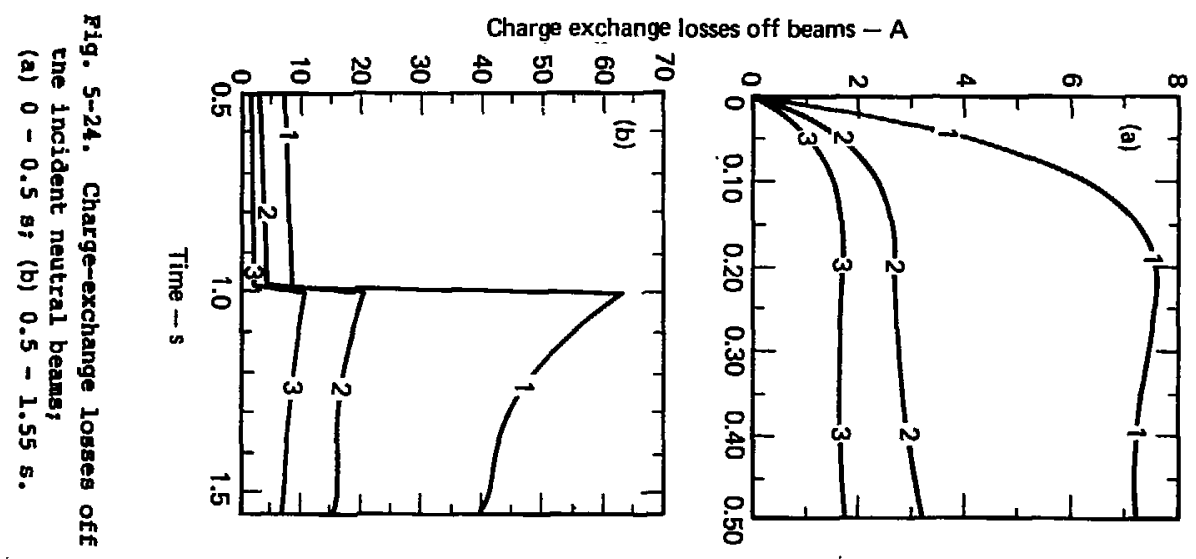


It is not yet clear that AIC instabilities will be of concern for reactor designs. They have not been obseryed in 2 XIIB, perhaps because of the small $\mathrm{L}_{\mathrm{p}} / \mathrm{a}_{i}$. Again, MFTP will permit this mode to be studied, if it is found to be present.

\section{Acknowledgments}

The calculation. described in Sec. 5.4 were carried out by John Boyd. Larry Hall offered helpful advice on the interpretation of the results. Some of the material in Sec. 5.5 was provided by Marvin Rensink. 


\section{References}

1. R. W. Moir, Ed., Standard Mirror Fusion Reactor Design Study, Lawrence Livermore Laboratory, Rept. UCID-17644 (1978).

2. R. W. Moir and C. E. Taylor in Proc. Cont. on Technology of Controlled Thermonuclear Fusion Reactors, Conf. 721111, p. 853 (1972).

3. D. E. Baldwin, Rev. Mod. Phys. 49, 317 (1977).

4. M. E. Rensink et al.. Proc. Intern. Conf. Plasma physics and Controlled Nuclear Fusion Res. 5th (IAEA, Vienna), vol. I, p. 311.

5. I. S. Hall, Phys. Fluids 15, 882 (1972).

6. I. S. Hall and B. McNamara, Phys. Fluids 18, 552 (1975).

7. H. Grad, Symp. in Appl. Nath 13, 162 (1967).

8. B. McNamara, Methods in Computational Physics 16, 211 (1976).

9. D. V. Anderson, J. Breazeal, and C. Sharp (to be published).

10. M. N. Rosenbluth and C. L. Longmire, Ann. Phys. 1, 120 (1957).

11. W. A. Newcomb, Large-Aspect-Ratio Equilibrium and Stability, Lawrence Livermore Laboratory, Rept. UCID-17182 (1976).

12. D. E. Baldwin and I. D. Pearlstein, Lawrence Livermore Laboratory, Internal Mernorandum (1977).

13. W. A. Perkins and D. FusB, MAFCO II-A Code for Calculating a Guidirg Center Trajectory of a Particle in Magnetic and Electric Fields, Iawrence Livermore Laboratory, Rept. UCRL-50438 (1968).

14. I. S. Hall, B. B. Hooper, and W. A. Newconb, Nucl. Fusion 15, 875 (1975).

15. J. Killeen, A. A. Mirin, and M. Rensink, "The Solution of the Rinetic Equations for a Multispecies Plasma," in Methods in Computational Physics, J. Killeen, Ed. (Academic Press, New York, 1976), vol. 16, p. 389.

16. T. A. Cutler, L. D. Pearlstein, and M. E. Rensink, Computation of the Bounce-Average Code, Lawrence Livermore Laboratory, Rept. UCRL-52233 (1977).

17. B. McNamara et al., "Theory of Mirror Machines at High Beta," in Proc. Intern. Conf. Plasma Physics and Controlled Nuclear Fision Res. 6th (IAEA, Vienna, 1977).

18. L. S. Hall, Electron Dynanics and Enhancement of 9 in Mirror Machines, Lawrence Livermore Laboratory, Rept. UCRL-78296 (1976).

19. R. H. Cohen and L. S. HaXI, Lawrence Livermore Laboratory, private comunication (1976). 
20. R. F. Post and M. N. Rosenbluth, Phys. Fluids 9, 730 (1966).

21. F. H. Coensgen et al., Phys. Rey. Lett. 35, 1501 (1975).

22. D. E. Baldwin, H. L. Berk and L. D. Pearlsteín, Phys. Rev. Lett. 36, 1051 (1976).

23. D. J. Rose and M. Clark, Plasmas and Controlled Fusion (MIT Press, 1961) p. 169.

24. B. W. Stallard, Radial Build Up Code for Neutral-Beam Injection into a Mirror Machine, Lawrence LIvermore Laboratory, Rept. UCRL-51784 (1975). R. S. Devoto, D. F. Faul and B. W. Stallard, 1978 IEEE International Conference on Plasma Science, Monterey (1978).

25. D. E. Baldwin et al., Present Status of Mirror Stabtlity Theory, Lawrence Livermore Iraboratory, Rept. UCID-17038, Rev. I (1976).

26. D. C. Hatson, L. D. Pearlstein, and L. L. Lodestro, Alfuén Ion-Cyclotron Instability in Mirror Machines, Lawrence Livermore Laboratory, Rept. UCID-17486 (1977). 


\section{FUSION COMPONENTS}

\subsection{Magnet Design}

The mirror plasma requires a minimum-B field configuration. In the present reactor design, this field is generated with the Yin-Yang conductor geonetry. The magnet is superconducting with an NbTi superconductor and copper stabilizer and is designed to a maximum field at the conductor of 8.5 T. The maximum current density in the bundle cross section is approxinately $1 \mathrm{kM} / \mathrm{cm}^{2}$; in the superconducting filaments it is $100 \mathrm{~A} / \mathrm{mm}^{2}$. The conductor cross section is sized to insure operation in the cryostatically stable regime.

The central field is $2.63 \mathrm{~T}$, and the mirror field is $5.95 \mathrm{~T}$, resulting in a vacuum mirror ratio of 2.26 and a coil efficiency (ratio of mirror field to maximum conductor field) of 0.70 . The coil is approximately $22 \mathrm{~m}$ in diameter, and the distance between mirror points is $13 \mathrm{~m}$. Analysis of the field with a three-dimensional $\mathrm{NWD}$ equilibrium code predicts a maximum plasma radius of $2.5 \mathrm{~m}$.

The reactor design reguired that the field at one mirror be 5 greater than the field at the other mirror, so as to create a selective leakage situation where the majority of the plasma leakage occurs through the weak mirror. This perturbation of the symmetric coil was not included in the magnet design.

\subsubsection{Magaet Desiga Considerations}

The main design constraints on the conductor bundle of a superconducting agnet are stability and protection. Stability refers to the situation where the superconductor temporarily reverts to a normal (nonsuperconducting) state, and the current jumps into the copper, causing joule heating. To mintain stability, the heat flux from the surface of the copper conductor must be lower than the value that will cause nucleate boiling of the liquid helium coolant. Boiling the helium will result in a comparativeIy low heat-transfer rate and will heat the conductor above its critical temperature. Protection deals with loss of conductor cooling due to, for exanple, blockage of a coolant channel. In this condition, the copper will heat up and cause the magnet to quench. The design of the magnet circuitry nust be such that the current can he dropped to zero before the windings are danaged by excessive temperature. In the following aiscussion, we focus on 
stability design considerations. Magnet protection is discussed in section 6.1 .6 .

\subsubsection{Conductor Design}

As a representative conductor design, we use the conductor (Fig. 6-1) proposed for the MX magnet ${ }^{l}$; it consists of three bars of stabilizing copper adjacent to a bar of copper containing the NbTi superconducting filaments. A cross section of the conductor with its associated turn-to-turn insulation, and its defining dimensions are shown in Fig. 6-2.

For cryostatic stability, the heat flux occurring when the current flows in the copper must be less than the critical heat flux $\dot{q}_{c}^{N}$ for the onset of nucleate boiling. This may be expressed as

$$
\frac{I^{2} R / L}{s / L}<\dot{q}_{c}^{n}
$$

where $I^{2} R / L$ is the joule heating in the copper per unit length and $s / L$ is the heat transfer area per unit length. Now using the relations $I=4 w^{2} j_{C u^{\prime}}, R / L=p / 4 w^{2}$, and $s / L=(4)(4 w) f_{q^{\prime}}$, where $w$ is defined in Fig. 6-2, $j_{\mathrm{Cu}}$ is the current density in the copper, $\rho$ is electrical resistivity, and $f_{q}$ is the fraction of the total surface that is available for heat transfer. With these, Eq. (1) is written as

$$
\frac{\rho w j_{C u}^{2}}{4 f_{q}}<\dot{q}_{c}^{n}
$$

The current density $j_{\mathrm{Cu}}$ denotes abnormal operation, $i . e .$, when the superconductor has gone normal, and the current has jumped into the copper. To relate $j_{\mathrm{Cu}}$ to the design operating condition (where the current flows in the superconductor), we have the relation

$$
j_{b}=j_{s c} f_{s c}=j_{c u} f_{c u}
$$

where $j_{b}$ is the bundle current density (that is, the current carried by the conductor divided by the total cross section $\left.I / h^{2}\right), j_{g c}$ is the current density when the current is carried by the NbTi, and $f_{s c}$ and $f_{C u}$ are the area fractions of superconductor and copper, respectively. Because 


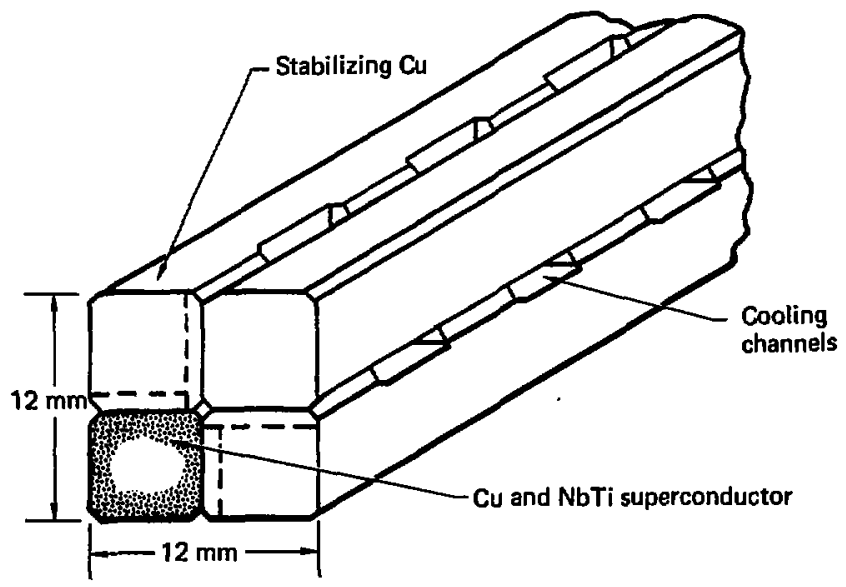

Fig. 6-1. Conductor design proposed for MX magnet. ${ }^{1}$

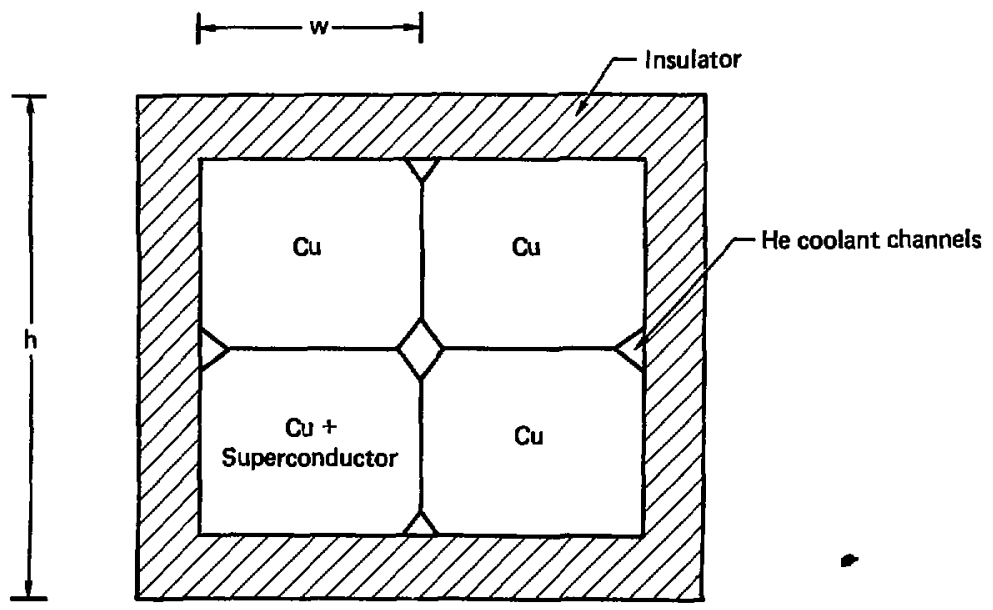

Fig. 6-2. Conductor cross section. 


$$
f_{S C}+f_{C u}+f_{H e}+f_{i}=1 \text {, }
$$

where $f_{H e}$ and $f_{i}$ are the area fractions for the helium coolant and insulation, respectively, we obtain the relation

$$
j_{C u}=\frac{j_{b}}{1-f_{i}-f_{H e}-j_{b} / j_{s c}} .
$$

Typically, $f_{i} \simeq 0.1$ and $f_{H e} \simeq 0.3$

Our next step is to select a current density for the superconductor. Here we refer to Fig. 6-3, which shows the critical current density in filamentary $\mathrm{NbTi}$ as a function of temperature and magnetic induction. ${ }^{2}$

Large, complex superconducting magnets like those required for the present reactor design will use forced convection cooling, and, thus, the liquid helium coolant will experience a pressure drop during its passage through the magnet. If we require the outlet pressure from the magnet to be $1 \mathrm{~atm}$, the inlet pressure will be above $1 \mathrm{~atm}$, and the inlet temperature will be greater than $4.2 \mathrm{~K}$. A design value for the helium coolant temperature of $4.5-5.0 \mathrm{~K}$ has been recommended. ${ }^{3}$ Therefore, from Fig. 6-3 we select

$$
\begin{aligned}
B_{c} & =8.5 \mathrm{~T}, \\
j_{s c} & =100 \mathrm{~A} / \mathrm{mm}^{2},
\end{aligned}
$$

where $B_{C}$ is the maximum field occurring in the magnet windings.

The conduction winding design described in sec. 6.1.3 resulted in a maximum bundle current density of $j_{b}=1057 \mathrm{~A} / \mathrm{cm}^{2}$. Using Eq. (5) yields the values

$$
\begin{aligned}
& j_{\mathrm{Cu}}=2138 \mathrm{~A} / \mathrm{cm}^{2}, \\
& f_{\mathrm{sc}}=0.106, \\
& f_{\mathrm{Cu}}=0.494 .
\end{aligned}
$$

The heat flux at the surface of the copper, when current is flowing in it, is

$$
\dot{q}^{n}=\frac{\rho w j_{\mathrm{Cu}}^{2}}{4 f_{q}} .
$$




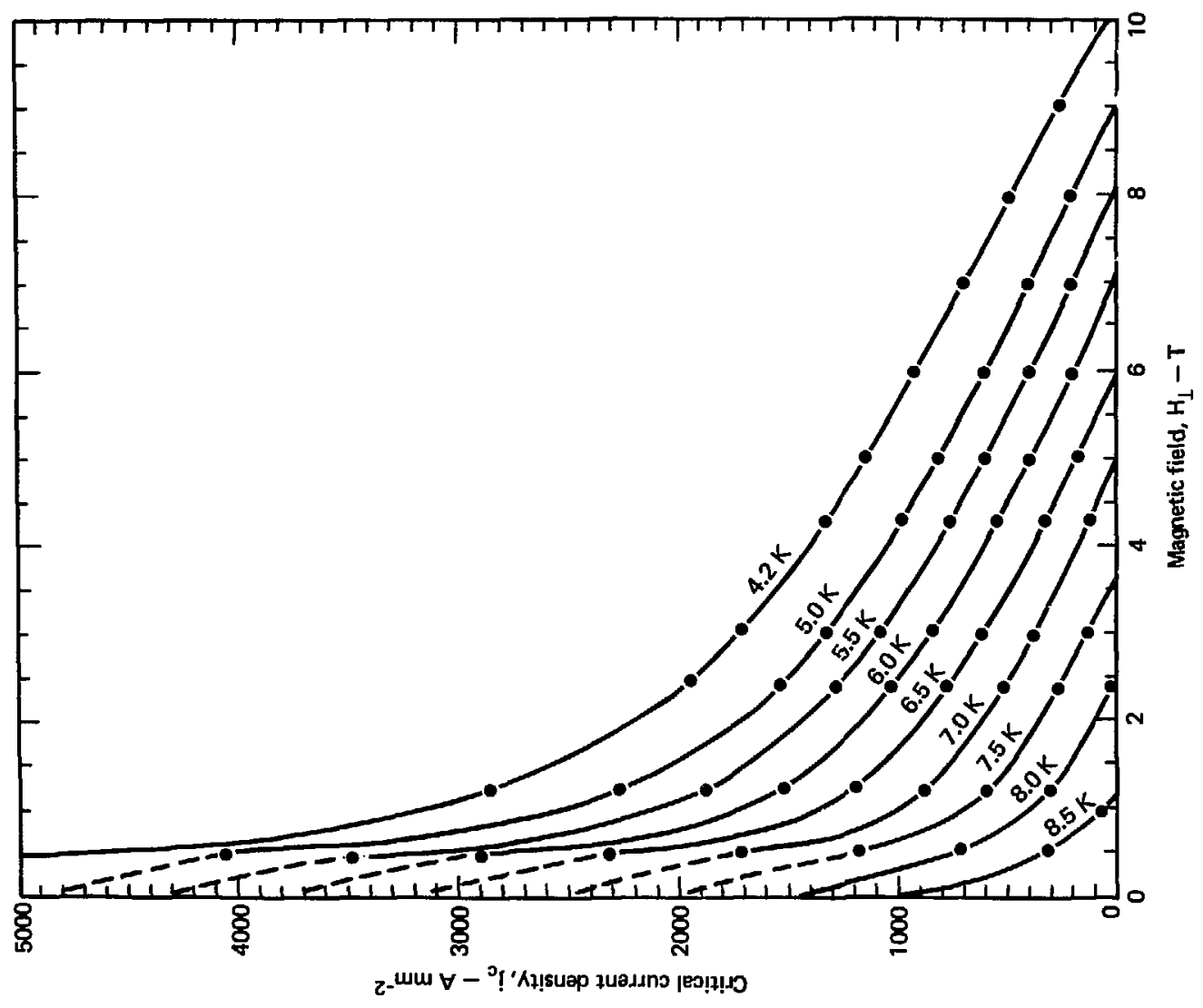

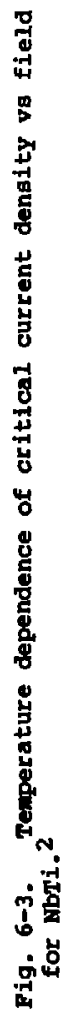


Using $w=0.6 \mathrm{~cm}($ Fig. $6-1), \rho=3 \times 10^{-8} \Omega-\mathrm{cm}$ at $8 \mathrm{~T}$ and $4 \mathrm{~K}$ for unirradiated copper ${ }^{4}$ (Fig. 6-4), and $f_{q}=0.5$ gives a heat flux of $\dot{q}_{2}^{n}=0.0412 \mathrm{w} / \mathrm{cm}^{2}$. Because the critical heat $f l u x$ is $q_{c}^{n}=0.1-0.2 \mathrm{~W} / \mathrm{cm}^{2},{ }^{7}$ we conclude that the conductor design is cryostatically stable. As the resistivity of the copper increases by only a factor of about 10 at a (comparatively high) neutron fluence of $10^{19} \mathrm{n} / \mathrm{cm}^{2}, 4$ adequate neutron shielding can insure the cryostability of the winding throughout the reactor lifetime.

parameters for the conductor design are summarized in Table 6-1.

\subsubsection{Winding Configuration and Fields}

The winding geometry is based on the Yin-Yang design, ${ }^{5}$ which produces a minimum-B magnetic well. Perspective views of the magnet windings are shown in

Table 6-1. Conductor parameters.

Superconductor

Stabilizer

Material volume fractions

NbTi

Cu

IHe

Insulator

Cu/superconductor ratio
NoTi

OFHC Cu

0.494

0.3

0.1

$4.62 / 1$

Superconductor environment

Maximum field

8.5 T

Coolant temperature

$4.5-5.0 \mathrm{~K}$

currents (maximum)

NbTi current density

$100 \mathrm{~A} / \mathrm{mm}^{2}$

Cu current density

Bundle current density

$2138 \mathrm{~A} / \mathrm{cm}^{2}$

Current per turn

$1057 \mathrm{~A} / \mathrm{cm}^{2}$

$1.69 \mathrm{kA}$

$\mathrm{I}^{2} \mathrm{R}$ loss in $\mathrm{Cu}$

$0.20 \mathrm{~W} / \mathrm{cm}$

Heat-transfer rate

$0.041 \mathrm{H} / \mathrm{cm}^{2}$

Dimension (Fig. 6-2)

width

$0.6 \mathrm{~cm}$

height 


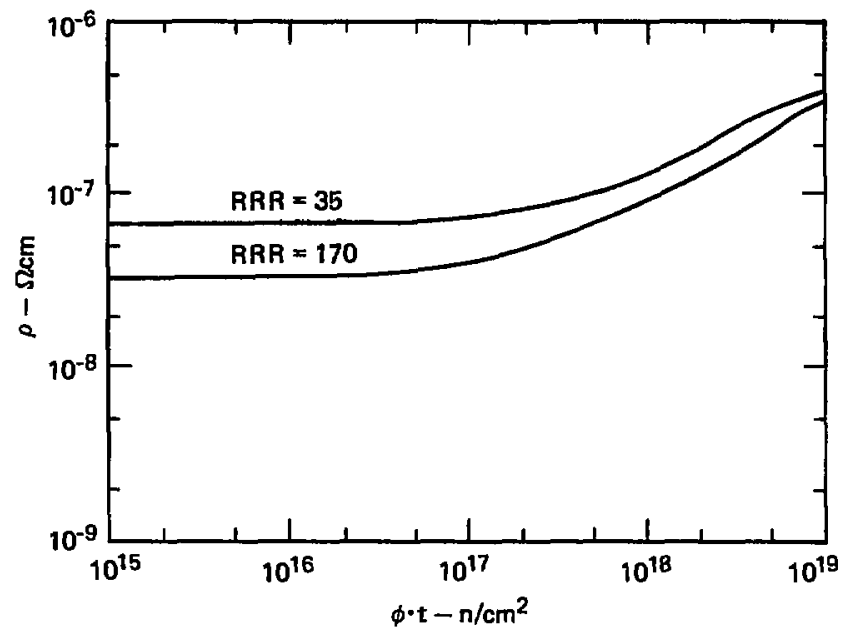

Fig. 6-4 Resistivity $\rho$ of copper at $4.2 \mathrm{~K}$ and $8 \mathrm{~T}$ as a function of neutron flux $\phi^{\circ} t$. Residual Resistivity Ratio (RRR) = $\rho(T=273 \mathrm{~K}) / \rho(T=4.2 \mathrm{~K}) . \quad($ Ref. 4) 
Fig. 6-5, and the winding dimensions are shown in Fig. 6-6. Some modifications to the basic Yin-Yang configuration have been made to accomodate reactor design constraints. The conductor bundle has been split into an inner or "mirror" coil and an outer or "main" coil. This split bundle moves the mirror point very near the inside diameter of the coil, which results in high fractional blanket coverage. The mirror coil has also been "spread" in the turnaround (minor arc) to ameliorate field concentration in this region. The main arc of the coil sweeps through a $75 \mathrm{deg}$ half-angle, as compared to the 90 deg half-angle in the basic Yin-Yang design; this was done to permit better access to the plasma region for the neutral-bean injectors.

Magnetic-field calculations were performed with the EFFI code, ${ }^{6}$ which models the current-carrying conductor as finite-size elements with a uniform current density and permits calculation of the field inside the conductor bundle. The coordinate system for the coil is shown in Fig. 6-7.

The coil has an outside diameter of $22.4 \mathrm{~m}$. The major arc of the mirror coil has a cross section of $1 \times 1 \mathrm{~m}$ and a bundle current density of $1057 \mathrm{~A} / \mathrm{cm}^{2}$ of the midpoint of the arc. The mirror coil expands to $1 \times 1.75 \mathrm{~m}$ cross section in the minor arc or turnaround. The main coil has a constant cross section of $3.7 \times 1.55 \mathrm{~m}$ with a bundle current density of $673 \mathrm{~A} / \mathrm{cm}^{2}$. These rather low current densities resulted from using large bundle cross sections to obtain a high coil efficiency $n_{c}$, where

$$
\eta_{c}=\frac{\text { field at the mirror point }}{\text { maximum field at the conductor }} \text {. }
$$

In the present design, a value of $\eta_{c}=5.95 / 8.49=0.701$ was achieved. Mod-B contours at the midpoint of the major arcs of the conductor bundles are shown in Figs. 6-8 and 6-9. The contours have been limited to the regions of maximum field concentration. For reference, the locations of the bundle cross sections used in Figs. 6-8 through 6-11 are indicated in Fig. 6-7. In Figs. 6-10 and 6-11, the mod-B contours at the midpoint of the minor arcs of the mirror and main coils are shown. The maximum field at the midpoint of the major arcs of the two coils increases from $7.79 \mathrm{~T}$ and $6.66 \mathrm{~T}$ (mirror and main coil, respectively) to a uniform value of $8.49 \mathrm{~T}$ at the minor arc midpoints. The smaller increase in the field in the mirror coil was achieved by spreading the windings in the minor arc.

A sumary of the winding parameters appears in Table 6-2. 


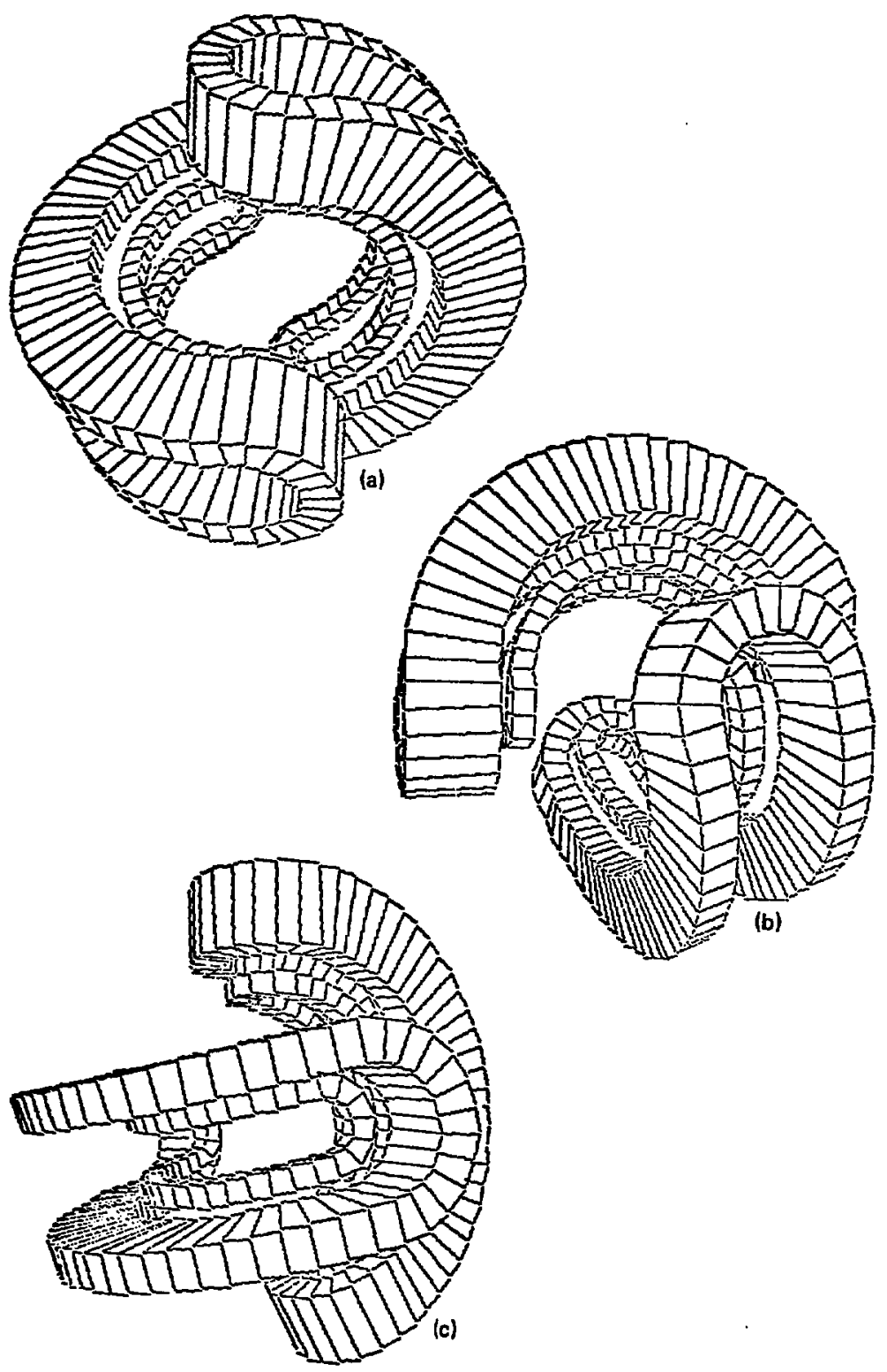

Fig. 6-5. Magnetic windings. 


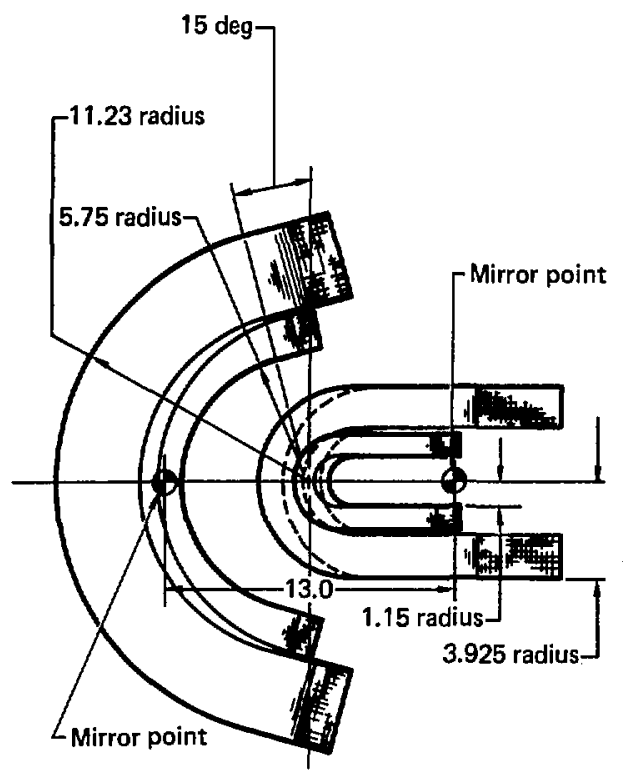

Fig. 6-6. Magnetic winding dimensions.

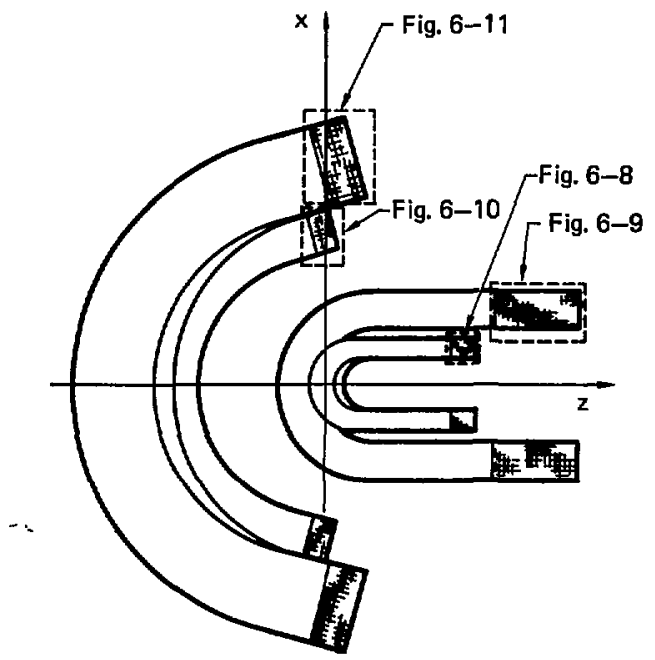

Fig. 6-7. Magnetic coordinate system and field plot locations. 


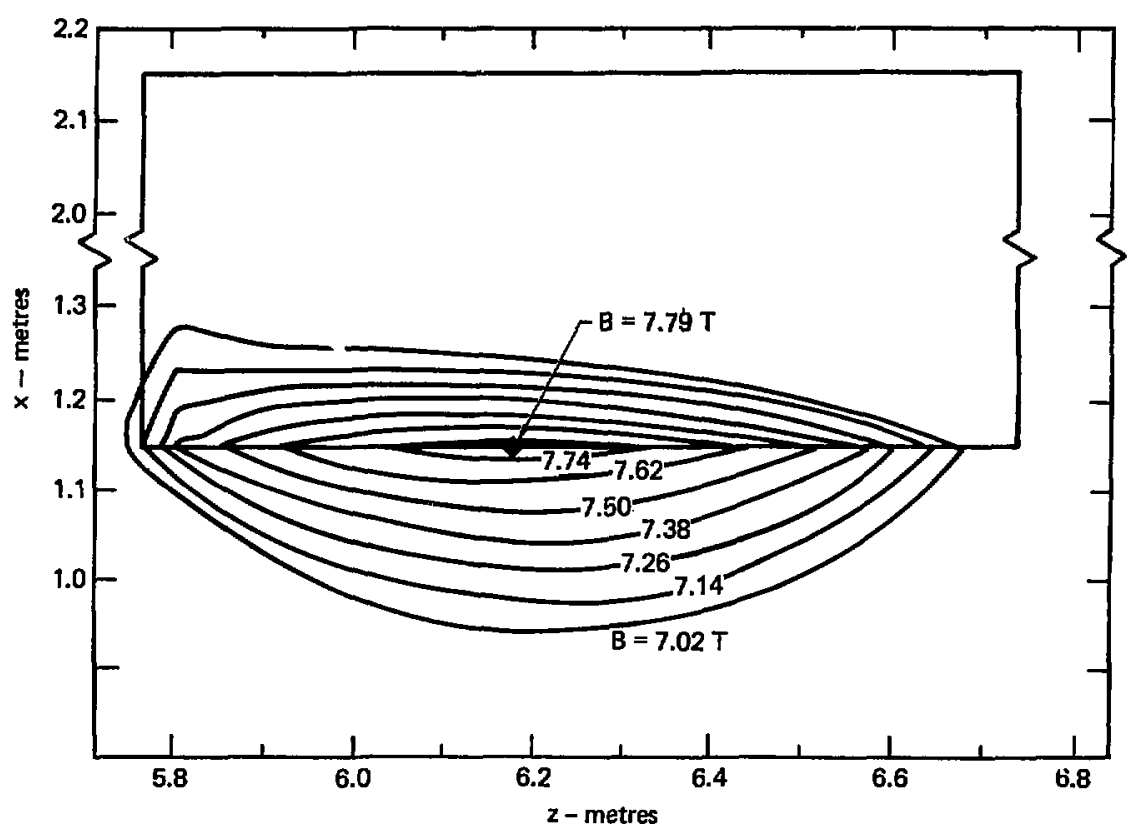

Fig. 6-8. Mod-B contours in the cross section of the major arc of the mirror coil (positive $x-y$ plane). 


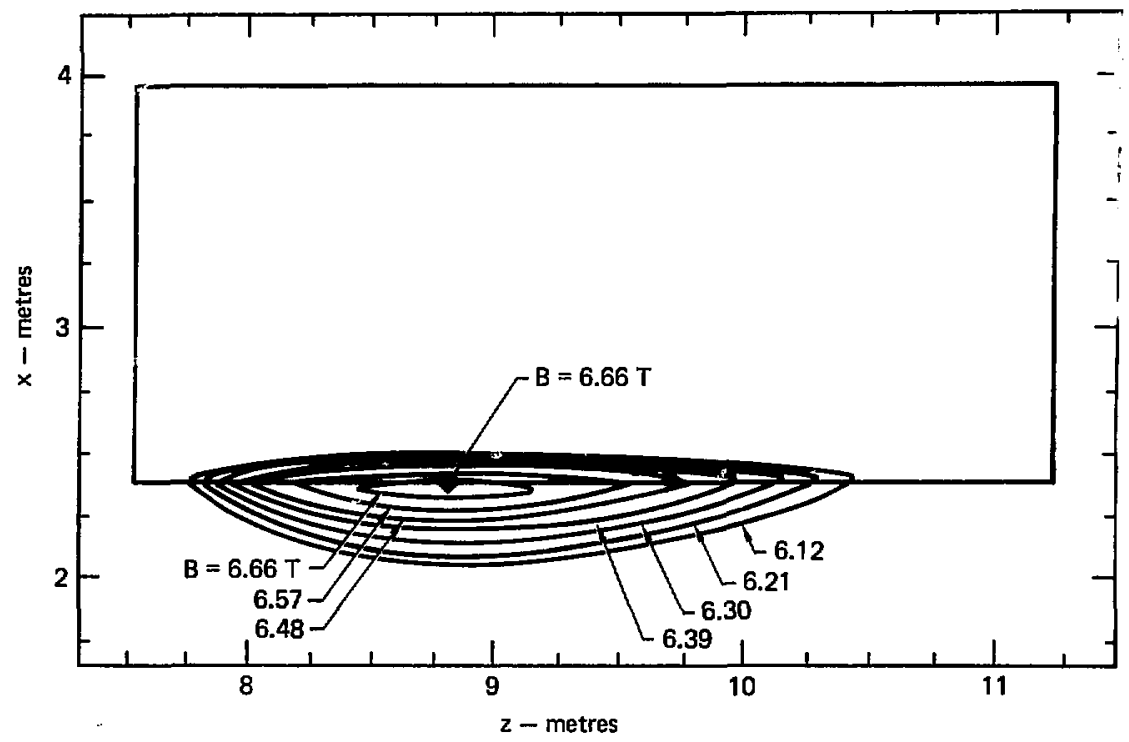

Fig. 6-9. Mod-B contours in the cross section of the major arc of the main coil (positive $x-y$ plane). 


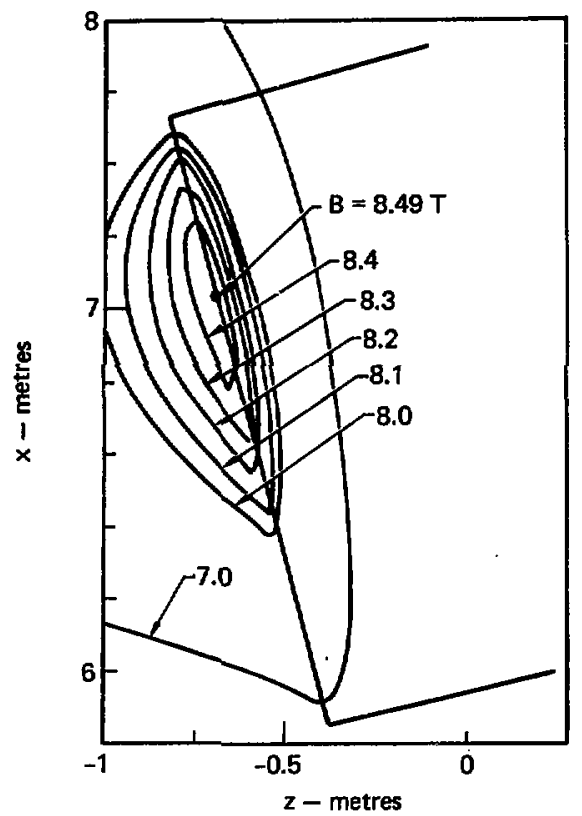

Fig. 6-10. Mod-B contours in the cross section of the minor arc of the mirror coil (negative $x-y$ plane).

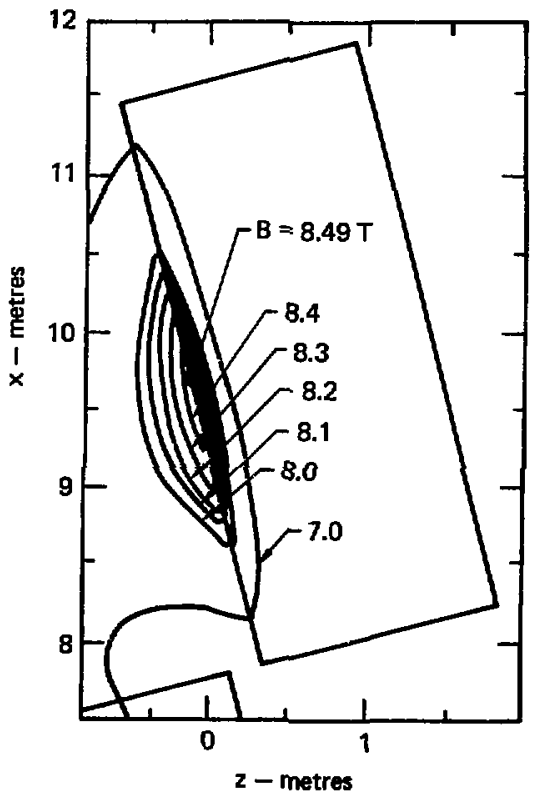

Fig. 6-11. Mod-B contours in the cross eection of the minor arc of the main coil ( $x-y$ plane). 
Table 6-2, Magnet winding paraneters.

\begin{tabular}{|c|c|c|}
\hline & $\begin{array}{l}\text { Mirror } \\
\text { coil }\end{array}$ & $\begin{array}{l}\text { Main } \\
\text { coil }\end{array}$ \\
\hline Major arc radius, ${ }^{a} \mathrm{~m}$ & 6.25 & 9.38 \\
\hline Minor arc radius, ${ }^{a} \mathrm{~m}$ & 1.65 & 3.15 \\
\hline Bundle width, ${ }^{b, c} \mathrm{~m}$ & $1.00(1.75)$ & 3.70 \\
\hline Bundle height, $m$ & 1.00 & 1.55 \\
\hline Maximum bundle current density, $\mathrm{A} / \mathrm{cm}^{2}$ & 1057 & 673 \\
\hline Amp-turns/coil ${ }^{d}$ & $1.06 \times 10^{7}$ & $3.46 \times 10^{7}$ \\
\hline Current per turn, $\mathrm{A}$ & 1691 & 1076 \\
\hline No. of turns/coil ${ }^{\mathrm{d}}$ & 6254 & 35844 \\
\hline Length/turn, ${ }^{d} \mathrm{~m}$ & 43.1 & 68.9 \\
\hline Anp-meters/coil ${ }^{\mathrm{d}}$ & $4.55 \times 10^{8}$ & $2.39 \times 10^{9}$ \\
\hline Maximun fields, $T:$ & & \\
\hline midpoint of major arc & 7.79 & 6.66 \\
\hline sidpoint of minor arc & 8.49 & 8.49 \\
\hline Total conductor leng th/coil, ${ }^{d} \mathrm{~km}$ & 270 & 2470 \\
\hline Bundle volume/coil, ${ }^{d} m^{3}$ & 64.5 & 395 \\
\hline Bundle mass/coil, ${ }^{d}$ tonne & 220 & 1990 \\
\hline Coolant volume/coil, ditres & $19.4 \times 10^{3}$ & $119 \times 10^{3}$ \\
\hline
\end{tabular}

$a_{\text {At }}$ center of bundle cross section.

binension parallel to $z$ axis at midpoint of major arc.

Chidth of the mirror coil expands from $1.0 \mathrm{~m}$ at midpoint of major arc to $1.75 \mathrm{~m}$ in the minor arc.

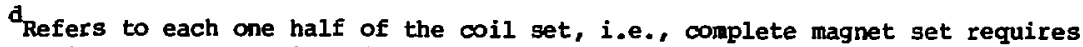
two nirror and two main coils.

\subsubsection{Confinement Field}

The magnet produces a central field of $2.63 \mathrm{~T}$ and a mirror field of $5.95 \mathrm{~T}$ for an axial (vacuum-field) mirror ratio of $R_{v}=2.26$. The wirror point is located $6.5 \mathrm{~m}$ from the center for a mirror-to-mirror length of $13.0 \mathrm{~m}$. The field intensity along the z-axis is plotted in Fig. 6-12 (symmetric about the $x-y$ plane).

Hod-B contours and fields lines in the $x-z$ plane are show in Fig. 6-13, and mod-B contours in the $r-2$ plane rotated 45 deg from the $x-2$ plane are shown in Fig. 6-14. (Both of these plots are symetric about the $z$ axis.) In 


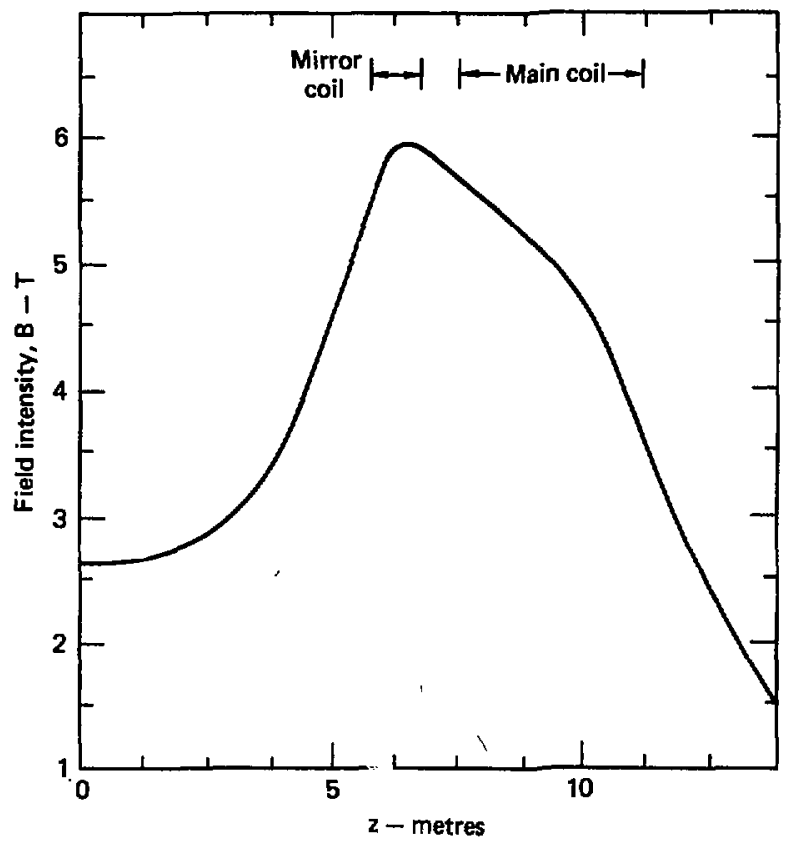

Fig. 6-12. Field intensity along the $z$ axis (symetric about the $x-y$ plane). 


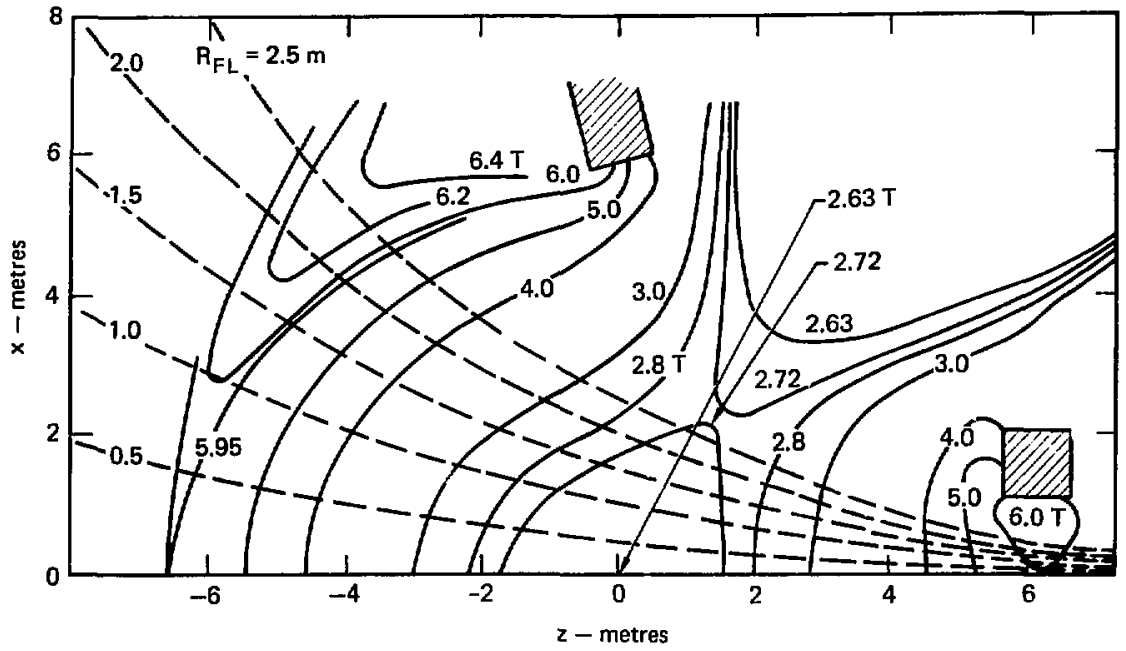

Fig. 6-13. Confinement field mod-B contours and field lines in the $x-y$ plane.

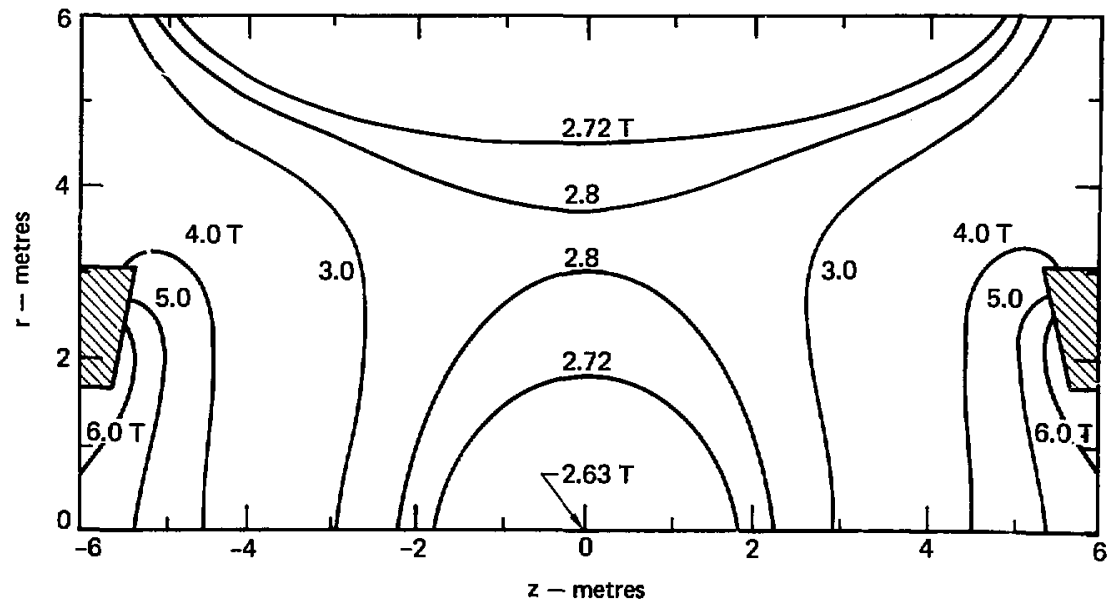

Fig. 6-14. Confinement field mod-B contours in an $r-z$ plane (rotated 45 deg from the $x-z$ plane). 
the $x-z$ plane, mod-B contours are closed out to a field of $2.72 \mathrm{~T}$, giving a radial mirror ratio of $R_{v}=2.72 / 2.63=1.034$. The field lines are labeled by their radius at the midplane $(z=0) R_{F L^{-}}$In $x-z$ plane, field lines lie entirely in this plane. The field along two of the field lines $\left(R_{F L}=0\right.$, $2.5 \mathrm{~m}$ ) are shown in Fig. 6-15. In the 45-deg plane (Fig. 6-14), the mod-B contours are closed to a field of $2.8 \mathrm{~T}$, greater than in the $x-z$ plane. Here, field lines are not shown because none lie entirely in the plane.

The outermost field line in the $x-z$ plane that passes through the region of closed mod-B contours corresponds to a radius of 2.5 to $3.0 \mathrm{~m}$. An empirical condition in the determination of plasma volume is that plasma can be stably confined on field lines that pass through the region of closed mod-B contours. Analytically, the condition for stable confinement is that $\int \mathrm{dl} / \mathrm{B}$ along the field line (where the limits of integration are taken from one mirror point to the other) decreases as $R_{F L}$ increases. These integrals are plotted in Fig. 6-16 for the field lines in the $x-z$ plane. It can be seen that $\int \mathrm{dl} / \mathrm{B}$ decreases out to $\mathrm{R}_{\mathrm{FL}}=2.5 \mathrm{~m}$, implying stability for these field lines. The analysis using a three-dimensional, finite- $\beta$ MFD stability code (See Sec. 5.4) also predicted a maximum plasma radius of $2.5 \mathrm{~m}$. The plasma radius (in the midplane, $2=0$ ) is related to the vacuum field line that forms the radial plasma boundary by the relation

$$
R_{F L}=R_{p}(1-\beta)^{1 / 4} \text {, }
$$

where $R_{p}$ is the plasma radius. Using $R_{p}=2.5 \mathrm{~m}$ and $B=0.7$, we obtain $R_{F L} \times 1.85 \mathrm{~m}$. The field envelope for the plasma in the $x-z$ plane is plotted in Fig. 6-17. The plasma volume can be calculated from

$$
v_{p}=\pi R_{p}^{2} L_{p} G^{0}
$$

where $L_{p}$ is the plasma length (mirror-to-mirror distance) and $\mathrm{G}^{0}$ is a volumetric shape factor, ${ }^{5}$ equal to $\sim 0.55$ for a plasma mirror ratio of 4.15 , giving a plasma volume of $140 \mathrm{~m}^{3}$. Confinement field parameters are listed in Table 6-3.

The mod-B contours outside the magnet are plotted in Fig. 6-18. The field along the $z$ axis is shown in Fig. 6-19 and decreases to a value of $0.01 \mathrm{z}$ at a distance of about $60 \mathrm{~m}$ from the center of the magnet. For regions outside those shown in Fig. 6-18, the far field may be approximated by the dipole field expressions ${ }^{7}$ 


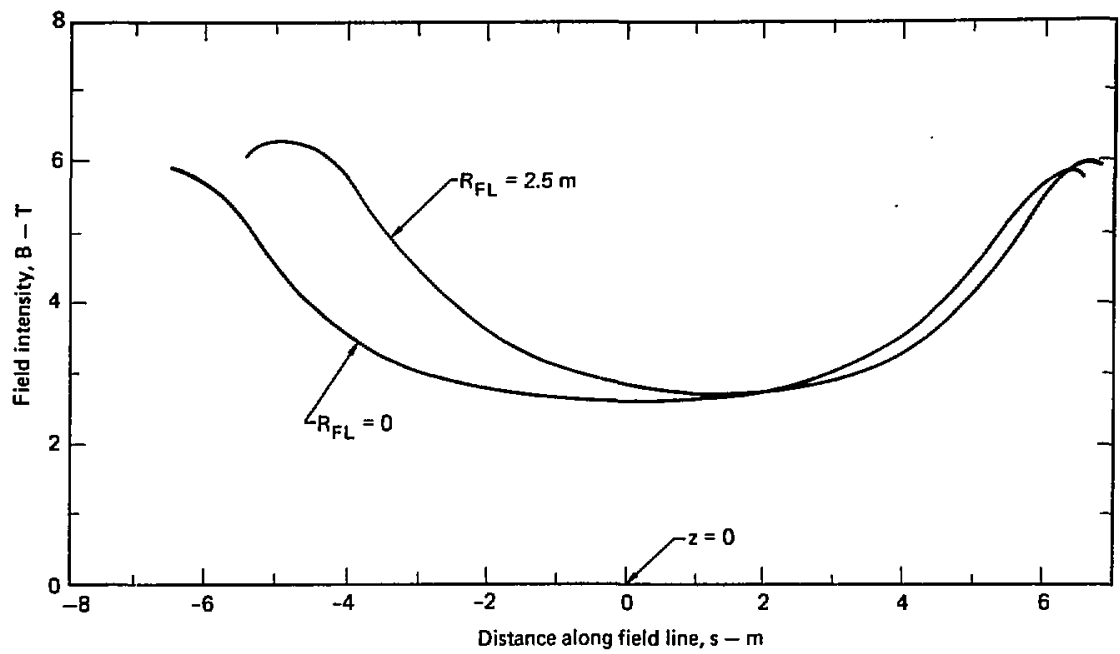

Fig. 6-15. Field intensity on field lines in the $x-z$ plane.

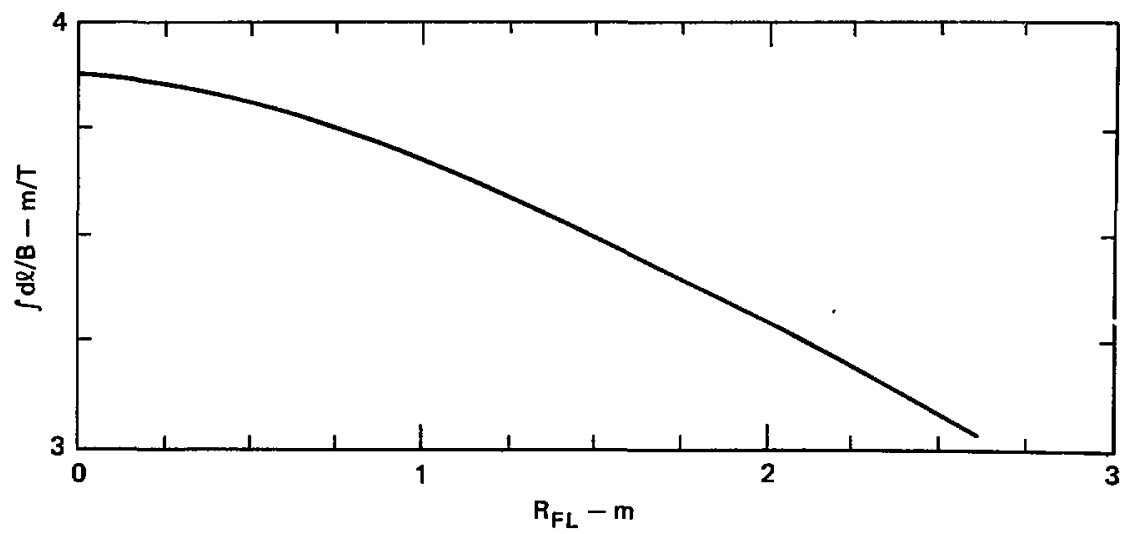

Fig. 6-16. Stability integral for field lines in the $x-z$ plane. 


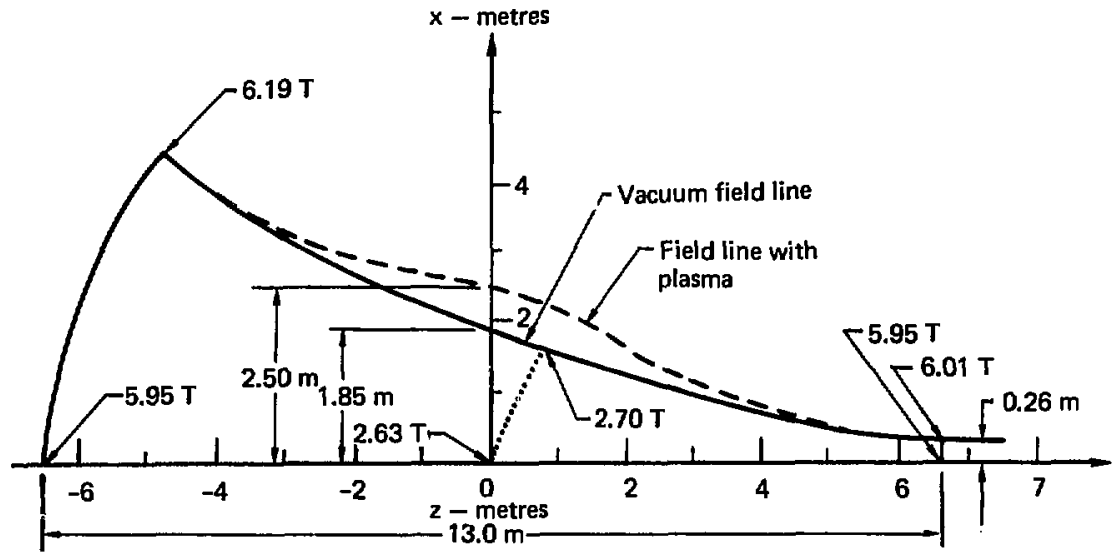

Fig. 6-17. Field envelope for $2.5 \mathrm{~m}$ plasma. (Field Intensities are vacuum values.)

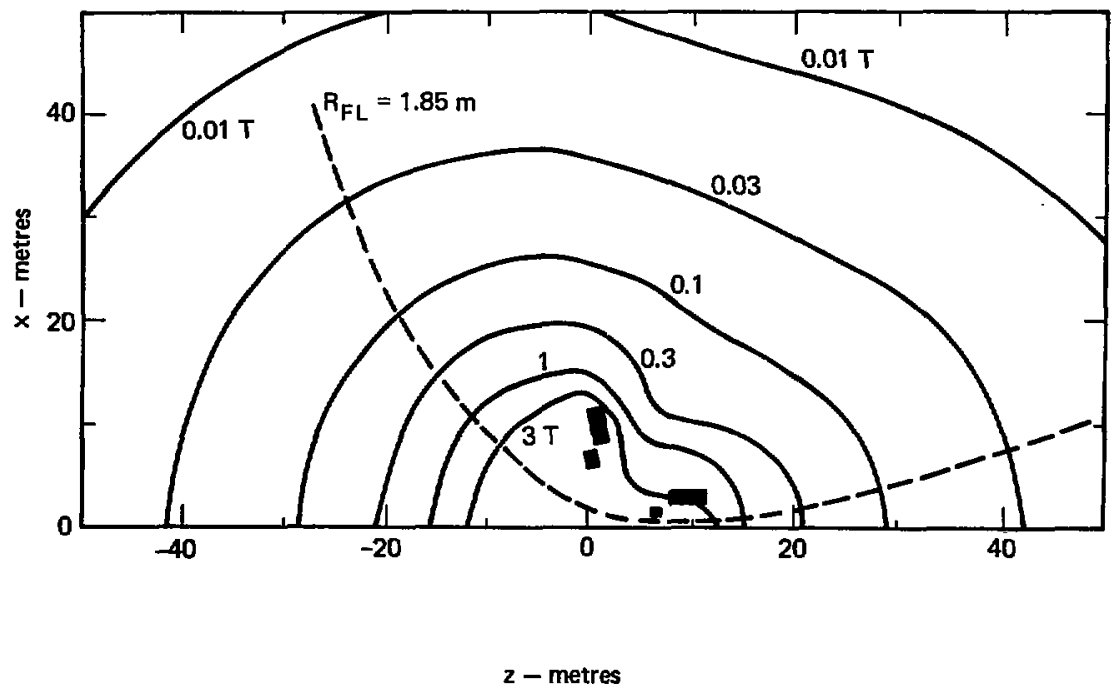

Fig. 6-18. Mod-B contours outside the magnet. 


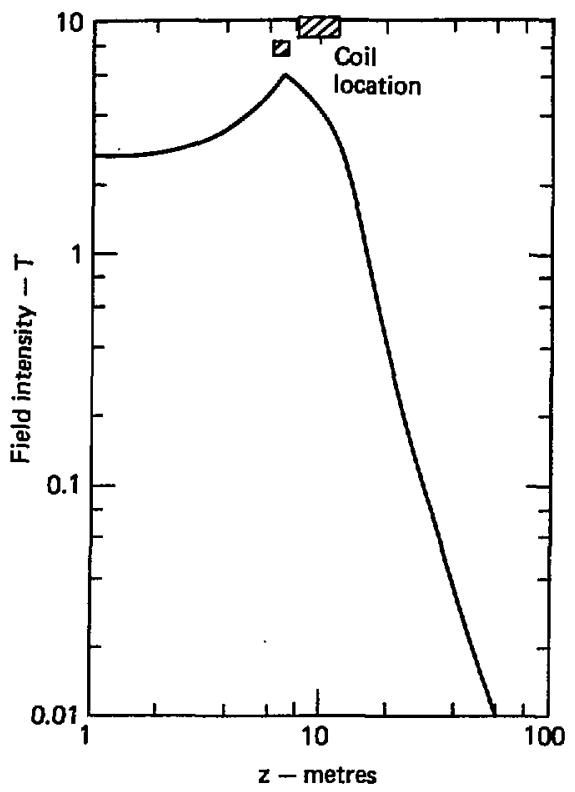

Fig. 6-19. Field intensity along the $z$ axis showing far-field region. 
Table 6-3. Confinenent field parameters.

Central field

Mirror field (on axis)

Axial mirror ratio

Radial mirror ratio

Radius of plasma boundary at midglane:

Vacuum

Plasma

Kirror-to-mirror distance

Plasma volune ( $R_{p}=2.5 \mathrm{~m}$ )
$2.63 \mathrm{~T}$

$5.95 \mathrm{~T}$

2.26

1.03

$1.85 \mathrm{~m}$

$2.50 \mathrm{~m}$

$13.0 \mathrm{~m}$

$140 \mathrm{~m}^{3}$

$$
\begin{aligned}
& B_{r}=\frac{\mu_{0}}{4 \pi} \times \frac{2 m}{r^{3}} \cos \theta, \\
& B_{\theta}=\frac{\mu_{0}}{4 \pi} \times \frac{m}{r^{3}} \sin \theta, \\
& B_{\phi}=0 .
\end{aligned}
$$

Here, spherical coordinates have been used; $m$ is the magnetic moment, and $\theta$ is the angle relative to the $z$ axis.

\subsubsection{Coil Forces and Magnet Structure}

The magnetic forces on the conductor bundles were also calculated with the EFFI code. 6 The coordinate system used to express the coil force distribution is shown in Fig. 6-20. The forces have two components perpendicular to the two faces of the bundle cross section (perpendicular to the current vector), and the torque about the axis of the arc is defined as positive in the direction of the current flow. The angular location $\alpha$ of the forces is measured from the transition point, $i, e$, where the major and minor arcs meet; $a$ is greater than zero in the major arc and less than zero in the winor arc.

The force distribution on the conductor bundles is shown in Fig. 6-21, where the quantity plotted is "coil case pressure" (the force per unit length divided by the bundle cross-sectional dimension perpendicular to the force). It is the average pressure transmitted to the coil case by the conductor. The torques on the bundies are plotted in Fig. 6-22. The net force on the 


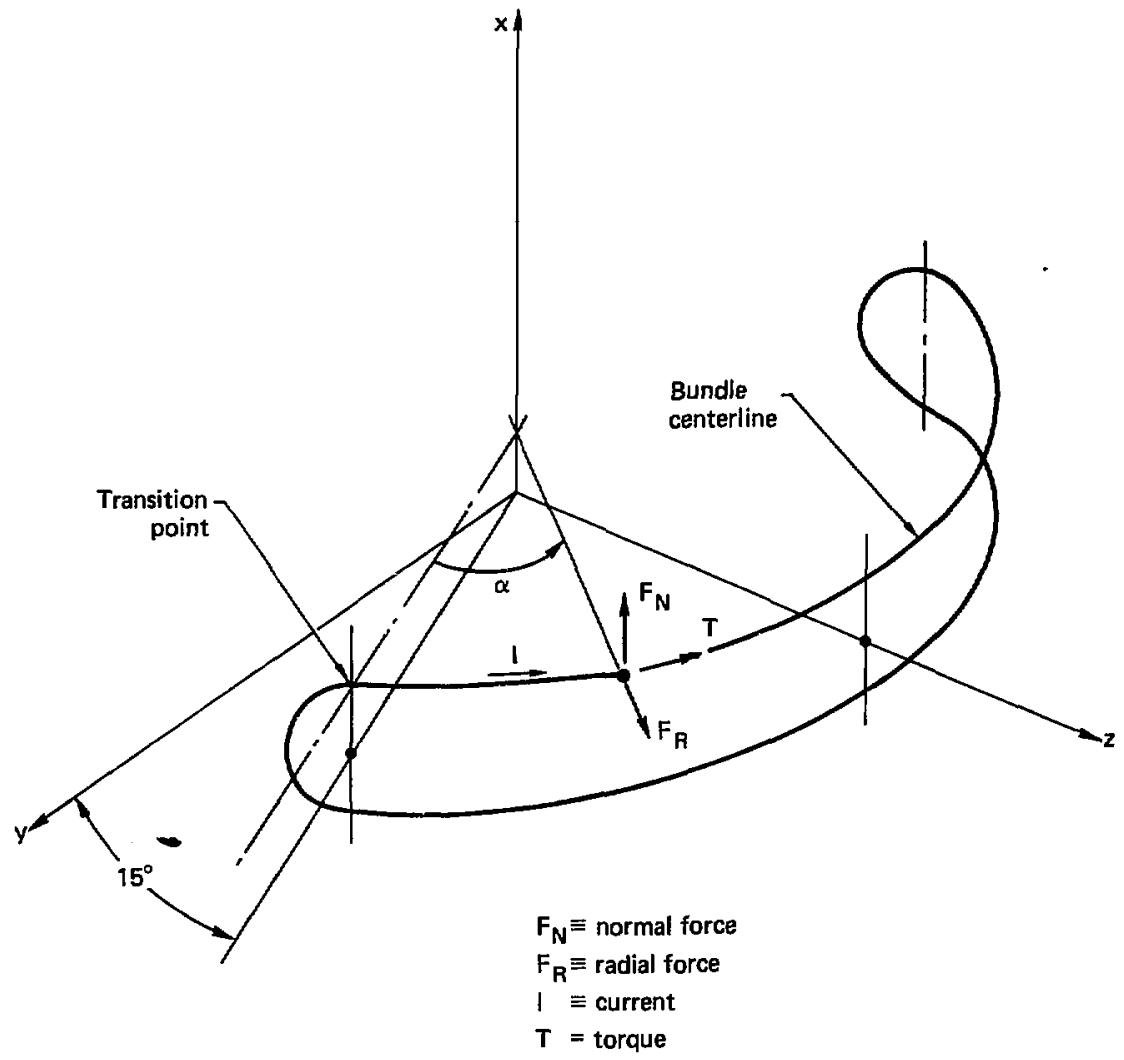

Fig. 6-20. Coordinate system for magnet force distribution. 


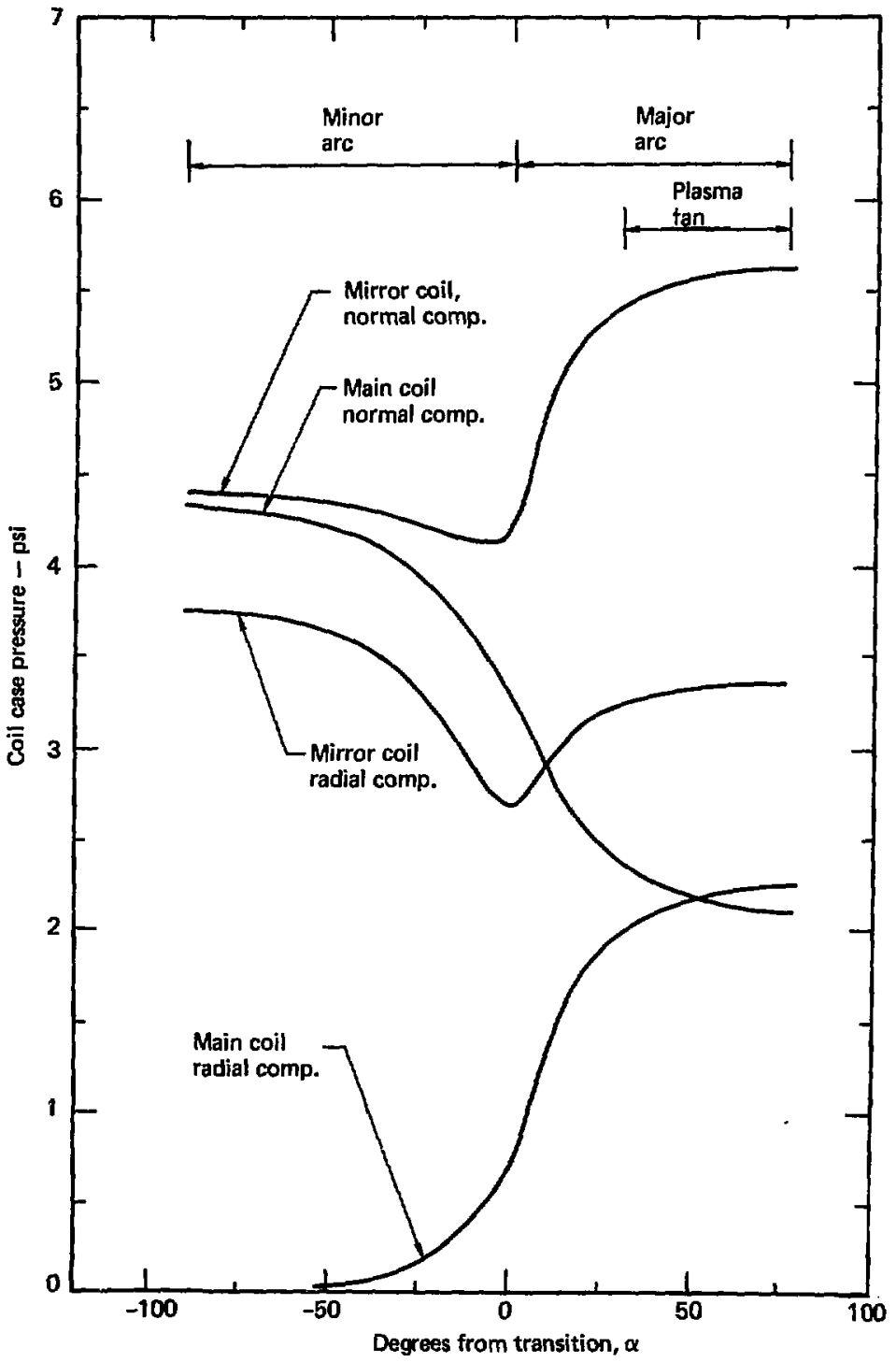

Pig. 6-21. Force distribution on conductor bundles. 


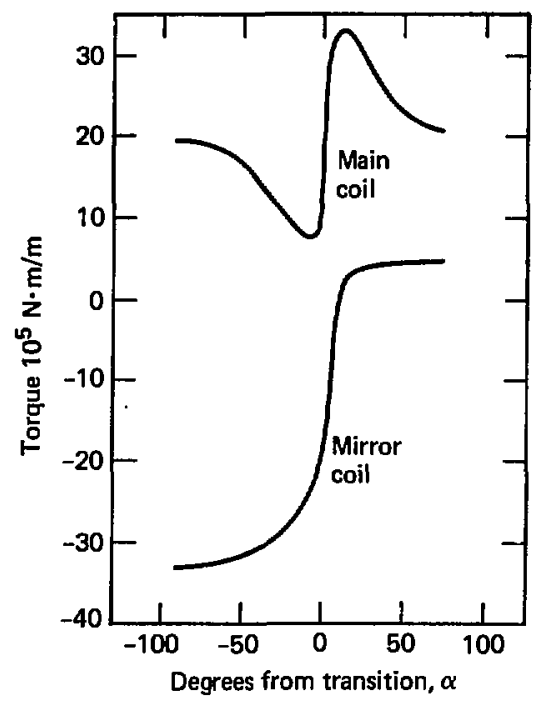

Fig. 6-22. Torque distribution on conductor bundles. 
Net force on $1 / 4$ of bundle located

in positiive quadrant, $M N$

(i) Mirror bundle

F

421. 0

20.5

68.5

(ii) Main bundle

$F_{X}$
$F_{y}$
$F_{z}$

1071.7

36.6

$-128.5$

Net force between argnet pair

(attractive), MN

239.6

Mass per $1 / 2$ of magnet set, tonnes

Coil case

750

Conductor

2210

Total

2960

Surface area per $1 / 2$ of magnet

set, $\mathrm{m}^{2}$

conductor bundles is found by integrating over the distribution. The net forces for the $1 / 4$ of each bundle $(-90 \mathrm{deg} \leq \alpha \leq 75 \mathrm{deg})$ lccated in the positive quadrant $(x>0, y>0, z>0)$ are listed in Table 6-4.

The magnet is constructed by enclosing the two bundles (mirror and main) in the positive-z region in one coil case and the two bundles in the negative-z region in another. The magnet thus consists of two halves. From Table 6-4, the (attractive) force between the two halves is $240 \mathrm{MN}$, which is reacted by the PCRV through load-bearing thermal insulation (see Sec. 8.1). Note that the gravitational force on each half of the coil, 29 m (see Table 6-4), is anall compared to the attractive force. The attractive force is trassmitted to the PCRV in the region of the minor arcs, requiring the loadbearing insulation to sustain an average stress of about 50 pgi.

The coil case is a stainless steel structure that is designed to react the torques and the "C-opening" forces $\left(F_{Y}\right.$ in Table 6-4). The normal forces are reacted by tension structure in the region outside the plasma fan. In the region of the plasma fan, the normal forces are transmitted by 
the coil case to the PCRV via load-bearing thermal insulation. These two regions for reacting the normal forces are shown in Fig. 6-23. This approach to the coil case design has been recommended by Bechtel Corporátion in a previous mirror reactor study. 8

A view of the coil case in the plasma fan region is shown in Fig. 6-24. The conductor bundles are framed with a box structure constructed of plates and groups of four gusset plates are located at regular intervals. The loadbearing thermal insulation is show in position on the outside faces of the main coil and transmits the normal forces to the PCRV, The normal force on the mirror coils is transmitted through the coil case to the insulation, requiring the loading-bearing insulation to sustain a pressure of about 3800 psi. The coil case outside the plasma fan is shown $i$ i Fig. 6-25. Here, the load-bearing insulation has been replaced by tension structure between the bundles, the plates labeled $A$ and $B$ in the figure.

In the present design, a structural analysis of the coil case was not performed. Rather, the mass of the case was scaled from the previous design. ${ }^{8}$ This scaling resulted in a mass per coil case of about $750 t$.

The magnet parameters are summarized in Ta' le 6-4.

\subsubsection{Circuit Design}

We consider the simplified magnet circuit shown in Fig. 6-26. Here, I is the coil inductance, $I_{0}$ is the steady state current per turn, $R_{L}$ is the load resistance used to dissipate the magnet energy during deenergizing of the magnet, and $R_{S}$ is the operating resistance of the circuit $\left(R_{S} \ll R_{L_{0}}\right)$. When a fault is detected in t.le magnet, the power supply is switched out and the load resistance in, resulting in the current

$$
I=I_{0} e^{-t / \tau}
$$

Here, $t$ is time, and $\tau$ is the circuit time constant,

$$
\tau=\frac{L}{R_{L}} \text {. }
$$

The voitage developed across the magnet is

$$
V=-L \frac{d I}{d t}=\frac{I_{0} L e^{-t / \tau}}{\tau},
$$

which has a maximum value at $t=0$ of

$$
v_{0}=\frac{I_{0} L}{\tau} \text {. }
$$




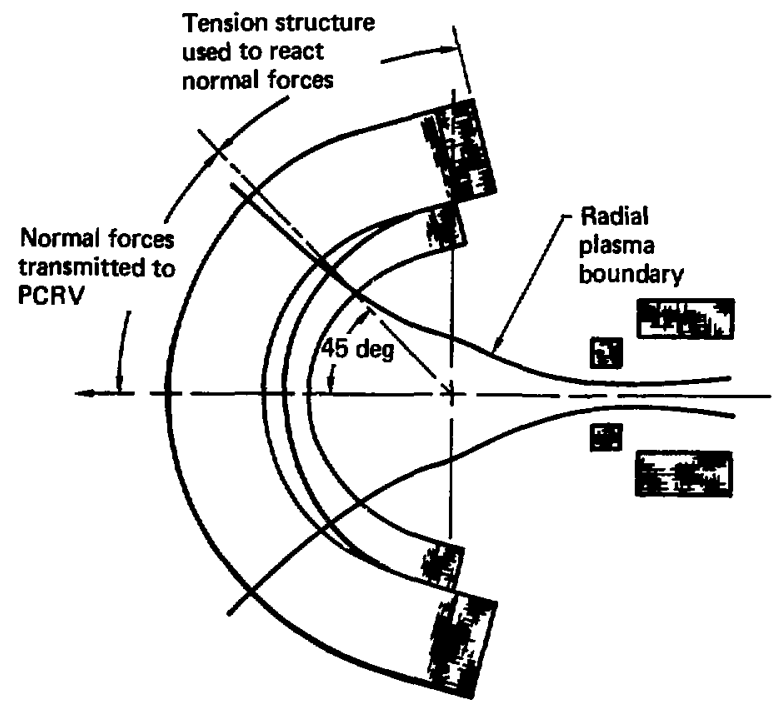

pig. 6-23. Regions of tension structure for the nagnet coil case. 


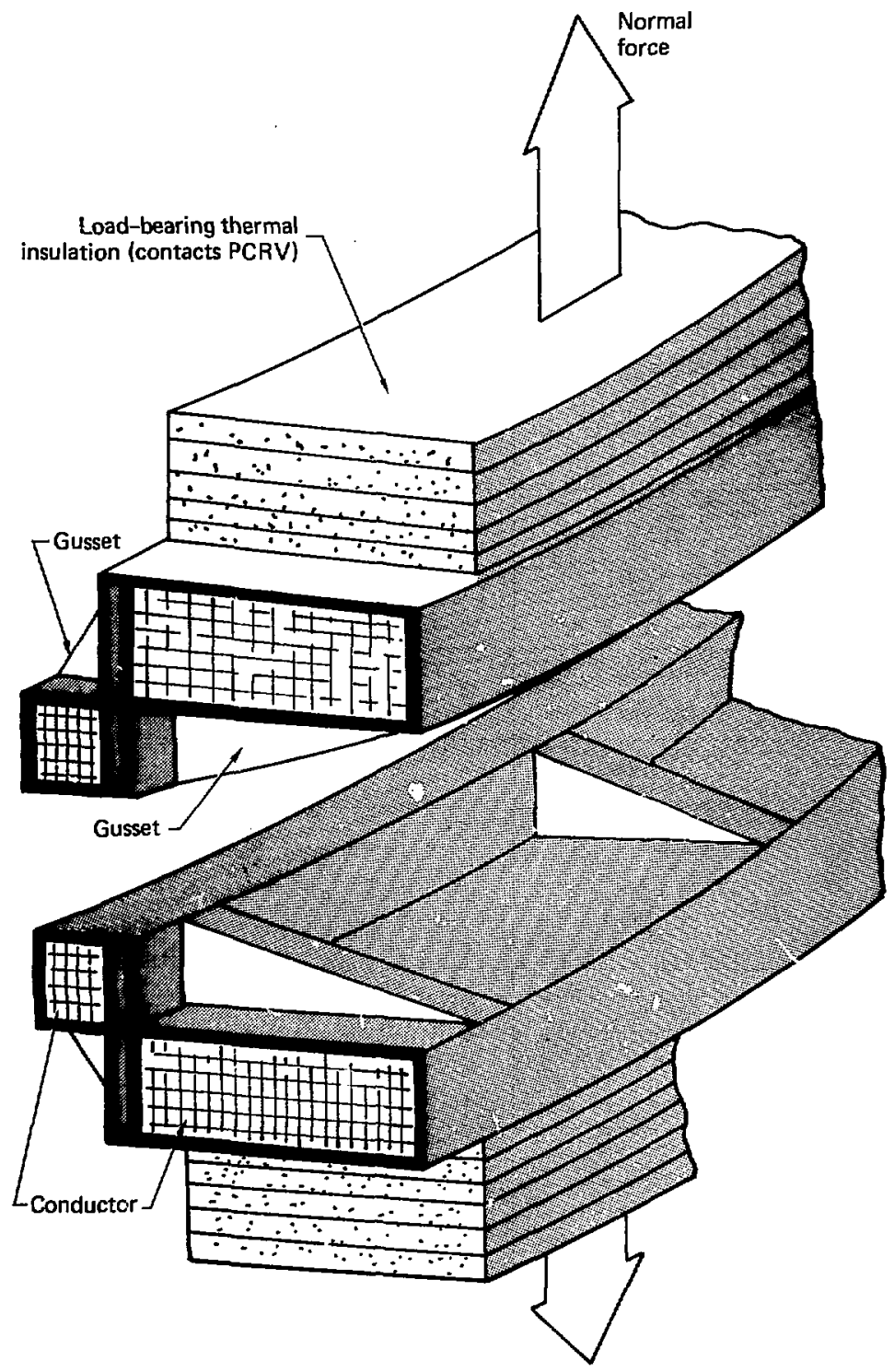

Fig. 6-24. Coil case in the region of the plasma fan. 


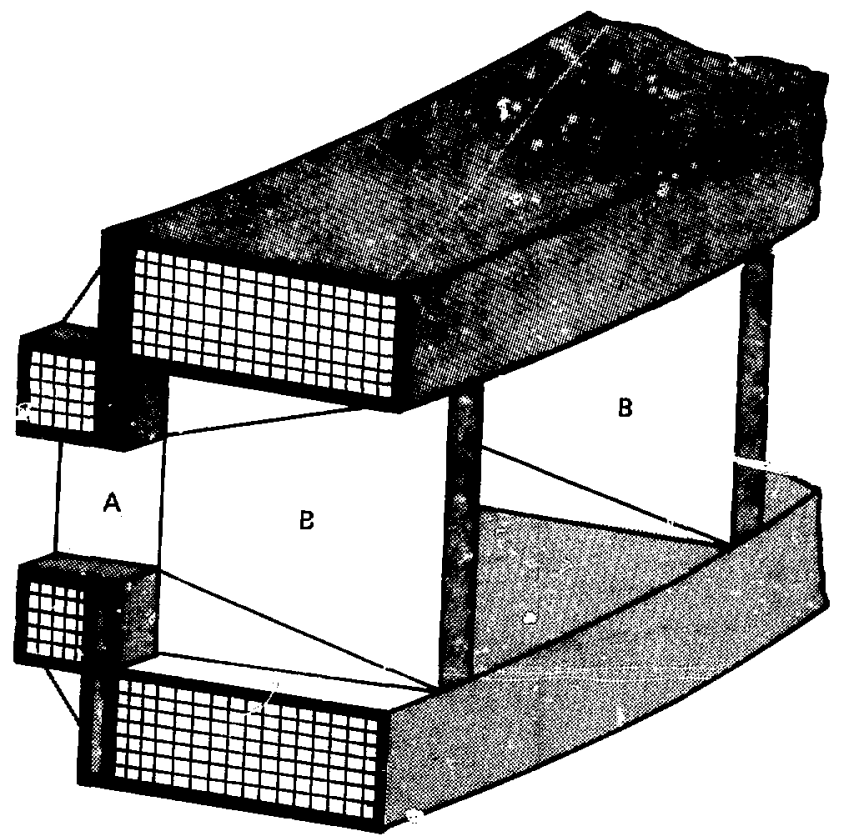

Fig. 6-25. Coil case for the major arcs in the region outside the plasma fan.

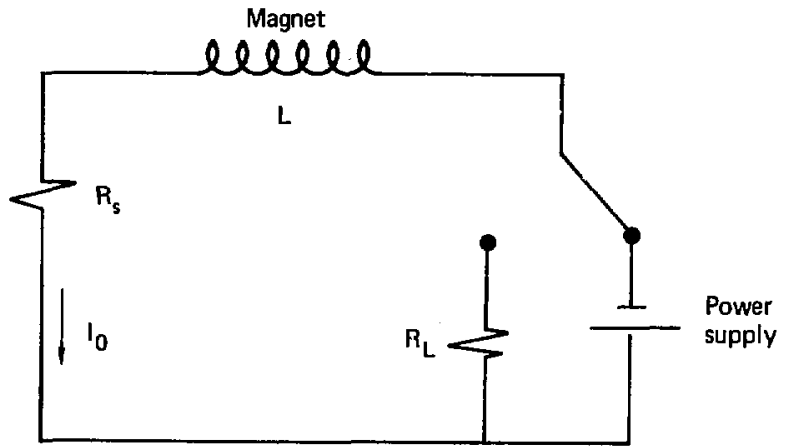

Fig. 6-26. Simplified magnet circuit. 
The two major design constraints on the circuity are:

- $\mathrm{v}_{0}<1000 \mathrm{v}$, to avoid arcing in the coil windings,

- : is small enough to keep the windings from being damaged by excessive temperature.

To develop a quantitative criteria for $\tau$, we consider adiabatic heating of the copper when the coil has gone normal, and current is flowing in the copper. A heat balance on the copper yields

$$
\hat{\rho} c_{p} \frac{d T}{d t}=\rho j_{C u}^{2}
$$

where $T$ is : imperature, $\hat{\rho}$ is mass density, $\hat{C}_{p^{\prime}}$ is specific heat, $\rho$ is electrical resistivity, and $j_{\mathrm{Cu}}$ is the current density in the copper. The current density is

$$
j_{\mathrm{Cu}}=j_{\mathrm{Cu}, 0} \mathrm{e}^{-\mathrm{t} / \mathrm{\tau}} \text {. }
$$

so that Eq. (15) can be written as

$$
\frac{\partial T}{d t}-\rho j_{C u, 0}^{2} e^{-2 t / \tau} \hat{\rho} c_{p}=0 \text {. }
$$

Because $\rho=\rho(T)$ and $c_{p}=c_{p}(T)$, the solution must be obtained numerically. By specifying a maximum final value for $T$, we can determine a maximum value of the time constant $\tau$. Assuming the ratio $\rho / c_{p}$ is approximately constant with temperature, the scaling is

$$
\Delta T \sim \frac{2 \tau \rho j_{C u_{,} 0}^{2}}{\hat{\rho} c_{p}},
$$

where $\Delta T$ is the temperature change in the copper. Selecting $\Delta T=300 \mathrm{~K}$, using $j_{\mathrm{Cu}, 0} \simeq 2100 \mathrm{~A} / \mathrm{cm}^{2}$ for the mirror winding (Table 6-1), and scaling from the $\mathrm{MX}$ coil design ${ }^{1}$ gives a time constant of $\tau=750 \mathrm{~B}$. Using the same circuit time constant for the main conductor bundle will result in $a$. lower conductor temperature rise $(\Delta \mathrm{T} \simeq 120 \mathrm{~K})$.

The stored energy in the coil is given by the volume integral

$$
u_{m}=\frac{1}{2} \int \vec{j}_{b} \cdot \vec{A} d v \text {, }
$$

where $\vec{j}_{b}$ is the bundle current-density vector and $\vec{A}$ is the vector potential $(\vec{B}=\vec{V} \times \vec{A})$. Thus, for coils of similar geometry, the stored energy will scale as 


$$
\mathbf{u}_{\mathbf{m}} \sim \mathbf{B}_{\mathbf{C}} \mathbf{J}
$$

where $B_{C}$ is the maximum field at the conductor, and $J$ is the number of amp-meters. Using the $\mathrm{MX} \operatorname{coil}^{\mathrm{l}}$ as a reference, we obtain $860 \mathrm{MJ}$ for each mirror winding and $4500 \mathrm{~kJ}$ for each main winding.

The stored energy in the coil can also be expressed as

$$
U_{m}=\frac{1}{2} I_{0}^{2} L \text {, }
$$

where $I_{0}$ is the current per turn and $L$ the inductance. With $I_{0}=1.69 \mathrm{kA}$ for the mirror bundle and $I_{0}=1.08 \mathrm{kA}$ in the main bundle, inductances are 602 and $7790 \mathrm{H}$, respectively, for the two windings. Evaluating Bq. (14) with $v_{0}=10^{3} v$ and $t=750 \mathrm{~s}$ restricts the inductance to 144 and $694 \mathrm{H}$ per circuit in the mirror and main windings, respectively. Thus, to stay within the voltage constraint, each mirror bundle requires two circuits and each main coil 12 circuits.

The circuit parameters are sumarized in Table 6-5.

\subsubsection{Masnet Heat Loads}

The thermal energy deposited in the cryogenic magnet is primarily from four sources:

- Neutron heating.

- Thermal heat transfer through the surface of the coll case theat leak).

\begin{tabular}{|c|c|c|}
\hline & $\begin{array}{c}\text { Each } \\
\text { mirror } \\
\text { winding }\end{array}$ & $\begin{array}{l}\text { Each } \\
\text { main } \\
\text { winding }\end{array}$ \\
\hline $\begin{array}{l}\text { Stored energl, WJ } \\
\text { Total for magnet, } 1.07 \times 10^{4}\end{array}$ & 860 & 4500 \\
\hline Inductance, $\mathbf{H}$ & 602 & 7790 \\
\hline Number of circuits & $\mathbf{2}$ & 12 \\
\hline Discharge time constant, 8 & 750 & 750 \\
\hline Conductor temperature rise, $\mathrm{K}$ & 300 & 120 \\
\hline Maxinum discharge voltage, $v$ & 680 & 935 \\
\hline Load resistance, $\Omega$ & 0.40 & 0.86 \\
\hline Inductance per circuit, $\mathbf{H}$ & 301 & 649 \\
\hline Stored energy per circuit, MJ & 430 & 375 \\
\hline Current per circuit, kA & 1.69 & 1.08 \\
\hline
\end{tabular}

Table 6-5. Circult parameters. 
Table 6-6. Magnet heat loads, $\mathrm{kw}$.

\begin{tabular}{lc}
\hline Neutron heating & 9.6 \\
Heak leak & 2.4 \\
Lead losses & 0.08 \\
Superconducting joints & $\underline{0.07}$ \\
Total & 12.2 \\
\hline
\end{tabular}

- Lead losses.

- Joule heating in the conductor joints.

The values for these individual heat loads are listed in Table 6-6, giving a total of $12.2 \mathrm{~kW}$ deposited in the magnet.

The heat load is dominated by neutron heating and the value of $9.6 \mathrm{kh}$ is based on a fusion power of $402 \mathrm{MH}$. For evaluation of this heat load, the reader is referred to the shielding discussion in Sec. 7.2.

The heat leak was calculated ${ }^{1}$ by assuming a heat flux through the coil surface of $0.8 \mathrm{w} / \mathrm{m}^{2}$ and a total surface area for the coil of $3000 \mathrm{~m}^{2}$ (Table 6-4).

The lead losses were calculated by assuming a pair of leads will dissipate $2.5 \mathrm{H} / \mathrm{kA} .^{1}$ As the coil has four sets of leads at $1.69 \mathrm{kA}$ and 24 pairs at $1.08 \mathrm{kA}$ (Table 6-5), the heat load is $0.08 \mathrm{~kW}$.

Losses in the joints of the superconductor were calculated assuming $10^{3} \mathrm{~m}$ between joints and a joint resistance of $10^{-8} \Omega .^{9}$ From Table 6-2, each mirror coil thus contains 270 joints and each main coil, 2470 joints. At currents of $1.69 \mathrm{kA} / \mathrm{turn}$ in the mirror windings and $1.08 \mathrm{kA} / \mathrm{turn}$ in the main windings, the total $I^{2} R$ loss in the magnet circuits from joint resistance is $0.07 \mathrm{~kW}$.

\subsection{Neutral Beam Injectors}

\subsubsection{Introduction}

A series of neutral-beam injector studies have been conducted at ILL to provide data from which the performance of various mirror fusion reactor concepts can be evaluated.

Because these studies concern future reactor designs, we must allow for the technological advances of the next few decades. As a consequence, our 
conclusions are estimates at best. Nevertheless, we attempt to be credible by including only those advances that seem consistent with today's technology.

Although injector design should be, and ultimately will be, done by computer, we have used a less precise analysis that more clearly illustrates our thinking, makes it easy to identify our assumptions, and provides a means to readily visualize the consequences of any changes or possible corrections. In no way do we mean to imply that this technique is the only way to generate these designs.

The conceptual design of the mirror hybrid reactor calls for two neutral-beam injectors, each continuously delivering the equivalent 2600 A of a mixture of 125-keV deuterium and 188-kev tritium atoms. Because the requirements of this machine are similar to those considered in previous mirror hybrid reactor designs, 10,11 we use a modified version of the earlier injector that includes the following features:

- Inpregnated tungsten cathodes for long life.

- High-voltage Insulators and power supplies, all shielded from the neutron and gamma flux.

- Cesiun-vapor curtains to reduce the flow of low-temperature gas along the beam lines.

- Stationary cryopump panels that are periodically outgassed.

- Injectors built of many ion sources to establish system reliability through redundancy.

Although sone fraction of the many ion sources in each injector must deliver tritiun to the reactor, in this study we are concerned only with an injector of deuterium beams. Because beams of 125-keV deuterium and 188-keV tritium travel at the same velocities and behave similarly, to have included tritium in this analysis would have added complexity without significantly changing the conclusions.

\subsubsection{Injector Description}

Each injector consists of 216 ion sources arranged in 18 columns of 12 units spread over an area of 6 by $9 \mathrm{~m}$. To confine the injected beam within a waximum half-angle of $15 \mathrm{deg}$, the distance from the source to the beam entrance aperture in the reactor blanket $1 \mathrm{~s} 17 \mathrm{~m}$.

Only 180 sources need operate at any one time to deliver the required $2600 \mathrm{~A}$ of neutral deuterium current. Thus, three columa of sources are available as standbys, to be activated if needed. Each column of 12 sources is supported by large alumina insulators above and below the beam line. 
Surrounding the sources are perforated electrostatic shields at ground potential. In the event of a high-voltage breakdown, an entire column of sources may have to be turned off. However, should an arc occur, we assume that the safety circuits (crowbars, fast interrupts, and arc snubbers) can prevent damage to the electrodes. As a result, a faulted column (having been turned off) wijll be available for subsequent reactivation.

High-voltage, low-pressure insulation about each column is provided by making the interelectrode spacing between the sources and the grounded shields less than the zaschen minimum for breakdown. The high-voltage insulators outside of the beam line are shielded from neutron and gamma radiation. Low-voltage insulators holding $20 \mathrm{kV}$ or less need not be shielded. Their dimensional change from radiation is small, and any consequential reduction in resigtivity wi. not be critical.

Figure 6-27 shows a schematic of the components along the beam line, along with the corresponding variations in the potential and pressure profiles. The ions extracted from the sources by the acceleration grids are neutralized in a gas cell that is terminated by a cesium-vapor curtain. Mercury ejector pumps maintain the pressure around the ion sources and in the gas cell at $2 \times 10^{-3}$ Torr. The low-temperature gas escaping from the ion sources fills the gas cell. Not being capable of penetrating the cesium-vapor cell (which acts as a curtain), the gas is removed at relatively high pressure and good efficiency via the large pump ducts behind the array of sources. Thereby, the gas load is reduced on the cryopanel pumps further down the beam line. Beyond the cesium cell, the high-energy ions, which are traveling with the beam, are removed by the energy-recovery electrodes.

\subsubsection{Ion Source}

The proposed ion source (see Fig. 6-28) is a modified version of the positive-ion source ${ }^{12,13}$ developed by Lawrence Berkeley Laboratory (LBL) and LLI. It uses a magnetic picket fence about the periphery of the arc chamber to sustain a high presaure of atomic deuterium ions in the output beam. We expect an output of $908 \mathrm{D}^{+}, 78 \mathrm{D}_{2}^{+}$, and $38 \mathrm{D}_{2}^{+}$.

The extraction grid, with an area of 8 by $35 \mathrm{~cm}$ and a grid transparency of 50\%, draws 28 of ions from the source plasma at a current density of $0.2 \mathrm{~A} \cdot \mathrm{cm}^{-2}$. The arc requires $3000 \mathrm{~A}$ at $45 \mathrm{~V}$ i tl.s filament requires 3000 $A$ at $5 \mathrm{v}$. The two large, hollow cathodes made of tungster-irpregnated enitters ${ }^{14}$ should provide thousands of hours of continuous operation. 
Component profile

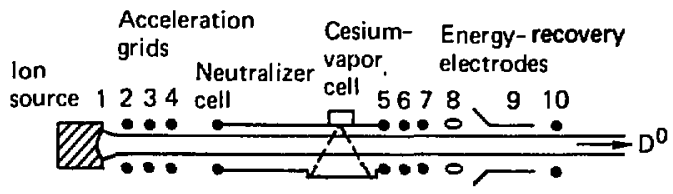

Potential profile

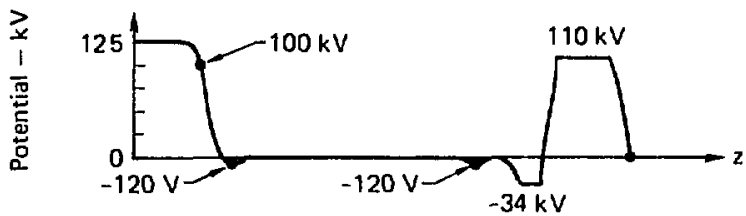

Pressure profile Cesium

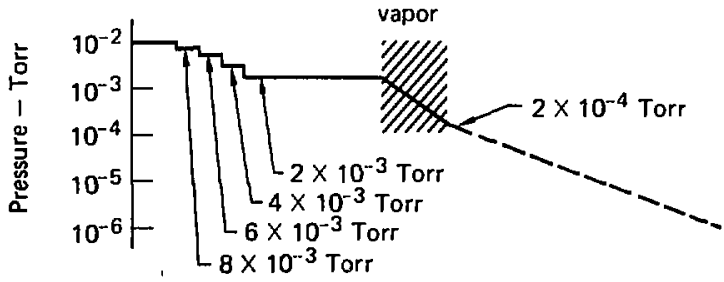

Fig. 6-27. Component and corresponding potential and prassure profiles along the beam line of the proposed neutral-bean injector (not to scale). 


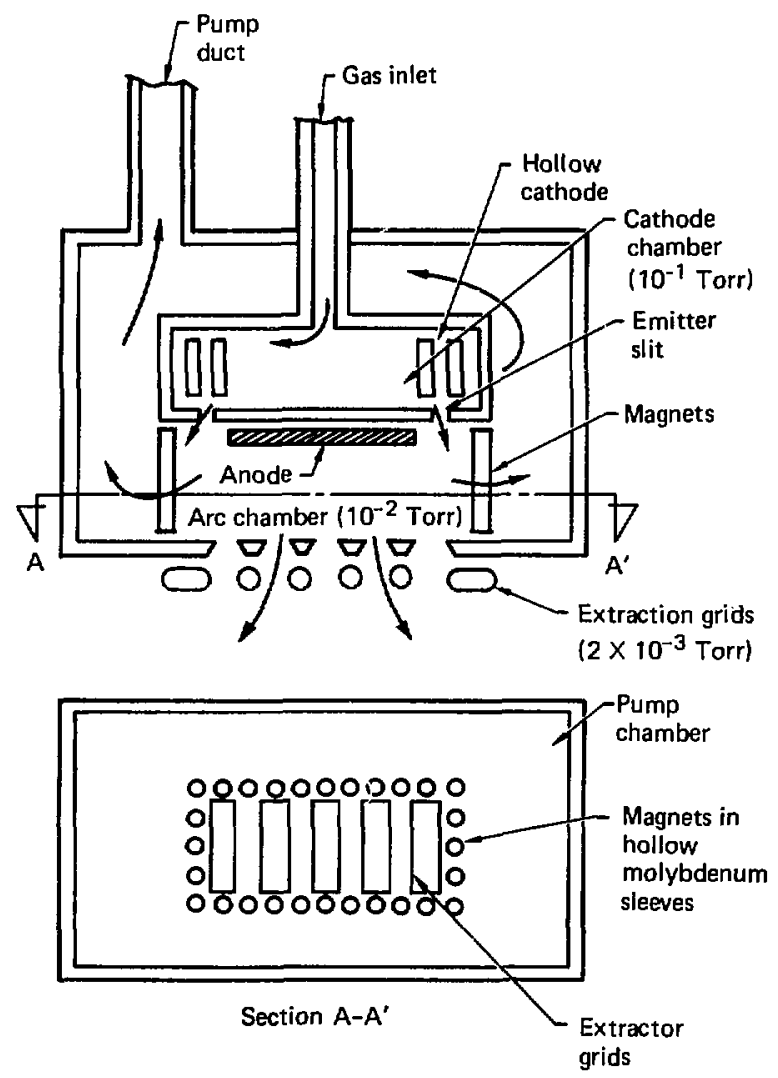

Fig. 6-28. Long-life ion source. 
These cathodes are mounted in an auxiliary chamber that is maintained at about $10^{-1}$ Torr; the arc current, approximately $3000 \mathrm{~A}$, is drawn through two narrow slits into the arc chamber.

The arc plasna is bounded on four sides by a magnetic picket fence, which is maintained by columns of permanent magnets that are individually housed in hollow, molybdenum cylinders. The low-tenperature gas, flowing into the arc chamber via the slits in the cathode chamber, is pumped away through the gaps between adjacent magnets. In this way, the arc pressure is held at $10^{-2}$ Torr.

We estimate the pumping requirements as follows: In the cathode chamber, the electron density $n_{e}$ equals the ion density $n_{1}$; therefore, the percent ionization is

$$
\zeta_{i}=\frac{n_{i}}{n_{i}+n_{0}}
$$

where $n_{0}$ is the density of the neutral gas. If the temperature of the ions $T_{i}$ is approximately equal to that of the neutrals, the amblent pressure in the cathode chamber must be

$$
P_{c}=n_{e} k T_{e}\left[1+T_{i} /\left(r_{i} T_{e}\right)\right]
$$

For 10: ionization, an electron temperature of $10^{4} \mathrm{~K}$, an ion temperature of $600 \mathrm{~K}$, and $P_{c}$ equal to $10^{-1}$ Torr, the electron density $n_{e}$ is 6.3 $\times 10^{19} \mathrm{a}^{-3}$. This corresponds to electrons flowing through the slits into the arc chamber with a current density of

$$
J_{e}=1 / 4 n_{e} e\left(\frac{8 k T_{e}}{\pi m_{e}}\right)^{.1 / 2}=140 \mathrm{~A} \cdot \mathrm{cm}^{-2}
$$

To accommodate an arc current of $3000 \mathrm{~A}$, the total area of the slits between the two chambers must be $21 \mathrm{~cm}^{2}$; for this, the conductance of the slits to molecular deuterium gas at $600 \mathrm{~K}$ is about 9361 itre* $\mathrm{s}^{-1}$. With a pressure drop of $10^{-1}$ Torr, the 48 flow into the arc chamber is 93.6 Torr-1itre $\cdot \mathrm{s}^{-1}$. Neanwhle, $28 \mathrm{~A}$ of ion beam is extracted from the arc chamer. If we take into account the assumed molecular composition of the 
beam, this is the equivalent of 3 Torr.litre*s ${ }^{-1}$. At $30 \%$ gas efficiency, approximately 10 Torr litre's $^{-1}$ is lost to the arc chamber via the extraction grids. Therefore, the neutral gas that must be pumped away through the openings between the picket fence magnets is 03.6 Torr-litre"s ${ }^{-1}$. Thus, we estimate that a large, 15-kw blower will handle the gas load.

The 7 Torr-litre"s ${ }^{-1}$ of gas escaping through the grids is pumped back through the space between adjacent sources, to be removed by a mercury ejector pump. At a throat pressure of $10^{-3}$ Torr, this entails a pumping capability of $7 \times 10^{3}$ Torr $\cdot$ litre $\cdot s^{-1}$. A large mercury ejector pump, which can cope with 10 Torr'litre's ${ }^{-1}$ at $10^{-3}$ Torr, requires about $20 \mathrm{~kW}$ for the mercury boiler and an additional $10 \mathrm{~kW}$ for a mechanical backer pump.

\subsubsection{Beam Optics}

The beams from all of the sources in an injector are focused at the center of the aperture in the reactor blanket. One of the constraints on neutral-beam injector designs is the allowable power loading on the aperture wall. In this analysis, we assume that a wall loading of $P_{w}=250 \mathrm{w} / \mathrm{cm}^{2}$ is tolerable. Therefore, if $J_{w}$ were the current density of 125-kev deuterium atoms intercepted by the wall, no serious dainge will occur if $J_{w}=2 \times 10^{-3} \mathrm{n}^{\cdot} \mathrm{cm}^{-2}$. If we use a tungsten wall with a sputtering rate of $10^{-3}$ atoms per incident ion, this corresponds to an erosion of less than $30 \mu \mathrm{m}$ per year.

The beam optics system is shown schematically in Fig. 6-29. Each bearn from the source arcay is focused on a plane that lies in che aperture through the blanket. In that plane, the locus of points that corresponds to a uniform current density is an ellipse of major and minor radii $x$ and $y$, respectively. Thus, if the aperture wall can tolerate a current density $J_{w}$, the minimum permissible aperture dimensions $x$ and $y$ must be such that

$$
J_{w}=\hat{J}_{0} \exp \left[-\left(x / x_{e}\right)^{2}-\left(y / y_{e}\right)^{2}\right],
$$

where $x_{e}$ and $y_{e}$ corres; -und to the $x$ and $y$ coordinates in the plane of the aperture at which the current density of a beam falls off to $1 / \mathrm{e}$ of the peak axial value $\hat{J}_{0}$. Rearranging the above equation, we find that

$$
x=x_{e}\left[\log _{e}\left(\hat{J}_{0} / J_{w}\right)\right]^{1 / 2}
$$




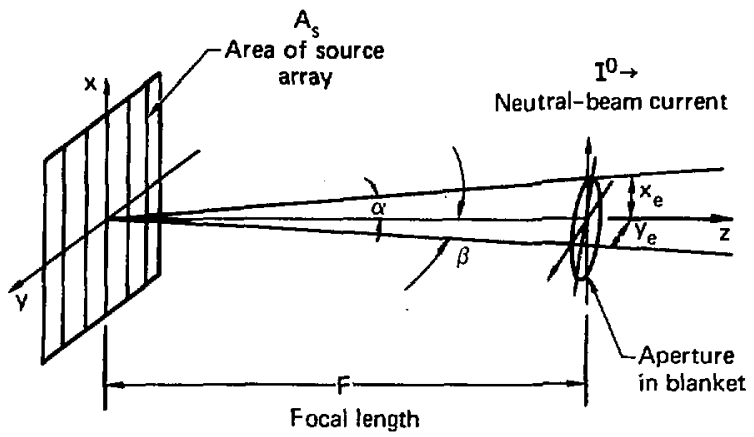

Fig. 6-29. Beam optics system, where $\alpha=x_{e} / F, \beta=y_{e} / F, \Omega_{0}=A_{g} / F^{2}$, $\Omega_{0 s}=\pi \propto \beta$, and $J_{s}=10 / A_{g}$. 
and

$$
y=y_{e}\left[\log _{e}\left(\hat{J}_{0} / J_{w}\right)\right]^{1 / 2}
$$

We can derive an expression firr the peak current density at the focus by assuming that the brightness of the beam at the focus is equal to the brightness at the source of the beam; i.e.,

$$
\frac{\hat{J}_{0}}{\Omega_{0}}=\frac{J_{s}}{\Omega_{g}} .
$$

The terms are defined as follows:

- $\Omega_{0}$ is the solid angle that encompasses all the neutrals that pass through the focal point; i.e., it equals the area of the source array divided by the square of the focal length:

$$
\Omega_{0}=A_{s} / F^{2} \text {. }
$$

- $J_{s}$ is the average equivalent current density of neutrals that appears to be emitted from the source array; i.e., it equals the neutral-beam current divided by the area of the source array:

$$
J_{s}=I^{0} / A_{s}
$$

- $\Omega_{s}$ is the solid angle of emission from a point on the source, whereby

$$
\Omega_{5}=\pi \alpha \beta \text {. }
$$

and

$$
\begin{aligned}
& \alpha=x_{e} / F \\
& \beta=y_{e} / F
\end{aligned}
$$

As a consequence,

$$
\hat{J}_{0}=\frac{I^{0}}{\pi F^{2} \alpha \beta} \text {. }
$$

In this injector, $I^{0}$ equals $2600 \mathrm{~A}$, the total injected neutral current; $E \approx 17 m$, the distance from the sources to the focal point; and $\alpha$ and $\hat{\beta}$ equal 26 and 8.7 mrad, respectively corresponding to the 0.5 and 1.5 deg observed in the neutral-beam studies conducted at LBL for the Tokanak 
Fusion Test Reactor (TFTR). 15 Thus, $J_{0}=1.27 \mathrm{~A} \cdot \mathrm{cm}^{-2}, x_{e}=44.2$ $c \mathrm{r}$, and $y_{e}=14.8 \mathrm{~cm}$; and the minimun acceptable aperture radii are $x=$ $112.2 \mathrm{~cm}$, and $y=37.6 \mathrm{~cm}$. The actual dimensions of the aperture should be about twice this size; e.g., $x=210 \mathrm{~cm}$, and $y=70 \mathrm{~cm}$ to accomnodate any structural flaws in the system. Less than 1 th of the incident beam should fall outside these coordinates.

\subsubsection{Ion Extraction}

The ion source is biased at $+125 \mathrm{kV}$ with respect to ground. The first grid (Gl) floats with respect to the discharge plasma, assuming a potential that results in a net zero grid current. Ions are extracted from the plasma by the second grid (G2), which is at $-25 \mathrm{kV}$ with respect to the source. The third grid (G3) is at $-120 \mathrm{~V}$ with respect to ground; the fourth grid is at ground. The 120-V bias between $\mathrm{G} 3$ and $\mathrm{G} 4$ causes some low-temperature ions to be drawn to $\mathrm{G3}$; however, it also prevents electrons from being accelerated from beyond $\mathrm{G3}$ to bombard $\mathrm{Gl}$ and $\mathrm{G2}$ at high energy. (Note: We assume that $G 3$ draws an ion current that is equal in magnitude to 108 of the highenergy beam current, i.e., $2.6 \mathrm{~A}$. Similarly, on the far side of the cesiumvapor cell, G6 is assumed to draw $2.6 \mathrm{~A}$ of ions.)

To be effective, the $\mathrm{G} 3$ bias must be sufficient to introduce a potential dip in the beam path between adjacent grid wires. With the grid geometry shown in Fig. 6-30, the required bias can be approximated by 16

$$
v_{3}=\frac{J a^{2}}{6 \varepsilon_{0} v}[(2 b / a)-1]=120 v \text {. }
$$

where $J$ is the current density of the ion beam, $a$ is the width of the ribbon-shaped beam passing between the grid wires $(\sim 0.3 \mathrm{~cm})$, b is the separation between grid wires $(0.4 \mathrm{~cm})$, and $v$ is the velocity of the beam ions at $125 \mathrm{keV}$. Because the preceding expression was derived for electrodes that are very long, the bias required for the present applicetion will be somewhat greater than the calculated value.

Even with perfect optics, grid loading results from bombarduent by ions and electrons formed in the wake of the ion beam. 17 The fraction of the high-energy beam $F_{10}$ that becomes neutralized in the grid region (by charge exchange with the background gas) constitutes a loss of current, that is conducted away via the newly formed, low-energy background ions. Keanwhile, the fraction of th incident ion beas $F_{1}$ that causes inpact ionization of the background gas creater two currents: a current of background 


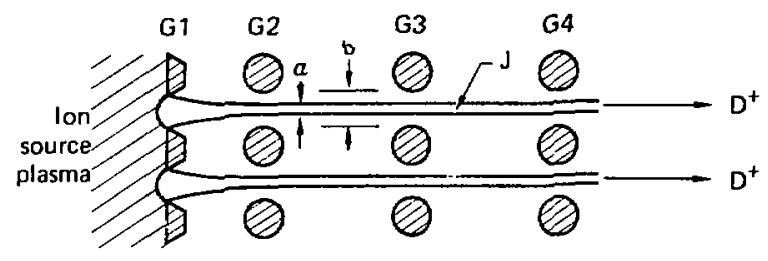

Fig. 6-30. Grid cross sections. 
ions that equals $F_{i}$ times the incident beam current and a current of background electrons of the same magnitude. It is these background ions, along with those created by charge exchange, that are drawn out of the bean path to bombard the electrodes. The electrons originating from the ionization, along with those secondary electrong created by ion bombardment of the grids, tend to follow the ion beam toward the source.

It is impossible to accurately determine the grid loading without knowing the trajectories of the background ions. However, we can make an estimate by assuning that all of the ions formed in a gap between any two grids originate in a plane midway between the grids and terminate at the wore negative grid. The secondary electron emission coefficient is assured to be 1 , and an estimated 10 of all the electrons generated along the ion beam line ultimately strike Gl, with the remainder passing through the grids into the arc chamber. 17

Let $n_{b}$ represent the background gas density and $\sigma_{10}$ the electroncapture cross section of the beam, so that the fraction of the ion beam that becomes neutralized over some path length $z$ in a region of uniform gas density is

$$
F_{10}=n_{b} \int_{0}^{z} \sigma_{10} d z
$$

Because the density of the extracted ions can be approximated by Child's Law, the potential along the beam path through the decelerating grids can be described by

$$
v=(J / q)^{2 / 3} z^{4 / 3}
$$

where the ion-beam current density $J=0.2 \mathrm{~A}^{\cdot} \mathrm{cm}^{-2}$, and the preveance $q=$ $3.2 \times 10^{-8} \mathrm{~A} \cdot \mathrm{v}^{-3 / 2}$ (taking the mixture of atomic and molecular ions into account).

By introducing the derivative of the potential with respect to the beam path into the previous equations, we find that the fraction of the primary ion bean that becones neutralized between two grids, one of which is at potential $v_{1}$ and the other at $v_{2}$, is

$$
F_{10}=(3 / 4) n_{b}(q / J)^{1 / 2} \int_{v_{1}}^{v_{2}} \sigma_{10} v^{-1 / 4} d v .
$$


From similar considerations, the fraction of the beam that causes impact ionication of the background gas is

$$
F_{i}=(3 / 4) n_{b}(q / J)^{1 / 2} \int_{v_{1}}^{v_{2}} \sigma_{i} v^{-1 / 4} d v .
$$

The solutions of the preceding integrals, wich are plotted in Fig. 6-31, were calculated from data given in Refs. 18 and 19 using values for $\mathrm{D}_{2}^{+}$and $\mathrm{D}_{3}^{+}$ions that were derived by assuming that $\mathrm{D}^{+}, \mathrm{D}_{2}^{+}$, and $\mathrm{D}_{3}^{+}$ ions of the same velocity have the same cross sections.

Meanwhile, we can estimate the density $n_{b}$ of the gas (at roughly 600 K) in the gaps separating the various grids by assuming an equal pressure drop acyoss each of the four grids separating the arc chamber, which is at $10^{-2}$ Trorr, from the region beyond $\mathrm{G} 4$, which is at $2 \times 10^{-3}$ Torr (see Fig. $(1-27)$.

The ion beam losses due to charge exchange in the G1-G2, G2-G3 regions are shown in Table $6-7$, as calculated for the total beam and for its $D_{l^{+}}$, $\mathrm{D}_{2}^{+}$, and $\mathrm{D}_{3}^{+}$components. The first two lines show the beam composition as extracted from the ion source, the next two lines show the fraction of the beam that was lost by charge exchange in the G1-G2 gap, and the last two lines show the beam fraction lost in the G2-G3 gap. In a similar manner, Table 6-8 shows the ion and electron currents generated by impact ionization in the background gas.

Table 6-7. Ion bean loss due to charge exchange in the acceleration grid region.

\begin{tabular}{|c|c|c|c|c|c|}
\hline Line & Item & $\begin{array}{l}\text { Total } \\
\text { beam }\end{array}$ & $\mathbf{D}_{1}^{+}$ & $\mathrm{D}_{2}^{+}$ & $\mathrm{D}_{3}^{+}$ \\
\hline 1 & Beam composition, $n(\%)$ & 100 & 90 & 7 & 3 \\
\hline 2 & Extracted beam current, $\mathrm{I}^{+}(\mathrm{A})$ & 28.0 & 25.2 & 2.0 & 0.8 \\
\hline 3 & G1-G2 charge exchange, $F_{10}(z)$ & - & 4.3 & 4.3 & 4.3 \\
\hline 4 & Ion beam leaving $\mathrm{G} 2, \mathrm{I}_{2}^{+}(\mathrm{A})$ & 26.8 & 24.1 & 1.9 & 0.8 \\
\hline 5 & G2-G3 charge exchange, $F_{10}(K)$ & - & 4.4 & 5.9 & 8.2 \\
\hline 6 & Ion beam leaving $\mathrm{G} 3, \mathrm{I}_{3}^{+}(\mathrm{A})$ & 25.5 & 23.0 & 1.8 & 0.7 \\
\hline
\end{tabular}




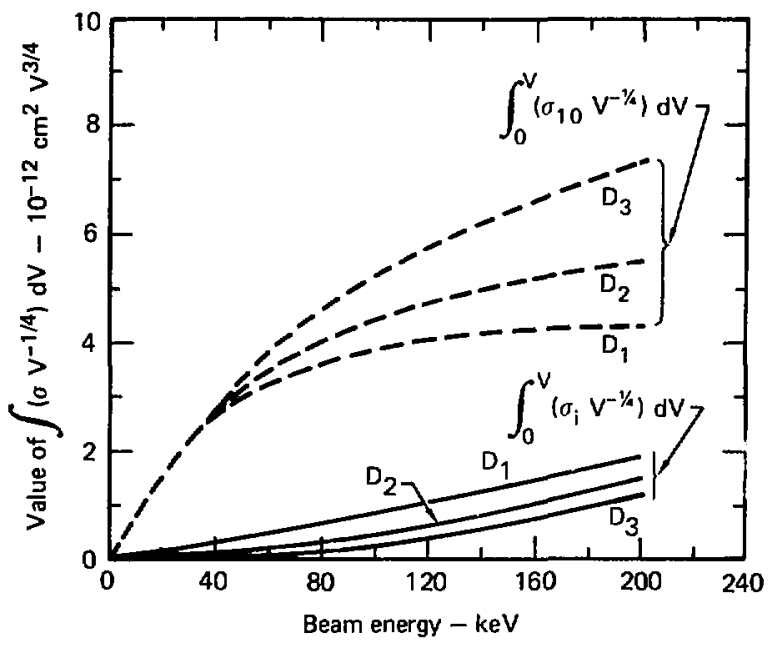

Fig. 6-31. Cross-section integrals used to determine the coefficienta for electron capture and background icnization. 
Table 6-8. Background ion and electron currents in the acceleration grid region.

\begin{tabular}{|c|c|c|c|c|}
\hline Line & Item & $\mathrm{D}_{1}^{+}$ & $\mathrm{D}_{2}^{+}$ & $\mathrm{D}_{3}^{+}$ \\
\hline 1 & Ionization between $\mathrm{Gl}-\mathrm{G} 2, \mathrm{~F}_{\mathrm{i}}(\mathrm{z})$ & 0.5 & 0.3 & 0.1 \\
\hline 2 & Electron current, $I_{e}$ (A) & 0.1 & $\sim$ & $\sim$ \\
\hline 3 & Ion current, $I_{i}(A)$ & 0.1 & $\sim$ & $\sim$ \\
\hline 4 & Ionization between $G 2-G 3, F_{i}(x)$ & 1.5 & 1.0 & 1.0 \\
\hline 5 & Electron current, $I_{e}(A)$ & 0.4 & 0.01 & 0.01 \\
\hline 6 & Ion current, $I_{i}$ (A) & 0.4 & 0.01 & 0.01 \\
\hline
\end{tabular}

Aside from the thermal loading, which we evaluate in a later section, ion bombardment results in sputtering that can limit the useful grid life. We can estimate the magnitude of this effect by assuming that all the ions that bombard a grid are formed in the adjacent grid gap. Thus, a beam of width a and current density $J$ will cause an ion sputtering current density of $J_{B}$ on a grid wire of radius $r$ (see Fig. 6-32); i.e.,

$$
J_{B}=J\left(F_{10}+F_{i}\right)\left(\frac{a}{\pi r}\right)
$$

In the unlikely circumstance that the more negative orid bordering the gap is bombarded by all of these ions at the same current density, the grid will sputter away at a rate of

$$
s=(3 / 4)(J q)^{1 / 2} \frac{a}{\pi r} n_{b} q_{s}\left(\frac{M}{\rho e}\right) \int_{v_{1}}^{v_{2}}\left(\sigma_{10}+\sigma_{i}\right) v^{-1 / 4} d v,
$$

where $M$ is the mass of the grid metal, $\rho$ is its density, $e$ is the electron charge, and $q_{g}$ is the sputtering coefficient of the bombarding ions. Although $q_{g}$ is not known for a wide range of energies, ${ }^{20}$ we do know that it is sensitive to the angle of ion impact. 21 Thus, for all angles at all energies, we have arbitrarily assigned to $q_{s}$ an average value of $10^{-3}$ atoms per incident ion of deuterium on tungsten. From this assumption, it appears that G2 and G3 will sputter away at a rate that is less than 25 $\mu \mathrm{m} /$ month. This could be minimized either by a more advantageous choice of 


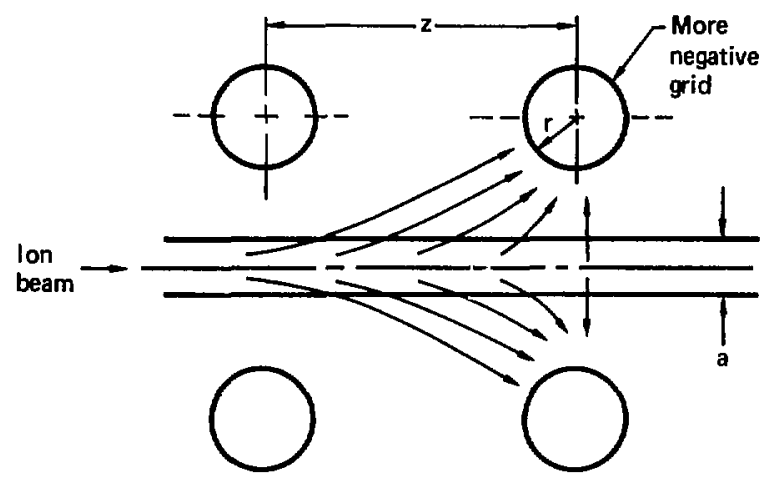

Fig. 6-32. Sputtering current density. 
grid contours and potentials or by reducing the extracted ion current density.

\subsubsection{Neutralization}

To inpede the flow of low-temperature gas along the beam line, we introduce a cesium-vapor cell between the acceleration grids and the energYrecovery electrodes. In this way, most of the gas escaping from the ion sources can be pumped away at relatively high pressure. This is advantageous because the low ambient pressure needed in the vicinity of the energyrecovery electrodes can then be maintained with cryopanels of considerably smaller area. As a result, the beam line is more compact and the pumping efficiency is enhanced.

We can estimate the performance of the cesium-vapor cell in the beam line by equating the rate at which the deuterium diffuses through the cesium with the gas flow $S$ on the low-pressure side of the cell. Thus,

$$
\underline{D} \frac{\mathrm{dn}}{\mathrm{dz}}=\mathbf{s} \text {, }
$$

where $\mathrm{n}$ is the deuteriun density, $\mathrm{z}$ is the beam path through the cesium and

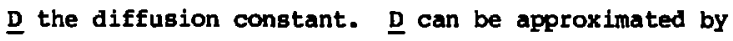

$$
\underline{\underline{D}}=\frac{\overline{\mathrm{c}}}{3 \mathrm{n}_{\mathrm{Cs}} \sigma_{\mathrm{Cs}}} \text {, }
$$

where $\bar{c}$ is the average velocity of a deuterium atom in the cesium vapor, and $\mathrm{n}_{\mathrm{cs}}$ is the density of the cesium vapor. The transport cross section of deuterium in cesium, $\sigma_{\mathrm{Cs}}$ is estimated to be $7 \times 10^{-15} \mathrm{~cm}^{2} /$ atom. 22

Meanwhile, the gas flow on the low-pressure side of the vapor cell, where the gas density is $n_{f}$, is roughly

$$
s=1 / 4 n_{f} \bar{c}
$$

By combining the last three expressions and setting the thickness of the vapor cell, $\pi_{\mathrm{Cs}}$ to $1.7 \times 10^{15}$ atoms $\mathrm{cm}^{-2}$, we find that the gas density on the far (low-pressure) side of the vapor cell is less than onetenth the density on the high-pressure gide.

The high-energy beam entering the cesium-vapor cell is composed of neutrals and ions such that

$$
\mathbf{F}_{0}+\mathbf{F}_{+}=1
$$


where $F_{0}$ is the iraction of the bean that is neutral, and $F_{+}$is the fraction that is ionized. As the beam passes through the vapor, the fraction of neutrals changes; 1.e.,

$$
\frac{d F_{0}}{d{ }_{C s}}=-F_{0} \bar{\sigma}_{01}+F_{+} \bar{\sigma}_{10} \text {, }
$$

where $\bar{\sigma}_{01}$ and $\bar{\sigma}_{10}$ are the respective cross sections of electron 105s and capture of deuterium in cesiun. Integrating this equation fram an initial neutral fraction $F_{01}$ to a final fraction $F_{0 f}$ reaults in

$$
F_{0 f}=\bar{F}_{000}+\left(F_{01}-\bar{F}_{000}\right) \exp \left[\pi_{C_{B}}\left(\bar{\sigma}_{10}+\bar{\sigma}_{01}\right)\right] \text {, }
$$

where $\overline{\mathrm{F}}_{0 \infty}$ is the fraction of deuterium neutrals that can be obtained in a cesiu-vapor cell of infinite thickness; 1.e.,

$$
\overline{\mathrm{E}}_{0_{\infty}}=\frac{\bar{\sigma}_{10}}{\bar{\sigma}_{10}+\bar{\sigma}_{01}}
$$

Unfortunately, all the cross sectiong pertinent to the passage of deuteriu through cesiui vapor are not avaliasle. As shown in Fig. 6-33, we have no data for the electron-10ss cross section $\vec{\sigma}_{01}$ at deuterium energies in excess of $30 \mathrm{kev},{ }^{23}$ although we have extensive data for deuteriun in $\mathrm{D}_{2}{ }^{18}$ However, we can make a crude estimate for a 125-kev deuteron, as shown by the dotted line in Fig. 6-33. From this extrapolation, we find that $F_{0 \infty}$ is approximately 0.45 for the atomic component of the ion bear. Because 63 is biased at $-120 \mathrm{v}$, the Ion beam energy in the vicinity of G3 is for all practical purposes at full energy. Thus, the G3-G4 gap can be considered part of the deuterium neutralizer cell. If $\pi_{0}$ represents the thickness of deuterium from $G 3$ to the cesiun-vapor cell, while $\sigma_{01}$ and $\sigma_{10}$ represent the electron-10ss and electron-capture cross sections of deuterium in deuteriun gas, then the fraction of neutrals in the high-energy bean that enters the cesium vapor must be

$$
F_{01}=\left(\frac{\sigma_{10}}{\sigma_{10}+\sigma_{01}}\right)\left\{1-\exp \left[-\pi_{0}\left(\sigma_{10}+\sigma_{01}\right)\right]\right\} .
$$




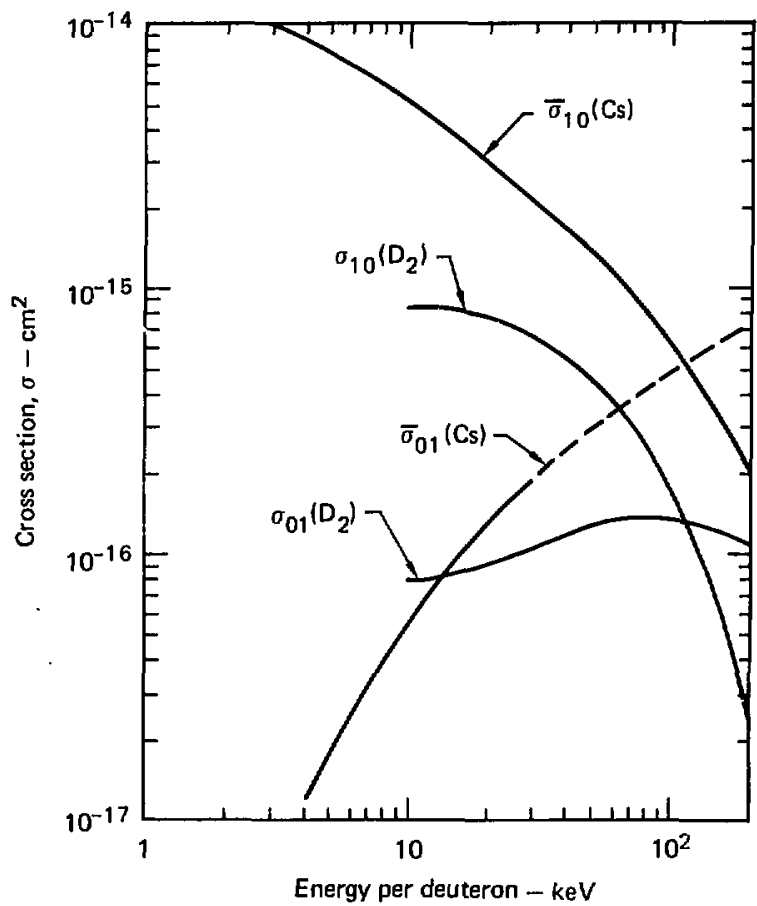

Fig. 6-33. Electron $108 \mathrm{~s}$ and capture cross sections of $\mathrm{D}$ in $\mathrm{D}_{2}$ and cesium. The dotted line represents extrapolated values. 
The gas thickness that results in 95 of the maximum possible neutralization (obtainable in a cell of infinite thickness) is

$$
\pi_{0}=\left(\frac{3}{\sigma_{10}+\sigma_{01}}\right) \text {. }
$$

From data in Fig 6-33, we find that for $\pi_{0}=1.2 \times 10^{16}$ eit $^{2}$.

$F_{0 i}=44$, for the atomic component of the beam. On the far side of the cesium-vapor cell, $F_{0 f}=454$.

The region beyond $\mathrm{G} 4$ is at a pressure of $2 \times 10^{-3}$ Torr (see

Fig. 6-27). Therefore, a gas temperature of $300 \mathrm{~K}$ requires a $1.8-\pi$-thick neutralizer cell.

If the cesiun-vapor cell were thicker, so that

$$
\pi_{C B}=\left(\frac{3}{\sigma_{10}+\sigma_{01}}\right)=3 \times 10^{15} \mathrm{~cm}^{-2},
$$

the neutral fraction of the beam $F_{0 f}$ leaving the cell would equal $\vec{F}_{0 \infty 0}$ (approximately 45). At the same time, it would be independent of $\mathrm{F}_{0 i}$ ' the fracion of neutrals in the incident beam. Thus, the $1.8 \mathrm{~m}$ of gas neutralization could be eliminated from the bean line if the ceslum-vapor cell were twice as thick. A typical beam line of this construction is shown in Fig. 6-34. However, in this study we have arbitrarily opted for the thinner cesium-vapor cell.

Table 6-9 shows an analysis of the beam in the vicinity of the cesiumvapor cell. As previously calculated, the gais thickness between 63 and the cesium-vapor cell. is $1.2 \times 10^{16}$ molecules $/ \mathrm{cm}^{2}$, whereas the cesiumvapor thickness is $1.7 \times 10^{15}$ atoras $/ \mathrm{cm}^{2}$. The first line in Table $6-9$ shows the components of the ion beam that pass through G3. The second line indicates the fraction of the beam that is neutral at the entrance to the cesilu-vapor cell, and the third line consists of the fraction of the bean that is neutral when it leaves the cesiun-vapor cell.

The $F_{0 i}$ and $F_{0 f}$ values for the molecular ions are calculated in the save wanner as above, using the corresponding $1 / 2$ - and $1 / 3-$ energy cross sections for the $D_{2}$ and $D_{3}$ wolecules.

The equivalent currents of neutral deuterium are also shown in Table 6-9, along with their associated energies. Because the high-energy molecular neutrals tend to break up into atomic neutrals at fractional energies, 


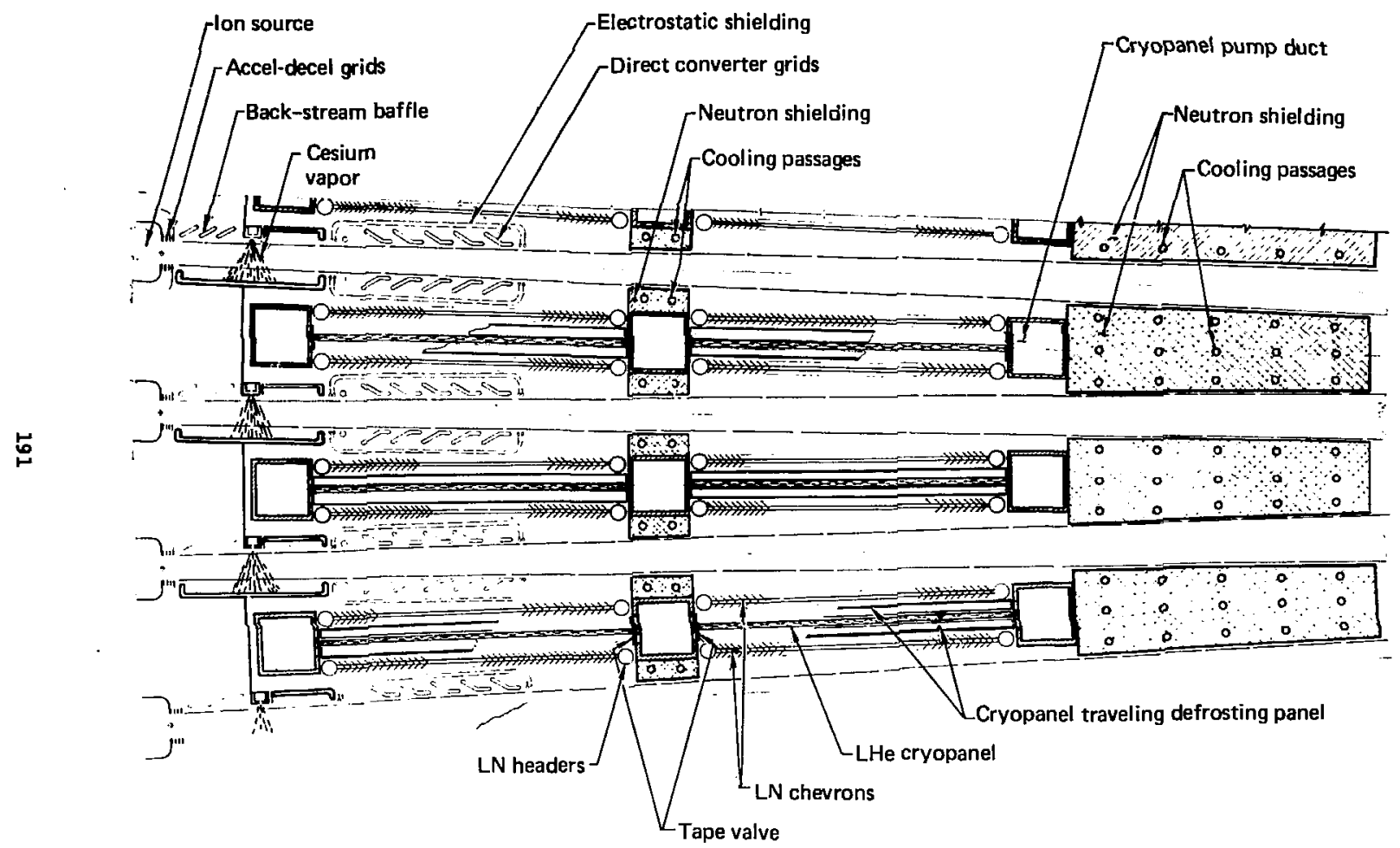

Fig. 6-34. Ion source for the positive-ion injector. 
Table 6-9. Beam components in the vicinity of the cesium-vapor cell.

\begin{tabular}{|c|c|c|c|c|c|c|}
\hline Line & Item & $\begin{array}{l}\text { Total } \\
\text { beam }\end{array}$ & $D$ & $\mathrm{D}_{2}$ & $\mathrm{D}_{3}$ & $\begin{array}{l}\text { Reference } \\
\text { (Table, IIne) }\end{array}$ \\
\hline 1 & $\begin{array}{l}\text { Iop beam leaving } \mathrm{G} 3 \text {, } \\
\mathrm{I}_{3}^{+}(\mathrm{A})\end{array}$ & 25.5 & 23.0 & 1.8 & 0.7 & $T 6-7$, \\
\hline 2 & $\begin{array}{l}\text { Neutral fraction } \\
\text { entering } C_{B} \text { ce } 11 \\
\mathrm{~F}_{\mathrm{Oc}}(\boldsymbol{x})\end{array}$ & - & 44.0 & 73.0 & 82.0 & \\
\hline 3 & $\begin{array}{l}\text { Neutral fraction leaving } \\
\text { Co cell, } F_{\text {of }}(z)\end{array}$ & - & 45.0 & 73.4 & 86.8 & \\
\hline 4 & $\begin{array}{l}\text { Neutral atomic beam }{ }^{0} \text { (A) } \\
\text { leaving } C_{s} \text { cell, } D^{0} \text { (A) }\end{array}$ & 14.8 & 10.4 & 2.6 & 1.8 & \\
\hline 5 & $\begin{array}{l}\text { Energy of atcmic beam } \\
\quad(\mathrm{keV})\end{array}$ & 104 (av) & 125 & 62.5 & 41.6 & \\
\hline 6 & $\begin{array}{l}\text { Ion current leaving Cs } \\
\text { cell, I } \mathrm{I}_{\mathrm{er}}^{\text {(A) }}\end{array}$ & 13.2 & 12.6 & 0.5 & 0.1 & \\
\hline
\end{tabular}

a In Tables 6-9 through 6-13, the Reference columns show the table and 11ne from which the data were obtained.

the atomic neutrals originating as $\mathrm{D}_{2}{ }_{2}$ are listed at twice their original neutral current and $1 / 2$ their original energy. Similarly, the atomic neutrals originating as $\mathrm{D}_{3}{ }_{3}$ are listed at three times their original neutral current and $1 / 3$ their energy.

\subsubsection{Energy Recovery and Beam Loss Along the Beam Line}

To recover the energy of the atomic ions that did not become neutral, the ions must be separated from the low-energy background electrons that travel with the beam. In a rectangular ion beam of width $a$, velocity $v$, and current density $J$, these electrons can be held back by suitably biased electrodes, separated by a distance $b$ on each side of the beam (see Fig. 6-35. 16 In the absence of electrons, the ions spread out because of their positive space charge and leave the neutral beam to be collected at almost the sawe energy with which the lons left the source. This ocnstitutes energy recovery, because it provides a net power savings. The bias on $G 8$ that is required to hold back the electrons is

$$
v_{8} \approx \frac{J a^{2}}{8 \varepsilon_{0} v}[(2 b / a)-1] \text {. }
$$




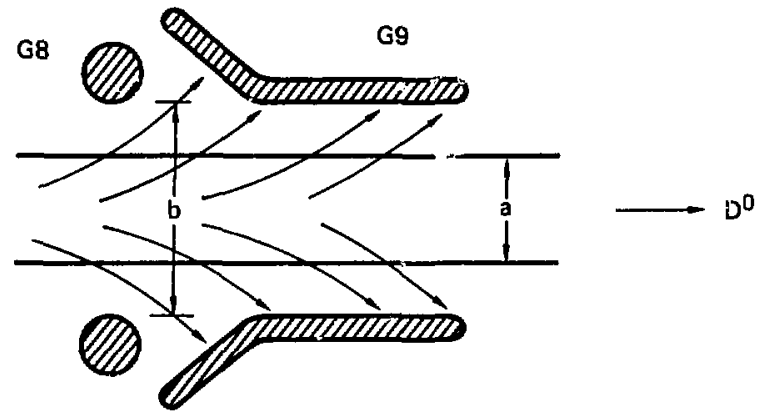

Fig. 6-35. Energy-recovery electrode. 
We can estimate the current density of the ions by considering that 28 A of ions were originaily extracted at $0.2 \mathrm{~A}^{\circ} \mathrm{cm}^{-2}$ through grids of 50 t transparency. As shown in Table 6-9, only $13.2 \mathrm{~A}$ are left in the vicinity of the energy-recovery electrodes. Hence, the curcent density of ions entering the region of the energy recovery is $\mathrm{J} \approx 0.047 \mathrm{~A} \cdot \mathrm{cm}^{-2}$. The beam width is $a \approx 10 \mathrm{~cm}$; the electrodes are separated at $b=14 \mathrm{~cm}$. Under these conditions, the the $125-\mathrm{keV}$ beam requires a bias of $\mathrm{v}_{8}=34 \mathrm{kV}$. We assume that an electron current equal to about 10 s of the incident ion beam (i.e., 1.3 3) is drawn from the background plasma to be collected at this electrode. According to Table 6-9, the ions traveling with the beam consist of $12.6 \mathrm{~A}$ of $\mathrm{D}^{+}, 0.5 \mathrm{~A}$ of $\mathrm{D}_{2}^{+}$, and $0.1 \mathrm{~A}$ of $\mathrm{D}_{3}^{+}$; however, only the atomic component $\left(12.6 \mathrm{~A}\right.$ of $\left.\mathrm{D}^{+}\right)$can be collected at the positive electrode G9, which is at $110 \mathrm{kV}$ (Bee Fig. 6-27). We assume that roughly $0.1 \mathrm{~A}$ of these ions do not arrive at $G 9$ and are collected at the injector walls. Nevertheless, all these ions eventually do collide either with electrodes or with the injector walls to form a neutral-gas load that must be punped away. Accordingly, for each source the gas load is

$$
q_{p}=0.19\left(1 / 2 D^{+}+D_{2}^{+}+3 / 2 D_{3}^{+}\right)=1.32 \text { Torr } 1 \text { litre } s^{-1},
$$

of 15.8 Torr*litre's ${ }^{-1}$ for the 12 sources mounted in each column.

As the beans delivered from each column of 12 ion sources flow past their respective neutralizer cells, they are isolated from the beams of the neighboring columns of sources by walls of cryopumps. These walls, which are roughly $5 \mathrm{~m}$ high and $5 \mathrm{~m}$ long (see Fig. 6-36), are divided into cryopump sections with roughing-pump ducts interspersed among them. During the outgassing cycle, while the cryopump surface is heated a few degrees and gas is being draw away through an open valve into the roughing-pump duct, sliding panels close off both sides of each pumping section. Before the panel moves on to the next punping section, the roughing valve is closed and the pump is re-refrigerated. As a consequence, only 508 of the available wall space is actively punping at any time, and we expect an average punping speed of about 4 itre. $\mathrm{s}^{-1} \cdot \mathrm{cm}^{-2}$. (A fully baffled cryopanel can handle 9 litre $\mathrm{s}^{-1}$.)

With pumps on both sides of each colum of beams, a pumping speed of 2 $\times 10^{6}$ litre $^{\cdot} \mathrm{s}^{-1}$ is available to each set of 12 ion sources. Therefore, a gas laad of 15.8 Tor $r \cdot 1$ itre $\cdot \mathrm{s}^{-1}$ will result in an average pressure of $B \times 10^{-6}$ Torr in the cryopung region. 


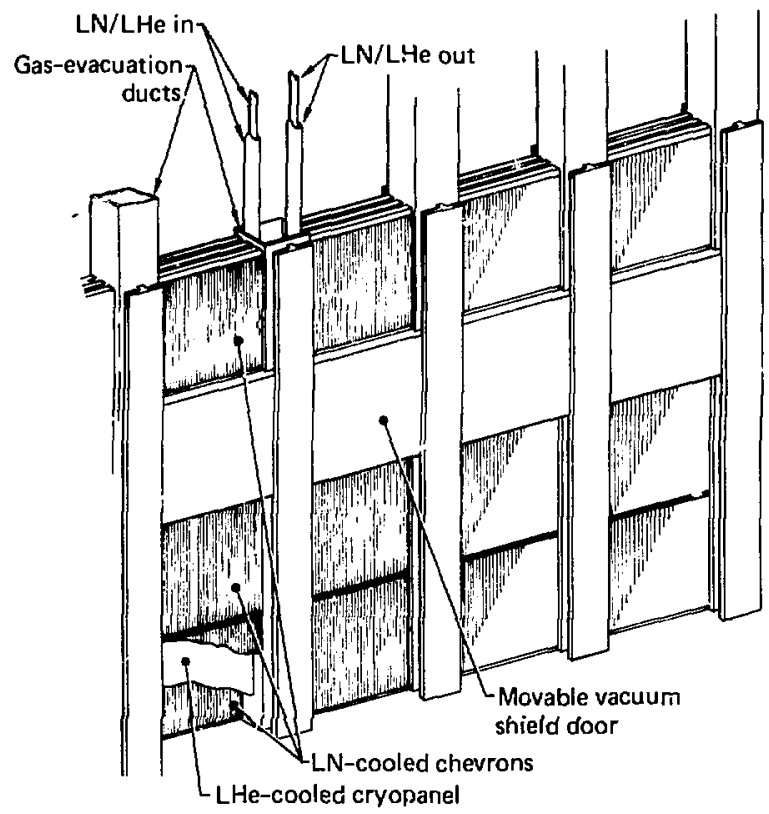

Fig. 6-36. Cryopanel assembly. 
As the beam passes through the cryopanels, the background pressure drop is not linear. Ii) the regions of highır pressure, the cryopumps take a larger share of the gas load; hence, the pressure just beyond the cesiumvapor cell can be about $2 \times 10^{-4}$ Torr, whereas the pressure at the exit from the cryopanels is considerably less than $10^{-6}$ Torr.

If we estimate the power requirements of the cryopumps ${ }^{24}$ at 0.7

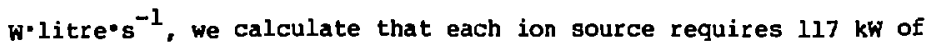
pumping power.

With an average pressure of $8 \times 10^{-6}$ Torr between 5-m-long cryopanels and a gas temperature of roughly.300 $\mathrm{K}$ [corresponding to the roomtemperature shields covering the liquid-nitrogen (LN) chevrons], the fraction of the neutral beam that suffers charge exchange in this region is

$$
F_{01}=n_{b} \sigma_{01} L=1.98 \text {. }
$$

Beyond the energy-recovery electrodes, over the next $10 \mathrm{~m}$ the average pressure is of the order of $10^{-6}$ Torr, with an average density of about $3 \times$ $10^{10} \mathrm{~cm}^{-3}$. Hence, there is an additional beam loss of about 0.58 .

Using the appropriate cross sections from Fig. 6-31 for the 1/2- and i/3- energy neutrals, we have calculated (see Table 6-10) the fraction of the neutral beam lost between the cryopanels along with the loss in the drift region beyond the cryopanels.

\subsubsection{Beam Transfer Through the Reactor Blanket}

Most of the ions traveling with the neutral beam are removed by the energy-recovery electrodes. Only a small fraction remains with the beam as it travels toward the reactor. However, this fraction is increased by ionizing collisions of the high-energy neutrals with the background gas.

As the beam gets closer to the reactor, the magnetic fhielding about the injector becomes less effective. Thus, all the ions are eventually deflected out of the neutral-beam path to bonbard the injector walls. Because the ion current density is so very small, the wall loading is $10 \mathrm{w}$ and any resulting gas release can be readily handled by the cryopanels in the injector. However, in the transfer section through the reactor blanket, there is neither magnetic shielding nor facilities for pumping. Hence the gas released by ion bombardment at the walls must be drawn away through the transfer tube either into the reactor or into the injector chamber. As a consequence, the transfer section itself acts as an impedence to the gas flow. 
Table 6-11. Injected neutral-beam components.

\begin{tabular}{|c|c|c|c|c|c|c|}
\hline Line & Item & $\begin{array}{l}\text { Total } \\
\text { beam }\end{array}$ & ${ }_{\mathrm{D}}^{125} \overline{0}^{\mathrm{keV}}$ & ${ }_{\mathrm{D}}^{62.5} \overline{0}^{\mathrm{keV}}$ & ${ }_{D}^{41.6} \overline{0}^{\mathrm{keV}}$ & $\begin{array}{c}\text { Reference } \\
\text { (Table, IIne) }\end{array}$ \\
\hline 1 & $\begin{array}{l}\text { Neutral current, } D^{0} \\
\text { (A) }\end{array}$ & & 10.4 & 2.6 & 1.8 & $T 6-9, \ell 4$ \\
\hline 2 & $\begin{array}{l}\text { Beam loss between } \\
\text { cryopanels, } F_{0 i}(z)\end{array}$ & & 1.9 & 1.9 & 1.9 & \\
\hline 3 & $\begin{array}{l}\text { Beam current leaving } \\
\text { cryopanels, } D^{0}(A)\end{array}$ & & 10.2 & 2.6 & 1.8 & \\
\hline 4 & $\begin{array}{l}\text { Beam loss beyond } \\
\text { cryopanels, } F_{\text {OF }}(z)\end{array}$ & & 0.5 & 0.5 & 0.5 & \\
\hline 5 & $\begin{array}{l}\text { In jected current, } D^{0} \\
(A)\end{array}$ & & 10.1 & 2.5 & 1.8 & \\
\hline 6 & $\begin{array}{l}\text { Injected leam power, } \\
\text { P(MW })\end{array}$ & 1.49 & 1.26 & 0.16 & 0.07 & \\
\hline
\end{tabular}

Further release of gas at the walls can increase the background gas density, resulting in a higher percentage of beam ionization, a more intense wall bombardment, and even greater gas release.

This process, as described elsewhere, ${ }^{25}$ need not be a problem if the bean current entering the transfer section is less than a critical value; i.e,

$$
I^{0}<\frac{C e}{\gamma L \sigma_{01}}=\left(1.23 \times 10^{-3}\right)(C / L) \text { amperes, }
$$

where' $\sigma_{01}$ is the cross section for ionization of the beam $\left(1.3 \times 10^{-16}\right.$ $c^{2}$ atom), $Y$ is the number of gas molecules released from the wall per incident ion (about 1 ), $e$ is the charge on an electron, $I$ is the length of the transfer section, and $C$ is the conductance of that section. If we assure that the transfer section has a uniform elliptical cross section of radii $x$ and $y$, corresponding to the ninimum tolerable radii at the focal plane (see Fig. 6-29), then $X=210 \mathrm{~cm}, Y=70 . \mathrm{cm}$. For nass $\mathrm{M}$ and temperature $T$,

$$
C=4.3 \times 10^{4}\left[\frac{x^{2} y^{2}}{L\left(x^{2}+y^{2}\right)^{1 / 2}}\right](T / M)^{1 / 2}
$$


For deuterium at $300 \mathrm{~K}$

$$
C=\left(36.4 \times 10^{20}\right) \frac{1}{\mathrm{~L}} \mathrm{~cm}^{3} \cdot \mathrm{s}^{-1} .
$$

From the above relationships, we find that for $I^{0}=2600 \mathrm{~A}$, the length of the unpumped transfer section must meet the following criterion:

$$
L \leq \frac{2.1 \times 10^{4}}{\left(I^{0}\right)^{1 / 2}}=414 \mathrm{~cm} \text {. }
$$

Because our design specifies a blanket thickness of $150 \mathrm{~cm}$, we anticipate a negligible beam loss in the transfer section.

\subsubsection{Beam Line Performance}

From the data in Tables 6-7 through 6-10, we can estimate the grid currents along the beam line, as shown in Table 6-11. For instance, line 1 of Table 6-11 shows $1.1 \mathrm{~A}$ of $\mathrm{D}^{+}$and $0.1 \mathrm{~A}$ of $\mathrm{D}_{2}^{+}$ions at $\mathrm{G2}$. These values were calculated as shown by the reference, from line 4 of Table 6-7. They represent the beam current loss in the G1-G2 gap that results from charge exhange with the background gas. All of this is presented as G2 current bysalse the approximation stipulates that all of the low-temperature ions generated in a gap between two grids be collected by the adjacent, more negative grid.

similarly, in line 2 of Table 6-11, an additional $0.1 \mathrm{~A}$ of ion current is collected at G2, while a minus $0.1 \mathrm{~A}$, signifying a current of electrons, flows into the ion-source plasma. This is explained by the reference to lines 2 and 3 of Table 6-8, which show these currents to be the result of b. ickground ionization.

From such considerations, Table 6-11 was completed, with the total e' $x$ trode currents presented in line 11 . The secondary electron emission coefficient is 1 for the high-energy Ion bombardment of G2 and G3. However, the low-energy ions drawn from beyond 63 , which bombard G3, are presumed to make a negligible contribution to the secondary electron current. Practically all the electrons in the ion acceleration region are accelerated back into the source plasma. For grid loading calculations, we assume that 10 t of the electrons have been collected at $\mathrm{Gl}$.

The power dissipated at the electrodes in Table 6-12 is derived from the data in Table 6-11. The ion energy was taken to be equal to the difference in potential between the points of origin and demise. Those ions 
Table 6-11. Distribution of electrnn and ion currents along a bean line.

\begin{tabular}{|c|c|c|c|c|c|c|c|c|c|c|c|c|c|}
\hline Line & $\begin{array}{l}\text { Processes along the } \\
\text { bead line }\end{array}$ & $\begin{array}{l}\text { Current } \\
\text { bean } \\
\text { (A) }\end{array}$ & Species" & $\begin{array}{l}\text { Source } \\
\text { Platema } \\
(A)\end{array}$ & $\begin{array}{l}\text { Gl } \\
(A)\end{array}$ & (A) & $\begin{array}{l}\text { Ca } \\
(A)\end{array}$ & $\begin{array}{c}64 / 65 \\
(A)\end{array}$ & $\begin{array}{l}66 \\
(A)\end{array}$ & $\begin{array}{l}\text { GB } \\
(A)\end{array}$ & ${ }_{(A)}^{\text {In }}$ & $\begin{array}{l}\text { njector } \\
\text { yall } \\
\text { (A) (T }\end{array}$ & $\begin{array}{c}\text { Reference } \\
\text { (Table, line) }\end{array}$ \\
\hline \multirow[t]{2}{*}{1} & Bean loss to charge & 1.1 & $0^{+}$ & & & 1.1 & & & & & & & $16-7$, \\
\hline & exchange in G1-G2 gap & 0.1 & $\mathrm{D}_{2}^{*}$ & & & 0.1 & & & & & & & \\
\hline \multirow[t]{2}{*}{2} & $\therefore$ ckground current & & & & & & & & & & & & \\
\hline & $\begin{array}{l}\cdots \text { ionization in } \\
\therefore .2 \text { gap }\end{array}$ & & $\mathrm{e}, \mathrm{D}^{+}$ & -0.1 & & 0.1 & & & & & & & $T 6-8,2 \ell 2,3$ \\
\hline \multirow[t]{3}{*}{ : } & $\because$ lost to churge & 1.1 & $D^{+}$ & & & & 1.1 & & & & & & I6-7, 26 \\
\hline & srchange in $\mathrm{C} 2-\mathrm{C} 3$ gap & 0.1 & $\mathrm{D}_{2}^{+}$ & & & & 0.1 & & & & & & \\
\hline & & 0.1 & $\mathbf{D}_{\mathbf{3}}^{+}$ & & & & 0.1 & & & & & & \\
\hline \multirow[t]{3}{*}{4} & Backgroutid current & & & & & & & & & & & & \\
\hline & from ionization in & & & & & & & & & & & & \\
\hline & G2-G3 gap & & $c, b^{+}$ & -0.4 & & & 0.4 & & & & & & T6-8. $\stackrel{0.5,00}{0}$ \\
\hline 5 & Ions from Gs-vapor cell & & $\mathrm{D}^{*}$ & & & & 2.6 & -5.2 & 2.6 & & & & \\
\hline \multirow[t]{2}{*}{6} & Secondary eniosion & & & & & & & & & & & & \\
\hline & from $62-63$ & & e & -2.6 & -0.3 & 1.3 & 1.6 & & & & & & \\
\hline \multirow[t]{3}{*}{7} & Ion current losa at & 12.6 & $\mathbf{D}^{+}$ & & & & & & & 0.1 & 12.5 & & \\
\hline & energy-recovery & 0.5 & $\mathrm{D}_{2}^{+}$ & & & & & & & & & 0.5 & $76-9,26$ \\
\hline & electrodes & 0.1 & $\mathbf{D}_{3}^{+}$ & & & & & & & & & 0.1 & \\
\hline \multirow[t]{2}{*}{$a$} & Dem lass between & & & & & & & & & & & & \\
\hline & cryopanels & 0.2 & $\mathrm{p}^{+}$ & & & & & & & & & 0.2 & I6-10, 23 \\
\hline \multirow[t]{2}{*}{9} & Deard loss beyond & 0.1 & $b^{+}$ & & & & & & & & & 0.1 & \\
\hline & cryopanels & 0.05 & $\mathrm{D}_{2}^{*}$ & & & & & & & & & 0.05 & $16-10,25$ \\
\hline \multirow[t]{3}{*}{10} & Injected neutral besa & 10.1 & $\mathrm{D}_{8}$ & & & & & & & & & & \\
\hline & & 1.25 & $\mathrm{D}_{2}^{0}$ (equiv.) & & & & & & & & & & T6-10, $\mathrm{LS}$ \\
\hline & & 1.8 & $D_{3}^{0}$ (equiv.) & & & & & & & & & & \\
\hline \multirow[t]{3}{*}{11} & Totels & 25.2 & $\mathrm{D}^{+}$ & -3.1 & -0.3 & 2.6 & 5.9 & -5.2 & 2.6 & 0.1 & 12.5 & 1.0 & $T 6-7,22$ \\
\hline & & 2.0 & $\mathrm{D}_{2}^{+}$ & & & & & & & & & & \\
\hline & & 0.8 & $\mathrm{D}_{3}^{+}$ & & & & & & & & & & \\
\hline
\end{tabular}

The $D_{2}^{0}$ and $D_{3}^{0}$ equivalent currents are $1 / 2$ and $1 / 3$ the1r equivalent $D^{0}$ currenta; e Indicatea a current of electroas.

and electrons originating in a gap between two grids were assumed to start a' a potential equal to the average in the gap.

The power loading of the electrodes, as shown in Table 6-13, is taken from Table 6-12. From estimates of the effective grid areas, we calculate an approximate value of the dissipated power density. If we allow 20 kW for the mercury diffusion pumps and $10 \mathrm{~kW}$ for the cesiun-vapor cell to be obtained directly as heat from the cooling lines around the ion bean line, $814 \mathrm{~kW}$ of heat is left for conversion to electric power. With an approxinate 30 e conversion efficiency, this results in a savings of about $250 \mathrm{kN}$, electric.

As stated in the introduction, the purpose of this study is to provide data from which the performance of the mirror hydrid reactor can be evaluated. To do this, however we had to consider the many constraints on the 


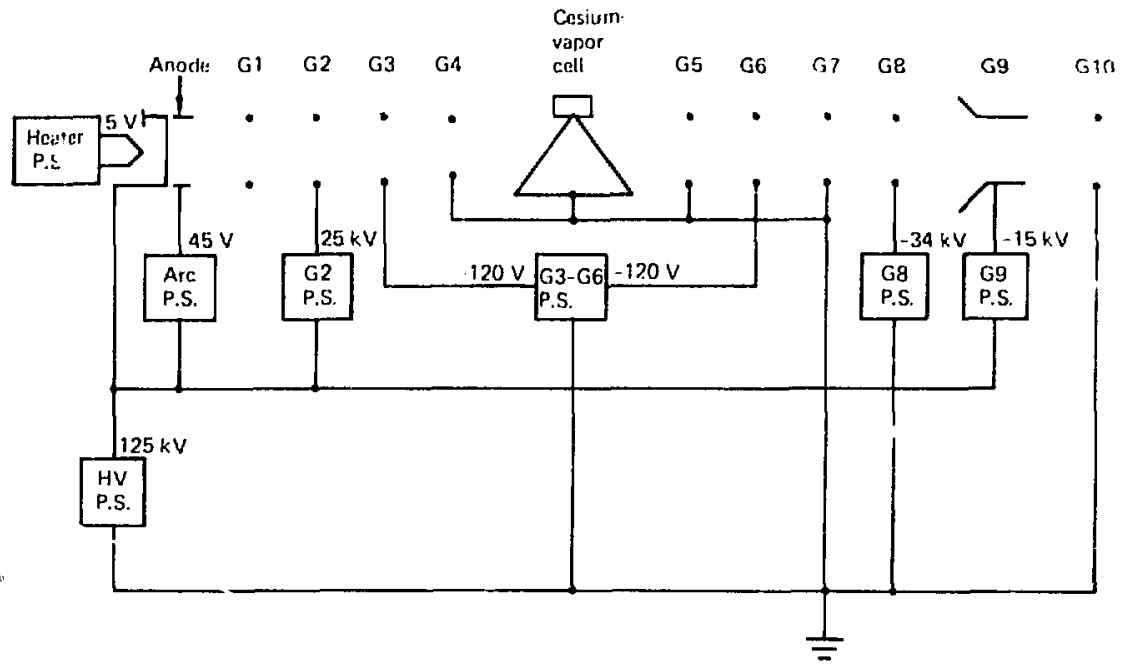

Fig. 6-37. Power supplies (P.S.) for the neutral-beam injector. 
Table 6-12. Power dissipation at electrodes in a beam line.

\begin{tabular}{|c|c|c|c|c|c|c|c|}
\hline Line & Electersit: & 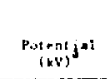 & $\begin{array}{l}\text { Electionde } \\
\text { curgent } \\
(A)\end{array}$ & $\begin{array}{l}\text { Auesagr } \\
\text { pursacle } \\
\text { eneray } \\
(k+v)\end{array}$ & Source of current & $\begin{array}{l}\text { biseipation } \\
\text { pouter } \\
\text { \{kHi }\end{array}$ & $\begin{array}{l}\text { Reference } \\
\text { (1ine of } \\
\text { Table } 6-11 \text { ) }\end{array}$ \\
\hline$i$ & $\begin{array}{l}\text { Ion } \\
\text { source }\end{array}$ & 123.0 & $\begin{array}{l}-0.1 \\
-0.6 \\
-1.2 \\
-1.4\end{array}$ & $\begin{array}{l}12.5 \\
75.0 \\
25.0 \\
175.0\end{array}$ & 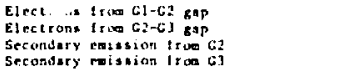 & 296.3 & $\begin{array}{l}2 \\
2 \\
6 \\
6\end{array}$ \\
\hline 2 & 61 & rloats & 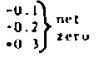 & $\begin{array}{c}23.0 \\
1<3.0 \\
\text { sesioibl. }\end{array}$ & 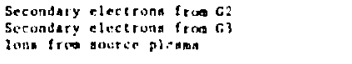 & 27.1 & 6 \\
\hline 3 & 02 & 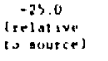 & $\begin{array}{l}1.2 \\
0.1 \\
1.3\end{array}$ & $\begin{array}{l}12.3 \\
12.3 \\
-\end{array}$ & 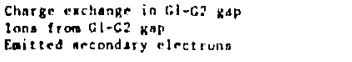 & 10.3 & $\begin{array}{l}1 \\
2 \\
6\end{array}$ \\
\hline$\therefore$ & 63 & $-6 \cdot 12$ & $\begin{array}{l}1.3 \\
0.4 \\
2.6 \\
1.6\end{array}$ & $\begin{array}{l}\text { so.0 } \\
\text { so.0 } \\
0.12 \\
-\end{array}$ & 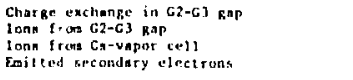 & B:3.3 & $\begin{array}{l}1 \\
4 \\
5 \\
6\end{array}$ \\
\hline s & $\mathrm{Gb}$ & 0.12 & 2.6 & 0.12 & I nnk (ton Ca-vapor cell] & 0.3 & 5 \\
\hline 6 & $\mathrm{Ca}$ & -34.0 & 0.1 & 159.0 & Stiay innd from bis & 15.8 & 1 \\
\hline 7 & 49 & -13.0 & 12.5 & 15.0 & $\begin{array}{l}\text { Alomic ions culleted at mergy-rocovery } \\
\text { elnetrodes }\end{array}$ & JRT.3 & 7 \\
\hline 8 & $\begin{array}{l}\text { Injector } \\
\text { walla }\end{array}$ & Cround & $\begin{array}{l}0.2 \\
0.2 \\
0.2\end{array}$ & $\begin{array}{l}125.0 \\
125.0 \\
125.0\end{array}$ & $\begin{array}{l}\text { Holecuiar ions not aubjecl to energy } \\
\text { tecovery } \\
\text { Charge exchange between cryopanela } \\
\text { Charge axchange beyond cryopanels }\end{array}$ & 125.0 & $\begin{array}{l}7 \\
B \\
9\end{array}$ \\
\hline
\end{tabular}

"Potentiala are relative to ground unlmas otheruiso noted.

this beam line, which delivers $1490 \mathrm{kw}$ via $14.4 \mathrm{~A}$ of neutrals into the reactor. IE we take into account the recovery of thermal energy, the injector efficiency $1490 / 2465=60.52$. With 180 beam lines in each injector, this results in a total of $269 \mathrm{MW}$ via 2600 a of neutrals.

\subsubsection{Conclusion}

As stated in the introduction, the purpose of this study is to provide data from which the performance of the mirror hydrid reactor can be evaluated. To do this, however we had to consider the many constraints on the neutral-beam injector. These include restrictions on size, the need for efficiency, and, in addition, the reliability and long life that is essential in a component of a commercial, power-producing systen.

Thus, we found it necessary to use components that have yet to be perfected. These include ion sources with long-life cathodes, grids designed for continuous operation, electrically insulating heat exchangers, recycling cesium-vapor cells, energy-recovery electrodes, and regenerating liquidhelium-coled cryopumps. To assure reliability and provide a minimum of six months of continuous operation, it is assumed that the electrical components and the high-voltage insulators are mounted in regions remote fron the laam lines, where the radiation is low. In addition, protective circuits are considered that will prevent danage should a high-voltage breakdown occur, while spare beam lines are included to guarantee continuous performance. 
Table 6-13. Electrode loading and thermal recovery along a beam line.

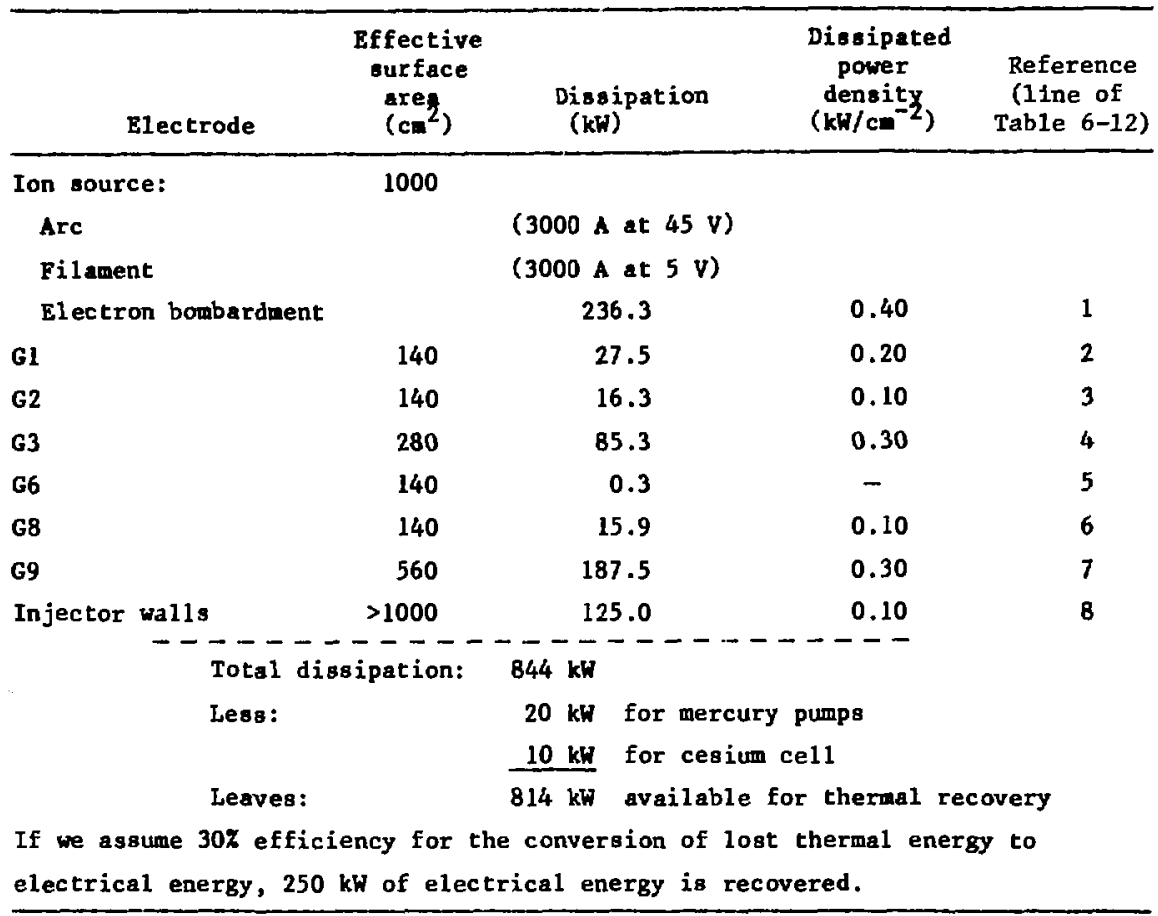

Table 6-14. Power supply requirements of a $28-\mathrm{A}$ ion beam 1 ine.

\begin{tabular}{lccccr}
\hline $\begin{array}{c}\text { Power } \\
\text { supply }\end{array}$ & $\begin{array}{c}\text { Voltage } \\
(\mathrm{kV})\end{array}$ & $\begin{array}{c}\text { Current } \\
\text { drain } \\
(\mathrm{A})\end{array}$ & $\begin{array}{c}\text { Power } \\
\text { drain } \\
(\mathrm{kW})\end{array}$ & $\begin{array}{c}\text { Power } \\
\text { supply } \\
\text { efficiency } \\
(\mathrm{z})\end{array}$ & $\begin{array}{r}\text { Input } \\
\text { powsr } \\
(\mathrm{kW})\end{array}$ \\
\hline Heater & 5.0 & 3000 & 15 & 90 & 16 \\
Arc & 45.0 & 3000 & 135 & 90 & 150 \\
High voltage & 125.0 & 16.0 & 2000 & 95 & 2105 \\
G2 & 25.0 & 2.6 & 65 & 90 & 72 \\
G3 G6 & 0.12 & 8.5 & 1 & 90 & 1 \\
G8 & 34.0 & 0.1 & 3 & 90 & 4 \\
G9 & 15.0 & 12.5 & 188 & 90 & 209 \\
& & & & & Total: \\
\hline
\end{tabular}


Table 6-15. Power and efficiency of a beam line.

\begin{tabular}{lr}
\hline & $\begin{array}{r}\text { Power } \\
(\mathrm{kW})\end{array}$ \\
\hline $\begin{array}{l}\text { Power requirement: } \\
\text { Power supplies } \\
\text { Mercury pumps (operated thermally) }\end{array}$ & 2557 \\
Blower pumps & 0 \\
Roughing pumps & 16 \\
Cryopumps & 20 \\
Cesium-vapor curtain & 117 \\
& 10 \\
Power recovery: & 2700 \\
Power input: & -250 \\
& 2450 \\
Power output: & 160 \\
10.1 A at $125 \mathrm{keV}$ & 70 \\
2.5 A at $62.5 \mathrm{keV}$ & 1490 \\
1.8 A at $41.6 \mathrm{keV}$ & - \\
\hline
\end{tabular}

To build such an injector will require considerable effort. However, despite the fact that not all of the desired components are part of today's development programs, no fundamental obstacle has been found that will prevent their ultimate development.

\subsection{Beam Line Layout}

The layout of the beam lines must satisfy the following constraints:

- Achieve the correct pitch angle to satisfy the plasma-physics analysis.

- Provide uniforn ion deposition on all drift gurfaces.

- Attenuate the injected beam as completely as possible.

- Conform to physical-access limitations.

The plasma requirenent of $5200 \mathrm{~A}$ of injected neutrals is satisfied witr. two injector arrays, each supplying $2600 \mathrm{~A}$; one injects near the plasma midplane ard the other in the plasin fan. The plasma represents a thick target at the idplane $\left(2 R_{p} n_{0}=4.5 \times 10^{16} \mathrm{~cm}^{-2}\right.$, where $R_{p}$ is the plasma 
radius and $n_{0}$ is the central ion density). The mean free path for interaction (charge exchange and ionization) 26

$$
\lambda=\frac{v_{0}}{n_{0}\left(\left\langle\sigma v_{c_{i}}+\langle\sigma v\rangle_{i}+\left\langle\sigma v_{e l}\right)\right.\right.}
$$

is $\lambda \simeq 70 \mathrm{~cm}$ for $125 \mathrm{keV} \mathrm{D}^{0}$. Here, $\mathrm{v}_{0}$ is the velocity of the injected deuterium atom, $n_{0}$ is the plasma midplane ion density, $\langle\sigma v\rangle_{c x}$ and $\langle\sigma v\rangle_{i}$ are the rate coefficients for charge exchange and lonization by the 1ons, respectively, and $\langle\sigma v\rangle_{\text {ei }}$ is the rate coefficient for ionization by electrons. Therefore, the midplane injector supplies ions only to the outer drift surfaces. To penetrate to the inner drift surfaces, the second injector is aimed at the plasma fan where the plasma is a comparativeiy thin target (nl $\simeq 1.9 \times 10^{16} \mathrm{~cm}^{-2}$ ).

The dimensions of the injector tanks are shown in Fig. 6-38. The beam apertures are located on the surface of a sphere with a 4-m radius and a center at the origin of the magnet coordinate system. Both tanks are 10cated in the positive $z$ region above the turnarounds of the lower half of the magnet as shown in Fig. 6-39. Note that the centerlines of the tanks are not coincident with the $x$ axis, but are offset with one side of each of the tanks approximately in the $x-z$ plane.

The attenuation of the beam as it penetrates the plasma is described by the relation 26

$$
\mathrm{dj} / \mathbf{j}=-\gamma \mathrm{d} \xi
$$

Here, $j$ is the beam current at a distance $s$ from the surface of the plasma, $\xi=s / l$, and $\ell$ is the path length through the plasma. The attenuation coefficient, $Y$, is

$$
r=l /
$$

where $\lambda$ is evaluated with the local ion density $n=n(s)$. The geometry is shown in Fig. 6-40. Thus, the current as the beam exits from the plasma is

$$
j_{e}=j_{0} \exp \left(-\int_{0}^{1} \gamma d \xi\right),
$$



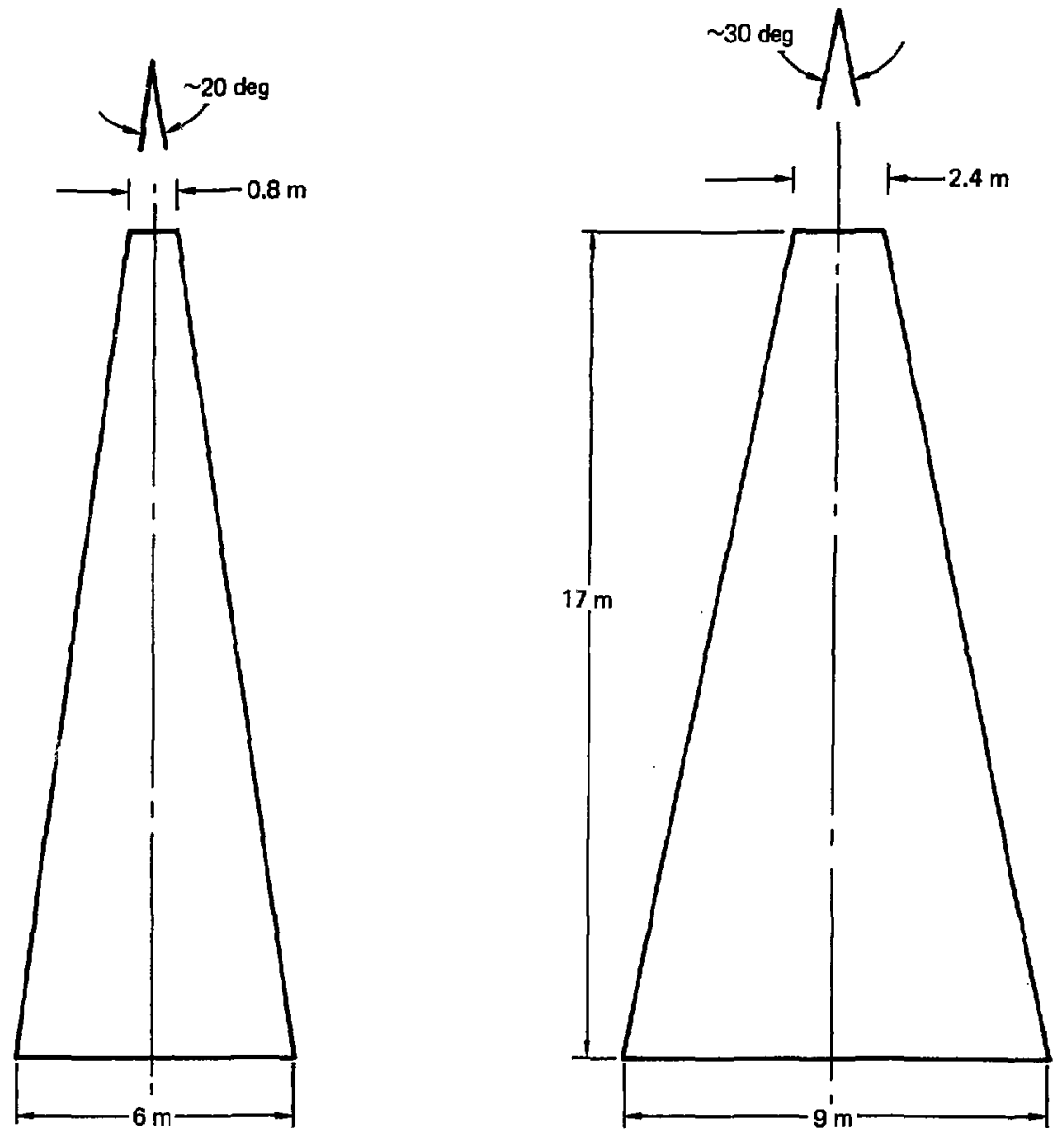

Fig. 6-38. Injector tank dimensions.

205 

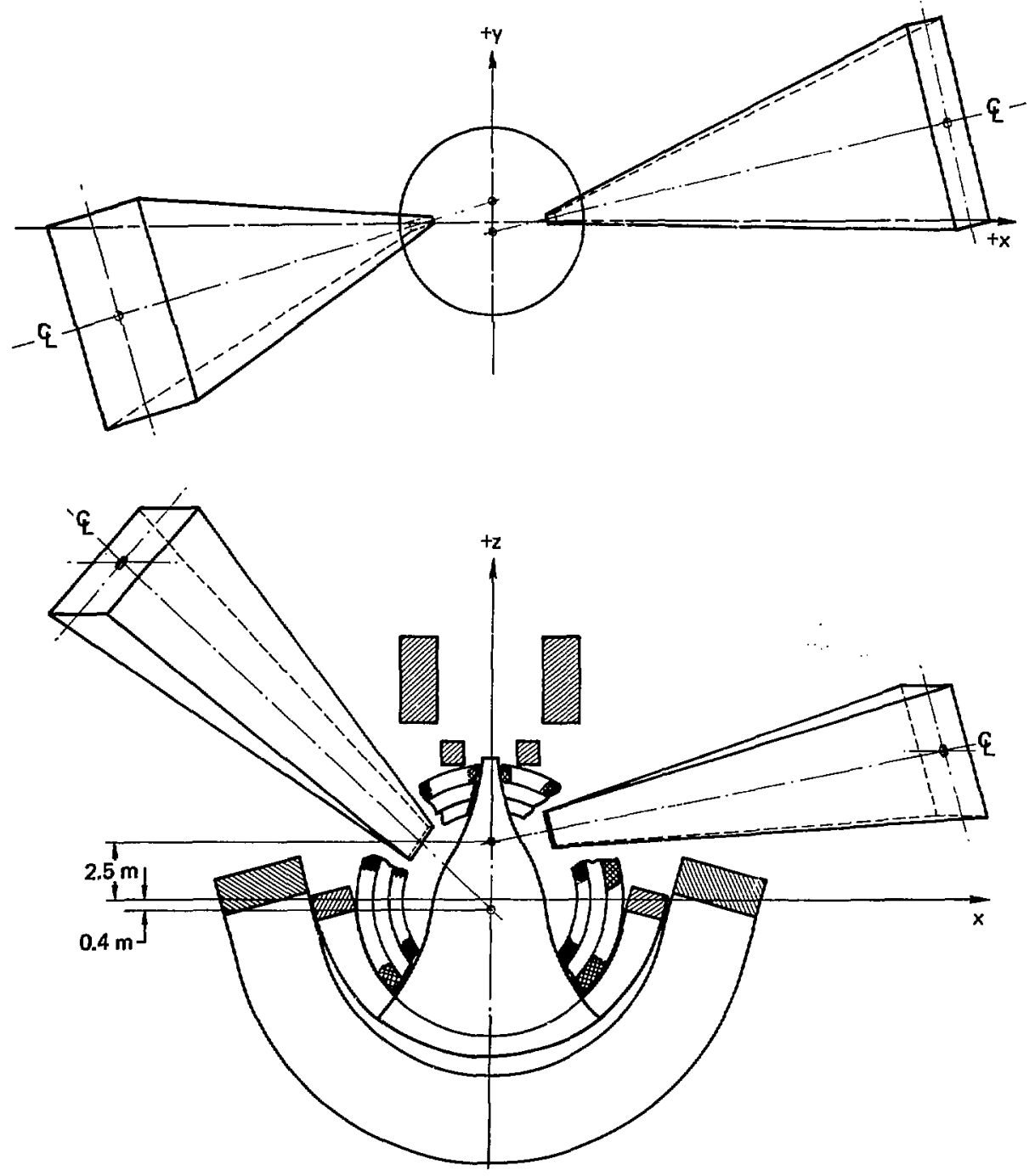

Fig. 6-39. Injector tank locations. 


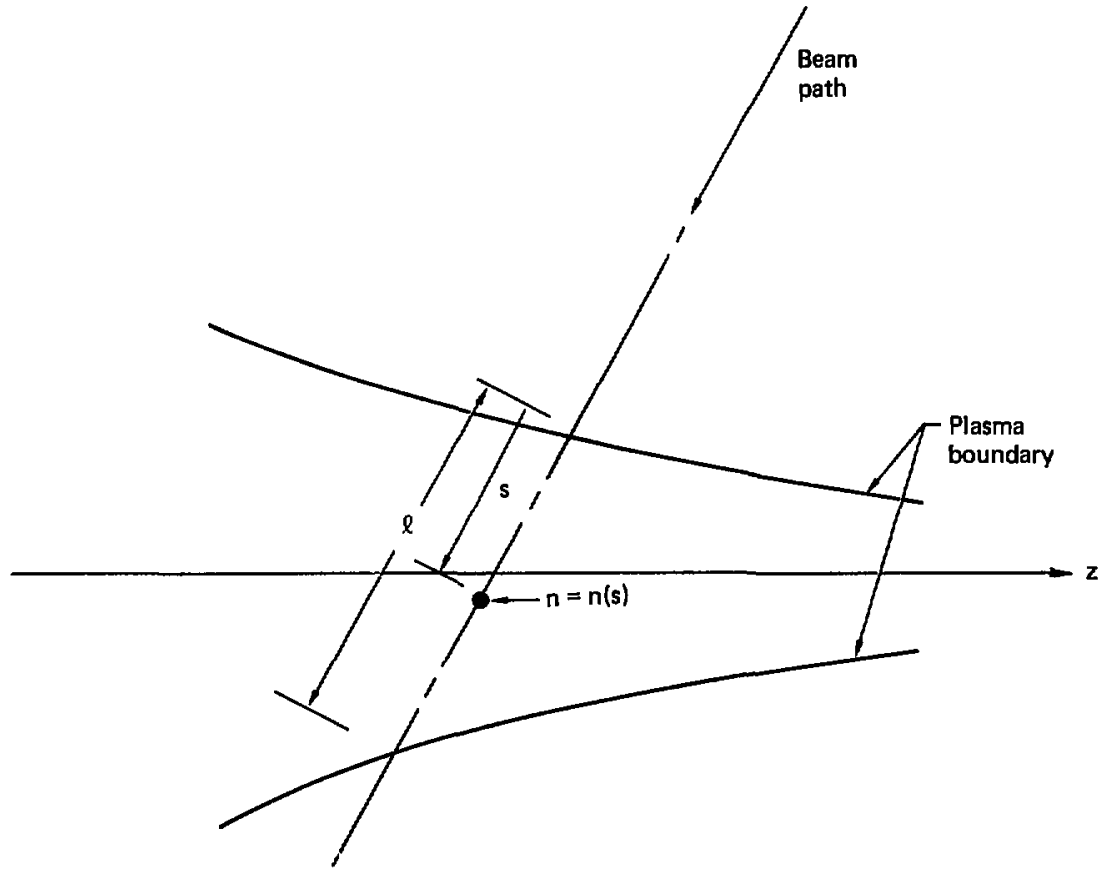

Fig. 6-40. Beam attenuation variables. 
where $j_{0}$ is the unattenuated current. In the layout of the beamline, it is desirable to make $j_{e} / j_{0}$ as small as possible (approximately 1 or less) so energy-expensive injected atoms will not be wasted and the first wall will be protected from bombardment by these high-energy particles. The ion pitch angle is defined as

$$
\theta=\arctan \left(\mathrm{v}_{\perp} / \mathrm{v}_{\|}\right) \text {, }
$$

where $v_{1}$ and $v_{1 l}$ are the perpendicular and parallel velocity components, respectively. Using conservation of kinetic energy

$$
v^{2}=v_{\perp}^{2}+v_{\|}^{2}
$$

and conservation of magnetic monent

$$
\mu=\frac{m v_{\perp}^{2}}{2 B}
$$

for an i'sn along a field line, the pitch angle at two different points along a field line are related by

$$
\theta_{2}=\arctan \left[\frac{\sin ^{2} e_{1}}{B_{1} / B_{2}-\sin ^{2} \theta_{1}}\right]^{1 / 2} \text {. }
$$

Therefore, using the subscripts " 0 " to denote the midplane and " $i$ " to denote the point of ion injection, the midplane pitch angle is related to the pitch angle at the point of injection by

$$
\theta_{i}=\arcsin \left[\frac{\left(B_{i} / B_{0}\right) \tan ^{2} \theta_{0}}{1+\tan ^{2} \theta_{0}}\right]^{1 / 2} .
$$

The plasma physics analysis specified a midplane injection angle of $\theta_{0} x$ $65 \mathrm{deg}$. Thus, the above equation specifies the orientation of the beam line relative to the field line at the injection point.

In Fig. 6-41, the pertinent ajection parameters are shown in the $x-z$ plane. These quantities include the outer plasma boundary $\left(R_{p}=2.5 \mathrm{~m}\right)$, the plasina density contours (evaluated from the Fokker-Planck calculation, Sec. 5.5), and the two injection patterns. The midplane injector is located in the region of maxinum density $\left(n / n_{0}=1.0\right)$ and the fan-injector centerline is near the $n / n_{0}=0.7$ density contour. 


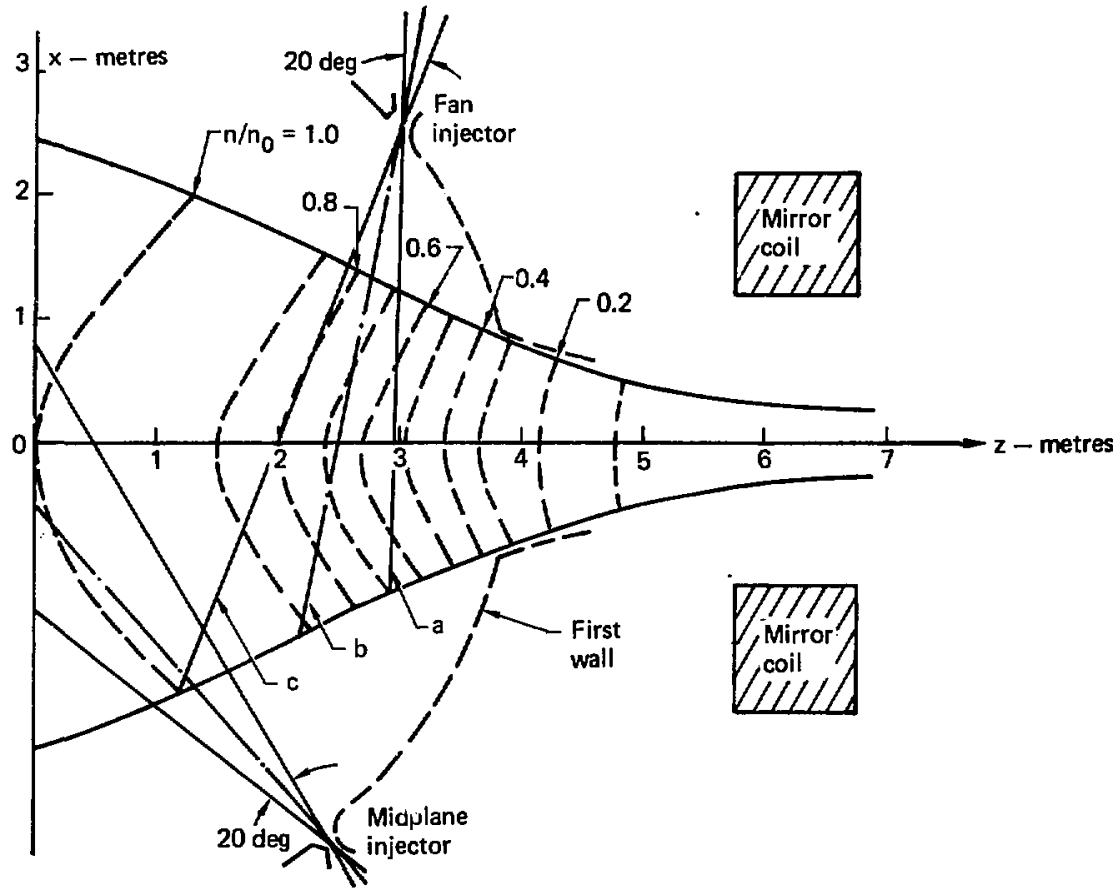

Fig. 6-11. Injection patterns. 
The fan injector was located as far from the midplane as possible within the constraint of $\theta_{0}=65 \mathrm{deg}$. That is, with reference to Eq. $(60)$, the maximum value of $\theta_{i}$ is $90 \mathrm{deg}$. Using the specified midplane pitch angle, $\theta_{0}=65 \mathrm{deg}, y$ ields $B_{i} / B_{0}=1.22$, which is the maximum value of the field at the injection point where $\theta_{0}=65$ deg can still be attained. Larger values of $B_{i} / B_{0}$ will result in $\theta_{0}<65 \mathrm{deg}$. The pitch angles for the fan injector are shown in Fig. 6-42. Here, three trajectories are plotted, labeled $a, b, c$ and are indicated in Fig. 6-41. In Fig. 6-42, the plot is $\theta_{i}$ along the trajectory, where the circles ( $\left.\theta\right)$ indicate the points where the beam enters the plasma. The attenuation analysis resulted in $j_{e} / j_{0}=0.008,0.001$ and 0.000 for trajectories $a, b$, and $c$, respectively. A cross section, in the $x-y$ plane, of the fan injector pattern is shown in Fig. 6-43. Here the off-set of the injector pattern is clearly shown; injection occurs just into the positive $y$ region. This arrangement is used to permit better penetration into the inner drift surfaces than would be obtained by having the beam centerline colinear with the $x$ axis.

The midplane injector is located near the maximum density contour, resulting in $\theta_{i} \simeq \theta_{0}$ for the part of the beam in the negative $x$ region. The beam was or iented such that $\theta_{i}=65 \mathrm{deg}$ for the field line having a midplane radius of $1.25 \mathrm{~m}$, resulting in a modest variation of the injection angle for the various trajectories about a mean value of 65 deg. Here, the beam is completed attenuated $\left(j_{\mathrm{e}} / j_{0}<0.001\right)$ before it reaches the $\mathrm{z}$ axis.

\subsection{End Tanks}

\subsubsection{Physical Processes and Functions}

In the mininum-B, or standard, mirror confinement configuration, plasma exits from the confinement volune along open magnetic field 1 ines via the two mirror regions. This plasma exhaust consists of unburned fuel ions $\left(D^{+}\right.$and $\mathrm{T}^{+}$), alpha particles generated in the fusion reactions $\left({ }^{4} \mathrm{He}{ }^{++}\right)$, and a flux of electrons equal to the total positive charge flux. Because the particle flow escapes from the magnetic well with considerable kinetic energy, there are both large mass and energy fluxes into 


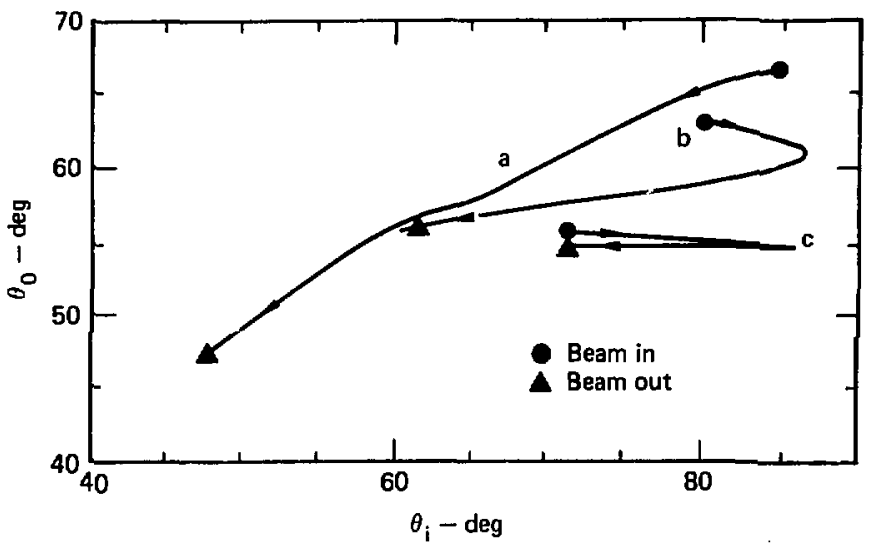

Pig. 6-42. Injection angles for fan injector.

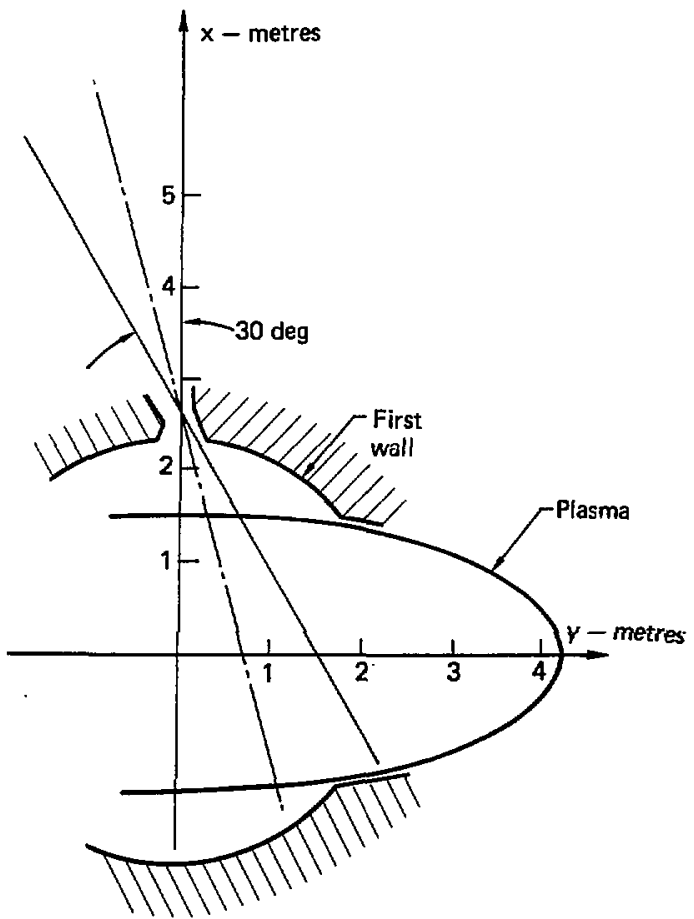

Pig. 6-43. Cross section of fan injector pattern in the $x-y$ plane, $z=2.8 \ldots$. 211 
the end tanks. Two end tanks are required, one for each leakage fan, and are located external to the confinement magnet enclosing the field lines that carry the plasma flow. Upon striking surfaces in the end tanks, the plasma is neutralized and transfers its energy to the various surfaces in the tanks. The end tanks must therefore perform two main functions:

- Pumping of the gas load resulting from the neutralized plasma exhaust.

- Recovery of the exhaust plasma energy.

In this reactor, the magnet is designed with one mirror field 5t stronger than the other. This magnetic field configuration causes a selective leakage situation, ${ }^{27}$ such that 90 s of the confined ions ${ }^{\left(D^{+}\right.}$and $\mathrm{T}^{+}$, flow out of the weak mirror and the remaining 10 out of the strong mirror. The alpha particles, because they do not behave adiabatically, are not magnetically confined. Rather, their nonadiabatic behayior causes them to rapidly scatter into the loss cone and stream out of the confinement volune along field lines into the end tanks. Thus the alphas are not subject to selective leakage, and equal currents of these ions flow out of the two mirrors.

The motivation for using selective leakage is that the size of the end tank is proportional to the amount of plasma flow into it. Thus the tank receiving the exhaust from the strong mirror is much smaller than the weakmirror end tank, and provides a convenient access path from outside the reactor into the plasma confinement chamber for blanket maintenance operations. The strong mirror is located on top of the reactor and the blanket maintenance remote handling equipment is lowered into the reactor from the top of the PCRV through the small end tank as described in Secs. 8.2 and 10.3 .

In the present study, a mechanical design of the end tasks was not undertaken. Rather, the end tank designs were scaled from a previous standard mirror reactor study, ${ }^{28}$ using the analytical models described in Refs. 29, 30, and 31. In the large tank, handling 908 of the fuel lons, a single stage direct converter ${ }^{31,32}$ is used to convert approximately 408 of the plasma kinetic energy directly to electrical energy; the remaining kinetic energy is deposited as thermal energy. In the small tank, all of the plasma kinetic energy is simply deposited as thermal energy. 


\subsubsection{End Tank Description}

The major features of an end tank are shown in Fig. 6-44. The plasma exhaust expands along the field lines of the external, or fringe, field of the magnet. The tank sidewalls (Fig. 6-45) enclose the fan, and the end wall is oriented perpendicular to the field lines. The plasma flow is terminated on the plasma collector, which, in the weak-mirror tank, is a single-stage direct converter and, in the strong mirror, is a plasma thermal dump, i.e., a grounded, convectively-cooled wall. The vacuum-pumping equipment, cryopanels, are located on the sidewalls where they are not subject to direct bombardment by neutrons from the plasma.

The dimensions of the tank are determined by the dimensions of the plasma fan, and the radial location of the plasma collector is determined by power flux considerations as follows. The plasma exhaust expands adiabatically in the flux tube defined by the radial plasma boundary in the confinement volume. By conservation of magnetic flux,

$$
A_{0} B_{0}=A_{c} B_{c} \text {, }
$$

where $A$ denotes the cross-section area of the flux tube and $B$ the magnetic field intensity; the subscripts " 0 " and " $c$ " denote the plasma midplane (z = 0 ) and plasma collector location in the end tank, respectively. If $P_{D C}$ is the power carried by the plasma exhaust into the end tank, then the power flux at the plasma collector is

$$
\dot{q}_{C}^{m}=\frac{P_{D C}}{A_{C}}
$$

or, using Eq. (61),

$$
\dot{q}_{c}^{n}=\frac{B_{c} P_{D C}}{A_{0} B_{0}}
$$

Recalling that the magnetic field decreases with distance from the magnet center, the radial location of the collector is determined by requiring that $B_{c}$ be low enough to yield a value of power (or heat) flux $\dot{q}_{c}^{n}$ that will meet heat-transfer constraints.

The details of the tank sidewalls are shown in Fig. 6-45. A set of water-cooled chevrons, at $\sim 300 \mathrm{~K}$, face the interior of the tank and remove incident heat loads resulting from the plasma collection process. Inside of 

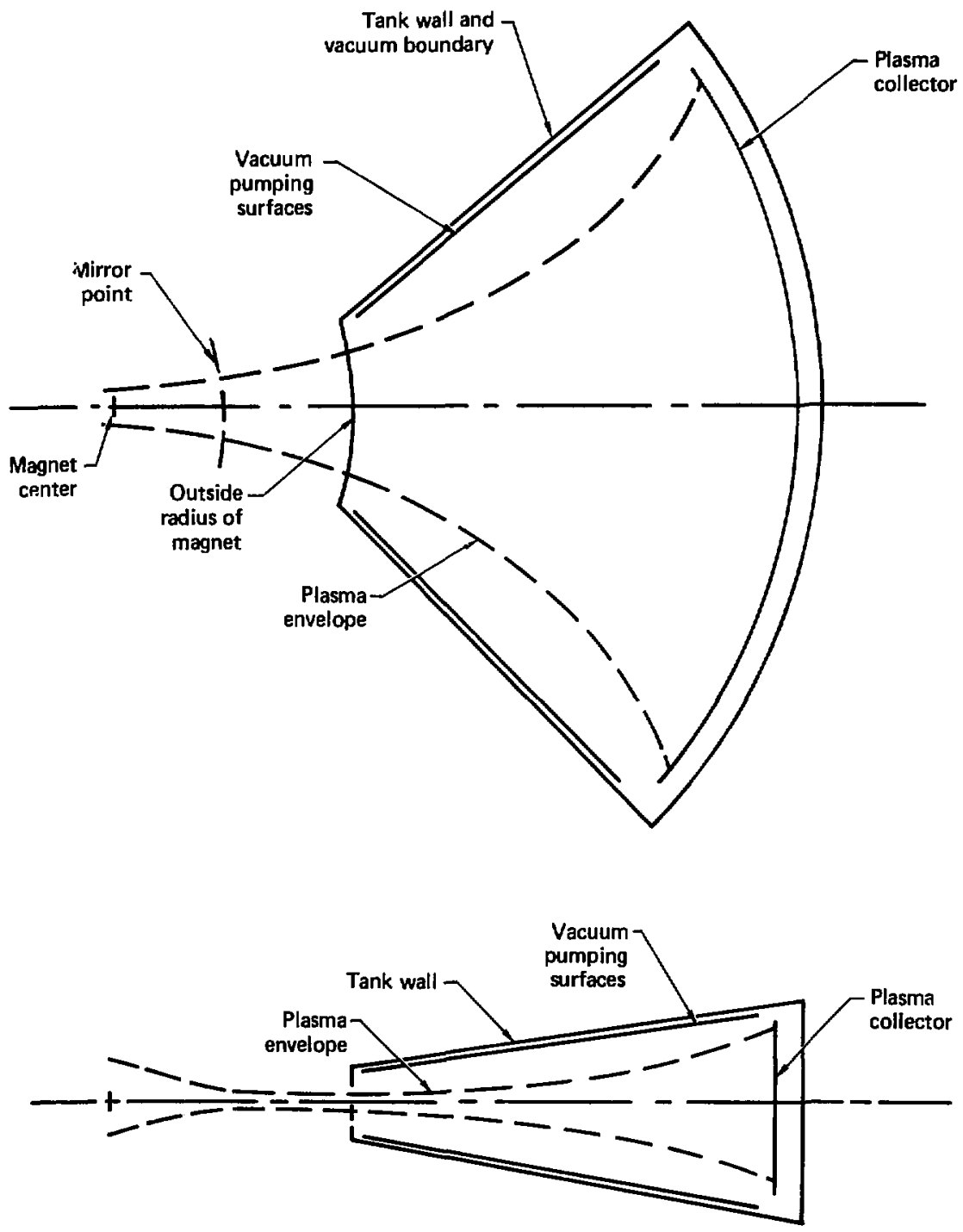

Fig. 6-44. End-tank features. 

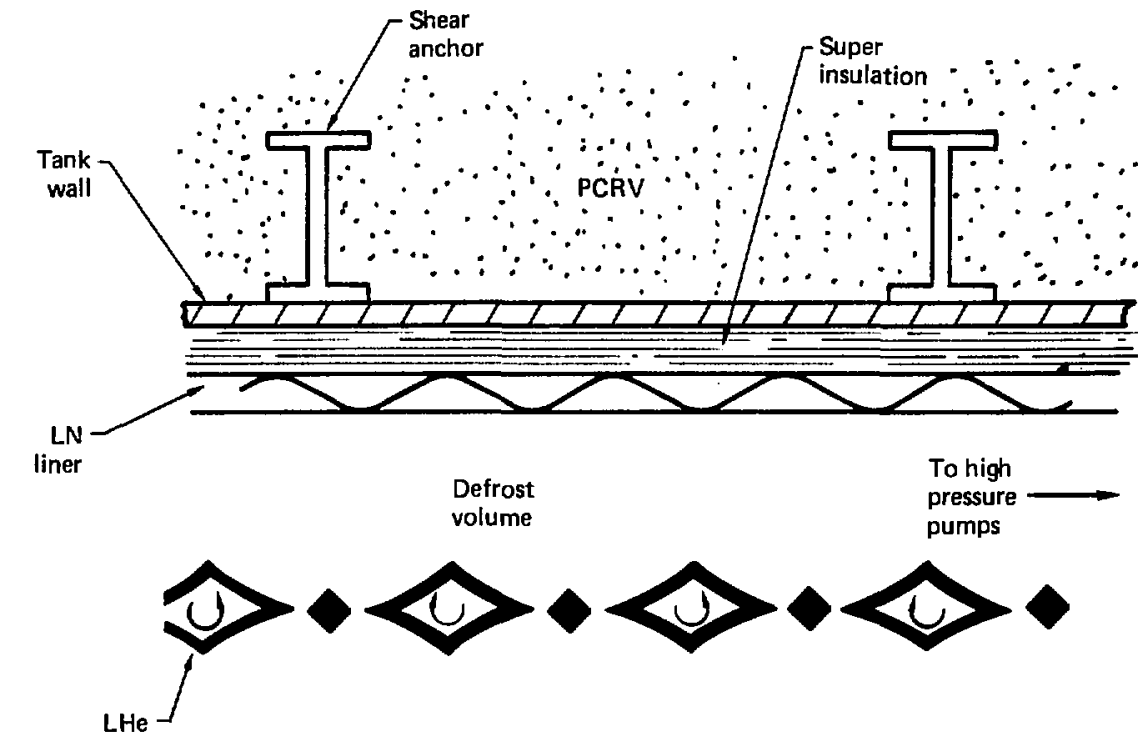
cryopanels
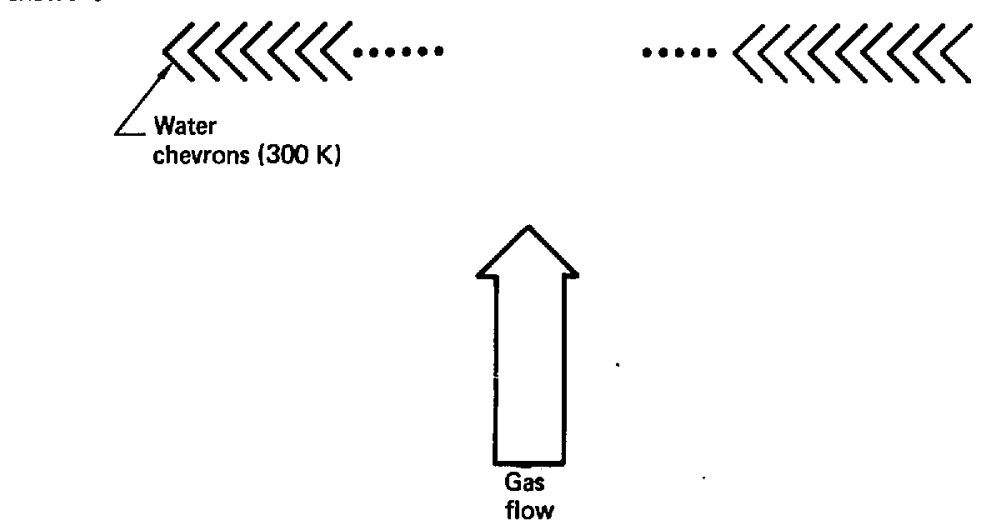

Fig. 6-45. Tank sidewall detalls. 
the water chevrons is a set of high-emissivity, liquid-nitrogen (LN) cooled chevrons $(77 \mathrm{~K})$ that protect the liquid helium (LHe) surfaces from an excessive radiative heat load. The high emissivity is used to provide a low transmission probability for photons from the water-cooled chevrons.

Punping of the $\mathrm{D}_{2}$ and $\mathrm{T}_{2}$ gas molecules that result from the neutralized plasma exhaust occurs by cryocondensation on LHe $(4.2 \mathrm{~K})$ panels. The cryopanels are mounted on rotatable valves (as proposed by Werner and Hoffmann) ${ }^{28,33}$ that, when full of condensed gas, are rotated 180 deg to face the high-pressure $\left(\sim 10^{-4}\right.$ Torr $)$ volume behind the cryopanels. In this position, the panels are defrosted and the gas removed by aercury ejector pumps, while the fresh panels on the other side of the valve continue to provide pumping capacity. A pumpiry speed of 4.3 litres $/ \mathrm{s}^{\cdot \mathrm{cm}^{2}}$ was used to calculate the cryopanel area. This speed is based on an average molecular weight of 4.7 and two sets of chevrons with a combined transmission coefficient of 0.15 .

The outside of the defrost region is bounded by a low-emissivity LN liner, a layer of superinsulation, and then the tank wall. The tank wall is a thin stainless steel membrane that provides vacuum integrity and derives structural integrity from the PCRV. The membrane is attached to the PCRV with shear anchors as shown in Fig. 6-45.

The pumping capacity in the present tank design is sized to accommodate the $\mathrm{D}_{2}^{0}$ and $\mathrm{T}_{2}^{0}$ gas load resulting from the injection of high-energy neutrals. Additional gas loads result from the alpha particles generated in the fusion reactions and cold $\mathrm{D}^{+}$ions required to stabilize the plasma boundary. As a IHe cryopanel will not pump heliun, alternate techniques must be used. Two promising methods are cryotrapping ${ }^{34}$ and cryosorption ${ }^{35}$; both are currently under development. Considerations for alpha-pumping capacity in the present design were, therefore, not included because of the absence of design information. The stabilizing cold ions flow into the end tanks along the surface of the plasma envelope shown in Fig. 6-44, It has been proposed in a previous study ${ }^{28}$ that this flow be scraped off and purped at a relatively high pressure with a comparatively amall pumping speed. However, the design of the equipment to produce and pump this particle flow was not included in the present study because of $t$ ime limitations and, therefore, is an important topic to be addressed in future studies. 
A schenatic of the sangle-stage direct converter, used in the (large) weak-mirror end tank, is shom in Fig. 6-46. It consists of a series of three electrodes. The grounded grid is composed of tubes cooled by high temperature helium and it intercepts a sall fraction of the ions and all of the electrons. The negative grid is a radiatively cooled graphite screen that serves to repel electrong from the collector. The collector is operated at a high positive voltages it consists of a contiguous wall of tubes that are convectively cooled by high-temperature helium.

In the (small) strong-mirror end tank, the grounded and negative grids are not used, and the collector is operated at ground potential. The wall end tank collector, therefore, functions simply as a thermal dump.

\subsubsection{Boundary Conditions for End Tank Design}

The input and output powers are shown schematically in Fig, 6-47. Input plasma power $P_{D C}$ is the kinetic energy of $\mathrm{D}^{+}, \mathrm{T}^{+}$, alphas, and electrons. Also, electrical power is input to the negative grid $P_{e, n}$ * The sum of these two $\left(P_{D C}+P_{e, n}\right)$ is removed in the form of low-temperature thermal energy $P_{t, w}$ from the water-cooled chevrons on the sidewalls, high-tenperature thermal energy from the grounded and collector grids $P_{t, g}$ and $P_{t, c}$, and electrical output from the collector $P_{e, c^{*}}$ The charged particle power leaving the confinement volume is listed in Table 6-16. The division of this power into the two end tanks is discussed in the subsequent sections. Of the 5204 A of energetic neutrals that are injected, $46 \mathrm{~A}$ or 0.88 are burned in the fusion reactions, resulting in $46 \mathrm{~A}$ of (doubly-charged) alphas, ${ }^{4} \mathrm{e}^{++}$.

The confined plasma, at the midplane, is at a high positive potential (ambipoiar potential in Table 6-16). This potential drops to 20 of its midplane value at the mirror and to ground at the grounded grid, as shown in Fig. 6-48. The power and energy listed for each plasma specie in Table 6-16 is at the grounded grid.

The magnetic field strength outside the magnet and along the $z$ axis is plotted in Fig. 6-49. The dimensions of the plasma fan in the $x-2$ plane appear in Fig. 6-50; the dimensions in the $y-z$ plane can be obtained from Fig. 6-50 by the transformaticin $(x, z) \rightarrow(y,-z)$. The cross section of the fan is elliptical.

The heliu-coolant flow conditions (inlet and outlet terparatures, pressure) in the end tanks are the sane as for the blanket wo that the high 


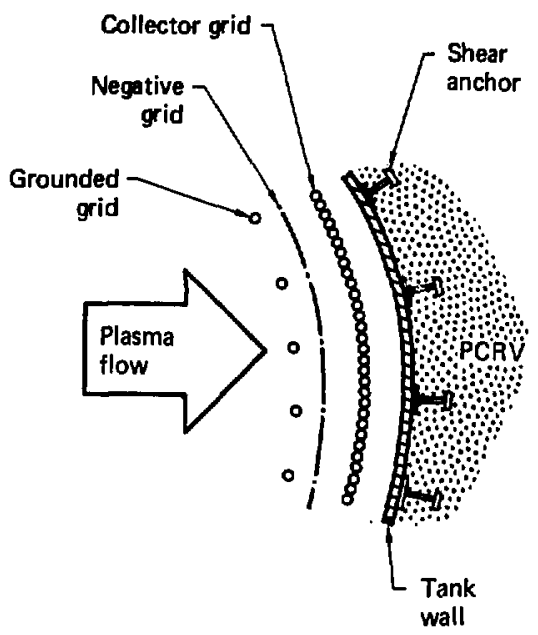

Fig. 6-46. Single-gtage direct converter.

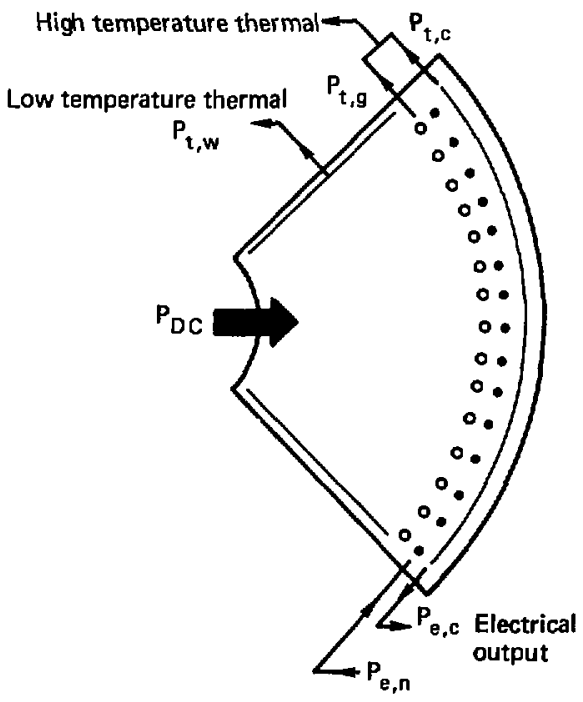

Fig. 6-47. End-tank power flow.

Electrical input 
Table 6-16. Plasma exhaust power flow.

\begin{tabular}{|c|c|c|c|}
\hline & Power, MA & Current, A & $\begin{array}{c}\text { Average } \\
\text { Energy, keV }\end{array}$ \\
\hline Fuel ions $\left(\mathrm{D}^{+}, \mathrm{T}^{+}\right)$ & 580.8 & 5158 & 112.6 \\
\hline Alphas & 80.5 & 46 & 3500 \\
\hline Electrons & 48.8 & 5200 & 9.4 \\
\hline Total & 710.1 & & \\
\hline \multicolumn{4}{|c|}{ Ambipolar potential, kV: } \\
\hline At midplane & 63.8 & & \\
\hline At mirror & 12.7 & & \\
\hline \multicolumn{4}{|c|}{ Magnetic field at the mirror, T: } \\
\hline Weak mirror & 5.66 & & \\
\hline strong mirror & 5.95 & & \\
\hline
\end{tabular}

temperature thermal output $\left(\mathrm{P}_{t, g}+\mathrm{P}_{t, c}\right)$ may be used in a common thermal conversion system.

\subsubsection{Weak Mirror End Tank}

The input powers and currents to the tank are listed in Table 6-17. The ion current consists of 90 of the fuel lons leaking from the confinement volume and 50 of of the alphas generated in the fusion reactions. The electron current is equal to the total ion current. The fuel ion selective leakage of 908 , as the result of a 58 mirror bias, was estimated to be a lower limit based on a simplified analytic model. 27

of the ions entering the tank at the mirror mouth, a fraction of them are lost by charge-exchange reactions on the cold background gas before they

\begin{tabular}{lcc} 
& Table 6-17. & Input to weak mirror end tank. \\
\hline & Power, & $\begin{array}{c}\text { Current, } \\
\text { A }\end{array}$ \\
\hline Fuel ions & 522.7 & 4641 \\
Alphas & 40.2 & 23 \\
Electrons & 43.7 & 4664 \\
Total & 606.7 & \\
\hline
\end{tabular}




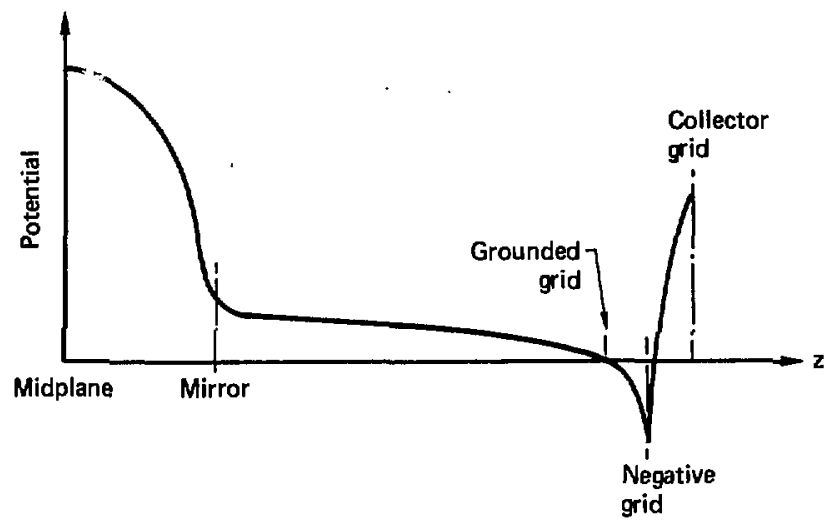

Fig. 6-48. Potential distribution.

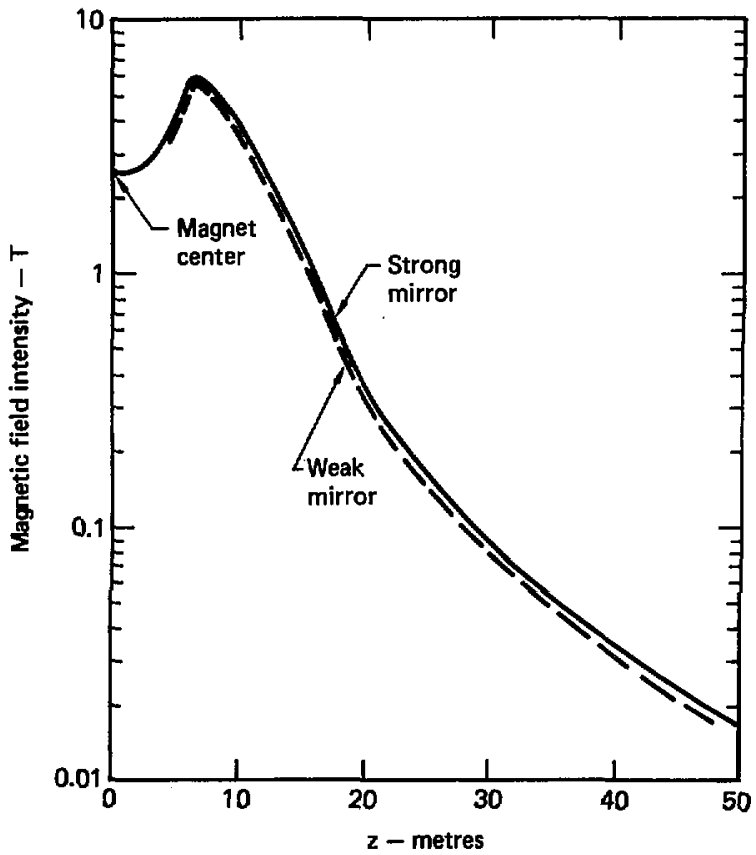

Fig. 6-49. Field intensity in the end tanks along the $z$ axis. 


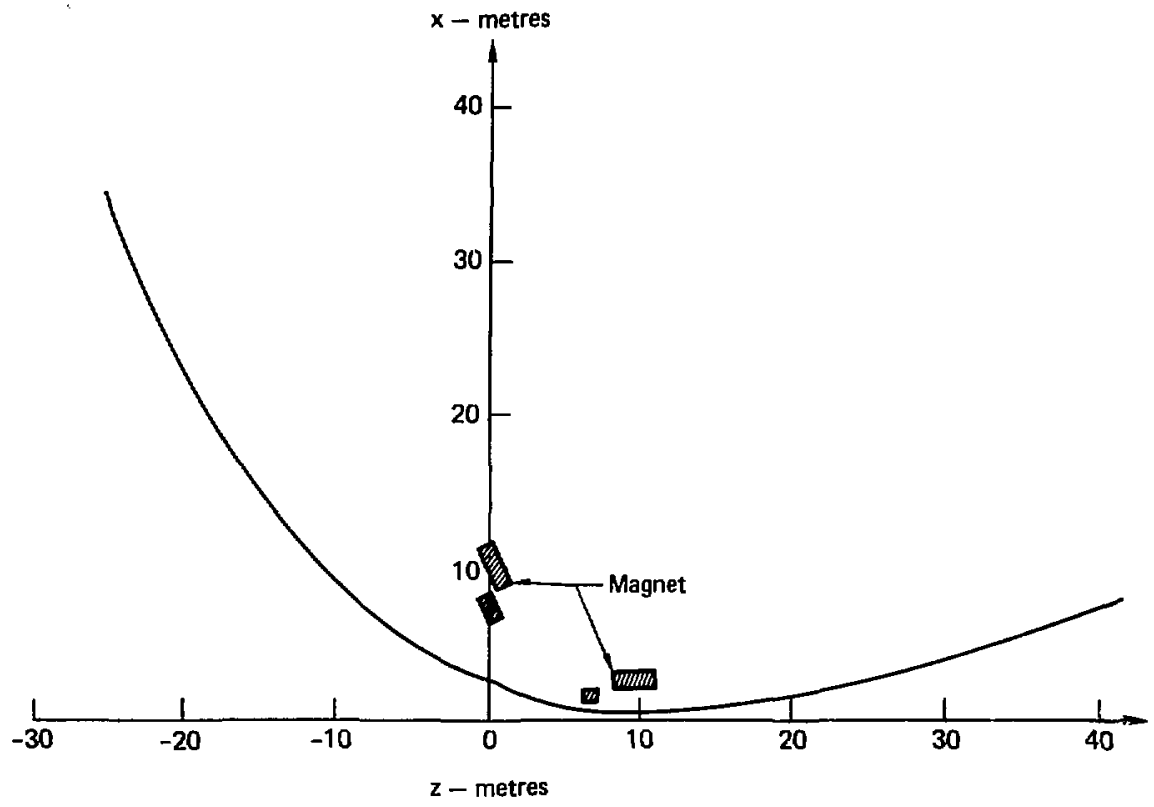

Fig. 6-50. Plasma-fan dimensions. 
reach the direct converter grids. The energy carried by these ions is ultimately deposited in the water-cooled chevrons on the tank sidewalls. All of the input electrons and the energy they carry are intercepted by the grounded grid; but because the transmission coefficient of the grounded grid is high, many passes by the electrons occur before they are collected. Some of the ions are also lost by interception as they pass through the grounded grid. In addition, the grounded grid receives thermionic and secondary electrons from the negative grid. All of the heat deposited in the grounded grid is removed by high temperature helium to be used in the thermal conversion system.

Beyond the grounded grid the flow consists only of ions $\left(\mathrm{D}^{+}, \mathrm{T}^{+}\right.$, alphas). A fraction of these ions are lost on the negative grid, ejecting secondary electrons as they impact the grid surface. Also, because the grid is radiatively cooled, it operates at a very high temperature and emits thermionic electrons. It is assumed that $1 / 2$ of the thermionic electrons and all of the secondary electrons flow to the grounded grid, and the other half of the thermionic electrons flow to the collector grid. The grid is assumed to radiate $1 / 2$ of $i$ ts thermal power to the cold sidewalls and $1 / 2$ to the collector grid. The net flow of negative charge from this grid requires electrical power input to the grid circuit.

The collector grid is a solid wall, operated at a positive potential equal to slightly less than the ambipolar potential at the midplane. The ions that are transmitted beyond the negative grid are decelerated and collected on the collector grid. The net current, equal to the ion current minus the thermionic current from the grounded grid, represents an electrical output from the direct converter. There is also a significant thermal input to the collector grid as some ions still have kinetic energy when they strike the grid, the thermionic electrons have significant kinetic energy, and the negative grid radiates thermal power to the collector. All of the thermal power is removed by the high temperature helium cooling circuit for the thernal cycle.

The grid characteristics are given in Table 6-18. The grounded grid transmission is comparatively low to permit sufficient flow area for the helium coolant. A previous study ${ }^{29}$ has shown that because the negative grid tends to be very lossy, it is best to make it radiatively cooled so that it is small and thus has a high transmission. The negative grid material properties are those for graphite. 
Table 6-18. Grid characteristics.

\begin{tabular}{ll}
\hline Grid transmission: & \\
Grounded & 0.95 \\
Negative & 0.98 \\
Negative grid material properties: & \\
Emissivity & 0.8 \\
Richardson constant, A/cm ${ }^{2}-\mathrm{K}^{2}$ & 60 \\
Work function, eV & 4.6 \\
Secondary electron emission coefficient & 2 \\
\hline
\end{tabular}

The direct converter model described by Hoffman ${ }^{29}$ was incorporated in the system model discussed in $\mathrm{Ch}$. 4. This tool was used to perform some optimization on the direct converter. The objective was to determine the direct converter parameters that would minimize the entire reactor unit capital cost, \$/kwe.

The two parameters varied were the fractional-ion, charge-exchange loss in the expander and the heat flux at the plasma collector which is varied by changing the radial location of the collector, see Eq. (63). In both cases, the tradeoff is between cost and efficienc:. The charge-exchange loss is decreased by decreasing the density of the background gas. The decrease in charge-exchange loss increase the efficiency but requires more pumping speed. The heat flux at the collector is decreased by increasing the distance from the mirror point to the collector. The decreased heat flux increases the efficiency by reducing thermionic electrons from the negative grid as a result of lower temperatures. However, the increased path length requires lowering the gas density because

$$
f_{c x} \sim n_{b} \ell \quad
$$

where $f_{c x}$ is the charge-exchange loss, $n_{b}$ is the background gas density, and $\ell$ is the distance from mirror to collector. Thus, increasing $\ell$ requires more puruing speed to keep $f_{c x}$ constant. Also, increased path length requires a larger and, thus, more expensive end tank and grid structure.

The results obtained from the optimization are shown in Figs. 6-51 and 6-52. In Fig. 6-51, the direct converter efficiency $\eta_{D C}$, which is defined as 


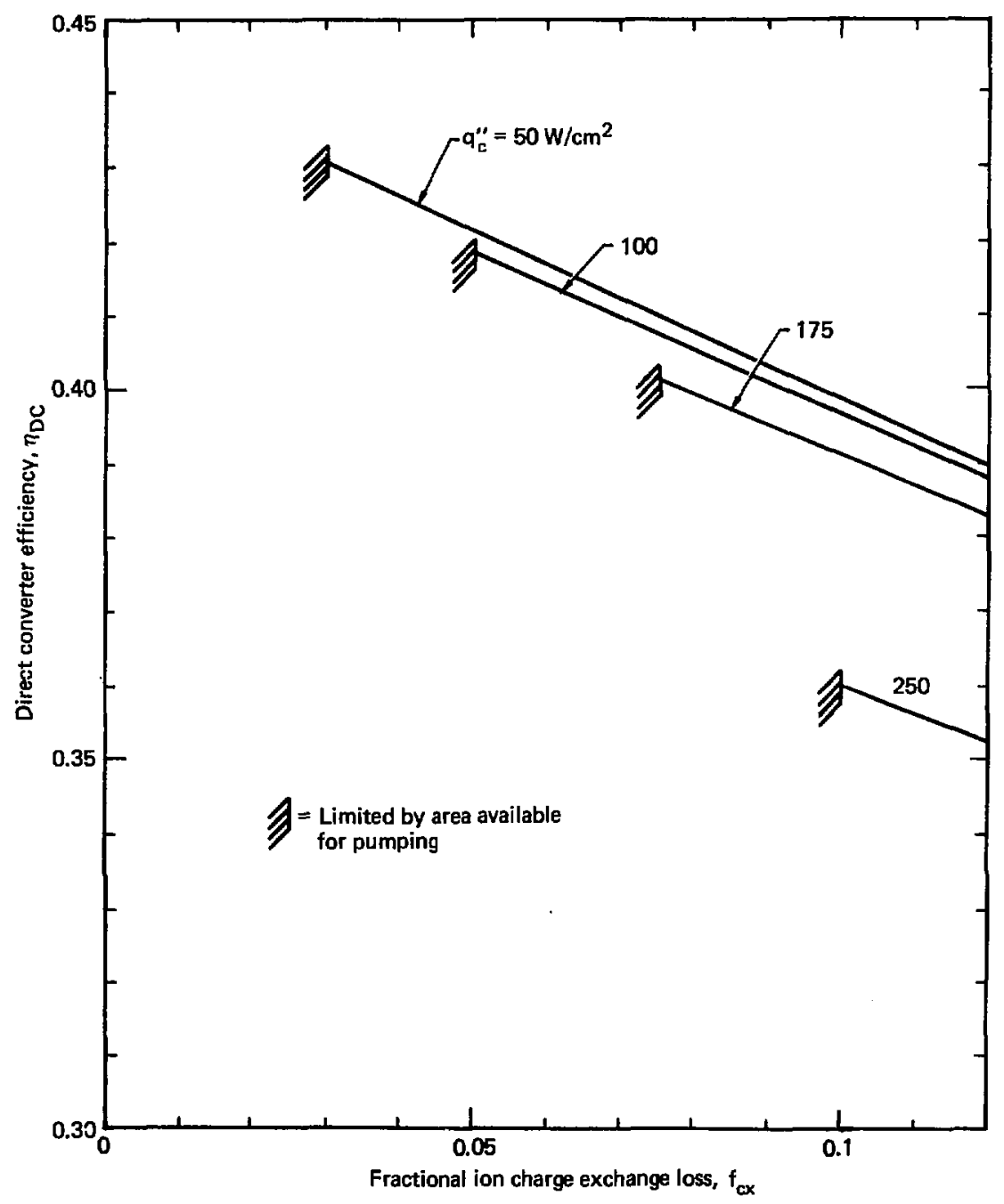

Fig. 6-51. Direct converter efficiency as a function of design paraneters. 


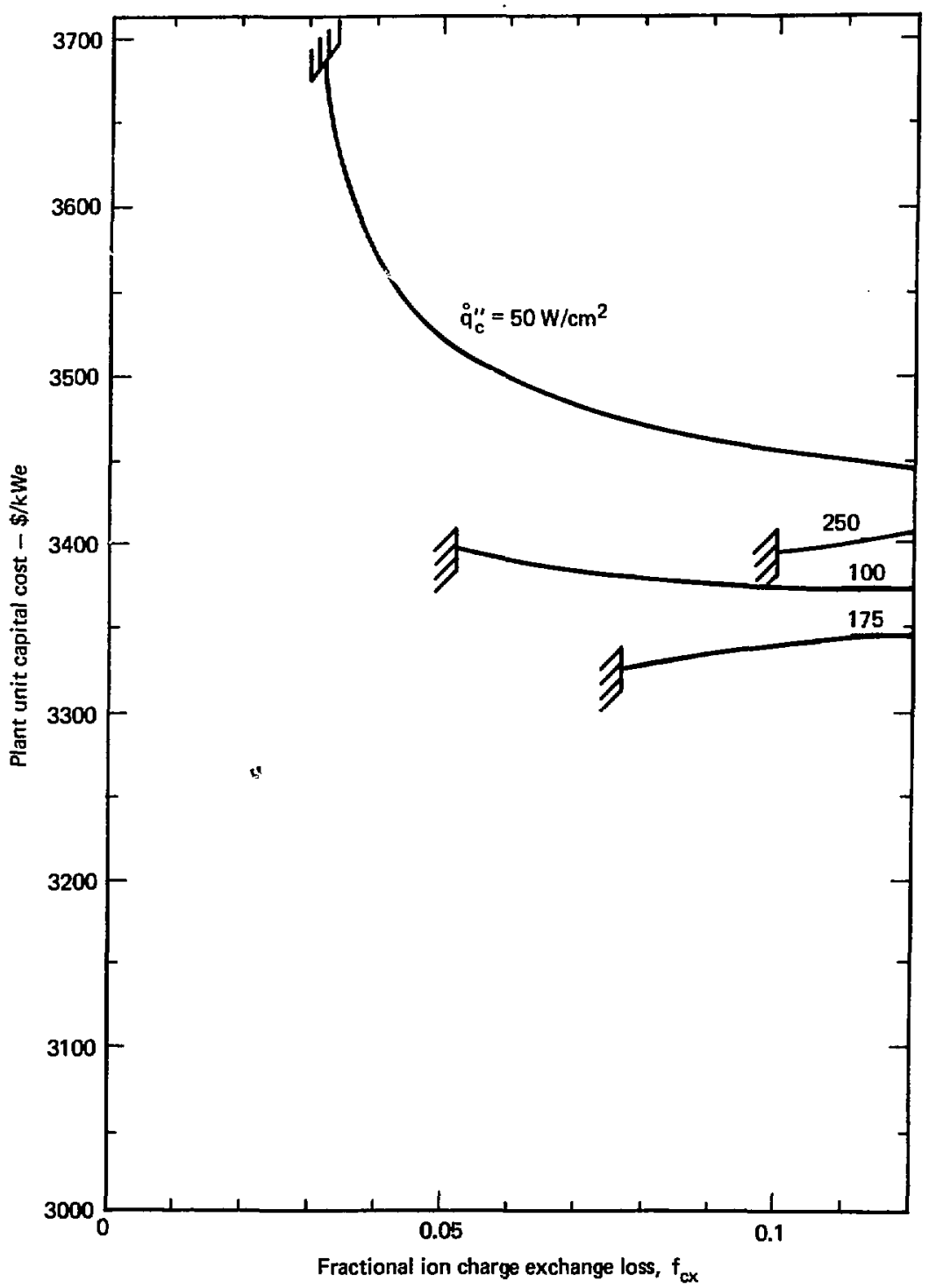

Fig. 6-52. Plant capital cost as a function of direct converter parameters. 


$$
n_{D C}=\left(P_{e, c}-P_{e, n}\right) / P_{D C},
$$

is plotted as a function of the fractional ion charge exhange loss $f_{c x}$ with the heat flux at the collector as a parameter. In all cases, the efficiency increases as $f_{c x}$ decreases. Also, at a fixed value of $f_{c x}$ the efficiency increases as the heat flux decreases because the negative grid temperature decreases and thereby decreases losses from thermionic emission. Below a value of $\dot{q}_{c}^{n} \simeq 100 \mathrm{~W} / \mathrm{cm}^{2}$ the thermionic loss is negligible but significant at $250 \mathrm{w} / \mathrm{cm}^{2}$. The left-hand limit of each curve is the point where the entire tank sidewall is used to provide the required pumping speed; thus values of $\mathrm{f}_{\mathrm{cx}}$ lower than this are not possible.

The corresponding plant unit-capital cost is plotted in Fig. 6-52. The optium conditions are seen to occur for $\dot{q}_{c}^{n}=175 \mathrm{~W} / \mathrm{cm}^{2}$ and $\mathrm{f}_{c x}=0.075$; these values were, therefore, selected for the final design. Also, the model was run with no direct converter and the resulting plant capital cost was $1975 \$ / k W e$, or 508 higher than the value of $3330 \$ / k W e$ with the optimized direct converter. Thus, the direct converter improves the plant economics but not by 50s, because plant electrical sales only provide about $1 / 3$ of plant revenues. The use of the direct converter was found to reduce the cost of producing fissile fuel by about $15 \%$.

The parameters for the optimized end tank are listed in Table 6-19. The magnetic expansion ratio (ratio of mirror field divided by collector field) is 68 . The collector potential of $63.1 \mathrm{kV}$ is set to collect all ions and is slightly less than the ambipolar potential of $63.8 \mathrm{kV}$ to accomodate the residual perpendicular velocity of the ions. The negative grid potential was taken to be $1 / 2$ the magnitude of the collector potential. 29 The direct converter efficiency was calculated to be $n_{D C}=0.40$, which is the fraction of the total plasma power $\left(\mathrm{D}^{+}, \mathrm{T}^{+}\right.$, alphas, electrons) converter to electrical power. The breakdown of the losses is listed in Table 6-19. The largest loss is at the collector and is a result of the ion energy distribution plus the high energy of the alphas. Also, the electron and charge exchange losses are significant. Parasitic losses at the negative grid (thermionic and secondary electrons) are negligible.

The net electrical output from the direct converter is 243 . of the therwal output, 315 is removed as high-tenperature heat and is trangferred to the thermal conversion system; $48 \mathrm{wh}$ is resoved from the sidewalls as low-tenperature heat and is rejected to ambient. 
Table 6-19. Large end tank parameters.

Tank dimensions

Radius to end wall, $m$

33.3

Height of end wall, $m$

10.3

Sweep angle, deg $_{2}$

sidewall area, $\mathrm{m}^{2}$
End wall area, $\mathrm{m}^{2}$

93

2100

560

Heat flux at grounded grid, $\mathrm{W} / \mathrm{Cm}^{2}$

175

Fractional ion charge exchange loss

0.075

Collector parameters

Radius to grounded grid, $m$

30.3

B field at collector, $T$

Collector grid potential, kV

0.083

Negative grid potential, kV

63.1

Collector height, $2^{m}$

$-31.5$

Collector area, $\mathrm{m}^{2}$

8.3

Magnetic expansion ratio

323

68.2

vacuun system

Tank background pressure, Torr

pumping speed, litgres/s

Cryopanel area, $\mathrm{m}^{2}$

$5.8 \times 10^{-6}$

$7.85 \times 10^{7}$

1790

Direct converter performance (single stage)

Efficiency losses

Electrons

0.072

Charge exchange

0.070

Grounded grid

0.043

Negative grid

0.033

Interception $(0.021)$

Thermionic $(0.003)$

2nd electron (0.009)

Collector

0.382

Total

0.600

Net efficiency

0.400

Power output, MT.

Thermal

Sidewall (low temperature)

48.5

Grounded grid

75.5

Collector grid

239.8

Total high temperature

315.3

Electrical

Negative grid

$-8.8$

Collector grid

251.7

Net

242.9 
Table 6-20. Input to strong mirror end tank.

\begin{tabular}{lcc} 
Table 6-20. & Input to strong mirror end tank. \\
\hline & $\begin{array}{c}\text { Power, } \\
\text { A }\end{array}$ & $\begin{array}{l}\text { Current, } \\
\text { Fuel ions }\end{array}$ \\
Alphas & 58.1 & 515.7 \\
Electrons & 40.2 & 23 \\
Total & 5.1 & 538.7 \\
\hline
\end{tabular}

\subsubsection{Strong Mirror End Tank}

The input power to this end tank is listed in Table 6-20 and consists of 108 of the fuel lons, 1/2 of the alphas, and a neutralizing flow of electrons. Here, because only about $1 / 2$ of the energy is carcied by fuel ions, direct conversion would be quite inefficient. Also, because this tank should be as mechanically simple as possible (to facilitate blanket maintenance through the tank), the use of a simple thermal dump for the plasma is justified.

The tank parameters are listed in Table 6-2l. The tank is quite compact with a radius to the end wall of about $18 \mathrm{~m}$, recalling that the magnet outside radius is $11.5 \mathrm{~m}$. The heat flux at the collector was set at a high value of $250 \mathrm{k} / \mathrm{cm}^{2}$, near the practical limit for helium cooling, to obtain the small tank. The fractional-ion, charge-exchange loss was also specified as comparatively high (10\%) to obtain the modest cryopanel area of $55 \mathrm{~m}^{2}$. Approximately 90 of the input power is recovered as high quality heat for the thermal conversion system.

\subsubsection{Output Powers}

The output powers from the two end tanks are sumarized in Table 6-22. The input plasma power of $710 \mathrm{MA}$ and electrical input of $9 \mathrm{MA}$ are converted to 252 we of electrical output, $409 \mathrm{~N}$ of high quality thermal output, and 58 wh of low quality thermal output.

\subsection{Vacuum System}

Portions of the vacuum system have been discussed in Secs, 6.2 and 6.4. Here, we present a description of the entire vacuum system and gas flows to assemble this information in an integrated presentation. The major source of gas in the reactor vacuum systern is the gas feed to the neutral bean injectors. This gas is puruped in the injector tanks and in 
Table 6-2l. Small end tank parameters.

Tank dimensions

$\begin{array}{ll}\text { Radius to end wall, m } & 18.4 \\ \text { Height of end wall, m } & 4.1 \\ \text { Sweep angle, deg } & 86 \\ \text { Sidewall, m } & 282 \\ \text { End wall, }{ }^{2} & 112 \\ \text { flux at collector, } \mathrm{w} / \mathrm{m}^{2} & 250 \\ \text { tional ion charge exchange loss } & 0.10 \\ \text { ector parameters } & \\ \text { Radius to collector, m } & 15.4 \\ \text { B field at collector, } \mathrm{T} & 0.72 \\ \text { Collector potential, } \mathrm{kV} & 0 \\ \text { Collector height, m } & 2.1 \\ \text { Collector area, }{ }^{2} & 37.4 \\ \text { Magnetic expansion ratio } & \mathbf{8 . 3}\end{array}$

Vacuum system

$2.1 \times 10^{-5}$

Tank background pressure Torr

Cryopanel area, $\mathrm{m}^{2}$

Thermal power output, $M$

Collector (high temperature)

93.5

sidewalls (low temperature)

9.8

Table 6-22, Sumary of output power, MN.

\begin{tabular}{|c|c|c|c|}
\hline & \multirow[b]{2}{*}{$\begin{array}{c}\text { Gross } \\
\text { Electrical }\end{array}$} & \multicolumn{2}{|c|}{ Thermal } \\
\hline & & $\begin{array}{l}\text { High } \\
\text { Quality }\end{array}$ & $\begin{array}{c}\text { Low } \\
\text { quality }\end{array}$ \\
\hline Weak mirror & 251.7 & 315.3 & 48.5 \\
\hline Strong nirror & 0 & 93.5 & 9.8 \\
\hline Total & 251.7 & 408.8 & 58.3 \\
\hline
\end{tabular}

accounting for the electrical input of 8.8 we to the negative grid, the net electrical output is 242.9 kie. 
the end tanks. In addition, a gas flow is used to provide stabilization to the surface of the plasma and helium is generated in the plasma as a result of fusion reactions. The gas loads caused by outgassing and possible vacuun leaks have not been addressed.

The injector gas flow, simplified for a description of the pumping, is shown in Pig. 6-53 for a single source. The gas feed to the arc chamber is 93.6 Torr-litre/s of which 83.6 Torr-litre/s is pumped from this chanber by a blower. Assuming a pump inlet pressure of $5 \times 10^{-2}$ Torr, the required pumping speed is 1672 litres/s.

The remaining 10 Torr-litre/s passes through the accel-decel grids into the neutralizer; 7 Torr-litre/s is cold gas and 3 Torr-litre/s is the equivalent gas flow corresponding to the $28 \mathrm{~A}$ of ions extracted from the source (30t source gas efficiency). In the neutralizer, the remaining 7 Torr-litre/s of cold gas is removed along with 0.31 Torr-litre/s as a result of bean losses. Here, this gas flow is removed with a Hg ejector punp; assuming a pump inlet pressure of $10^{-3}$ Torr, the required pumping speed is 7310 litre/s.

In the beam direct converter and drift region, the gas load is $\mathbf{1 . 3 2}$ Torr-litre/s resulting from unneutralized ions in the beam that are collected by the direct converter and beam collisional losses in the drift region between the beam direct converter and the plasma chamber. primary pumping in this third chamber is with liquid helium cryopanels. Each source is provided with $4.17 \mathrm{~m}^{2}$ of cryopanel, with an effective speed of 4 litre $/ \mathrm{s}^{\circ} \mathrm{cm}^{2}$. The total cryopumping speed is thus $1.67 \times 10^{5} \mathrm{litre} / \mathrm{s}$ per source. Assuming the gas from the defrosted panels is remcved at a pressure of $10^{-3}$ Torr, the pump speed is 1320 litre/s.

The neutral atom current of $14.4 \mathrm{~A}$ injected into the plasma represents an equivalent gas flow of 1.37 Torr-litre/s. Of the gas introduced into the source, only about 1.58 is converted into injected neutrals.

The reactor requires two injector tanks, with 180 active sources per tank, or a total of 360 of the sources shown in Fig. 6-53, of these injectors, two-thirds, or 240 sources, operate with deuterium and one-third, or 120 sources, operate with tritiun. Thus the total gas flow into the injectors is $240 \times 93.6=2.25 \times 10^{4}$ Torr-litre/s of deuterium and 120 $\times 93.6=1.12 \times 10^{4}$ Torr-litre/s of tritium. The total injected current into the fusion chamber is $14.4 \mathrm{~A} \times 360=5184 \mathrm{~A}$ (nominally, 5204 A) corresponding to an equivalent gas load of 492.5 Torr-litre/s. 


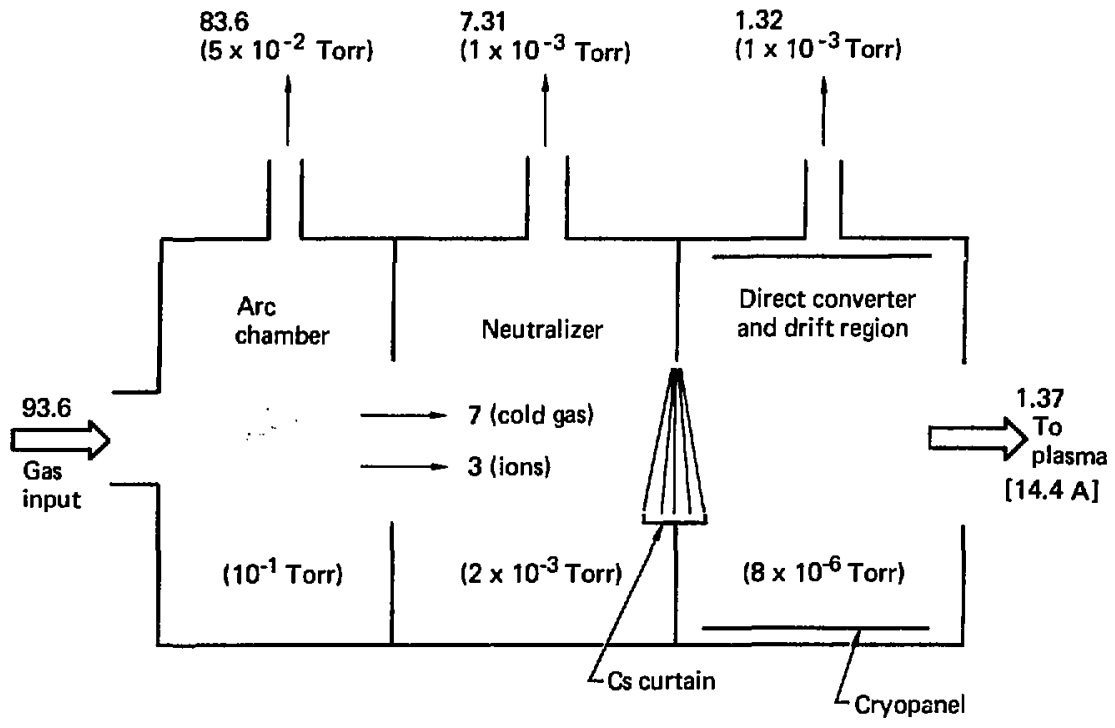

Fig. 6-53. Injector gas flow in Torr-1itre/s. 
The complete vacuum system gas flow is shown in Fig. 6-54. In addition to the injector gas flow, the warm plasma stabilizing stream is shown ("deuterium stream") and the flow of the 4.4 Torr-litre/s of "He generated in the fusion reaction. As was discussed in some detail in Sec. 6.4.2, the stream and 4 He pumping have not been explicitly treated in this study. Figure 6-54 illustrates our concept for supplying and pumping the stream plasma. We estimate about $15 \mathrm{kA}$ or 2425 Torr-litre/s of gas are required for boundary layer stabilization. This gas is introduced into the vacuum system in the small (upper) end tank around the surface of the plasma fan. The gas is ionized, flows through the confinement volume, and is scraped off of the surface of the plasma in the large (lower) end tank where it is neutralized and pumped. By collecting the gas in a chamber separate from the main end tank, it is proposed to operate the stream tank at a comparatively high pressure, e.g.. $10^{-4}$ Torr. Pumping this gas with cryopanels, at a speed of $4.3 \mathrm{litre} / \mathrm{s}^{*} \mathrm{~cm}^{2}$, will require $333 \mathrm{~m}^{2}$ of panel.

The helium production of 4.4 Torr-litre/s flows in equal amounts into the two end tanks, where it must be pumped. The helium pumping capacity has not been included in the end tank design because of the absence, at present, of a feasible helium pumping technology for reactor applications. Two potentially useful techniques presently under investigation are cryosorption and cryotrapping.

The injectors introduce $492 \mathrm{Tor}-1 \mathrm{itre} / \mathrm{s}$ into the confinement volume in the form of energetic neutral atoms. The plasma itself acts as a pump, in that all atoms incident on the plasma (energetic or cold) are ionized and eventually escape from the confinement volume along the open field lines into the end tanks. In the end tanks the ions strike solid surfaces, are neutralized, and pumed away.

of the injected energetic neutrals, approximately 0.98 undergoes fusion, and of the remainder, 90\% flows into the large end tank and 108 into the small end tank. The large end tank operates at a pressure of $5.8 \times$ $10^{-6}$ Torr; here, primary pumping is provided by cryopanels. Using an average puming speed for deuteriun and tritium of 4.31 itre $/ \mathrm{s}^{\circ} \mathrm{cm}^{2}$ for a double-baffled panel requires $1790 \mathrm{~m}^{2}$ of cryopanel. The small end tank operates at a pressure of $2.1 \times 10^{-5}$ Torr, requiring $55 \mathrm{~m}^{2}$ of panel.

The pressures in each chamber of the vacuum system are shown in Fig. 6-55 and the vacuum system parameters are listed in Table 6-23. 


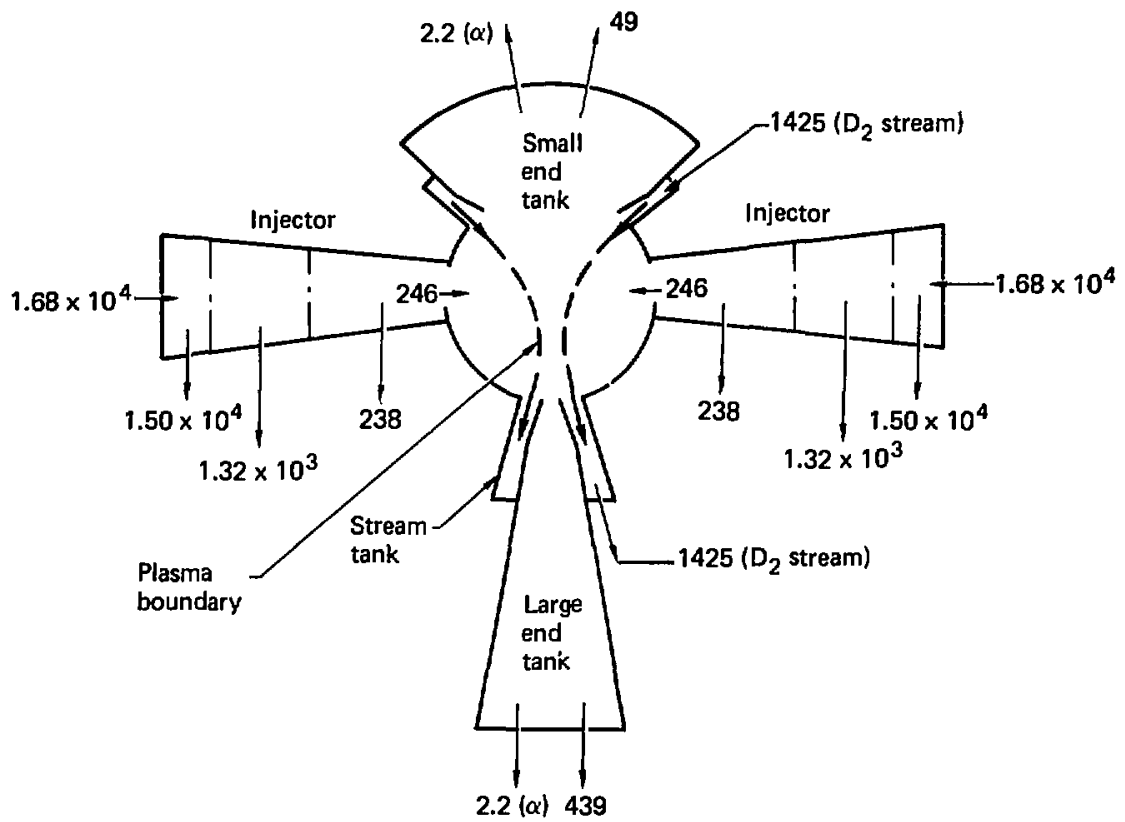

Fig. 6-54. Vacuum system gas flows in Torr-litre/s. 


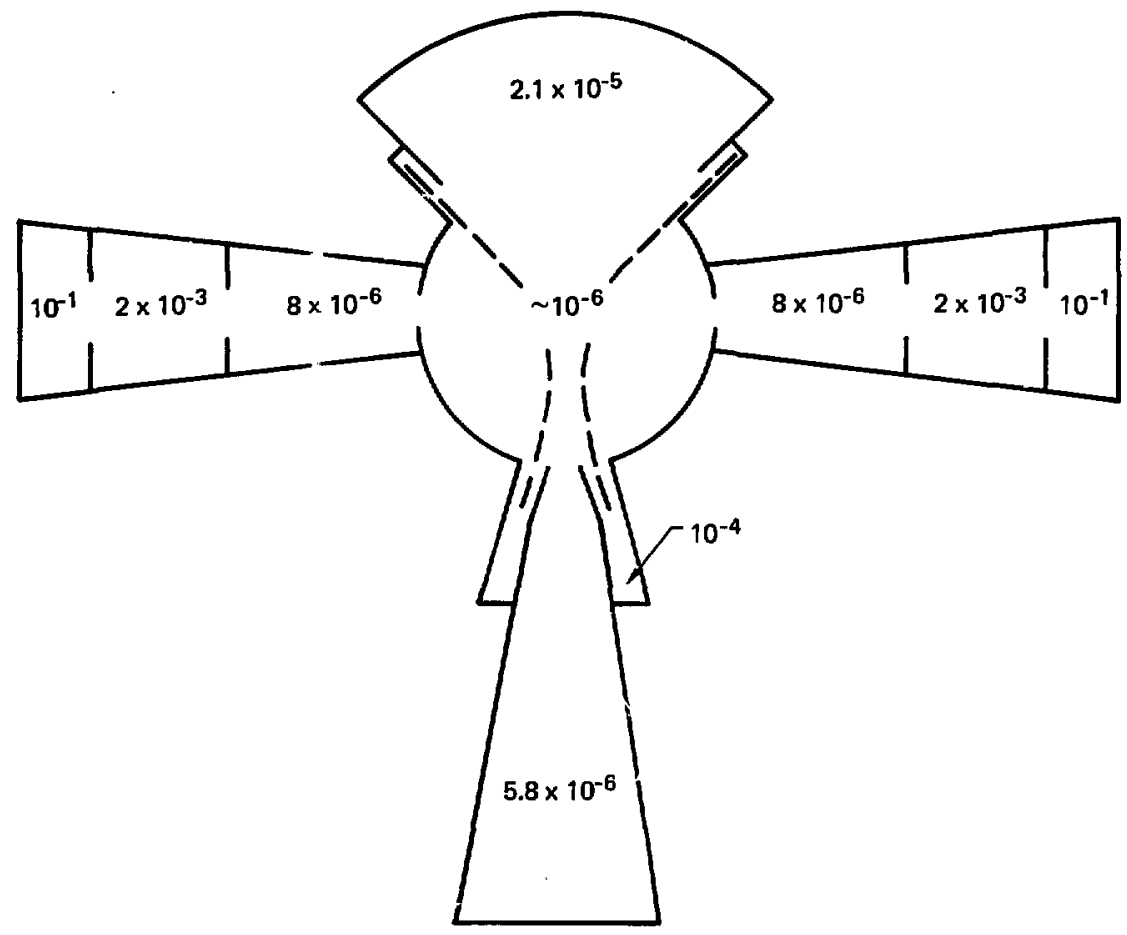

Fig. 6-55. Vacuun system pressures in Torr. 
Table 6-23. Vacuum system parameters.

\begin{tabular}{|c|c|c|c|c|c|}
\hline & $\begin{array}{l}\text { Chamber } \\
\text { pressure, } \\
\text { Torr }\end{array}$ & $\begin{array}{l}\text { Pumping } \\
\text { load, } \\
\text { Torr-litre/s }\end{array}$ & $\begin{array}{l}\text { Punp } \\
\text { inlet } \\
\text { pressure, } \\
\text { Torr }\end{array}$ & $\begin{array}{l}\text { Pumping } \\
\text { speed, } \\
\text { litre/s }\end{array}$ & $\begin{array}{c}\text { Cryopanel } \\
\arg ^{2} \\
m^{2}\end{array}$ \\
\hline \multicolumn{6}{|l|}{ Injector system } \\
\hline $\begin{array}{l}\text { Arc chamber } \\
\text { (source }=3.37 \times 10^{4} \text { Torr-litre/s) }\end{array}$ & $10^{-1}$ & $3.00 \times 10^{4}$ & $5 \times 10^{-2}$ & $6.02 \times 10^{5}$ & - \\
\hline Neutralizer & $2 \times 10^{-3}$ & $2.64 \times 10^{3}$ & $10^{-3}$ & $2.6 \times 10^{6}$ & - \\
\hline $\mathbf{D C}$ and drift region & $8 \times 10^{-6}$ & $4.76 \times 10^{2}$ & $10^{-3}$ & $6.0 \times 10^{7}$ & 1500 \\
\hline Confinement volume & $\sim 10^{-6}$ & - & - & - & - \\
\hline $\begin{array}{l}\text { (source }=4.4 \text { Torr-litre/s of } \mathrm{He} \text { ) } \\
\text { Strean tanks }\end{array}$ & $10^{-4}$ & 1425 & $10^{-4}$ & $1.43 \times 10^{7}$ & 333 \\
\hline $\begin{array}{l}\text { (source }=1425 \text { Torr } / \text { litre } / \mathrm{s} \text { of } \mathrm{D}_{2} \text { ) } \\
\text { Large end tank }\end{array}$ & $5.8 \times 10^{-6}$ & & & & \\
\hline$\left(D_{2}\right.$ and $\left.T_{2}\right)$ & & 439 & $5.8 \times 10^{-6}$ & $7.6 \times 10^{7}$ & 1790 \\
\hline $\begin{array}{l}\text { (He) } \\
\text { Snall end tank }\end{array}$ & $2.1 \times 10^{-5}$ & 2.2 & - & - & - \\
\hline $\begin{array}{l}\left(\mathrm{D}_{2} \text { and } \mathrm{T}_{2}\right) \\
(\mathrm{Be})\end{array}$ & & $\begin{array}{l}49 \\
2.2\end{array}$ & $\begin{array}{l}2.1 \times 10^{-5} \\
-\end{array}$ & $\begin{array}{c}2.3 \times 10^{6} \\
-\end{array}$ & $\begin{array}{l}55 \\
-\end{array}$ \\
\hline
\end{tabular}




\subsection{Tritium Containment and Handling}

\subsubsection{Suniary}

Tritium control in the mirror hybrid reactor is possible with enissions to the environment below $10 \mathrm{ci} / d$, while still maintaining worker safety and cost effectiveness. A number of new methods have been employed to achieve such low levels in a near-term machine with today's technology. Figure 6-56 sumarizes the various tritium leak source terms and the associated processing loops.

The tritium breeding pins are designed as tritium containment vessels filled with lithium deuteride and are constructed of a special lowpermeability, high-strength aluminum alloy. In the hybrid reactor these tritium breeding pins are "harvested" together with the plutonium pins. The tritium is extracted from these tubes in-plant and the lithium deuteridefilled tubes remanufactured and reinstalled for the next cycle. A breeding pin failure rate of $10^{-3}$ per year is assumed, thus releasing $448 \mathrm{ci} / \mathrm{d}$ into the helium coolant, which is then scavenged by a slip-stream processor handling 29 of the helium flow. The tritium-contaminated helium will permeate about $3 \mathrm{ci} / \mathrm{d}$ into the steam generators, which is in turn lost to the cooling towers and then to the environment.

The next major source of tritium leakage is from the tritium neutral beam injector, where $55 \mathrm{keV}$ energetic tritons implant tritium into the surface of the electrode tubing. Once within the tube metal, tritium will diffuse, producing a flux of tritium into the electrode helium coolant flow at about $70,560 \mathrm{ci} / \mathrm{d}$. A 10 fraction of the coolant flow is then processed to remove 99: of the tritium and the resulting residual tritium leaks into the stean generators at about $3 \mathrm{ci} / \mathrm{d}$, where it eventually passes into the cooling water and then to the enviroment. The deuterium injector uses deuterium with a maximum 1 tritiun impurity level and it creates a leak to the stean of $0.3 \mathrm{Ci} / \mathrm{d}$.

A similar tritium loss phenomenon occurs in the hybrid's direct converter where $55 \mathrm{keV}$ energy tritons produce a loss of $8,000 \mathrm{Ci} / \mathrm{d}$ into the helium flow. Again a 99.94 processor is used, handling $5 \%$ of the flow to reduce this source of tritium in the main helium coolant to less than $3 \mathrm{Ci} / \mathrm{d}$.

All of the remaining miscellaneous leaks are taken to be from the process equipment and piping or ducting directly into the reactor hall at a 


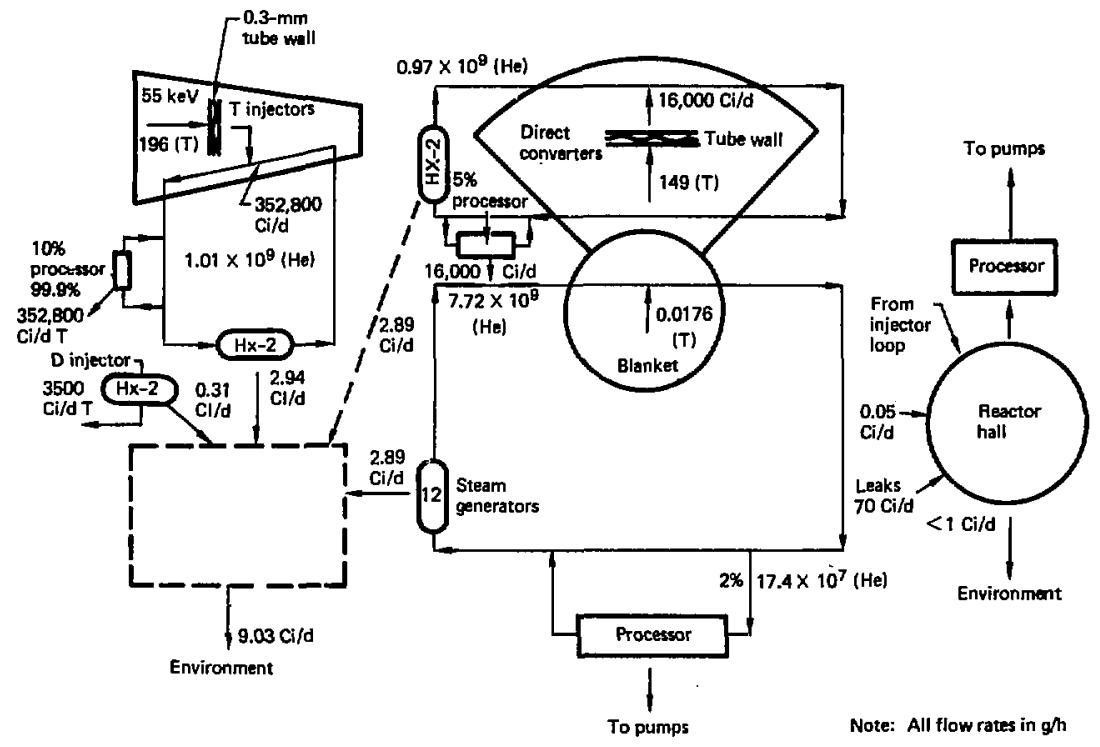

Fig. 6-56. Tritium source terms and processing Ioops. 
maximum total of $180 \mathrm{Ci} / \mathrm{da}$. The reactor hall atmosphere is processed and kept at low humidity to maintain tritiun gas levels of below $40 \mu \mathrm{Ci} / \mathrm{m}^{3}$ and tritiun water of below $5 \mu \mathrm{Ci} / \mathrm{m}^{3}$. This reactor hall processing design is expected to result in routine as well as credible accident losses through the reactor hall containment of less than $1 \mathrm{ci} / \mathrm{d}$.

In this way, the tritium loss to the environment is designed to be less than $3 \mathrm{ci} / \mathrm{d}$ from breeding pin losses, $3 \mathrm{ci} / \mathrm{d}$ from injectors, $3 \mathrm{ci} / \mathrm{d}$ from direct converter, and less than $1 \mathrm{Cl} / \mathrm{d}$ from reactor hall containment permeation. This is some 40 times lower than is required by NRC regulations for all nuclear fuel cycle operations, but is about the level suggested by the ALARA cost/benefit analysis amounting to an expenditure cutoff at the $\$ 66,000$ level. It was found no further reductions in emissions could be accomplished by an expenditure of $\$ 56,000$.

\subsubsection{Containment Limits on Reactor Hall Tritium}

6.6.2.1 Environment Impact. The DOE-policy for tritium releases from its facilities is to keep releases to levels as $10 \mathrm{w}$ as practicable. ${ }^{36}$ We will see that, for a near-term fusion reactor, this level comes out to below $10 \mathrm{ci} / \mathrm{d}$. The accumulated tritim enissions from 100 hybrid fusion reactors by year 2010 with $10 \mathrm{Ci} / \mathrm{d}$ emissions or from 1500 lGWe fusion reactor power plants at $1 \mathrm{Ci} / \mathrm{d}$ to be built in a world fusion economy by the year 2025 would not significantly $(<108)$ add to the prevailing tritium background (or a reduced background from a total nuclear device test ban), with a natural tritium production of 10,000 to $20,000 \mathrm{Ci} / \mathrm{d}^{37}$ Such low emission goals may not prove to be cost effective in the ALARA sense and this will be addressed in Sec. 6.6 .2 .2 .

For a reactor hall of the approximate volume of $150000 \mathrm{~m}^{3}$, losses could be kept to such a low level only if the tritium concentration within the gas cover (inside the reactor hall) were kept $10 \mathrm{w}\left(\mathrm{i}, \mathrm{e}_{,}, 40 \mu \mathrm{Ci} / \mathrm{m}^{3}\right.$ ) and if the entire wall surface were provided with a permeation barrier as the hermetic seal. A stainless-steel shell (alloys 304, 316, or 321) 2-m (1/16-in.) thick at ambient temperatures will provide such protection. 38 Today the technology exists to weld metal plates in place within a large concrete building structure. This shell will meet a criteria for low permeation (below $1 \mathrm{Ci} / \mathrm{d}$ ) even for the case of the most incredible accident where $26 \mathrm{~kg}$ of $\mathbf{T}_{2}$ from the meltdown of the breeding blanket is ignited and is dunged, at once releasing Hro and/or $\mathbf{T}_{2} \mathrm{O}$ into the reactor hall, and quickly increasing the concentration up to $600 \mathrm{ppm}\left(1500 \mathrm{ci} / \mathrm{m}^{3}\right)$. Release 
of $T_{2}$ gas inventory into the reactor hall is also serious because permeation losses to the environment of $T_{2}$ or $\mathrm{HT}$ will eventually result in the more hazardous Hro.

6.6.2.2 Fence Line Criteria for the General Public. In addition to maintaining a low permeation loss, the interior design of the hermetic reactor hall must also protect personnel just outside the building from experiencing HTO or $\mathrm{T}_{2} \mathrm{O}$ concentrations in excess of the maximum permissible concentrations (MPC) during both routine operations and extreme conditions. Current legislation does not exist that would set limits on accidental tritium releases. The most conservative approach is to assume that the occupational worker will continue working routinely outside the reactor hall and the general public will reside permanently about $1 / 2$ mile away from the concrete reactor hall following an ignition and explosion of $26 \mathrm{~kg}$ of $\mathrm{T}_{2}$. For protection of the unmasked, unsuited worker, routine work can continue as long as the $\mathrm{T}_{2} \mathrm{O}$ concentration will remain below $5 \mu \mathrm{ci} / \mathrm{m}^{3}(\sim 0.002 \mathrm{ppb})$ for a controlled access area. ${ }^{36}$

Members of the general public might reside continuously at the fence line of a 1-square-mile plant. Under worst case meteorological conditions $\left(x / Q=6.7 \times 10^{-4}\right)$ coincident with the accident, less than a 5-mrem dose would result at the fence line if leakage from the accident remained below $200 \mathrm{Ci}$. If the accident occurred under annusl average conditions $\left(X / Q=9 \times 10^{-6}\right), 100$ times better dispersion would result. This accident situation would be much less serious to the fence line populace (2 $\times 10^{-2} \mathrm{mrem}$ ). Light water reactor (LWR) routine operation legislation requires 5 mrem based on that industry's demonstration that this low level was currently achievable.

Other fission reactor legislation could be applied as well. The EPA $^{39}$ has suggested 25 mrem. But to date, the most applicable to fusion is the new $\mathrm{NRC}^{36}$ regulation of 170 mrem applied to all nuclear fuel cycle operations annually. This would allow $350 \mathrm{ci} / \mathrm{d}$ as routine tritium releases averaged over the year. One-time accidental doses to the general public are set by NRC ${ }^{36}$ to 5 ren for fuel cycle operations. If this is applied to fusion, an "incredible accident," where $26 \mathrm{~kg}$ is released into the reactor hall simultaneously with another accident that breaches the reactor hall and spills $26 \mathrm{~kg}$ Hro or $\mathrm{T}_{2} \mathrm{O}$ into the environment at ground level, would result in about a 70-rem fence-line dose under annual average meteorological conditions. 
Thus, designing a fusion plant with a maximum credible accident of 200 $\mathrm{Ci}$ and routine emissions around $10 \mathrm{ci} / \mathrm{d}$ is clearly extremely conservative. Now the question is whether this is too conservative in the cost benefit sense. Invoking the KRC test, ALARA ("as low as is reasonably achievable"). as $\$ 1000 /$ man-rem within a 50-mile radius, ${ }^{40}$ a $26-\mathrm{kg}-\mathrm{T}_{2}$ release from a 1-GWe mirror fusion plant located at LLL could result in a worst case (958 conf. 1imit) $6.5 \times 10^{7}$ man-rem exposure, which would justify an expenditure of $\$ 65$ billion to avoid the accident. Similarly, $\$ 56,000$ could be spent to avoid $200 \mathrm{Ci}$. NRC has also interpreted ALARA for LWR's as it of fuel pins failed or $5,000 \mathrm{Ci}$ dumped into the coolant system not to exceed 5 mrem at the fence, as a design limit to seek 0.05 mrem for routine operation if justified by NARA.

The design to provide the required protection for all of the above (except maybe the last recommendation) could consist of a space kept below 5 $\mu \mathrm{Ci} / \mathrm{m}^{3}$ between the concrete building shell and the hermetic seal liner. In other words, the hermetic liner is welded together on standoffs cast into the concrete shell. If we state that the facility will have the $T_{2}$, BT, HTO or $\mathbf{T}_{2} \mathrm{O}$ containment and removal capability to reduce this high accident level down to a level of $20,000 \mu \mathrm{Ci} / \mathrm{m}^{3}$ for reentry with ventilated tritium suits within the building after $7 \mathrm{~d}$ of reactor hall air processing, then we can compute the length of the standoff. If the $600 \mathrm{ppm}$ spill leaks for $7 \mathrm{~d}$ into this space at $0.007 \mathrm{Ci} / \mathrm{d}$ [the permeation through a 2-mm (1/16-in.) stainless-steel hermetic liner] and the level within this space must remain below $5 \mu \mathrm{Ci} / \mathrm{m}^{3}$, the standoff distance must be $79 \mathrm{~cm}$ and air circulated. This release under the worst case meteorological dispersion conditions $\left(X / Q<6.7 \times 10^{-4}\right)$ would result in only a $0.005-m r e m ~ e x-$ posure to the general public at the site boundary. This concept therefore would be economic, very safe, and would quslify as a secondary containment of the reactor hall.

It should be noted parenthetically that nothing we have done for tritiu itself requires a concrete reactor dome as in conventional fission plants. Since tritium releases are orders of magnitude less serious than the fission variety of radioisotopes, protection against earthquake, external missiles, airplanes, etc. does not seem necessary. No present U.S. tritiun facility or plant is constructed of such costly dome structures. It is the hybrid's actinides and fission products that require the concrete dome. 
6.6.2.3 Safety of the occupational worker. The routine safety of the worker must be the next topic in the reactor hall containment, involving normal ambient operating levels. We selected the nominal level of $40 \mu \mathrm{Ci} / \mathrm{m}^{3}$, as in the previous discussion, for very particular health and safety as well as state-of-the-art hardware reasons. An occupational worker can be exposed at 1008 MPC for $40 \mathrm{~h}$ per week without any clothing protection at these contaminaton levels of $40 \mu \mathrm{Ci} / \mathrm{m}^{3}$ if the HTO fraction remains below 128 by volume. Such environments (with once-through air) are not atypical of production facility work environments in the U.S. Mound ${ }^{41}$ is demonstrating $40 \mu \mathrm{Ci} / \mathrm{m}^{3}$ in a recirculating highly processed system and Los Alamos ${ }^{42}$ on a smaller scale processed glove box train. Los Alamos has also achieved $0.5 \mu \mathrm{Ci} / \mathrm{m}^{3}$ in a highly ventilated room surrounding this processed glove box while achieving stack enissions to the environment around $1 \mathrm{Ci} / \mathrm{d}$. Sandia Livermor $\mathrm{e}^{43}$ is constructing a system with similar goals. However, for routine work the design basis for exposure should be well below the 1008 MPC. ERDA recommends ${ }^{36}$ that for design purposes, levels at 208 MPC should be used. This would be quite feasible at work assignments of a $\mathrm{h}$ per week, or one could provide analytical evidence for HTO fractions below 28. Also, the occupational worker can be protected by lightweight suits and face masks that would not interfere with his needs for agility and dexterity. Such lightweight suits (two piece with overalls) with air supplied masks typically $y^{44}$ can provide protection factors of 100 or more if changed hourly or factors of 10 or more if not changed for $8 \mathrm{~h}$. Thus, it can be seen that this nominal level of $40 \mu \mathrm{Ci} / \mathrm{m}^{3}$ offers the largest number of operational options while still remaining close to the economic and safety optimum. Under accidental conditions or higher levels, ventilated suit technology has advanced to the stage where factors of 1400 or 1900 can be achieved. ${ }^{44}$ we are presently examining the different suit demands from routine and emergency egress points of view.

Occupational worker safety with respect to tritium within the reactor hall should be excellent, since he could work at the ambient levels of 40 $\mu \mathrm{Ci} / \mathrm{m}^{3}$ (less than $28 \mathrm{HrO}$ ) for a $40-\mathrm{h}$ work week without additional suits or breathing masks. We have also provided $i$; the design a full capability for worker survival from the "maximum credible accident," where $26 \mathrm{~kg}$ of $T_{2}$ explodes to form $600 \mathrm{ppm}\left(1500 \mathrm{ci} / \mathrm{m}^{3}\right)$ of $\mathrm{T}_{2} \mathrm{O}$ within the reactor hall. Bven though calculations show that such an explosion would not breach 
the integrity of the helium coolant containment, the "maximum credible failure" postulates 100 s escape of $T_{2} O$ into the reactor hall.

We propose that the workers normally wear overalls with a plastic suit underneath and outside as well, and carry a breathing mask with 3 min of air in a small belt tank. In addition, we propose to supply a large number of fresh air "breathing booths," where fresh air is flushed and exhausted through a large duct and manifold. This design would permit the worker to enter the "breathing booth," where not only protection from the high tritium levels is provided, but a safe egress port is available to him for escape to the outside world. These booths with escape ducts would be located conveniently at all building elevations to permit worker entry from all locations in less than $3 \mathrm{~min}$. The suit and breathing mask would permit the worker survival and egress during the "maximum credible failure." Normally upon hearing the alarm, within seconds the worker would install his breathing mask, run to a booth, avoid the elevated concentrations that would take many minutes to accumulate within the reactor hall. At $1500 \mathrm{ci} / \mathrm{m}^{3}$, a 0.5-s FrO exposure would result in $25 \mathrm{rem}$, a dose equal to that allowed to the general public under accident conditions, assuming a quality factor of unity for the radiation. 45 In the best tritium suit, a 20 -min exposure would give 25 rem. There is, of course, the unlikely possibility that the worker would be sprayed directly with $1008 \mathrm{~T}_{2} \mathrm{O}$ before he could mount his face mask. But we feel this design concept and operational procedure would provide excellent worker safety, well within even the most restrictive DOE guidelines that could be anticipated far into the future.

\subsubsection{Tritium Leakage and Reactor Hall}

6.6.3.1 Sources of Leakage. Now that it has been established that a tritium level of $40 \mu \mathrm{Ci} / \mathrm{m}^{3}$ is a safe working environment within the reactor hall, let's examine the constraints that this level places on the other components in the nuclear island. Experience in our facility at wL and at other tritium research and development facilities in the U.S. suggests that either in a continuous air recirculation or a once-through air exhaust system, the ambient rocm levels depend on the cleanliness of the worker. That is, it depends on how much outgassing of tritium occurs from leaking equipment and deadsorbing from room and hood surfaces. The ambient level for a given roos volume depends on the replacement rate of the air within this volune and the tritium outgassing rate. Operating practice in 
once-through air exhaust svstems suggests twenty changes of air per hour are needed to keep room levels about $15 \mu \mathrm{Ci} / \mathrm{m}^{3}$. When laboratory operations are not as careful, the level increases.

The mass balance is fundamental to these air flow-outgassing relationships. Table 6-24 gives results of the mass balance analysis relating the maximun outgassing rate of tritium that can be tolerated to meet the $40-\mu \mathrm{Ci} / \mathrm{m}^{3}$ level for a given air-flow rate in the reactor hall $\left(150000 \mathrm{~m}^{3}\right)$. In addition to the outgassing or leaking of tritium from the nuclear island, we have examined the extent to which the myriad of not large helium ducts will lose tritium into the reactor hall. If the duct walls were around $500 \mathrm{c}$, the tritium loss would not be acceptable. However, we utilize a double-wall duct design where the space only slowly communicates via holes with the main helium flow in such a way that the space is relatively stagnant and cool. In this way the enormous area of the ducts $\left(7100 \mathrm{~m}^{2}\right)$ will permeate less than $0.1 \mathrm{Ci} / \mathrm{d}$ into the reactor hall. For safety and accident reasons, the ducts should still be placed in a "pipe-race" isolated from the main reactor hall, purged with air, monitored and separately connected to the inlet of the reactor hall processor.

We should alsu mention here that the reactor hall processor provides a large source of leaks itself and is contained in a vault, separately purged (possibly with argon), and processed in case of an accident. The molecular sieves would normally have as much as $1000 \mathrm{Ci}$ in the loaded state before regeneration or under maximum credible accident situations, as much as $26 \mathrm{~kg}$.

These sources of leaks can be compared favorably with available estimates $^{45}$ for tritium leakage loss (including permeation) from state-of-theart energy recovery system (process piping, pumps, heat exchangers,

Table 6-24. Maximum tolerable outgassing within reactor hall to maintain a $40-\mathrm{Ci} / \mathrm{m}^{3}$ asymptote (infinite processing time).

\begin{tabular}{|c|c|c|c|}
\hline \multicolumn{2}{|c|}{ Air flow } & \multirow{2}{*}{$\begin{array}{l}\text { Volume changes } \\
\text { per hour }\end{array}$} & \multirow{2}{*}{$\begin{array}{c}\text { Outgassing tolerated, } \\
\mathrm{Ci} / \mathrm{d}\end{array}$} \\
\hline$m^{3} / s$ & CFM & & \\
\hline 0.36 & 607 & $1 / 24$ & 1.0 \\
\hline 5.88 & 10,000 & 0.05 & 18.0 \\
\hline 58.8 & 100,000 & 0.5 & 180.0 \\
\hline 2352 & $4,000,000$ & 20.5 & 7200.0 \\
\hline
\end{tabular}


turbines, etc.). For example, a conventional energy recovery system constructed of stainless steel for a 470-MWe plant would release about $70 \mathrm{Ci} / \mathrm{d}$ of tritium gas into the reactor hall.46 Thus, typical for a 1-GWe reactor design, $180 \mathrm{Ci} / \mathrm{d}$ might be expected and thus air flows of around $60 \mathrm{~m}^{3} / \mathrm{s}$ (100 $000 \mathrm{cfm})$ are required. This rate is typical of moderate-size office buildings with conventional off-the-shelf air conditioning hardware. Approximate cost would be $\$ 30$ million for complete processor from Englehard 47 including $\mathbf{\$ 3}$ million for precious metal catalyst.

It is clear that there will be an optimum trade-off between the size of the reactor hall waste gas handling system and the design of the thermal energy recovery system relative to tritium leakage and permeation. Doubly or triply contained process piping and heat exchanges represent one extreme and thin steel at high temperature, the other. A compromise may well be the use of a single tube unit with triple layers: stainless steel, low permeabililty layer, and stainless steel. A triple sandwich using copper as the middle layer substantially reduces the high temperature tritium permeation. The feasibility of such a copper-containing design has been explored ${ }^{48}$ and indeed looks favorable. Further reductions may be possible with a ceramic inner layer.

Also indicated in Table 6-24 is the air flow of $607 \mathrm{cfm}$ which would transport $1.0 \mathrm{Ci} / \mathrm{d}$ at $40 \mu \mathrm{ci} / \mathrm{m}^{3}$. This fact tells us that the operation of conventional "air flush" air locks through the reactor hall wall to the outside would be a problem. There are two air locks: one large lock for moving blanket segments and other large equipment out of the reactor alal, and several smaller ones for personnel. For special conservatism, one could operate both personnel and equipment locks upon entering and exiting the reactor hall as opposed to just exiting. If one expects to change 16 blanket segments per year, this would represent a flow of only $2 \mathrm{~m}^{3} / \mathrm{h}$ (l cfm) if the lock were the pump-down variety and for 100 personnel accesses per day. This release would amount to $8 \mathrm{~m}^{3} / \mathrm{h}(4 \mathrm{cfm})$. This is excellent. But if a conventional lock is used where air velocities past a moving person are required to be $0.6 \mathrm{~m} / \mathrm{s}(120 \mathrm{ft} / \mathrm{min})$, it rapidly becomes excessive. Thus, a double lock variety, where one isolated chamber is pumped down, must be used to avoid excessive tritiun release to the environment. A pumped double air lock design could also pung from its exhaust air to the reactor hall waste gas handling system and no substantial increase in system load would result. Thi: operation would also allow for fresh air makeup. The total 
amount of air makeup, however, will still be limited to under $0.36 \mathrm{~m}^{3} / \mathrm{s}$ $(607 \mathrm{cfm})$, depending on both the actual tritium leakage out to the reactor hall and the low level achieved by the molecular sieve just before the waste air is stacked. More makeup air can be admitted if molecular sieve-bed performance achieves less than the $40-\mu \mathrm{Ci} / \mathrm{m}^{3}-1 \mathrm{mit}$. With an "air curtain," it might be possible to reduce back-mixing to such a low point that the pumpdown feature could be deleted.

6.6.3.2 Accidental Release. We have illustrated our discussion with a maximum credible accident involving a $26-\mathrm{kg}$ HTO release resulting from a blanket melt-down. There can also be a fallure in the direct converter vacuum vessel where frozen DT (about $0.25 \mathrm{~kg}$ ) is formed and oxidized (ignited) by the incoming air (oxygen). Actually, however, there is a whole spectrum of accidents leading to tritium releases into the reactor hall or to the environment - each with a different probability as shown in Fig. 6-57. As indicated, this release probability vs release size can be broken into a "routine" release pattern resulting from normal, operational leakage and small maintenance releases and into a "catastrophic" release portion where some accident has occurred. The experimental data used to obtain this distribution is based on about 20 years of tritium experience at the LIL Tritium Facility. It has been assumed that the normal release experience is simply scaled by the differences in inventory handled.

Table 6-25 is a compilation of our best collective guesses as to the accident mode and tritium release quantity. The largest tritium accident would involve a melt-down of tritium breeding pins and the release of 26.2 $\mathrm{kg}$; however, to release this huge quantity of tritium to the environment, a simultaneous rupture of the reactor hall containment is required - a most unlikely occurrence. The explosive force of this quantity of hydrogen is many orders of magnitude too small to rupture the reactor halj. structure by shock wave and over-pressure energy release; therefire, only an external accident exceeding a 747 plane crash could accomplish this. A dual accident such as this is termed an "incredible accident." Releasing this $26 \mathrm{~kg}$ of tritium to the reactor hall under melt-down conditions would, therefore, be considered the maximum credible accident. For a hybrid reactor, however, this tritium accident would most probably be associated with a uranium pin meltdown as well, thus dwarfing the tritium spill by the much more hazardous actinides. Since this $k$ ind of accident would probably require a total shutdown and closure of the reactor, the tritium processor for the reactor 


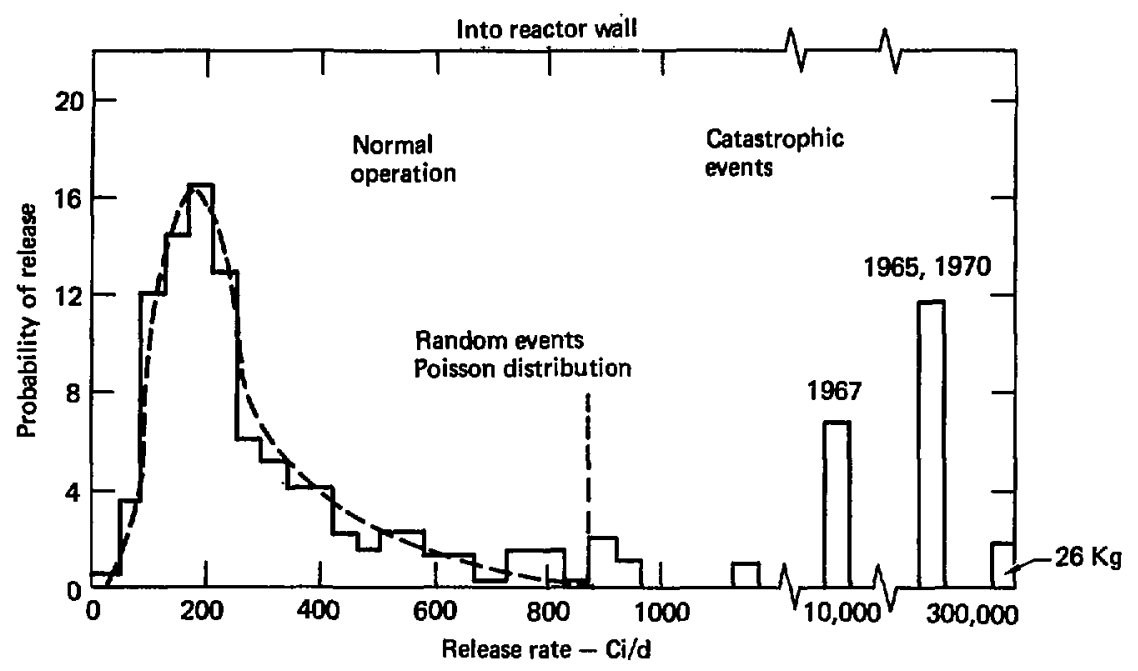

Fig. 6-57. Tritiun release statistics. 
Table 6-25. Risk analysis for tritium release accidents.

Accident mode

Tritium release

1. Breeding blanket melt down simultaneous with reactor hall rupture

$262 \mathrm{MCi}(26.2 \mathrm{~kg})$ to outside

2. Breeding blanket melt down

$262 \mathrm{MCl}(26.2 \mathrm{~kg})$ to hall

3. Cryogenic distillation column rupture

$14 \mathrm{MCl}(1.4 \mathrm{~kg})$

4. Tritium fuel storage tanks

$81 \mathrm{MCi}(8.1 \mathrm{~kg})$

5. Netural beam direct converter cryopanel

$2.5 \mathrm{MCi}(0.25 \mathrm{~kg})$

6. Direct converter cryopanel

$20 \mathrm{MCi}(0.20 \mathrm{~kg})$

7. Neutral beam processor sieve line

$282,000 \mathrm{Cl}$

8. Direct converter processor sieve line

$50,000 \mathrm{Ci}$

9. Reactor hall processor sieve line

$1800 \mathrm{Ci}$

10. Helium coolant loop rupture

$60 \mathrm{Ci}$

hall (discussed in Section 6.6.3.5) will not be sized for this accident. Although the processor will be sized for the steady-state operational level of $40 \mu \mathrm{Ci} / \mathrm{m}^{3}$, it will be capable of reducing the tritium levels following this maximum credible accident down to 400 MPC in about 7 days, if there is not interference or poisoning effects from the actinides.

6.6.3.3 Surface Outgassing. Now that we described the various sources exuding and releasing tritlum into the reactor hall, surface outgassing must be carefully considered as well as its consequences for reactor hall and the waste gas prucessing systems. Ionger worker re-entry times will result and these must be estimated as well. This problem is a difficult one because the transient behavior is difficult to predict. However, asymptotic, limiting analytical solutions are obtained. A tritium concentration spike will drive $\mathrm{T}_{2}$ and $\mathrm{HT}$ into many materials and create adsorbed layers of $\mathbf{T}_{2} \mathrm{O}$ on all exposed surfaces. As the reactor hall concentration drops, these walls act as sources.

Pirst, the complexing of the gas or water (1500 Ci/m $\mathrm{m}^{3}$ tritiun) from the "maximum credible accident" with the metal parts will be estimated. If the metal available is 300000 tons of stainless steel and at room temperature, the tritium dissolved will reach about $200 \mathrm{Ci}$ and the amount adsorbed onto the surfaces in the form of a monolayer will be around 50006 Ci. Thus, sone $52000 \mathrm{Ci}$ will become tied up with the metal parts as a result of this $1500-\mathrm{Cl} / \mathrm{m}^{3}$ spike. Iittle is known about the deadsorption 
of tritium from such surfaces, but practical experience tells us that the outgassing rate is very slow. It is very difficult to decontaminate tritium-fouled equipment. Our present practice at small scale says as a practical rule 49 an additional 40 changes of air are required to reduce the surface accunulations on small parts. This added processing time will be treated later in this report.

The consequences of this lack of knowledge about tritium surface complexing, tritium forms, outgassing, and rates from equipment are substantial. The waste-gas processing time needed to clean up this spike and restore the normal $40-\mu \mathrm{Ci} / \mathrm{m}^{3}$ level is difficult to predict and may be unacceptably long in large-scale practice. We can predict the processing time to reduce the $1500 \mathrm{ci} / \mathrm{m}^{3}$ to the $20000-\mu \mathrm{Ci} / \mathrm{m}^{3}$ re-entry levels in the presence of 180-Ci/d outgassing, and can roughly estimate the additional time needed when real deadsorption unsteady-state outgassing occurs, but cannot now treat surface complexing, tritium forms, and associated rates. There are procedures for surface cleaning designed to enhance tritium outgassing, such as steam cleaning a smooth, polished surface, ion discharge cleaning, electrocleaning, etc. This is the one area that must be studied more carefully and on an urgent basis. If outgassing occurred at the processor maximu capacity $(180 \mathrm{Ci} / \mathrm{d})$, about 150 days would be needed to remove the $52000 \mathrm{Ci}$ of tritium that is driven onto and into the surfaces.

6.6.3.4 Radiolytic Oxidation Within Reactor Hall. There must be sone assurance that in this operational design the level of 28 or less HTO can be feasibly mintained. It is this question that is critical to the practicability of our proposed design. The HTo can be produced from pure $\mathrm{T}_{2}$ or HT by several chemical routes: isotopic exchange with $\mathrm{H}_{2} \mathrm{O}$ in the water-contaminated air within the reactor hall, autoradioylsis of $T_{2}$ in air (oxygen), catalytic conversion of $\mathrm{T}_{2}$ or HY via active or hot metal surfaces exposed, and radiolysis of $\mathrm{T}_{2}$ or $\mathrm{HT}$ in air via radiation field escaping the mirror machine. It is for these reasons that, in the past, the use of air has been rejected from fusion reactor halls. However, careful review of contemporary research and demonstrated technology shows that the use of air must be reconsidered. If care is taken to avoid hot, precious wetal (catalytic) surfaces, excess water vapor, and a buildup of $T_{2}$, then the formation of Hro can be kept at nanageable levels. Estimates of the conversion rates have been made by fitting available data ${ }^{50-52}$ with a rate equation. The results are as follows: 


$$
\text { HYO }=T_{2}(0)\left[1-\exp \left(-k T_{2}(0) \theta\right)\right] \text {, }
$$

where $\theta$ is time for conversion in hours, $T_{2}(0)$ is the initial $T_{2}$ concentration in $\mu \mathrm{Ci} / \mathrm{m}^{3}, k$ is the rate constant (i.e., $k=29 \times$ $10^{-13} \mathrm{~m}^{3} / \mu \mathrm{Ci}-\mathrm{Hr}$ at 1002 humidity or $\mathrm{k}=6.24 \times 10^{-13} \mathrm{~m}^{3} / \mathrm{Ci}-\mathrm{Hr}$ for dry airj, and HrO is the concentration of tro found in $\mu \mathrm{Ci} / \mathrm{m}^{3}$. At initial levels of $\mathrm{T}_{2}(0)$ of $40 \mu \mathrm{Ci} / \mathrm{m}^{3}$, the rate of formation of $\mathrm{HrO}$ would always be less than $1.0 \times 10^{-4} \mu \mathrm{Ci} / \mathrm{m}^{3}$ per hour, small indeed. The conversion rate does not become significant until levels of $65 \mathrm{ci} / \mathrm{m}^{3}$ are approached, over $10^{6}$ larger than planned for in the design. The important point from this analysis is to keep $\mathrm{T}_{2}$ at low concentrations with maximum dedication.

The above model predictions have been made for $\mathbf{T}_{2}$ conversion by isotopic exchange and autoradiolysis to HTO in humid air. The effects of the availability of substantial areas of active metals surfaces on this conversion rate have been examined. 51 using this accelerated rate constant for steel surfaces, a comparison of oxidation rates is made in Table 6-26.

Table 6-26. Oxidation of $T_{2}$ to Bro in reactor hall (initial concentration $40 \mathrm{Ci} / \mathrm{m}^{3}$, Bro conc. in $\mu \mathrm{Ci} / \mathrm{m}^{3}$ ).

\begin{tabular}{|c|c|c|c|c|}
\hline Time & Dry air & Hunid air & $\begin{array}{c}\text { Steel } \\
\text { catalyzed }^{a}\end{array}$ & $\begin{array}{l}\text { Radiation field } \\
\text { on steel }\end{array}$ \\
\hline $1 \mathrm{hr}$. & $9.96 \times 10^{-10}$ & $4.01 \times 10^{-9}$ & $9.75 \times 10^{-8}$ & $0.6 \times 10^{-6}$ \\
\hline 2.5 & $2.49 \times 10^{-9}$ & $1.00 \times 10^{-8}$ & $2.44 \times 10^{-7}$ & $1.58 \times 10^{-6}$ \\
\hline 50 & $4.98 \times 10^{-8}$ & $2.01 \times 10^{-7}$ & $4.88 \times 10^{-6}$ & $3.1 \times 10^{-5}$ \\
\hline 2,000 & $1.99 \times 10^{-7}$ & $8.00 \times 10^{-6}$ & $1.92 \times 10^{-4}$ & $5.0 \times 10^{-3}$ \\
\hline 10,000 & $\begin{array}{l}9.38 \times 10^{-6} \\
=\left(1500 \mathrm{cl} / \mathrm{m}^{3}\right)\end{array}$ & $\begin{array}{l}4.00 \times 10^{-5} \\
\text { (Ero conc. in }\end{array}$ & $\begin{array}{l}0.96 \times 10^{-3} \\
\left.\mathrm{~m}^{3}\right)\end{array}$ & $25.0 \times 10^{-3}$ \\
\hline $12 \min$ & 0.01 & 0.03 & 0.78 & 5.0 \\
\hline $\mathrm{l} \mathrm{hr}$ & 0.04 & 0.16 & 3.90 & 24.0 \\
\hline $2.5 \mathrm{hr}$ & 0.10 & 0.40 & 9.76 & 63.2 \\
\hline $50 \mathrm{hr}$ & 1.99 & 8.40 & 195.2 & 1240 \\
\hline
\end{tabular}

With hunid air.

badiation field taken to be $10,000 \mathrm{R} / \mathrm{hr}$, on steel catalyzed surface in hunid air. 
Also shown are the enhancement effects of a superimposed radiation field ${ }^{53}$ on top of the heterugeneous catalytic surface and the humidity effects. Although the mirror plama flux is only $4000 \mathrm{R} / \mathrm{h}$, the spectrum is much harder and is expected to compensate. It can be seen that the overall enhancement effect is some 500 times over that formed in dry air. However, it is clear that when the concentrations are kept low (i.e., $\left.40 \mu \mathrm{Ci} / \mathrm{m}^{3}\right)$, and when the air within the reactor hall is continuously processed and not allowed to sit stagnant for very long periods of time, the percentage of HTO present will remain well below the 28 figure desired for the design case (208 MPC HTO). For a worse case accidental release of $20 \mathrm{~kg} \mathrm{~T} 2$ gas that is left isolated within the reactor hall at $150 \mathrm{c} / \mathrm{m}^{3}$ then the HTO concentration would build up to the $\mathrm{MPC}$ of $5 \mu \mathrm{Ci} / \mathrm{m}^{3}$ in $12 \mathrm{~min}$, or 20000 $\mu \mathrm{Ci} / \mathrm{m}^{3}$ in $5 \mathrm{~d}(\mathrm{i} . \mathrm{e}, 400 \mathrm{mpc}$ ). Free water volumes should be avoided

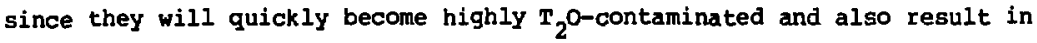
high humidity in the reactor hall atmosphere.

It should be noted in passing that an evacuated reactor hall (i.e., containing no air) would not have HT or $T_{2}$ comparable to HTO problems in the presence of ordinary water humidity. Although from this standpoint the differences are not large, because in the operation visualized here for the air-containing reactor hall, the tritium gas levels are kept is enough so that HTO formation is not significant. Accident situations, however, can be better handled in an evacuated reactor hall since HTO formation is nearly negligible and the tritium mole fraction concentration levels are some $10^{6}$ times higher and much more easily processed without air diluents. The lower volunetric flows woula significantly reduce the catalyst bed heating costs and the catalyst volume. The other improvements in structural features of the first wall and added costs in the reactor hall building must be carefully considered in future designs. But at this early stage, our brief look indicates advantages from the tritium standpoint.

6.6.3.5 Scavenging Tritium from Reactor Hall. Such a reactor hall containment processing system with pump-down locks has been depicted in Fig. 6-58 where one of the concepts for the mirror reactor reference design $^{54}$ is shown with such a processing concept added. Fresh air is contained within both personnel locks Nos. 1 and 2. In egress operation, both doors to No. 1 are closed and No. 2 is opened. Personnel enter lock No. 2 and its reactor hall door is closed. The center door is opened and personnel move from No. 2 to 1 . Lock No. 2 is fumped down with its waste 


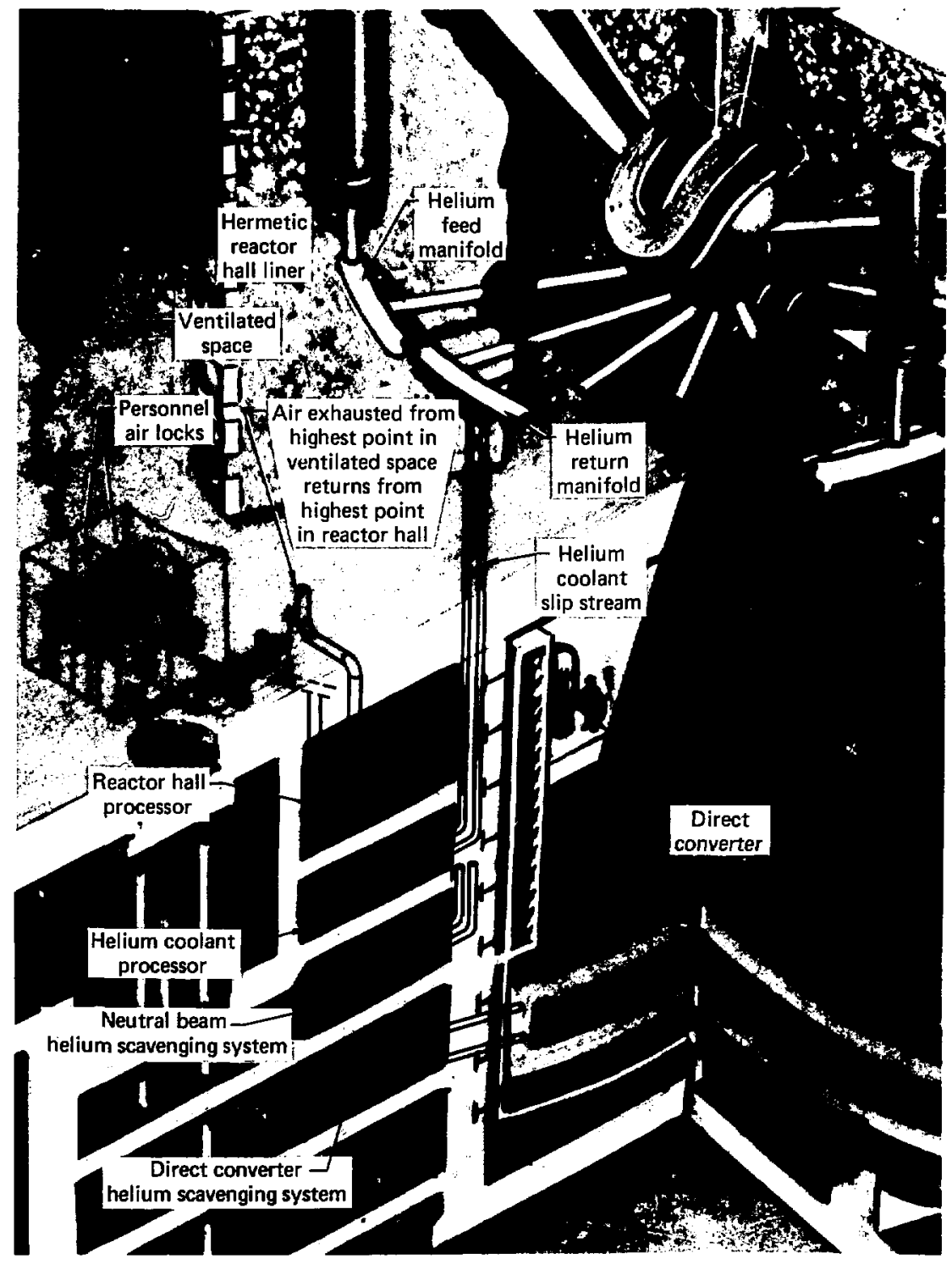

Pig. 6-58. Reactor hall containment processing system with punp-down locks. 
gas discharged in the reactor hall. Fresh air is exchanged through No. 1 into No. 2 until No. 2 air pressure matches atmospheric. Personnel move from No. 1 to outside. The doors on lock No. 1 are closed and its contents are pumped down and exhausted into the reactor hall and lock No. 1 is filled with fresh air. Personnel could enter or leave without any pump-down.

The hermetic seal secondary containment space inside the reactor hall structure is flushed with fresh air (about $0.36 \mathrm{~m}^{3} / \mathrm{s}$ or $600 \mathrm{cfm}$ ) when the air locks are not in operation, maintaining in this space levels of EIO below $2 \mu \mathrm{Ci} / \mathrm{m}^{3}$ into the waste gas processing system.

This combined low and high-temperature processing system shown in Fig. 6-59 would catalytically oxidize in alr the $T_{2}$ or Hr to Hro. Operation at high temperature $\left(350^{\circ} \mathrm{C}\right)$ would permit oxidation of tritiated hydrocarbons. The higher boiling refractory organics would be trapped by the activated carbon columns. Helium impurities will also be removed by a bleed air purge system, but these problems of nontritium impurities will be treated later in the project. Waste air would be recycled for the most part into the reactor hall itself, with a small bleed stream exhausted to the stack, hopefully below $40 \mu \mathrm{Ci} / \mathrm{m}^{3}$. The processed air would enter the reactor hall near floor level where occupational workers would be concentrated. The bulk flow of this reprocessed air would be expected to be $58.8 \mathrm{~m}^{3} / \mathrm{s}(100,00 \mathrm{cfm})$ or better, depending on how leaky or permeable the blanket tritium processing systems turned out to be. The air velocity near the worker might be in excess of $3.3 \mathrm{~m} / \mathrm{min}(10 \mathrm{fpm})$; however, the net velocity upward through the interior of the reactor hall would be only 0.33 $\mathrm{m} / \mathrm{duin}$ ( $1 \mathrm{fpm}$ ) or so. Thus, no credit of the velocity could be attributed to safety $(<120 \mathrm{fpm})$.

Spill Cleanup. Neglecting these adsorption-controlled outgassing problems discussed above for metal surfaces in the reactor hall air and for any free water volumes present, we can estimate the processing time required to reduce the $1500 \mathrm{ci} / \mathrm{m}^{3}$ from the "maximum credible failure," to the $20,000 \mu \mathrm{Ci} / \mathrm{m}^{3}$ reentry level or to the $40 \mu \mathrm{Ci} / \mathrm{m}^{3}$ operating level. Using the study at Mound Laboratory ${ }^{44}$ to determine the catalytic oxidation kinetic rate data, a plug flow reactor can be designed.

A schematic for such a recirculating tritium removal system has been shown in Fig. 6-60 with a low-temperature processor for handing the refractory tritium compounds discussed above. The tritium concentration (grans of $\mathrm{T}_{2}$ per cubic metre of total gas) is given as $T$, subscripted according to 


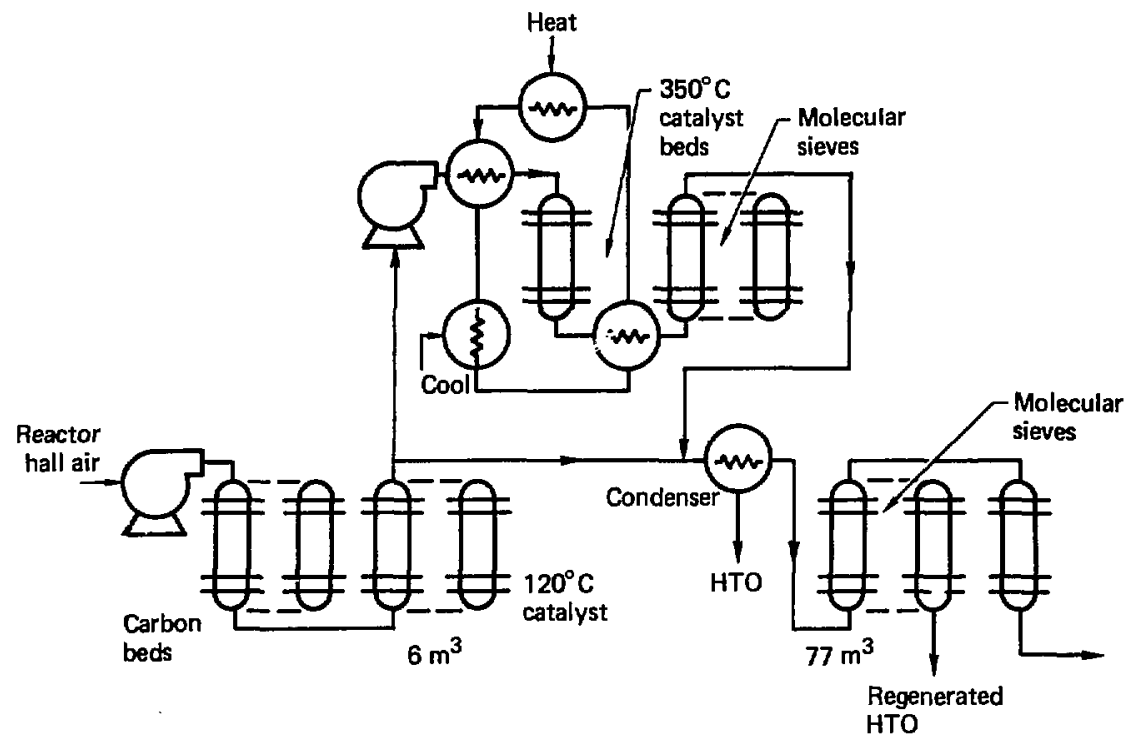

Fig. 6-59. Reactor hall processing system. 


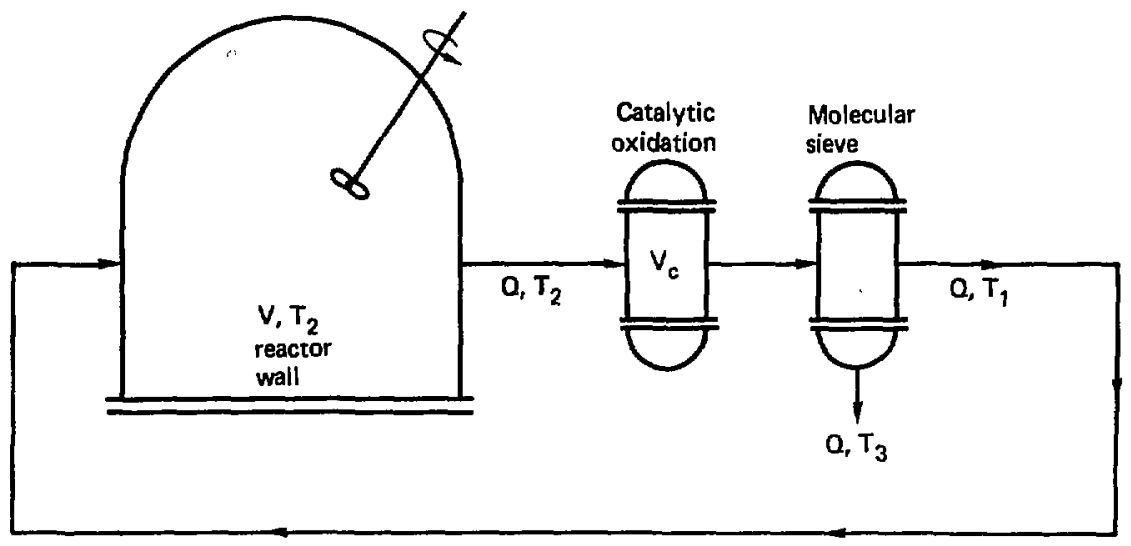

(1) $T_{2}(\theta)-T_{1}(\theta)=-\frac{V}{0} \frac{d T_{2}(\theta)}{d \theta}$

(2) $T_{1}(\theta)=T_{2}(\theta)\left\{\exp \left[-k \frac{V_{c}}{Q}\right]\right\}$

(3) $k=2.27 \times 10^{5} \exp (-7100 / R T)$

Solution: $T_{2}(\theta)=T_{2}(0) \exp \left\{-\left[1-\exp \left(-k \frac{V_{c}}{Q}\right)\right] \frac{Q}{V} \theta\right\}$

Fig. 6-60. Spill cleanup (apike of $2 \mathrm{~kg} T_{2}$ or $T_{2} 0$ ) . 
location. It is assumed that the reactor hall is well mixed and that the volunetric removal of gas (mostly tritium) designated as $q$, is negligible compared to the recirculating flow, $Q$, and is exuding tritium from the walls, leaks, etc. at $5 \mathrm{ci} / \mathrm{d}$.

A mass balance on the reactor hall can be made by expressing the difference between inlet and outlet concentration and the decrease in its prevailing level:

$$
T_{2}(\theta)-T_{1}(\theta)=-\frac{v}{Q} \frac{d T_{2}(\theta)}{d \theta}+\frac{s}{Q},
$$

where $\theta$ is the process time and $v$ the reactor hall volume. A similar balance can be made around the removal process unit:

$$
Q\left[T_{2}-T_{1}\right]=\mathrm{qT}_{3}
$$

or

$$
\frac{\mathrm{T}_{1}}{\mathrm{~T}_{2}}=1-\frac{\mathrm{qT} \mathrm{T}_{3}}{\mathrm{QT}_{2}}
$$

The plug flow reactor design equation can be written ${ }^{55}$ as:

$$
\mu=1-\frac{T_{1}(e)}{T_{2}(\theta)}=1-\exp \left[-k \frac{V_{c}}{Q}\right] \text {. }
$$

where $\mu$ vs the fraction converted, $v_{c}$ is the catalyst volume, and $k$ is the first order kinetic rate constant. Thus,

$$
k=2.4 \times 10^{9} \mathrm{~s}^{-1} \exp [-12300 / \mathrm{RT}] \text {. }
$$

with the activation energy in cal/mole. 56 This was obtained by correcting Mound's result $t^{57} ; k=2.27 \times 10^{5} \exp [-7100 / \mathrm{RT}]$ for tenperature-

dependent pore diffusion and obtaining new Arrheius parameters. 56 The Mound surface-controlled, plug-flow approach is adequate in neglecting pore diffusion and dispersion effects. For low-temperature (300 k) operation, these effects can be significant but can lead to significant errors at high temperatures (i.e., $>600 \mathrm{k}$ ). When these additional high-temperature effects are rigorously taken into account, the predicted performance is consistently worse. 56

Accordingly, Eq. (67) must be modified ${ }^{55}$ as:

$$
\mu=1-\frac{T_{1}(\theta)}{T_{2}(\theta)}=1-\left[1+\left(\frac{k v_{c}}{Q}\right)^{2} \frac{d p}{2 L}\right] \exp \left(-\frac{K v_{c}}{Q}\right),
$$


for moderately small axial dispersion or small peclet numbers, approximated by small catalyst-particle-to-bed-length ratio, dp/L. This axial dispersion correction in Eq. (69) typically reduces conversion by 25 for 99.94 conversion for $L=100 \mathrm{dp}$; it rapidly becomes more important for higher conversion and shorter bed operation. Note that at a given operating temperature, conversion $\mu$ is enhanced by increased residence time $v_{c} / Q$. For fixed-flow rate, this must be balanced against increased catalyst and pumping costs. Pumping costs are reduced at lower bed velocity, but mass transfer and dispersion limitations will then reduce conversion. Eliminating $T_{1}(\theta)$ between Egs. (65) and (68) results in

$$
\mu \mathrm{T}_{2}(\theta)=-\frac{\mathrm{v}}{\mathrm{Q}} \frac{\mathrm{dT}_{2}(\theta)}{\mathrm{d} \theta}+\frac{\mathrm{s}}{Q}
$$

If we integrate

$$
\int_{T_{2}(0)}^{T_{2}(\theta)} \frac{d T_{2}(\theta)}{\frac{S}{Q}-\mu T_{2}(\theta)} \times \int_{0}^{\theta} \frac{Q}{v} d \theta,
$$

the result is

$$
T_{2}(\theta)=T_{2}(0) \exp \left(-\frac{\mu Q \theta}{V}\right)+\frac{S}{\mu Q}\left[1-\exp \left(\frac{-\mu Q \theta}{V}\right)\right] .
$$

This result is valid for the case where the outgassing rate, S, remains constant with time; thus, it will be termed the "steady-state outgassing cleanup mode1."

Table 6-27 and Fig. 6-61 show the results of the "steady-state outgassing cleanup model," relating safe (400 MPC with light suit) "re-entry times" as a function of required catalyst volunes.

We must now carefully examine the consequences of the intensity of the phenomena of driving the tritium pike into the wall surfaces (i.e., "soaking") and the associated unsteady outgoing source that results.

A. E. Sherwood ${ }^{58}$ has corrected the simple exponential clean-up nodel for the unsteady-state mass diffusion effect of tritium gas into the wall surfaces. The simple "ideal" cleanup can be expressed as

$$
c / c_{0}=\exp (-\lambda t),
$$




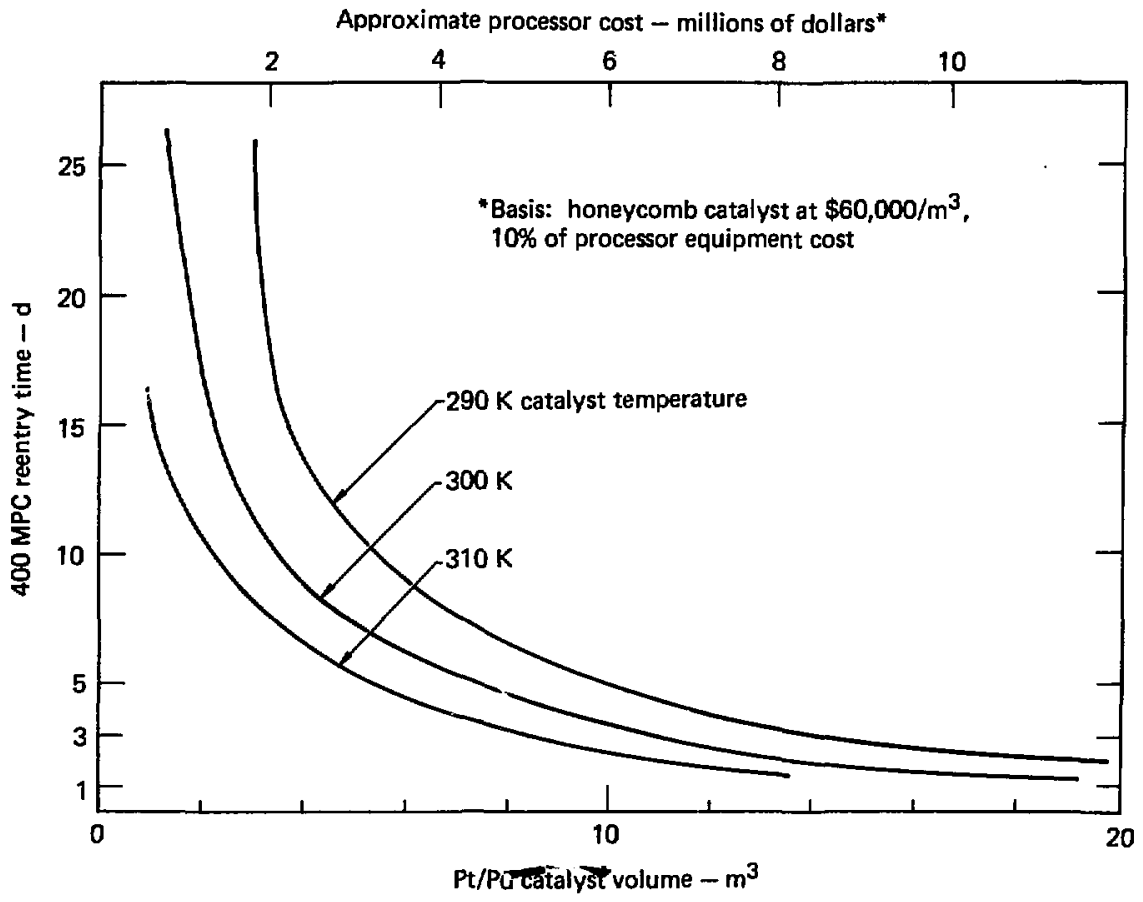

Fig. 6-61. Results of steady-state outgassing cleanup model. 
Table 6-27. Incredible accident: tritium reduction by the "steady-gtate outgassing cleanup mode1" $\left(Q=58.8 \mathrm{~m}^{3} / \mathrm{sec}(100,000 \mathrm{cfr})\right.$. $V=150,000 \mathrm{~m}^{3}$, temp $=300 \mathrm{~K}, \mathrm{~T}_{2}(0)=1500 \mathrm{ci} / \mathrm{m}^{3}$, steady source $=180 \mathrm{cl} / \mathrm{d}$ ).

\begin{tabular}{|c|c|c|c|c|}
\hline \multirow[b]{2}{*}{$\begin{array}{l}\text { Re-ent:y times, } \\
\text { days }\end{array}$} & \multicolumn{4}{|c|}{$\begin{array}{l}T_{2}(\theta) \text {, reactor hall tritium, } \mu \mathrm{Ci} / \mathrm{m}^{3} \text {, } \\
\text { at four values of } v_{c} \text {, catalyst volune }\end{array}$} \\
\hline & $2 m^{3}$ & $4 m^{3}$ & $6 m^{3}$ & $8 \mathrm{~m}^{3}$ \\
\hline 1 & $4.50 \times 10^{9}$ & $1.41 \times 10^{8}$ & $4.59 \times 10^{7}$ & $1.56 \times 10^{7}$ \\
\hline 2 & $1.35 \times 10^{8}$ & $1.32 \times 10^{7}$ & $1.41 \times 10^{6}$ & $1.61 \times 10^{5}$ \\
\hline 3 & $4.05 \times 10^{7}$ & $1.24 \times 10^{6}$ & $4.34 \times 10^{4}$ & $1.95 \times 10^{3}$ \\
\hline 4 & $1.22 \times 10^{7}$ & $1.17 \times 10^{5}$ & $1.66 \times 10^{3}$ & $1.80 \times 10^{2}$ \\
\hline 5 & $3.67 \times 10^{6}$ & $1.14 \times 10^{4}$ & $3.85 \times 10^{2}$ & $2.63 \times 10^{2}$ \\
\hline 6 & $1.13 \times 10^{6}$ & $1.53 \times 10^{3}$ & $3.45 \times 10^{2}$ & $2.63 \times 10^{2}$ \\
\hline 7 & $3.37 \times 10^{5}$ & $6.04 \times 10^{2}$ & $3.44 \times 10^{2}$ & $2.63 \times 10^{2}$ \\
\hline 8 & $9.92 \times 10^{4}$ & $5.16 \times 10^{2}$ & $3.44 \times 10^{2}$ & $2.63 \times 10^{2}$ \\
\hline 9 & $3.05 \times 10^{4}$ & $5.08 \times 10^{2}$ & $3.44 \times 10^{2}$ & $2.63 \times 10^{2}$ \\
\hline 10 & $9.83 \times 10^{3}$ & $5.07 \times 10^{2}$ & $3.44 \times 10^{2}$ & $2.63 \times 10^{2}$ \\
\hline 11 & $3.65 \times 10^{3}$ & & & \\
\hline 12 & $1.79 \times 10^{3}$ & & & \\
\hline 13 & $1.23 \times 10^{3}$ & & & \\
\hline 14 & $1.07 \times 10^{3}$ & & & \\
\hline 15 & $1.02 \times 10^{3}$ & & & \\
\hline
\end{tabular}

where

$\lambda=$ forced decay constant $=\mu \mathrm{Q} / \mathrm{N}, \mathrm{h}^{-1}$,

$\mu=$ tritium fractional removal in cleanup system,

$Q=$ Elow tate in cleanup loop, $\mathrm{m}^{3} / \mathrm{h}$,

$t=t i m e$ after accident, $h$,

$c=T_{2}$ concentration at time $t, \mu C i / m^{3}$,

$c_{0}=$ initial $\mathbf{T}_{2}$ concentration, $\mu \mathrm{Ci} / \mathrm{m}^{3}$,

$v=$ reactor hall volume, $\mathrm{m}^{3}$.

The wall soaking diffusion nodel can be solved rigorously, however, for our purposes; two asymptotic solutions adequately serve as follows:

for small time $(\lambda t \ll 1)$

$$
(\alpha / \lambda \ll 1) \text {, }
$$




$$
\frac{c}{c_{0}} \cong 1-\frac{2}{\sqrt{\pi}}(\alpha t)^{1 / 2}-\lambda t+\ldots ;
$$

and for large time $(\lambda t \gg 1)$

$$
\begin{gathered}
(\alpha / \lambda \ll 1), \\
\frac{c}{c_{0}} \approx \frac{1}{2 \sqrt{\pi}} \frac{(\alpha / \lambda)^{1 / 2}}{(\lambda t)^{3 / 2}}+\ldots .
\end{gathered}
$$

where

$\alpha=$ diffusion parameter $=1 / 4\left(D S^{2}\right)(\mathrm{A} / \mathrm{N})^{2}, \mathrm{~h}^{-1}$

$D=T_{2}$ diffusion coefficient in solid material, $\mathrm{m}^{2} / \mathrm{h}$,

S = solubility coefficient, a pseudo-Henry's Law constant,

$A=$ wall surface area for assumed semifinite solid, $\mathrm{m}^{2}$.

The results of the wall soaking and diffusion model are depicted in Fig. 6-62 for the parameters of $\lambda=1.0 \mathrm{~h}^{-1}$ and $\alpha$ ranging from $10^{-7}$ to $10^{-5}$. This illustration would apply for our reactor hall of $150,000 \mathrm{~m}^{3}$ with air flows of $58.8 \mathrm{~m}^{3} / \mathrm{s}$ and conversion $\mu$ of 78 per pass $\left(4 \mathrm{~m}^{3}\right.$ of $300 \mathrm{~K}$ catalyst). Note that after 10 hours or so, the tritium removals are considerably better than the "simple ideal cleanup," owing to the loss or disappearance of the tritium into the wall surfaces (i.e., "soaking"). However, for long times (over 100 hours or so), the walls begin to exude tritium back out into the reactor hall, thus making the processor cleanup task considerably more difficult.

The diffusion parameter, $\alpha \equiv 1 / 4\left(D S^{2}\right)(A / V)^{2}$, is important for the long-term cleanup. Polymeric (plastic, vinyl or alkyd paints) coatings on wall surfaces typically have $\alpha \equiv 10^{-5}$, whereas stainless steel may have $\alpha \approx 10^{-2}$. Therefore, from this model, polymeric coatings appear to behave better from the dynamics standpoint, although polished stainless steel surfaces are known to respond better to automated surface cleaning methods.

For our "maximum credible accident" case, Fig. 6-62 illustrates that we cannot achieve the desired 400 MPC in the 7-day period that our "steady-state outgassing cleanup" model predicts. In fact, this wall soaking and diffusion model says that 4000 MPC is more likely after 7 days, depending on $\mu$ and $\alpha$. This is indeed the maximum level that we would consider sending in a worker with an advanced ventilated tritium suit to undertake the maintenance. And we would restrict his work hours accordingly. Note that this comparison illustrates well the extreme importance of considering wall effects in tritium cleanup systems. 


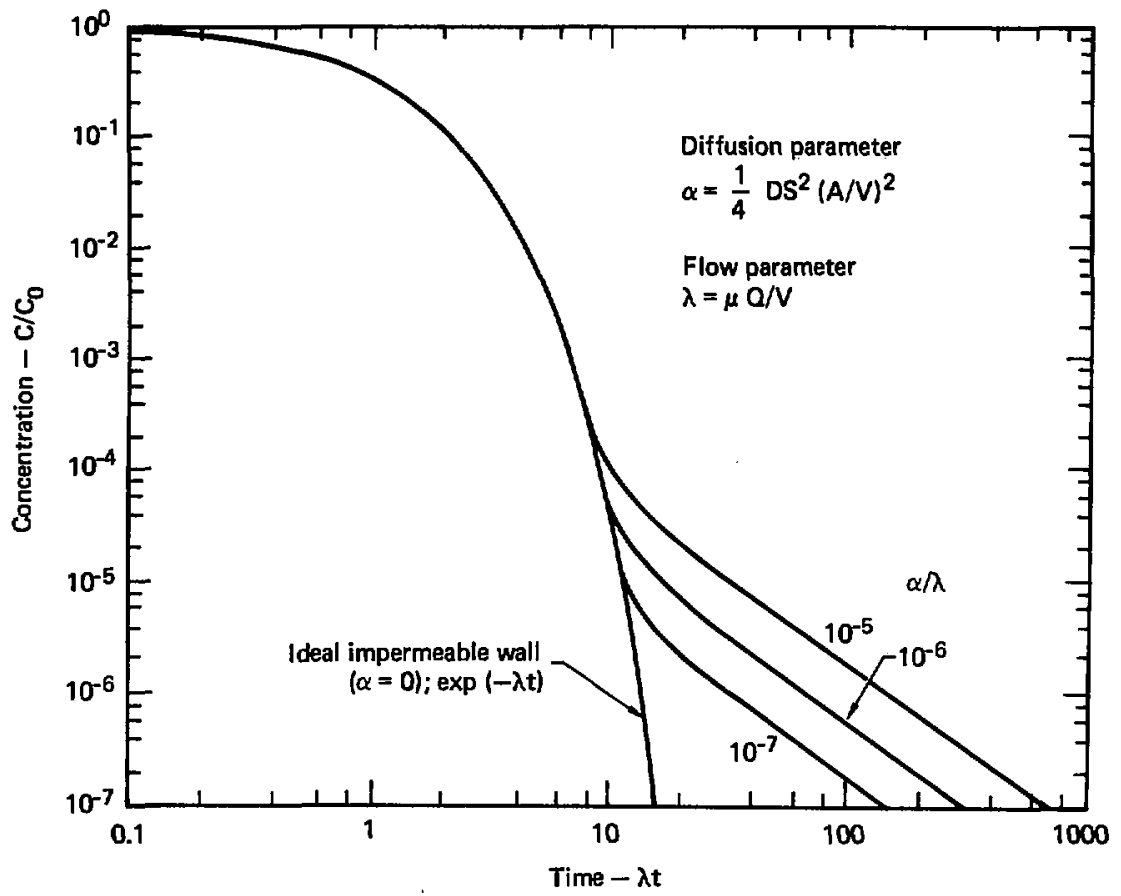

Fig. 6-62. Fusion reactor hall tritium cleanup with wall diffusion. 
There are additional mechanisms that need to be considered in considerably more detail before we can accept into the results of this wall soaking and diffusion model based on tritium gas solubility into, and diffusion within, these surfaces. Certain effects on these surfaces are important: conversion of the gas ( $\mathrm{HT}$ or $\mathrm{T}_{2}$ ) to water ( $\mathrm{HTO}$ or $\mathrm{T}_{2} \mathrm{O}$ ) on the surface, adsorption in the form of the monolayer, deadsorption, reconversion, etc. Maroni ${ }^{59}$ has made a first attempt to consider such water adsorption effects. The model parameters of surface conversion to water, adsorption and description rates were obtained by fitting experimental data taken on a small cleanup-system operation on a 50-litre plastic-walled chamber.

Maroni has used his model to predict the cleanup response for our system, assuning only wall adsorption effects and neglecting the wall-soaking and diffusion effects and the continuous $180-\mathrm{Ci} / \mathrm{d}$ outgassing source. The results are shown in Fig. 6-63. The model predicts similar effects for short and long times as does our wall soaking and diffusion model in Fig. 6-62, except larger conversion parameters are required to obtain effects as pronounced as ours. The long time asymptotes vary from 200 to $6000 \mu \mathrm{Ci} / \mathrm{m}^{3}$, depending on whether the water conversion rate on the surface is that from Table 6-26, or 100 times that amount. Needless to say, both our models predict extenc ad reentry times well in excess of the simple ideal cleanup model.

clearly, catalyst beds much larger than $10 \mathrm{~m}^{3}$ are expensive and impractical; thus, reentry times range from around 3 days to 2 weeks for reasonable bed designs. An interesting note can be made here regarding the estimated the $\$ 30$ million cost from a catalyst supplier. Their be $z$ volume was over $100 \mathrm{~m}^{3}$ since their conservative approach (as catalyst supplier) is to mer:t the efficiency requirements in a single pass. This is equivalent to a reentry of minutes.

Reentry times of the order of days to a week or so would be acceptable. Catalyst volumes from 2 to around $8 \mathrm{~m}^{3}$ are called for with catalytic reactor cost from $\$ 150000$ to $\$ 600000$. It is clear that there are sany areas where costs can be cut if further optimization of the design is made to suit the particular specialized requirements for the fusion reactor hall processing.

Now that we have exarined the design constraints on the catalytic reactor we must examine the molecular sieve. The design basis for molecular sieve beds that can achieve the lowest possible outlet tritium water concentration has been reviewed by vermeulen. ${ }^{60}$ In essence, one desires low water loading per unit mass of mole sieve - the lowest possible temperature down to $5^{\circ} \mathrm{C}$ 


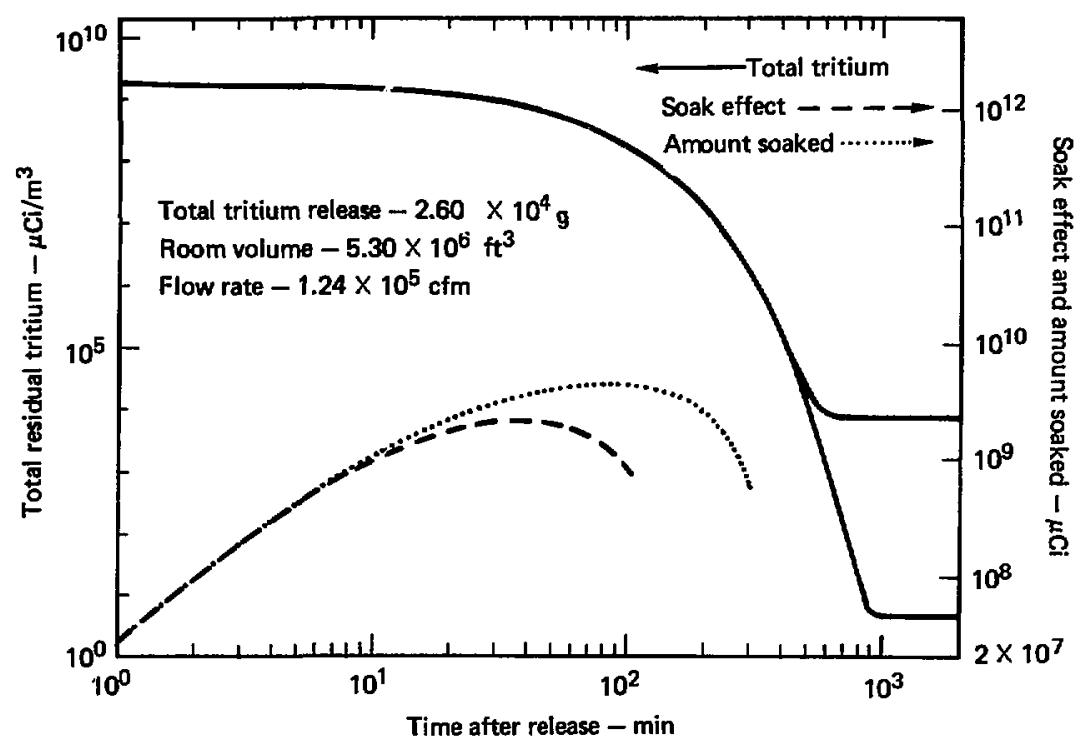

Fig. 6-63. Atmospheric cleanup analysis. Curves represent total residual tritiu, anount soaked, and soak effect. 
$\left(40^{\circ} \mathrm{F}\right)$ - and operation well before breakthrough. For tritium applications in inert gases, the HTO concentrations are so low that before breakthrough all beds examined here are loaded below $0.001 \mathrm{wt}$ - low indeed. The reactor hall processor sieves handiling $0.5 \%$ relative humidity in the reactor hall air are always loaded below 28. The temperature is held close to $50^{\circ} \mathrm{C}$ $\left(40^{\circ} \mathrm{F}\right)$, by conventional chilled water systems. No further vapor pressure reductions are practically achieved below $0^{\circ} \mathrm{C}\left(32^{\circ} \mathrm{F}\right)$, because of blinding of the molecular sieve pores with ice. Consequently, the only design variable important to these designs is the breakthrough time.

A convenient representation of available data ${ }^{60}$ for sieve breakthrough times is given as follows:

$$
t_{B}=\frac{307,000}{1+37.1 r} \frac{v_{s}}{Q} .
$$

where $r$ is the relative humidity fraction, and $v_{s}$ the volume of the sieve and the volumetric flow rate through the sieve. Operating below breakthrough guarantees HTO outlet concentrations as low as the previous regeneration, which can be performed by mole sieves around $5,000 \mu \mathrm{Ci} / \mathrm{m}^{3}$.

Noted that with the reactor hall atmosphere maintained at a ary $0.5 \%$ relative humidity, there is not a significant quantity of ordinary water to effectively realize a swamping effect on tritium water more than a mere factor of five. Consequently, a mcleular sieve freshly regenerated to around 0.18 moisture will produce output HTo levels around $5,000 \mu \mathrm{ci} / \mathrm{m}^{3}$ without swamping, and around $1,000 \mu \mathrm{Ci} / \mathrm{m}^{3}$ with swamping.

To achieve a further water reduction to $208 \mathrm{MPC}$ or $1 \mu \mathrm{Ci} / \mathrm{m}^{3}$, a cryogentic polishirig step is needed on the last molecular sieve. We envisage a cryogenic exchange-condenser operating at around $-150^{\circ} \mathrm{C}$. The vapor pressure of Hro over the solid would be sufficiently $10 \mathrm{w}^{61}$ to effect the final goal of $1 \mu \mathrm{Ci} / \mathrm{m}^{3}$ tro. Normally, this approach would be far from feasible because a thick build-up of j,ce on the exchanger finned/surfaces would quickly spoil the operation. However, as a final polishing step - removing only around $10^{-7} \mathrm{~g} / \mathrm{s}$ or about $3 \mathrm{~g}$ ErO per year - the performance should remain high. This polishing cryogenic condenser can opurate at reasonable conditions such as $-150^{\circ} \mathrm{C}$, handle the full $58.8 \mathrm{~m}^{3} / \mathrm{s}$ flow, and utilize a reasonable surface area around $40 \mathrm{~m}^{2}$.

Part of the catalytic oxidation-molecular sieve adsorption system described above involves the probleat of handling the spent molecular sieve 
from an occupational hazard standpoint. The reactor hall is planned to be operated at a relative humidity of $0.5 \%$. This level was selected as the lowest demonstrated level routinely used in a worki.ag environment within the DOE system (the thermal battery program at Sandia Albuquerque). Workers have been routinely operating in this environment for 15 years with no bad experience. There are presently no OSHA guidelines on this topic.

with this low level of humidity, the liquid stream collected on molecular sieves will amount to $750 \mathrm{lb} / \mathrm{d}$ with a tritium contamination level of $0.5 \mathrm{Ci} / 1$ itre. Disposal would ir.rolve several 55-gal drums buried per day, or deep well injection in a dry, stable geclogical deposit. Thus, this problem does not appear to be insurmountable.

The molecular sieve beds are operated in the dynamic breakthrough mode with a cycle time from loading to regeneration of 4 days for molecular sieve 5A. This corresponds to a sieve volume of $77 \mathrm{~m}^{3}$ or a sieve cost of $\$ 38,000$ per bed for the 3 sieve beds shown the total capital cost is expected to be $\$ 250,000$ plus $\$ 100,000$ for instruments and controls.

before we leave this topic, we should comment about the ability of the abo design to handle the routine processing task. As indicated in Table 6-24, a processing systen scaled at around $58.8 \mathrm{~m}^{3} / \mathrm{s}(100,000 \mathrm{cfm})$ could handle tritium outgassing from the blanket, as well as process piping and contaminated parts, etc., at levels of $180 \mathrm{Ci} / \mathrm{d}$ if the catalytic reactor operated at complete conversion.

Unfortunately, the economic constraints on the catalytic reactor design forces us to accept something less than complete conversion. Consequently, we will be forced to modify the limits of Table 6-24 as follows using the source term, $S / \mu \mathrm{Q}$, in $\mathrm{Eq} .8$, where $S$ is the source strength of the tritium outgassing and leakage, $\mu$ is the catalytic oxidation conversion rate, and $Q$ is the volumetric flow of reactor hall air being processed. Now we demand that the reactod tritiug gas fraction remain at or below $40 \mu \mathrm{Ci} / \mathrm{m}^{3}$; thus, we :equire that

$$
\frac{5}{\mu Q} \leq 40 \mu \mathrm{Ci} / \mathrm{m}^{3}
$$

The system performance predicted in Table 6-27 comes close to this, judging from the long time asymptotes slightly more than 40 . These asymptotes are close enough to weet the $40-\mu \mathrm{Ci} / \mathrm{m}^{3}$ limit simply by adjusting the catalyst teaperature slightly as indicated in makls $6-29$. Depending on the 
Table 6-28. Catalyst temperature to hold $40 \mu \mathrm{Ci} / \mathrm{m}^{3}$.

\begin{tabular}{ccccccc}
\hline $\begin{array}{c}\text { Plant } \\
\text { size, } \\
\text { Gie }\end{array}$ & $\begin{array}{c}\text { Estimated leakage } \\
\text { and } \\
\text { outgassing rate, } \\
\text { Ci/d }\end{array}$ & $\mu_{\min }$ & $2 \mathrm{~m}^{3}$ & $4 \mathrm{~m}^{3}$ & \multicolumn{3}{c}{$\begin{array}{c}\text { Catalyst temperature, } \mathrm{K}, \\
\text { at four values of } \mathrm{m}_{\mathrm{c}}\end{array}$} \\
\hline 1 & 180 & 0.885771 & 437 & 403 & 385 & 373 \\
0.47 & 70 & 0.344466 & 364 & 340 & 327 & 319 \\
\hline
\end{tabular}

plant's actual leakage and outgassing rates, a $6-\mathrm{m}^{3}$ catalytic reactor would require only from 27 to $85^{\circ} \mathrm{C}$ highex temperature operation to meet the $40-\mu \mathrm{Ci} / \mathrm{m}^{3}$ requirement.

In addition to the recirculation scheme discussed above, the costs could be iurther reduced by introducing the traojeoff between processor capital cost and operating cost. For energy consumptions around $0.5 \mathrm{HW}$, the air could be heated another $10^{\circ} \mathrm{C}$. Catalyst volume would be reduced by $34 \mathrm{q}$, thus saving about $\$ 1$ million, as indicated in Fig. 6-61. There is a similar tradeoff by compressing the air to 4.5 atm, a $180^{\circ} \mathrm{C}$ heat of compression. ${ }^{42}$ About $\$ 2$ million would be saved at an operating cost of $6.8 \mathrm{MW}$.

As indicated earlier in this report, this processing capability is safely above the estimated ${ }^{46}$ leakages around $70 \mathrm{Ci} / \mathrm{d}$. Thus, the system presented is nearly optimally configured for both the routine leakage task and the "incredible cailure." Now we have to ensure that our blanket containment scheme can meet this goal of below $180 \mathrm{ci} / \mathrm{d}$.

6.6.3.6. Dose to the Occupational Worker. Now that we have established the reactor hall processing characteristics, we can estimate the dose to the occupational worker. As stated in section 6.6.2.3, the work assignments within the reactor hall can be made according to the HTO level. If HTO has averaged $5 \mu \mathrm{Ci} / \mathrm{m}^{3}$ or $1.00 \mathrm{MPC}$, this work assignment should be restricted to $8 \mathrm{~h}$ nar week or 12 a per quarter. If, however, the HTo level is around $1 \mu \mathrm{Ci} / \mathrm{m}^{3}$, he can work a full 40-h work week. This approach will keep worker dose below 208 MPC as DOE desires. 36 If the worker wears a lightweight suit, reducing skin air exposures and skin contact a factor of 10 or so, his HTO would be reduced by about 498 to levels that depend mostly on inhalation. This should allow reactor hall work assignments to average 23 days per quarter.

In the maximum credible accident situation where the reactor hall reaches $1500 \mathrm{ci} / \mathrm{m}^{3}$ of HTO before the worker hears the emergency tritiun "claxon", 
the worker has $0.5 \mathrm{~s}$ to flip the suit hood over his head and pull the emergency air rip-cord on his belt. This slow response would give the worker 25 rem, the accident dose allowed to the general public. The worker can, of course, receive up to 100 rem before he is likely to suffer long-term radiation effects. At $100 \mathrm{rem}$, local power-plant management under emergency conditions could authorize fellow workers to enter the $1500-\mathrm{Ci} / \mathrm{m}^{3}$ HTOcontaminated reactor to carry out rescue operations.

The possibility of a worker receiving a $1500-\mathrm{Ci} / \mathrm{m}^{3}$ HTO exposure could result from alarm failures or slow worker response to the alarms. During an incredible accident of a $26-\mathrm{kg}$ HTO (or $\mathrm{T}_{2} \mathrm{O}$ ) dump, the concentration within the reactor hall could take some time to increase from around $5 \mu \mathrm{Ci} / \mathrm{m}^{3} \mathrm{HTO}$ to $1500 \mathrm{Ci} / \mathrm{m}^{3}$ HTO; long before $1500 \mathrm{Ci} / \mathrm{m}^{3}$ would be reached, the claxon alarms would have sounded. For example, HTO alarm levels could be set as $20 \mu \mathrm{Ci} / \mathrm{m}^{3}$ HTO, and claxon levels at $75 \mu \mathrm{Ci} / \mathrm{m}^{3}$ HTO. This will require the development of reliable and economic monitoring instrumentation that will provide "real-tine" $\mathrm{Hr}$ gas and HTO water discrimination (or $\mathrm{T}_{2}$ from $\mathrm{T}_{2} \mathrm{O}$ ) The possibility of the worker zeceiving more thun a $1500-\mathrm{Ci} / \mathrm{m}^{3}$ HTO exposure could occur in a situation where the spill takes place near the worker before the release plume has time to disperse and dilute in the reactor hall. The likelihood of this occurrence can be reduced by holding HTO concentrations within ducts, piping, and some process vessels below $19,000 \mu \mathrm{Ci} / \mathrm{m}^{3}$ or provide air hood shrouding to provide a $1-\mathrm{m} / \mathrm{s}$ (150 fpm) air velocity inward and away from the worker. Small air flow features such as this will help to cut down the annual dose to the worker in a developina U.S. fusion economy.

\subsubsection{Tritium Breeding Blanket}

6.6.4.1. Tritium Profuction in Lithium Deuteride. A careful study of the Eeasibility of the various candidate solid lithium compounds for tritium rzsovery from fusion blankets has been recently completed at Brookhaven National Laboratory. ${ }^{62}$ A detailed review and analysis of these results suggests lithium deuteride would be the best for this application owing to its high solubility, low varor pressure and high breeding ratio. This contrasts with the beryllium metal-lithium beryllate $\left(\mathrm{Li}_{2} \mathrm{Be}_{2} \mathrm{O}_{2}\right)$ mixture which is ideal for a purged blanket situation owing to its high melting point $\left(1250^{\circ} \mathrm{C}\right)$, low tritium solubility, and sustained breeding ratio (BR > 1.2 ). $\mathrm{Li}_{2} \mathrm{O}$ and $\mathrm{LiAlO}_{2}$ would qualify except for their low solubility or capacity. For this sealed-pin hybrid blanket, lithium deuteride salt was selected. 
Tritium can be produced at $1.76 \mathrm{~g} / \mathrm{h}(0.422 \mathrm{MCi} / \mathrm{d})$ by neutron breeding in the lithium deuteride salt packed into 85,000 aluminum alloy pins. The breeding pins will have a residence time within the blanket of 3.4 years and at the end of life will contain tritium at a concentration of $5200 \mathrm{ppm}$ or $13,416 \mathrm{Ci} / \mathrm{m}^{3}$. The breeding pins will have a diameter of $3.0 \mathrm{~cm}$ and a length of $54 \mathrm{~cm}$. They will be placed in an hexagonal configuration with a pitch (center-to-center) distance of $3.1 \mathrm{~cm}$. The total pin surface area available for tritium permeation is $4.3 \times 10^{7} \mathrm{~m}^{2}$. The average temperature of the pin over its life is $350^{\circ} \mathrm{C}$.

Consequently, the containment of tritium within these pins to achieve reasonable loss levels is indeed a challenge. We selected lithium deuteride (LiD) for its high breeding ratio and low tritium vapor pressure. From the experimental work of Heumann and Solomon ${ }^{63}$ the vapor pressure of $T$ over LiD is presented as a function of the concentration of the salt. With the addition of a few percent of lithium metal, a flat plateau exists where the vapor pressure becomes independent of salt concentration. This is an excellent region in which to carry out the breeding reaction, since the vapor pressure will remain nearly constant over the life of the pin.

The tritium inventory within these 85,000 pins held for 3.4 years is $23.3 \mathrm{~kg}$. If this quantity as well as the $20 \mathrm{z}$ loss by the 3.4 out of $12.3-\mathrm{yr}$ decay to hejium is a concern, then helium-purged pins are required. Each pin must be fitted with a porous cap and a vacuum connection to a common manifold at the bottom. Helium coolant (at $60 \mathrm{~atm}$ ) would leak through the porous cap down into the pin, stripping tritium from the lithium compound within. The next challenge is to identify and develop an appropriate lithium compound that will release its breed tritium at $350^{\circ} \mathrm{C}$. For lithium aluminate, owen 64 showed that below $650^{\circ} \mathrm{C}$, significant quantities of tritiun could not be removed simply by evacuation. Johnson ${ }^{65}$ showed that for low density pressed, sintered lithium aluminate, significant yields (i.e., 28\%) could be obtained by in-reactor venting to vacuum around $100^{\circ} \mathrm{C}$. For low-temperature in-reactor purge extraction of the breed tritium at significant yields, more experimental work involving rates will be required. The concept tentatively appears feasible at low temperature if small pellets are used. If low rates are a problem, the coolant flow past these pins could be reduced to achieve elevated tenperatures when necessary.

Helium gas production within these pins at the low neutron flux this far out in the blanket has been estimated by the neutronics code and found to be 
acceptably small relative to concern about pressure buildup and over-stressing the breeding pin strlicture.

6.6.4.2. Tritium Permeation Through Breeding Pins, The preferred blanket concept for breeding tritium involves sealed pins fabricated out of a high-temperature aluminum alloy. Our first task is to estimate the permeation of tritium to the helium gas coolant stream in order to properly size the wall thicknesses and obtain tritium loss estimates into the helium flow.

Available permeation data ${ }^{66-71}$ for various metals and alloys have been displayed in Fig. 6-64. It is obvious for this application at $350^{\circ} \mathrm{C}$ that: aluminum is an excellent choice owing to its low tritium permeation. There is a concern, however, that an abnormal temperature excursion in the reactor might melt some of the pins in-place and release tritium in large quantities into the helium flow. For this reason, high-temperature aluminum alloys appear especially attractive.

The tritium production $(0.62 \mathrm{~g}$ per pin) is then released by melting the pins anu the LiD in a inert gas furnace at 700 to $750^{\circ} \mathrm{C}$ and releasing the deuterium and tritium as the gas. DOE's Savannah River Plant ${ }^{69}$ has developed this technology to a high level of reliability. The gases are fed to the fuel purification system for reuse in the plant. The details of this breeding pin on-site reprocessing facility will be discussed later.

Materials compatibility is also important in maintaining pin integrity over the long lifetime of the pins. To prevent material dissolution of the aluminum alloy with the LiD a thin coating of iron is applied to the interior of these pins. The pins are crushed and shredded and then melted around $700-750^{\circ} \mathrm{C}$ and the IiD-LiAl system will result ${ }^{64}$ where the tritium vapor pressure and the diffusion rates become large enough to economically extract the tritium. The IiD-LiAl mixture is reprocessed and fabricated into pins as discussed later.

We have considered lockalloy and SAP as the leading candidates. Lockalloy is available and can be machined and welded by conventional methods, 72 however, it contains 38\% beryllium. The beryllium is excellent from a neutronics point of view, but can be criticized from a resource limitations and human hazards standpoint. The SAP (sintered aluminum product) alloy ${ }^{73}$ is machjnable with some added care (owing to tool wear) and is welded by special methods. The alloy contains small quantities of alumina, $\mathrm{Al}_{2} \mathrm{O}_{3}$, to provide added high temperature strength nearly to its melting point $\left(624^{\circ} \mathrm{C}\right)$. SAP alloys are manufactured with aluminum as the continuous phase. 


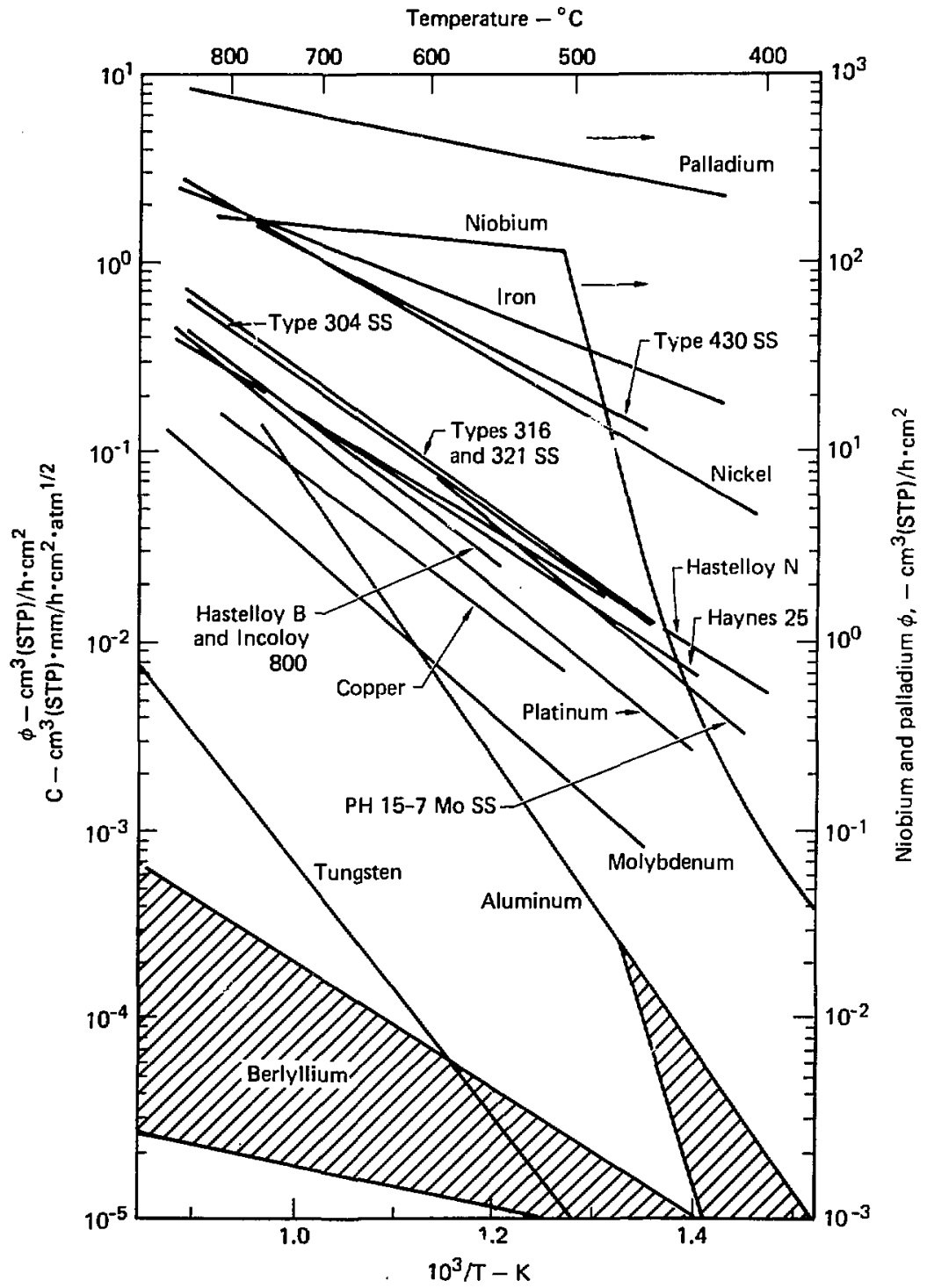

Fig, 6-64. Permeation reduction in oxidized Incoloy-800. 
Some tritium permeation measurements were made with several coupons of the aluminum-beryllium alloys, with the conclusion that the permeation was not significantly higher than pure beryllium or aluminium, although the experimental uncertainties were large owing to the extreme difficulties of obtaining a coupon seal and detecting the low levels. Full-scale tube tests are now underway where the full diameter and wall thickness has been used for the pin. The prototype tubes have been filled with the hydrogen isotopes to measure the actual permeation under fully realistic conditions.

Therefore, with the above reservations, we proceed to estimate tritium permeation through the breeding pins as if they behave as a pure aluminum. First, we compute the plateau vapor pressure of tritium over the $\mathrm{LiD} / \mathrm{Li}$ system and obtain $4.05 \times 10^{-6}$ atm at $350^{\circ} \mathrm{C}$. This will be the partial pressure driving force for permeation loss of tritium and will be independent of time or concentration of tritium within the pin. For 85,000 pins with a 1-mm wall thickness, the net permeation will be $20 \mathrm{ci} / \mathrm{d}$.

Now that the routine, normal operating permeation loss rate of tritium from the breeding pins has been determined, we must estimate the loss from pin failure. A standard failure rate of fission reactor fuel pins that is commly used in designs today is 18 failure. The failure rate is this large because the pins run very hot, are subjected to a high radiation flux, and cladding swelling forces from within. The tritium breeding pins in a fusion hybrid reactor design are not subjected to such extreme conditions. They operate at low temperatures in a low radiation flux, are simple in mechanical design, and need not be subjected to large swelling forces. Consequently, we have selected a failure rate of 0.18 .

A breeding pin failure rate of 0.18 of the tritium production rate results in a flux into the helium coolant of $422 \mathrm{Ci} / \mathrm{d}$. This design condition, unfortunately will be contrc:.'ing until we can obtain sound evidence for smaller failure rate in the 85,000 pins or improved means for containing and scavenging this loss.

6.6.4.3. Helium Scavenging system. A tritium scavenging processor has been provided that will remove tritium from a 28 slip stream of the main helium coolant flow. Tritium losses from the pins involve $20 \mathrm{ci} / \mathrm{d}$ from routine permeation and $422 \mathrm{ci} / \mathrm{d}$ from an anticipated failure rate of 0.18 , for a total of $448 \mathrm{ci} / \mathrm{d}$.

The problem now becomes how to size the slip-stream tritium processor as well as specify its performance to keep the tritium levels in the recirculating 
Table 6-29. Physical features of energy recovery system affecting permeation.

\begin{tabular}{lccc}
\hline Exchanger & $\begin{array}{c}\text { Average wall } \\
\text { temp, }{ }_{\mathrm{C}}\end{array}$ & $\begin{array}{c}\text { Wall } \\
\text { thickness, mm }\end{array}$ & $\begin{array}{c}\text { Surface } \\
\text { area, } \mathbf{m}^{2}\end{array}$ \\
\hline Economizers & 250 & 1.6 & 15960 \\
Evaporators & 300 & 1.6 & 4008 \\
Superheaters & 425 & 1.8 & 5652 \\
Resuperheaters & 475 & 2.5 & 5532 \\
\hline
\end{tabular}

nelium coolant loop low enough to obtain acceptable losses to the environment through the energy recovery system heat exchangers. The properties that control permeation are the area, permeability of the alloy, wall thickness, and average wall temperature. These factors have been tabulated in Table 6-29. The heat exchangers are constructed of Incoloy 800 to take advantage of lower tritium permeation through the inherent oxide film. ${ }^{69-71}$ Although there has been considerable controversy as to the extent and mechanism for this oxide barrier formation and its effectiveness in tritium permeation reduction, there has been verification of the laboratory studies 69,70 in the full scale power plant at Peach Bottom. This experimental verification was made by Ling Yang of General Atomics ${ }^{71}$ by taking actual samples of the Incoloy-800 from the steam generators and measuring the tritium permeation through the oxide barrier coated alloy and through the cleaned alloy. Substantial agreement with Strehlow's measurements ${ }^{70}$ was obtained.

In applying these results to our design, there is the problem in deciding on the partial pressure driving force functional dependence, varying fron square root to linear. The oxide barrier-sontrolled permeation is characterized by a near-linear pressure dependence. When temperature transients occur this barrier is temporarily reduced in its effectiveness owing to the formation of cracks or other imperfections in the coating. ${ }^{69}$ For this reason we are reluctant to take full credit for a linear pressure dependence, which would result in exceedingly low (i.e.. $0.05 \mathrm{ci} / \mathrm{d}$ ) tritiun permeation losses into the stream. We feel it is risky to extrapolate the experimental data, with around 1 Torr driving forces, back to $10^{-3}$ Torr using the linear pressure dependence.

For this design, therefore, it was decided to use a conservative extrapolation procedure as follows: take the fuli credit for the oxide barrier permeation reduction observed as $200 \times$ reduction at around 1 Torr, ${ }^{69}$ but 
extrapolate to the lower driving forces using the conservative square root dependence as shown in Fig. 6-65.

Now using the above criteria for obtaining the oxide protected Incoloy-800 permeation rates for tritium into the stream, we can now size the helium coolant flow slip stream process in order to reduce these tritium losses to acceptable levels. Figure 6-66 shows the schematic relationship of the slip-stream processor. Such system designs are frequently incorrect because the buildup of tritium in a recirculating system processed by a small slipstream flow is neglected. The problem results from the fact that the tritium leakage into the flow system will bulld up the tritium concentration, $T$, to high levels where there is obtained at steady state a mass balance between the leakage source strength and the removal rates by permeation to the stream and the slip stream processor.

The solution for $T$ is obtained by trial and error in the relationship

$$
R+P=0.99(0.02 T)+B T^{1 / 2}=448 \mathrm{Ci} / d
$$

where $B$ involves the geometric factors for permeation as summarized in Table 6-28. The salution obtained for $T$ is $22,439 \mu \mathrm{\mu Ci} / \mathrm{m}^{3}$. Neglecting this buildup condition would have resulted in an error of a factor of 50 in tritium concentration in the helium flow. This results in a tritium loss to the stream system of $3 \mathrm{ci} / \mathrm{d}$.

There is another source of tritium into the helium flow that must be estinated, and this involves the energetic triton implantation into the first wall. As discussed previously ${ }^{40}$ this mechanism effectively produces a source of tritium within the first wall at a shallow depth of around 1 micron which will then permeate into the helium stream. We have estimated this triton implantation of tritium and consequent permeation loss into the helium previously $\mathrm{y}^{72}$ and found it to be $96 \mathrm{ci} / \mathrm{d}$. In comparison to the $448 \mathrm{Ci} / \mathrm{d}$ source of tritium from the 0.18 pin leakage, this $96 \mathrm{Ci} / \mathrm{d}$ is significant. with this additimal source of tritium added to the $448 \mathrm{Ci} / \mathrm{d}$, there is obtained a $3 \mathrm{ci} / \mathrm{d}$ tritium leakage to the steam.

The slip stream processer configured as a catalytic oxidizer, condenser and nolecular sieve adsorber has been designed and sized as in Fig. 6-67. The catalyst bed has a small volume of 12 litres, an the molecular sieve beds of $18 \mathrm{~m}^{3}$. The process cost is estimated at $\$ 100,000$,ncluding instruments and automatic control and safety systems. 


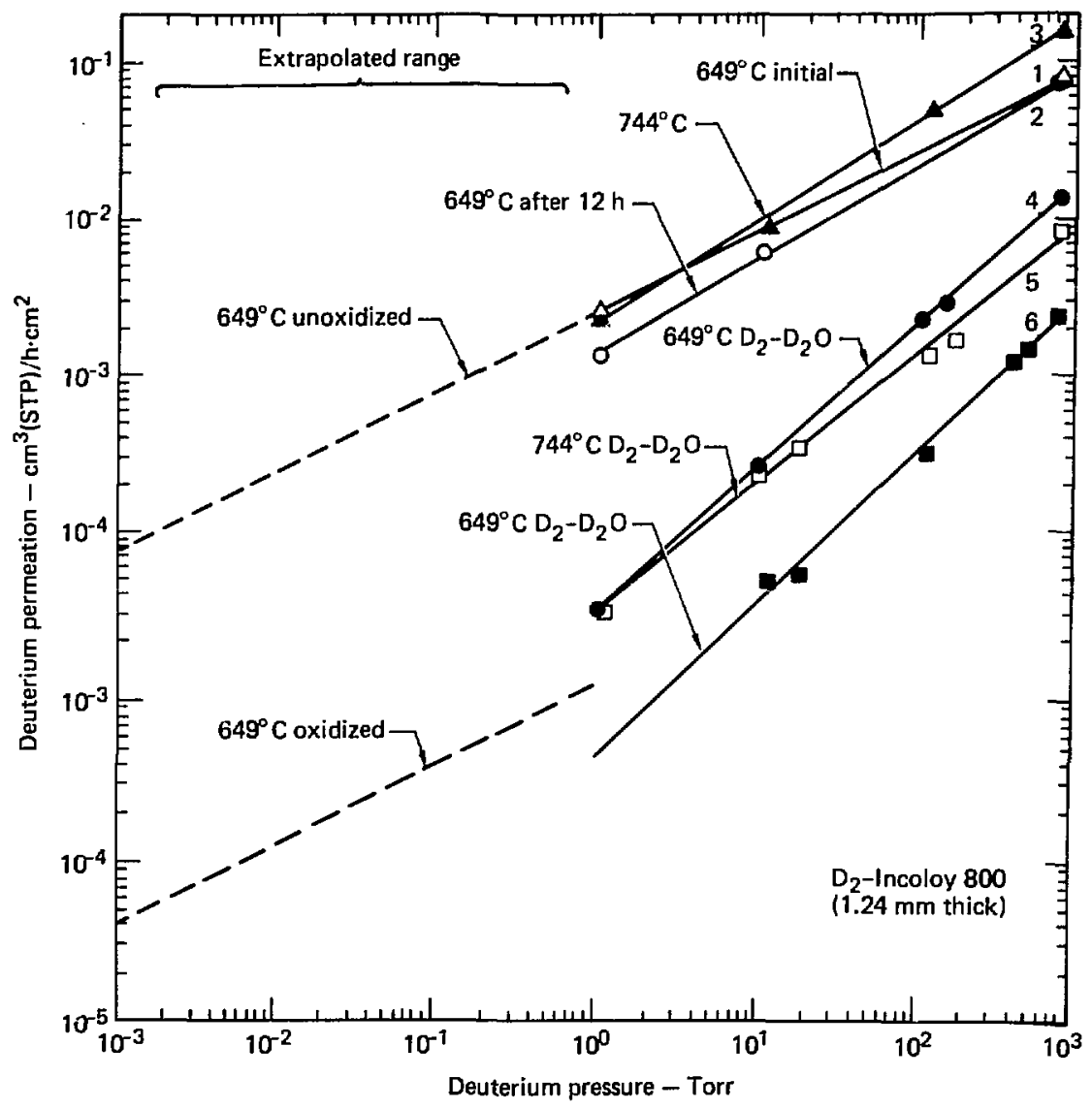

Fig. 6-65. Permeation rate vs temperature. 


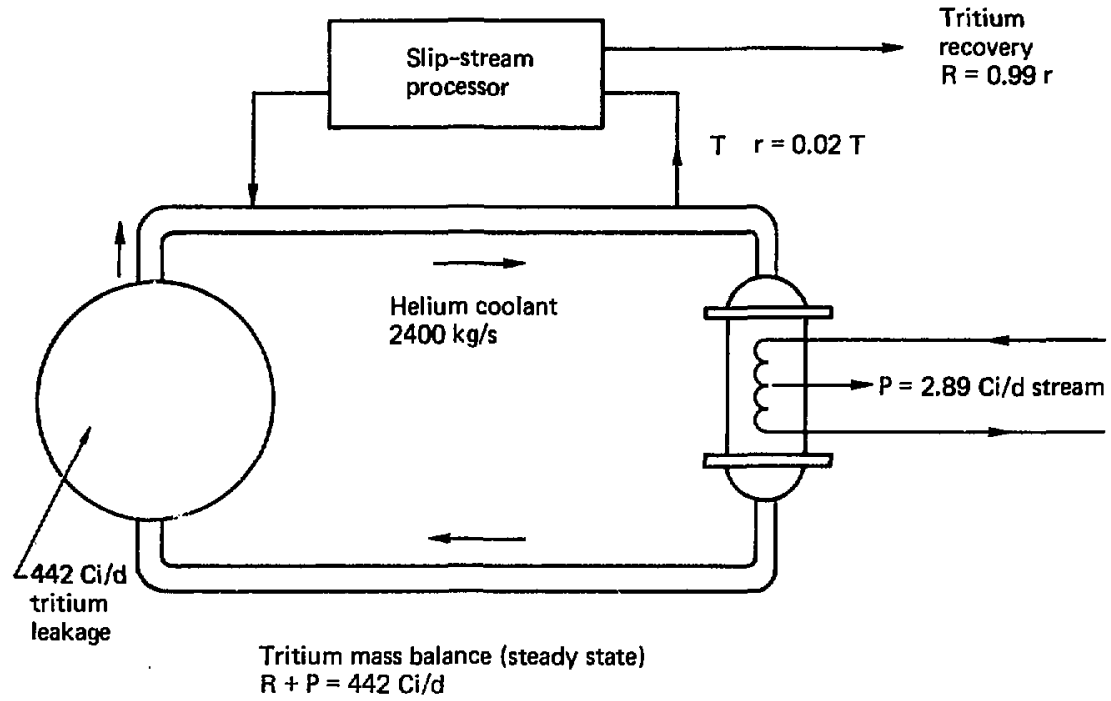

Fig. 6-66. Slip stream processing in a recirculating system.

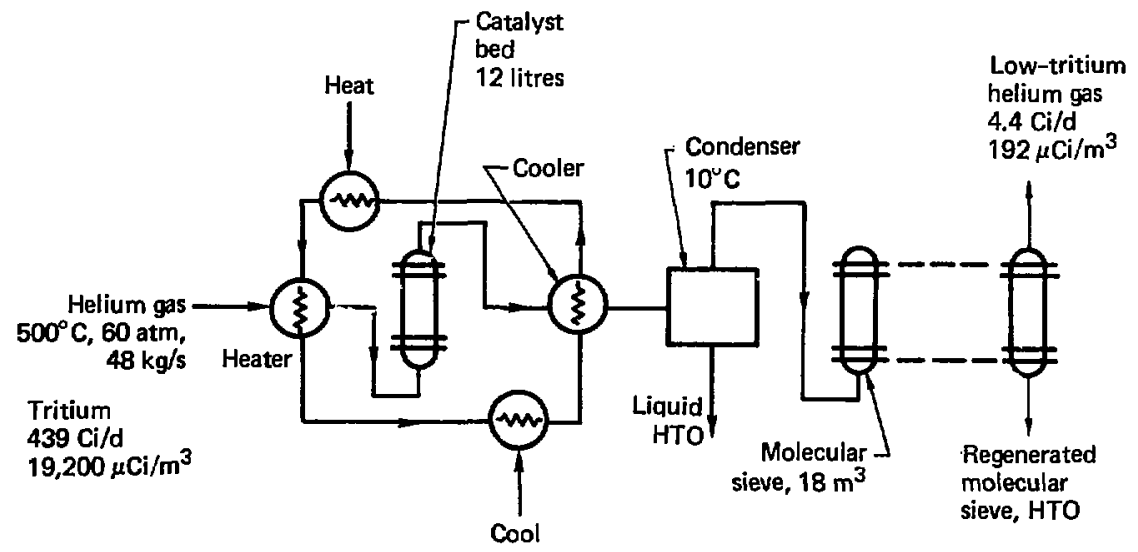

Fig. 6-67. Helium coolant slip stream processci. 
An evacuated $\left(10^{-6}\right.$ Torr) reactor hall (i.e., containing no air) could be scavenged of tritium more easily ard at far less cost, because the mole fraction tritium concentration would be raised from the maximum credible accident level of $600 \mathrm{ppm}\left(1500 \mathrm{(i} / \mathrm{m}^{3}\right)$ in the air-containing reactor hall to essentially unity. Similarly for a $750 \mathrm{ppt}\left(2,000 \mathrm{uCi} / \mathrm{m}^{3}\right)$ level raised to 36 moles, or $0.37 \mathrm{ppt}\left(1 \mu \mathrm{Ci} / \mathrm{m}^{3}\right)$ raised to 0.03 moles. Thus, the tritium processor requirements and costs for maintaining low-level reactor hall tritium would indeed be trivial. There remains the cost tradeoff between constructing and maintaining the reactor hall structures at $10^{-6}$ Torr and the cost of the reactor hall processor and operation and maintenance of the the large, tritium contaminated vacuum pumps. Evidently there must be other additional reasons for justifying or evacuating the reactor hall since the tritium processor savings is likely to be small in comparison.

As an alternate to the catalytic-oxidation, molecular-sieve adsorption technique moving bed getter ${ }^{43}$ of zirconium pellets can be used to selec-ively remove tritium from the helium coolant flow slip stream taken off the main flow. Such getter systems ${ }^{69}$ typically have capacities of $60 \mathrm{cc} \mathrm{T}_{2} / \mathrm{gm}$ of getter at STP, thus this moving getter bed would remove at 808 per pass (loading at $3.8 \mathrm{wppm}$ ) the tritium that would leak into the helium coolant flow. The getter material would move at $176 \mathrm{~g} / \mathrm{d}$, tying up less than $1 \mathrm{~g}$ of tritium as inventory in the 7-litre bed. In railroad-car lot quantities, zirconium (with up to 10 hafnium as impurity) as getter would cost less than $\$ 1000$.

Breeding Pin Reprocessing Facility. The process concept for the onsite breeding pin processing facility is based on a shredder and a vacuum furnace. The tritium pins are handled remotely in the reactor hall and then passed into the tritium hot cell where they are crushed and shredded into snall pieces. This operation fractures the thin iron coating within the pins. Upon melting of the aluminum alloy at $700-750^{\circ} \mathrm{C}$, the iron fraction is extreme?y small in comparison to the beryllium in Lockalloy or the alumina in the SAP. Its presence does not effect the mechanical properties of the alloy.

Once the iron coating is broken, the molten aluminum reacts with the LiD to form Iißl which has a tritium vapor pressure many orders of magnitude larger than tid. ${ }^{64}$ The tritium is nearly quantitatively driven from the melt at high rates because of the greatly enhanced vapor pressure and diffusivity. ${ }^{62}$ The deuterium gas is purified in the fuel purification system and fed back into newly fabricated breeding pins. 
The LiAl melt is augmented with fresh aluminum and new aluminum pins are extruded, cut to length, filled with powdered LiD, and fitted with end caps (preferably by special welding techniques). It is expected that the tritium contamination and low-level radiosotopes and activation products will allow single storage and handling methods.

An inexpensive re-equilibrium process step may permit LiAl to decompose into the elements so that an acceptabie fraction of the Li can be recovered, rehydrided, and used again for the LiD powder that fills the breeding pins. Such a process might involve electrochemical selective deposition in a cell designed similarly to that cumercially used to produce aluminum from the molten salt system. Carbon extraction also appears feasible. A selective vacuum distillation of the more volatile lithium from the melt may be possible. Also carbon may be used in a selective adsorption process.

\subsubsection{Newtral Beam Injectors}

6.6.5.1. Tritium Injection. The tritium neutral beam injectors accelerate $395 \mathrm{~g}$ of tritium per hour. Triton implantation on 0.3-mm-thick filament surfaces will result in about 1 leakage into the coolant flowing within the filaments or 70,560 Ci/d. The helium coolant flows from the neutral beam injectors into two steam generator units where the waste heat is recovered. The helium coolant flow at $300 \mathrm{~kg} / \mathrm{s}$ will be processed in a 108 slip stream mode where the main coolant flow will be carrying $798 \mu \mathrm{Ci} / \mathrm{l}$.

6.6.5.2. Tritium Contamination and Backstreaming in the Deuterium Injector. The deuterium neutral beam injector will become tritium contaminated both by deuterium that contains 18 tritium and by exposure to the plasma. Both mechanisms result in the grids being bombarded by the energetic tritons that will implant into the first layer of grid filaments. The surface area of these grids is approximately the same as the first wall, and the triton implantation will therefore produce a tritium permeation leakage into the helium coolant of about $802 \mathrm{ci} / \mathrm{d}$. Compared to the tritium neutral-beam-injector permeation leakages of $70,560 \mathrm{Ci} / \dot{\alpha}$, this is indeed a regligitle problem and will not require a deuterium neutral-beam helium coolant processor syster. The direct leakage into the stream is expected to be only $0.31 \mathrm{ci} / \mathrm{d}$.

6.6.5.3. Heliun Scavenging System. The tritiun neutral-beam heliurcoolant flow processor, therefore, will need to be of very high performance and sized to handle the $70,560-\mathrm{Ci} / \mathrm{d}$ load as in Fig. 6-68, with a 10 slip strean to reduce the tritiun leakage to acceptable levels. If we ain for proceas efficiency around 99.997 at 10 flow, the catalyst bed will be sized at 


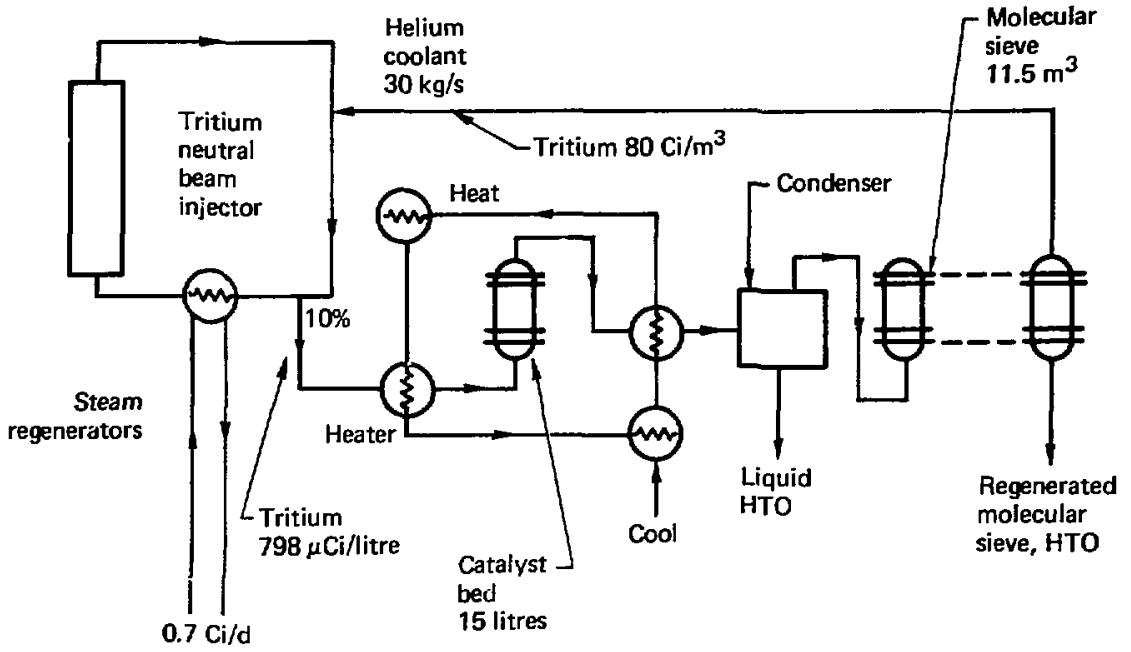

Fig. 6-68. Tritium neutral beam injector processor. 
15 litres. The concentration of tritium flowing into the two steam generators will be $798 \mu \mathrm{Ci} / \mathrm{l}$. If we use Incoloy-800 gteam generators as before with oxide barriers, we would predict a tritiun loss to the steam around $2.94 \mathrm{Ci} / \mathrm{d}$. The mole sieve beds again will be sized for a breakthrough time of $d$, thus, requiring a volume of $11.5 \mathrm{~m}^{3}$. As before we will install three beds for a sieve cost of $\$ 18,000$ and capital cost of $\$ 10,000$. The beds will hold a maximum inventory of $282,000 \mathrm{Ci}$.

The total aystem cost of catalyst, catalytic reactor, mole sieve and beds, instruments and controls is estimatsa at $\$ 50,000$.

\subsubsection{Director Converter}

6.6.6.1. Tritium Implantation. Trittum implantation in the direct converter is somewhat less of a problem thsn it is in the tritium neutral beam injector, since the direct converter fjlament wall thicknesses are around $1 \mathrm{~mm}$ and the triton implantation energy is $55 \mathrm{keV}$. The permeation leakage is estimated at $16,000 \mathrm{Ci} / \mathrm{d}$. With 5 slip stream processor, the tritium contamination level in the helium coolant can be held to $728 \mu \mathrm{Ci} / \ell$. This would result in a loss to the steam of $2.89 \mathrm{Ci} / \mathrm{d}$.

6.6.6.2. Eelium Scavenging System. The processor design in Fig. 6-69 that will handle this tritium permeation leakage is sized to process 58 of the helius coolant flow of $15 \mathrm{~kg} / \mathrm{s}$. The inlet tritium concentration therefore would be $728 \mu \mathrm{Ci} / \mathrm{l}$. A 99.98 processor would cut this to $728 \mu \mathrm{Ci} / \mathrm{m}^{3}$ and would result in a permeation loss of $2.89 \mathrm{Ci} / \mathrm{d}$ through the two stream generators.

The catalytic oxidizer reactor would be sized at $6 l$ and the 4-d holdup molecular sieves at $6 \mathrm{~m}^{3}$. The sieves would hold a maximum of $65,000 \mathrm{Ci}$ before regeneration. The total processor cost is estimated at $\$ 50,000$.

\subsubsection{Fuel Purification System}

6.6.7.1. Cryogenic Distillation Plant. This portion of the reference design study was subcontracted to Dr. Williant R. Wilkes at Mound Iaboratory for rough equipment design, sizing, and cost analysis. He has made a number of valuable suggestions to reduce equipment cost and tritiun inventory, one of which was to utilize LiD instead of LiH for the breeding pin solid contents, thus saving a catalytic equilibrator and another oryogenic distillation colun. The text which follows is for the most part reproduced directly from his repori to $u s$.

Introduction. There are four streams of gas that leave the reactor and that require processing. Stream A is approxisately 918 deuteriun with the 


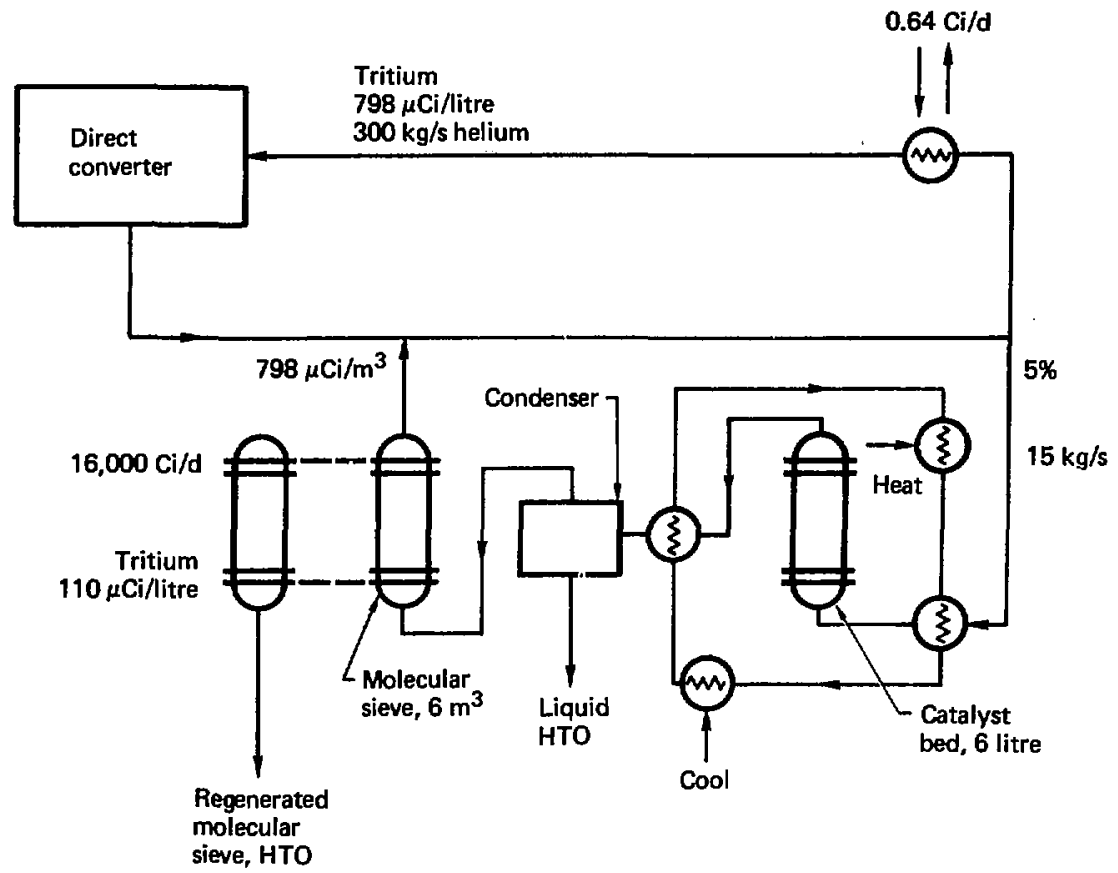

Fig. 6-69. Direct convertor tritium processor. 
remainder tritiun and a small inclusion of helium. This stream comes from the direct conversion unit collectors. A second stream, B, containing about 988 deuterium and 27 tritium, comes from the deuterium neutral beam injectors. Strean $C$, from the tritjum neutral beam injectors, is about 98 tritium and 28 deuterium. Finally, the reactor has a tritium breeding blanket from which a stream of tritiun gas comes, carried in a much larger stream of deuterium. The tritiun concentration in this stream, D, is about $1.5 \%$.

These four streams must be processed to provide a flow of fuel to the injectors of at least 998 deuterium. In addition, a supply of cold deuterium gas of 998 purity is required. The approximate concentrations and flow in the reactor as they were originally specified are shown graphically in Fig. 6-70.

The necessary fuel processing (other than removing the helium and other nonhydrogenic impurities) is provided in Fig. 6-70 by a system of five interconnected distillation columns, operating at temperatures near $26 \mathrm{k}$. Two coluwns provide a primary separation into 958 purity streams, e.g., $958 \mathrm{D}_{2}$, 58 Dr. In addition, the two columns combined with an external catalytic equilibrator removed most of the DT component. Two more columns refine the deuterium and tritium-rich streams to the required purity, while a fifth column removes a small protium component from the deuterium stream.

Determining Flow Rates and Compositions. The flow rates and compositions are determined in the following way:

Each neutral beam injector requires the amount of gas (deuterium or tritium) specified in Table 6-30 at 998 purity. of this gas, only 13.088 is injected $(13.08$ is the value obtained by dividing injected quancities by feed to the injectors). The 678 of the gas not injected becomes isotopically contaminated and must be reprocessed.

We assume the injected gas has the same isotopic composition as the feed. We also assume that there will be back flow into each injector from the plasma at the plasma concentration at a rate equal to $5 \mathrm{~g}$ of the injected quantity. This gas flow thus tends to contaminate the uninjected side stream from the injectors. We further assume a forward leakage of cold gas from the injector to the plasma at the same $5 \%$ rate, but at the isotopic composition (e.g., 998 deuterium, 17 tritium) of the injected gas.

Furthermore, the deuteriun ani tritium injectors are located close enough to each other their contents intermix. We assume the mixing occurs at a rate of 18 of the average injected flow, and that 99 deuterium enters the tritium injector and vice versa. 


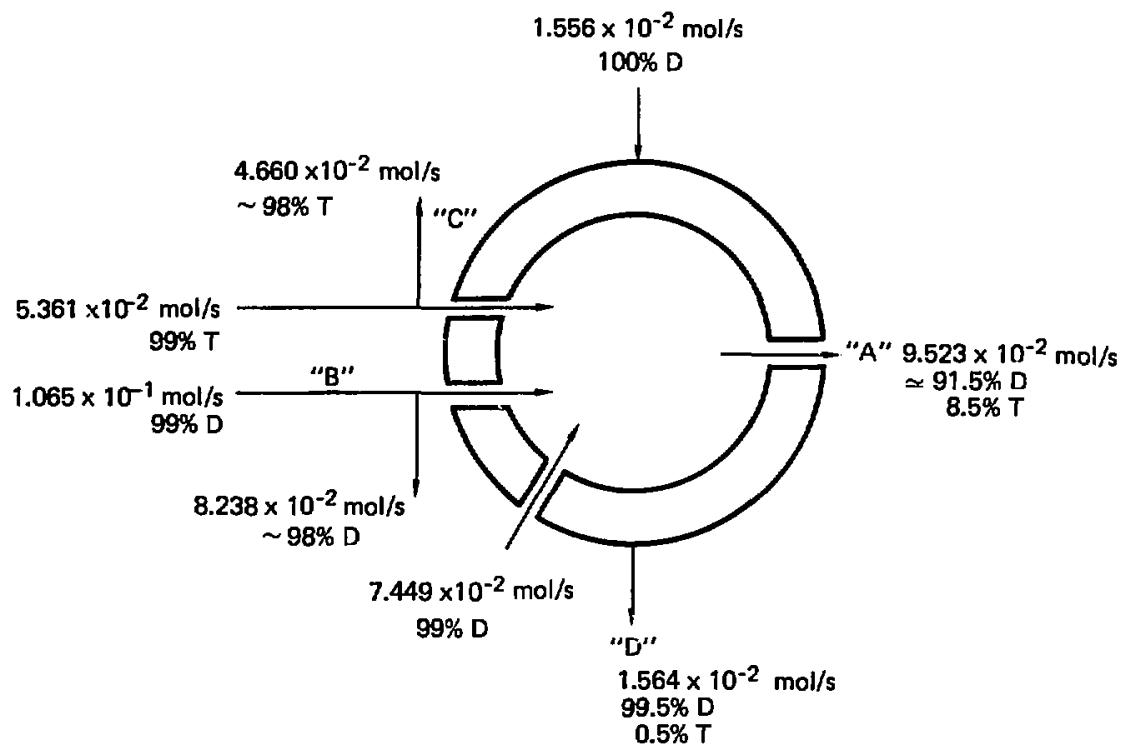

Fig. 6-70. Approximate initial specifications of flows and compositions. 
Table 6-30. Fusion reactor supply requirements.

\begin{tabular}{|c|c|c|c|}
\hline & $\begin{array}{c}\text { Deuterium, } \\
\end{array}$ & $\begin{array}{c}\text { Tritium, } \\
\text { 8 }\end{array}$ & $\begin{array}{l}\text { Flow, } \\
\text { mol/s }\end{array}$ \\
\hline Deuteriun injector & 99 & 1 & $1.063 \times 10^{-1}$ \\
\hline Tritium injector & 1 & 99 & $5.361 \times 10^{-2}$ \\
\hline Cold deuterium gas feed & 99 & 1 & $7.449 \times 10^{-2}$ \\
\hline Tritiun breeding pins & 99 & 1 & $1.556 \times 10^{-2}$ \\
\hline
\end{tabular}

The isotopic composition in the plasma, in turn, is determined by the compositions of the material that enters from the injectors and from the cold gas stream; and is modified slightly by the small (0.0858 of the fuel flow) amount of material that is burned ip in the reactor. The gas leaving the direct converters has the same composition as the plasma. The flow rate is the injection rate minus the burnup.

The blanket fuel breeding pins were specified to generate a flow of $56 \mathrm{~mol} / \mathrm{h}$ of hydrogen and $0.293 \mathrm{~mol} / \mathrm{h}$ of tritium. This has been changed for two reasons. First, there is no need to deliberately introduce protium into the system; it is a useless impurity. Instead, by using deuterium to carry the tritium out of the breeding material, this impurity is eliminated. Secondly, the stated flow rates correspond to more than 998 deuterium concentration. This is already more pure than required for the deuterium fuel. This deuterium-tritium mixture would have to be stripped of virtually all the tritium, say to 99.9958 deuterium purity to recover the necessary flow of tritium.

Instead, we have assumed that deuterium that contains 18 tritium is put into the breeding pins, and that the material removed after breeding is 1.58 tritium. Thus only 338 recovery is required, and the deuterium supply to the breeding pins can come from the injector supply.

Finally, a small amount of protiun will be generated by deuteriumdeuterium reactions in the plasma, and some protium will certainly diffuse or leak into the fuel system. This protium influx is arbitrarily assumed to occur at a rate equal to the tritium burnup rate, $\sim 0.3 \mathrm{~mol} / \mathrm{h}$. The protium will be allowed to accumulate to a concentration at which a 508 recovery rate in the fuel processor will result in a steady state concentration of protium. Thus, the protium fraction will rise to $2 \times 0.3 \mathrm{~mol} / \mathrm{h} \times(843.7 \mathrm{~mol} / \mathrm{h})^{-1}$ or $7 \times 10^{-4}$. 
It is assumed that all gases entering the fuel processor have room temperature equilibrium values of the concentrations of the six molecular species of hydrogen: $\mathrm{H}_{2}, \mathrm{HD}, \mathrm{HT}, \mathrm{D}_{2}, \mathrm{DT}, \mathrm{T}_{2}$. Thus, the four s-reams $\mathrm{A}-\mathrm{D}$ have the concentration and flow rates shown in Table 6-31.

Determining the Column specifications - The four gas streams are fed to the five distillation columns of the fuel processor as shown in Fig. 6-71. The five columns are labeled $\alpha$ through $\varepsilon$, and the box labeled EQ is a catalytic equilibrator inserted for the purpose of converting DT into deuterium (as $D_{2}$ ) and tritium (as $T_{2}$ ).

The problem is to determine the six concentrations and one flow rate in each of 15 process systems. Ignoring $B, C$, and $D$, and $\alpha, \beta$, and $\varepsilon$ for now, the two-column cascade $\gamma$ and $\delta$ can be solved. End concentrations, i.e., the overhead project, $J$, from $\lambda$ and the bottoms, $M$, from $\delta$, are assumed to contain the same $D / T$ ratio as streams $B$ and $C$, respectively. There is so little protium in the system that $\mathrm{H}_{2}, \mathrm{HD}$, and HT constitute minor perturbations.

Assuming such a solution is possible, the feed rates to $\alpha$ and $B$ are altered by the addition of $J$ and $N$, but the compositions are still those of $B$ and $c$.

The requirement of 998 purity in the final product streams $\mathrm{E}$ and $\mathrm{H}$ coupled with the known input concentrations permits the calculation of the flow rates from both top and bottom of both $\alpha$ and $\beta$. In addition, approximate values of concentrations in $F$ and $G$ may now be calculated. Because $F$ and $G$ together only add about $6 \%$ to the flow from $A$, the values of concentrations in $I$ can be estimated closely. Flows in $J$ through $P$ are determined from those in $I$.

Starting from stream $I$, and the concentrations of certain key components in $J, K$, and $N$, distillation column $\gamma$ is simulated by the computer code. The simulation is repeated, while various column parameters such as the number of stages of reflux ratio are varied in an empirically determined way to optimize the solution. Optimizing here means achieving the desired degree of separation with a minimum of refrigeration power and tritium inventory. capital costs of the system are relatively independent of column size; thus, the tradeoff between minimum reflux ratio and minimum number of stages generally is biased toward the minimum reflux ratio, hence minimum refrigeration roquirement. 
Table 6-31. Isotopic compositon of hydrogen streams before processing.

\begin{tabular}{cccccccc}
\hline Stream & $\mathrm{H}_{2}$ & HD & HT & $D_{2}$ & DT & $T_{2}$ & Flow, mmol/s \\
\hline A & $5 \times 10^{-7}$ & $1.302 \times 10^{-3}$ & $1.19 \times 10^{-4}$ & 0.8786 & 0.15367 & $7.046 \times 10^{3}$ & $9.523 \times 10^{-2}$ \\
B & $5 \times 10^{-7}$ & $1.394 \times 10^{-3}$ & $2.7 \times 10^{-5}$ & 0.96098 & 0.03727 & $3.61 \times 10^{-4}$ & $9.238 \times 10^{-2}$ \\
C & $5 \times 10^{-7}$ & $4.8 \times 10^{-5}$ & $1.373 \times 10^{-3}$ & $1.131 \times 10^{-3}$ & 0.06494 & 0.93250 & $4.660 \times 10^{-2}$ \\
D & $1.2 \times 10^{-7}$ & $6.39 \times 10^{-4}$ & $1.0 \times 10^{-5}$ & 0.97023 & 0.02891 & $2.18 \times 10^{-4}$ & $1.5637 \times 10^{-2}$ \\
\hline
\end{tabular}




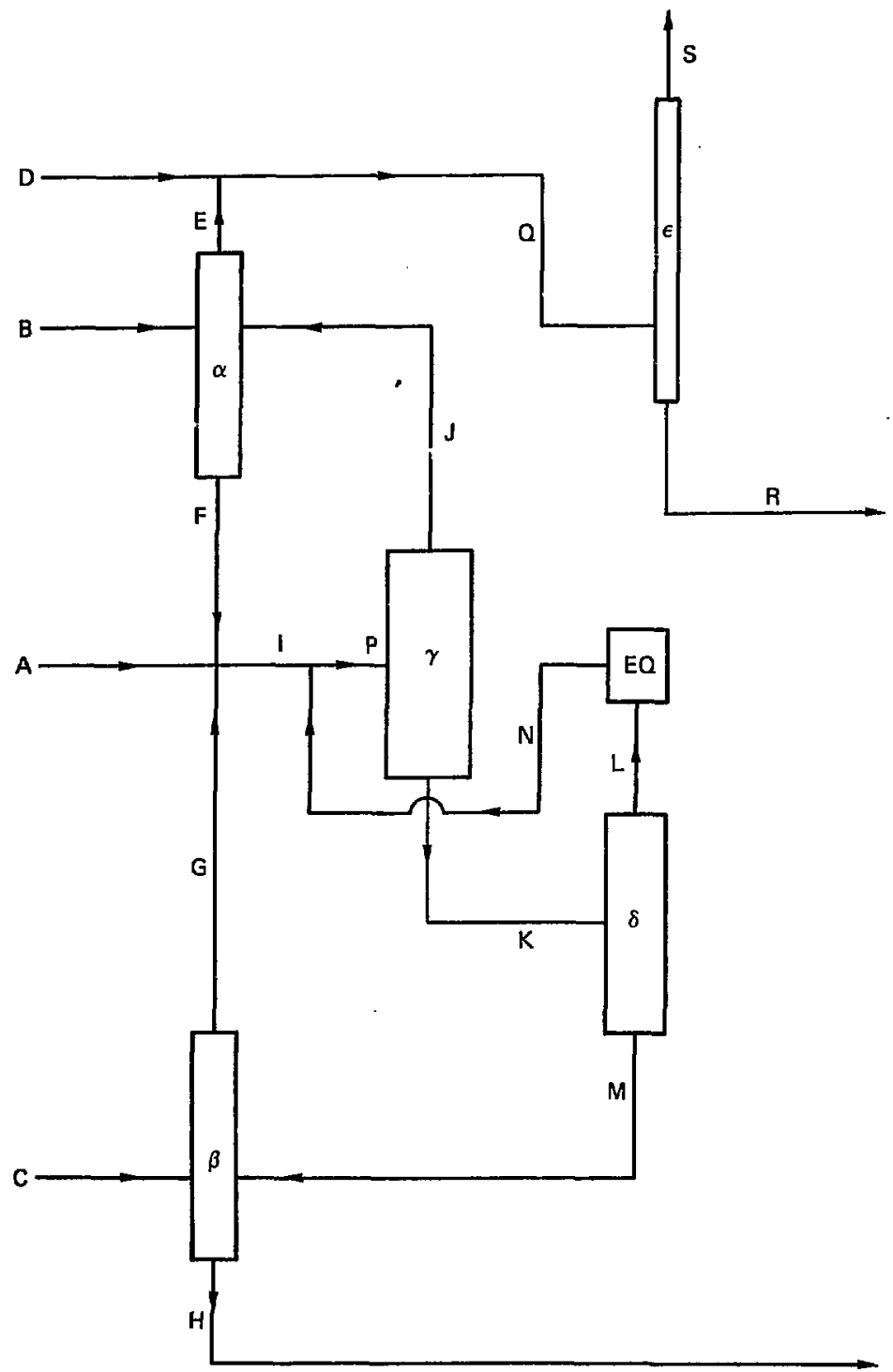

Fig. 6-71. Schematic of the distillation columns. 
The computer-determined concentrations in $\mathrm{K}$ are then used as feed and the p) edure is repeaced. After $Y$ and $\delta$ have been separately optimized, a two-column simulation is done in which the "product" $L$ is equilibrated and returned to stream as shown in Fig. 6-70. The values of concentrations in stream $P$ deciermined in this way should equal those which are used as feed to $Y$.

The computer-determined concentrations in $J$ and $M$ are now added to $B$ and $C$ respectively, and colunns $\alpha$ and $B$ are $s$ imulated in the same manner as before. Product $\mathrm{A}$ may be used airectly, but product E contains virtually all the protium that enters the fuel processor. This protium must be removed in colum $\varepsilon$.

Colunn $\varepsilon$ has an extremely small overhead product, only 0.078 of the feed. This causes about half the protium (which is nearly entirely in the form of $\mathrm{HD}$ ) to be removed in the overhead. The remainder of the material which is 99 deuteriun, is fer partially into the breeding blanket (about 78) and partially into the plasme fueling systems. A small amount of pure $D_{2}$ from an external source is added to the fuel stream at this point to replenish that small amount of deuterium either lost by burnup or discarded.

A very small quantity of tritium is discarded in the waste stream. This loss amounts to less than $1 \mathrm{Cl} / \mathrm{d}$. The quantity of waste gas in which the tritium is contained, only about 200 sccm, can easily be processed by a small air or glovebox detritiation system, so no estimate has been made of the necessary processing equipment required to handle this material.

Results - There are two principal results of the simulation. First, a flow rate and set of concentrations can be determined for every point in the syster. Secondly, the physical requirements, e.g., refrigeration power and colum sizes are estimated, and from these, costs may be estimated.

The flow rates and isotopic compositions externa:. to the fuel processor are shown in Fig. 6-72. If one makes a detailed mass balance for a given isotope around one component in the fuel cycle, the sums of the flows do not total exactly zero. The errors, which are of the order of 0.18 , are a result of lack of computational precision and are not signiflcant. The flow rates and isotopic composition within the fuel processor are given in Table 6-32. The letters A through S refer to flow streans in Fig. 6-72.

The physical characteristics of the isotope separation system are listed in Table 6-33. Here certain further assumptions have been made. First, the height of a single stage is taken to be $5.0 \mathrm{~cm}$, and the maximum allowable 


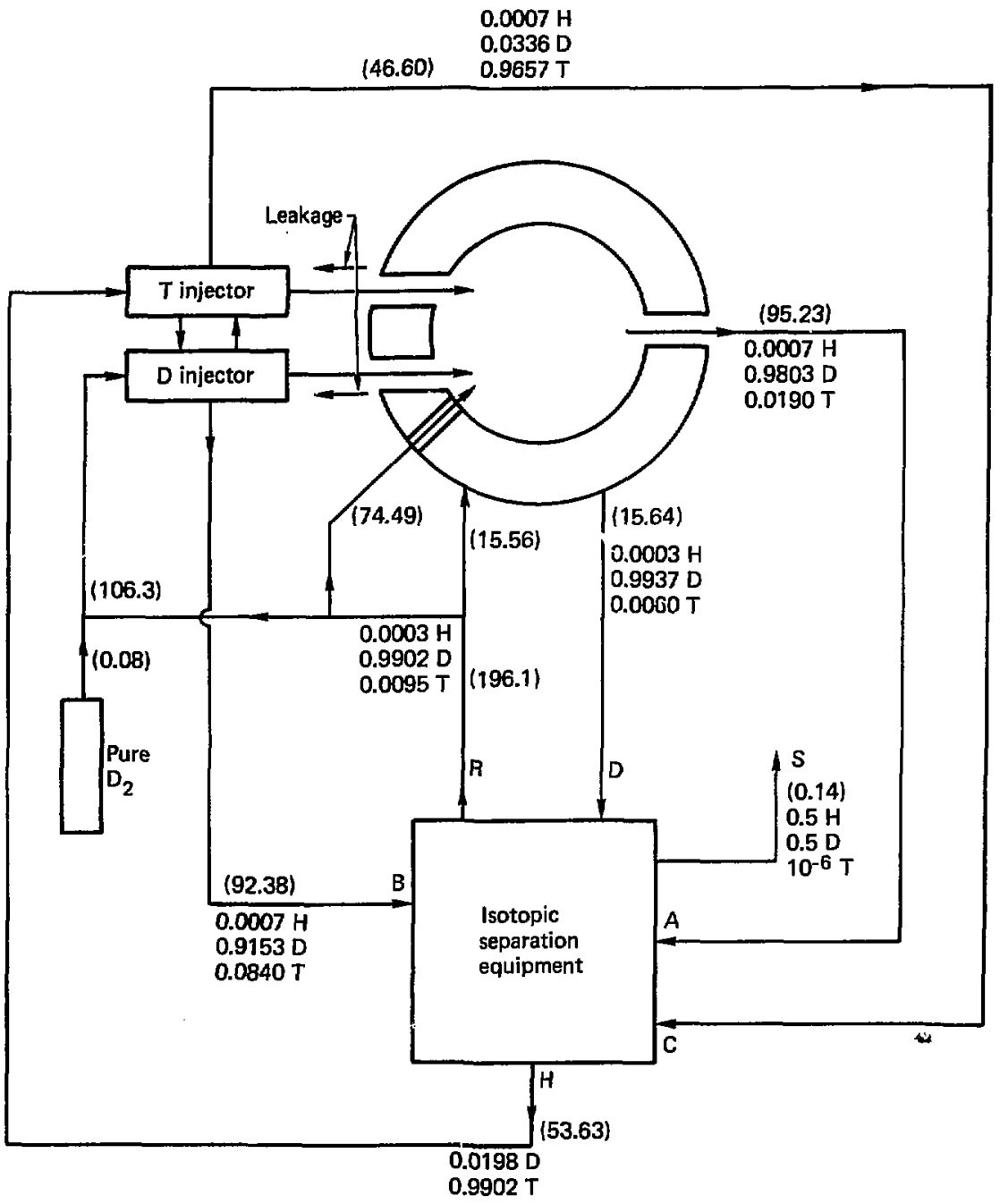

Fig. 6-72. Fuel flow rates in the fusion reactor. Flows (in parenthesis) are in $01 / 8$ and concentrations are atomic fractions. 
Table 6-32. Concentrations and flow rates in the fuel processor.

\begin{tabular}{|c|c|c|c|c|c|c|c|}
\hline Sticeam & $\mathrm{H}_{2}$ & ED & HT & $D_{2}$ & $\mathbf{D T}$ & $\mathbf{T}_{2}$ & $\begin{array}{l}\text { Flow, } \\
\text { monol/s }\end{array}$ \\
\hline A & $5 \times 10^{-7}$ & 0.00130 & 0.00011 & 0.83786 & 0.15267 & 0.00705 & 95.23 \\
\hline B & $5 \times 10^{-7}$ & 0.00139 & $2.7 \times 10^{-5}$ & 0.96098 & 0.03727 & 0.00036 & 92.38 \\
\hline c & $5 \times 10^{-7}$ & $4.8 \times 10^{-5}$ & 0.00137 & 0.00113 & 0.06494 & 0.93250 & 46.60 \\
\hline D & $10^{-7}$ & 0.00054 & $6 \times 10^{-6}$ & 0.97020 & 0.02891 & 0.00022 & 15.64 \\
\hline E & $5.2 \times 10^{-7}$ & 0.00144 & $2.8 \times 10^{-5}$ & 0.98056 & 0.01792 & $4.1 \times 10^{-5}$ & 180.01 \\
\hline $\mathbf{F}$ & $2 \times 10^{-8}$ & $10^{-10}$ & $10^{-9}$ & 0.38728 & 0.60299 & 0.00974 & 6.151 \\
\hline G & $6.2 \times 10^{-6}$ & 0.00060 & 0.01715 & 0.01388 & 0.58644 & 0.38185 & 4.664 \\
\hline H & $10^{-14}$ & $10^{-9}$ & $7 \times 10^{-7}$ & $2.2 \times 10^{-5}$ & 0.01959 & 0.98039 & 53.63 \\
\hline I & $7 \times 10^{-7}$ & 0.00120 & 0.00086 & 0.77584 & 0.19877 & 0.02389 & 106.04 \\
\hline $\mathbf{J}$ & $8 \times 10^{-7}$ & 0.00134 & 0.00080 & 0.96075 & 0.03707 & $2.5 \times 10^{-5}$ & 93.82 \\
\hline K & $10^{-19}$ & $10^{-11}$ & $10^{-8}$ & 0.03664 & 0.71836 & 0.24503 & 52.47 \\
\hline $\mathbf{L}$ & $10^{-20}$ & $10^{11}$ & $10^{-8}$ & 0.04686 & 0.90572 & 0.04746 & 41.02 \\
\hline M & $10^{-18}$ & $10^{-18}$ & $10^{-15}$ & $4.4 \times 10^{-6}$ & 0.04703 & 0.95296 & 11.45 \\
\hline $\mathbf{N}$ & $10^{-16}$ & $10^{-8}$ & $10^{-8}$ & 0.24970 & 0.50000 & 0.25030 & 41.02 \\
\hline $\mathbf{P}$ & $5 \times 10^{-7}$ & 0.00864 & 0.00511 & 0.62918 & 0.28144 & 0.08797 & 146.29 \\
\hline 2 & $5 \times 10^{-7}$ & 0.001327 & $3 \times 10^{-5}$ & 0.97970 & 0.01889 & $6 \times 10^{-5}$ & 196.20 \\
\hline $\mathbf{R}$ & $10^{-10}$ & 0.00063 & $5 \times 10^{-5}$ & 0.98039 & 0.01890 & $6 \times 10^{-5}$ & 196.06 \\
\hline $\mathbf{s}$ & 0.00066 & 0.99934 & $2 \times 10^{-6}$ & $5 \times 10^{-6}$ & $10^{-11}$ & $10^{-17}$ & 0.14 \\
\hline
\end{tabular}


Table 6-33. Fuel processor characteristics.

\begin{tabular}{|c|c|c|c|c|c|}
\hline & $\alpha$ & $\beta$ & $Y$ & $\delta$ & $\varepsilon$ \\
\hline Refrigeration power, $W$ & 600 & 465 & 1028 & 540 & 730 \\
\hline $\begin{array}{l}\text { Refrigeration power/ } \\
\text { quadrant }\end{array}$ & 200 & 155 & 343 & 180 & 243 \\
\hline Diameter, cm & 5.3 & 4.0 & 6.4 & 4.9 & 5.4 \\
\hline Number of stages & 55 & 50 & 75 & 50 & 60 \\
\hline Reflux ratio & 2.75 & 73 & 9.0 & 10.0 & 5000 \\
\hline Length, cm & 275 & 250 & 375 & 250 & 300 \\
\hline Volume, litres & 25.5 & 12.5 & 48 & 18.8 & 27.5 \\
\hline Tritium fraction & 0.067 & 0.93 & 0.25 & 0.73 & 0.002 \\
\hline $\mathrm{T}$ inventory, $\mathrm{g}$ & 63 & 425 & 444 & 500 & 2.7 \\
\hline Operating pressure, Torr & 1500 & 1500 & 2500 & 1200 & 1500 \\
\hline Condenser temperature, $\mathbf{K}$ & 26.3 & 7.2 & 26.3 & 26.1 & 24.7 \\
\hline $\begin{array}{l}T_{2} \text { heat input per } \\
\text { quadrant, } W\end{array}$ & 5 & 34 & 36 & 40 & $0.2^{\circ}$ \\
\hline
\end{tabular}

vapor velocity in the column is $10 \mathrm{~cm} / \mathrm{s}$. These choices are significant for two reasons. First, a large stage height or low vapor veiocity leads to more column in each column, hence to more inventory. In addition, however, in columns with large tritium concentrations, the heat added to the column by tritium decay can be significant. The additional heat, added along the entire length of the column, causes the vapor flow rate at the top of tha colum to be greater than that at the bottom. If enough tritium heat is added, either the column must be expanded to accommodate the increased flow near the top, or the flow at the bottom must be reduced.

There is some correlation between columa diameter and stage height, with large diameters leading to greater stage heights. My measurements indicate easily attainable stage heights of $<2 \mathrm{~cm}$ for a $1-\mathrm{cm}-\mathrm{diam}$ column and vapor velocity ug to $20 \mathrm{~cm} / \mathrm{s}$; hence, the figures of $5 \mathrm{~cm}$ and $10 \mathrm{~cm} / \mathrm{s}$ are probably conservative.

Another assumption is that the fuel processor is divided into four units of equal capacity. This provides redundancy, as each unit can be slightly oversized to permit operation at full flow with only three units functioning. secondly, it provides greater safety, as the failure or destruntion of a single unit will involve the potential release of only one fourth of the trit.: ium inventory. Finally, there is likely to be more reduction of tritium inventory, as the use of smaller-diameter columns leacis to shorter stage heights. 
The refrigeration power required per gradient, column dianeter, and volune are calculated assuming that each unit is sized to provide one third of the entize system capacity. The tritium inventory is that which would prevaij with all four columns operating simultaneously. No benefit has been taken for reduced stage height in a smaller column.

Equipment costs - The capital equipment costs (in current dollars) are given in Tabie 6-34. Because the distillation apparatus is not expected to contribute more than a few percent to the total cost of the Mirror Hybrid Reactor, detailed cost estimates of this apparatus were not made. These cost figures assume the purchase of four separate, identical processing systems with some cost savings resulting from duplication. Furthermore, these costs assume that refrigeration for this equipment is independent of that used for superconductive magnets and other cryogenic equipment. This is probably a conservative estimate. The emergency storage containers are simply four or more large evacuated tanks connected through rupture disks to each distillation system.

Discussion - This present design raises the blanket inventory from $24.2 \mathrm{~kg}$ to $86.2 \mathrm{~kg}$. This can be reduced to $32.2 \mathrm{~kg}$ by reducing the tritium impurity level in the deuterium that is used in IJD. In the original analysis we have utilized $18 \mathrm{~T}$, 998 into fuel plus with 1.58, $98.58 \mathrm{D}$ out. Protium is removed in distillation column No. 5 which is used solely for "polishing" the deuterium.

We prefer option 1 in Fig. 6-73 where we can used 0.18 T, 99.98 D into fuel pins and $0.68 \mathrm{~T}, 99.48 \mathrm{D}$ out. This will reduce $\mathrm{T}_{2}$ inventory by 668 .

Table 6-34. Capital costs for hydrogen: ope separation for mirror hybrid fusion reactor.

2 distillation columns and controls

(4 identical 5-column systems)

1100

4 control systems

4 containment systens 800

4 energency storage systems 50

aefrigeration power is that required for 4 systems, each with capacity of 338 of full flow. 


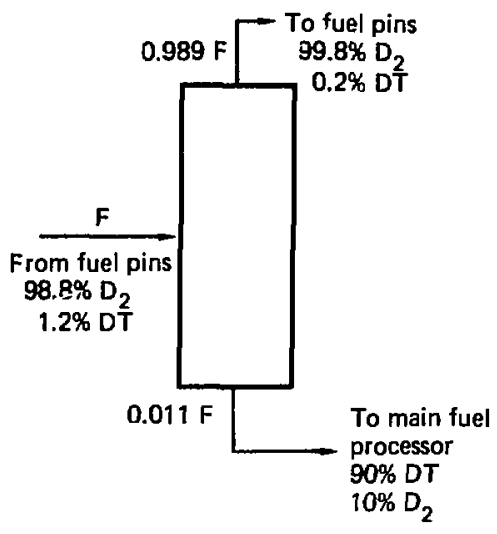

Fig. 6-73. Inventory reduction by decreased tritiun impurity in LiD. 
Addition is required of a 6 th distillation column. It will have large refrigeration requirements but low T inventory. Refrigeration requirements of columns 4 and 5 (the ones with highest $D_{2}$ content) may be reduced somewhat.

For contrast we mention option 2 in Fig. 6-74 where we use voll T, 99.94 $\mathrm{H}$ into fuel pins. Addition is required of a 6 th and 7 th column. This does not eliminate the need for the 5th "polishing" column.

6.6.7.2 Inventory Accidents. As shown in Table 6-35, there are two sources of tritium in the fuel purification and supply system that could be released under accident conditions. The cryogenic distillation columns, 1.44 $\mathrm{kg}$, and in the 6-mo full storage network, maximum $8.1 \mathrm{~kg}$. Three philosophies can be employed in order to reduced the 11 kelihood of releasing these inventories to the environment.

First, the storage tanks can be remotely distributed away from the reactor hall proper in an underground network, in order to reduce the release probability of the whole inventory. Eight tanks could be employed in all, in order to reduce the single accident spill to avoid $1 \mathrm{~kg}$ where fence line doses to the public would be below $25 \mathrm{rem}$.

The second approach is to carry out all breeding pin processing, fuel purification and fuel storage within the reactor hall containment so that any spill from the system could be handled by the large reactor hall processor. For a $8.1-\mathrm{kg}$ spill from the storage tank network, the processor is sized to clean up the accident in about one week.

The third approach, presented earlier in the report, involves helium purging the breeding pin. A lithium compound such as lithium aluminate can

Table 6-35. Tritium plant inventory.

\begin{tabular}{lcc}
\hline & Mass & Curies \\
\hline Tritium breeding blanket pins & $32.2 \mathrm{~g}$ & $322 \mathrm{MCi}$ \\
Cryogenic stills & $1.44 \mathrm{~g}$ & \\
Tritium storage tanks (6-mo production) & $8.1 \mathrm{~g}$ & $162 \mathrm{MCi}$ \\
Neutral beam direct converter cryopanel & $0.25 \mathrm{~g}$ & $2.5 \mathrm{MCi}$ \\
Direct converter cryopanel & $0.20 \mathrm{~g}$ & $2.0 \mathrm{MCi}$ \\
Neutral beam processor sieve (max) & $28.2 \mathrm{~g}$ & $282.000 \mathrm{Ci}$ \\
Direct converter processor sieve (max) & $6.5 \mathrm{~g}$ & $65,000 \mathrm{Ci}$ \\
Reactor hall processor sieve (max) & $0.18 \mathrm{~g}$ & $1800 \mathrm{Ci}$ \\
Helium coolant loops inventory & $6 \mathrm{mg}$ & $60 \mathrm{Ci}$ \\
\hline
\end{tabular}


$0.6 \%$ T, $99.4 \%$ H out:

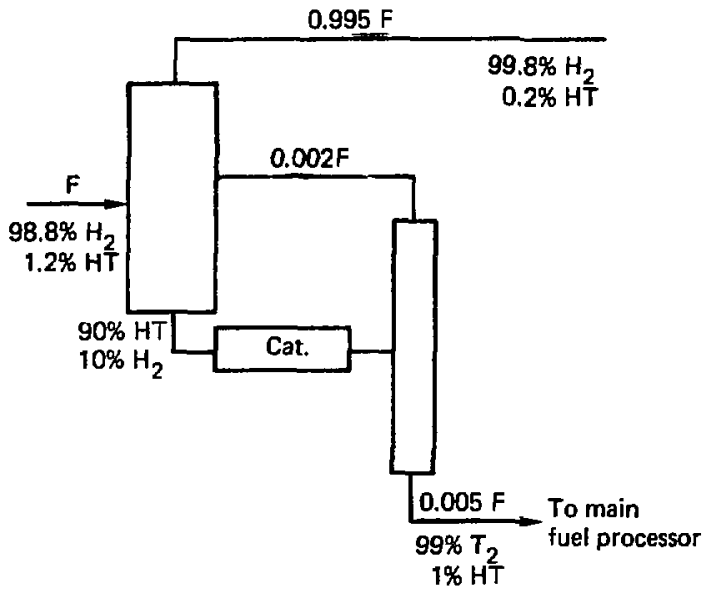

Fig. 6-74. Inventory reduction by decreased tritiun impurity in LiD. 
probably be used at $350^{\circ} \mathrm{C}$, but further feasibllity examinations are required before this approach can be used in a design.

\subsubsection{Tritium Plant Inventory and Component Costs}

The tritium plant inventory, 1 temized in Table 6-35, totals around $42 \mathrm{~kg}$ at the maximum, mainly resulting from the breeding pin concept and opezation. Although this tritium inventory is large, thi hazard from a reactor accident will be dominated by the uranium fuel pin actinides. The breeding pin approach very substantially simplifies the blasket piping system and avoids costly and complex secondary and tertiary piping and processed contairment piping shells. The storage tanks for tritiun must be sized to handle the annual "harvest" of tritium from the breeding pin hydride $(16.2 \mathrm{~kg})$. Although the average tank Inventory over the year wi.l. be $8.1 \mathrm{~kg}$, the maximum could be credibly released. There does not appear to be any possible reductions in tritium inventory.

A summary of the component costs is given in Table 6-36. The total tritium systems cost of $\$ 8.3$ million is only 18 of the total plant cost. of the tritium systems cost, only $\$ 3.6$ million is spent for safety or environmental protection, which is well below the $2 \%$ plant cost figure typical of present industrial plants.

\subsubsection{Technology Assessment}

As a result of this reference design study the following areas need further work:

\section{Low-level tritium detection instrumentation:}

(a) evaluate and recommend the best available instrumentation for lowlevel real-time tritium gas/water detection down to limiting levels of around $\pm 0.1 \mu \mathrm{Ci} / \mathrm{m}^{3}$;

Table 6-36. Tritiun plant component costs.

Reactor hall processor

Coolant processor

Neutral beam processor

Direct converter processor

Cyogenic distillation plant

Cryopumps

Total
3,600

100

50

50

4,000

500

8,300 
(b) assess reliability a.xd failure modes;

(c) explore methods of isolating other interfering, beta-emitting radioisotopes.

2. Protective suits for tritium contaminated environment:

(a) extend present suit technology tc $\sim 1 \mathrm{Cl}_{1} \mathrm{~m}^{3}$ tritium levels for long exposures and $1500 \mathrm{ci} / \mathrm{m}^{3}$ for short emergency escape periods;

(b) develop suit safety performance monitors;

(c) develop emergency egress attachments for suits;

(d) demonstrate suit.

3. Tritium removal by catalytic oxidation:

(a) obtain kinetic data for precious metal and other proprietary catalysts for tritium oxidation together with temperature limitations;

(b) assess mass transfer limitation in flow reactor configurations

(c) develop design equation;

(d) assess extent of adsorptive holdup interference from catalysts support materials;

(e) identify problem contaminants affecting conversion (i.e., moisture, poisons, inhibitors):

(f) identify catalyst activators;

(g) develop low pressure drop catalyst geometries suitable for high volume, recirculating systems (i.e., honeycomb catalyst).

4. Capture of tritium water on adsorbents:

(a) obtain isotherm data for tritium on various adsorbents;

(b) assess radiolytic decomposition and adsorbent damage;

(c) model and develop design equations for dynamics of adsorbent bed;

(d) evaluate the potential of low temperature operations;

(e) identify the applicable regeneration schemes to produce; economic recovery of tritium for ultimate reuse or effective disposal;

(f) contrast difference between adsorbent operation in the unsaturated versus the saturated mode with respect to ordinary water.

5. Capture of tritium by active metal getters:

(a) develop a candidate list of getters with advantages and disadvantages and ranges of conditions;

(b) assess possibility of developing a getter for tritium capture from contaminated air streams;

(c) obtain lower limits of tritium removal (i.e., $\sim 200 \mu \mathrm{Ci} / \mathrm{m}^{3}$ ) capabilities of the best getters; 
(d) develop economic regeneration schemes.

6. Surface contamination by tritium:

(a) determine the key dynamic characteristics of tritium contarination (i.e., adsorption) on important reactor materials;

(b) assess surface cleaning methods.

7. HTO formation rate $f$ rom the gas phase:

(a) in practical reactor hall environments, determine range of possible conversion rates;

(b) determine sensitive materials or conditions which should and can be avoided in reactor designs.

8. Cryogenic aistillation:

(a) obtain the economic range of low level concentration for recovery;

(b) assess schemes for holdup inventory reduction;

(c) develop and or evaluate catalytic packing to achieve in-column isotopic shift (2DT $\mathrm{D}_{2}+\mathrm{T}_{2}$ );

(d) develop dynamic simulation control models for multiple column systems.

9. Tritium removal from aqueous wastes:

(a) access the potential of several processes (e actrolysis, gas exchange, reduction, laser isotope separation, stc.) for tritium water removal from low level aqueous waste streams;

(b) identify interferring contaminant levels which could disable the procegs.

10. Tritium process control:

(a) identify specialized control transducers and functions needed for handling tritium (i.e., humidity, surface adsorbed layers, forms of discrimination, etc.);

(b) develop control and accountability systems capable of real time in-. ventory control and its reduction;

(c) assess sicatus of present dynamic control for the key process unit operations listed above;

(d) develop fault-tolerant, redundant control schemes peculiar to tritium;

(e) assess impact of safe-guarding tritium as a strategic material.

11. Tritiun accident analysis:

(a) develop use of fault tree and risk analysis in process design with quality assurance; 
(b) develop data base of tritium component hardware failure mode and rate;

(c) cover total population commitment economic guidelines.

12. Tritium neutral beam injectors:

(a) examine redesign possibilities in reducing triton implantation losses to filament coolant;

(b) assess extent of damage or interference of tritium with electrical and electronic component operation;

(c) develop decontamination and maintenance methods for these injectors under the presence of tritium;

(d) test injector prototypes with tritium.

\subsubsection{Conclusions and Recommendation}

Tritium emission goals for $10 \mathrm{ci} / d$ seem reasonable but challenging for mirror hybrid power stations coming on-line early in 2000 . Hermetically sealed reactor hall atmospheres (continually processed) also appear to be quite feasible from a tritium-handling, worker-safety and envirunmental-impact point of view. We feel that spills of $26 \mathrm{~kg}$ of tritium as gas or water can be cleaned up within reasonable operational time periods for worker reentry.

The concept of utilizing low-permeation, aluminum-alloy tritium-breeding fuel pins with an average lifetime of $3.4 \mathrm{yr}$ appears to be feasible with acceptably low tritiun losses of $20 \mathrm{ci} / \mathrm{d}$. The helium coolant tritium contamination levels of $19,200 \mu \mathrm{Ci} / \mathrm{m}^{3}$ can be handled by an inexpensive 28 slip strean processor costing around $\$ 100,000$, cutting the tritiun loss rate to the cooling water below $3 \mathrm{ci} / \mathrm{d}$.

The tritium neutral beam injectors release tritium into the helium coolant at $70,560 \mathrm{Ci} / \mathrm{d}$ via the energetic triton implantation mechanism. The cilaments appear to be too thin-walled and small in diameter for installation of permeation barriers. A 108 helium flow slip-stream processor can reduce those tritium losses into the cooling water below $3 \mathrm{ci} / \mathrm{d}$. The direct converter releases tritium similarly to the injectors above but at only $16,000 \mathrm{Ci} / \mathrm{d}$. A 28 processor can cut this source of tritium to below $3 \mathrm{Ci} / \mathrm{d}$ into the coolant water.

The leakage and outgassing rate from the reactor hall nuclear island at a maximum of $180 \mathrm{ci} / \mathrm{d}$ - controls the size of the reactor hall processor and not the $26 \mathrm{~kg}$ accident. The precious-metal catalyst bed is the single most expensive item at $\$ 3.6$ million for tritium handling. 
It appears that the NRC guideline of 170 mrem at the fenceline can be used to roughly size the containment tritium processing systems, with ALARA used to suggest how much lower levels would be cost effective.

\subsection{Cryogenic Systems}

Cryogens, liquid helium (LHe) at $4.2 \mathrm{~K}$ and liquid nitrogen (LN) at $77 \mathrm{~K}$ are required for the superconducting magnet, the vacuum cryopumping equipment, and the D-T isotopic separation equipment.

\subsubsection{Liquid Helium Refrigeration}

Ifquid helium is necessary to remove the heat loads from the superconducting magnet and the cryopanels in the injectors and end tanks. The deuterium-tritium isotopic separation equipment requires cooling at $25 \mathrm{~K}$; this cooling requirement will also be provided with the helium refrigerator as described below.

The (ideal) refrigeration cycle is shown on a T-s diagram in Fig. 6-75, and the refrigeration equipment in Fig. 6-76. The working fluid is first isothermally compressed, and then transfers heat to the returning flow via the heat exchangers in the cold box. The cold gas is then expanded through a Joule-Thompson valve into the two-phase region. The liquid produced flows to the heat load where it is boiled. The resulting saturated vapor is combined with the saturated vapor from the separator, used to cool the inlet flow and returned to the compressor. The refrigerator operates between $300 \mathrm{~K}\left(\mathrm{~T}_{1}\right)$ and $4.2 \times\left(T_{4}\right)$. The (limiting) Carnot coefficient of performance (COP) is

$$
\begin{aligned}
& (C O P)_{c}=\frac{Q_{R}}{T_{c}}=\frac{T_{4}}{T_{1}-T_{4}}, \\
& (C O P)_{c}=\frac{1}{70.4}=0.0142 .
\end{aligned}
$$

As was described in Sec. 6.1, the heat load on the magnet is $12.2 \mathrm{~kW}$, all of which is deposited in the LHe refrigeration system.

The cryopanels in the injectors face another whe cryopanel on the back and a set of IN chevrons on the front. The heat laad consists of thermal radiation from the chevrons, sensible heat of the pumped gas, and latent heat for freezing (gas to solid) the gas. The radiative heat load is

$$
\dot{q}^{n}=\dot{q}_{\mathrm{BB}}^{n}\left(1 / \varepsilon_{1}+1 / \varepsilon_{2}-1\right)^{-1},
$$




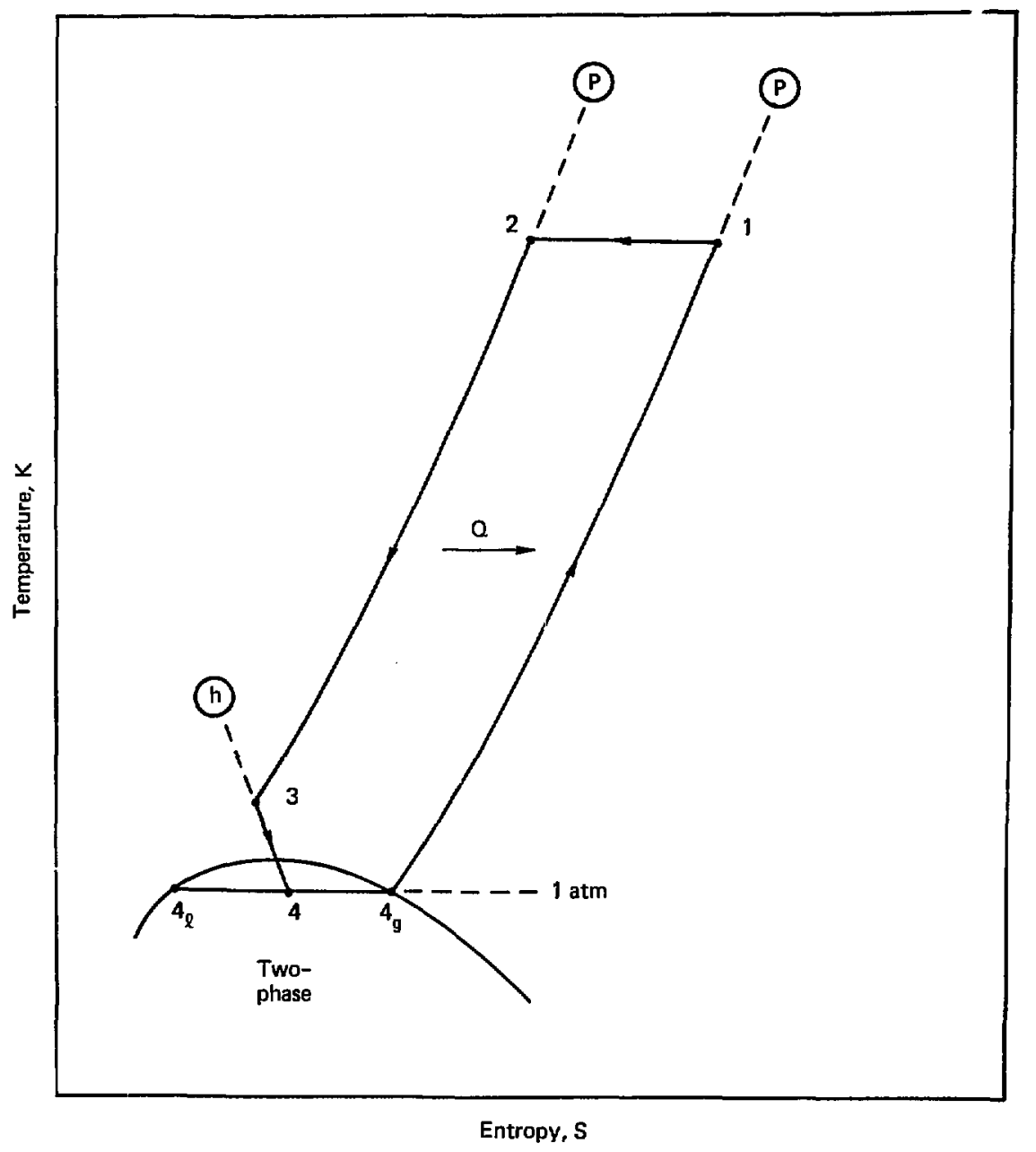

Fig. 6-75. Ideal refrigeration cycle. 


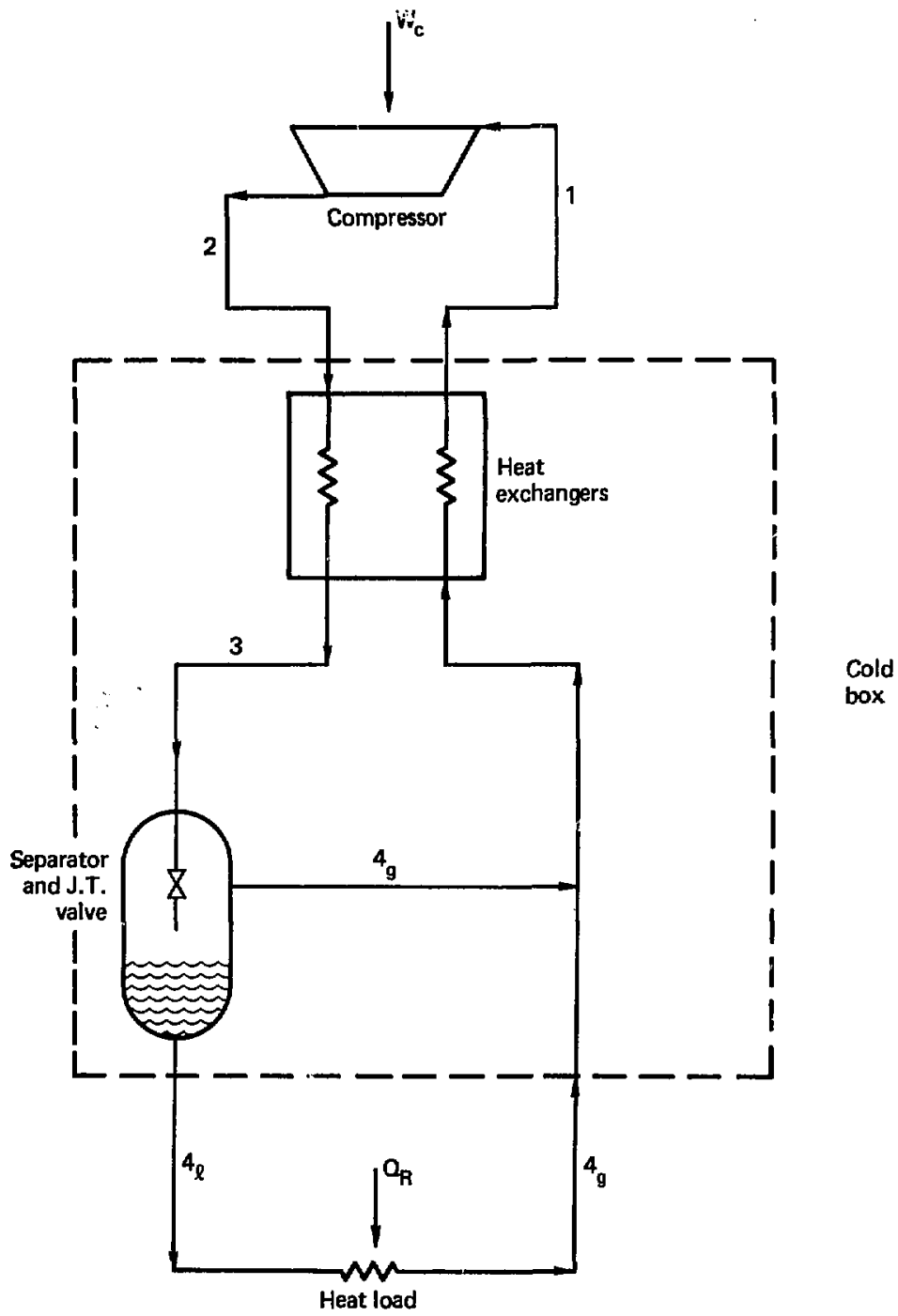

Fig. 6-76. Refrigeration equipment. 
where $\dot{q}_{\mathrm{BB}}^{\prime \prime}$ is the blackbody radiation, and $\varepsilon_{1}$ and $\varepsilon_{2}$ are the emissivities of the two surfaces. The IN chevrons have high enissivity $(\varepsilon \simeq 1)$ to minimize the transmission of high-energy photons; the cryopenels are taken to have $\varepsilon=0.1$. Referring to Table 6-37, the resulting heat load is $0.2 \mathrm{w} / \mathrm{m}^{2}$; we increase this by $25 \%$ to $0.25 \mathrm{w} / \mathrm{m}^{2}$ to accont for hanger (conduction) $108 s e s$. As the injectors have a total of $1500 \mathrm{~m}^{2}$ of cryopanel, the total radiative heat load is $375 \mathrm{w}$. The gas load of 476 Torr-litre/s in the injectors represents an additional heat load die to sensible heat and latent hea: (see Table 6-37) of $82 \mathrm{w}$. Thus, the total heat load on the injector cryopanels is 457 W.

The cryopanels in the end tanks have an $L N$ liner on the back $(\varepsilon \simeq 0.14)^{74}$ and a set of $\mathrm{LN}$ chevrons on the front, for a unit heat load of $(0.20+0.12)$ $=0.33 \mathrm{w} / \mathrm{m}^{2}$; this is increased to $1.25 \times 0.33=0.41 \mathrm{w} / \mathrm{m}^{2}$ to account for hanger losses. The large end tank has a cryopanel area of $1790 \mathrm{~m}^{2}$ and the small end tank $55 \mathrm{~m}^{2}$ for total radiative heat loads of $734 \mathrm{w}$ and $23 \mathrm{w}$ in the large and small tanks, respectively. The gas loads in the two tanks are 439 Torr-litre/s and 49 Torr-litre/s resulting in additional heat loads of

Table 6-37. Cryogenic engineering constants.

Blackbody radiation, $w / \mathrm{m}^{2}$

$293 \mathrm{~K} \rightarrow 77 \mathrm{~K}$

$77 \mathrm{~K} \rightarrow 4.2 \mathrm{~K}$

Sensible Heat of $\mathrm{H}_{2}, \mathrm{D}_{2}, \mathrm{~T}_{2}, \mathrm{~J} /$ Torr-litre

$293 \mathrm{~K} \rightarrow 77 \mathrm{~K}$

$77 \mathrm{~K} \rightarrow 4.2 \mathrm{~K}$

Iatent heat of freezing at $4.2 \mathrm{~K} \mathrm{~J} /$ Torr-1itre

$\mathrm{H}_{2}$
$\mathrm{D}_{2}$

Latent heat of evaporation at $1 \mathrm{~atm}, \mathrm{~J} / \mathrm{g}$
0.245

417

0.0831
0.0560

0.0799
IN

LHe 
$76 \mathrm{~W}$ and $\mathrm{g} \mathrm{W}$ in the large and small tanks, respectively. Thus, the total liguid helium heat loads are $515 \mathrm{~W}$ and $58 \mathrm{~W}$ in the two tanks.

similarly, pumping the plasma stabilizing stream of 1425 Torr-litre/s requires $333 \mathrm{~m}^{2}$ of cryopanel, resulting in an the heat load of $136+246=382 \mathrm{~N}$.

The D-T isotopic separation equipment has a heat load of $3.36 \mathrm{kH}$ at $25 \mathrm{~K}$. This refrigeration will be supplied by bleeding off some of the He return flow in the refrigerator at the appropriate temperature (between points 49 and 1 in Figs. 6-75 and 6-76). To estimate the necessary refrigeration input power, we reduce the heat load to an approximate equivalent value at $4.2 \mathrm{~K}$ by multiplying by the ratio of the carnot refrigeration cop's:

$$
4.2 /(300-4.2) \times(300-25) / 25=(1 / 70.4) \times 11.0=0.16
$$

for an equivalent value of $0.16 \times 3.36=0.54 \mathrm{~kW}$.

The individual heat loads are sumarized in Table 6-38 for a total of $14.15 \mathrm{~kW}$, which is dominated by the neutron heating in the magnet; in the present study neutron heating in the cryopanels has not been evaluated. From Ref. 75, it is estimated that an IHe refrigerator with a 14-kw heat load will achieve 25t of the Carnot $C O P$, for a resulting $C O P$ of $0.25 \times 0.0142$ $=0.00355$. The input power required to drive the refrigerator is thus $14.15 \times 10^{-3} / 0.00355=4.0 \mathrm{MW}$.

\subsubsection{Liquid Nitrogen Refrigerator}

The heat load on the IN refrigerator occurs in the cryopumping system: the chevrons, liners and gas sensiv? heat. The magnet does not have IN cooling as its $4.2 \mathrm{~K}$ case is interfaced to ambient temperature with load-bearing thermal insulation.

Table 6-38. LHe refriçeration system.

\begin{tabular}{cc} 
Heat loads, $\mathrm{kW}$ & \\
Magnet & 12.2 \\
Cryopanels & 1.41 \\
Injectors & 0.457 \\
Large end tank & 0.515 \\
Small end tank & 0.058 \\
Plasma stream & 0.382 \\
Isotopic separator & 0.54 \\
Total & 14.15 \\
Input power, & 4.0 \\
\hline
\end{tabular}

aquivalent value at $4.2 \mathrm{~K}$; see text. 
In the injectors, only IN cilevrons are used (emissivity, $\varepsilon \simeq 1$ ). If we assume these chevrons view a $300 \mathrm{~K}$ surface with $\varepsilon=0.5$, the unit heat load 18 $0.5 \times 417 \times 1.25=260 \mathrm{w} / \mathrm{m}^{2}$, where we have included a $25 \mathrm{~s}$ loss for conduction. For the $1500 \mathrm{~m}^{2}$ of panel, this is $391 \mathrm{kw}$ heat load. The sensible heat of the pumped gas (476 Torr-litre/s) adds an additional (negligible) 117 พ.

In the end tanks, both chevrons and LN linerg are used. The large end tank has $1790 \mathrm{ni}^{2}$ of chevrons for a heat $10 \mathrm{c}$ of $200 \times 10^{-3} \times 1790$

$=465 \mathrm{~kW}$. He neglect the sensible heat of the pumped gas. The LN liners $(E=0.14)$ are interfaces to the ambient temperature tank liner wi.th superinsulation. He estimate the heat leak as equivalent to the radiative transfer from a $300 \mathrm{~K}$ surface with $E=0.5$, or a heat $f 1 u x$ of $0.12 \times 417=51 \mathrm{w} / \mathrm{m}^{2}$. The total is $1.25 \times 51 \times 10^{-3} \times 1790 \times 114 \mathrm{kw}$. Thus, the total $\mathrm{Ls}$ heat load in the large end tank is $505 \mathrm{kw}$. similarly, for the $55 \mathrm{~m}^{2}$ of pumping surface in the small end tank, the heat load is $16 \mathrm{kw}$ and the $333 \mathrm{~m}^{2}$ of pumping surface for the plasma stream has a heat load of $94 \mathrm{kw}$. The heat loads are summarized in Table 6-39.

The LN refrigerator will work on the same cycle as the LHe refrigerator, shown in Figs. 6-75 and 6-76. The limiting Carnot coefficient of performance is

$$
(\operatorname{COP})_{C}=\frac{77}{300-77}=\frac{1}{2.90}=0.345 .
$$

From Ref. 75, for a 1-MA heat load, it is estimated that $30 \%$ of the Carnot value can be achieves, or $C O P=0.104$. Thus, the required input power to the refrigerator is $9.7 \mathrm{MH}$.

Table 6-39. UN refrigeration system.

\begin{tabular}{cc}
\hline Heat loads, $\mathrm{kN}$ & \\
Cryopumping equipment & 391 \\
Injectors & 505 \\
Large end tank & 16 \\
Small end tank & 94 \\
Plasma stream & 1006 \\
Total & 9.7 \\
input power, w
\end{tabular}




\section{References}

1. F. H. Coensgen, Ed., MX Major Project Proposal, Lawrence Livermore Laboratory, Rept. LLL-Prop-142, Appendix F (1976) .

2. R. Hanpshire, J. Sutton, and M. T. Taylor, "Effect of Temperature on t:he Critical Current Density of Nb-44 wt. Ti Alloy," Proc. Conf. on Low Temperatures and Electric Power (The Royal Society, London, Mar., 2969), p. 69 .

3. D. Cornigh, Lawrence Livermore Laboratory, private communication (1977).

4. H. Ullmaier, "Radiation Damage in CTR Magnet Components," Proc. Int'I Conf. On Radiation Effects and Trittum Technology for Fusion Reactors (Gatlinburg, Tenn., Oct., 1975), CONF-750989, Vol. II, p. 403.

5. R. W. Moir and C. E. Taylor, "Magnets for Open-Ended Fusion Reactors," Proc. Conf. on Technology of Controlled Thermonuclear Fusion Experiments and the Engineering Aspects of Fuston Reactors (Austin, Texas, Nov., 1972), CONF-721111, p. 853; also, Lawrence Livermore Laboratory, Rept. UCRL-74326 (1972) .

6. S. J. Sackett, "Calculation of Electromagnetic Fields and Forces in Coil Systems of Arbitrary Geometry," Proc. 6th Sympos. on Engineering Problems of Fusion Research (San Diego, CA., 1975), p. 935; also, Lawrence Livermore Laboratory, Rept, UCRL-77244 (1975).

7. R. H. Moir, Lawrence Livermore Laboratory, private communication (1977).

B. An Evaluation of the Technical and Economic Feasibility of Mirror Fusion Devices, prepared for Lawrence Livermore Laboratory by Bechtel Corporation, San Francisco, CA; Lawrence Livermore Laboratory, Rept. UCRL-13695 (1976).

9. D. H. Cornish, D. H. Deis, A. R, Harvey, D. G. Hirzel, J. E. Johnston, R. L. Leber, R. L. Nelson, and J. P. Zbaznik, "Development Work on Superconducting Coils for a Large Mirror Fusion Test Facility (MFTF)," Proc. 6th Int'l Conf. on Magnet Technology (Bratislava, Czechoslovakia, 1977); also Lawrence Livermore Laboratory, Rept. UCRL-78891 (1977).

10. J. H. Fink, W. L. Barr, and G. W. Hamilton, "A 225 MW Neutral Injection Systen for a Mirror Fusion-Fission Hybrid Reactor," Nucl Fusion 15, 1067 (1975) .

11. J. H. Fink, W. L. Barr, and G. W. Hamilton, A Study of Efficient, High Power, High Energy Neutral Beams for the Reference Mirror Reactor, Lawrence Livermore Laboratory, Rept. UCRL-52173 (1976). 
12. K. H. Berkner et al., "Performance of LBL $20 \mathrm{kV} \mathrm{10-A}$ and 50-A Neutral Beam Injectors," Proc. Second Symp. On Ion Sources and Formation of Ion Beams (Berkeley, CA, 1974), VI-12.

13. K. W. Ehlers et al., "Large Area Plasma Sources," Proc. Second Symp. on Ion Sources and Eormation of Ion Beams (Berkeley, CA, 1974), I-5.

14. J. H. Fink and L. A. Biagi, "A Long Life Cathode for the Berkeley.Type Ion Source," Proc. Seventh Symp. on Engineering Problems of Eusion Research (Knoxville, TN, 1977).

15. R. . Cooper, Lawrence Livermore Laboratory, personal conmunication (1977).

16. G. W. Hamilton, A $120 \mathrm{keV}$ Beam Direct Conversion System for TFTR Injectors, Lawrence Livermore Laboratory Rept. UCRL-52137 (1976).

17. J. H. Fink and C. E. McDowell, "Ionization, Charge Exchange, and Secondary Emission in the Extractor of an LBL/LWL Neutral Beam Source," Proc. Sixth Symp. On Engineering Problems of Fusion Research (San Diego, CA, 1975). p. 161 .

18. K. H. Berkner, R. V. Pyle, and J. W. Stearns, "Intense, Mixed Energy Bydrogen Beams for CTR Injection," Nucl. Fusion 15, 243 (1975).

19. E. W. McDaniel, Collision Phenomena in Ionized Gases (John Wiley and Son, New York, 1964), p. 282, Fig. 6-7-4.

20. C. F. Barnett et al., Atomic Data for Controlled Fusion Research, Oak Ridge National Laboratory Rept. ORNL-5207 (1977), P. D.1.17.

21. P. D. Townsend, J. C. Kelly, and N. E. W. Hartley, Ion Implantation, Sputtering, and Their Applications (Academic Press, 1976), p. 132.

22. . A. Anderson, A Compact High-Energy Neutral Beam System, Lawrence Livermore Laboratory Rept. UCID-16914 (1975).

23. H. Tawara and $\mathrm{A}$. Russek, "Charge Changing Processes in Hydrogen Beam," Rev. Nod. Phys. 45, 223 (1973).

24. G. Klipping, "Relations between Cryogenics and Vacuum Technology, Up to Now and in the Future," Jap. J. Appl. Phys. Suppl. 2, Pt. 1 81 (1974).

25. A. C. Riviere and J. Sheffield, "Transfer Efficiency of Intense Neutral Beams," Nucl. Fusion 15, 944 (1975).

26. D. J. Bender and G. A. Carlson, Fusion Plasma Losses Due to the Charge Exchange of Injected Neutrals, Trans. ANs 21, 44 (1975); also, Lawrence Livermore Laboratory, Rept. UCRL-76438 (1975).

27. G. A. Carlson, Selective Leakage of Charged Particles in Mirror Machines, Lawrence Livermore Laboratory, Rept. UCRL-51434 (1973). 
28. R. H. Moir, st al, Standard Mirror Fusion Reactor Design Study, Lawrence Livermore Laboratory, Rept, UCID-17644 (1978).

29. M. A. Hoffman, Electrostatic Direct Energy Converter Performance and Scaling Laws, Lawrence Livermore Laboratory, Rept. UCID-17560 (1977).

30. A. S. Blum, Advanced Fueled Fusion Reactors Suitable for Direct Energy Conversion. Project Note: The Expander Structure, Lawrence Livermore Laboratory, Rept, UCRL-79061 (1977).

31. A. S. Blum, Advanced Fueled Fusion Reactors Suitable for Direct Energy Conversion. Project Note: The Electrode Structure, Lawrence Livermore Laboratory, Rept, UCRL-79062 (1977).

32. W. I. Barr and R. W. Moir, "A Review of Direct Energy Conversion for Eusion Reactors," Proc. 2nd ANs Toptcal Meeting on the Technology of Controlled Nuclear Fusion (Richland, HA, 1976), CONF-760935-P4, p. 1181.

33. R. W. Werner and M. A. Hoffmann, "Combination Cryogenic Panel and Valve System for Quasi-DC Pumping of Condensable Gases," AEC Record of Invention, Case IL-6119 (Feb., 1976).

34. "Exploratory Studies of High-Efficiency Advanced-Fuel Fusion Reactors," Electric Power Research Inst., Annual Report (Feb., 1978), EPRI ER-581.

35. P. พ. Fisher and J. S. Watson, "Cryosorption Pumping of 958 Deuterium 5* Helium on Nolecular Sieve-5A at $4.2^{\circ} \mathrm{K}, "$ Proc. 7th Symposium on Engineering Problems of Fusion Research (Knoxville, TN, 1977).

36. I. D. Hangborough, "Tritium Inventories and Leakage: A Review and Some Additional Considerations," Symposium of CTR Technology, U.S.A.E.C.. Series 31 pp. 92-104 (1974).

37. D. J. Jacobs, Sources of Tritium and its Behavior Upon Release to the Environment, U.S.A.E.C. (1968).

38. T. S. Ellemen and K. Verghese, "Surface Effects on Tritium Diffusion in Niobium, zirconlum, and stainless steel," ․․ ㅆuc. Mat. 프, 229-306 (1974).

39. U.S. Federal Regigter 40 (104), 23420 (1975).

40. U.S. Federal Register 40 (158), 40816 (1975).

41. C. J. Kerschner and J. C. Bixel, "Tritium Effluent Contr " Laboratory," Proc. of the 13th ADC Air Cleaning Conference, San Francisco, August 12-15, 1974. U.S. AEC Conf. 740807, vols. I and II, PP. 948-971 (1975).

42. J. L. Anderson, et al,, "Tritium Handling Facilities at the Los Alamos Scientific Laboratory," in Proc. 23ra Conf. on Remote Systems Techno. (1975), and J. L. Anderson, LOB Alamos Scientific Laboratory, private communications, February 3, 1976, and September 21, 1977. 
43. W. A. Swansiger and L. A. West, "Current Sandia Program and Laboratory Facilities for Tritium Research," Proc. of the Symposium of Tritium Technology Related to Fusion Reactor Systems, U.S. ERDA, June 1976;

R. พ. Werner, Lawrence Livermore Iaboratory, private communication (1972).

44. R. V. Osborne, "Adsorption of Tritiated water Vapor by People," Health Physics 12, 1527-1537 (1966).

45. National Commission on Radiation Protection, Handbook 39, January 15, 1971, p. 83.

46. J. F. Draley and S. Greenberg, "Some Features of the Environmental Impact of a Fusion Reactor PCwer Plant," in AEC Symposium on Tritium Technology, AEC No. 31 (1974).

47. Quotation from Engelhard Industries Division, Dec. 9, 1975, and followup meeting on December $30,1975$.

48. V. A. Maroni, "An Analysis of Tritium Distribution and Leakage Characteristics for Two Fusion Reactor Reference Designs," Proc. Fifth Symposium on Engineering Problems of Fusion Research. IEEE Publication No. 73 CHO 843-3NPS, Princeton Univ., No. 5-9, 1974.

49. R. W. Moir, et al., Progress on the Conceptual Design of a Mirror Hybrid Fusion-Fission Reactor, Lawrence Livermore Laboratory, Rept. UCRI-51797 (1975).

50. G. J. Casellato, et al., The Self-Radiation oxidation of Tritium in oxygen and Air, U.S. Naval Radiological Defense Laboratory, San Francisco, Dept. US NRDL-TR-565 (1962).

51. A. A. Moghissi and M. W. Carter, "Tritium," Messenger Graphics, Phoenix, Arizona (1973).

52. L. F. Belovodskii, V. K. Gaevoi, V. I. Grishmanovski and N. V. Nefedov, "Oxidation of Tritium in Air Under the Action of Intrinsic Radiation," At. Energ. 38, PQ. 379-381 (1975).

53. J. Y: Yang and L. H. Gevantman, "Tritium B-Radiation-Indnsed Isotopic Exchange in the $T_{2}-H_{2} O$ Systems," U.S. Manual Defense Lab., $R \& D$ Tech Rept. USNRDL-TR-471 (1960) .

54. G. A. Carlson and R. W. Moir, "Mirror Machine Reactors," in Eroc. of the 2nd Topical Meeting on the Technology of Controlled Nuclear Fusion, Richland, Washington, Sept. 1976, p. 55-574.

55. O. Levenspiel, Chemical Reaction Engineering (John Wiley, N.Y., 1972). 56. A. E. Sherwood, Tritium Removal from Air streams by Catalytic Oxidation and Water Adsorption, Lawrence Livermore Laboratory, Rept. UCRL-78173 (1976). 
57. J. C. Bixel and C. J. Kerschner, "A Study of Catalytic Oxidation and Oxide Adscrption for the Removal of Tritium from Air," in Proc. of the Second AEC Environmental Protection Conference, Albuquerque, New Kexico (1974).

58. A. E. Sherwood, private communication, Lawrence Livermore Laboratory, oct. 19. 1967.

59. R. G. Clemer, R. H. Land, W. A. Maroni, and J. M. Mintz, "Simulation of Large Scale Air Detritiation Operations by Computer Modeling and Bereh-Scale Experimentation," in 7th Sympogium on Engineering Problems of Fusion Research, Knoxville, Tennessee, 1977.

60. T. Vermuelen, G. Klein and N. K. Hiester, "Process Design Methods for Adsoxption and Ion Exchange," American Institute of Chemical Engineers, Aiche Today Series, 345 E. 47th St., New York, N.Y., 10017.

61. K. K. Kelley, Ed., Perry's Chemical Engineering Handbook, U.S. Department of Interior, Bureau of Mines, contributions to the data on theoretical metallurgy, Bulletin 383 (1935).

62. J. R. Powe11, R. H. Wiswall and F. Wirsing, Tritium Recovery from Eusion Blankets Using Solid Lithium Compounds, Brookhaven National Laboratory, Informal Rept. No. BNL 20563 (1975).

63. F. K. Hetmann, O. N. Solomon, The Lithium Hydride, Deuteride, and Tritide Systens, USAEC Rept. KAPL-1667, Knolls Atomic Power Laboratory, Schenectady, N.Y. (1956).

64. J. Harding Owen, "Equilibrium and Kinetic Studies of Systems of Hydrogen Isotopes, Lithium HYdrides, Aluminum, and LiALO ${ }_{2}, "$ in Proc. Conference on Radiation Effects and Tritium Technology for Fusion Reactorg, ERDA, ANS and AIME Conference CONF-750989, Vol. 3, Tp. 433-457 (1975).

65. A. B. Johnson, Tritium Release from Lithium Sllicate and Lithium Aluminate, In-Reactor and Out-of-Reactor, Battelle Pacific Nor thwest Laboratories, Rept. BNWL-2116 (1976).

66. R. H. Webb, Permeation of Hydrogen Through Metal, Atomics International, Div. North American Aviation, Inc., Tech Rept. NAA-SR-10462 (1965).

67. V. A. Karoni, An Analysis of Tritium Distribution and Leakage Characteriatics for Tro Fusion Reactor Reference Designs, Argonne National Isaboratory, Rept. CEN/CTR/TM-9 (1974).

68. V. A. Maroni, "Some Perspective on Tritium Permeation in Fusion Reactors Based on Existing Data and Experience," in Proc. 1977 Annual Keeting of Amer. Nuc. Soc., New York, June 12-17, 1977. 
69. J. T. Bell and J. D. Redman, Tritium Permeation Through Metals Under Stean Conditions, ORNL Draft Paper (1975).

70. R. A. Strehlow and H. C. Sausage, "The Permeation of Hydrogen Isotopes Through Structural Metals at Low Pressures and Through Metals with Oxide Film Barriers," Nuc. Technol. 22, 127 (1974).

71. L. Yang, H. A. Baugh, and N. L. Baldwin, Study of Tritium Permeation Through Peach Bottom Steam Generator Tubes, General Atomic Co., Draft paper (1977).

72. R. พ. Fenn, et al., "New Ductile Beryllium-Aluminum Wrought Alloys," in Proc. 6th Annual Meeting of ASTM, Chicago, Illinois, June $21,1964$.

73. K, R. Van Horn, Ed., "Aluminum-Vol. I. Properties, Physical Metallurgy, and Phase Diagrams," ASTM (1967).

74. M. A. Hoffman, Electrostatic Direct Energy Converter Performance and Cost Scaling Laws, Lawrence Livermore Laboratory, Rept. UCID-17560 (1977).

75. T. R. Strobridge, Cryogenic Refrigerators, An Updated Survey, National Bureau of Standards, Tech. Note 655 (1974). 


\section{NUCLEAR ANALYSIS}

\subsection{Blanket Nuclear Analysis}

\section{Nomencinture and Units}

BS number of blanket segments

BU burn-up (fission) of heavy metal

$c_{1} \quad$ kilomoles of neutrons per $\mathrm{M}$-year of fusion neutrons 10.0232 for DT fusion)

I inventory of tritium

$k$ 's constants used in analytical model of blanket performance

$\mathrm{L}_{\mathrm{f}} \quad$ length of fission zone

M energy multiplication: energy produced per fusion neutron divided by kinetic energy of fusion neutron

$\ddot{P}$ power density

P/D pitch-to-diameter ratio

$\overline{P_{n}} \quad$ power carried by fusion neutrons, averaged in time

pu plutonium buildup

$\mathrm{Pu} / \mathrm{n}$ net plutonium breeding ratio: ${ }^{239} \mathrm{Pu}$ per fusion neutron

$T_{b} \quad$ tritium contained in blanket

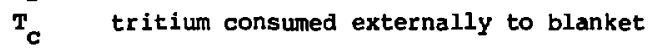

$T / n$ tritium breeding ratio: tritons per fusion neutron

$t_{p} \quad$ time between consecutive reactor shut-downs for blanket segment replacement

$\alpha \quad$ conical angle of unit cell used for transport calculations

$\lambda \quad$ tritium decay constant: $0.0562 \mathrm{y}^{-1}$

$\Phi$ exposure in terms of integrated kinetic energy current of fusion neutrons through the first wall; units: $\mathrm{MN}-\mathrm{y} / \mathrm{m}^{2}$

$\phi \quad$ "wall loading," kinetic energy current of fusion neutrons through the first wall; units: $\mathrm{m} / \mathrm{m}^{2}$

$\Omega$. fraction of fusion neutrons entering blanket (blanket coverage)

Unless otherwise specified, units used in this section are: metres years kilograms kilonoles megawatts watts per cubic centimetre 


\subsubsection{Objectives}

The overall objective of this hybrid reactor study is to conceptually develop and analyze a conservative design based on standard minimum-B plasma containment, first generation magnet and beam technology, and present-day fission technology. The design is optimized to minimize the power cost of the hybrid and the fission reactors that consume the ${ }^{239} \mathrm{Pu}$ produced by the hybrid.

To meet our overall study objective we specify the following set of blanket nuclear design objectives:

- Large net ${ }^{239} \mathrm{pu}$ breeding in depleted uranium.

- Production of all tritium needed by the plant after start-up.

- As much energy multiplication as possible without greatly affecting net ${ }^{239}$ pu breeding.

- Subcritical under all conditions.

- Limitation of power density and burnup in the uranium fuel to levels consistent with the thermohydraulic design and fuel materials.

Based on our past work, ${ }^{1-10}$ we believe the best way to meet the combined blanket objectives of large ${ }^{239}$ pu breeding, tritium breeding, and energy multiplication is to maximize neutron and energy multiplication by fusion-neutron-induced fission of ${ }^{238} \mathrm{U}$. We therefore chose the fast fission blanket concept for this hybrid study.

To analyze blanket performance, we specify the following blanket nuclear analysis objectives:

- Use multidimensional modeling and transport methods to account for neutron and gamma streaming around and through the blanket modules. The model is to be a good approximation of the actual mechanical design of the module blanket.

- Account for both spatial and time effects of compositional change (principally, buildup of ${ }^{239} \mathrm{Pu}$ and burnup of heavy metal) and power density in the uranium fuel.

- Include effects of tritium decay in determining required tritiumbreeding ratios and purchase requirements.

- Develop scaling methods to determine blanket performance as changes in reactor parameters nove blanket off the design point used for the transport calculations. 


\subsubsection{Procedure}

The steps used to determine blanket nuclear performance are as follows:

1. Construct a geometric model of the blanket and 14-MeV-neutron source with which to perform multidimengional neutron and gamma transport calculations.

2. Perform Monte Carlo transport calculations to determine local "o" exposure blanket performance trade-offs [tritium-breeding ratio ( $\mathrm{T} / \mathrm{n}$ ) vs ${ }^{239} \mathrm{Pu}-$ breeding ratio $(\mathrm{Pu} / \mathrm{n})$ and $14-\mathrm{MeV}-$ source-neutron energy multiplication (M)]. Local blanket performance refers to blanket response to a 14-MeV-source neutron that interacts with the blanket. If the blanket occupies 100\% of the spherical area surrounding the fusion neutron source, overall blanket performance equals local blanket performance. The " 0 " exposure refers to the start of blanket life when no nuclear transmutations have occurred.

3. Determine local exposure-dependent performance by running a series of transport calculations in which isotopic compositions are adjusted by the numbers of nuclear reactions calculated in the previous step.

4. Use results of steps 2 and 3 to formulate analytic expressions for the exposure-dependent performance parameters.

5. Using the analytic expression for exposure-dependent tritium bretding ratio, determine the local zero-exposure tritium breeding ratio needed for the reactor to be self-sufficient in tritiun after start-up, and the quantity of tritiun that must be purchased for start-up.

6. Combine results of steps 23 , and 5 and calculate a self-consistent: set of blanket performance parameters.

\subsubsection{Transport Model and Method}

7.1.3.1 Geometry. The blanket is a spherical shell surrounding the plasma neutron source. The spherical shell has penetrations through it to allow for plasma leakage and injection. The blanket is made up of approximately 600 cylindrical pressure vessels. Each is aligned on a ray from the plasma center. Blanket layout and design of an individual cylindrical blanket module are shown in Figs. 7-1 and 7-2, respectively. For more details, see Section 8 on blanket mechanical design.

7.1.3.2 Unit cell. For calculation of neutron and gamma transport, a single blanket cell is considered. This cell consists of a cone whose apex is at the plasma center and contains one blanket module. A diagram of this unit cell is given in Fig. 7-3. The conical angle of the unit cell is sized to have a spherical area equal to that of the actual perimidal unit cell. The 



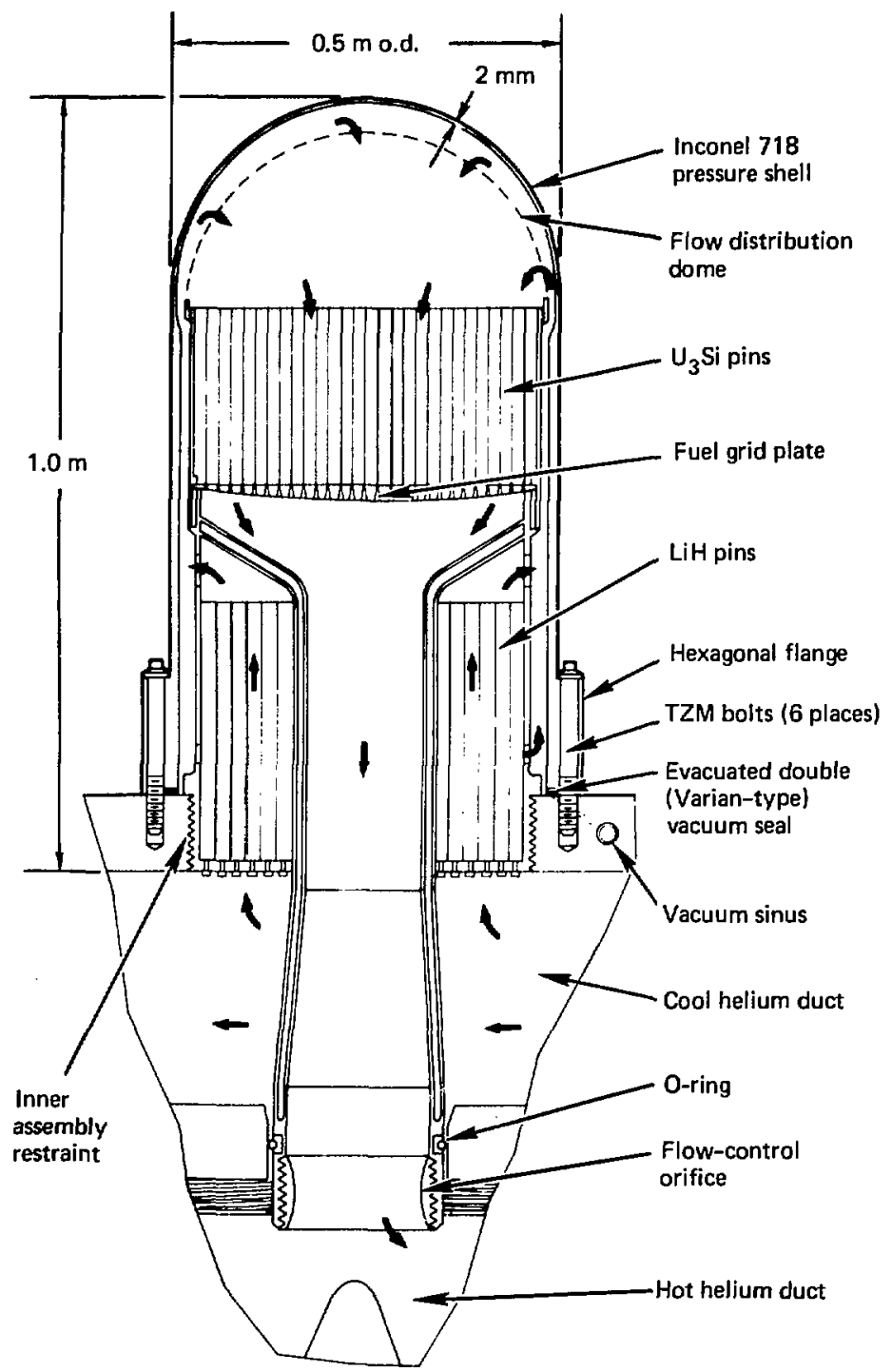

Fig. 7-2. Blanket module. 


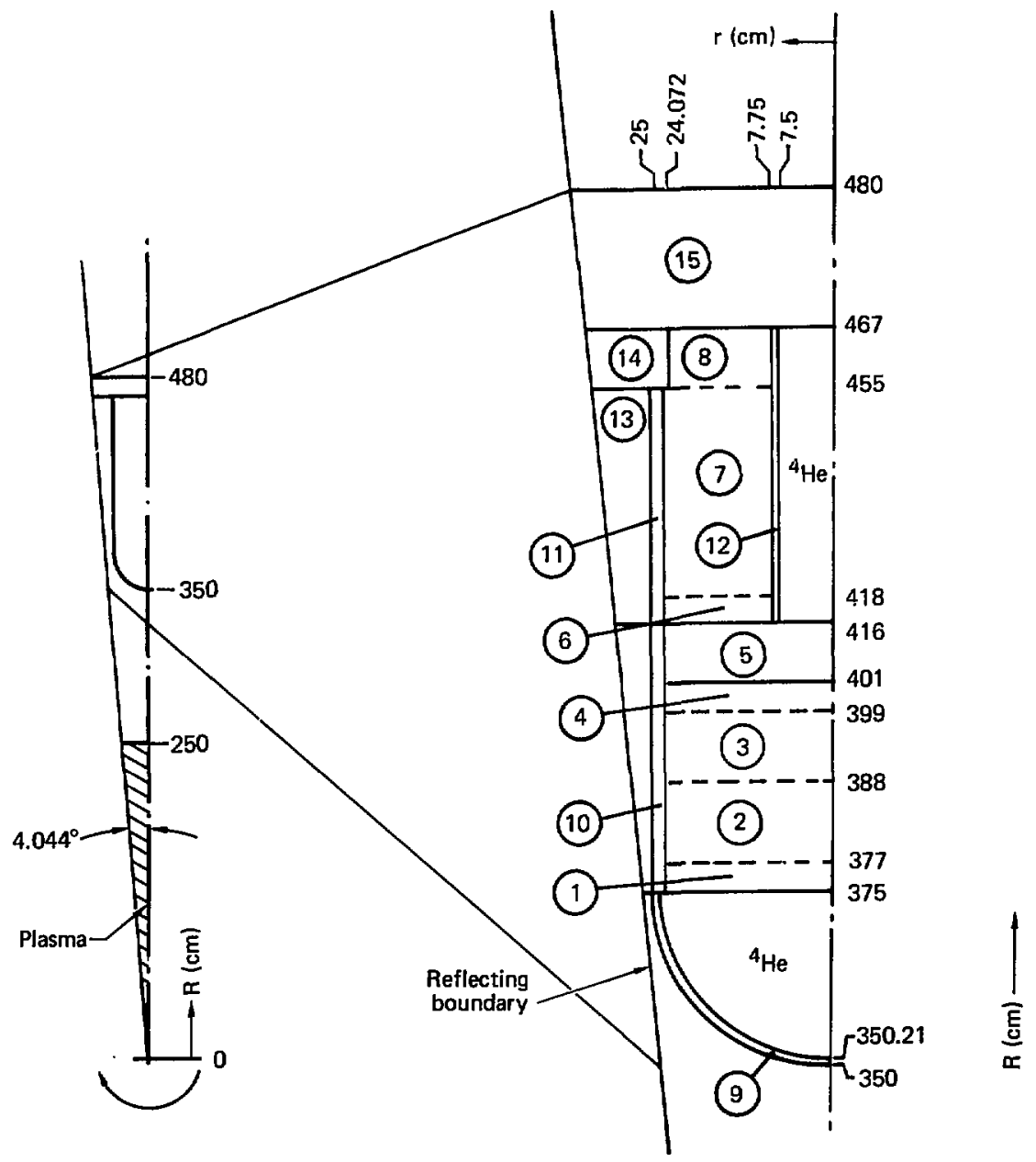

Fig. 7-3. The model used for the neutron and gama Monte Carlo transport calculations. zones of interest are numbered and described in Table 7-1. zones labeled He contain only helium coolant; unlabeled zones are vacuum zones. Except for zone 13, zones with material volume fractions less than 1 contain helium coolant in the renaining volume. 
sides of the unit cell are reflecting $[$ (rebound angle) $=\pi$ - (incident angle)] to account for neutrons and gammas scattering into and out of the cell. This cell model was chosen so that streaming around and through the individual blanket modules is accounted for.

Dimensions defining the boundaries of the unit cell are listed on Fig. 7-3.

The conical angle (a) of the cell boundary is dependent on the firstwall radius and on the pitch of the modules (where pitch is the distance between module centers). A pitch-to-diameter ratio (P/D) of 1.01 at the interface of the cylindrical and hemispherical portions of the module (located $3.75 \mathrm{~m}$ from plasma center) defines the conical boundary of the calculational unit cell.

The calculational model of the blanket module contained in the unit cell is divided into zones (Fig. 7-3). The zones (described in Table 7-1) contain different materials and/or are used to study spatial effects.

Table 7-1. Descriptions of calculational model zoning shown in Fig. 7-3.

\begin{tabular}{|c|c|c|c|c|}
\hline Zone No. & Description & Material & $\begin{array}{l}\text { Material } \\
\text { volume fraction(s) }\end{array}$ & Volume, $\mathrm{cm}^{3}$ \\
\hline \multirow[t]{4}{*}{$1-4$} & Fission zones & $\mathrm{u}_{3} \mathrm{si}+$ (Inconel & $0.692+(0.073)$ & 3641 \\
\hline & & clad) & & 20025 \\
\hline & & & & 20025 \\
\hline & & & & 3641 \\
\hline 5 & $\begin{array}{l}\text { Fission zone support } \\
\text { and piping }\end{array}$ & Inconel & 0.0933 & 27306 \\
\hline \multirow[t]{3}{*}{$6-8$} & T-breeding zones & $\mathrm{LiH}+(\mathrm{Al}-\mathrm{Fe}$ clad $)$ & $0.716+(0.106)$ & 3263 \\
\hline & & & & 60374 \\
\hline & & & & 19581 \\
\hline 9 & $\begin{array}{l}\text { First wall and coolant } \\
\text { baffle }\end{array}$ & Inconel & 1.0 & 818 \\
\hline $10-11$ & $\begin{array}{l}\text { Cyl. pressure vessel } \\
\text { and coolant baffle }\end{array}$ & Inconel & 0.448 & $\begin{array}{l}5866 \\
5580\end{array}$ \\
\hline 12 & He outlet pipe & Inconel & 1.0 & 611 \\
\hline 13 & Graphite insert & Graphite & 0.4 & 39666 \\
\hline 14 & Module support & Inconel & 1.0 & 18208 \\
\hline 15 & Fixed blanket structure & Inconel & 1.0 & 45777 \\
\hline
\end{tabular}


The mass and atom densities of the materials are listed in Table 7-2.

7.1.3.3 Source. The geonetry of the 14.1-MeV, D-T fusion neutron solyzis was taken to be a spherical volume source with a radius of $2.5 \mathrm{~m}$. To save computer time, the spherical volume source was converted to $\rightarrow$ spherical o...face source at a radius of $3 \mathrm{~m}$; a reflecting spherical surface w also placed at a radius of $3 \mathrm{~m}$. Computer time is saved $(\sim 20 \%)$ bec $\cdots$. es all source neutrons are yenerated heading in the positive radial 2 - ection and all secondary neutrons and gammas are reflected; thr-ecore, no wonte Carlo particles spend time inside a radius of $>$ :.., a region of the unit cell where nothing of interest nappens. The angular spectra of the 3 m-radius, spherical-surface source is listed in Table 7-3.

7.1.3.4 Method. A Monte Carlo code called TARINP, ${ }^{11}$ using nuclear data from the ENDL ${ }^{11}$ library, was used for the neutron and gamma transport problems. The nuclear data has a 176-group, flat-weighted energy structure. Problems were run with a maximum sample size of 4000 source neutrons. Maximum

Table 7-2. Materials (at a volume fraction of 1.0 ).

\begin{tabular}{|c|c|c|c|}
\hline Material & Mass, $\mathrm{g} / \mathrm{cm}^{3}$ & Atom densities, $10^{22}$ & atoms $/ \mathrm{cm}^{3}$ \\
\hline \multirow[t]{4}{*}{$\mathrm{U}_{3} \mathrm{Si}$} & 16.1 & U (depleted) & \\
\hline & & ${ }^{235} 5_{U}$ & 0.00981 \\
\hline & & $23 \mathrm{~B}_{\mathrm{U}}$ & 3.912 \\
\hline & & si & 1.307 \\
\hline \multirow[t]{4}{*}{ Inconel } & 8.19 & $\mathrm{Fe}$ & 1.839 \\
\hline & & $\mathrm{Cr}$ & 1.830 \\
\hline & & $\mathbf{N i}$ & 4.593 \\
\hline & & $\mathrm{Nb}$ & 0.276 \\
\hline \multirow[t]{3}{*}{ LiE } & 0.82 & ${ }^{6} \mathrm{Li}$ & 0.470 \\
\hline & & ${ }^{7} \mathrm{Li}$ & 5.75 \\
\hline & & H & 6.22 \\
\hline \multirow{2}{*}{$\begin{array}{l}\text { Lit cladding (10\% Fe } \\
+90 \% \text { Al by vol) }\end{array}$} & 3.22 & Al & 5.42 \\
\hline & & $\mathrm{Fe}$ & 0.848 \\
\hline Helium & 0.00518 & ${ }^{4} \mathrm{He}$ & 0.078 \\
\hline Graphite & 2.26 & C & 11.3 \\
\hline
\end{tabular}


Table 7-3. Fraction of source neutrons between direction cosines 1 and 2 .

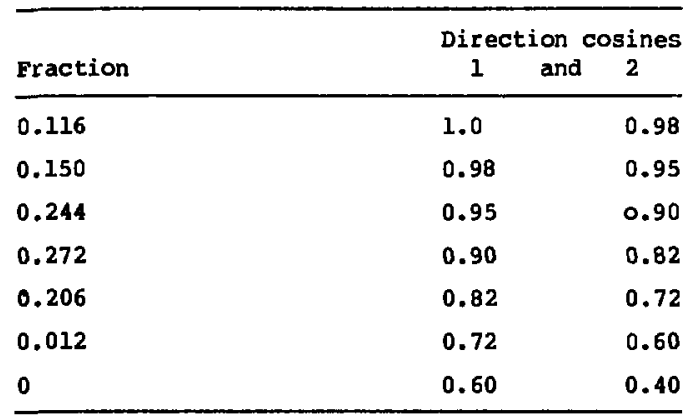

running time on the $C D C-7600$ was 4 min. Results have a statistical uncertainty (standard deviation) of approximately 58. Uncertainties in nuclear data were not considered.

\subsubsection{Transport Results}

7.1.4.1 Performance vs Fission zone Thickness. The independent blanket variable available for adjusting blanket tritium-breeding performance to match that required for a specific set of reactor parameters is fission $\left(\mathrm{U}_{3} \mathrm{Si}\right.$ ) zone length. In the range of interest, rast fission and, thus, neutron multiplication increase as the fission zone length increases, but the number of neutrons leaking into the tritium breeding zone decreases. The objective is to make the fission zone as thick as possible while still breeding the required tritium.

A series of Monte Carlo calculations were performed to determine the effects on blanket performance as fission-zone thickness is varied; total blanket length is held constant. Results of these calculations are listed in Table 7-4 and graphically displayed in Fig. 7-4.

7.1.4.2 Performance vs Exposure. With exposure to a current of 14-MeV neutrons, the isotopic composition of the blanket changes. This effect is particularly important in the fission zones where ${ }^{238} \mathrm{U}(n, y)$ and fission reactions result in the buildup of ${ }^{239} \mathrm{Pu}$ and fission products. Other transmutations also nccur but are of lesser importance. The isotopes followed are listed in Table 7-5 with the expressions used to calculate their atom densities at each exposure. 


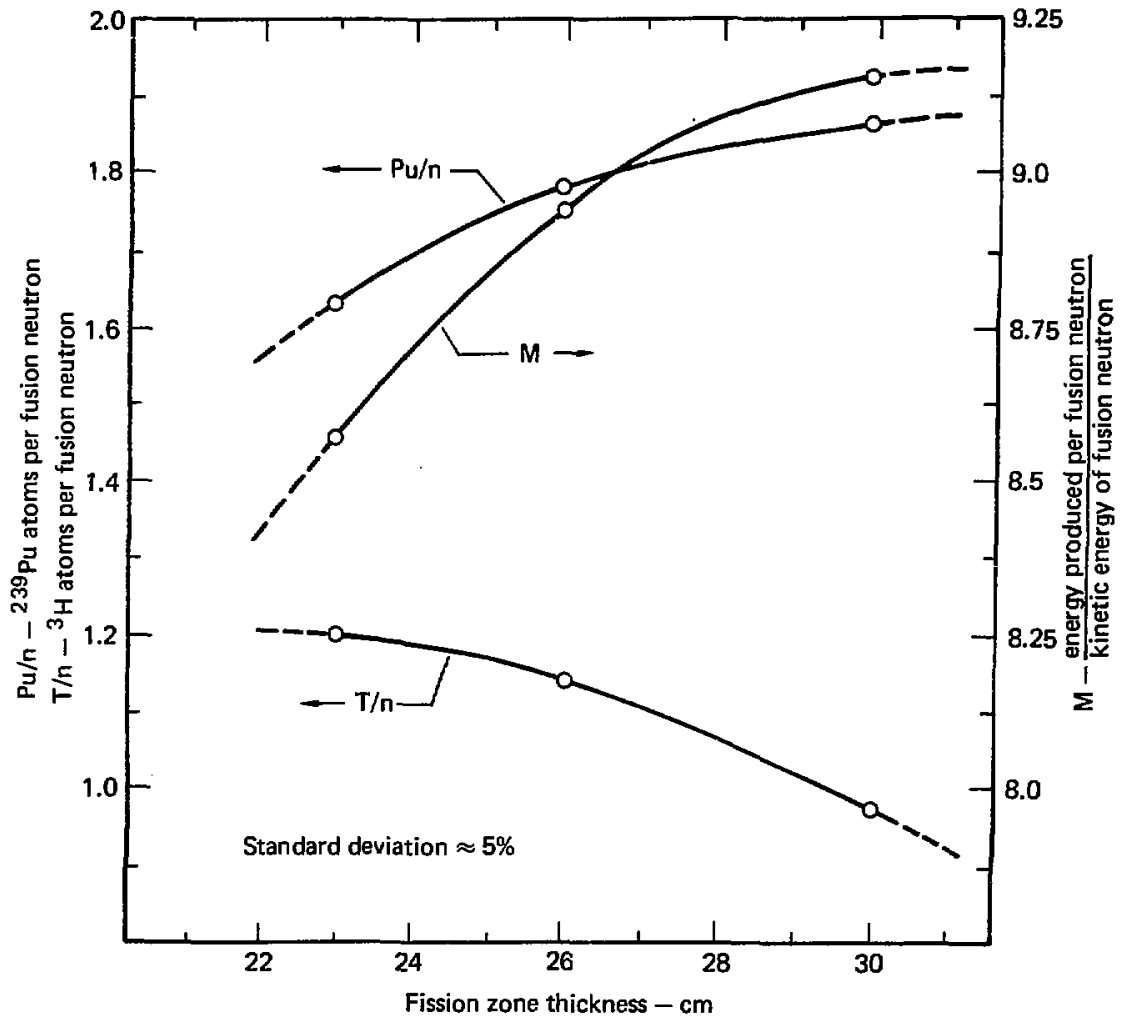

Fig. 7-4. Blanket performance tradeoffs - local start-of-life values for net plutonium breeding ratio $(\mathrm{Pu} / \mathrm{n})$, trittum breeding ratio $(\mathrm{T} / \mathrm{n})$, and energy multiplication $(M)$. 


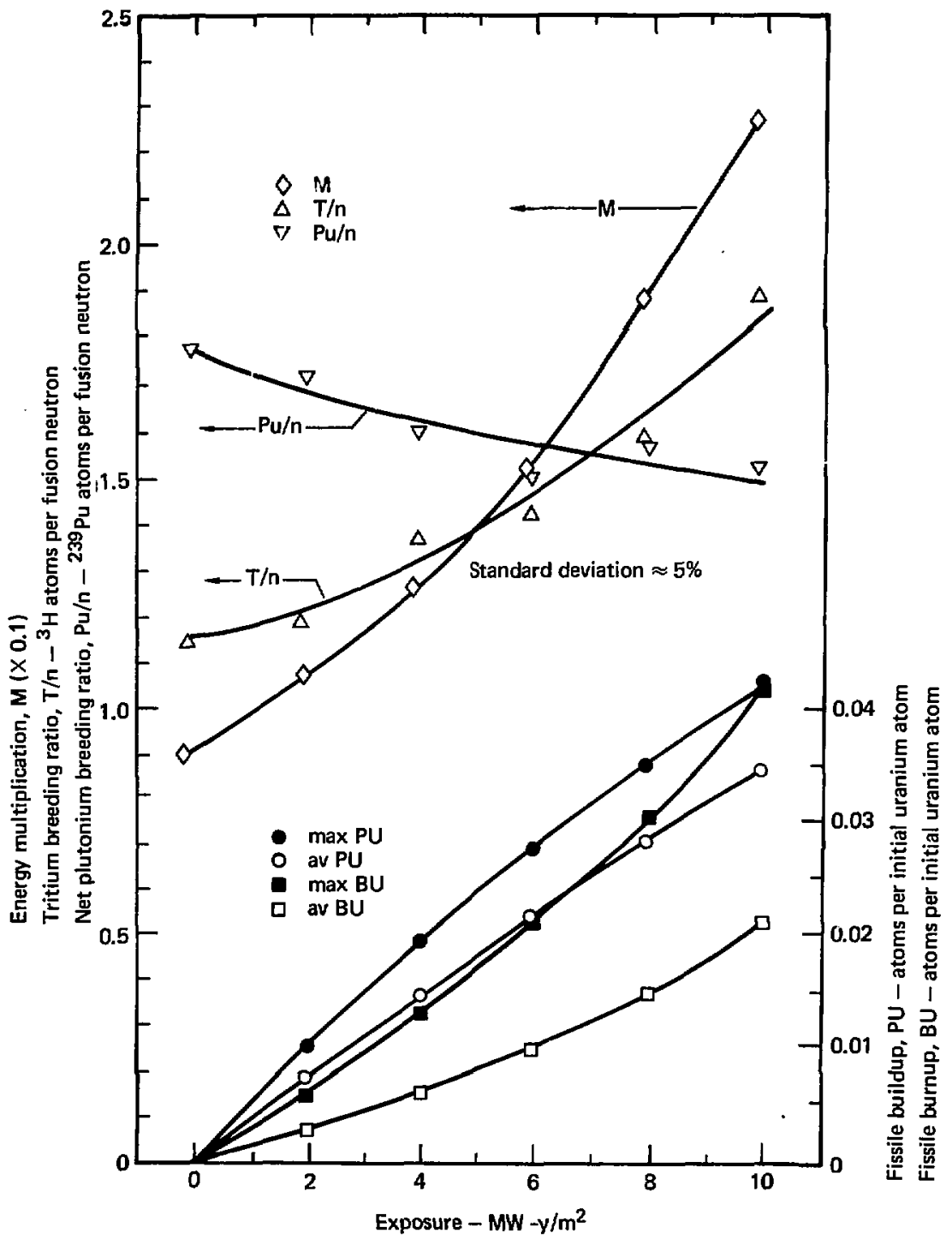

Fig. 7-5. Blanket performance (local) vs exposure. (The blanket has a 26-cin fission zone.) 
Table 7-4. Local " 0 " exposure blanket performance vs fission zone length $\left(L_{f}\right)$.

\begin{tabular}{lccr}
\hline$L_{\mathrm{f}}$ (metres) & $\mathrm{T} / \mathrm{n}(0)$ & $\mathrm{Pu} / \mathrm{n}(0)$ & M(0) \\
\hline 0.23 & 1.20 & 1.63 & 8.57 \\
0.26 & 1.14 & 1.78 & 8.94 \\
0.30 & 0.97 & 1.86 & 9.14 \\
\hline
\end{tabular}

(standard deviation $\approx 5 q$ )

Table 7-5. Isotopes and expressions to calculate their atom densities.

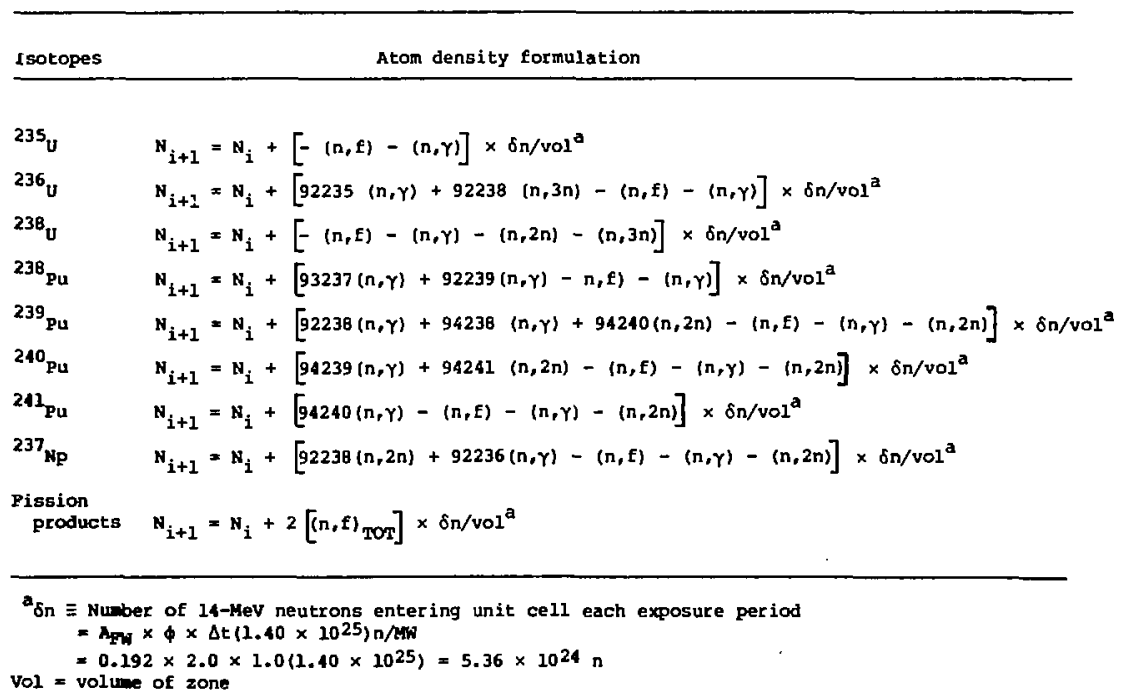

A series of Monte Carlo calculations were performed over an exposure range of 0 to $10 \mathrm{w}-\mathrm{y} / \mathrm{m}^{2}$ in steps of $2 \mathrm{k}-\mathrm{y} / \mathrm{m}^{2}$. After each step, the isotopic composition in each of the four $\mathrm{U}_{3} \mathrm{~S}_{1}$ zones are adjusted as shown in Table 7-5. Results of this procedure are listed in Table 7-6 and displayed graphically in Fig. 7-5. Exposure is in terms of integrated energy current [kinetic energy of the DT neutrons (14 MeV per neutron)] flowing through the surface of a sphere centered at the plasma center with a radius determined by 
Table 7-6. Results of exposure-dependent transport calculations.

\begin{tabular}{|c|c|c|c|c|c|c|}
\hline $\begin{array}{l}\text { Exposure, } \\
M N-y / m^{2}\end{array}$ & $\mathbf{T} / \mathbf{n}$ & $\mathrm{Pu} / \mathrm{n}$ & $\begin{array}{l}\qquad{ }^{239} \mathrm{Pu}(\mathrm{s} \\
\text { of } \mathrm{u} \text { initial), } \\
\text { peak/average }\end{array}$ & $\begin{array}{l}239_{\mathrm{pu}} \\
\mathrm{kg} / \mathrm{m}^{2}\end{array}$ & $\mathbf{M}$ & $\begin{array}{c}\text { Burnup ( } \% \text { of } U \text { initial) } \\
\text { peak/average }\end{array}$ \\
\hline 0 & 1.14 & 1.78 & $0 / 0$ & 0 & 8.94 & $0 / 0$ \\
\hline 2 & 1.18 & 1.72 & $1.00 / 0.1 / 8$ & 19.7 & 10.7 & $0.594 / 0.270$ \\
\hline 4 & 1.37 & 1.60 & $1.92 / 1.47$ & 38.7 & 12.6 & $1.30 / 0.600$ \\
\hline 6 & 1.42 & 1.48 & $2.72 / 2.14$ & 56.5 & 15.1 & $2.08 / 0.988$ \\
\hline 6 & 1.58 & 1.56 & $3.45 / 2.76$ & 72.9 & 18.6 & $3.01 / 1.47$ \\
\hline 10 & 1.88 & 1.51 & $4.17 / 3.41$ & 90.1 & 22.6 & $4.13 / 2.06$ \\
\hline
\end{tabular}

Table 7-7. Isotopic composition of plutonium after an exposure of $10 \mathrm{M}-\mathrm{y} / \mathrm{m}^{2}$.

\begin{tabular}{lc}
\hline Isotope & Percent \\
\hline 239 Pu & 96.7 \\
$240_{\text {Pu }}$ & 2.11 \\
$238_{\text {Pu }}$ & 1.23 \\
$241_{\text {Pu }}$ & 0.014 \\
\hline
\end{tabular}

the minimum distance of the blanket-module end cap from the plasma center (3.50 $\mathrm{m}$ in this case). This energy current is commonly called "wall loading" or "first wall loading" (WL) and usually has units of ( $\mathrm{HN} / \mathrm{m}^{2}$ ).

7.1.4.3 Plutonium Composition, The isotopic composition of the plutonium calculated for this blanket after an exposure of $10 \mathrm{~m}-\mathrm{y} / \mathrm{m}^{2}$ is listed below (Table 7-7).

7.1.4.4 Thermal Power Density. Volumetric heating varies with location and exposure. Spatial effects occur because the DT neutron source is external to the blanket and also because the blanket is composed of various materials. Exposure effects occur because the 1sotopic composition of the $\mathrm{U}_{3} \mathrm{si}$ fuel material changes.

Energy deposition per source neutron in each module zone was calculated at " 0 " exposure and after $10-1 w-y / m^{2}$ exposure using coupled neutron-gama transport. Then, the zone energy dispositions were used to calculate the average zone power densities in the materials in each zone at a given wall loading $(\phi)$ of $1.0 \mathrm{kN} / \mathrm{m}^{2}$. Results are listed in Table 7-8. 
Curves of power density profiles in the $\mathrm{U}_{3} \mathrm{Si}$ at 0 and $10 \mathrm{MH}-\mathrm{y} / \mathrm{m}^{2} \mathrm{ex}-$ posure are displayed in Fig. 7-6. These curves are fit to the zone-average values listed in Table 7-8. At start of life, peak power density in the $\mathrm{U}_{3} \mathrm{Si}$ is $105 \mathrm{~W} / \mathrm{cm}^{3}$ at $1.0 \mathrm{w} / \mathrm{m}^{2}$ wall loading. Peak-to-average $\mathrm{U}_{3} \mathrm{Si}$ power density at start of life is 2.17 . After an exposure of $10 \mathrm{NN}-\mathrm{y} / \mathrm{m}^{2}$ peak $\mathrm{U}_{3} \mathrm{si}$ power density is $244 \mathrm{~W} / \mathrm{cm}^{3}$ and the peak-to-average is 1.93 . The slight drop in the $\mathrm{U}_{3} \mathrm{si}$ peak-to-average power density with exposure is most likely due to the nearly even buildup and subsequent fission of ${ }^{239} \mathrm{Pu}$.

7.1.4.5 Neutron Flux and Fluence. Neution flux and fluence were calculated for three blanket-module zones over an exposure range of 0 to $10 \mathrm{~m}-\mathrm{y} / \mathrm{m}^{2}$. The three zones are:

1. The 2-mm-thick hemispherical first wall (Zone 9).

2. The $U_{3} \mathrm{Si}$ fuel-bundle base plate (zone 5) located behind $\mathrm{U}_{3} \mathrm{si}$ fuel zones.

3. The module support $r$ ing (zone 14), which is located at the rear of the module and is representative of the permanent structure on which the module is mounted.

Table 7-8. 'patial and exposure-dependent energy deposition and power tensities. $a$

\begin{tabular}{|c|c|c|c|c|c|}
\hline \multirow[b]{2}{*}{ zone } & \multirow[b]{2}{*}{ Description } & \multicolumn{2}{|c|}{$E / \pi, \operatorname{MeV}$} & \multicolumn{2}{|c|}{$\because \mathrm{P}, \omega / c^{3}$} \\
\hline & & at " 0 " exposure & at $10 \mathrm{~ms}-\mathrm{y} / \mathrm{m}^{2}$ & at $=0=$ exposure & at $10 \mathrm{~m} * \mathrm{y} / \mathrm{m}^{2}$ \\
\hline 1 & $\mathrm{v}_{3}$ Si zone 1 & 18.6 & 43.2 & 101.0 & 234.0 \\
\hline 2 & $v_{3}$ Si zone 2 & 63.9 & 170.0 & 62.9 & 167.0 \\
\hline 3 & $v_{3}$ si zone 3 & 27.4 & 80.1 & 27.4 & 78.8 \\
\hline 4 & $v_{3}$ si zone 4 & 3.0 & 10.0 & 16.1 & 53.8 \\
\hline $\mathbf{5}$ & Fission core structure & 0.236 & 0.382 & 1.26 & 2.04 \\
\hline 6 & LiH zone 1 & 1.09 & 1.89 & B.78 & 15.2 \\
\hline 7 & LiH zone 2 & 5.17 & 8.85 & 1.63 & 2.79 \\
\hline 8 & LiH zone 3 & 0.19 & 0.33 & 0.19 & 0.32 \\
\hline 9 & Pirst wall & 0.422 & 0.641 & 7.03 & 10.7 \\
\hline 10 & Inser pressure vessel & 0.574 & 0.892 & 2.98 & 4.63 \\
\hline 11 & Outer pressure vessel & 0.120 & 0.193 & 0.65 & 1.05 \\
\hline 12 & Be outlet pipe & 0.020 & 0.041 & 0.45 & 0.91 \\
\hline 13 & Graphite insert & 0.416 & 0.588 & 0.36 & 0.51 \\
\hline 14 & rodule support & 0.228 & 0. 707 & 0.17 & 0.23 \\
\hline \multirow[t]{2}{*}{15} & Fixed blanket structure & 0.106 & 0.157 & 0.03 & 0.05 \\
\hline & & 122.000 & 317.600 & & \\
\hline
\end{tabular}

wherial volumetric heating rates (average for xone) at a wil looding of $1.0 \mathrm{~m} / \mathrm{m}^{2}$;

$$
\ddot{p}=n \times A \times \frac{1}{v} \times \frac{E / n}{14.06} \times \frac{1}{v g}=13,630 \times E / n \times \frac{1}{v} \times \frac{1}{V}
$$


An axial profile of the total neutron flux at the beginning and end of life $\left(10 \mathrm{~m}-\mathrm{y} / \mathrm{m}^{2}\right)$ is given in Fig. 7-7. Neutron energy spectra in the three representative spatial zones at the beginning and end of life are show in Fig. 7-8. Figures 7-9 and 7-10 show total neutron flux and total neutron fluence vs exposure. Peak flux and fluence occur in the first wall at peak exposure. Fluence in the first wall after an exposure of $10 \mathrm{w}-\mathrm{y} / \mathrm{m}^{2}$ is $2.8 \times 10^{23} \mathrm{n} \cdot \mathrm{cm}^{-2} ; 17$ of this is supplied by neutrons with energies greater than $1.0 \mathrm{MeV}$. At the module's other end, the permanent aodule-support ring recelves a fluence of $1.8 \times 10^{22} \mathrm{n} \cdot \mathrm{cm}^{-2}$ during a $10 \mathrm{~m} / \mathrm{y} / \mathrm{m}^{2} \mathrm{ex}-$ posure; 20 of this is above $1.0 \mathrm{MeV}$. If the reactor operates for 40 years with a wall loading of $2 \mathrm{~m} / \mathrm{m}^{2}$ and a duty factor of $75 \%$, and the blanket wodules are removed after $10 \mathrm{w}-\mathrm{y} / \mathrm{m}^{2}$ exposure, the module support rings would have an end-of-life, fast neutron ( $E>1.0 \mathrm{MeV}$ ) fluences of $2.2 \times 10^{22} \mathrm{n} \cdot \mathrm{cm}^{-2}$.

The neutron flux and fluence results have standard deviations ranging from 2 at the first wall to 17 at the module support $r i n g$.

\subsubsection{Tritium}

7.1.5.1 Tritium Breeding Requirements. The required local tritiumbreeding ratio must be determined in order to determine the other principal blanket nuclear-performance parameters.

We require the hybrid to be self-sufficient in tritium, except for startup requirements. After equilibrium is established, blanket tritium breeding must fuel the D-T fusion reaction and make up for losses. The only significant loss anticiplated is by tritium decay. Tritium decays with a 12.33-year half-life, so tritium inventory (I) plays a significant role in setting tritium breeding requirements. The tritium inventory is comprised of two principal components, the inventory in the blanket system and that tied up in the rest of the reactor. The blanket tritium inventory is dependent on the blanket tritium removal and containment scenario employed. Two blanket tritium removal and containment scenarios are considered in this study: batch, and purge. With the batch scenario, tritium produced in the blanket is contained within the lithium-bearing fuel pins. After a blanket segment reaches the desired exposure, it is removed from the reactor, and the lithium fuel pins are processed to remove the tritium. With the purge scenario on the other hand, tritium is continuously removed from the blanket as it is produced via a purge gas system. In both scenarios the blanket is divided into refueling 


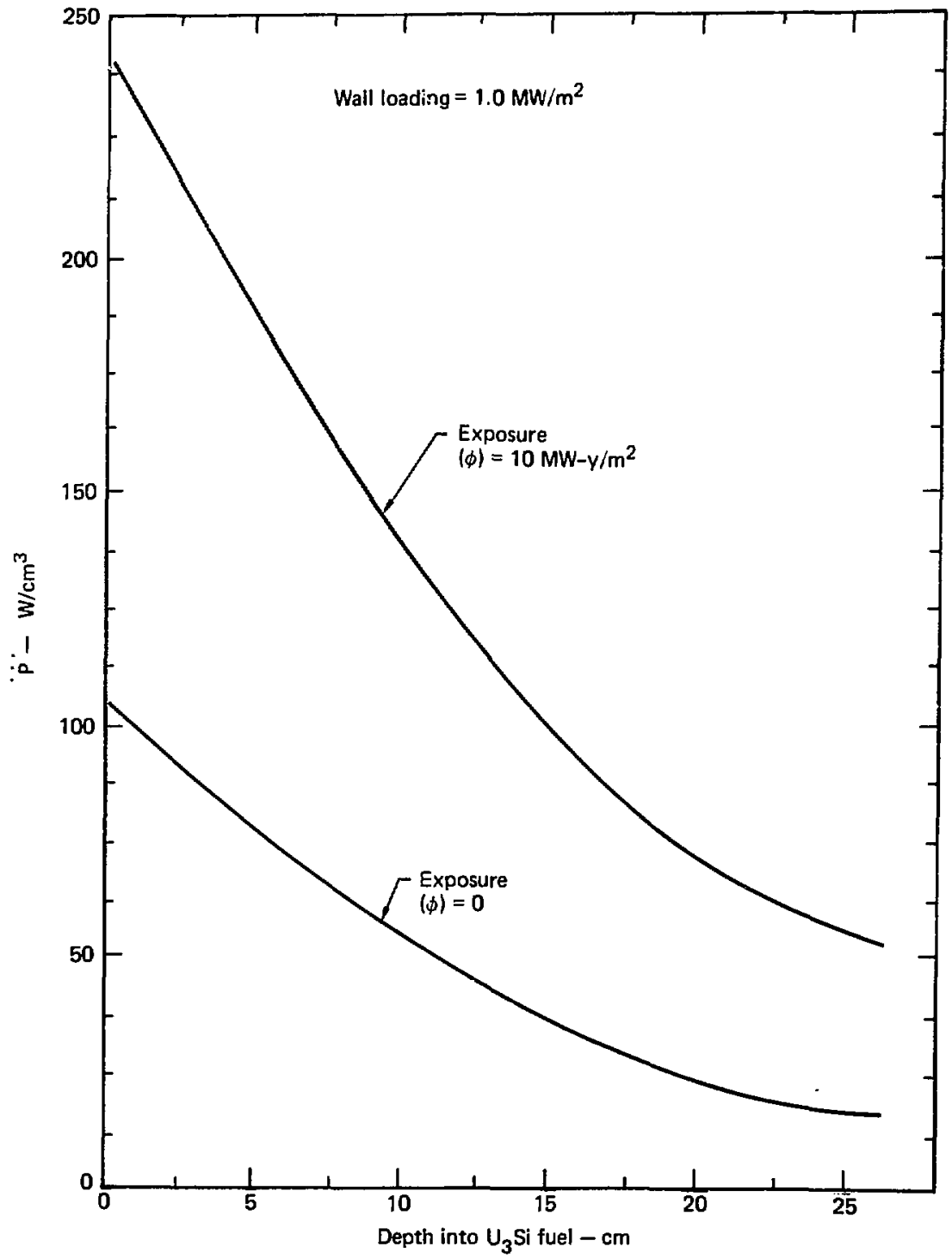

Fig. 7-6. Power density profiles in $\mathrm{U}_{3} \mathrm{si}$ fuel. 


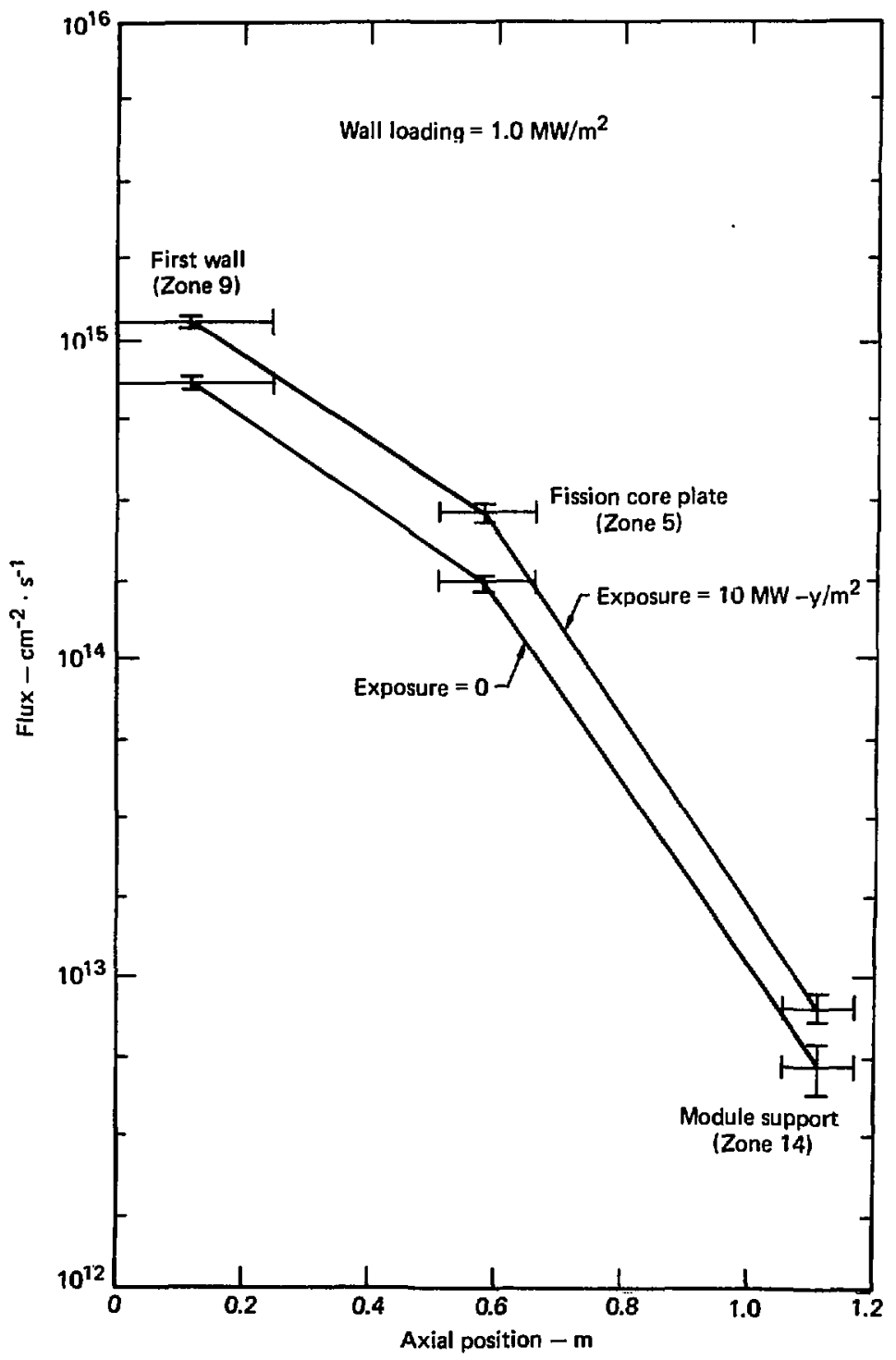

Fig. 7-7. Neutron flux (total) profile with a wall loading of $1.0 \mathrm{w} / \mathrm{m}^{2}$. 


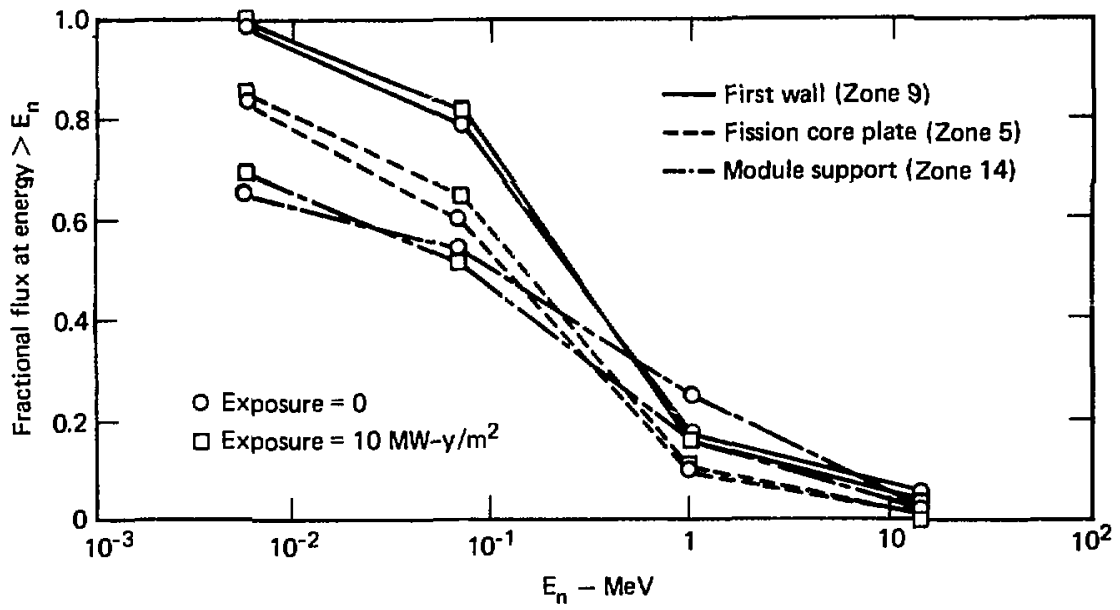

Fig. 7-8. Neutron spectra (fractional flux at energy $>E_{n}$ ). 


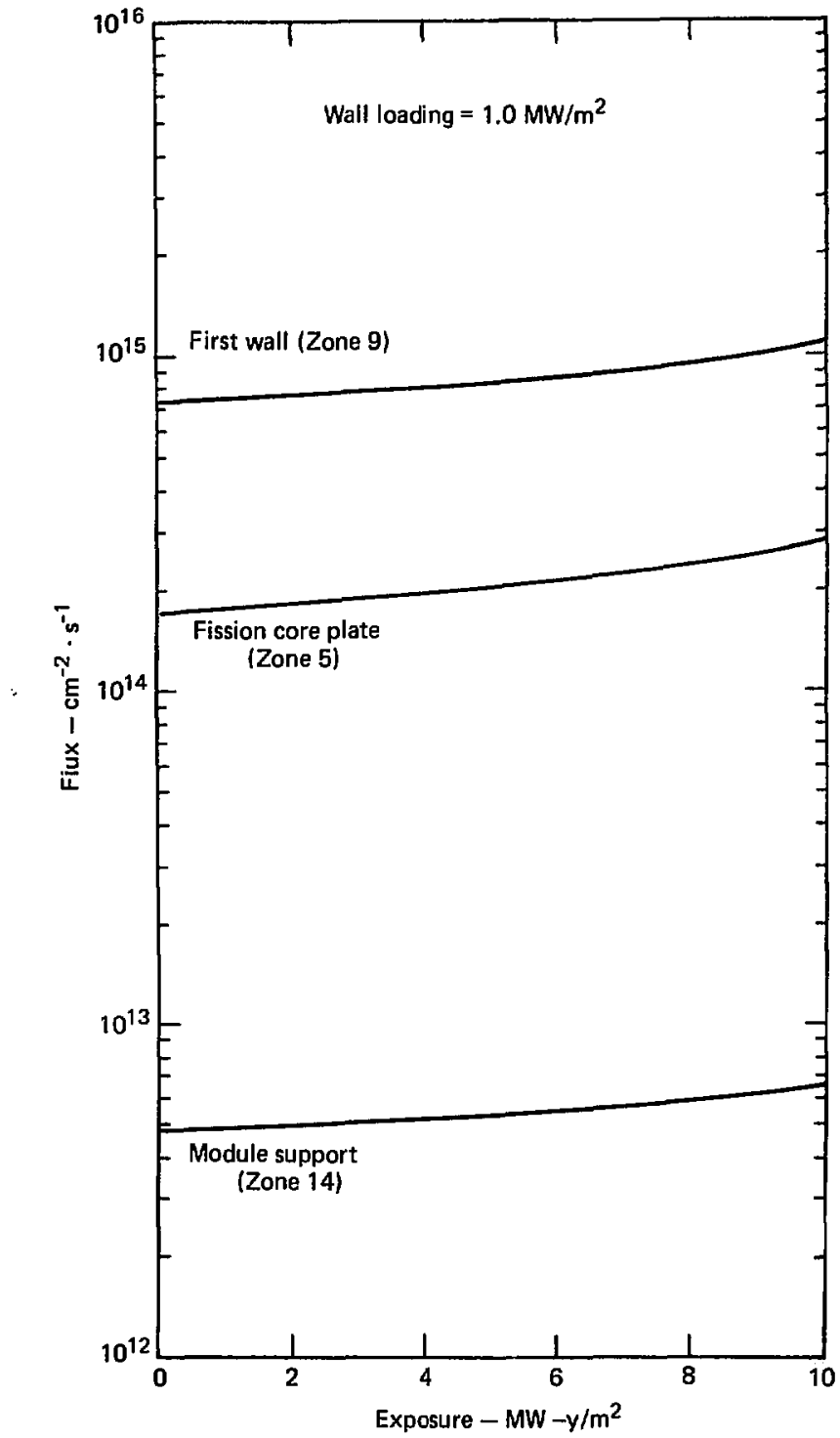

Fig. 7-9. Neutron flux (total) vs exposure with a wall loading of $1.0 \mathrm{~m} / \mathrm{m}^{2}$. 


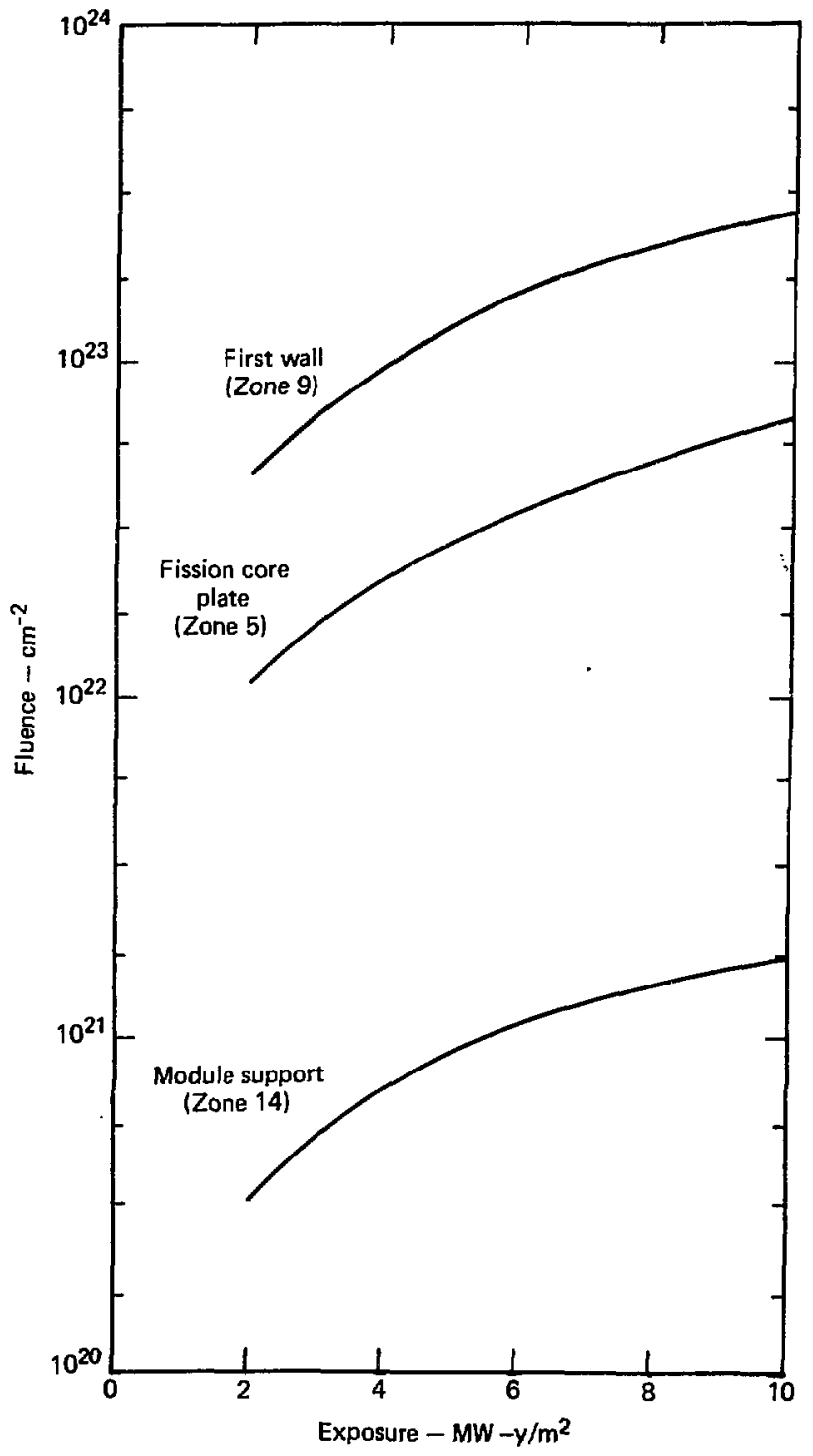

Fig. 7-10. Neutron fluence (total) vs exposure. 
segments. Blanket replacement is accomplished by sequentially replacing segments, one per operating period.

7.1.5.1.1 Batch. The method developed to calculate tritium breeding ratio needed for batch processing is derived below.

Tritium conta ined in a blanket segment at removal ( $\mathrm{T}_{b}$ ). must equal tritium consumed $\left(T_{c}\right)$ outside of the blanket during the time period ( $t_{p}$ ) until the next blanket segment is removed.

$$
\begin{aligned}
& \mathbf{T}_{\mathbf{b}}=\mathbf{T}_{\mathbf{c}} \\
& \mathbf{T}_{\mathbf{b}}=\mathbf{T}\left(t=t_{\mathbf{p}} \times \mathrm{BS}\right) .
\end{aligned}
$$

The rate of change of tritium contained in a blanket segment is

$$
\begin{aligned}
\frac{d T}{d t} & =\{T \text { breeding rate }\}-\{T \text { decay rate }\} \\
& =\left\{3 c_{1} \bar{P}_{n} \Omega \frac{1}{B S} \quad\left(T / n(0)+k_{4} \bar{\phi} t\right)\right\}-\{\lambda T\} .
\end{aligned}
$$

Solving this differential equation and applying the boundary condition $\mathbf{r}(0)=0$ gives

$T_{b}=\frac{3 C_{1} \bar{P} n^{\Omega}}{B S}\left\{T / n(0) \frac{\left(1-e^{-\lambda t} p^{B S}\right)}{\lambda}+\frac{k_{4} \bar{\phi}}{\lambda^{2}}\left[\left(\lambda t_{p} B S-1\right)+e^{-\lambda t_{p} B S}\right]\right\}$.

Tritium consumed oltside of the blanket $\left(T_{c}\right)$ during one fueling period ( $t_{p}$ ) equals the tritium on hand (excluding blanket) at $t=0$ minus the tritium remaining at $t \times t_{p^{*}}$. The rate of change of the tritium inventory (T) external to the blanket is

$$
\frac{d T}{d t} \times-\{T \text { burn rate }\}-\{T \text { decay rate }\}=-\left\{3 c_{1} \overline{\mathbf{P}}_{\mathrm{n}}\right\}-\{\lambda \mathrm{T}\}
$$

Solving this cifferential equation and applying the boundary condition $T\left(t_{p}\right)=I$ gives

$$
T_{c}=T(0)-I=\left(\frac{3 C_{1} \bar{P}_{n}}{\lambda}+I\right)\left(e^{\lambda t} p-1\right)
$$


Now since $T_{b}$ must equal $T_{c}$ for equilibrium,

$$
\underset{T / n(0)}{\text { (batch) }}=\frac{\frac{T_{c} B S}{3 C_{1} \bar{p}_{i 1}}-\frac{\bar{\phi} k_{4}}{\lambda^{2}}\left\{\left(\lambda t_{p} B S-1\right)+e^{-\lambda t_{p}^{B S}}\right\}}{\frac{\left(1-e^{\left.-\lambda t_{p}^{B S}\right)}\right.}{\lambda}} .
$$

This is the zero-exposure, local tritium-breeding ratio needed for a batchprocessed blanket to break even on tritium after blanket equilibrium is achieved. Equilibrium means that the average blanket exposure at the star.i of each operational period is the same.

7.1.5.1.2 Purge. The tritium breeding ratio needed for tritium selfsufficiency at equilibrium with a purged blanket is derived below.

The rate of change of the total tritium inventory (T) is

$$
\begin{aligned}
\frac{\mathrm{dT}}{\mathrm{dt}} & =-\left\{\begin{array}{c}
\mathrm{T} \text { burn } \\
\text { rate }
\end{array}\right\}-\left\{\begin{array}{c}
\mathrm{T} \text { decay } \\
\text { rate }
\end{array}\right\}+\left\{\begin{array}{c}
\mathrm{T} \text { breeding } \\
\text { rate }
\end{array}\right\} \\
& =-\left\{3 C_{1} \overline{\mathrm{P}}_{\mathrm{n}}\right\}-\{\lambda \mathrm{T}\}+\left\{3 \mathrm{C}_{1} \overline{\mathrm{P}}_{n} \Omega\left[\mathrm{T} / \mathrm{n}(0)+k_{4} \bar{\phi}\left(t+\mathrm{f}_{\mathrm{n}} \mathrm{t}_{\mathrm{p}}\right)\right]\right\},
\end{aligned}
$$

where $f_{n} t_{p}=$ average blanket exposure at beginning of each exposure period. For a blanket in equilibrium, $f_{n}=\frac{B S-1}{2}$

Solving for T gives:

$$
T(t)=A B / \lambda+A D / \lambda^{2}(\lambda t-1)+C e^{-\lambda t},
$$

where $A=3 C_{1} \vec{P}_{n}$

$$
\begin{aligned}
& B=-1+\Omega[T / n(0)]+D f_{n} t_{p} \\
& D=\Omega k_{4} \Phi \\
& C=\text { constant of integration. }
\end{aligned}
$$

The constant of integration $\mathrm{C}$ is evaluated by applying the equilibrium condition; total tritiun inventory at the beginning and end of each blanket refueling period must be equal: 


$$
\left(T(t=0)=T\left(t=t_{p}\right)\right) \text {. }
$$

Therefore,

$$
T(t)=A B_{e q} / \lambda+A D / \lambda^{2}\left\{(\lambda t-1)+\lambda t_{p} \frac{e^{-\lambda t}}{1-e^{-\lambda t} p}\right\} .
$$

The next step is to solve for $B_{e q}$ and, thus, $T / n(0)$. This is done by using the fact that the total tritium inventory $T(t)$ cannot drop below the reactor tritium hold-up inventory I' (I' is all the tritium held up in various reactor systems, such as crsppanels and distillation column, plus that in the blanket). Therefore,

$$
T_{\min }=I^{\prime}
$$

$T_{\text {min }}$ occurs (at $t_{\min }$ ) when the time derivative of $T(t)$ equals 0 ; therefore,

$$
t_{\text {min }}\left(\frac{d T}{d t}=0\right)=\frac{1}{-\lambda} \ln \left(\frac{1-e^{-\lambda t} p}{\lambda t_{p}}\right) \text {. }
$$

Now set $T\left(t_{\text {nin }}\right)=I$ ' to solve for the value of $B$ needed for equilibrium. Call this value of $B, B_{\text {eq }}$ (purge):

$$
B_{e q}=\frac{\lambda}{A}\left\{I^{\prime}-A D / \lambda^{2}\left[\left(\lambda t_{n}-1\right)+\frac{\lambda t_{p} e^{-\lambda t_{m}}}{1-e^{-\lambda t_{p}}}\right]\right\} \text {. }
$$

Now solve for the zero-exposure, local tritium-breeding ratio $[\mathrm{T} / \mathrm{n}(0)]$ needed for equilibrium:

$$
\underset{\text { (purge) }}{T / n(0)}=\frac{1}{\Omega}\left\{\left(B_{\text {eq }}+1\right)-D\left(\frac{B S-1}{2}\right) t_{p}\right\} .
$$

7.1.5.2 Tritiun Purchase Requirements. Tritium must be supplied from an external source for the initial phase of reactor operation before blanket equilibrium is achieved. Once blanket equilibrium is achieved, the reactor produces all its ow tritium. (Recall that tritium self-sufficiency at equilibrium is the condition used to set the tritium breeding ratio needed.) The method of blanket tritium rewoval (batch or purge) strongly influences the 
tritium purchase requirements. The derivation of expressions used to determine tritium purchase requirements for batch and purge blanket tritium renoval are outlined below.

7.1.5.2.1 Batch. With batch tritium removal, tritium is held up in the blanket segments until the segments are replaced. One blanket segment is replaced after each operational period. Under equilibrium conditions, a blanket segment contains on removal just enough tritium to supply the reactor for the next operational period. During start-up, this is not the case, and tritium must be purchased. The scenario used to determine tritium purchase requirements is:

- Tritium on hand at the start of each period must equal all tritium consumed $\left(T_{C}\right.$ ) during that period.

- Tritium purchase $T_{p}(n)$ needed for period (n) equals $T_{c}$ minus the tritium $\left(T_{b}\right)$ contained in the blanket segment removed after the preceding period. The first period requires an additional purchase of $I$, the plant tritium hold-up inventory.

- Tritium purchases take place only at the beginning of a period. The start-up blanket segments are physically identical to the equilibrium segments.

Therefore, tritium purchase needed for the first period is

$$
\mathbf{T}_{\mathrm{p}}(\mathrm{n}=\mathrm{l})=\mathbf{T}_{\mathrm{c}}+\mathrm{I} \text {. }
$$

Period 2 through BS requires:

$$
T_{p}(n)=T_{c}-T_{b}(n-1)
$$

where

$$
T_{c}=\left(\frac{3 c_{1} \bar{P}_{n}}{\lambda}+I\right)\left(e^{\lambda t_{p}}-1\right)
$$

and

$$
\begin{gathered}
T_{b}(n-1)=\frac{3 C_{1} \bar{P}_{n} \Omega}{B S}\left\{T / n(0)\left(\frac{1-e^{-\lambda t_{p}(n-1)}}{\lambda}\right)+\frac{\bar{\phi}_{k}}{\lambda^{2}}\left(\left[\lambda t_{p}(n-1)-1\right]\right.\right. \\
333 \\
\left.\left.+e^{-\lambda t_{p}(n-1)}\right)\right\}
\end{gathered}
$$


The derivation of $T_{c}$ and $T_{b}$ are outlined in Section 7.1.5.1.1.

7.1.5.2.2 Purge. Fith a purge tritium system, tritium is purged from the blanket as it is being produced, and thus is imnediately available for use. All other aspects of the blanket handling are the same as with the batch case.

The scenario for deternining tritium purchase requirements is:

- Tritium left over at the end of each period $\left\{T_{n}\left(t=t_{p}\right)\right\}$ is set equal to tritlum left over at the end of each equilibrium period $\left\{T_{\text {eq }}\left(t=t_{p}\right)\right\}$.

- Tritium needed at the beginning of each period $\left\{T_{n}(t=0)\right\}$ can now be solved by using the boundary condition

$$
T_{n}\left(t=t_{p}\right)=T_{e q}\left(t=t_{p}\right) .
$$

- The tritiun purchase requirement $\left\{T_{n} \mid\right.$ for period $n$ is

$$
T P_{n}=T_{n}(t=0)-T_{\text {left over }(n-1)} \text {. }
$$

By using the expression $T(t)$ derived in Section 7.1.5.1.2 and the boundary condition $T_{n}\left(t=t_{p}\right)=T_{\text {eq }}\left(t=t_{p}\right)$ to solve for $T_{n}(t=0)$,

$T_{n}(t=0)=\frac{A B_{n}}{\lambda}\left(1-e^{\lambda t_{p}}\right)+\frac{A D}{\lambda^{2}}\left[-1-\left(\lambda t_{p}-1\right) e^{\lambda t_{p}}\right]+\left[T_{e q}(t=t)\right] e^{\lambda t_{p}}$.

For first period $(n=1), T_{\text {left over }(n-1)}=0$.

For periods $n>1, T_{\text {left over }(n-1)}=T_{\text {eq }}\left(t=t_{p}\right)$.

To solve for $T_{e q}\left(t=t_{p}\right)$, we use Eq. (9) for $T(t)$ :

$$
T_{e q}\left(t=t_{p}\right)=\frac{A B}{\lambda}+\frac{A D}{\lambda^{2}}\left\{\left(\lambda t_{p}-1\right)+\frac{\lambda t_{p}}{\lambda t_{p}-1}\right\} \text {. }
$$

Substituting into Eq. (17) for $\mathrm{TP}_{n}(1<\mathrm{n}>\mathrm{BS})$ gives

$$
\mathbf{T P}_{\mathbf{n}}=\frac{\mathbf{A}}{\lambda}\left(B_{n}-B_{e q}\right)\left(1-e^{\lambda t} p\right)
$$


Because $B_{n}=-1+(T / n(0))+D f_{n} t_{p}$

$$
f_{n}=\frac{B S(B S-1)-(B S-n)(B S-n+1)}{2 B S},
$$

and $B_{\text {eq }}=B_{n}$, where $n=B S$ (from Section 7.1.5.1.2)

For $n=2$ through BS,

$$
T_{n}=\frac{A}{\lambda}\left(-\frac{(B S-n)(B S-n+1)}{2 B S}\right) D t_{p}\left(1-e^{\lambda t_{p}}\right),
$$

and, for $n=1$,

$$
T P=\frac{A}{\lambda}\left(-\frac{(B S-1)}{2}\right) D t_{p}\left(1-e^{\lambda t_{p}}\right)+T_{e q}\left(t=t_{p}\right)
$$

\subsubsection{Scaling}

Fusion power, first-wall radius, blanket coverage, blanket segmentation, blanket life time, tritium handling, tritium inventory, and plant-capacity factors all influence blanket performance. These reactor variables are adjusted in a system code to find the optimum design point. To optimize blanket performance for a given reactor design point, an snalytic model approxinating blanket performance is used. The model is based on results from the transport calculations. The model consists of analytical expressions fit to the transport results. They are:

- Iocal energy multiplication vs exposure:

$$
M(\Phi)=M(0)+k_{1} \Phi+k_{2} \Phi^{2}
$$

- Local net ${ }^{239} \mathrm{Pu}$ breeding ratio vs exposure:

$$
\mathrm{Pu} / \mathrm{n}(\Phi)=\mathrm{Pu} / \mathrm{n}(0)+\mathrm{k}_{3} \Phi
$$

- Local tritium breeding ratio vs exposure:

$$
T / n(\Phi)=T / n(0)+k_{4} \Phi \text {. }
$$

- Maximun thermal power density in $\mathrm{U}_{3} \mathrm{Si}$ fuel vs exposure and wall loading $\left(\mathrm{U}_{3} \mathrm{Si}\right.$ power density in zone 1$)$ : 


$$
\ddot{P}_{\text {fuel max }}(\Phi, \phi)=\left(k_{5}+k_{6} \Phi+k_{7} \Phi^{2}\right) \Phi
$$

- Maximum burnup in $\mathrm{U}_{3} \mathrm{Si}$ fuel vs exposure (ratio (t) of fissions to initial uranium atoms in zone 1 ):

$$
\mathrm{BU}_{\max }(\Phi)=k_{18} \Phi+k_{19} \Phi^{2}
$$

- Fission zone length vs local-"0" exposure tritium breeding ratio (evaluation of $T / n(0)$ discussed in previous section):

$$
L_{f}[T / n(0)]=k_{B}+k_{g} T / n(0)+k_{10} T / n(0)^{2}
$$

- Local "0" exposure net ${ }^{239} \mathrm{Pu}$ breeding ratio, energy multiplication and uranium loading vs fission zone length:

$$
\begin{aligned}
& \mathrm{Pu} / \mathrm{n}(0)=k_{11}+k_{12} L_{f}+k_{13} L_{f}^{2} \\
& M(0)=k_{14}+k_{15} L_{f}+k_{16} L_{f}^{2} \\
& \text { J loading }=k_{17} L_{f} \pi R_{F f}^{2} \Omega
\end{aligned}
$$

Average fractional burn-up (* fission) of heavy metal in $\mathrm{U}_{3} \mathrm{SI}$ fuel bundle vs exposure:

$$
\overline{B U}(8)(\Phi)=\frac{11.03}{k_{17_{f}} L_{f}}\left\{[H(0)-1] \Phi+\frac{k_{1}}{2} \Phi^{2}+\frac{k_{2}}{3} \Phi^{3}\right\} .
$$

- Average fractional buildup of (tenrichment) ${ }^{239} \mathrm{Pu}$ in $\mathrm{U}_{3} \mathrm{si}$ fuel bundle vs exposure:

$$
\overline{\mathrm{Pu}}(\mathrm{\varepsilon})(\Phi)=\frac{554.5}{\mathrm{k}_{17} \mathrm{~L}_{f}}\left\{\mathrm{Pu} / \mathrm{n}(0) \Phi+\frac{\mathrm{k}_{3}}{2}, \Phi^{2}\right\} \text {. }
$$

- ${ }^{239} \mathrm{Pu}$ production $(\mathrm{kg} / \mathrm{y})$ at equilibrium conditions:

$$
\mathrm{Pu} / Y=69.68 \frac{\mathrm{R}_{\mathrm{EH}}^{2} \Omega}{\mathrm{BSt}_{\mathrm{p}}}\left\{\mathrm{Pu} / \mathrm{n}(0) \phi^{\prime}+\frac{\mathrm{k}_{3}}{2} \phi^{\prime 2}\right\}
$$




$$
\text { where } \Phi^{\prime} \equiv \begin{aligned}
& \text { exposure of blanket } \\
& \text { segment at removal }
\end{aligned}=\frac{P_{f^{0.8}}}{4 \pi R_{F w}^{2}} \text { BSt duty factor. }
$$

- Average blanket energy multiplication (M), net ${ }^{239}$ pu breeding ratio $(\mathrm{Pu} / \mathrm{n})$, and tritium breeding ratio $(\mathrm{T} / \mathrm{n})$ at equilibrium:

$$
\begin{aligned}
& M_{a v}=\left\{M(0)+\frac{k_{1}}{2} \Phi^{\prime}+\frac{k_{2}}{3} \Phi^{\prime}\right\}^{2} \Omega \\
& (P u / n)_{a v}=\left\{P u / n(0)=\frac{k_{3}}{2} \Phi^{\prime}\right\} \Omega \\
& (T / n)_{a v}=\left\{T / n(0)+\frac{k_{4}}{2} \Phi^{\prime}\right\} \Omega .
\end{aligned}
$$

Peak blanket energy multiplication loccurs at end of each equilibrium period) :

$$
M_{\max e q}=\Omega\left\{M(0)+\frac{1}{B S} \sum_{n=1}^{B S} k_{1}\left(\frac{\Phi^{\prime} n}{B S}\right)+k_{2}\left(\frac{\Phi^{\prime} n}{B S}\right)^{2}\right\} .
$$

- Minimum equilibrium blanket energy multiplication (occurs at beginning of each equilibrium period):

$$
M_{\min } \text { eq }=\Omega\left\{M(0)+\frac{1}{B S} \sum_{n=1}^{B S-1} k_{1}\left(\frac{\Phi^{\prime} n}{B S}\right)+k_{2}\left(\frac{\Phi^{\prime} n}{B S}\right)^{2}\right\} \text {. }
$$

- Minimun blanket energy multiplication (occurs at reactor start-up):

$$
M_{\min }=\Omega M(0)
$$

The constants $\left(k_{1}-k_{19}\right)$ are unique to a particular blanket module design; $k$ values for this design are listed in Table 7-9. With these constants, the analytic model of blanket performance is valid for exposures up to $10 \mathrm{wh}-\mathrm{y} / \mathrm{m}^{2}$ and for $\mathrm{U}_{3} \mathrm{St}$ bundle length in the range 0.23 to $0.30 \mathrm{~m}$.

\subsubsection{Sample Cases}

Blanket performance is calculated for two sample cases using the scaling relationships in the previous section. The reactor parameters for case 1 are show in Table 7-10. 
Table 7-9. Constants (k's) for analytical model of blanket.

$$
\begin{aligned}
& k_{1}=0.503, k_{2}=0.0867 \\
& k_{3}=-0.0306 \\
& k_{4}=0.0537 \\
& k_{5}=101, k_{6}=3.80, k_{7}=0.95 \\
& k_{8}=-0.7444, k_{9}=2.193, k_{10}=-1.151 \\
& k_{11}=2.083, k_{12}=26.00, k_{13}=-42.86 \\
& k_{14}=-0.5314, k_{15}=63.67, k_{16}=-104.8 \\
& k_{17}=10,182 \\
& k_{18}=0.247, k_{19}=0.0166
\end{aligned}
$$

Table 7-10. Reactor parameters for sample case 1.

Fusion power

Radius of blanket first wall

First wall loading

Blanket refueling segments

Time between shut downs for refueling

Blanket tritium handling

Tritiun inventory ( $I$ ')

Plant capacity factor (life-time average)

Blanket coverage
$400 \mathrm{MH}$

$3.57 \mathrm{~m}$

$2.0 \mathrm{mw} / \mathrm{m}^{2}$

4

$1.5 \mathrm{Y}$

off-line batch

$4 \mathrm{~kg}$

0.7

0.85

The reactor parameters for case 2 are the same as for case 1, except that tritiun bred in the blanket is continuously removed by an on-line purge systen. The results for both sample cases are shown in Table 7-11. 
Table 7-11. Results of sample cases.

\begin{tabular}{|c|c|c|}
\hline & $\begin{array}{l}\text { Case } 1 \\
\text { (T-batch) }\end{array}$ & $\begin{array}{l}\text { Case } 2 \\
\text { (T-purge) }\end{array}$ \\
\hline $\begin{array}{l}\text { Local zero-exposure tritium-breeding } \\
\text { ratio }[\mathrm{T} / \mathrm{n}(0)] \text { needed for tritium } \\
\text { self-sufficiency after start-up. }\end{array}$ & 1.21 & 0.97 \\
\hline $\begin{array}{l}\text { Fission zone length }\left(L_{f}\right\rangle \text { needed to } \\
\text { get }[T / n(0)], \text { m }\end{array}$ & 0.23 & 0.30 \\
\hline \multicolumn{3}{|l|}{$\begin{array}{l}\text { Tritium purchase requirements for } \\
\text { start-up periods, } \mathrm{kg}\end{array}$} \\
\hline Period: 1 & 28.4 & 7.78 \\
\hline 2 & 18.4 & 1.75 \\
\hline 3 & 12.3 & 0.58 \\
\hline 4 & 6.14 & $\underline{0}$ \\
\hline Totals & 65.2 & 10.1 \\
\hline \multicolumn{3}{|l|}{$\begin{array}{l}\text { Local instantaneous blanket performance } \\
\text { at zero exposure: }\end{array}$} \\
\hline${ }^{239}$ Pu-breeding ratio $[\mathrm{Pu} / \mathrm{n}(0)]$ & 1.62 & 1.86 \\
\hline Energy multiplication $\mathbf{M}(0)$ & 8.57 & 9.14 \\
\hline Tritium-breeding ratio $T / n(0)$ & 1.21 & 0.97 \\
\hline Maximum exposure, $M w_{n}-y / m^{2}$ & 8.40 & 8.40 \\
\hline \multicolumn{3}{|l|}{$\begin{array}{l}\text { Local instantaneous blanket performance } \\
\text { at maximum exposure: }\end{array}$} \\
\hline Tritium-breeding ratio & 1.66 & 1.42 \\
\hline Net ${ }^{239}$ Pu-breeding ratio & 1.37 & 1.60 \\
\hline Snergy multiplication & 18.9 & 19.5 \\
\hline Uranium loading, kg & $3.9 \times 10^{5}$ & $4.16 \times 10^{5}$ \\
\hline
\end{tabular}




\begin{tabular}{|c|c|c|}
\hline & $\begin{array}{l}\text { Case } 1 \\
\text { (T-batch) }\end{array}$ & $\begin{array}{l}\text { Case } 2 \\
\text { (T-purge) }\end{array}$ \\
\hline \multicolumn{3}{|l|}{$\begin{array}{l}\text { Burn-up at maximum exposure } \\
\text { (8 of heavy metal fissioned) }\end{array}$} \\
\hline Average & 1.73 & 1.39 \\
\hline \multicolumn{3}{|l|}{$\begin{array}{l}\text { Build-up of }{ }^{239} \mathrm{Pu} \text { at maximum } \\
\text { exposure, of initial heavy metal }\end{array}$} \\
\hline Average & 2.99 & 2.64 \\
\hline Peak (in $\mathrm{U}_{3} \mathrm{Si}$ zone 1 ) & 3.65 & 3.65 \\
\hline \multicolumn{3}{|l|}{$\begin{array}{l}\text { Maximum fuel power density } \\
\left(\mathrm{U}_{3} \mathrm{Si} \text { zone } 1\right), \mathrm{H} / \mathrm{cm}^{3}\end{array}$} \\
\hline At zero exposure & 202 & 202 \\
\hline At maximum exposure & 400 & 400 \\
\hline \multicolumn{3}{|l|}{ Blanket energy multiplication: } \\
\hline At initial start-up & 7.28 & 7.77 \\
\hline At start of equilibrium periods & 10.1 & 10.6 \\
\hline At end of equilibrium periods & 12.0 & 12.5 \\
\hline \multicolumn{3}{|l|}{$\begin{array}{l}\text { Effective equilibrium blanket } \\
\text { performance (time-averaged values) }\end{array}$} \\
\hline Tritiun-breeding ratio & 1.22 & 1.01 \\
\hline Net ${ }^{239}$ pu-breeding ratio & 1.28 & 1.47 \\
\hline Energy multiplication & 10.8 & 11.3 \\
\hline Net ${ }^{239} \mathrm{Pu}$ production, $\mathrm{kg} / \mathrm{y}$ & 1590 & 1830 \\
\hline
\end{tabular}




\subsection{Shielding}

Magnets, injectors, direct converters, and personnel must be protected from the intense radiation produced by the DT plasma. Various forms of shielding are provided for this purpose.

\subsubsection{Magnet Shielding}

The superconducting Yin-Yang colls must be shielded to:

- Limit total heating in the cryogenic coils and structure.

- Limit local maximum heating density in the coil windings to a value that can be removed by the coil coolant.

- Limit radiation damage of the coil materials. The geometry of the mirror reactor calls for a shield configured as a spherical shell behind the blanket plus anular disks extending through the mirror slots. Shielding is also needed around neutral beam lines as they pass through the coil structure.

Because of the dependence of coil size and blanket coverage on coil-shield thicknesses, an analytic model of the coil shield is included in the reactor- system computer code. In this model, shielding for the Yin-Yang magnet consists of a spherical shell located between the blanket and the coils plus disks extending through the coils on the sides of the mirror leakage fans. These shields are sized to optimize the trade-off between cryogenic power requirements and blanket coverage. Total heating in the cryogenic coil and structure optimized at $\sim 3 \times 10^{-5}$ of the fusion neutron power; $1 / 3$ of this heating occurs behind the spherical shield and $2 / 3$ behind the disk shields. The analytical expressions used to size these shields are

$$
\begin{aligned}
& t_{s}=-\mu_{s-1} \log \left(\frac{P_{s} / P_{n}}{A_{b} \alpha / 4 \pi}\right) \\
& t_{d}=-\mu_{d-1} \log \left(\frac{P_{s} / P_{n}}{\beta / 4 \pi}\right),
\end{aligned}
$$


$t_{s}$ and $t_{d} \equiv$ shield thicknesses needed to limit magnet heating (coil and cold structure) behind the shields to $P_{g} / P_{n}$ and $P_{d^{\prime}} / P_{n}$.

$\mu_{s-1}$ and $\mu_{d-1} \equiv$ shield thicknesses needed to give 1 decade of attenuation.

$P_{n} \equiv$ fusion neutron power level (MT).

$\mathbf{P}_{\mathbf{s}} \equiv$ magnet $n+\gamma$ heating behind the spherical shield (Mif).

$\mathbf{P}_{\mathbf{d}} \equiv$ magnet $\mathrm{n}+\boldsymbol{\gamma}$ heating behind the disk ahield (wW).

$A_{b} \equiv$ blanket attenuation (0.1 is a conservative value).

$\alpha \equiv$ solid angle subtended by the magnet (coil and cold structure) behind the spherical shield.

$B \equiv$ solid angle subtended by the magnet (coil and cold structure) behind the disk shield.

The coil shield thicknesses are checked by the following expressions to see if they limit local heating and neutron fluences to acceptable levels. Maximum specific power densities:

$$
\begin{aligned}
& \dot{\mathrm{P}}_{s}=\frac{B_{n}}{4 \pi R_{c}^{2}} K_{s-2} A_{b} \cdot 10^{-\left[\left(t_{s}+t_{c a s e}\right) / \mu_{s-2}\right]} \\
& \dot{\mathrm{p}}_{\mathrm{d}}=\frac{\mathrm{P}_{\mathrm{n}}}{4 \pi \mathrm{R}_{\mathrm{c}}^{2}} k_{\mathrm{d}-2} \cdot 10^{-\left[\left(t_{\mathrm{d}}+\mathrm{t}_{\text {case }}\right) / \mu_{\mathrm{d}-2}\right]} \text {. }
\end{aligned}
$$

Maximum fluences:

$$
\begin{aligned}
& \Phi_{s}=\frac{P_{n} T}{4 \pi R_{c}^{2}} K_{s-3} A_{b} \cdot 10-\left[\left(t_{s}+t_{c a s e}\right) / \mu_{s-3}\right] \\
& \Phi_{d}=\frac{P_{n}^{T}}{4 \pi R_{c}^{2}} K_{d-3} \cdot 10-\left[\left(t_{d}+t_{c a s e}\right) / \mu_{d-3}\right]
\end{aligned}
$$


where

$R_{c} \equiv$ coil inner radius $(m)$.

$k_{s}$ and $k_{d} \equiv$ proportionality constants

$T \equiv$ effective operating time.

$t_{\text {case }} \equiv$ thickness of magnet coil case $(\mathrm{cm})$.

The $\mu$ and $K$ values used with these expressions are listed in

Table 7-12. They are derived from the coil-shield calculations done for the FY 1976 Mirror Reactor Study. ${ }^{12}$ The shleld modeled by these values consists of iron, graphite and lead-loaded concrete. To demonstrate this model, parameters for the reference hybrid reactor are outlined in Table 7-13.

Both the local $n+\gamma$ heating and end-of-life neutron-fluence levels are well within the anticipated materials capabilities and coil design. Ullmaier ${ }^{13}$ reports that after a fluence of $1.3 \times 10^{18} \mathrm{n} / \mathrm{cm}^{2}$, the critical current in NbTi drops only about 208, while resistivity of the copper increases about six times. Since $j^{2} p=0.13 \mathrm{w} / \mathrm{cm}^{3}$ in the unirradiated copper and the heat-transfer limit is $2.6 \mathrm{~W} / \mathrm{cm}^{3}$, an increase of six times in copper's resistivity is well within the design capability of the coil. Direct local $n+\gamma$ heating is insignificant.

Table 7-12. Values of $\mu$ and $k$ used in expressions that check coil-shield thicknesses for their ability to limit local heating and neutron fluences.

\begin{tabular}{|c|c|c|c|}
\hline Expression & $\mu, \mathrm{cm}$ & K & \\
\hline$t_{s}$ & 17.3 & - & \\
\hline$t_{d}$ & 17.5 & 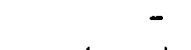 & \\
\hline$\dot{\mathrm{P}}_{\mathrm{s}}$ & 17.3 & $8.86\left(\frac{\mathrm{w} / \mathrm{cm}^{3}}{\mathrm{MW} / \mathrm{m}^{2}}\right)$ & \\
\hline$\dot{\mathrm{P}}_{\mathrm{d}}$ & 17.5 & $0.309\left(\frac{\mathrm{W} / \mathrm{cm}^{3}}{\mathrm{wW} / \mathrm{m}^{2}}\right)$ & \\
\hline$\Phi_{s}$ & 18.1 & $1.24 \times 10^{22}$ & $\left(\frac{n / \cos ^{2}}{\sin ^{2} y / m^{2}}\right)$ \\
\hline$\Phi_{d}$ & 15.1 & $2.96 \times 10^{21}$ & $\left(\frac{n / c m^{2}}{n-y / m^{2}}\right)$ \\
\hline
\end{tabular}


Reactor parameters:

Fusion neutron power $\left(P_{n}\right)$

$322 \mathrm{MTF}$

Coil inner radius $\left(R_{c}\right)$

$5.75 \mathrm{~m}$

Blanket attenuation $\left(A_{b}\right)$

0.1

Coil case thickness ( $t_{\text {case }}$ )

$15 \mathrm{cII}$

Coil shield parameters:

Spherical shield

$P_{B} / P_{n}$

$1 \times 10^{-5}$

Thickness ( $t_{s}$ )

$61.4 \mathrm{~cm}$

Disk shields

$\mathbf{P}_{\mathbf{d}} / \mathbf{P}_{\mathbf{n}}$

$2 \times 10^{-5}$

Thickness $\left(t_{d}\right)$

$55.8 \mathrm{~cm}$

Haximum local $n+\gamma$ heating in winding

Behind spherical shield ( $\left.P_{s}\right)$

Behind disk shields $\left(\dot{P}_{d}\right)$

$2.63 \times 10^{-5} \mathrm{~W} / \mathrm{cm}^{3}$

$2.16 \times 10^{-5} \mathrm{~W} / \mathrm{cm}^{3}$

Maximum neutron fluence after 30 years

operation with 748 duty factor

Behind spherical shield $\left(\Phi_{s}\right)$

$1.28 \times 10^{18} \mathrm{n} / \mathrm{cm}^{2}$

Behind disk shield $\left(\Phi_{a}\right)$

$1.04 \times 10^{18} \mathrm{n} / \mathrm{cm}^{2}$

A note of caution: this shielding model is quite primitive. While it is hopefully conservative, detailed shielding analysis and optimization are needed.

\subsubsection{Irjector and Direct Comverter Shielding}

wo shield analysis was carried out on these systems. Based on our earlier work on similar systems, it is clear that sensitive components such as cryopanels must be shielded from direct view of the plasma. 12,14 Accordingly, the beam lines were layed out with shadow shields in front of sensitive components. Analysis of the effectiveness of shadow shields in these beam lines is planned for FY 1978. Cryopanels in the mirror-leakage direct converter are protected by locating them on the chamber sides out of "view" of the plasma. The effectiveness of this procedure must also be exanined. 


\subsubsection{Biologieal Shielding}

Since the whole reactor, including beam lines, is surrounded by a concrete monolith, the PCRV, and an additional containment structure, we assume no additional shielding will be required. This assumption should of course be checked, especially at the end of beam lines, if and when a more detailed design study of this reactor is performed. Potential radiation exposure to workers during maintenance is another obvious area requiring analysis. 
1. J. D. Lee, "Neutronics of Sub-Critical Fast Fission Blankets for D-T Fusion Reactors," in Proc. 7th Conf. Intersociety Energy Conversion Engineering, 1972 (American Chemical Society, 1972), p. 1294.

2. J. D. Lee, "Neutronic Analysis of a $2500 \mathrm{MH}$ th Fast Fission Natural Uranium Blanket for a Dr Fusion Reactor," in Proc. First Topical Meeting on the Technology of Controlled Nuclear Fusion, 1974 (San Diego, CA, 1974) CONF-740402-P1, Vol. 1, p. 233; Lawrence Livermore Labora tory, Rept. (UCRL-75304).

3. R. C. Haight and J. D. Lee, "Calculations of a Fast Fission Pianket for D-T Fusion Reactors with Two Evaluated Data Libraries," in Proc. First Topical Meeting on the Technology of Controlled Nuclear Fusion, 1974 (San Diego, CA, 1974), CONF-740402-P1, Vol. 1, p. 271; Lawrence Livermore Labora tory, Rept. (LCRL-75627).

4. R. W. Moir, et al., Progress on the Conceptual Design of a Mirror Hybrid Fusion-Figsion Reactor, Lawrence Livermore Laboratory, Rept. UCRL-51797 (June 1975).

5. R. C. Haight et al., Reaction Rates in a Uranium Pile Surrounding a 14-MeV Neutron Source: Calculations of the Feale Experiment. Lawrence Livermore Laboratory, Rept. UCRL-77364 (October 1975). Also, NS\&E 61, 53(1976).

6. J. D. Lee et al., Optimizing the Mirror (Fusion-Fission) Hybrid Reactor for Plitonim Production, Lawrence Livermore Laboratory, Rept. UCRL-76986 (November 1975). Presented at 1975 Winter ANS Meeting.

7. K. R. Schultz et al., Conceptual Design of the Blanket and Power Conversion System for a Mirror Hybrid Fusion-Fission Reactor, General Atomic Co., Rept. GA-Al4021 (July 1976).

8. J. D. Lee, "Blanket Design for the Mirror Fusion/Fission Hybrid Reactor," in Proc. of Fission/Fusion Conference, LLL, Livermore, CA, July 13-16, 1976. CONF-760733 Lawrence Livermore Laboratory, Rept. (UCRL-78515).

9. D. J. Bender, "Mirror Hybrid Reactor Optimization Studies," Proc. of the US/USSR Symp. on Fusion-Fission Reactors, Livermore, CA, July 13-16, 1976 , COAR-760733; Lawrence Livermore Laboratory, Rept. (UCRL-78614).

10. J. D. Lee, "Nuclear Design of Fast Hybrid Blanket," Proceedings of 2nd DMFE Hybrid Rector Meeting, Nov. 2-3, 1977. Also Lawrence Livermore Laboratory, Rept. (UCRL-80651). 
11. An Integrated System for Production of Neutronics and Photonic Calculational Constants, Lawrence Livermore Laboratory, Rept. UCRL-50400, vol. 14, TARTNP (February 1976) and vol, 16, EMDL (April 1976).

12. R. W. Moir, Ed., Standard Mirror Fusion Reactor Design Study, Lawrence Livermore Laboratory, Rept. UCID-17644 (1978).

13. H. Ullmaier, "Radiation Damage in CTR Magnet Components," in proc. of the Conf. on Radiation Effects and Tritium Technology for Fusion Recctors (Gatlinburg, Tennessee, 1976), CONF-750989, Vol. II, p. 403.

14. T. H. Batzer, R. C. Burleigh, G. A. Carlson, W. L. Dexter, G. W. Hamilton, A. R. Harvey, R. G. Hickman, M. A. Hoffman, E. B. Hooper, R. W. Hoir, W. J. Silver, B. H. Smith, C. E. Taylor, R. W. Werner, and T. P. Wilcox, Conceptual Design of a Mirror Reactor for a Fusion Engineering Research Facility (FERF), Lawrence Livermore Laboratory, Rept. UCRL-51617 (1974). 


\section{MECHANICAL DESIGN}

\subsection{Prestressed Concrete Reactor Vessel}

The first gas-cooled nuclear power plant built in the United States was Peachbottom I, with an electrical output power level of $40 \mathrm{MN}$. The high-pressure helium-coolant system was a distributed array of high temperature metallic piping and pressure vessels similar to that used in IwR nuclear-steam electric power plants. The second gas-cooled nuclear power plant built in the United states was Fort st. Vrain. Along with its eightfold increase in dasign power-output rating came the first use in the United states of a PCRV, a high-pressure-system confinement technology that had been extensively used in European nuclear programs. We propose to use this technology on the mirror hybrid reactor (MHR) plant with only a slight extension in current capability.

A PCRV, a prestressed concrete reactor vessel, is a monolith cast in place with integral reinforcing steel, prestressing tendon tubes and circunferential wire-wrap channels, gas-tight liner, and coolant piping. The entire structure may be very large and is held in triaxial compression by an appropriate combination of linear tendons and peripheral wire ......... wrapping. The PCRV can, therefore, withstand internal pressures, such as those that arise in power plants from gas-coolant pressure, thermal gradients, and, in the case of the MHR, from magnetic confinement pressure. Thus the function of the MHR PCRV is to restrain the Yin-Yang magnets and support the reactor blanket, to confine all of the primary coolant helium and its equipment, such as steam generators, and circulators, and to provide cavities and ducts within the structure to house such equipment. Steel plate and thermal barrier structures line the inner surfaces of the cavities within the monolith to prevent the escape of the coolant gas or conduction of its heat to the concrete structure itself. The concrete is water-cooled or heated to control the thermal profile within the monolith and for energency cooling as well. Figures 8-1 and 8-2 illustrate early ideas of how a PCRV might provide for the major equipment and magnet cavities, embedded gas ducting, and injection and leakage ports to the central reactor plasma chamber. A desirable feature of embedded ducting relates to the PCRV's inherent dead weight and seismic load capabilities without need of 


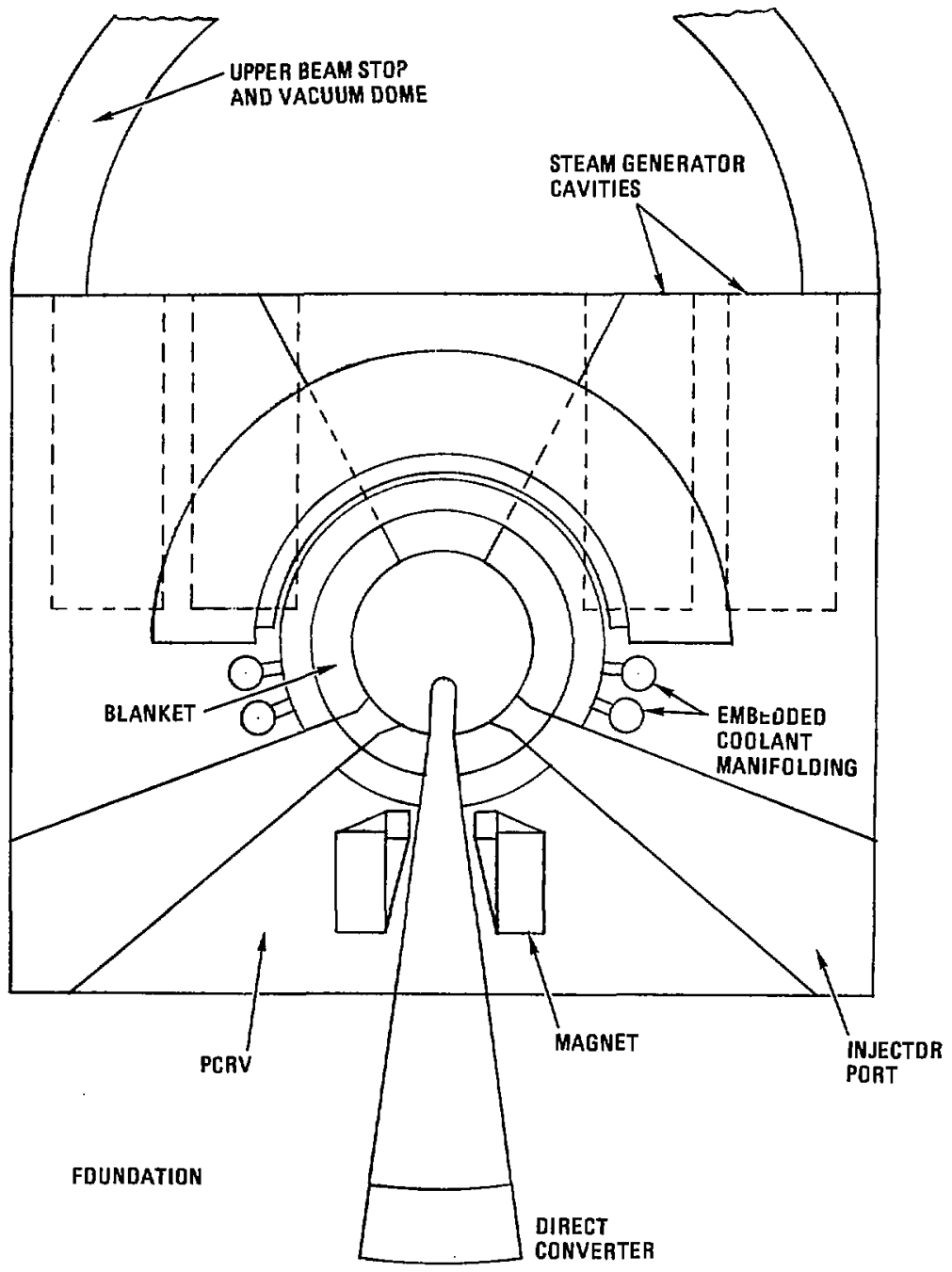

Fig. 8-1. Prestressed concrete reactor vessel - elevation view. 


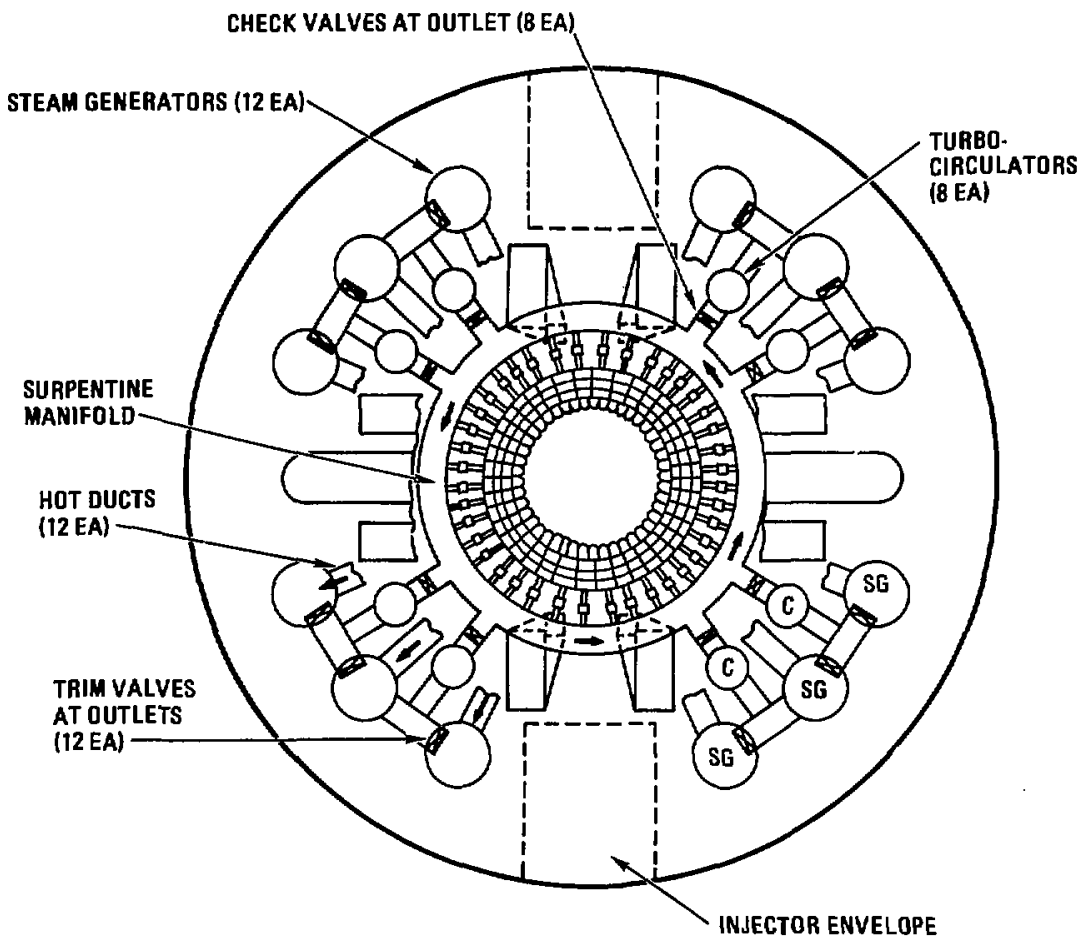

Fig. 8-2. Prestressed concrete reactor vessel - plan view. 
snubbers, supports, rollers, and related maintenance. The PCRV would be surmounted by a plasma dump, surrounded by a secondary containment building and would sit upon a foundation containing a deep fan-shaped well housing a direct converter and associated cryogenic pumping equipment.

When energized, the large superconducting Yin-Yang magnets develop large reaction loads at their mechanical supports, which correspond to the plasma magnetic-confinement forces. The magnets tend to move apart from each other, open up, and flatten out. Local maximum case pressures as high as 3000 to $5000 \mathrm{psi}$ have been estimated for the reference design. Restraint is a significant problem at this pressure level because the devices are large and the magnet coils operate at liquid-helium temperature. The coefficient of performance (COP) of cryogenic conditioning equipment is on the order of $1 / 500$, so that heat leaks to the magnets through mechanical supports are expensive from a facility-cost and power-balance viewpoint as well as from thermal stress considerations. Magnet forces are generated over the entire surface of the coil case, and they must thus be received over a large area of the lined, PCRV inner surfaces. Therefore, if the PCRV is to support the magnet case directly, it must operate at cryogenic temperatures and contain expensive stainless steel reinforcement that remains ductile at cryogenic temperature. Alternately, a load-bearing insulation must be used between the magnet case and the room-temperature, conventional ferro-cement PCRV.

Bechtel Corp. ${ }^{1}$ evaluated PCRV-type magnet restraint for the mirror reactor and determined that a cryogenic PCRV support would be more cost-effective than an austenitic steel-truss structure. clearly, a room-temperature, ferritic-steel-reinforced PCRV is more cost-effective than a cryogenic one. In addition, the heat load of a cryogenic PCRV would be large because of the large surface area of the interface between the cold PCRV and the environment. If the PCRV were to contain the hot helium as well as support the magnet forces, the heat flow to the PCRV from the internal duct and cavity surfaces that carry the heated-helium coolant would be large. Further, there would be direct conduction of heat from the earth through the PCRV foundation. Finally, the thermal-gradient-induced stress by a cryogenic PCRV would use up most of the structure's strength, and little useful load-carrying capability would remain. For example, a thermal 
gradient from liquid-nitrogen to liquid-helium temperature across the PCRV would produce about a 4000-psi thermal stress in the 6009-psi concrete. Bechtel advocated 10,000-psi concrete to golve this problem, but this strength is beyond current, normal commercial-production capabilities.

Load-bearing thermal insulation blankets were investigated to render the current PCRV technology fully compatible with MHR reguirements. A 6-m-thick, layered structure of honeycomb stainless steel panels would be required to provide adequate structural and thermai-resistance features between the PCRV vacuum liner and the magnet coll case. Fortunately, however, an attractive alternate was also discovered in fused silica insulation. This material has a nearly ideal combination of properties that will allow an 0.3- to 0.5-m-thick layer to properly insulate and support the magnets. Unfortunately, commercially avai.table fused-silica insulation has wide variations in strength level between samples. However, Lockheed Aerospace Co. has developed a fibrous silica product for the ispace-shuttle reentry-vehicle skin, which, at a higher density than currently produced, appears to be capable of providing the resiliency and property repeatability lacking in today's products (Ref. 2). The expected cost of the silica load-bearing insulation is 10 to 20 of the stainless steel honeycomb structure and represents a cost-effective plant design option.

Figures 8-3 and 8-4 display the geometry of the reference design PCRV, including the access penetrations to the plasma chambers, the upper and lower fan ports, twin injector ports, and injector cavity access closures in the top head. Other top head cavities with closures include the 12 steamgenerator and eight circulator cavities. The major PCRV cavities house the twin magnets and the central blanket assembly. The PCRV is $64 \mathrm{~m}$ in diameter and $30.5 \mathrm{~m}$ tall. These dimensions were determined by satisfying both the geometric requirements of the many systems contained within the PCRV and the primary structural requirements of the monolith. Sufficient ligament area between cavities is given to allow for concrete placement and a compressive stress within all allowable values. The number of vertical prestress tendons needed to react vertical cavity forces caused by magnetic effects, gas pressures, and thermal stresses were estimated, and the resulting tendon density is compatible with current design practice. Horizontal loads are resisted by hoop-wrapped steel wire at the PCRV periphery, much as a pipe wall resists internal pressure loads. The LPS and CPS (longitudinal and 


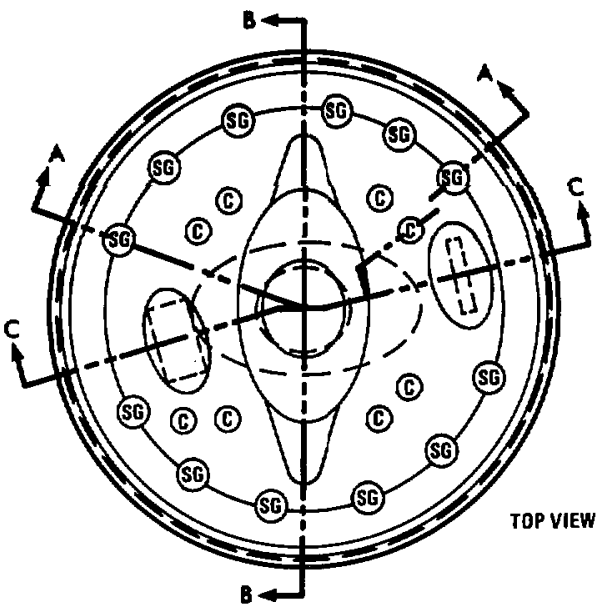

Fig. 8-3. MHR PCRV - top view. 
SECTION A A THRU STEAM GEN. AND HELIUM CIRC.

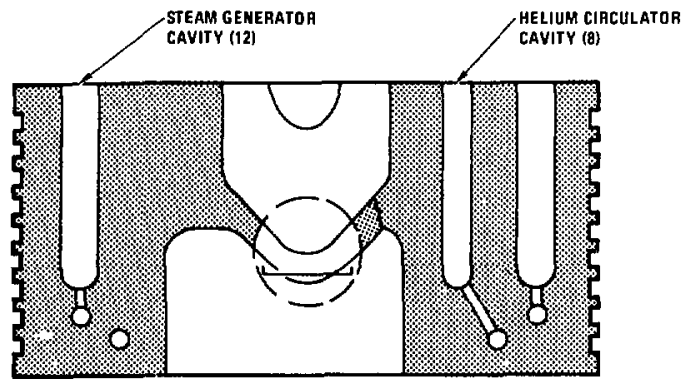

SECTION B B

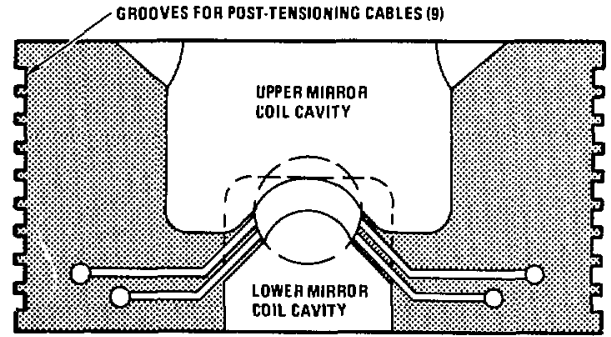

SECTION C C THRU BEAM IMJECTORS

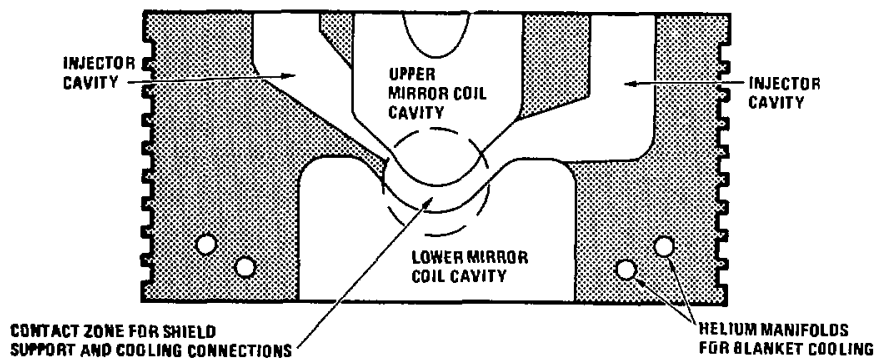

Fig. 8-4. MHR PCRV - side views. 
circumferential prestress systems) together maintain the concrete monolith in triaxial compression at values below the allowable strength, whether the system is pressurized, magnetized, and thermally loaded or not.

\subsubsection{Primary Loop Containment}

Cavity and gas-duct sizes, having been determined by equipment gize and gas dynamic considerations, are used to size the gas-tight, water-cooled liner system requirements. These data are also useful in determining liner thermal barrier cost takeoff for the high and low temperature sections of the power conversion system. A system of helium flow control valves and gas ducts connect the varlous cavities (Fig. 8-5).

Cooled helium exits the steam-generator cavities through flow trim values and enters the circulators. The trim valves control circulator power requirements between loops. From the steam-driven, turbo-circulator outlets, the coolant is transferred through check valves to a large diameter embedded ring manifold surrounding the reactor itself. These check valves allow plant operation with inoperative circulators. Coolant is bled from the ring manifold through 50 radial ducts containing blanket-flow-control values; the valves match the coolant flow to the age (and, thus, heat load) of the blanket modules in that portion of the blanket. Helium then moves through the vertical final-distribution channels within the permanent blanket structure, through the modules themselves, and outward into the outlet ring manifold. The heated coolant is directed toward the 12 steam generators to complete the primary power conversion loop circuit. An attractive feature of the PCRV is that the primary power conversion 100p (PCL) helium never leaves the concrete monolith, which gives the primary loop great integrity and renders a sudden depressurization impossible. The permanent blanket structure is also embedded in the PCRV, which eliminates a maintainable interface between the PCL and the blanket. All PCL maintenance is done either on the inner face of the reactor sphere by replacing blanket modules remotely as described in Section 8.2 .2 or on equipment located in top-head cavities with standard gas-cooled reactor technology. The containment building crane is used to remove and replace top-head cavity closures and PCL equipment within the cavities, including neutral beam injectors, circulators, and steam generators, if necessary. The MHR plant 


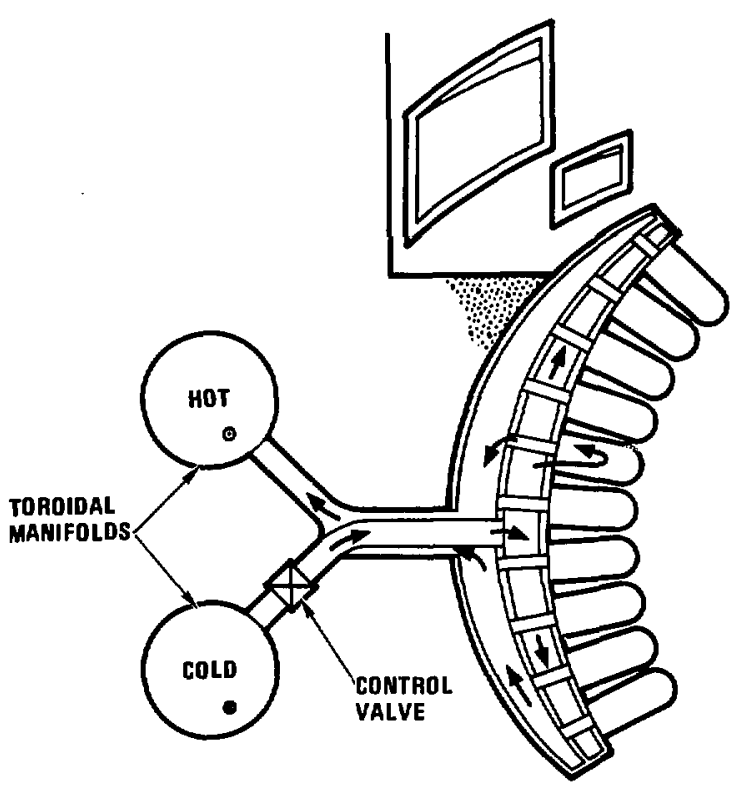

Fig. 8-5. MHR manifolding. 
will be designed to allow removal of either mirror magnet, but the overall design philosophy is based on superconducting-magnet experience that shows the magnets are expected to be very reliable and normally will not fail in such a way that they cannot be repaired in situ. The power-plant downtime caused by a major magnet failure would be about three years. Thorough magnet testing before installation during the construction phase should preclude the uneconomic magnet failure event during porer plant operating lifetime.

\subsubsection{Magnet Restraint}

The coil forces are described in Sec. 6.1. The cavity in the PrEV to position the coils would be rectangular if it followed the coil surfaces that must be restrained from motion. At present it is believed unlikeiy that the PCRV could withstand the stress concentration resulting fror "sharp" reentrant voids. This rectangular void cross section is encompassed with an elliptical internal contour which should prove to be a very small stress intensifier compared to cylindrical shapes already known to be acceptable. The space between the elliptical internal concrete surface and the flat side of the coil housing must be occupied by a steel (or composite) spacer that acts separately from but in concert with, the PCRV : len the magnets are energized. We plan that the spacers will also ope ate at room temperature.

The neutral-beam-injector array can be arranged to form an elliptical beam cross section. This allows the beam-line void in the PCRV to have an elliptical cross section tapered toward the center of the PCRV to form an elliptical cone. This shape will result in the lowest possible stress concentration in the PCRV.

It must be emphasized that this PCRV must be modeled, instrumented, tested, and exhaustively stress-analyzed by finite-element methods to predict its satisfactory performance under preload anc. operating stress conditions.

The superconducting Yin-Yang coil is two separzte C-shaped assemblies, one below and one above the horizontal plane through the plasma confinement zone. The superconducting winding is accomplishe $\bar{c}_{i}$ on a steel form, which, after winding, is closed permanently, forming the jacket that contains the helium at $4.2 \mathrm{~K}$ and appropriate plumbing for circulation of the refrigerant. This jacketed magnet assembly is supported on thermal insulators 
within the coil case, another major steel fabrication. In addition to providing mechanical support to the coil through load-bearing insulators, the space between the case and coil jacket is completely filled with radiation barriers to minimize the steady refrigeration load on the coil. A material must be found that maintains good thermal insulating properties at loads of about 5000 pgi. Fused silica, such as that manufactured by Glasrock Products, Inc., has appropriate strength and thermal conductivity in modest sizes (dimensions $\approx 10 \mathrm{~cm}$ ) but larger pieces will be needed for our purpose, and more consistency in the manufacture of large pieces must be achieved. Also, engineers at Lockheed have developed laboratory samples of such insulators and feel that five years of development will easily yield the solution to manufacture of bulky pieces with reliable physical and thermal properties.

Differential thermal expansion between the coil and its case can cause two intolerable effects:

- Kovement of the coil producing position error.

- Space between case and coil allowing excessive coil strains before contact is achieved and the load supported.

One method of employing Glasrock, or its equivalent, is to encapsulate two wedges of the material in thin, stainless steel envelopes. As the two wedges, constituting one load pad, are moved against each other (by an electrical linear actuator or hydraulic cylinder), the inickness of the pad can be varied to accommodate differences in clearance. Because of very large friction forces, such adjustments can be made only in the unloaded condition. Fig. 8-6 illustrates this concept and the location of such load pads.

\subsubsection{Blanket Support Structure}

One function of the PCRV is to support the spherical blanket structure. Several views of the ranv (Figs. 8-4) show the cavities for the Yin-Yang mirror coil set. The space occupied by the coils prevents cooling duct access to the plasma containuent chamber in all regions except a serpentine band. This band is shown on the surface of a sphere representing the outside of the spherical shield (Fig. 8-7). The serpentine band nust include hoth inlet and exit cooling-gas duct openings. Routing of the ducts from the sphere to the two ring manifolds has been considered, and typical 


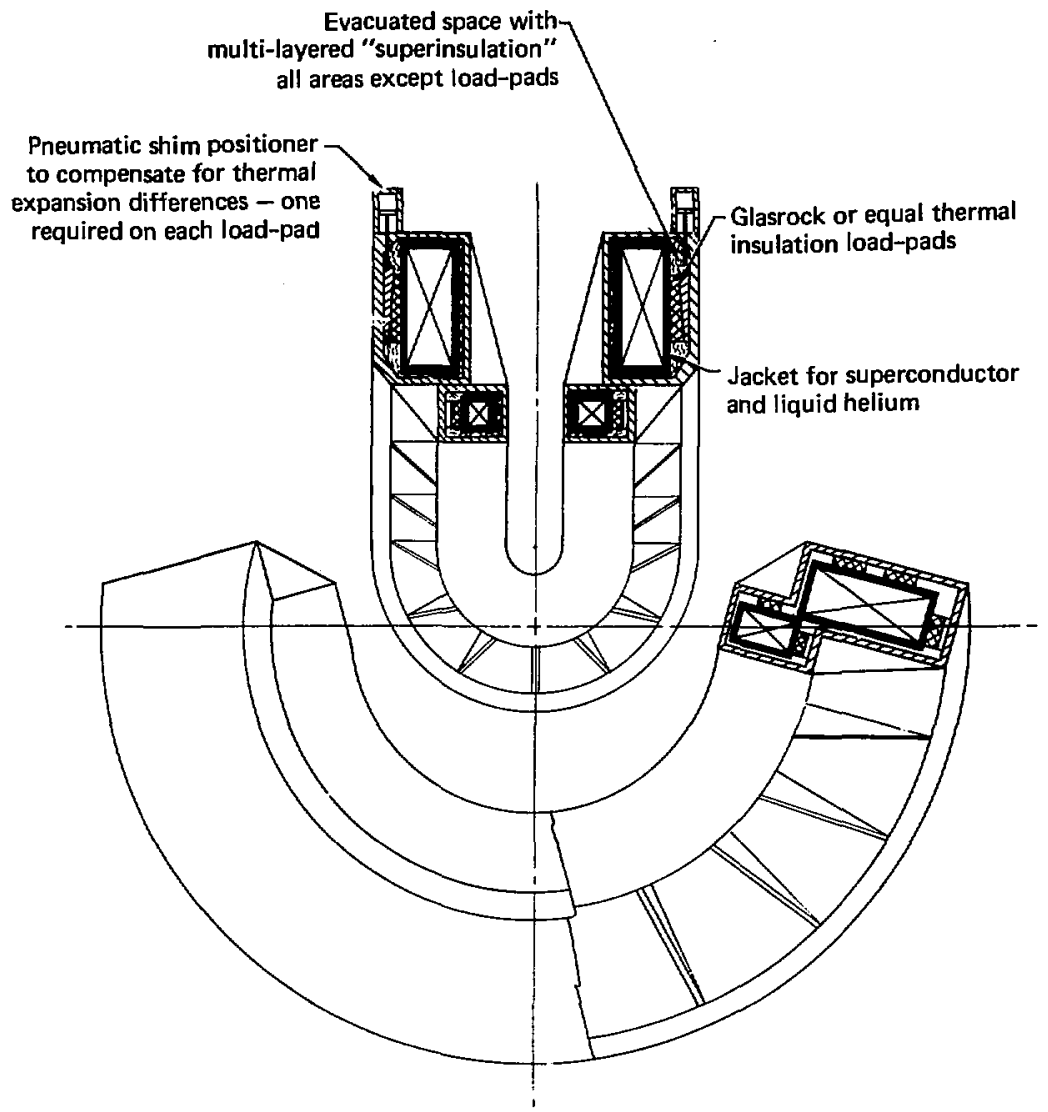

Fig. 8-6. Hagnet coil case and insulation. 


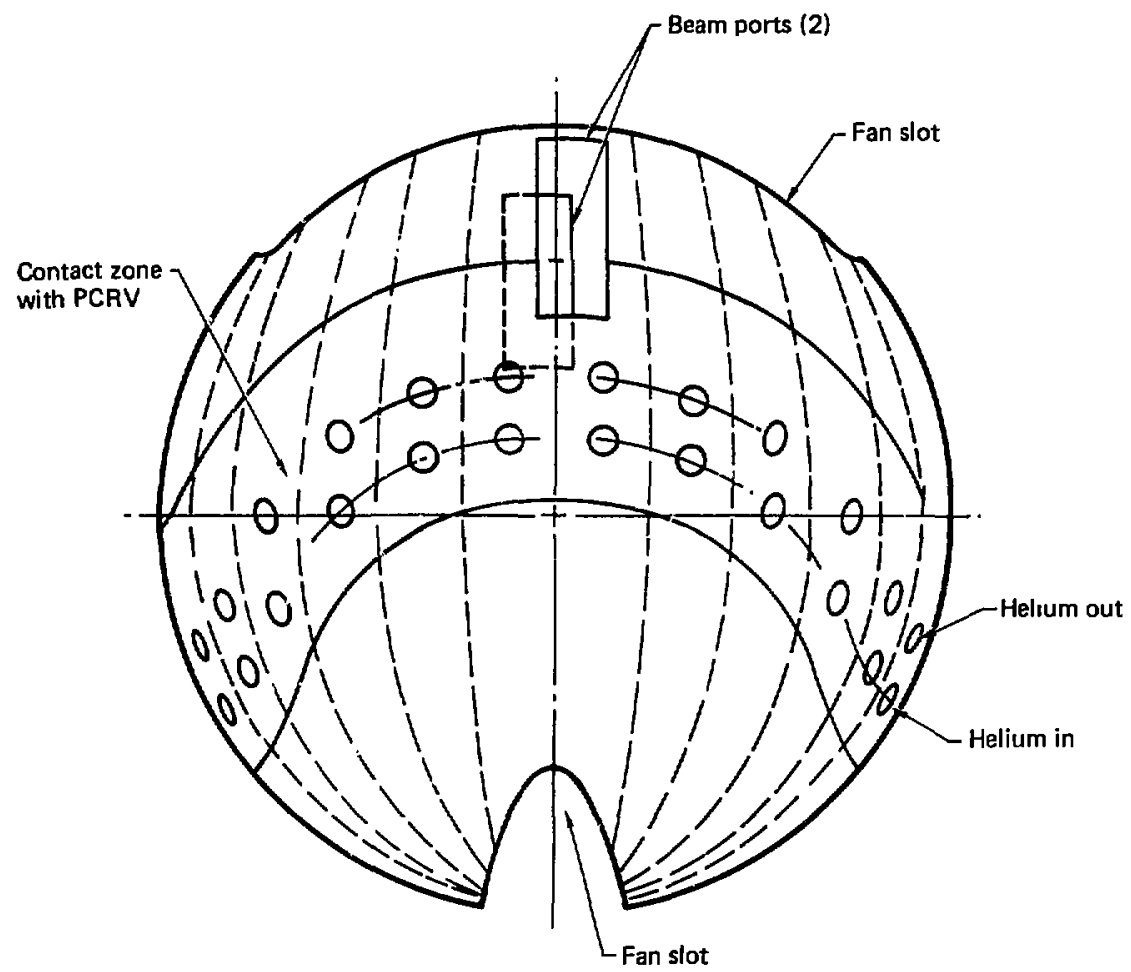

Fig. 8-7. Blanket/8hield support and cooling ports. 
"runs" are shown in Fig. 8-4. The ring manifolds - one supply and one return -. are located in the lower portion of the PCRV and are, in turn, connected to the steam generators and helium circulators.

A cut-away view of the spherical blanket and shield is Fig. 8-8. A group of blanket modules extending from the "north" pole to the "south" pole and constituting one segment of the sphere are serviced by one inlet and one exit duct. (Certain blanket modules will be missing because the space is needed for flux fan slots and beam entrance ports.)

The outer shield will be about $50 \mathrm{~cm}$ thick. It will be in contact with the PCRV in that serpentint-.snaped zone where the cooling ducts have access to the sphere. Two concentric spherical annuli distribute and collect helium from the blanket modules. Since helium pressure of 60 atm is required, the shield and the shells that form the annuli must be integrated to make a pressure vessel. The PRCV will provide direct support only in the serpentine zone. Chamber sections above and below that zone must be self-supporting.

\subsection{Blanket Module Design}

\subsubsection{Materials Selection}

The blanket neutronics calculations done as part of the MHR studies have shown that the plutonium and tritium production rates in the blanket are very sensitive to the fraction of structural material in the blanket and to the thickness of the first wall between the plasma and the blanket fuel. 3 Because of this sensitivity, the module design has attempted to minimize the amount of structural material required. This has been done by using a curved first wall shape that results in pure tension pressure stresses, by avoiding bending movements to minimize the stresses, and by using high strength first wall materials.

The materials environment of the first wall in the mirror hybrid reactor is quite harsh. The first wall is the primary pressure and vacuum boundary and must contain the reactor helium coolant at $6 \mathrm{MPa}$ pressure. It will experience approximately $16 \mathrm{w} / \mathrm{cm}^{3}$ of internal heating due to the neutron and gamma radiation incident upon it and will operate at elevated temperatures because of this heating. It must withstand a total fast 


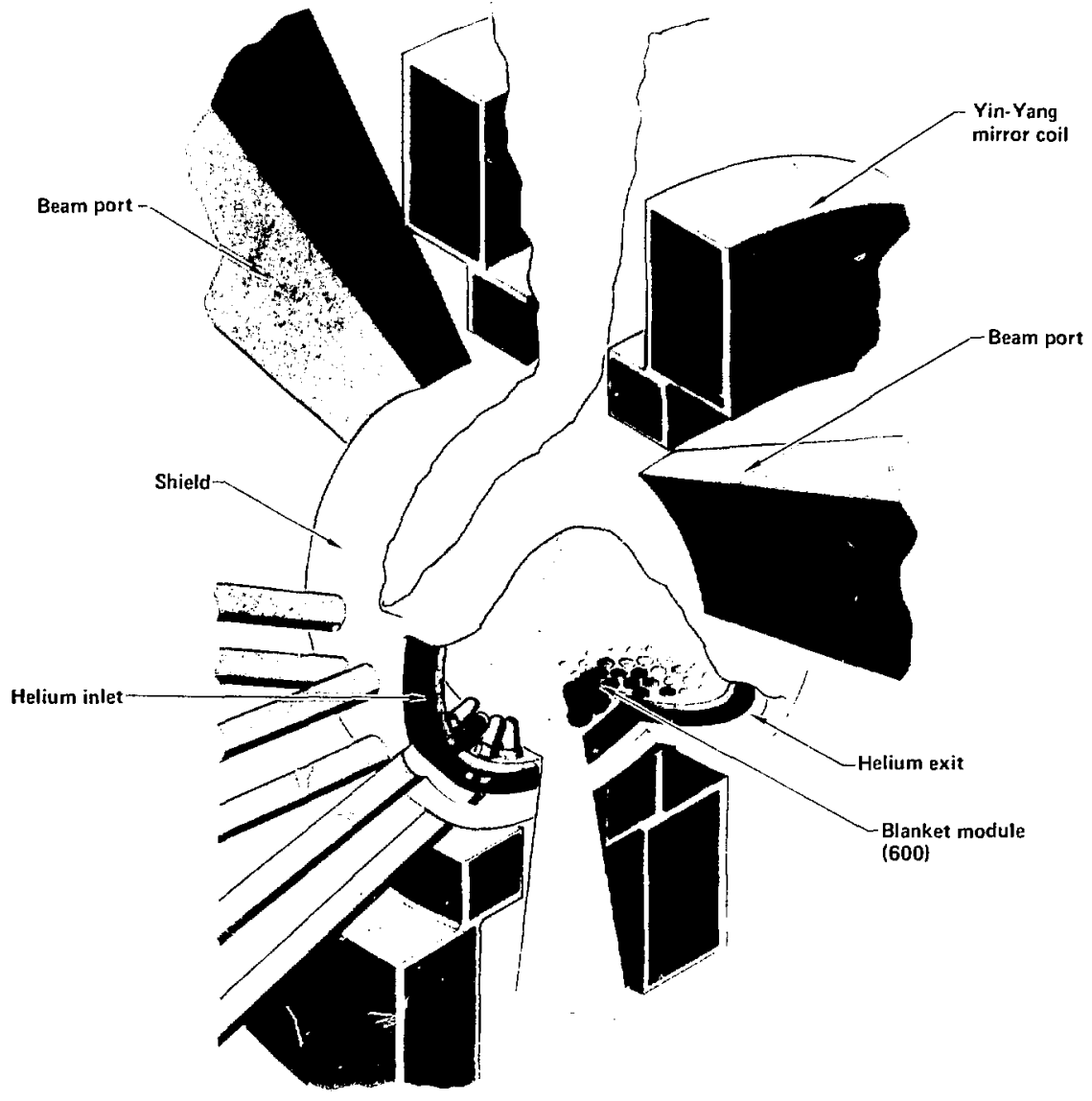

Fig. 8-8. Blanket and shield cutaway. 
neutron fluence of about $8 \times 10^{22} \mathrm{n} / \mathrm{cm}^{2}$. The material selected for the first wall structural material will have to operate reliably in this environment.

Interest has generally been concentrated on the 300-series stainless steels for near-term fusion technology applications; the refractory metals are of interest for longer term applications. 4 Although 316 stainless steel is a well known material with an excellent nuclear data base, its strength is modest. Increased interest has been shown recently in nuclear applications of the nickel-base alloys because of the high strength capabilities available with some of these alloys. 5 The precipitationstrengthened alloy Inconel 718 was chosen for use as the MHR module structural materfal because of its high strength at temperatures up to $600^{\circ} \mathrm{C}$ and because of its good resistance to radiation daruge. The allowable design tensile stress for precipitation-strengthened Inconel 718 is shown on Fig. 8-9. The design allowable stress is taken to be the minimur ultimate tensile strength, or two-thirds of the minimum creeprupture strength for $2 \times 10^{4} \mathrm{~h}$, as obtained from Ref. 6 . The tensile strength of Inconel 718 appears to increase with irradiation at the expense of decreased ductility. ${ }^{7}$ The swelling of Inconel 718 under EBR-II irradiation to about $5 \times 10^{22} \mathrm{n} / \mathrm{cm}^{2}$ is shown on Fig. 8-10 from Ref. 8. These data indicate that for irradiation between 470 and $650^{\circ} \mathrm{C}$, very little swelling will be expected. Simulated CRT irradiation in HFIR to the equivalent of $6.4 \mathrm{MW}-\mathrm{y} / \mathrm{m}^{2}$ exposure of the similar PE-16 alloy confirms the prediction for low swelling. 9 Loss of ductility with irradiation is a concern since some residual ductility is needed at the end of irradiation to accomodate the strain of normal design loading; the strain of maximum design stress loading is 0.228 . Residual ductility of the PE-16 alloy as a function of irradiation temperature after HFIR irradiation to the equivalent of about $6 \mathrm{~N}-\mathrm{y} / \mathrm{m}^{2}$ is shown on Fig. 8-11 from Ref. 10. These data indicate that, for irradiation temperatures between 320 and $700^{\circ} \mathrm{C}$, ample residual ductility will be available at the end of exposure. EBR-II irradiation data from Ref. 7 confirm this trend but suggest that perhaps $630^{\circ} \mathrm{C}$ would be a safer irradiation temperature limit.

On the basis of the data and discussion presented above, it appears that Inconel 718 will be able to withstand the radiation damage, 


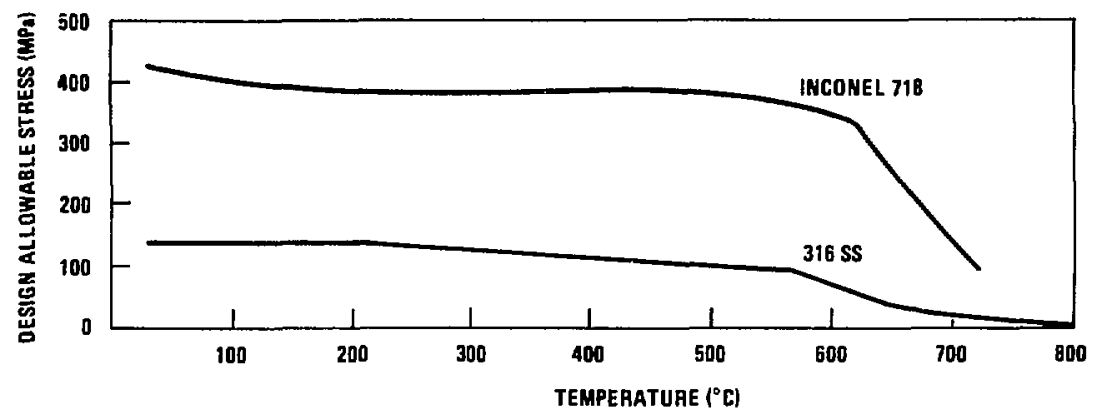

Fig. 8-9. Allowable design stresses. 


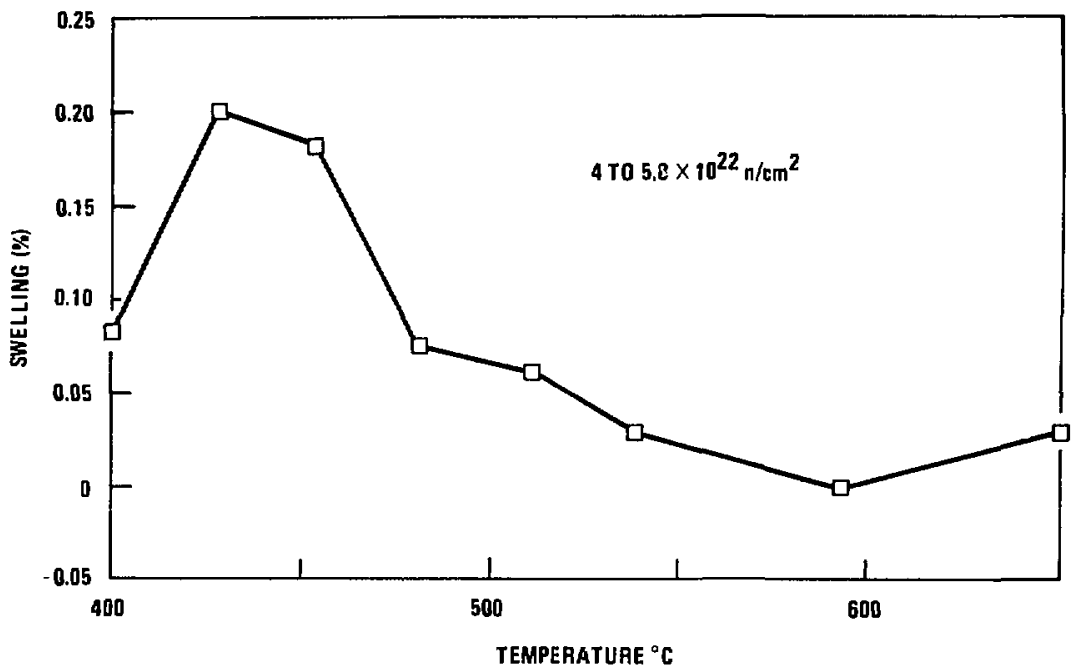

Fig. 8-10. Swelling of solution-treated and aged Inconel 718 as a function of temperature after EBR II neutron irradiation to $5 \times 10^{22} \mathrm{n} / \mathrm{cm}^{2}$ $(\mathrm{E} \geq 0.1 \mathrm{MeV})$. 


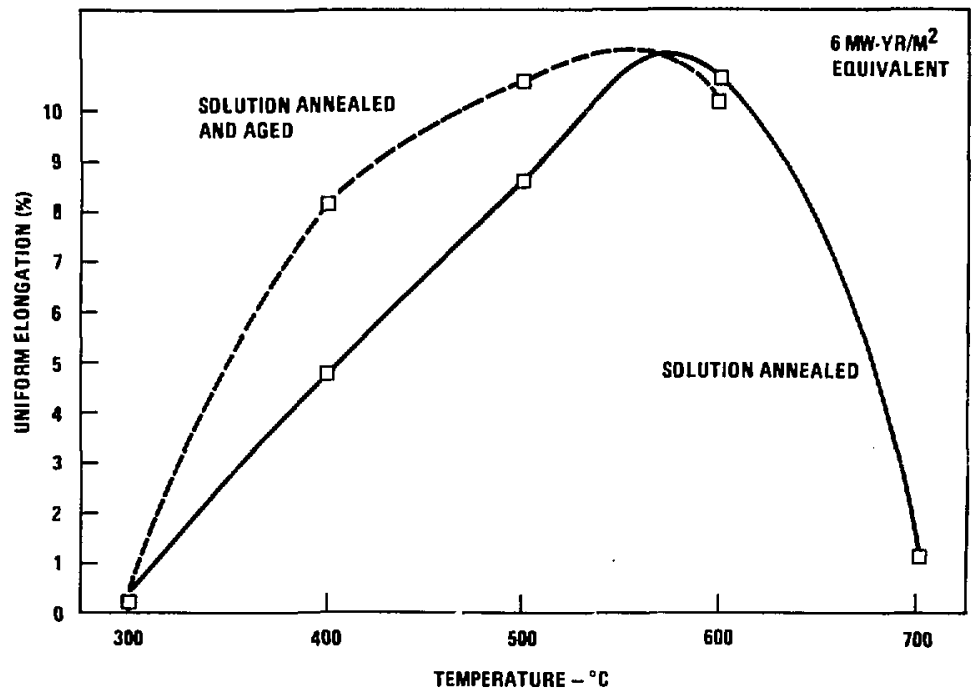

Fig. 8-11. Residual ductility of alloy PE 16 as a function of temperature after neytron irradiation in HFIR to the equivalent of approximate] $y$ $6 \mathrm{MH}-\mathrm{y} / \mathrm{m}^{2}$. 
temperature, and stress environment at the first wall of the MHR. Although data is still being determined for irradiation-increased creep, compatibility with impurities, and other materials concerns, Inconel 718 appears to be an excellent choice of material for the module structural material and no unexpected difficulties are anticipated.

Welding and machining of Inconel 718 are more difficult than 316 stainless steel. Machining appears to be approximately four times as diffirult, so intricate mach; ned parts should be avoided. TIG welding of up to 1-cm-thick sections appears to be readily accomplished, bit thick sections should be avoided. The strength of a welded section is only rated at $60 \%$ of the parent metal. To regain the full strength capability of Inconel 718 , the structure will have to be heat treated after welding. This makes it highly desirable that the modules be small enough to allow them to be accomnodated in a heat treating oven.

Because of the cost of the raw materials that constitute nickel-base alloys and the cost of fabrication, Inconel 718 is expensive. It is estimated that fabricated Inconel 718 will cost twice as much as fabricated 316 stainless steel. A class of structural materials that may have economic advantages over Inconel 718 is martensitic stainless steel. Martensit _c stainless is produced by precipitation-hardening ferritic stainless steel. The ferritic steels are magnetic, having only 9 to $13 \%$ chrome and very low nickel content. Because of the steady state nature of the magnetic fields in the MHR, the magnetic properties of ferritic steel may not cause problems. The material cost of ferritic steel is about two-thirds that of 316 stainless steel and one-third that of Inconel 718. The martensitic materials appear to be capable of yield strengths comparable to those of Inconel 718 ( 1200 MPa) and compared to 316 stainless steel, the ferritic alloys have lower neutron absorption and appear to exhibit less swelling and higher residual ductility under irradiation. Irradiation data on martensitic alloys appears to be sparse, but the LMFBR materials program is considering 400-series stainless steels, so more data may be forthooming. The marte: sitic stainless steels may deserve future study for potential cost reduction in the MHR modules.

\subsubsection{Module Concepts and Support}

The starting point for CY-1977 blanket design work was that reported in Ref. 11. That design involved 16 lune-shaped modules, each containing its 
htilum coolant distribution system and blanket fuels. Four lunes were to be replaced by removal of the upper cryogenic magnet at approximately yearly intervals, and they were assumed to be freestanding assemblies. Details of collant and mechanical interfaces had not been delineated. Investigation of lune module support schemes during 1977 revealed that the large structures were subject to significant thermal growth during operation and to sizable seismically induced loads because of their shape. The natural frequency for vibration of the large lune as calculated to be $1.1 \mathrm{~Hz}$. This low frequency would be susceptible to excitation by earthquakes, so restraint of the modules to prevent this vibrational mode is essential. A support scheme was developed that attached the module to the reactor structure (PCRV) at the large diameter coolant interface and provided sliding supports at the tips of the lunes. The free-standing module shown in Fig. 8-12 was, thereby, centrally supported and seismically unloaded in most orthogonal directions while thermal expansion was allowed. The lune was to be constructed with a 165-mn-thick strongback to prevent excessive distortion during a design-basis earthquake.

The blanket support system will probably become subject to In Service Inspection (ISI) requirements so the elements of the support system should be:

1. Removable with the module for inspection, or

2. Easily inspected in-situ, or

3. Not subject to ASME Code, Section XI, Div. 1 ISI requirements

Another method of restraining a distribution structure, mindful of earthquake loads, is to grasp it in two or three locations through pinned or ball joints that form the end of "links" or "A frames." The links should be snubbed with rate-sensitive dampers.

choice of this scheme, as with the former, depends in part on other system restrainte, wch as possible ultimate anchor point locations, access to joints and snuobers, and other required interfaces (coolant pipes, etc.).

The former support method is favored, especially inasmuch is the existence of a lune strongback does reduce the "fixed" shielding requirements to a degree, and it interfaces in a superior way with the blanket coolant system. Figure $5-12$ shows removable shield rings which provide support for the modules but which mist themselves be supported by an external system. 


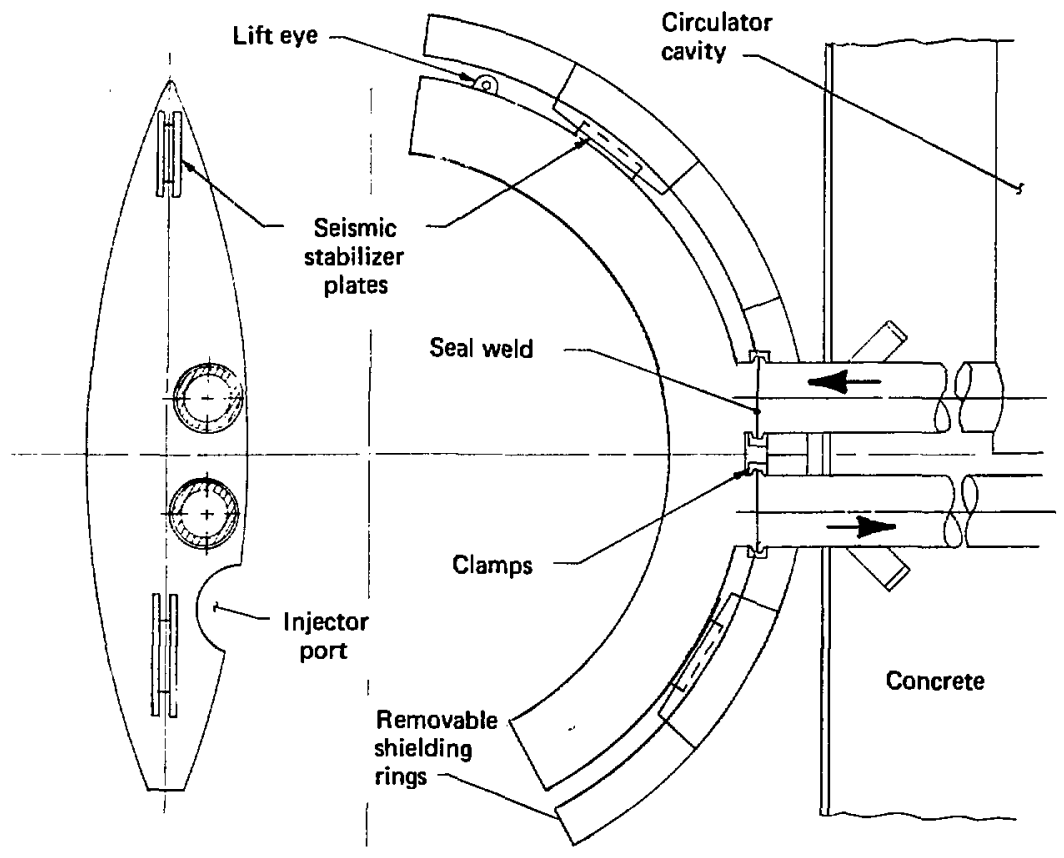

Fig. 8-12. Free-standing lune module. 
It was decided to use a Prestressed Concrete Reactor Vessel (PCRV) (see section 8.1), which could also house the entire helium-coolant power-conversion system. Load-bearing thermal insulation had been identified that wolid allow a cryogenic magnet to be restrained by a room temperature structure with an acceptable heat leak to the cryogenic refrigeration system. It was, therefore, conceptually necessary to warm the magnet only for removal from the PCRV, and this was envisioned as a lengthy procedure. Furthermore, lifting the 3000-t magnet was viewed as marginally feasible on a routine basis. Because of these factors, a module maintenance scheme was actively sought that would eliminate the need for routine magnet movement.

The first fixed-magnet blanket-maintenance scheme retained the 16 lune concept and allowed the lunes to be passed upward or downward through magnet-leakage ports on the ends of hydraulic rams. Removal of neutron shielding from the leakage ports rendered them wider than the module midsections. Subsequently (refer to Fig. 8-13), removal of several of the 16 shielded, PCRV-access-cavity plugs would allow the helium hot ducts to be removed and replaced by maintenance rams. Translation of the ram by an ex-vessel machine attached to the PCRV periphery with the aid of a Reactor Containment Building annulus crane would bring the module into the leak port window for grappling by the RCB crane hook. Subsequent rotation and lifting or lowering of the lune would remove it from the reactor. Notice that the PCRV geometry allows permanent neutron shielding to be embedded into the monolith, and that the original, basic lune support system is retained and interstitially insulated to guarantee blanket-module cooling continuity during powered operation.

A second, fixed-magnet blanket scheme was devised that made use of more numerous, smaller blanket modules; they are more easily handled by remote maintenance machinery and can be more easily passed through the magnet leakage poits than the large lunes. Furthermore, the modules contribute sone neutron shielding, so that a pernanent support structure containing coolant manifolding and shielding can be embedded into the concrete monolith for the reactor lifetime.

The geometry of the spherical hybrid reactor blanket was analyzed, and it was folnd possible to divide it rather uniformly into 600 hexagonal and 


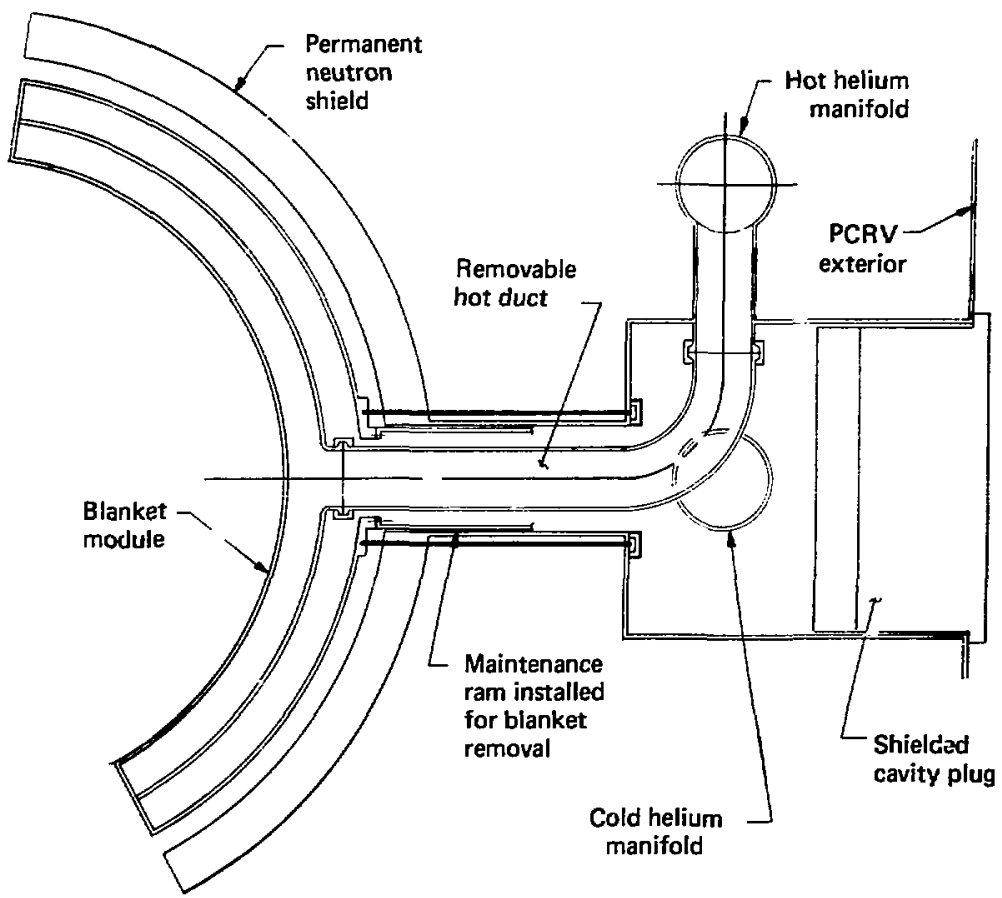

Fig. 8-13. Wal1-mounted lune concept. 
eight pentagonal regions or cells, on the basis of a spherical icosahedron pattern, which is intersected by two leakage and two injector ports. The resulting hexcell size is $0.50 \mathrm{~m}$ across the flats at the first wall radius of $3.75 \mathrm{~m}$; it tapers to a larger size at the permanent structure radius of about $4.5 \mathrm{~m}$.

Three generic nodule degigns were investigated: the hexagonal module, the compound module with side pipes, and the cylindrical module with hexagonal flange. For all module designs it was assumed that remote connection to the permanent steel structure from within the reactor sphere was requiced and that such connection was to be made leak-free to the integrated 60-atm-pressure coolant manifolds, which are arranged concentrically in the spherical shell. The modules required an inner assembly of fuel pins and a lithium-deuteride pin array near the coolant inlet, as well as an outer pressure cover to contain the coolant. The inner assembly included the hot heliun outlet duct.

The hexagonal module design (Fig. 8-14), resulted from a desire to maximize blanket coverage with the modules. A hexagonal pressure vessel was used to transport coolant radially inward from the lithium-deuteride array to the inner coolant-distribution dome. A seal weld was required at the first wall to seal the leakage of coolant to the plasma chamber and, secondarily, to inhibit rotation of the mechanically loaded vacuum seal at the base of the module, preventing loss of seal pressure during operation. This design was eventually discarded because of the structurally inefficient nature of the hexagonal pressure vessel and because both a first wall weld and a rear mechanical seal were required.

A second-generation degign attempted to maintain good blanket module coverage with a cylindrical module by utilizing small diameter side pipes to transport helium coolant radially inward to the first wall (see Fig. 8-15). The design also reduced the number of modules reguired from 600 to 150 by use of concentric fuel assemblies with a 1-m o.d. The first-wall thickness was retained at $0.24 \mathrm{~cm}$ by use of torotdal first wall of $0.125 \mathrm{~m}$ radius in the outer assenbly and a hemisphere of $0.25 \mathrm{~m}$ radius in the inner one. Therefore, only 150 fuel assemblies, rather than 600 , were required per reactor, but $3 / 4$ of the fuel weight was located in the annular arrays. Also, two seals were still required, a welded one at the first wall and a 


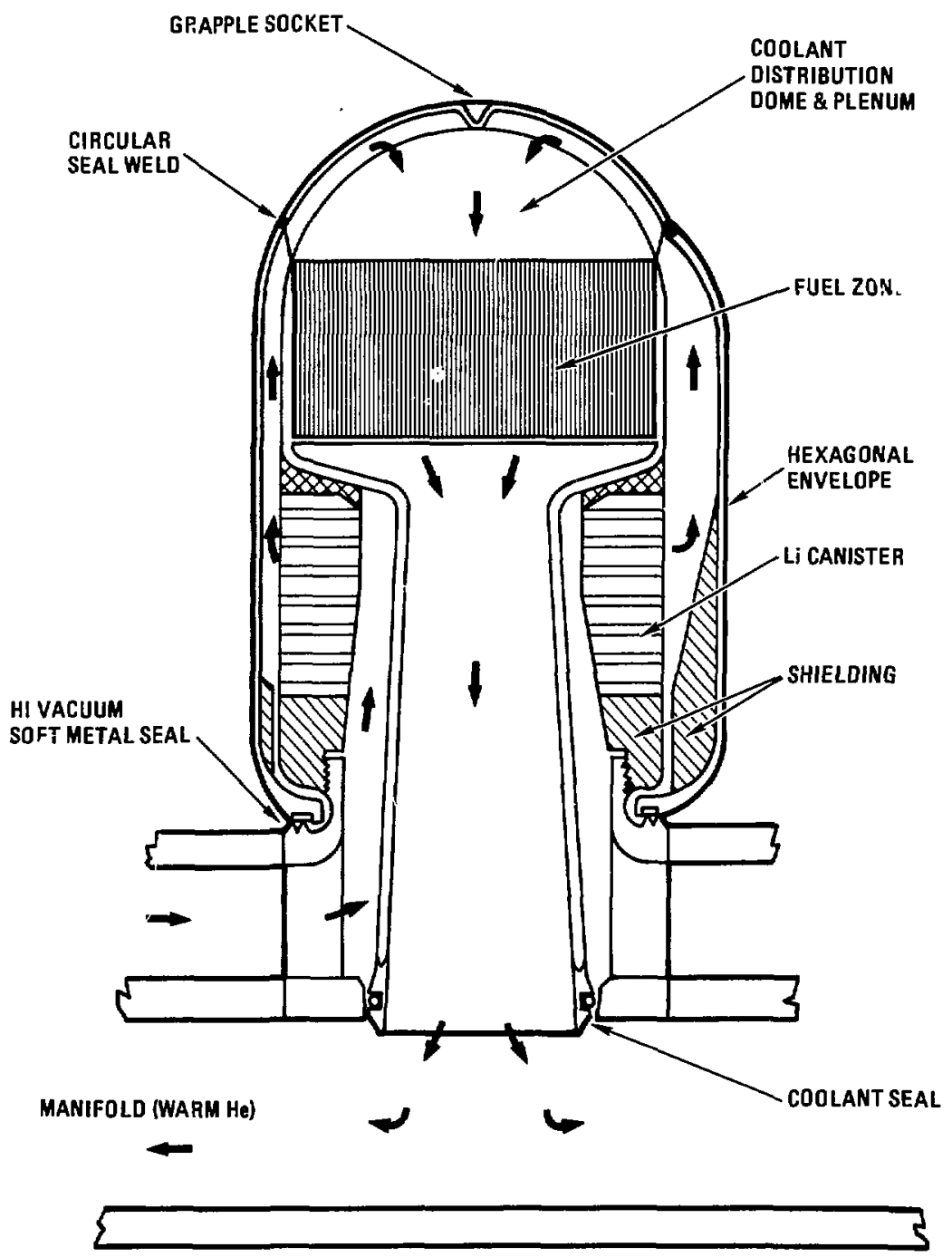

Fig. 8-14. Hexagonal module concept. 


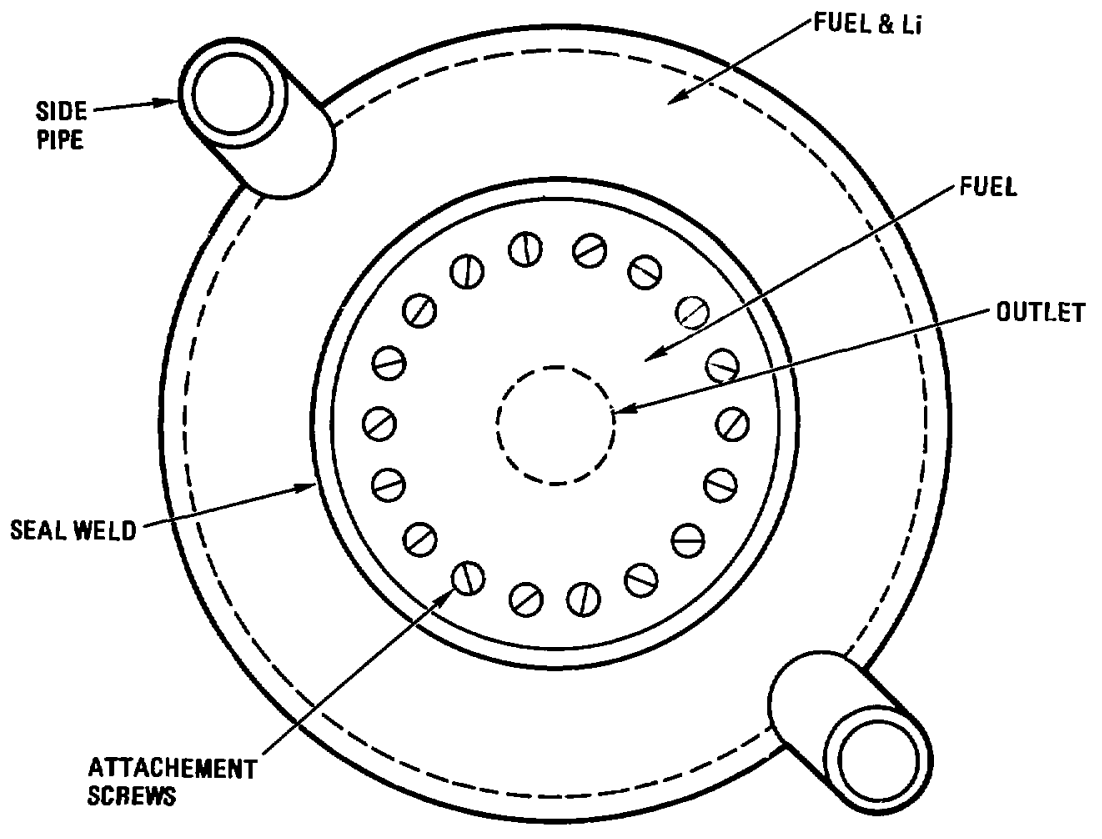

Fig. 8-15. Side pipe module - top view. 
mechanical one at the structure interface. It was also found, finally, that no net increase in fuel loading was allowed by this design, because of the presence of three fuel-lattice/cylindrical-wall interfaces rather than one per module, a very inefficient geometric condition.

The cylindrical module design was ultimately chosen as the reference blanket concept, and its more fully explored configuration is shown in Fig. 8-16. The design uses a thick hexagonal flange at the permanent structure interface with a double (Beries) high-vacuum mechanical seal. The annular region between the seals is vacuum pumped so that a high coolant leakage rate to the plasma chamber is prevented even if the primary vacuum seal itself leaks. In addition, the vacuum system can be used to detect leaks during installation of a module. Six large, high-temperature bolts hold the seal load on the module pressure shell. Large-diameter acme profile threads hold the heavy fuel assembly to the permanent reactor structure. The six bolts are accessible from the plasma chamber by direct line of sight when the tri-cusp graphite neutron shields have been removed from between the modules. These thick shields reduce neutronic bolt heating and damage to the interstitial permanent structure; they are radiation cooled by thermal radiauion to the module side walls. Bolt material will be chosen for its high-temperature strength, low thermal-expansion coefficient, and neutron damage resistance at temperature, the molybdenum alloy TaY is a candidate material. Because the thermal expansion coefficient of TZM is lower than that of Inconel 718 , as the module heats up the vacuum seal will become tighter.

Coolant flow through the module of Fig. 8-16 is as follows: Helium enters the module at $280^{\circ} \mathrm{C}$, flows through the lithium-deuteride pin array support plate and "forward" through the pin array, emerging at $300^{\circ} \mathrm{C}$. It flows along the module side wall to the flow distribution dome, by the first wall, and through the flow distributor to the $\mathrm{U}_{3} \mathrm{si}$ pin array. Flowing radially outward through the fisgion fuel, the helium is rapidly heated until it passes through the fuel support plate at $540^{\circ} \mathrm{C}$ and outward to the exhaust plenum through the central coolant duct, containing a flow control trim orifice and internanifold seal.

Figure 8-17 shows how the hot helium manifolds in the permanent structure are shielded with thermal barrier and water-cooled rear 


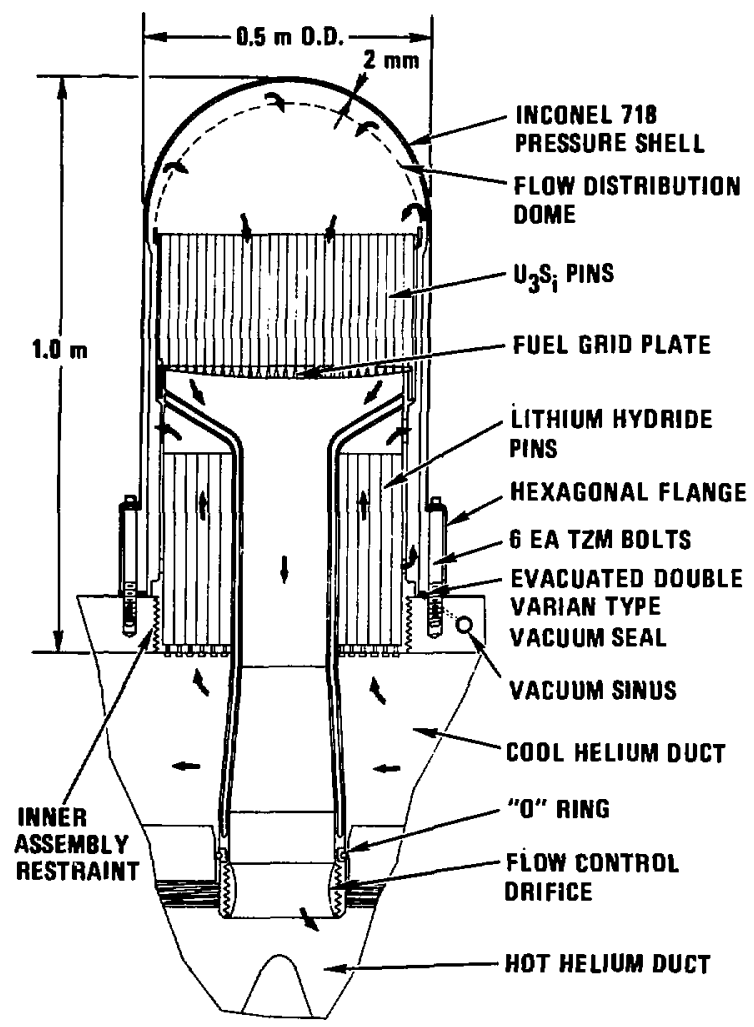

Fig. 8-16. HR blanket module. 


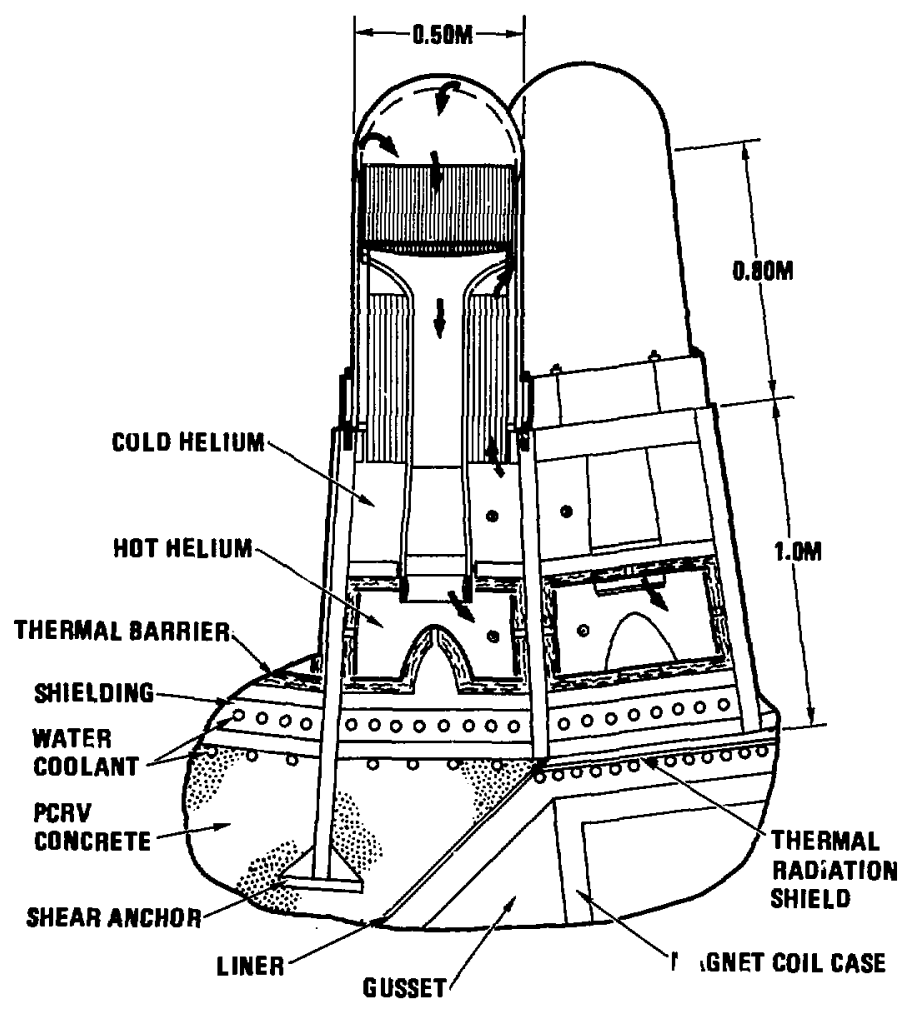

Fig. 8-17. MHR blanket manifold structure. 
shielding. Modules "nest" within the structure, and shear anchors hold the structure within the PCRV monolith.

A conceptual module-handling machine is show in Fig. 8-18. This multifunctional machine seals the upper end tank while it removes module pressure vessels. It also allows depressurized coolant at approxinately 1 ato pressure to flow into and circulate through the plasma and end-tank chambers. The coolant flowing throuyn he module fuel zone removes the module afterheat during the handling operation. An ex-vesgel module-handling machine receives spent modules through the doubled door transfer port in the upper end tank (not show in Fig. 8-18) and returns new modules. The in-vessel machine must be capable of indexing modules to proper locations, threading them into position on large, coarse threads, and finally torquing the six pressure-vessel bolts accurately before sniffing for vacuun seal leaks and moving to the next module.

The concept of requiring a nearly zero-leak mechanical seal across a 60-ata gradient with helium gas was arrived at in two ways. Firstly, we believe that technology exists currently to do this task at temperature up to abc it $250^{\circ} \mathrm{C}$ with a single varian-type mechanical seal. The technical development to $350^{\circ}$ to $400^{\circ} \mathrm{C}$ appears feasible with a double, differentially pumped design. Furthermore, such a development project appears to be a very-low-cost alternative to other systems, which require:

- Routine 3000-t cryogenic magnet movement, as with the original lune concept.

- Development of extreme-weight-handling maintenance equipment, integrated into the PCRV with accurate geometric control, as with the alternate lune/ram concept.

- Renote first wall welding and cutting with attendant leak checking, debris rewoval, and extended maintenance time requirements for routine procedures, as with other module concepts.

To support neutronic calculations done at Livermore, comparative fuel and structure fraction calculations were made for the reference 1976 lune module blanket and the reference 1977 cylindrical blanket module. The results are listed in Table $8-1$. They support the use of the small cylindrical module design. 


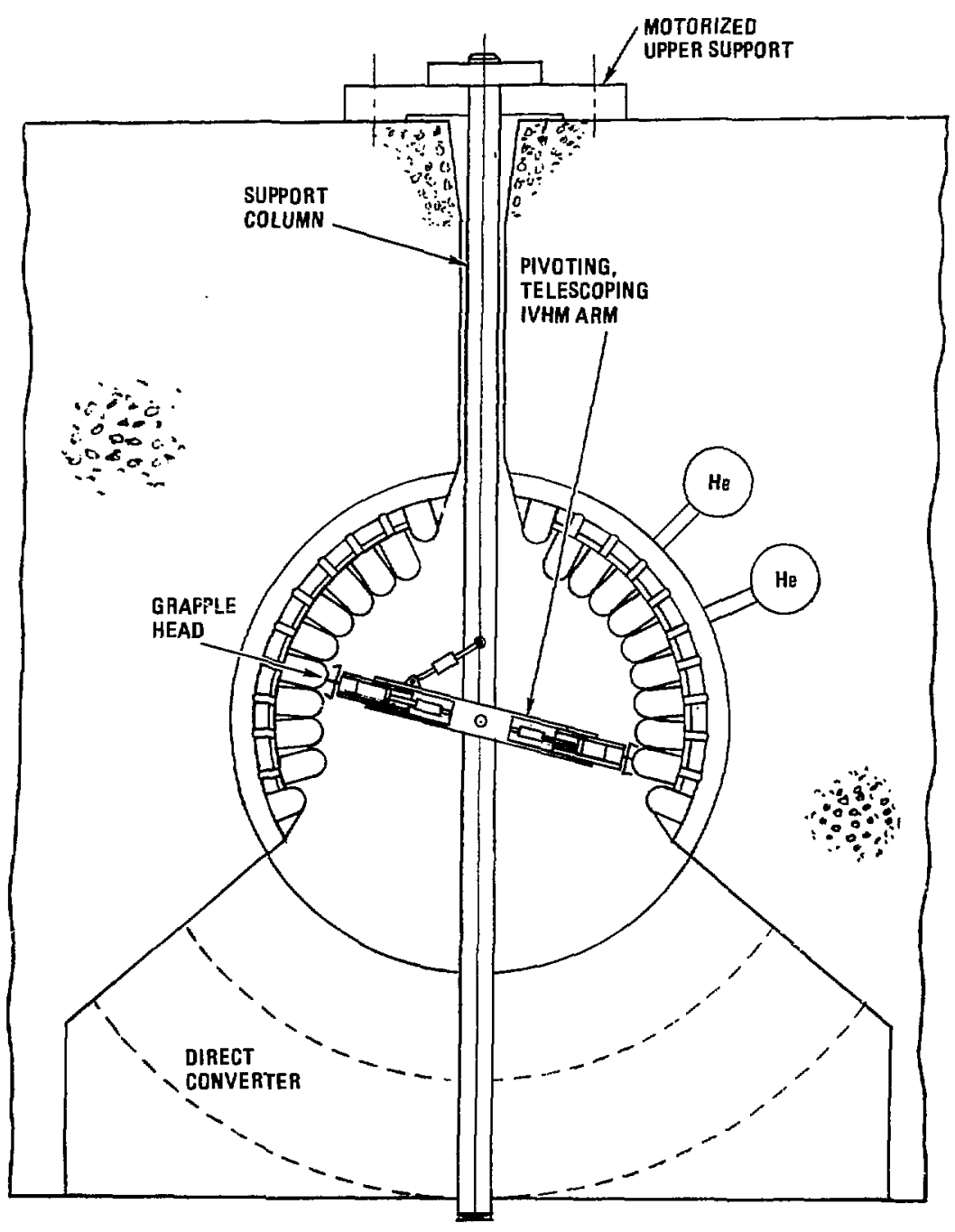

Fig. 8-18. MHR module-handling machine. 
Table 8-1. MHR module comparison.

Cylindrical module

Lune module

Fuel/structure ratio

$$
5.90
$$

5.50

Fuel volune fraction

0.57

0.61

Stru ture volume frawtion

0.10

0.11

Coolant and vold fraction

0.34

0.28

First wall thicknesss, $\mathrm{mm}$

2.1

3.7

Effective wall thickness, $\mathrm{mm}$

4.8

6.7

Effective grid plate thickness, ${ }^{b} \mathrm{~mm}$

14

21

\footnotetext{
ancludes flon distributor and curvature effects.

bincludes flow shrouds and fuel rod end plugs.
}

Several module-seal alternatives were considered including structurally welded seals, mechanical attachment with a thin, nonstructural seal weld, and the mechanical seal chosen for the reference design. Handing-time estimates indicated thai, even for simple weld shapes, the time required to remotely mill or grind off the weld dominated the required module maintenance time; the rewelding time would also be excessive. It was estimated that each module would require approximately 2 hours for weld removal and up to 1 hour for rewelding while the operations required with the mechanical seal design woird take only one-guarter as long.

A major advantage of the sirail-module concept is that it allows the nechanical seal design to be developed on a full scale in the laboratory. The size of the module was selected on the basis of currently available lift capability for remote manipulators with tactile feedback. Because of favorable experience with computer-controlled remote manipulators of this type in fuel randiing in the Fort st. Vrain HTGR, it is expected that the mechanical-seal, small-moäule concept selected for the MrR reference design will enjoy a straightforward and successful development. 
1. An Evaluacion of the Technical and Economic Feasibility of Mirror Fission Devices, Bechtel Corp. Report (Aug 1976).

2. K. R. Schultz, personal communication to J. T. Lloyd, Lockheed Missiles - Space Co. (Dec. 7, 1976).

3. R. W. Moir, et al., Progress on the Conceptual Design of a Mirror Hybrid Fusion-Fission Reactor, Lawierce Iivelmore Laboratory, Rept. UCRL-51797, (Jun. 1975).

4. D. W. Kearney, et al., Conceptual Design Study of a Noncircular Tokamak Demonstration Fusion Power Reactor, General Atomic. CC., Rept. GA-A13992 (Nov. 1976), pp 4-56 - 4-62.

5. Report of the Annual Information Meeting of the National Advanced Alloy Development Program, Hanford Engineering Development Laboratory, Rept. HEDL-TC-721 (Oct. 20, 1976).

6. Nuclear Systems Materials Handbook, Vol. 1, Design Data, Section 5 , Banford Engineering Develogment Laboratory, Rept. TID 26666.

7. A. L. Ward, J. M. Steicher and R. L. Knecht, "Irradiation and Thermal Effects on the Tensile Properties of Inconel $718, "$ presented at the ASTM 8th International Symposium on Effects of Radiation on structural Materials (St. Louis, MO, May 4-6, 1976); Hanford Engineering Development Laboratory, Rept. HEDL-SA-853.

8. D. S. Gelles, "Postirradiation Examination of Neutron Irradiation of Commercial Alloys," National Advanced Alloy Development Program, Annual Information Heeting, Hanford Engineering Development Laioratory, Rept. HEDL-TC-721, (Oct, 20, 1976).

9. F. \%. Wiffen, Swelling of Nickel-Base Alloys Under Simulated CTR Conditions, Draft Report, Oak Ridge National Laboratory (Dec. 9, 1976).

1.0. F. W. Wiffen, The Tensile Properties of Nickel-Base Alloys Irradiated Under Conditions that Sinulate CTR Service, Draft Report, Oak Ridge National Laboratories (Dec. 1976).

11. K. R. Schultz, et al., Conceptual Design of the Blanket and Power Conversion System for a Mirror Hybrid Fusion-Fission Reactor, General Atonic Co., Rept. GA-Rl4021, July, 1976. 


\section{HEAT TRANSPORT SYSTEMS}

\subsection{Fuel Design}

\subsubsection{Fuel Materials Selection}

9.1.1.1 Uranium zone. The candicate fuel materiais that have been evaluated for use in the MHR inc.lude uranium metal, alloys, oxide, carbide and nitxide. During FY 1976, a comparative evaluation was made in terms of important parametiars such as fuel density, neutron economy; physical, mechanical and corrosion properties; Irradiation effects; compatibility with cladding and with impurities in the relium coslant, particularly tritium; and fabrication cost (Ref. 1). The results of this evaluation were reviewed and confirmed; they will be sumariand briefly here.

The 10w-burnup and modest-temperature operational conditions of the MHR tend to favor the use of metallic uranium alloys, particularly uranium silicide $\left(\mathrm{U}_{3} \mathrm{Si}\right)$ fuel, which has been under development in Canada for the CANDU reactor program during the past decade. Uranium oxide is less desirable because of the significantly lower fuel density, lower neutron economy, and higher fabrication cost compared to uraniun silicide. Uranium carbide and uranium nitride also are less desirable, for similar reasons. However, uranium carbide may be a suitable alternative fuel if the silicide should not qualify becauge of temperature limitations. Table 9-1 compares some of the pertinent properties of candidate fuel materials.

Metallịc uranium fuels are generally limited to operation below approximately $600^{\circ} \mathrm{C}$ maximum tenperature and to relatively low burnups of about 5000 MND/HWU (0,5) burnup) because of 1rradiation damage. Swelling and growth become excessive primarily because of fission gas bubbles at high temperatures and the formation of lattice defects (vacancles, Interstitials, dislocation loops, etc.) at low temperatures. Irradiation creep is also a problem at low temperatures. There is little swelling below about $400^{\circ} \mathrm{C}$. The growth reaches a maximum in the range 400 to $600^{\circ} \mathrm{C}$. Above approximately $700^{\circ} \mathrm{C}$, fission gas swelling predominates. The performance of modified metallic uranium fuels has been greatly inproved by providing voidage with an axial hole (Ref. 2). Teat elements with 5 vold have been irradiated to 18,000 MND/MrU (2.2 at. $t$ burnup) at fuel temperatures of up to $610^{\circ} \mathrm{C}$. The available voidage in the fuel was consumed at a rate of 34 per at. burnup. 
Table 9-1. Properties of fuels.

\begin{tabular}{|c|c|c|c|c|c|c|}
\hline & $\begin{array}{c}\text { U } \\
\text { (modi- } \\
\text { fied) }\end{array}$ & $\begin{array}{c}\mathrm{U}-10 \\
\text { Mo }\end{array}$ & $\begin{array}{l}\text { U-5Fs } \\
\text { (EBR-II } \\
\text { Fuel! }\end{array}$ & $\mathrm{U}_{3} \mathrm{Si}$ & $w C$ & $\mathrm{w}_{2}$ \\
\hline $\begin{array}{l}\text { U density, } \\
\mathrm{g} / \mathrm{cm}^{3}\end{array}$ & 19.0 & 17.1 & 18.0 & 15.5 & 13.0 & 9.65 \\
\hline $\begin{array}{l}\text { Melting } \\
\text { point, }{ }^{\circ} \mathrm{C}\end{array}$ & 1133 & 1150 & 1002 & 930 & 2400 & 2800 \\
\hline $\begin{array}{l}\text { Max operating } \\
\text { temperature, }\end{array}$ & 610 . & 700 & 660 & 900 & 2000 & 2400 \\
\hline $\begin{array}{l}\text { Thermal } \\
\text { conductivity, } \\
\text { w/cm }{ }^{\circ} \mathrm{C}\end{array}$ & 0.40 & 0.29 & 0.32 & 0.21 & 0.25 & 0.029 \\
\hline $\begin{array}{l}\text { Thermal expansion } \\
\times 10^{-6} /{ }^{\circ} \mathrm{C}\end{array}$ & 19 & 12.3 & - & 14 & 11.6 & 10.1 \\
\hline $\begin{array}{l}\text { Irradiation } \\
\text { swelling at } \\
\text { 18 burn-up, volume }\end{array}$ & 38 & 68 & $4 \%$ & 2.48 & $\begin{array}{l}18 \\
\left(<900^{\circ} \mathrm{C}\right)\end{array}$ & $\begin{array}{l}0.48 \\
\left(<1500^{\circ} \mathrm{C}\right)\end{array}$ \\
\hline
\end{tabular}

During the past decade, extensive work has been carried out at Chalk River (Atomic Energy of Canada, Limited) on the development of uranium silicide $\left(\mathrm{U}_{3} \mathrm{si}\right.$ has $96.2 \mathrm{wt.8} \mathrm{U}$ and $3.8 \mathrm{wt.8} \mathrm{si}$ ) fuel for t..e CANDU reactors (Ref. 3). The interest in $\mathrm{U}_{3} \mathrm{Si}$ as a fuel arose from its unique conbination of high uranium density $\left(15.58 \mathrm{~g} / \mathrm{cm}^{3}\right)$ with low parasitic absorption, excellent corrosion resistance (especially with $1.58 \mathrm{Al}$ additions), and high thermal conductivity $\left(0.2 \mathrm{w} / \mathrm{cm}^{\circ} \mathrm{C}\right)$. A peritectoid phase change takes place in $\mathrm{U}_{3} \mathrm{Si}$ at $930^{\circ} \mathrm{C}$, which is taken as the upper limit of operation. The $\mathrm{U}_{3} \mathrm{Si}$ fuel was estimated to have a cost advantage of $10 \%$ to $20 \%$ over $\mathrm{WO}_{2}$ fuel because of lower fabrication costs and higher uranium density.

The ir -adiation behavior of $\mathrm{U}_{3} \mathrm{si}$ has been studied extensively (Refs. $4,5,6)$. The results indicate that this fuel has good irradiation stability in reasonably high burnups, up to about $900^{\circ} \mathrm{C}$. The swelling car be very effectively restrained by the cladding and coolant pressure that forces the fuel to swell into the axial or peripheral voidage machined into the fuel rods. The maximum swelling occurs at $500^{\circ} \mathrm{C}$ and decreases both above and below this temperature. However, above $900^{\circ} \mathrm{C}$ rapid swelling occurs during postirracistion annealing.

Three firel-material candidates were salected as viable candiates for finel evaluation: uraniun-molybdenum alloy, uranium silicide, and uranium 
carbide. Since any of these materials would be an acceptable choice for the MHR, the final selection was based on cost estirates for the system economics that each alternative would dictate.

The economics of the various fuels are affected by four factors:

- Basic tuel cost.

- Fabrication cost.

- Revanue from power produced.

- Revenue from fissile production.

The estimated blanket costs and revenues for the $1976 \mathrm{MHR}$ design with the three fuel options are shown on Table 9-2. Fabrication cost will depend upon fuel-rod size, which is dictated by the allowable fuel rod centerline

Table 9-2. Economics of candidate fertile materials fot MHR.

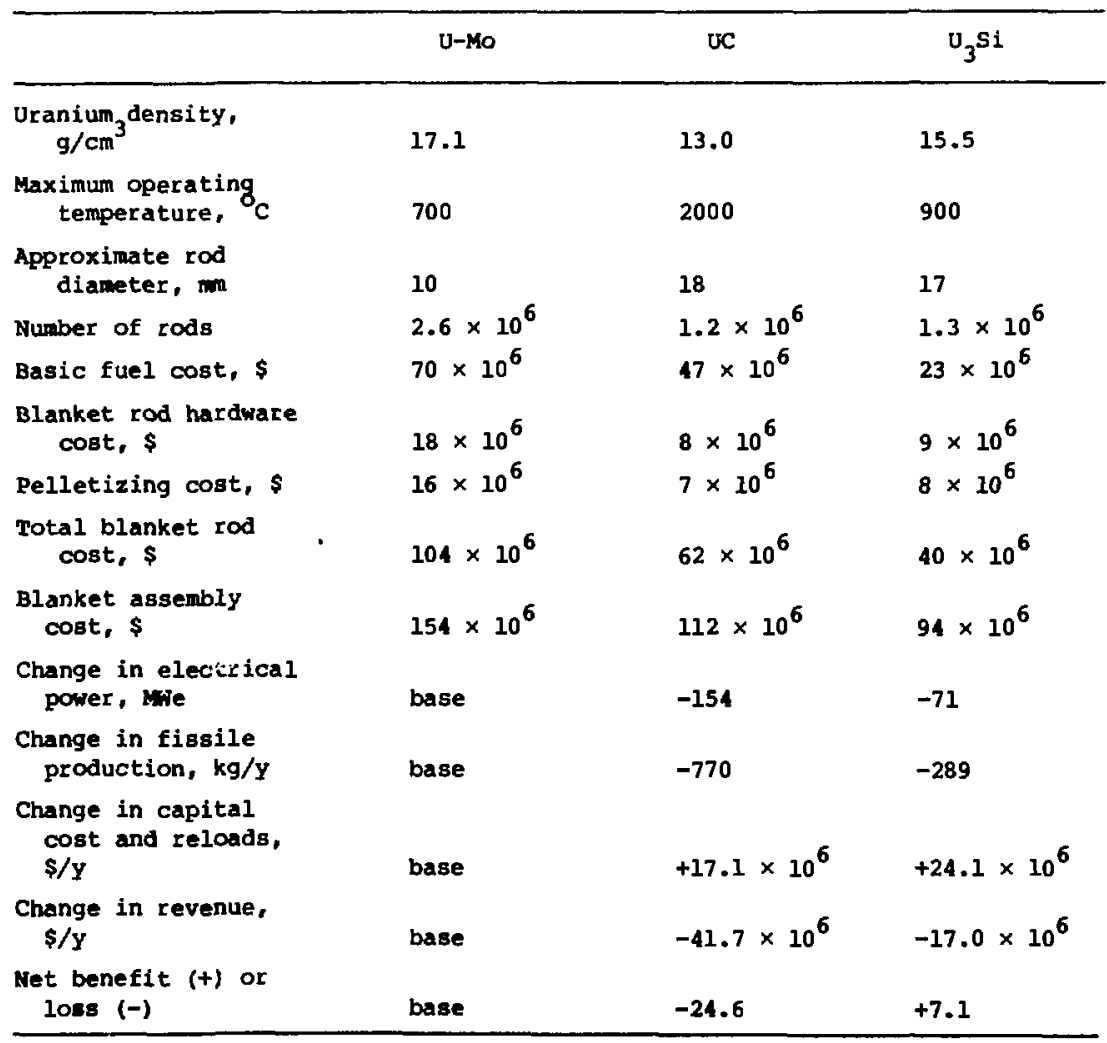


temperature, up to about $17 \mathrm{~mm}$ in diameter when the cladding temperature becomes limiting. Revenues from power and fissile production are roughly proportional to uranium density. Estimates of changes in these revenues are shown with uranium-molybdenum as a reference.

The $\mathrm{U}_{3} \mathrm{Si}$ offers the advantages of improved system economics, ease of fabrication, lower parasitic absorption than uranium-molybdenum, and higher fuel density than uranium carbide. For these reasons $U_{3} S i$ was chosen for the MHR; this choice was reviewed and reaffirmed.

9.1.1.2 Tritium Breeding Material. A unique aspect of hybrid reactors is the desire to breed two products in the blanket: both fissile fuel and tritium to fuel the fusion reactor. We have thosen lithium hydride (Lif) for the MHR design; LiH has a high lithium density plus hydrogen to moderate the neutrons. It also appears to have excellent irradiation stability as radiation damage anneals out at temperatures above $300^{\circ} \mathrm{C} .^{7}$ A potential problem with LiH is its high hydrogen vapor pressure. In a system designed to release the bred tritium by use of vented fuel rods or hydrogen-permeable cladding, dehydriding the LiH would be a serious concern. We have opted to contain the tritium in the fuel rods by using hydrogen-retentive aluminum-alloy cladding and low lithium-zone temperatures. The aluminum-beryllium alloy, Lockalloy-43, wa: used as LiH cladding because of the additional strength and temperature capability that beryllium adds to aluminum alloys.

\subsubsection{Fuel Configuration}

The following configurations were evaluated for the blanket fuel of the MRR $^{1}$ : honeycomb, plates, tangential rods with cross flow, radial rods with solid pellets, and radial rods with annular pellets.

Evaluation of these configurations from thermal-hydraulic, as well as manufacturing considerations indicated that, with $\mathrm{U}_{3} \mathrm{Si}$ as the blanket fuel, radial rods with annular pellets represent the best configuration. Flat plates oriented with their edges towards the plasma and radially oriented helium flow may offer potintial advantages in that the flow gap may be made smaller than the gaps between a tight-packed bundle of cylindrical rods. This would allow a higher coolant velocity with better cooling and could allow a higher fuel packing fraction. However, the structural concerns associated with use of Elat plates, especially in the small cylindrical modules chosen for the MR, weigh against the flat-plate configuration. Thus, the radially oriented cylindrical rods, with a helium flow parallel to the axis of the rods, were chosen for the MHR fuel design. 
The rods (made of Inconel $71 \kappa$ ) will be sealed at atmospheric pressure for operation in a pressure of $6.5 \mathrm{MPa}(60 \mathrm{~atm})$. Because of the small burnup to which the fuel is subjected (about 18 peak and 0.50 average) and the highly retentive nature of the fuel, fission-product release will be negligible and will not contribute to any significant increase is the rod plenum pressure. During operation, the coolant pressure will cause the cladding to creepcollapse onto the fuel. With proper design of voidages, the swelling force of the fuel on the cladding can be minimized so that, even if the reactor experiences a depressurization accident at operating temperature, the stresses in the cladding will be acceptably low.

\subsubsection{Fuel Design and Performance}

9.1.3.1 Uranium zone. The objective of this task was to determine a fuel design that did not exceed any safe operating limits for temperature or stress.

The material selected for clad and blanket structure is Inconel 718. The cladding was fixed at a $0.15-\mathrm{mm}$ thickness. The fuel material is uranium silicide $\left(\mathrm{U}_{3} \mathrm{Si}\right)$. The temperature limits were fixed at:

- Maximum hot-spot clad temperature of $700^{\circ} \mathrm{C}$.

- Maximum hot-spot fuel temperature of $900^{\circ} \mathrm{C}$.

- Maximum pressure-vessel temperature of $500^{\circ} \mathrm{C}$.

The inlet and outlet coolant temperatures for the blanket module were fixed at $280^{\circ} \mathrm{C}$ and $540^{\circ} \mathrm{C}$. These temperatures enable a safe design of the blanke'c fuel while achieving a reasonable thermal efficiency in the steam generator.

The distribution of the power-generation rate (approximated at the end of life) in the blanket is shown in Fig. 9-1. The coolant flow was treated as a one-dimersional incompressible flow. The heat-transfer coefficient for the wire-wrapped bundle is 108 to 308 larger than the standard turbulent-flow correlation. 8 as a conservative approach, we assumed that the heat-transfer coefficient in the MHR blanket is 10\% larger than turbulent flow in a tube.

Fuel design was based on hot-spot temperatures obtained by applying the hot-spot factors to the nominal temperatures calculated in an average channel. The hot-spot factors account for the effects on the thermohydraulic analysis of the uncertainties in experimental correlations, manufacturing tolerances, instrumentation accuracy, material properties, and multidinensional flow-distribution effects. A semistatistical method ${ }^{9}$ adopted for FFrF, CRBRP, and GCFR analyses was used in the present analysis. Using this method, 


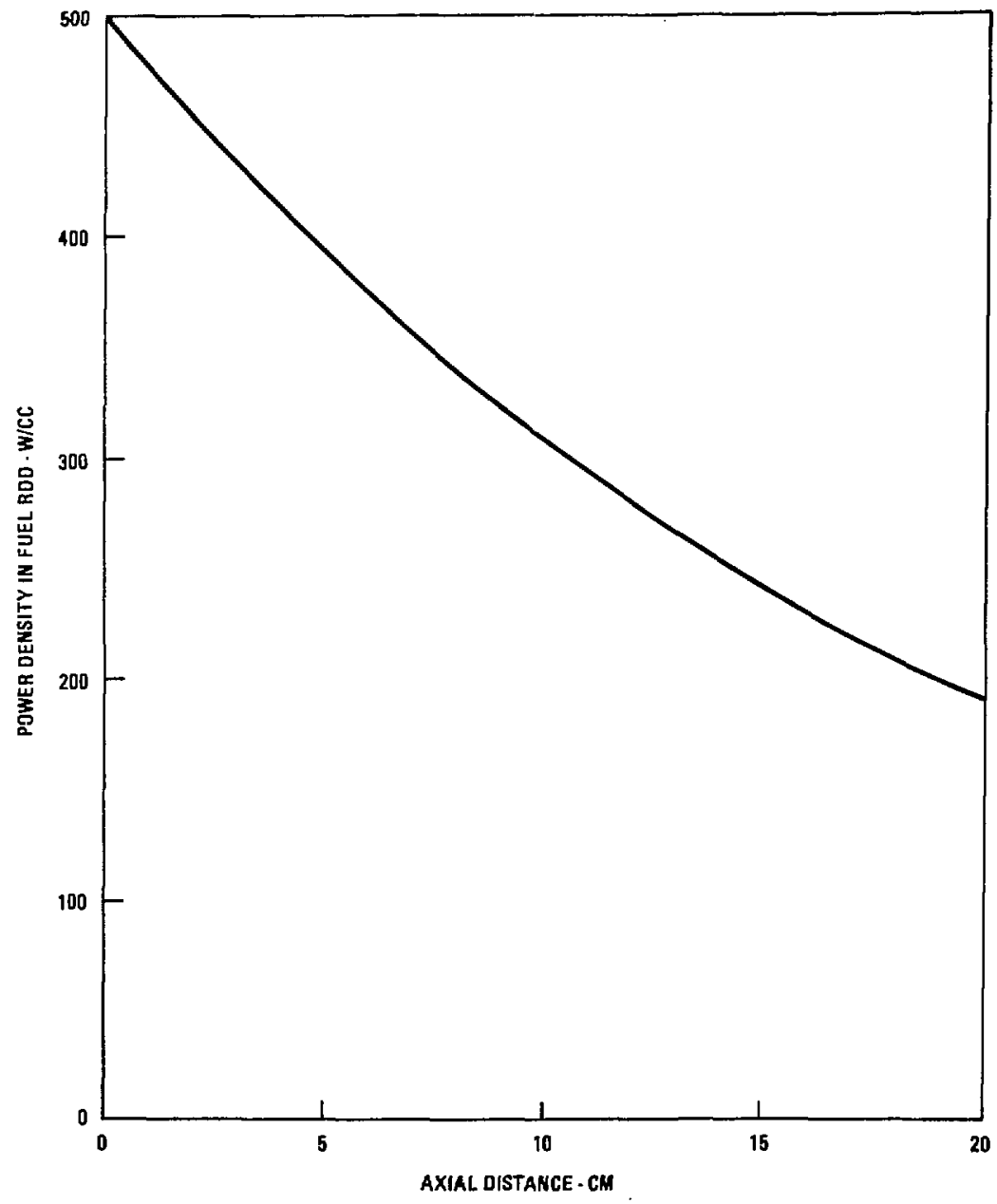

Fig. 9-1. Power distribution in MRR blanket fuel at the end of life. 
hot-spot factors for the MHFFR blanket were evaluated. These are sumarized in Table 9-3.

The hot-spot factors in Table 9-3 are used to calculate the hot-spot temperatures as follows:

$$
T_{c}^{\prime}=T_{I}+\Delta T_{B} F_{b}+\Delta T_{f} \stackrel{F}{F}_{f}+\Delta T_{c} F_{c}
$$

and

$$
\begin{aligned}
\mathrm{T}_{\mathrm{F}}^{\prime}=\mathrm{T}_{\mathrm{I}} & +\Delta \mathrm{T}_{\mathrm{B}} \mathrm{F}_{\mathrm{b}}+\Delta \mathrm{I}_{\mathrm{f}} \mathrm{F}_{\mathrm{f}}+2 \Delta \mathrm{T}_{\mathrm{c}} \mathrm{F}_{\mathrm{C}} \\
& +\Delta \mathrm{T}_{\mathrm{g}} \mathrm{F}_{\mathrm{g}}+\Delta \mathrm{T}_{\mathrm{F}} \mathrm{s}_{\mathrm{R}},
\end{aligned}
$$

where,

$$
\begin{aligned}
T_{C}^{\prime} & =\text { mid-wall hot-spot cladding temperature } \\
T_{I} & =\text { nominal inlet temporature of the coolant } \\
\Delta T_{B} & =\text { nominal bulk temperature rise } \\
F_{b} & \approx \text { hot-spot factor for bulk coolant rise } \\
\Delta T_{f} & =\text { nominal film drop } \\
\vec{F}_{f} & =\text { hot-spot factor for film drop (clad) } \\
\Delta T_{C} & =\text { nominal temperature drop through half the clad thickness } \\
F_{C} & =\text { hot-spot factor for cladding } \\
T_{F}^{\prime} & =\text { hot-spot fuel temperature } \\
t_{f} & =\text { hot-spot factor for film drop (fuel) } \\
\Delta T_{G} & =\text { nominal temperature drop through the clad pellet gap } \\
F_{g} & =\text { hot-spot factor for gap temperature drop } \\
\Delta T_{F} & =\text { nominal temperature drop in the fuel } \\
F_{R} & =\text { hot-spot factor for fuel } \Delta T \text { unse:tainty. }
\end{aligned}
$$

Table 9-3. Hot-spot factors for MRR fuel.

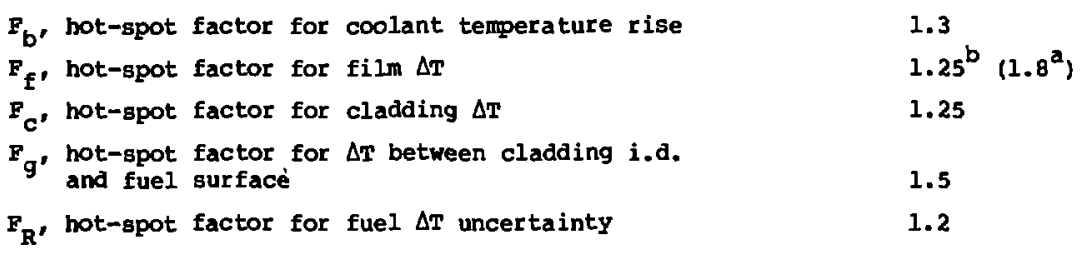

aor hot-spot cladding temperature.

$b_{\text {For fuel temperature calculations. }}$ 
The various temperatre rise or drop terms were calculated using a twodimensional heat-transfer model to represent axial coolant flow along the fuel rod and radial heat transfer from the rod to the hellum. ( $\mathrm{Axial}$ conduction in the rod is small and has been neglected.)

Their expressions are:

$$
\begin{aligned}
& \Delta T_{B}(l)=\frac{1}{\dot{m}_{p}} \cdot \pi\left(r_{p}^{2}-r_{h}^{2}\right) \int_{0}^{l} q^{\prime \prime}(x) d x . \\
& \Delta T_{f}(l)=\frac{1}{h_{f}} \dot{q}^{\prime} \cdots(l) \frac{\left(r_{p}^{2}-r_{h}^{2}\right)}{2 r_{r}} \\
& \Delta T_{c}(l)=\frac{t}{k_{c}} \dot{q} \cdots, \quad(l) \frac{\left(r_{p}^{2}-r_{h}^{2}\right)}{4\left(r_{r}-t\right)} \\
& \Delta r_{g}(l)=\frac{1}{h_{g}} \dot{q} \cdots, \quad(l) \frac{\left(r_{p}^{2}-r_{h}^{2}\right)}{r_{p}} \text {. } \\
& \Delta T_{F}(l)=\dot{q} \cdots(l) \frac{r_{p}^{2}}{4 k_{F}}\left[1-\left(\frac{r_{h}}{r_{p}}\right)^{2}-\left(\frac{r_{h}}{r_{p}}\right)^{2} \ln \left(\frac{r_{p}}{r_{h}}\right)^{2}\right] \text {, }
\end{aligned}
$$

where

$$
\begin{aligned}
\dot{m} & =\text { helium flow rate per rod } \\
c_{p} & =\text { helium specific heat } \\
r_{p} & =\text { radius of fuel pellet } \\
r_{h} & =\text { radius or central fuel hole } \\
\dot{q}_{(x)}^{\prime} & =\text { local heat generation rate in the fuel rod } \\
h_{f} & =\text { heat transfer conductance for helium film drop } \\
& =1.1\left(0.0201 \frac{k_{h}}{d_{H}} R^{0.8} f_{e}\right) \text { (turbulent) } \\
& =1.1\left(3.66 \frac{k_{h}}{d_{H}} f_{e_{l}}\right) \text { (laminar) } \\
k_{h} & =\text { conductivity of helium } \\
d_{H} & =\text { hydraulic diameter of flow path } \\
R_{e} & =\text { Reynolds number } \\
& =\text { entrance effect correction for laminar or turbulent } \\
f_{e_{\ell}} & \text { flow }
\end{aligned}
$$


$r_{r}=$ radius of the fuel rod

$k_{c}=$ conductivity of the clad

$k_{F}=$ conductivity of fuel

\pm clad thickness

$h_{\underline{L}}=$ heat transfer conductance for the clad-pellet gap.

Equations (1) and (2) may be used to calculate the nominal temperatures by simply using hot-spot factors of 1.0 .

Thermal analysis was performed for an average flow channel of the rod bundle to obtain the nominal temperatures at full power. Hot-spot factors were applied to these temperatures to obtain the maximum temperatures.

The first wall of the MHR blanket with the baffle is designed so that the velocity of flow remains constant as the flow turns and part of the flow yues through the baffle. This is achieved by taparing the gap between the pressure dome and the flow baffle toward the center and by providing holes in the baffle that increase is diameter toward the center. The first wall temperature could be limited to $342^{\circ} \mathrm{C}$ with this arrangement. Concer $\mathrm{x}$ about the effect of flow stagnation at the center point of the module dome was addressed by a simple analysis that assumed no heat transfer to the helium at the centerpoint. All heat was removed by conduction through tis Inconel along the dome. It was found that the central, uncooled stagnai;on-point region could be as large as $3 \mathrm{~cm}$ in diameter without causing the $500^{\circ} \mathrm{C}$ pressurevessel temperature limit to be exceeded. The relative ease of first wall cooling is due to the lack of a significant heat flux on the first wall from the plasma. Because of the low $Q$ or "leaky" nature of the standard mirror plasma, no alpha or impurity radiation heating is expected. First wall heating will be due to backscatter from the neutral-beam injectors (approximately $\left.2 \mathrm{H} / \mathrm{cm}^{2}\right)$ and neutron heating $\left(13.8 \mathrm{w} / \mathrm{cm}^{3}\right)$.

The thermal performance of the fuel was calculated using Eqs. (1) through (6), and a number of feasible blanket designs were generated for different peak powers at the end of life (Table 9-4). For safe partial-power operation away from laminar flow regimes, it is desirable to have high Reynolds numbers at full power. For manufacturing and structure-to-fuel ratios, large rod diameters are desirable. The design point was therefore selected at $500 \mathrm{w} / \mathrm{cm}$ peak power.

The axial distribution of nominal temperatures for the chosen 7-mmdiameter rod size is plotted in Fig. 9-2. The cladding and the fuel temperatures are almost uniform except for the first few centigeters where the 


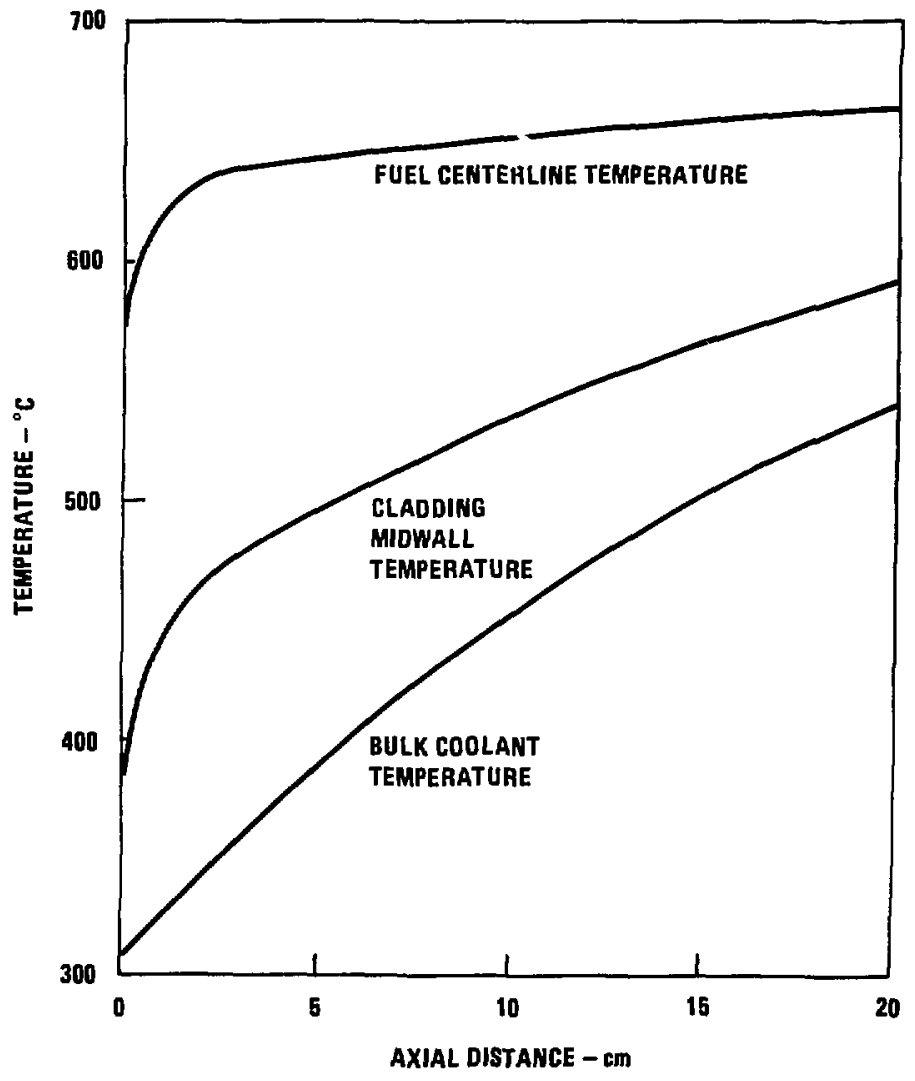

Fig. 9-2. Nominal temperatures in the MHR $u_{3}$ si fuel rods. 

Table 9-4. Thermal performance of fuel calculated with Eces
for several peak powers at the end of life.

(1) through (6)

\begin{tabular}{|c|c|c|c|c|c|c|c|c|}
\hline \multirow{2}{*}{$\begin{array}{l}\text { Peak } \\
\text { power, } \\
\mathrm{w} / \mathrm{cm}^{3}\end{array}$} & \multirow{2}{*}{$\begin{array}{c}\text { Rod } \\
\text { diam, } \\
\mathrm{cm}\end{array}$} & \multirow{2}{*}{$\begin{array}{c}\text { Pellet } \\
\text { diam, } \\
\mathrm{cm}\end{array}$} & \multirow[b]{2}{*}{$\begin{array}{r}R e \\
\times \quad 10^{4}\end{array}$} & \multirow{2}{*}{$\begin{array}{c}\text { Fuel rod } \\
\text { inlet } \\
\text { temp, } \\
{ }^{\circ} \mathrm{C}\end{array}$} & \multicolumn{2}{|c|}{ Max temp } & \multicolumn{2}{|c|}{$\begin{array}{l}\text { Infinite lattice } \\
\text { volume fraction }\end{array}$} \\
\hline & & & & & $\begin{array}{c}\text { Cladding, } \\
{ }_{{ }^{\circ}}\end{array}$ & $\begin{array}{l}\text { Fuel, } \\
\text { oc }\end{array}$ & Fuel & $\begin{array}{l}\text { Clad ano } \\
\text { wire wrap }\end{array}$ \\
\hline 600 & $\begin{array}{l}0.7^{b} \\
0.8^{c}\end{array}$ & $\begin{array}{l}0.66 \\
0.76\end{array}$ & $\begin{array}{l}1.02 \\
1.18\end{array}$ & $\begin{array}{l}306 \\
326\end{array}$ & $\begin{array}{l}700 \\
736\end{array}$ & $\begin{array}{l}799 \\
900\end{array}$ & $\begin{array}{l}0.692 \\
0.700\end{array}$ & $\begin{array}{l}0.073 \\
0.064\end{array}$ \\
\hline 500 & $\begin{array}{l}0.7 \\
0.9\end{array}$ & $\begin{array}{l}0.66 \\
0.86\end{array}$ & $\begin{array}{l}0.85 \\
1.12\end{array}$ & $\begin{array}{l}310 \\
316\end{array}$ & $\begin{array}{l}700 \\
738\end{array}$ & $\begin{array}{l}775 \\
900\end{array}$ & $\begin{array}{l}0.692 \\
0.706\end{array}$ & $\begin{array}{l}0.073 \\
0.057\end{array}$ \\
\hline 450 & $\begin{array}{l}0.7 \\
0.9\end{array}$ & $\begin{array}{l}0.66 \\
0.86\end{array}$ & $\begin{array}{l}0.77 \\
1.00\end{array}$ & $\begin{array}{l}312 \\
354\end{array}$ & $\begin{array}{l}700 \\
774\end{array}$ & $\begin{array}{l}755 \\
900\end{array}$ & $\begin{array}{l}0.692 \\
0.706\end{array}$ & $\begin{array}{l}0.073 \\
0.057\end{array}$ \\
\hline 400 & $\begin{array}{l}0.7 \\
1.0\end{array}$ & $\begin{array}{l}0.66 \\
0.96\end{array}$ & $\begin{array}{l}0.68 \\
1.00\end{array}$ & $\begin{array}{l}314 \\
326\end{array}$ & $\begin{array}{l}700 \\
759\end{array}$ & $\begin{array}{l}756 \\
900\end{array}$ & $\begin{array}{l}0.692 \\
0.711\end{array}$ & $\begin{array}{l}0.073 \\
0.051\end{array}$ \\
\hline 350 & $\begin{array}{l}0.8 \\
1.1\end{array}$ & $\begin{array}{l}0.76 \\
1.06\end{array}$ & $\begin{array}{l}0.69 \\
0.97\end{array}$ & $\begin{array}{l}302 \\
308\end{array}$ & $\begin{array}{l}700 \\
753\end{array}$ & $\begin{array}{l}755 \\
900\end{array}$ & $\begin{array}{l}0.700 \\
0.715\end{array}$ & $\begin{array}{l}0.064 \\
0.047\end{array}$ \\
\hline 300 & $\begin{array}{l}0.8 \\
1.2\end{array}$ & $\begin{array}{l}0.76 \\
1.16\end{array}$ & $\begin{array}{l}0.59 \\
0.91\end{array}$ & $\begin{array}{l}305 \\
304\end{array}$ & $\begin{array}{l}700 \\
760\end{array}$ & $\begin{array}{l}744 \\
900\end{array}$ & $\begin{array}{l}0.700 \\
0.719\end{array}$ & $\begin{array}{l}0.064 \\
0.043\end{array}$ \\
\hline
\end{tabular}

aconditions:

$\Delta$ T Blanket $=230^{\circ} \mathrm{C}$

Cladding thickness $=0.015 \mathrm{~cm}$

Maximum allowable

fuel temperature $=900^{\circ} \mathrm{C}$

$P / d=1.05$.

${ }^{b}$ cladding limit $700^{\circ} \mathrm{C}$.

cladding limit $800^{\circ} \mathrm{C}$.

entrance effect is dominant. The hot-spot temperatures for the reconmended rod diameter are plotted in Fig. 9-3.

Regeneration effects due to heat transfer between hot and cold helium in the blanket module are quite small because of the small heat-transfer areas available, the large mass flow of helium, and the use of double walls in the module. The regeneration effect was estimated to be less than $5^{\circ} \mathrm{C}$.

Since the Mach number of flow in the MHR blanket is much smaller than 1, the flow was treated as incompressible. The following relations were used to calculate the pressure drop through the MiR blanket module:

$$
\begin{aligned}
& \Delta P_{f}=\frac{f L}{d_{H}} \frac{\rho v^{2}}{2 g_{c}}, \\
& \Delta P_{1}=K \frac{\rho v^{2}}{2 g_{c}},
\end{aligned}
$$




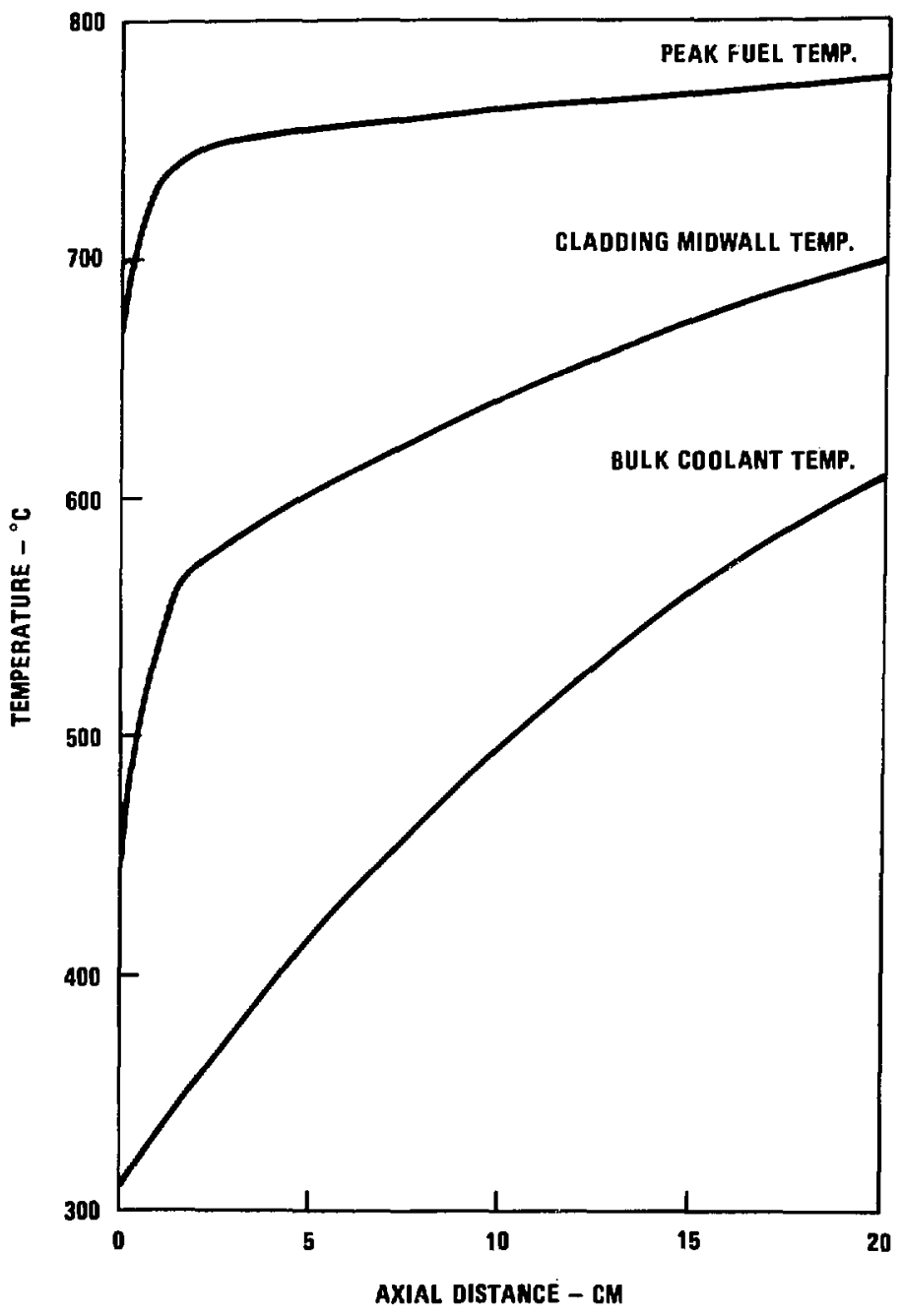

Fig. 9-3. MRR blanket $\mathrm{U}_{3} \mathrm{Si}$ fuel hot-spot temperatures. 


$$
\Delta P_{W}=K \frac{f L}{d_{H}} \frac{\rho v^{2}}{2 g_{c}},
$$

where

$$
\begin{aligned}
& \Delta P_{f}=\text { pressure drop for flow through passages } \\
& \Delta P_{1}=\text { pressure drop due to losses (e.g., turning, expansion, } \\
& \text { contraction, etc.) } \\
& \Delta \mathrm{P}_{\mathrm{w}}=\text { pressure drop in wire-wrapped bundle } \\
& f=\text { friction factor } \\
& I=\text { length of passaje } \\
& D=\text { diameter of fuel rod } \\
& d_{H}=\text { hydraulic diameter } \\
& \rho=\text { average density of coolant } \\
& V=\text { velocity of flow } \\
& g_{c}=\text { gravitational constant } \\
& K=\text { loss coefficient } \\
& M=\text { friction factor multiplier for wire-wrapped bundle } \\
& M=\left[\frac{1.034}{(P / D)^{0.124}}+\frac{29.7(P / D)^{6.94} \mathrm{Re}^{0.086}}{(\mathrm{H} / \mathrm{D})^{2.239}}\right]^{0.885} \\
& P \times \text { rod-to-rod pitch } \\
& \text { H }=\text { wire-wrap pitch } \\
& \text { Re }=\text { Reynolds number of flow }
\end{aligned}
$$

The fraction-factor correlations and loss coefficients in Egs. (3) to (5) were obtained from Ref. 10, and the friction-factor multiplier in Eq. (5) is a Novendstern correlation. ${ }^{11}$

The blanket submodule was modeled as a flow-resistance network. Using Eqs. (8) to (10), the following pressure drops were calculated:

- Total pressure drop in module $=4.1 \times 10^{4} \mathrm{~Pa}(6.0 \mathrm{psi})$

- Pressure drop in blanket region $=1.32 \times 10^{4} \mathrm{~Pa}(1.9 \mathrm{psi})$

- Pressure drop in module during shutdown (depressurized) cooling $=0.2 \times 10^{4} \mathrm{~Pa}(0.3 ; \mathrm{si})$

The significant dimensions and thermal-hydraulic parameters for the blanket are sumarized in Table 9-5.

9.1.3.2 Partial Power Operation. The radial-flow configuration chosen for the MFR blanket appears to be ideally suited to hybrid-reactor blankets, which exhibit a very steep radial power-density gradient. Because of the 
Blanket rod dimensions, $\mathrm{mm}$

Rod 0.d. 7.0

Clad thickness

0.15

Rod i.d.

6.70

Pellet 0.d.

6.60

Annular hole

1.87

Rod pitch

Wire-wrap pitch 100.0

Temperatures, ${ }^{\circ} \mathrm{C}$

Helium at inlet to fuel

Helium at outlet from fuel

Maximum hot-spot cladding temperature

Maximum hot-spot fuel temperature

Elow

Average Reynolds number in blanket

Average velocity of $\mathrm{flow}, \mathrm{m} / \mathrm{s}$

51

Heat-transfer coefficient, $H / \mathrm{m}^{20} \mathrm{C}$

Pressure drop. Pa (psi)

In the blanket

$1.32 \times 10^{4}(1.9)$

Total module

Burnup, o

Reak

short fuel-rod length, however, the coolant flow per channel is modest, and even with very tight rod packing (pitch/diameter $=1.05$ ), good cooling is difficult to achieve. The flow Reynolds number is only about $10^{4}$, which leads to modest heat-transfer conductances and, thus, small fuel-rod diameters. In a hybrid reactor, the peak fuel power density occurs at the end of the iuel life because of the buildup of bred fissile material. At the beginning of fuel life, the local power density in the MHR at full power is only 48 s of the design power that will occur at the end of fuel life. If the helium temperature rise across the blanket is kept constant by reducing helium flow so as to maintain steam-generator temperatures and steam conditions, the heliun-flow 
conditions at partial power drop into the transition flow zone, and the fuel cladding temperature exceeds the $700^{\circ} \mathrm{C}$ hot-spot design limit as shown on Fig. 9-4. Although this condition is undesirable, the design is acceptable because this is a hot-spot limit and because the limit is set by timeintegrated irradiation embrittlement considerations.

9.1.3.3 Lithium zone. Radially oriented rods were also selected for the lithium zone. The lithium hydride will not need a central void since swelling problems are not anticipated with $\mathrm{LiH}$ at $\mathrm{T}>300^{\circ} \mathrm{C}$. The roc's are made of Lockalloy-43, with a 30-mm diameter and a 1.0-mi-thick wall.

The lithium zone generates 6 of the total blanket power, and the peak power is $30 \mathrm{w} / \mathrm{cm}^{3}$. To minimize tritium release, the average operating temperature of the cladding is limited to $350^{\circ} \mathrm{C}$. Figure 9.5 shows the peak temperature of the lithium and cladding as a function of the cladding dianeter. The peak hot-spot temperatures are also shown using the factors from Table 9-3. The significant dimensions and parameters are sumnarized in Table 9-6.

Table 9-6. MHR blanket data lithium zone.

Blanket rod dimensions, $\pi m$

Rod o.d. 30.00

cladding thickness 1.00

Rod i.d. 28.00

Pellet diameter 27.9

Rod pitch 31.5

Temperatures, ${ }^{\circ} \mathrm{C}$

Inlet helium 282

Outlet helium 296

Peak cladding miduall 356

Peak hot spot midwall 393

Peak centerline 511

Peak hot-spot centerline 557

Flon

Average Reynolds number 43,630

Average velocity, $\mathrm{m} / \mathrm{s}$ 6.46

Heat-transfer coefficient, $w / m^{20} \mathrm{C}$ 4745

Pressure drop, Pa (psi)

$1.01 \times 10^{4}(1.46)$ 


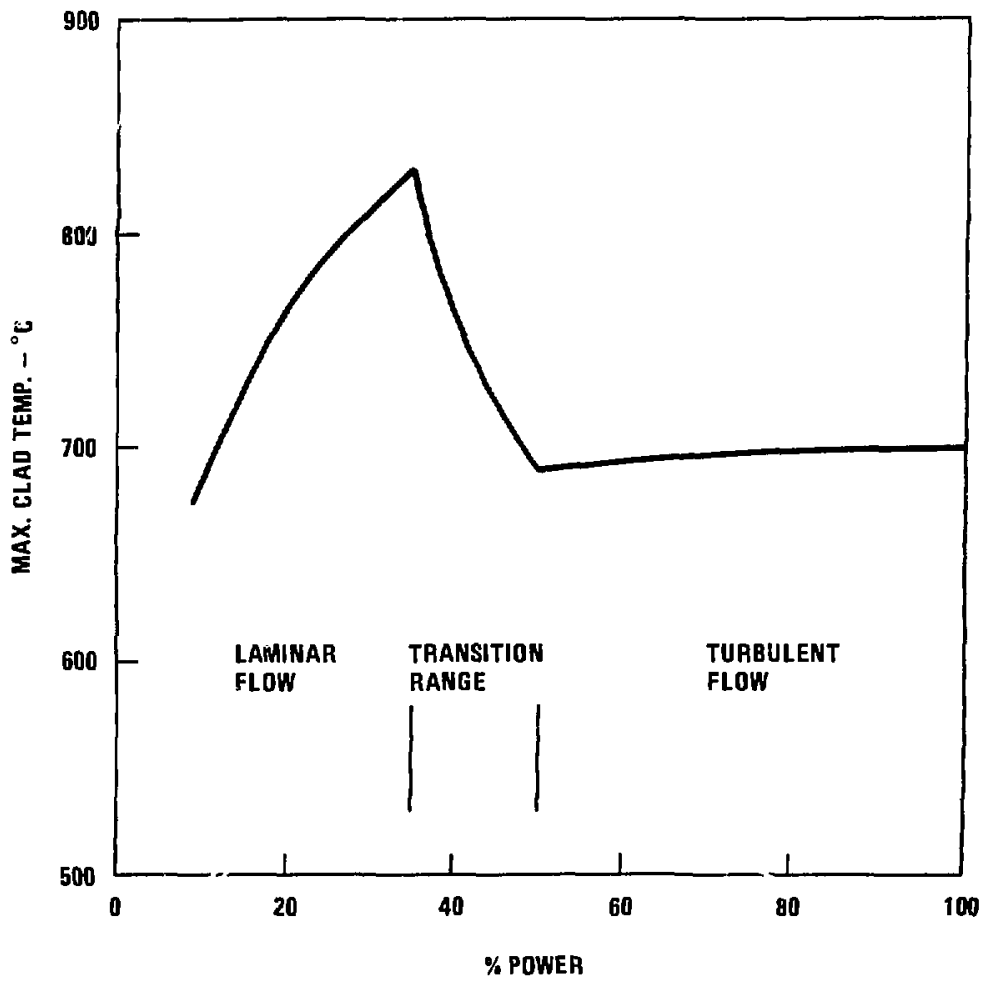

Fig. 9-4. MHR hot-spot cladding temperature at fartial power. 


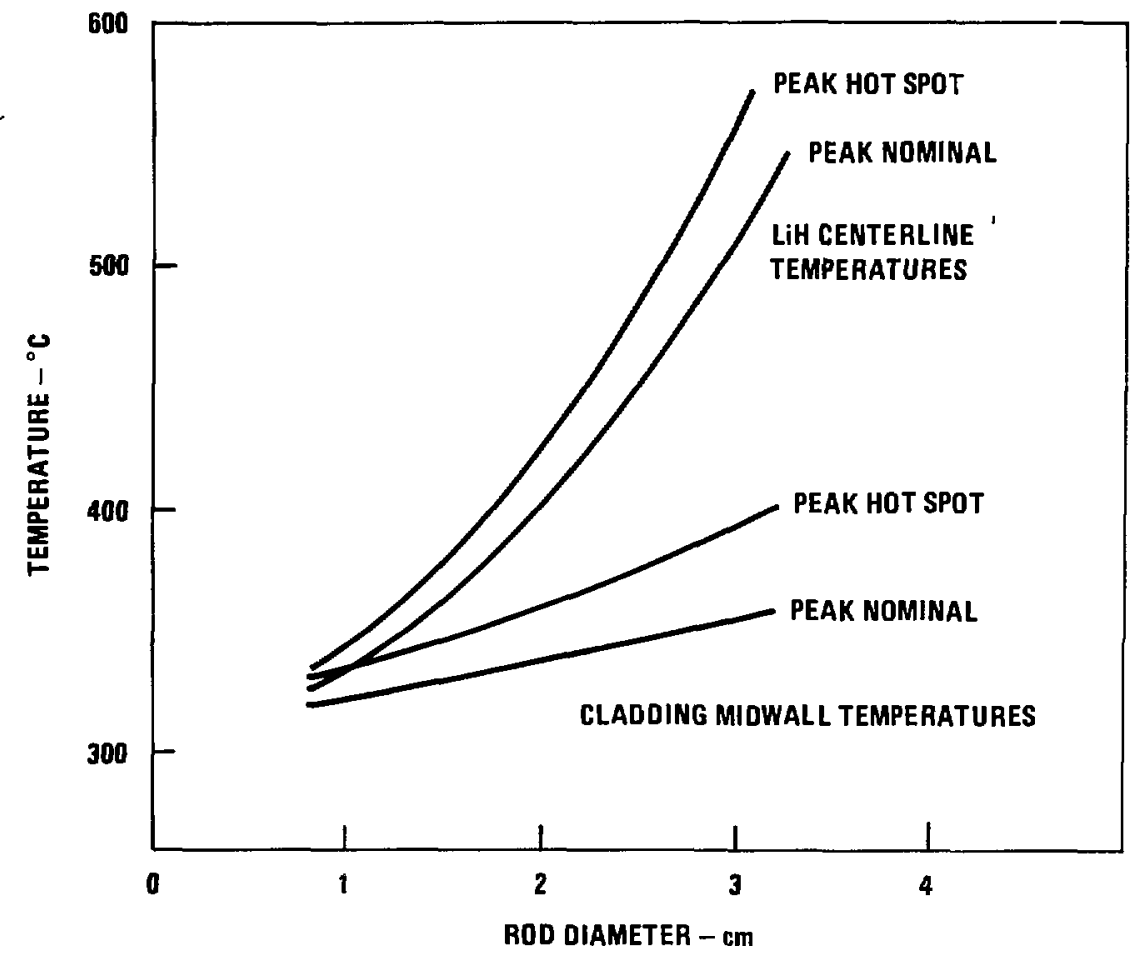

Fig. 9-5. MHR LiH fuel rod temperatures. 


\subsubsection{Fuel Mechanical Design}

The design of the $\mathrm{U}_{3} \mathrm{Si}$ and $\mathrm{LiH}$ fuel rods are shown on Fig. 9-6. The designs follow conventional nuclear practice. Unusual features are the thin end caps and the lack of internal springs or pellet constraints. The end-cap volume is minimized because of the sensitivity of the nuclear performance to structural material between the plasma and the fuel. Internal fuel-pellet constraints in the form of springs are frequently included in nuclear fuel-rod designs. A void space must be left in mott designs to accommodate fission-gas pressure and to allow for expansion of the pellets. To prevent motion and possible damage to the pellets during shipping, a spring restraint may be used. In the MHR, no fission-gas plenum is needed because of the low burnup and the highly retentive nature of the $\mathrm{U}_{3} \mathrm{Si}$ and $\mathrm{LiH}$. An axial hole is left in the $\mathrm{U}_{3} \mathrm{Si}$ pellet to accommodate fuel irradiation swelling. The differential thermal expansion between the fuel and the clad as the fuel is heated to operating conditions is very small, and, thus, close pellet-clad axial tolerance can be used. This close tolerance and the ductile nature of the $\mathrm{U}_{3} \mathrm{Si}_{\text {, }}$ which resists chipping and cracking, allows us to dispense with the pellet restraint spring.

The fuel rods are not vented in this design, although both the $\mathrm{U}_{3} \mathrm{Si}$ rods and the $\mathrm{L}$ il rods could easily be vented through a pressure equalization system to fission product and tritium recovery systems. The venting could be easily accomplished thzough passages, drilled in the grid plates, connecting to the individual fuel pins as is done in the GCFR.

The fuel rods are not prepressurized and, although the cladding is freestanding, it is expected that the clad may creep down on to the fuel pellet during its approximately four-year life. The $\mathrm{U}_{3} \mathrm{Si}$ and $\mathrm{LiH}$ should be able to support the cladding although the rods could be prepressurized if necessary.

The structure of the modules is shown in some detail on Figs, 9-7 and 9-8. The fuel rods are located in a close-packed triangular array with wire wrapping to maintain proper spacing. The rods are short and are cantilevered from the grid plate. The thin, close-fitting scalloped flow shroud directs the incoming helium flow to the first wall and also helps support the rods against lateral motion. All flow paths have been sized for minimum area consistent with acceptable pressure drop to maximize the volume fraction of fuel in the moivle.

The $U_{3} S i$ fuel grid plate is located between the uranium and tritium breeding zones. The blanket neutronic performance is quite sensitive to 
U3SI FUEL ROD

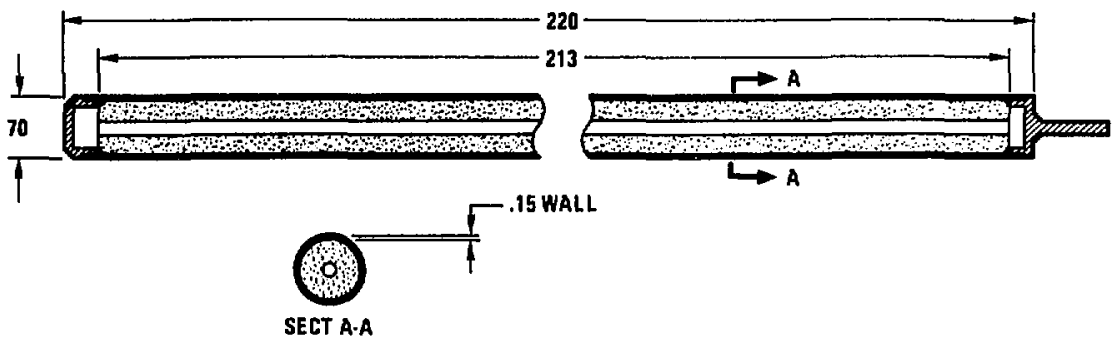

LiH FUEL ROD

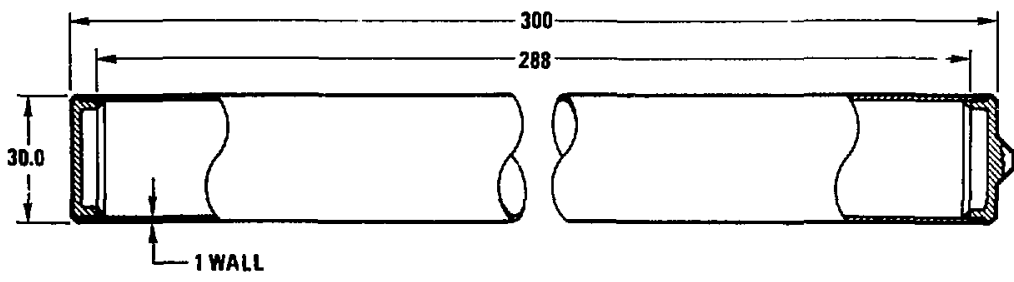

Fig. 9-6. MHR fuel rod designs. 


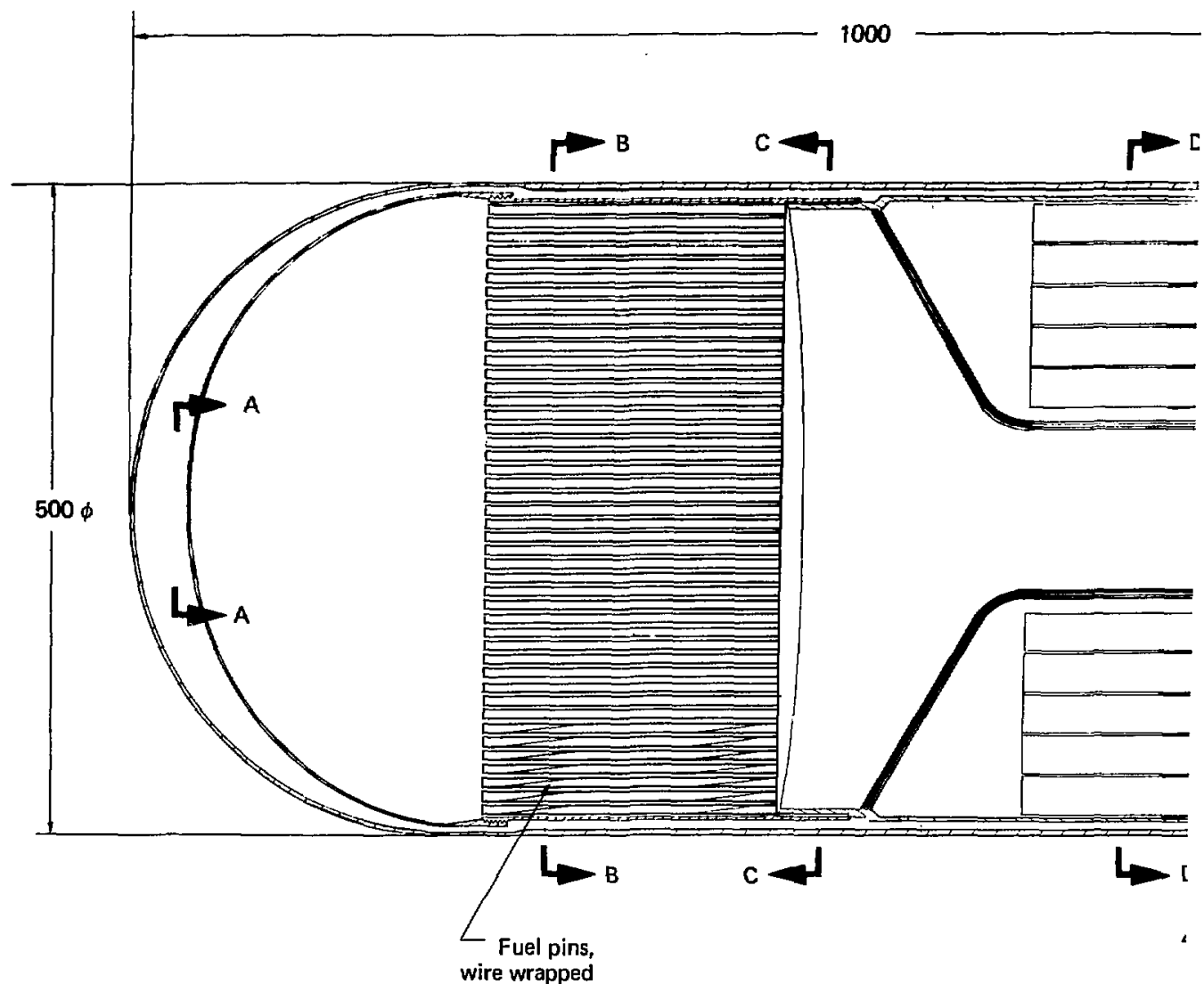




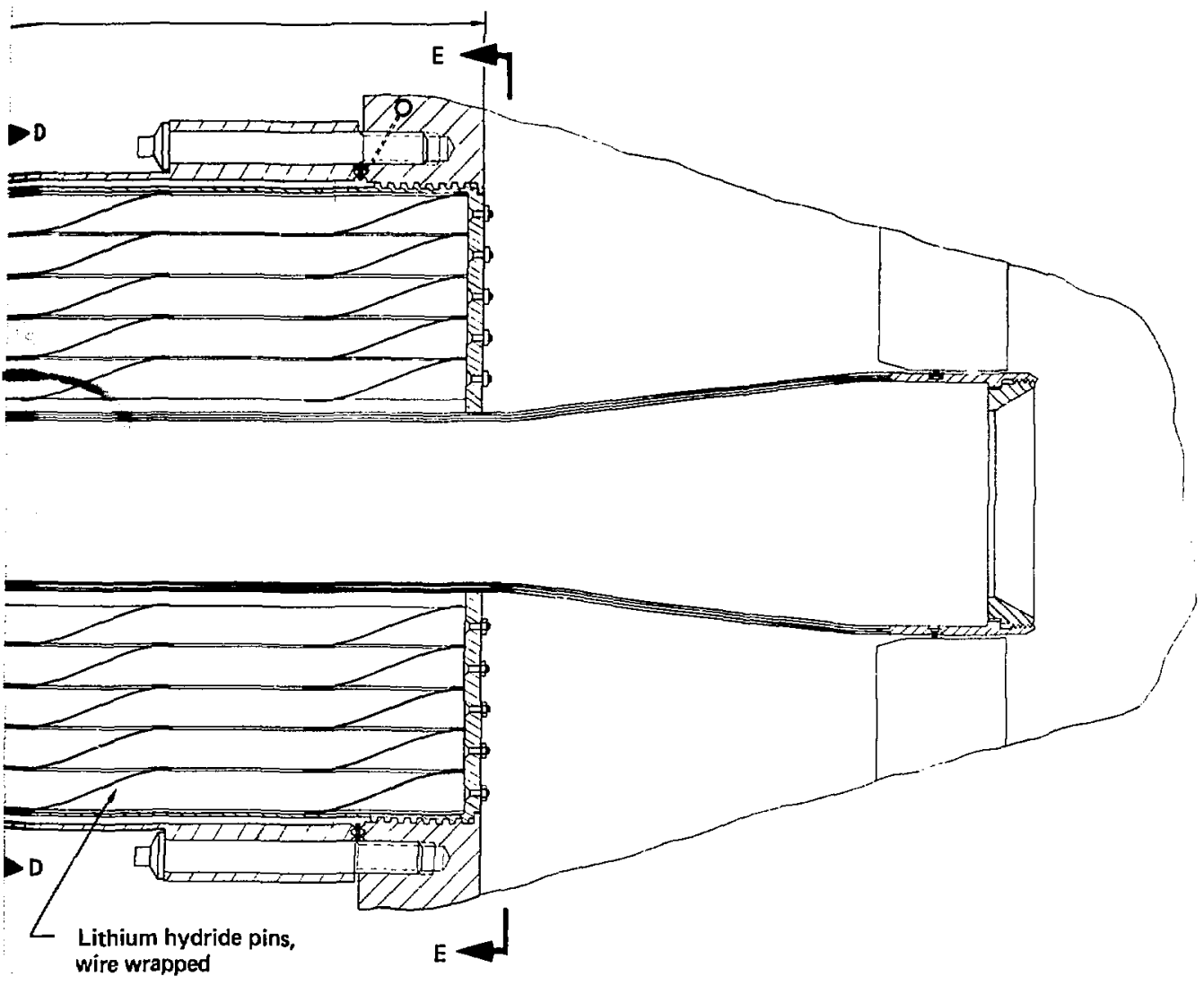

Fig. 9-7. MFR blanket module. 

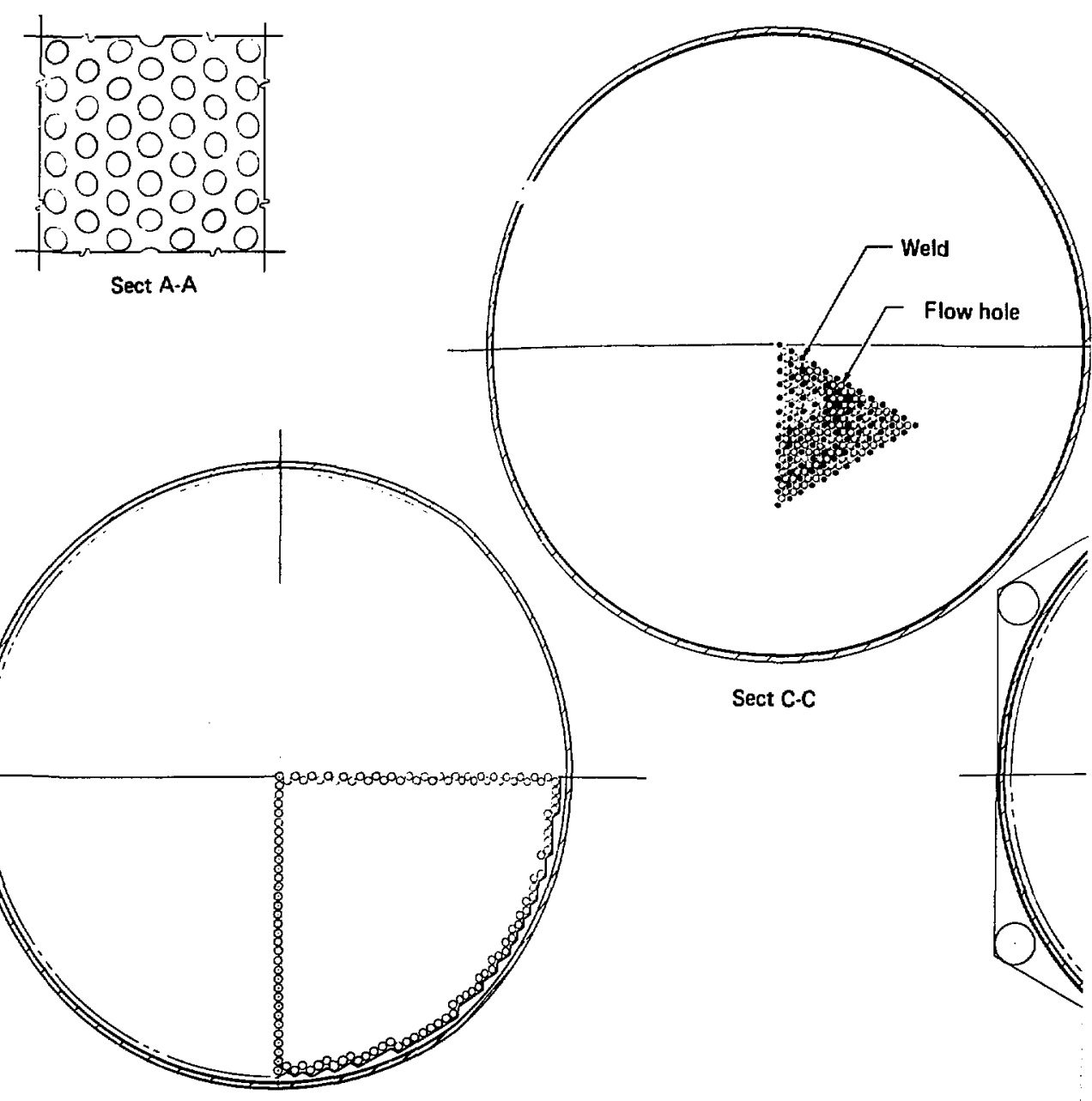

Sect B-B 

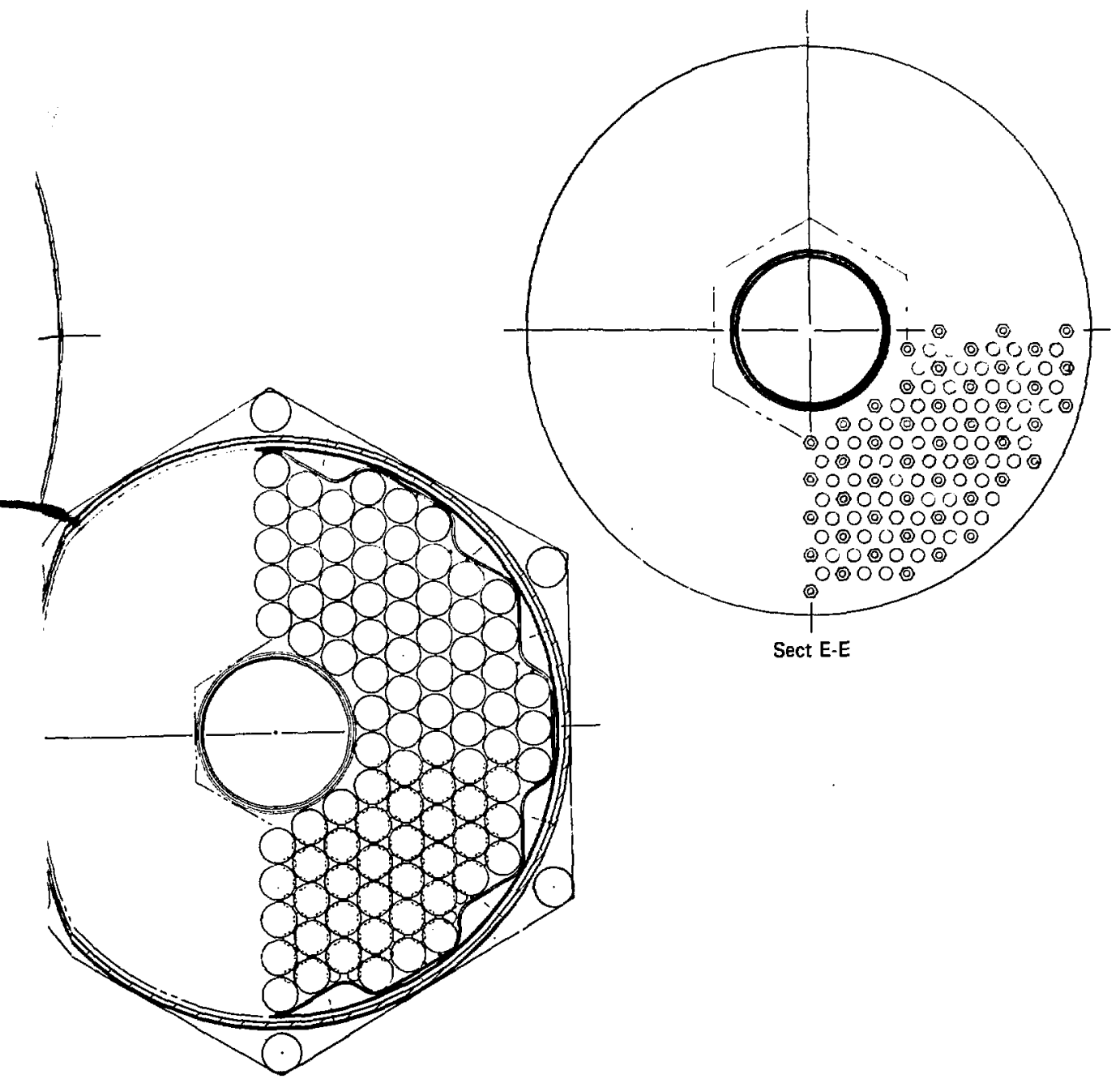

Sect D-D

Fig. 9-8. MHR blanket module details. 
structure in this zone. As a consequence, the grid-plate thickness was minimized by using a constant stress design with a cross section that varies in thickness. The neutronic performance is also quite sensitive to the firstwall thickness. For this reason, the wall thickness was minimized by using a hemispherical pure tension design; the thickness of this wall is only $2.4 \mathrm{~mm}$. Subsequent analysis indicates that an ellipsoidal head with a two-to-one majorto-minor diameter ratio would exhibit the same first-wall thickness but would allow the module height to be reduced by $12.5 \mathrm{~cm}$.

A douhle wall is used between the inlet and outlet helium. Although this reduces heat conduction from the outlet slde to the inlet side, the primary purpose of the double wall is to insure that a single failure of the flow Juct cannot short circuit the coolant flow and leave the $\mathrm{U}_{3} \mathrm{Si}$ zone without cooling. Differential thermal expansion of this double wall structure is a concern but can easily be accommodated within the elastic limit of the flow duct material. At the botton of the flow duct is a removable flow orifice. This orifice allows the flow characteristics of the module to be trimned before installation to match its particular position on the helium distribution manifold. This ablitity to trim the module flow insures that each module will receive equal flow and makes adjustable flow valves for each module unnecessary. Valves are only required to adjust flow to the helium distribution manifolds to compensate for blanket - segment age and, thus, power differences.

The seal between the hot and cool helium ducts is a simple metal 0-ring. A Grafoil graphite slip ring is provided to prevent galling when the inner module assembly is screwed into its socket. This simple seal arrangement is adequate since the pressure differential across the seal is only $41 \mathrm{kPa}$ (6 psi).

The throat size of the hot-coolant outlet duct was selected so that it has a smaller flow area than the $U_{3} S i$ fuel zone. This insures that, should the module cover fail, the flow would choke in the duct and not in the fuel zone. This prevents excessive forces from building up on the fuel and insures that, even in the unlikely event of module-cover failure, the fuel can be retained in a coolable geometry. 


\subsection{Power Conversion System}

\subsubsection{Primary Coolant Loop}

9.2.1.1 Design Conditions and Requirements. The primary coolant loop is designed to distribute the coolant in accordance with the power distribution in the fuel modules contained in the fission blanket assembly of the mirror hybrld reactor (MHR). The power levels in the fuel modules vary, depending on the fuel residency period in the blanket as plutonium is bred in the $\mathrm{u}_{3} \mathrm{si}$ fuel and the blanket energy multiplication increases.

Economic analysis of the entire MHR system indicates that because of their high cost it is most cost effective to operate the fusion components at full power at all times, producing a constant fusion power level. Because the blanket multiplication changes with time, this means that the blanket power will vary with time during the blanket lifetime. The power of an individual blanket module will more than double from beginning to end of life as its multiplication increases from 8.76 to 18.39 . To minimize the variation in reactor power outpuc, the blanket is divided into setveral sugments, which are replaced on staggered intervals. In the present blanket design, the fuel modules are grouped in four quadrants. The fuel management scheme selected for the MHR utilizes yearly refueling, resulting in each quadrant being replaced with fresh fuel once in four years.

Figure 9-9 shows the power distribution in each quadrant of the blanket assembly on a normalized scale as a function of fuel age during the equilibrium cycle. The total peak blanket thermal power (obtained by sumaing the power in each quadrant at the end of the year during the equilibriu cycle) is estinated at 3957.0 wt. The power conversion system (PCS) components from the hot manifold to the cold manifold, which include ducts in and out of the steam generator units and circulators, are sized for this reactor average peak power. The ducting components in each quadrant lying between the blanket assembly and the hot and cold manifolds are sized considering the local peak power expected at the end of the fourth year of fuel residency.

The PCS receives heat from two sources, the blanket and a direct converter attached to the reactor. Helium gas is used as the primary coolant in the reactor to transport the heat for power conversion. Figure 9-10 schematically represents the power flow for the reactor. 


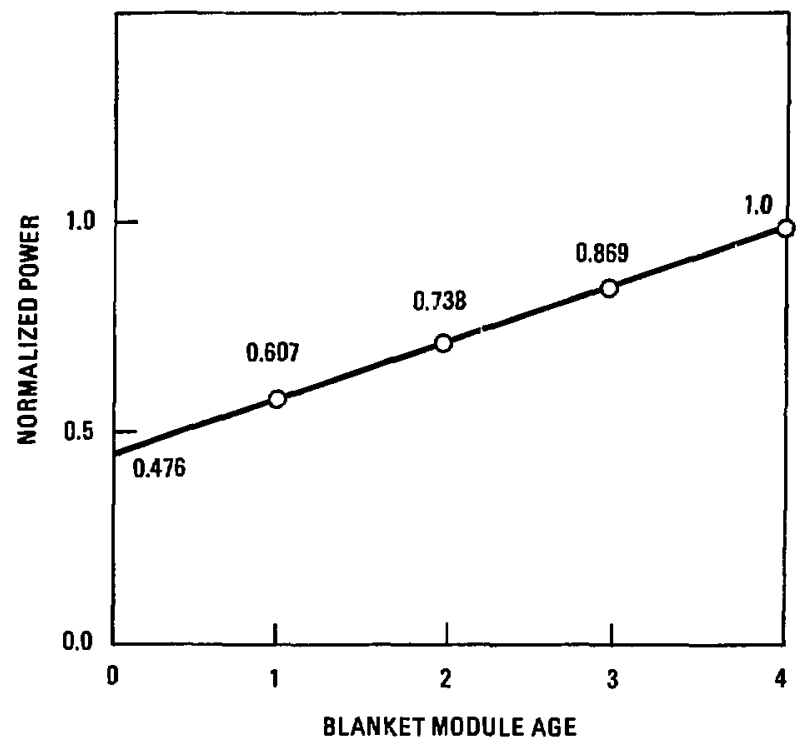

Fig. 9-9. Normalized blanket module power. 


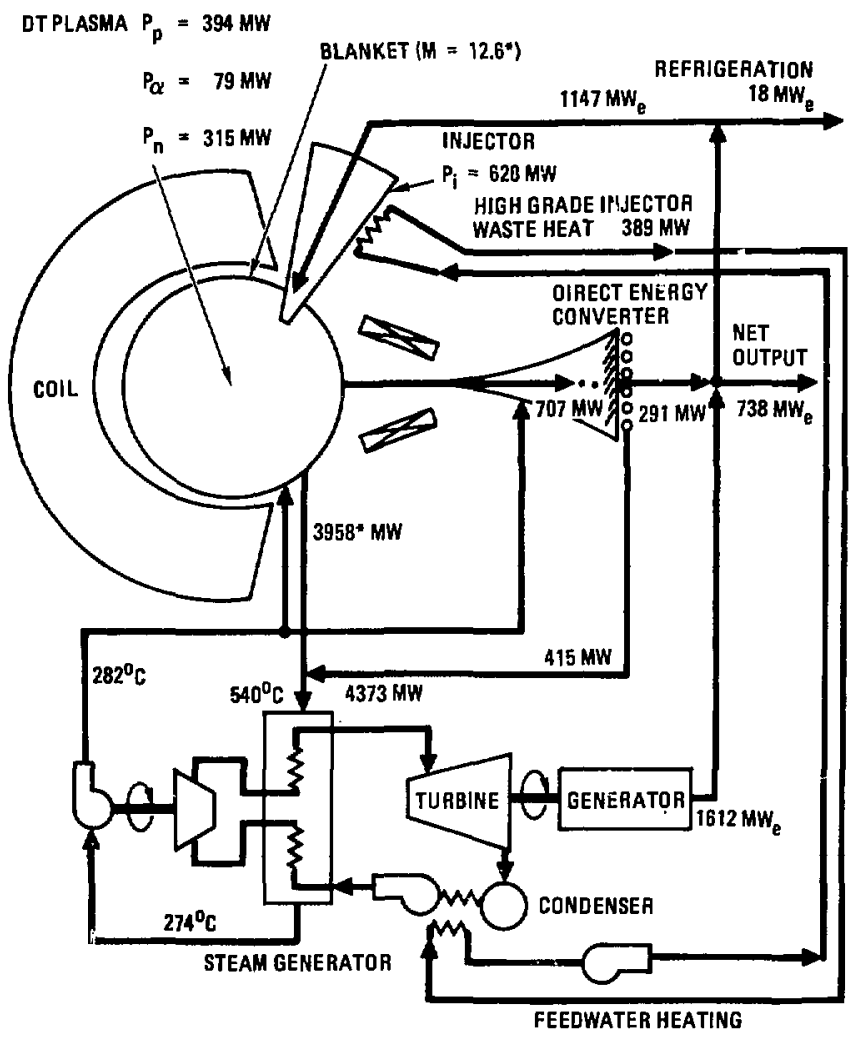

* NOTE: VALUES SHOWN CORAESPOND tO END-OF-CYCLE PEAK POWER CONDITIONS.

Fig. 9-10. MRR power flow schematic. 
The reactor blanket consists of 600 cylindrical modules. Twelve modules are arranged in a vertical pattern (see Fig. 8-5), and are connected to a common ducting system. The ducting system has contoured flow passages, designed to carry adequate helium flow in and out of the respective fuel module. Two large pipe connections are provided at the back panel of each module assembly for helium gas entry and return. Helium circulation inside the blanket fuel submodules is described in Sec. 8.2. The primary loop design discussed here covers aspects of the loops external to the blanket module.

\subsubsection{Reactor Coolant System. The reactor coolant system consists} of an integrated primary loop and an independent auxiliary 100p. The primary loop is used for normal power operation and for shutdown or depressurized cooling. The auxiliary loop is provided for reactor decay-heat removal following normal or emergency shutdown of the primary loops. The work reported here builds upon the work done during FY'76 and reported in Ref. 1.

A conceptual arrangement of the primary loop is shown in Fig. 9-11. The principal components of the primary loop are the steam generators, the main helium circulators, the auxiliary heat exchangers and auxiliary helium circulators, and the helium ducts and valves. A discussion of these components is given in greater detail in this section, including design bases, descriptions and, where applicable, design evaluations for the particular component or system involved.

\subsubsection{Design Base Requirements. The principal function of the} reactor coolant system is to transfer heat from the blanket fuel modules and leakage-fan end tanks to the steam generators through the primary loop. The steam produced in the steam generators is used to drive the plant turbine generator system. Helium, the reactor coolant, is circulated at a flow rate, temperature, and pressure consistent with the functions of the fission blanket, the steam generators, and the helium circulators. Under full-1oad normal operating conditions, the heat transferred from the reactor is approximately 4400 wit. The reactor coolant system is required to operate at full-load power and also under decay-heat removal conditions following a reactor shutdown. It will also be capable of confining any radioactive material and linit its accidental release within acceptable limits. The pressure-retaining boundary of the reactor coolant system and its associated components is a steel liner kept in compression by prestressed concrete. 


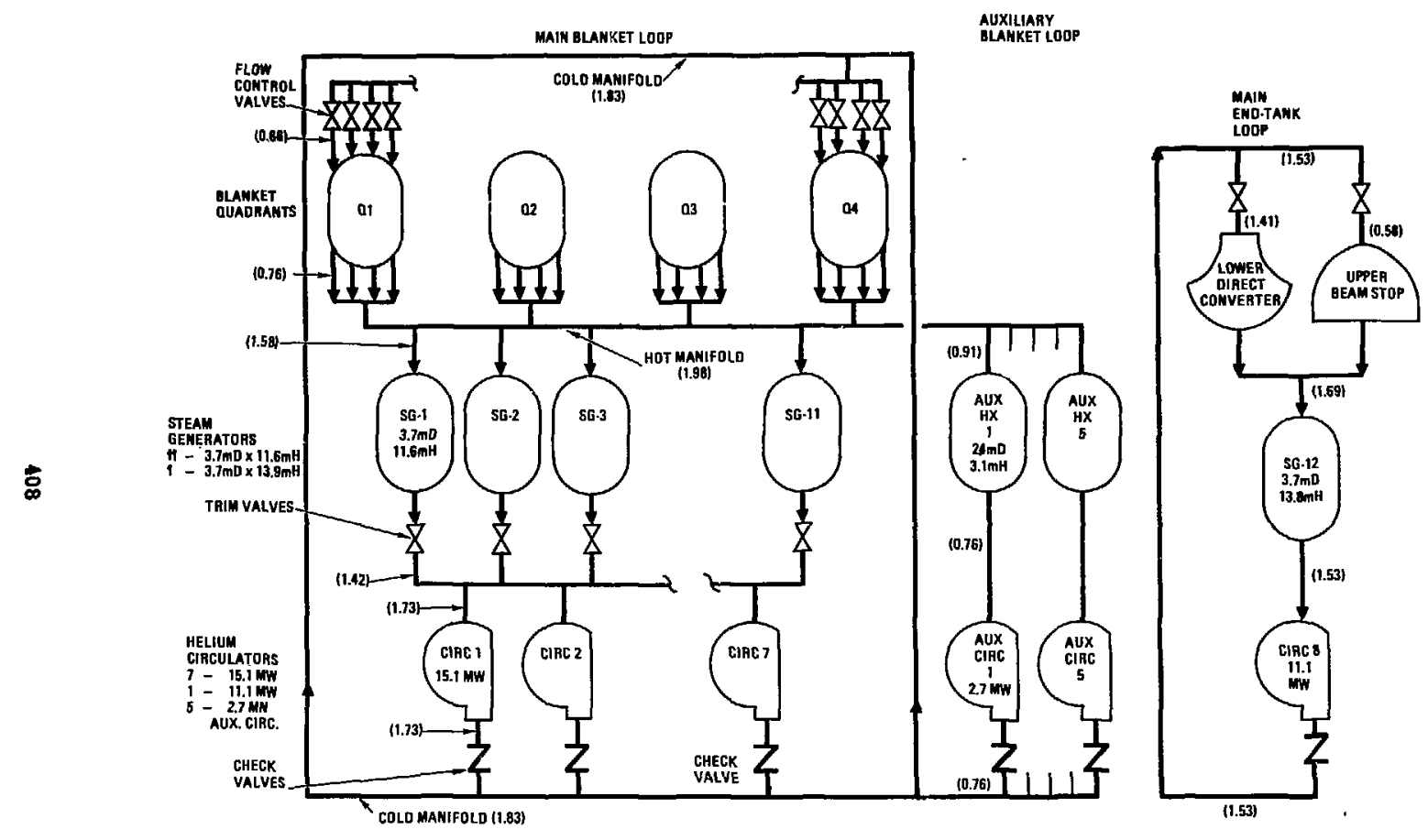

HOTE: DUCT DIAMETERS SHOWN IN METERS（ ।

Fig. 9-1l. HHR helium loOp schematic. 
The design requirements considered for the reactor coolant system are primarily based on the normal steady-state performance, and can perform satisfactorily against a postulated worst accident condition caused by depressurization. The effects due to plant transients, such as part load operation, startup, and normal shut down, were not considered in detail.

\subsubsection{Primary Loop Design. A schematic primary loop design} arrangement for the MHR is shown in Fig. 9-11. The primary loop consists of the main blanket 100p, the auxiliary blanket loop and the main end tank loop. There are 50 12-module blanket units which are interconnected by ducts and the hot and cold helium manifolds which encircle the reactor. There are 12 steam generators, 8 helium turbocirculators, 5 auxiliary heat exchangers, and 5 auxiliary helium circulators. The end tank loop does not require auxiliary cooling.

The steam generator unit consists of two sections; viz., an economizer-evaporator-superheater (ESS) section and a separate resuperheater section (RSH). Each steam generator unit is of monotube helical-coil design with upward boiling for stability. Hot helium enters at the top of the steam generator and passes downward in a single pass over the resuperheater and EES bundle.

Steam from the superheater tubesheet headers drives the single-stage axial flow compressor. Upon exiting the turbine, the stean reenters the steam generator at the resuperheater unit. The resuperheated steam is then used to drive the main power turbine.

At full power operation all main loop components are in operation. In the event of failure of any main loop component, the fusion power plasma will be shut down, although operation at reduced power could be continued using the remaining components if desired. Manifolding of all main loops provides the capability to continue to cool all the modules in the blanket assembly following a reactor shutdown. Details of decay-heat removal procedures will be discussed later.

The main helium circulator at full-load conditions provides a pressure rise of approximately $1.52 \times 10^{5} \mathrm{~Pa}(22 \mathrm{psi})$. The circulator outlet pressure at the circulator diffuser is about $62 \times 10^{5} \mathrm{~Pa}(900 \mathrm{psi})$ at full power under pressurized conditions.

The approximate pressure losses around the helium circuit, based on the helium flow rates of the preliminary heat balance for normal full-load conditions are: 


\begin{tabular}{ll}
\hline $\begin{array}{l}\text { Blanket } \\
\text { Steam generator }\end{array}$ & $4.13 \times 10^{4}(6.0)$ \\
EEs section & $4.14 \times 10^{4}(6.0)$ \\
RSH section & $1.38 \times 10^{4}(2.0)$ \\
Helium ducts, hot and cold & $5.50 \times 10^{4}(8.0)$ \\
including bends, expansion & \\
and contractions, values & \\
Total & $15.15 \times 10^{4}(22.0)$
\end{tabular}

9.2.1.5 Primary Ioop Functional Evaluation. The primary loop not only provides the cooling during full-load normal operating conditions, but also provides the "first line of defense" cooling during shutdowns and following a depressurization accident. All four quadrants are interconnected via manifolds, and the isolation of one steam generator or circulator will not affect the function of the other components, and all the modules will receive adequate coolant flow. The failure of one blanket loop components thus need not require shutdown of the entire reactor. The end tank loop is separate to avoid use of radioactive helium in the end tanks. Failure of its steam generator or circulator would require reactor shutdown. Because of this, in future designs it may be advantageous to provide the end tank 100 with several smaller steam generators and circulators operating in parallel. In the event the auxiliary cooling system (ACS) is required due to multiple or common-mode failures in the primary loop, the ACs is designed to provide the required cooling of the fuel modules in the blanket via the manifolding.

9.2.1.6 Helium Ducting Design. Reference 12 suggested a low system pressure of 20 atw for the KHR in order to have a thin first wall for the submodule. To reduce pumping power requirements, to size the helium ducting within practical limits, and to design the helium turbocirculator with $\mathrm{GA}^{\prime} \mathrm{s}$ current gas-cooled technology practice, a higher system pressure was preferred; a pressure of $6.078 \mathrm{MPa}(60 \mathrm{~atm})$ was selected, with wLL concurrence. Duct sizes calculated with this high pressure were found to be reasonable, as shown in Fig. 9-11. Hot helium ducts will be designed to ASiE code case 1592. Duct interior surfaces may be coated with a tritium barrier, if required, and outside surfaces will be insulated. The 
insulation may have a small gap kept under a light vacuum, if necessary, to contain any possible tritium release from the helium stream. The containment of the ducting in the PCRV is expected to help reduce tritiun release.

The primary loop layout scheme is significantly dependent upon the geometry of the Yin-Yang magnets which surround the plasma. The structural massiveness and support requirements of the magnets and large neutral beam injectors restrict the access, ducting and support area available for the blanket. Design of the module system was discussed in Sec. 8.2. The use of a PCRV for blanket and magnet support integrates well with the primary $100 p$ component support and containment requirements. The PCRV totally encloses the ducts, steam generators, helium circulators, and auxiliary loop components, resulting in a safe and effective containment that is inherently redundant. A massive primary loop rupture is completely impossible. The helium ducting to the top leakage fan end tank must leave the PCRV. This 0.58-m-diameter duct is the only free-standing helium pipe in the MHR system. The end tank loop is isolated from the blanket loop to prevent failure of the end tank duct from affecting cooling of the blanket.

In order to keep the parasitic pressure arop to a minimum in the ducts, a dynamic head of $3447 \mathrm{~Pa} \mathrm{(} \frac{1}{2}$ psi) was selected. The net pressure drop in the primary helium ducts is estimated to be approximately $55 \mathrm{kPa}$ (B pgi), accounting for bends, contraction/expansion losses, valves and Tee-connections. Table 9-7 shows the principal design data for the power conversion loop design.

\subsubsection{Main Helium Circulator.}

Design Bases. The function of the main circulators is to provide helium flow through the reactor. Each circulator unit consists of a single-stage axial-flow compressor and a single-stage steam turbine driven by superheated steam before it is reheated and passed to the main turbine. These units are similar to those designed for GCFR-HTGR plants.

Operating Requirements. The circulators will be designed to operate under various normal and abnormal conditions, including i) normal plant operation at full load, 2) plant startup, 3) routine plant shutdown for refueling or maintenance, and 4) postulated accidents, including the design-basis depressurization accident. The circulator operating paraneters for pressurized conditions are given in Table 9-8. 
Table 9-7. Power conversion loop principal design data.

\section{Power data}

Net PCL output power, Me

1612

Net power input to PCL, MAt

4762

Power input from blanke:, wift

3958

Power input from direct conver: :, wit

415

Power input from injector waste heat, wt

389.0

Helium circulator punping power, sint

116.5

Turbine-generator output, we

1612

PCI efflciency,

33.84

Direct converter output, wife

291

Net station output power, we

737.5

Total nuclear power - fusion plus fission, wht

4037

Net station efficiency,

18.27

Primary coolant (helium) data

Average pressure, HPa (atm)

$6.08(60)$

$\Delta \mathrm{P} / \mathrm{P}, 8$

2.50

Total flow rate, $\mathrm{kg} / \mathrm{s}(1 \mathrm{bm} / \mathrm{h})$

Inlet temperature at steam generator, ${ }^{\circ} \mathrm{C}$ ( ${ }^{\circ} \mathrm{F}$ )

$3246\left(25.79 \times 10^{6}\right)$

outlet temperature at steam generator, ${ }^{\circ} \mathrm{C}\left({ }^{\circ} F\right)$

540 (1004)

Temperature rise through circulator, ${ }^{\circ} \mathrm{C}\left({ }^{\circ} \mathrm{F}\right)$

274 (525)

8.33 (15)

Secondary coolant (steam/water)

Feedwater inlet pressure, MPa (psia)

10.3 (1497)

Net flow rate, $\mathrm{kg} / \mathrm{s}(1 \mathrm{bm} / \mathrm{h})$

$1611.5\left(12.8 \times 10^{6}\right)$

Superheater outlet pressure, MPa (psia)

$8.42(1222.0)$

Resuperheater inlet pressure, MPa (psia)

5.37 (779)

Resuperheater outlet pressure, MPa (pgla)

$5.26(764.0)$

Feedwater inlet temperature, ${ }^{\circ} \mathrm{C}$ ( $\left.{ }^{\circ} \mathrm{F}\right)$

171 (339)

Resuperheater outlet temperature ${ }^{\circ} \mathrm{C}\left({ }^{\circ}\right)$

504 (939)

Net pressure drop in stean generator, HPa (psid)

$2.00(290)$

at peak-power, blanket average end-of-life conditions.

Design Requirements. The helium circulators will be designed to meet the requirements shown in Table 9-8 under 1002 load condition and will be capable of operating without surge or surge instability at all operating conditions. 
Table 9-8. Circulator operating parameters at full power.

\begin{tabular}{|c|c|}
\hline Compressor type & single stage \\
\hline He flow rate, $\mathrm{kg} / \mathrm{s}(1 \mathrm{~b} / \mathrm{sec})$ & $406(896.0)$ \\
\hline He inlet pressure, MPa (psia) & $6.08(882.0)$ \\
\hline He inlet temperature, ${ }^{\circ} \mathrm{C}\left({ }^{\circ} \mathrm{F}\right)$ & $274(525)$ \\
\hline He pressure rise, MPa (psi) & $0.152(22)$ \\
\hline He outlet pressure, MPa (psia) & $8.66(904)$ \\
\hline Speed, rpm & 5400 \\
\hline Blade tip diameter, $m$ (in.) & $1.32(52)$ \\
\hline Turbine type & Single stage \\
\hline Steam flow rate, $\mathrm{kg} / \mathrm{s}$ (lb/sec) & $201(444.0)$ \\
\hline Steam inlet temperature, ${ }^{\circ} \mathrm{C}\left({ }^{\circ} \mathrm{F}\right)$ & $\$ 44(832.0)$ \\
\hline Steam inlet pressure, MPa (psia) & $8.42(1222)$ \\
\hline Steam outlet pressure, MPa (osia) & $6.15(779)$ \\
\hline speed, rpm & 5400 \\
\hline
\end{tabular}

Normal circulator control will be achieved by means of an inlet throttle valve. A steam bypass will be provided to obtain balance between circulators. The circulator will be provided with a service system to supply high-pressure water for lubricating the bearings and to supply purified buffer helium for preventing inleakage of the helium coolant.

Design and Operation Description. The steam turbine for each main helium circulator is located between the superheater outlet and the resuperheater inlet. Thus the full flow of steam generated in the plant drives the circulator turbines before passing to the main steam power turbine. In this flow arrangement, the steam-driven helium circulators tend to be inherently self-regulating, since the helium cooling requirements of the reactor are directly related to the supply of stean produced. Analysis indicates that the best location for the circulator turbine is between the superheater and the resuperheater. Elimination of the resuperheater reduces the plant efficiency by about 2 and therefore affects the plant economics. The principal design data and performance characteristics of the main heliun circulator and its steam turbine are given in Table 9-8. 


\subsubsection{Steam Generators.}

Functional Requirements. The purpose of the steam generators is to transfer the heat from the reactor coolant to the secondary coolant.

High-temperature $\left[540^{\circ} \mathrm{C}\left(1004^{\circ} \mathrm{F}\right)\right]$ helium from the blanket and direct converter enters the steam generators and gives up its heat to the resuperheater section and main steam section to produce superheated steam at $444^{\circ} \mathrm{C}\left(830^{\circ} \mathrm{F}\right)$ and resuperheated steam at $505^{\circ} \mathrm{C}\left(940^{\circ} \mathrm{F}\right)$.

Design and Operating Requirements. The steam generators will be designed for continuous operation and also for conditions during reactor shutdown when the main loops are used for decay-heat removal.

The steam generators will be designed to accommodate, without operational impairment, all normal and emergency operating conditions. The design bases are, in general, similar to those for HTGR-GCFR plants and include the following:

- Allowance will be made in sizing the steam generator to permit full-load operation with a certain number of tubes plugged in addition to a certain degree of internal tube fouling.

- The allowances on the heat-transfer bundle tubing will include provision for imbalances, expected in the circuit flow due to tube plugging, gas-flow imbalances fabrication tolerances, heat-transfer-coefficient uncertainties, and friction-factor uncertainties.

- The steam generators and their components will be designed so that no danage or malfunction is caused by internally generated vibrations, flow-induced vibrations, or environmental vibrations. The design, material, fabrication and inspection of the steam generators will be in accordance with applicable ASME codes.

Description and Operation. The plant has twelve steam generators: eleven in the main blanket loop and one in the end tank loop. Each blanket loop stean generator module is about $3.66 \mathrm{~m}(12 \mathrm{ft})$ in diameter and approximately $11.6 \mathrm{~m}$ (38.7 ft) high. The end tank steam generator module is $3.66 \mathrm{~m}(12 \mathrm{ft})$ in diameter and $13.4 \mathrm{~m}(44.7 \mathrm{ft}) \mathrm{tall}$. The module is an axial-flow heat exchanger that is made up of two separate helically wound tube bundles; these are the resuperheater tube bundle and the main steam-generator tube bundle, which consists of the combined economizer, evaporator, and superheater sections. In the steam-generator main bundle, the incoming high-pressure feedwater is converted into superheated stean 
that is then used to drive the main helium-circulator series turbine. The steam is subsequently returned to the steam generator and resuperheated before it flows to the main turbine-generator get of the power plant. The water-stean flow on the tube side is upward; the helium flows downward across the tube bundles. The blanket loop steam-generator major design parameters are summarized in Table 9-9.

Helium coolant gas carries the reactor heat via ducts from the blanket and direct converter to the steam-generator. The hot gas flows downard

Table 9-9a. Steam-generator design data in metrlc units (data are for each generator).

\begin{tabular}{|c|c|c|c|c|}
\hline & \multirow{2}{*}{$\begin{array}{l}\text { Resuper heater } \\
\text { section }\end{array}$} & \multicolumn{3}{|c|}{ Stean-generator sections } \\
\hline & & Superheater & Evaporator & Economizer \\
\hline Stean generator diameter, $\pi$ & 3.658 & 3.658 & 3.658 & 3.658 \\
\hline Heat duty, & 34.93 & 67.66 & 178.6 & 92.0 \\
\hline $\begin{array}{l}\text { Apparent logarithmic mean } \\
\text { temperature difference, } \\
{ }_{C}\end{array}$ & 51.0 & 93.9 & 57.6 & 40.0 \\
\hline $\begin{array}{l}\text { Apparent overall heat- } \\
\text { tragsfer coefficient, } \\
W / m^{2} \cdot K\end{array}$ & 992 & 1572 & 1682 & 1568 \\
\hline $\begin{array}{l}\text { Heat-transfer surface area, } \\
m^{2}\end{array}$ & 512 & 386 & 1409 & 1018 \\
\hline Heliv side & & & & \\
\hline Flow rate, kg/s & 265 & 265 & 269 & 269 \\
\hline Inlet temp., ${ }^{\circ} \mathrm{C}$ & 540 & 515 & 467 & 340 \\
\hline Outlet tenp., ${ }^{\circ} \mathrm{C}$ & 514 & 466 & 338 & 274 \\
\hline Av pressure, MPa & $6: 02$ & 6.00 & 5.99 & 5.98 \\
\hline Pressure drop; MPa & 0.013 & 0.012 & 0.013 & 0.011 \\
\hline Water-stean side & & & & \\
\hline Flow rate, $\mathrm{kg} / \mathrm{s}$ & 133.7 & 133.7 & 133.7 & 133.7 \\
\hline Inlet temp... ${ }^{\circ} \mathrm{C}$ & 396 & 301 & 312 & 171 \\
\hline Outlet teap.., ${ }^{\circ} \mathrm{C}$ & 505 & 445 & 301 & 312 \\
\hline Av pressure, MPa & 5.32 & 8.54 & 9.41 & 10.25 \\
\hline $\begin{array}{l}\text { Pressure drop, MPa } \\
\text { Tubes }\end{array}$ & 0.103 & 0.22 & 1.52 & 0.163 \\
\hline Nunber of tubes & 653 & 323 & 323 & 323 \\
\hline $\begin{array}{l}\text { Tube size, } \\
\text { Tube bundle height, }\end{array}$ & $\begin{array}{l}25.4 \times 2.54 \\
1.39\end{array}$ & $\begin{array}{l}25.4 \times 1.8 \\
1.14\end{array}$ & $\begin{array}{l}19 \times 1.6 \\
4.55\end{array}$ & $\begin{array}{l}19 \times 1.6 \\
3.28\end{array}$ \\
\hline
\end{tabular}


Table 9-9b. Steam-generator design data in U.S. unlts (data are for each generator).

\begin{tabular}{|c|c|c|c|c|}
\hline & \multirow{2}{*}{$\begin{array}{l}\text { Resuperheater } \\
\text { section }\end{array}$} & \multicolumn{3}{|c|}{ Stean-generator sections } \\
\hline & & Superheater & Evaporator & Economizer \\
\hline $\begin{array}{l}\text { Steam generator diameter, } \\
\text { ft }\end{array}$ & 12.0 & 12.0 & 12.0 & 12.0 \\
\hline Heat duty, Btu/h & $119 \times 10^{6}$ & $230 \times 10^{6}$ & $610 \times 10^{6}$ & $314 \times 10^{6}$ \\
\hline $\begin{array}{l}\text { Apparent logar ithmic mean } \\
\text { temperature difference, } \\
\text { F }\end{array}$ & 91.8 & 169.0 & 103.7 & 72.0 \\
\hline $\begin{array}{l}\text { Apparent overall heat- } \\
\text { transfer }{ }^{2} \operatorname{coffficient,~} \\
\text { Btu/h-ft }{ }^{-}{ }_{\mathrm{F}}\end{array}$ & 175 & 277 & 296 & 276 \\
\hline $\begin{array}{l}\text { Heat-transfer surface area, } \\
\mathrm{ft}^{2}\end{array}$ & 5508 & 4149 & 15,167 & 10,952 \\
\hline Helium side & & & & \\
\hline Flow rate, $1 b / h$ & $2.106 \times 10^{6}$ & $2.106 \times 10^{6}$ & $2.106 \times 10^{6}$ & $2.106 \times 10^{6}$ \\
\hline Inlet temp.., ${ }^{\circ}$ & 1004 & 959 & 873 & 645 \\
\hline Outlet temp.., ${ }_{F}$ & 956 & 871 & 869 & 867 \\
\hline Av pressure, psia & 873 & 871 & 869 & 867 \\
\hline Pressure drop, psi & 2.0 & 1.76 & 2.63 & 1.62 \\
\hline Water-steam side & & & & \\
\hline Flow rate, $1 \mathrm{~b} / \mathrm{h}$ & $1.06 \times 10^{6}$ & $1.062 \times 10^{6}$ & $1.062 \times 10^{6}$ & $1.062 \times 10^{6}$ \\
\hline Inlet temp., ${ }^{\circ} \mathbf{F}$ & 744 & 573 & 594 & 340 \\
\hline Dutlet temp., ${ }_{F}$ & 940 & 832 & 573 & 594 \\
\hline Av pressure, gsia & 771 & 1238 & 1364 & 1485 \\
\hline $\begin{array}{l}\text { Pressure drop, psi } \\
\text { Tubes }\end{array}$ & 15 & 31.9 & 219.5 & 23.6 \\
\hline Number of tubes & 653 & 323 & 323 & 323 \\
\hline $\begin{array}{l}\text { Tube size, in. } \\
\text { Tube bundle height, ft }\end{array}$ & $\begin{array}{l}1.0 \times 0.10 \\
5.22\end{array}$ & $\begin{array}{l}1.0 \times 0.070 \\
3.74\end{array}$ & $\begin{array}{l}0.75 \times 0.062 \\
14.91\end{array}$ & $\begin{array}{l}0.75 \times 0.062 \\
10.76\end{array}$ \\
\hline
\end{tabular}

over the resuperheater, superheater, evaporator, and economizer tube bundles. After passing through the stean-generator bundle, the cooled gas then passes into the main circulator inlet. Gas leaving the circulator passes through the diffuser, and reenters the blanket and direct conver cer, completing the main loop circuit.

The feedwater for each stean-generator module enters through feed-water tubesheets and then through lead-in tubes connscting the tubesheets to the bottom of the econonizer section. 
The economizer and evaporator sections have $0.02 \mathrm{~m}(0.75 \mathrm{in}$ ) dianeter tubes, whereas the superheater section of the steam-generator tube bundle has $0.0254 \mathrm{~m}$ (1.0 in.) diameter tubes. Variation in the tube diameter in the economizer, evaporator, and superheater sections will provide pressure drop in the system and, if necessary orifices will be installed in each feedwater tube to assure stability at low flow. Tube plugging to isolate defective tubes is done at the tubesheets, which are readily accessible.

\subsubsection{Secondary Coolant Loop}

9.2.2.1 Steam-Cycle. The reference steam-cycle for full-load operation, which is the basis of the deslgn described in this report, is shown in Fig. 9-12. The major equipment in the cycle consists of twelve once-through steam generators, including resuperheating sections, the turbine generator, steam-turbine drives for the helium circulators, condensers, feedpumps, feedwater heaters, and associated piping.

Feedwater enters the economizer section of the steam generators at a net flow rate of $1611 \mathrm{~kg} / \mathrm{s}\left(12.8 \times 10^{6} \mathrm{lb} / \mathrm{hr}\right)$ at $10.22 \mathrm{MPa}$ (1485 psi) and $171^{\circ} \mathrm{C}\left(340^{\circ} \mathrm{F}\right)$ and is converted to superheated steam at $8.27 \mathrm{MPa}$ $(1200 \mathrm{psia})$ and $443^{\circ} \mathrm{C}\left(830^{\circ} \mathrm{F}\right)$. The steam from each steam generator is directed to a helium-circulator series steam turbine, from which it leaves at $5.35 \mathrm{MPa}(779 \mathrm{psia})$ and $396^{\circ} \mathrm{C}\left(745^{\circ} \mathrm{F}\right)$. To reduce the moisture content in the last stages of the main turbine, the steam is reheated to $505^{\circ} \mathrm{C}\left(940^{\circ} \mathrm{F}\right)$ at $5.27 \mathrm{MPa}(764 \mathrm{psia})$ and then it proceeds to the high-pressure turbine. The extraction steam for the high-pressure Ceedwater heater is taken from the crossover between the high-pressure and intermediate-pressure turbines and, together with the waste heat from the injectors (see Sec. 9.2. for a discussion) derived in the form of hot water, is used to heat the feedwater to its final temperature of $171^{\circ} \mathrm{C}$ $\left(340^{\circ} \mathrm{F}\right.$ ). Extraction steam at approximately $6.2 \times 10^{5} \mathrm{~Pa}$ (90 psia) drives the boiler feedpumps. Additional extractions are made from the low-pressure turbine and are directed to the low pressure feedwater heaters. The remaining steam flow is exhausted to the main turbine condenser. Condensate from the hot well of the main condenser is pumped at low pressure through three stages of feedwater heating.

Gross electrical output of this plant is 1611.5 M. Net heat rate is $10.63 \times 10^{6} \mathrm{~J} / \mathrm{kWh}(10085 \mathrm{Btu} / \mathrm{kWh})$, which corresponds to a power conversion system thermal efficiency of 33.848. The heat balance was based on a helium-10og design pressure drop of $1.52 \times 10^{5} \mathrm{~Pa}(22 \mathrm{psia})$, circulator 


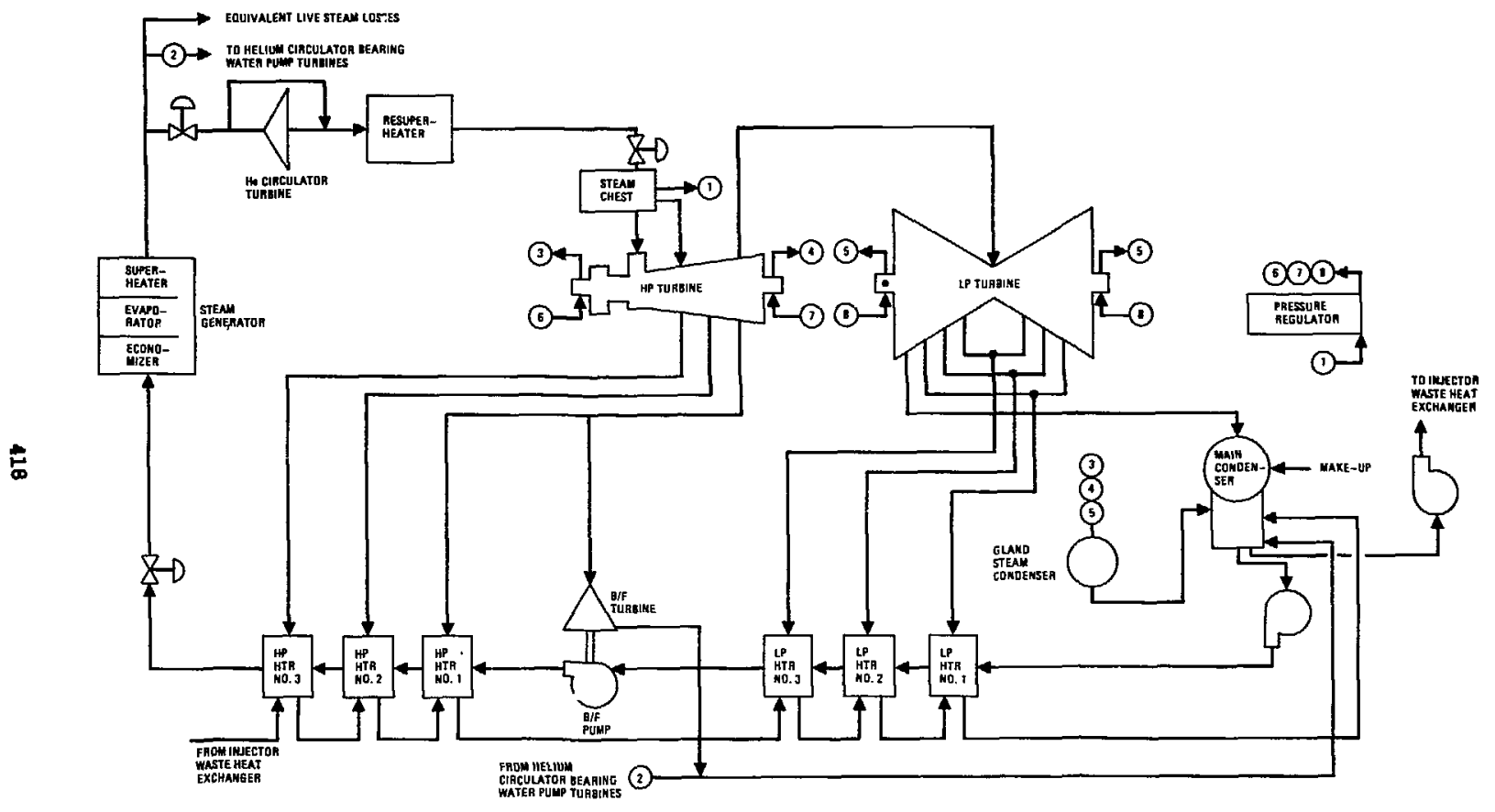

Fig. 9-12. Secondary coolant (steam) loop arrangement. 
and circulator turbine overall efficiency of 808 , and reactor coolant inlet and outlet temperatures of $282^{\circ} \mathrm{C}\left(540^{\circ} \mathrm{F}\right)$ and $540^{\circ} \mathrm{C}\left(1004^{\circ} \mathrm{F}\right)$ respectiveily. The steam is reheated after it passes through the series circulator turbines, thereby providing throttle conditions of $5.06 \mathrm{MPa}(734 \mathrm{psia})$ and $504^{\circ} \mathrm{C}$ $\left(939^{\circ} \mathrm{F}\right)$, which are acceptable to conventional nonreheat steam turbines. The moisture content of the exhaust steam is $10 \mathrm{w}$.

9.2.2.2 System Design.

Turbine Generator. The turbine selected is a tandem-compound machine comprising a high-pressure section, an intermediate-pressure section, and an eight-flow low-pressure section exhausting downward to the condenser. Inlet steam conditions are $5.27 \mathrm{MPa}(764 \mathrm{psia})$ and $503^{\circ} \mathrm{C}\left(937^{\circ} \mathrm{F}\right)$. The turbine generator unit has a design rating of 1618 MA with the throttle valve wide open, corresponding to a backpressure of 3 in. Hg absolute.

The entire turbine is steam-sealed and a bypass arrangement from the main steam line to the main condenser is provided for startup and shutdown.

Condenser and Circulating-Water System. The main steam condenser will be capable of rejecting heat to the circulating-water system from the main turbine, low-pressure heater drains, and the auxiliary condenser. In addition, during startup and plant upset conditions, the main condenser will provide the heat sink for secondary coolant from the flash tank.

An auxiliary condenser provides a heat sink for the boiler feedpump turbines and the circulator bearing-water pump turbines. Water from this condenser is returned to the condensate system through the main condenser. Startup and Shutdown Auxiliaries. Datails and sequential procedures concerning the plant startup and shutdown controls are discussed in $\mathrm{Ch} .11$. The following summarizes the principal operations.

The main cooling loop has two redundant auxiliary boilers to provide steam for the circulator turbine and circulator bearing-water-pump turbin: during initial startup and during decay-heat-removal operations. The boilers will be sized to provide the steam required for operation with the reactor coolant system depressurized.

An auxiliary boiler feedpump will be provided for each auxiliary boiler to supply feedwater to it from the emergency water supply system. These pums will be electrically driven and sized to accomodate the auxiliary boilers.

During startup, shutdown, and decay-heat-removal operations, the feedwater flow to the steam generators is provided by electrically driven 
shutdown boiler feedpumps. These pumps supply high-pressure feedwater to the steam generators from the emergency water supply system. They will be sized to maintain sufficient water level in the steam generators and to supply feedwater to the desuperheaters during shutdown and decay-heatremoval operations.

The bypass line from the circulators to the main condenser will also contain a flash tank that will supply a source of steam, which can be used to drive the main boiler feedpumps when extraction steam is not available from the main turbine and can algo be used for feedwater heating. During normal operation, the flash tank will be pressurized with extraction steam from the main steam turbine.

\subsubsection{Sy. tem Operation.}

Normal Power Operation. During normal operation there are five steam loops operating in parallel, the end tank loop and four blanket loops, each consisting of three resuperheaters, two circulator turbines, three EES sections, and high-pressure heaters connected to a feedpump heater. The resuperheater oltlets are joined in a common main steam pipe leading to the high-pressure tirbine. Steam is extracted in each loop prior to the circulator turbines to drive the circulator bearing-water pump turbines and is exhausted to the auxiliary condenser along with steam from the main boiler feedpump curbines. Condensate from the auxiliary condenser flows to the main condenser and is recycled with the main condensate flow.

Normal shutdown and Reactor Trip. The control features concerning the normal shutciown are covered separately in Ch. 1l. Normal shutdown or reactor trip refers to the plant shutdown operations required to get the plant load decreased to decay-heat level. Removal of decay heat is described in Sec. 9.2.2.4.

For a programmed shutdown, the power level will be decreased gradually down to a minimum load with the main turbine on line. At this load, the main turbine will be tripped and the reactor power further decreased. The main steam will be bypassed to the desuperheater and flash tank, where steam will be extracted to continue to drive the main boiler feedpumps until the load decreases :o about 6\%. At that time, the shutdown boiler feedpumps will be started and the main boiler feedpumps shut down. The load will then continue to be decreased to the decay-heat level. During a reactor trip, the intermediate step of driving the main feedpumps with steam from the flash tank may be optional. 
The shutdown boiler feedpumps supply feedwater for low flows during all shutdown operation. The inventory of the steam generators is aufficiently large to supply steam to the main circulators during startup of these feedpumps or during temporary loss of electric power to the pumps without. significantly degrading the steam conditions.

During normal shutdown or reactor trip with all equipment available, the bypassed steam will be desuperheated and sent to the main condenser via the flash tank. The water from the condenser is then pumped and heated with steam from the flash tank. It then flows to the shutdown boiler feedpunps, completing the heat-sink portion of the cycle. The principal safety-related characteristics of this system are these:

- The transition from normal operation to shutdown requires only a few, simple control actions (see plant Controls, Ch. 11).

- The system can operate for sufficient time after loss of any single component to initiate the decay-heat-removal phase with either main or auxiliary loops.

- The water inventory in the steam generators is sufficient so that the shutdown boiler feedpumps do not have to be started rapialy and could be interrupted to allow changeover to emergency electric power supply.

9.2.2.4 Decay-Heat-Removal Operation. The control features governing this cperation are given separately in Ch. 1l. The shutdown procedures described above will be capable of providing adequate cooling as the plant load is reduced to about 2\%. Thereafter, additional operating procedures will be required to continue the use of the main circulators, or a switch to the alixiliary loops will be made.

As the decay heat decreases further, the quantity of stean produced by the stean generator will be insufficient to supply the circulators with enough power to maintain blanket flow stability. In order to maintain the proper steam flon to the main circulator turbines, auxiliary stean will be supplied by auxiliary boilers. The steam from the steam generators will be bypassed around the circulator turbines to a desuperheater and then to the main condenser. The auxiliary boilers will be started at the beginning of shutdown.

stean from the auxiliary boilers will flow through the main circulators and then bypass the resuperhesters to the desuperheater. From there it flows through the flash tank to the main condenser. As in nornal shutdown, water from the condenser is then punped and heated with the stean from the 
flash tank. It then flows to the shutdown boiler feedpumps, completing the heat-sink portion of the cycle.

Each main 100p has connections to the auxiliary boiler system to provide steam for the circulator turbine and the circulator bearing-waterpump turbine uuring decay-heat-removal operation. The end tanis will not have significant decay-heat levels but will require auxiliary steam for start-up operations.

Energency water can be supplied to the shutdown boiler feedpumps from the condensate storage system in case the main condenser is lost. This system thus gives a means for long-term decay-heat removal completely independent of the auxiliary core-cooling loops. Much of the equipment is used for normal operation and thus is already in operation.

\subsubsection{Auxiliary Cooling System}

\subsubsection{System Description.}

Design Bases: Functional Reguirements. The auxiliary cooling system provides an independent means of cooling the shutdown reactor and removing the decay heat produced by the blanket. The system will be used 1) when the main cooling loops are not available, or 2) when it is desired not to perform decay-heat removal with the main cooling loops. The auxiliary cooling system must provide adequate cooling and prevent the temperatures of the fuel, the cladding, and the reactor coolant from exceeding prescribed limits, so that safe cool-down of the reactor and the reactor cooling system is ensured after any credible system failure.

The auxiliary cooling system is designed to perform the engineered safety function of providing adequate energency reactor cooling after shutdown as an independent backup to the main cooling loops. Figure 9-11 schenatically shows the conceptual arrangement of the auxiliary cooling systen (ACS).

The auxiliary loop consists of five heat exchangers and five circulators. The loop circuitry is so arranged that if one circulator or one heat exchanger failed, the remaining operable units would provide adequate cooling.

The auxiliary loops will be designed to provide cooling in either a pressurized or a depressurized condition.

In addition to the engineered safety function, the auxiliary cooling loops provide decay-heat removal and cooling during refueling. For these uses of the auxiliary cooling loops, the transfer from the main cooling 
loops to the auxiliary loops will be done sufficiently long after shutdown to minimize the auxiliary power requirements. Iong-term cooling by the auxiliary cooling system is described in sec. 9.2.4.

In addition to the above functions, the auxiliary cooling system must also be capable of providing safe cooldown of the reactor with a mixture of gases in the reactor coolant system, such as could result from accidents involving steam or air inleakage.

Design Criteria. To ensure that the auxillary cooling system adequately meets performance objectives under all normal operating or credible combinations of accident conditions, the general design criteria for the system will be in accordance with the criteria presented for the reactor coolant system and with the specific requirements applicable to the essential system components, viz., the auxiliary circulator and the auxiliary heat exchanger.

The auxiliary loop design performance discussed in this report does not include effects ingosed by plant transients. The transient conditions and effects will be considered in the future.

Description. The auxiliary cooling loop contains five auxiliary heat exchangers, five auxiliary circulators, and a cooling-water system for supplying the proper quantity of pressurized cooling water to the heat exchangers. The auxiliary cooling system is designed as a class I system and as an engineered safeguard. It will be capable of cooling the reactor with the reactor coolant system either pressurized or depressurized and with one of the heat-exchanger/circulator systems out of service. Each auxiliary circulator is electrically driven by a motor supplied by an independent power supply.

The auxiliary heat exchangers employ helically coiled tubes with a single-pass helium cross-flow passing over the tubes. The water side of the heat exchanger is divided into parallel subsections.

In the external part of each auxiliary cooling system there are two circulating pumps in parallel. One is a low-capacity pump that is used to circulate water through the heat exchangers during normal station operation when the auxiliary circulators are not in operation. The larger pump is used to provide the higher flow rates required for auxiliary cooling. Pumping power is available from multiple sources, including the essential power system. The closed-lcop cooling system rejects heat to the atmosphere through an air-cooled heat exchanger. 
Operation of the heat exchanger will be continuous at full water-side temperature differential $82^{\circ} \mathrm{C}\left(180^{\circ} \mathrm{F}\right)$ inlet, $204^{\circ} \mathrm{C}\left(400^{\circ} \mathrm{F}\right)$ outlet except for two conditions: 1) in the depressurized mode, the heat exchanger outlet temperature will be held at $260^{\circ} \mathrm{C}\left(500^{\circ} \mathrm{F}\right)$, and 2) during the latter stages of decay-heat removal or during refueling, the outlet temperature will decrease because of decreased heat-removal requirements. Operation of the auxiliary circulator service system will be continuous during plant life-time, but at a reduced heat load during the periods in which auxiliary circulator operation is not required.

The following principles govern actions upon startup of an auxiliary core-cooling loop:

1. The auxiliary circulator drive motor is energized to produce a $\mathbf{f}^{*}$. uw corresponding to that of the reactor coolant, and to establish coolant flow through the blanket and the auxiliary heat exchanger.

2. Cooling-water flow in the auxiliary heat-dump system is switched to the large-capacity circulating pump by administrative controls.

3. The power supply, and thus the auxiliary circulator speed, is automatically adjusted until the set point of the helium temperature at the blanket inlet is achieved. This provides for the increase in speed needed for cooling when the reactor is depressurized or for the decrease in speed that should accompany repressurization.

The auxiliary loops will be designed and instrumented to facilitate periodic testing of operability while the plant is on load. The heat exchanger and related heat-removal system will be in continuous service with circulation of cooling water.

\subsubsection{Auxiliary Circulators.}

Functional Requirements. The auxiliary circulators are part of the auxiliary cooling system and provide helium flow for decay-heat removal. Five circulators are provided for the primary loop and will supply the necessary flow in the most severe operating condition. Each auxiliary circulator unit will consist of an electric-motor-driven centrifugal compessor and diffuser. The auxiliary circulators are to be operable at all pressure levels from full helium inventory down to refueling (depressurized) status.

The normal use of the auxiliary circulators will be during the following conditions:

1. Plant shutdown. 
2. Re iueling (approximately atmospheric pressure).

3. F ictional checkout with the reactor at full load.

The emergency-cooling mode of auxiliary circulator operation occurs in the event of trouble in any one main loop. Because of the wide range of required operating conditions, the electric-motor drives will be capable of variable speeds and each motor will be operated by an independent control system and be supplied from an essential power supply.

Performance Requirements. The design of the auxiliary loop is based on a heat-removal capacity of 5 of the normal thermal reactor power. The helium-side design conditions, required for the emergency cooling model of operation, are for a pressure of $1.52 \times 10^{5} \mathrm{~Pa}$ (1.5 atm) and blanket inlet and outlet temperatures of $100^{\circ} \mathrm{C}\left(212^{\circ} \mathrm{F}\right)$ and $500^{\circ} \mathrm{C}\left(932^{\circ} \mathrm{F}\right)$. respectively. These conditions require the following characteristics for each circulator:

Mass flow rate

Circulator outlet pressure

Conpressor pressure rise

Compressor inlet temperature
$23.44 \mathrm{~kg} / \mathrm{s}$

$1.714 \times 10^{5} \mathrm{~Pa}$

$0.193 \times 10^{5} \mathrm{~Pa}$

$100^{\circ} \mathrm{C}$
$186,212 \mathrm{lb} / \mathrm{hr}$

24.87 psia

2.8 psia

$212^{\circ} \mathrm{F}$

General Design Requirements. Equipment, piping, and the electrical wiring associated with the operation of the auxiliary circulators will be designed to operate under environmental conditions resulting from the design basis depressurization accident.

The auxiliary circulator will be capable of startup or shutdown against normal system pressure and temperature and will have a provision for functional checkout with the main circulators operating.

The normal speed control of the circulator will be by means of the variable-frequency power available to the drive motor. The control system and power supply for each circulator will be independent of the others. The motors will be capable of restarting following any voltage interruption.

The single-failure criterion will be met in that failure in any one control system or power supply will not cause a failure or inhibit operation of the other auxiliary cooling subloop.

Description. The design of the auxiliary circulator is conceptual, and only a prelininary design has been made to date.

The preliminary design data for the auxiliary belium circulator is sumarized in Table 9-10. 
Table 9-10. Auxiliary helium circulator preliminary design data.

Type

Drive

Fluid

Speed, rpm

Inlet temperature, ${ }^{\circ} \mathrm{C}\left({ }^{\circ}\right)$

Inlet pressure, Pa (psia)

Outlet pressure, Pa (psia)

Mass flow, $\mathrm{kg} / \mathrm{s}$ (lb/sec)

Efficiency, *

Power, MW (hp)
Centrifugal

Electric motor

Helium

5000

100 (212)

$1.52 \times 10^{5}(22.07)$

$1.71 \times 10^{5}(24.87)$

23.44 (51.72) per circulator

82

2.75 (3700)

The speed required for the auxiliary circulator is a strong function of the primary circuit pressure; quite low speed is sufficient with the reactor coolant pressurized, whereas high speed is required in the depressurized condition. Each circulator is driven by a squirrel-cage induction motor supplied with variable-frequency power from independent essential power supplies for each machine.

Design Evaluation. An electric-motor drive is provided for the auxiliary circulators as a different motive source from the steam turbine drive used on the circulators for the main loops. The design of the auxiliary circulators is based on well-established technology. Due to its fundanental simplicity, the electric-motor-driven centrifugal comprsssor of moderate size and power poses no particular new problems $;$ i development requirements.

Five circulators are provided where four are adequate to meet performance requirements in the event of a depressurization accident. Each circulator is self-contained, and failure of one cannot cause failure in the other. The service systems will be based on the modular concept for each unit for essential services.

Electric power for the motors may be obtained from either the normal or the emergency power sources. Availability of the auxiliary circulators can be assured in the time available in the event of loss of a main loop.

9.2.3.3 Auxiliary Heat Exchangers.

Functional Requirements. The purpose of the auxiliary heat exchangers is to remove heat from the reactor helium coolant when the main cooling loops are shut down and unavailable for this purpose. Helium flows 
through the heat exchanger, where heat is transferred to the cooling water inside the tubes. Cooled helium leaving the top of the heat exchanger enters the auxiliary circulator and is circulated through the blanket modules to repeat the cycle.

Design and Operating Requirements. The auxiliary heat exchangers will be designed to provide cooling for both pressurized and depressurized conditions. Each heat exchanger will have a heat-removal capacity of about 1.258 of the normal reactor power. For the depressurized design case, the required auxiliary loop will provide adequate cooling following a reactor scram. At present, transfer of reactor cooling to the auxiliary loop (if ACS service is required), is expected in about 85 seconds, worst case following plasma shutdown, based on GCFR plant practice. Within a few seconds or almost instantaneously after the reactor shutdown, the auxiliary circulator could be brought to speed if there is not a loss of offsite power. The parameters for the depressurized design conditions are summarized in Table 9-11.

Boiling in the auxiliary heat exchangers will be prevented by maintaining the water under a pressure sufficient to assure approximately $28^{\circ} \mathrm{C}\left(50^{\circ} \mathrm{F}\right)$ subcooling at the maximum expected operation temperature.

Safety relief valves will be provided for the auxiliary heat exchangers to protect then from overpressurization when an exchanger is isolated on the water side.

Since the depressurized condition limits the design of the auxiliary heat exchanger, the pressurized condition performance was assumed to be satisfactory.

Other considerations that govern the design of the heat exchangers are discussed below.

The tubes in the heat-exchanger tube bundle will be segregated into subgroups, each with approximately the same number of tubes. Separate cooling-water supply (and return) lines will connect each of these tube subgroups to the cooling-water headers. The arrangement of the tubes will be such as to minimize the effect of inleakage from a tube failure.

The heat exchangers will be designed so that they will not be damaged or be caused to malfunction either by internally generated vibrations, such as flow-induced vibrations, or by environmental vibrations. 
Table 9-11. Design data for an auxiliary heat-exchanger. a

\section{General}

Helium frontal flow area, $\mathrm{m}^{2}\left(\mathrm{ft}^{2}\right.$ )

$3.48(37.5)$

Heat duty, $(B t u / h)$

$48.71\left(166.39 \times 10^{6}\right)$

Logarithmic mean temperature difference, ${ }^{\circ} \mathrm{C}\left({ }^{\circ} \mathrm{F}\right)$

68 (154.52)

overall heat transfer coefficient, $w / m^{2} \cdot k$

$$
\text { (Btu/h-ft }{ }^{2}-{ }^{\circ} \text { ) }
$$

$549(96.7)$

Heat transfer surface area, $m^{2}\left(f t^{2}\right)$

$1034.5(11,136)$

Helium aide

Flow rate, $\mathrm{kg} / \mathrm{s}(1 \mathrm{~b} / \mathrm{h})$

$23.44(186,212)$

Inlet temperature, ${ }^{\circ} \mathrm{C}\left({ }^{\circ}\right)$

500 (932)

outlet temperature, ${ }^{\circ} \mathrm{C}\left({ }^{\circ}\right)$

100 (212)

Average pressure, Pa (psia)

$1.52 \times 10^{5}(22.07)$

Pressure drop, Pa (psi)

$13,788 \quad(2.0)$

Water side

Flow rate, $\mathrm{kg} / \mathrm{s}(\mathrm{lb} / \mathrm{h})$

$62.63(497,500)$

Inlet temperature, ${ }^{\circ} \mathrm{C}\left({ }^{\circ} \mathrm{F}\right)$

82 (180)

outlet temperature, ${ }^{\circ} \mathrm{C}\left({ }^{\circ} \mathrm{F}\right)$

$260(500)$

Average pressure, Pa (psia)

$14.48 \times 10^{6}(2,100)$

Pressure drop, Pa (psi)

$2.068 \times 10^{5}(30)$

Tubes

Tube size, min (in.)

$19 \times 2.1$

$(0.75) \times(0.083)$

Longitidinal tube pitch, mu (in.)

$25(1.0)$

Transverse tube pitch, mm (in.)

$31(1.246)$

Tube bundle height, m (ft)

$3.048(10)$

a Depressurized design conditions.

The design, fabrication, inspection, and materials specified for the component parts of the auxiliary heat exchangers shall be in accordance with the requirements of the appropriate approved codes where applicable.

Description. The auxiliary-loop coolant is pressurized water. Each heat exchanger is a helically wound, axial-flow tube bundle with an integral shroud. The tube bundle is approximately $2.43 \mathrm{~m}(8 \mathrm{ft}) 0 . d$. and $3.05 \mathrm{~m}$ (10 ft) high and is made of carbon or low-alloy steel. Preliminary design data for the auxiliary heat exchanger are summarized in Table 9-11. 
The helium flow path from the blanket through the auxiliary cooling loop is similar to that through the main cooling loop. During normal reactor plant operation, there is a small bleed of helium from the blanket through the closed auxiliary loop. A small flow of cooling water through the heat exchangers maintains the auxiliary cooling loops at close to the cold helium temperature. This reduces thermal transients on loop startup and minimizes parasitic heat losses. During use for shutdown cooling (depressurized), the design inlet water temperature is $82^{\circ} \mathrm{C}\left(180^{\circ} \mathrm{F}\right)$ and the outlet water temperature to the heat-dump system is $260^{\circ} \mathrm{C}\left(500^{\circ} \mathrm{F}\right)$.

Design Evaluation. The auxiliary heat exchanger is conventional as to design, material, and duty. Dynamic flow instabilities are not anticipated, since no boiling occurs. The performance of the heat exchanger under transient concitions will be evaluated later.

\subsubsection{ACS Response and Depressurization Accident Elfects}

9.2.4.1 Punctional Aspects. The general response of the blanket during a depressurization accident is that the maximum fuel temperature is reached in about 24 seconds (see Sec. 12.1) after depressurization is comp:ete and no coolant flow is established. The main circulators have considerable power and speed capability, so the depressurized conditions or the effects of air ingress do not impose undue requirements. Should a depressurization accident occur, the main cooling loops would continue to provide shutdown cooling, and the auxiliary cooling system (ACS) would provide an independent and diverse backup if the main loops should fail. Presently the ACS is designed to operate immediately following signals from a main loop failure to function. The depressurized condition provides the design basis for the ACS circulator and its electrical drive motor. If the main loops fail and the cooling function is transferred to the ACS, the initial ACS water temperatures and circulator speed are dependent upon the timing of main loop failure.

9.2.4.2 Auxiliary Loop cooler. The auxiliary loop cooler transfers heat to the ultimate heat sink, which is air. Its required performance is defined by the loop heat duty and the maximum auxiliary heat exchanger water inlet and outlet temperatures, but the actual design is not yet definitely specified at this conceptual stage. The auxiliary loop cooler is a counter cross flow heat exchanger with water on the tube side and air on the shell side. 
9.2.4.3 Auxiliary Cooling system Response and Performance Following Depressurization. The auxiliary cooling system is made up of the auxiliary heat exchanger (AHE), the auxiliary loop cooler (ALC), the auxiliary circulator, and the pressurized water loop with pumps and pressurizer. $A$ schematic of this system is shown in Fig. 9-13. Heat is transferred from the reactor coolant to the flcwing water system in the AHE and is rejected from the flowing water system to the ambient air in the ALC. The auxiliary circulators are of centrifugal compressor design and each is driven by a squirrel-cage induction motor which derives variable frequency power from separate essential buses. The design of the auxiliary circulator is conceptual, and only a preliminary design has been made. The torque required of the circulator arive motor is directly related to the system pressure drop. The circulators are nearly constant volume flow machines, implying that the mass flow is nearly proportional to the product of the motor speed and the coolant density. For a low system pressure drop characteristic, as would be expected for full depressurization with pure helium as the reactor coolant, the maximum speed limit would be encountered. If significant air ingress occurs in a depressurized reactor, the system density increases, and the motor torque limit may be reached and the motor speed would be reduced.

During normal plant operation, the ACs loops are in a standby mode with the auxiliary circulators shut down and the auxiliary-loop isolation valves closed, and a small leakage flow from the blanket inlet manifold will expose the auxiliary heat exchanger to a helium temperature of $282^{\circ} \mathrm{C}\left(540^{\circ} \mathrm{F}\right)$. In order to prevent boiling in the auxiliary heat exchangers should the water flow stop, the system will be pressurized to $14.67 \mathrm{mPa}$ (2100 psia), which is the saturation pressure at $340^{\circ} \mathrm{C}\left(643^{\circ} \mathrm{F}\right)$. The main circulating pumps and the large air fans are shut down. Only small auxiliary circulating pumps operate in each loop to provide a small flow through the coolant system to maintain the equipment near operating temperature and prevent thermal shock to the system when the main pump starts. The auxiliary pump flow is small enough so that it does not constituts a major thermal loss for the reactor. The initial water temperature prior to the depressurization accident can be set by the helium leakage flow, the auxiliary pump flow, and the small air flow in the ALC.

When a reactor trip or a scram signal is generated, the ACs controls are activated for starting the large air fans and the main circulating 


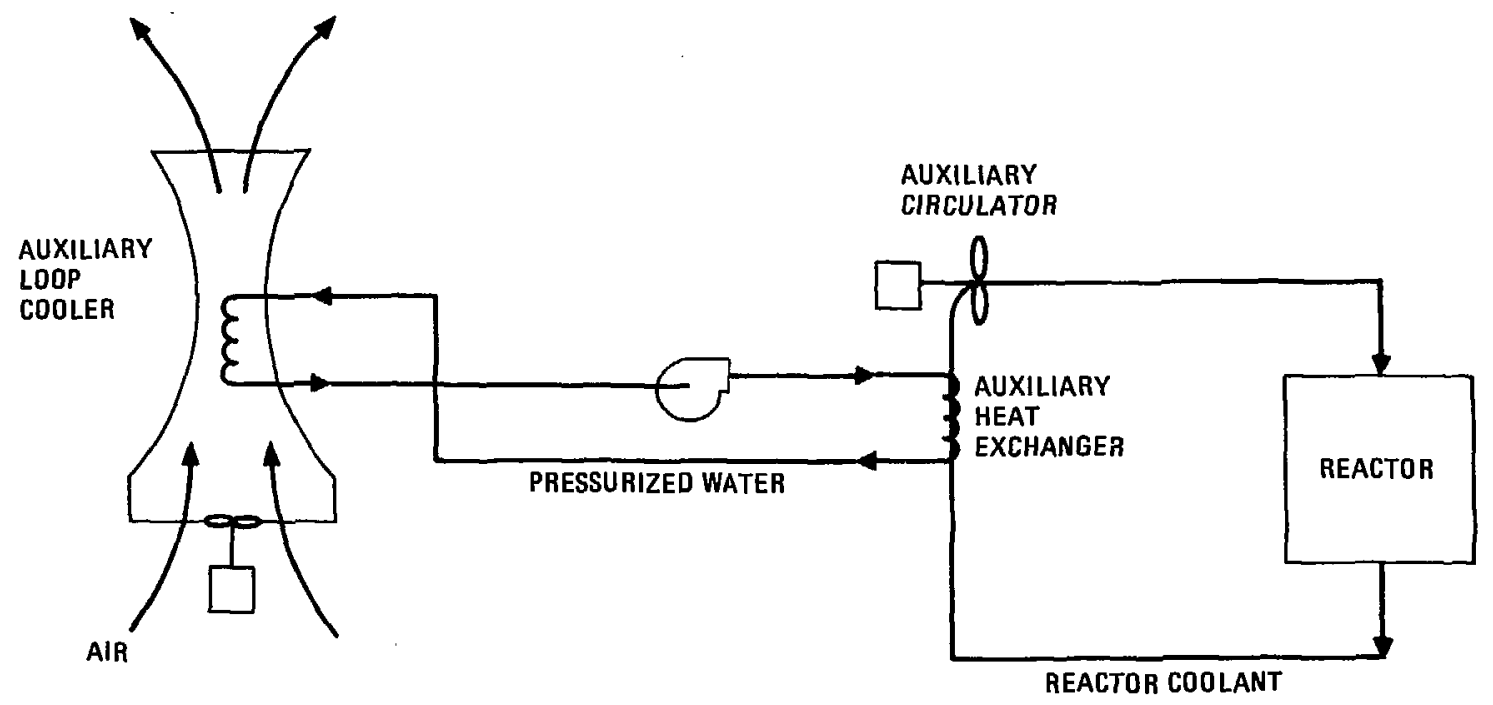

Fig. 9-13. Auxiliary cooling system schematic. 
pumps. Speed controls of the auxiliary circulator will respond to the main cooling loop performance and the auxiliary circulator is in a ready condition to transfer cooling to the ACS, in case of main loop functional problems.

It should also be noted that the ACs controls, while switching over the cooling function from the main loops to the auxiliary loops, will not impose serious thermal transient effects on the loop components.

A depressurization accident may cause the helium inventory from the power conversion system to be mixed with the air in the secondary containment. The ACS design and its performance are based on this possibility. It is estimated, based on GCFR experience, that significant air ingress effects on the cooling will be introduced approximately 10,000 seconds following a depressurization accident. The thermal power at this time is approximately 18 of the full thermal power.

9.2.4.4 Sequential and Emergency Use of ACS. The ACs, if required for the cooling operation following a depressurization accident, could be used either in conjunction with the cooling by main loops after a deferred time interval, or within a few seconds after the accident. The modes of its possible operations are summarized as follows.

The ACs has two basic objectives: one, to serve as a diverse backup with its service reserved for assistance in case of unavailability of the main loops to cool the reactor following an accident, and second, to supplement the long-term blanket decay-heat removal at low thermal power ( $\leq 18$ full power).

Subsequent to an accident or a trip of the reactor, steam from the main steam generators will be stored in separate pressure vessels and the fusion plasma power will be instantaneously terminated, resulting in blanket power being dropped to 58 of its full thermal power. The stored steam will then be used to drive the main circulator turbines for helium circulation, and blanket cooling will be performed through the primary cooling loops. It is estimated that the stored steam energy will be sufficient to arive the circulator turbines for about 30 minutes following the accident, accounting for the decrease in the helium flow rate with the transient decrease in the af terheat.

At the signal of a reactor accident or a trip, the auxiliary boilers will be operated to capacity and auxiliary drive steam is expected to be available within a few minutes. When signs of the insufficiency of the 
stored main steam energy to drive the main circulator turbines are indicated, the auxiliary steam is supplied to the circulator turbines for continuing the cooling through the primary loop. The auxiliary boiler system is capable of providing the steam for cooling for shutdown and also for long-term decay-heat removal on the main circulators.

Thus, the need to the ACs does not arise for this mode of afterheatremoval. The ACS may be used for a long-term decay-heat removal at a low thermal power (such as $\leq 18$ full power), if 1 t is economically favorable over the auxiliary boiler operation at such conditions. This use in conjunction with the main loop cooling will be the logical use of the ACS following a normal or accidental shutdown.

However, if for some reason the main loops have totally failed to provide cooling and the auxiliary boilers could not come on line in time, then the ACs will be used to provide the emergency cooling. Within a few seconds, the electrically driven ACs circulators will be brought to speed and the system will be ready to cool the reactor.

Figure 9-14 shows the sequential and emergency points of operation of the ACS and the standard fission-product decay heat curve as a tunction of time.

Preliminary analysis, using the conservative assumption of adiabatic heating, shows that in case of total loss of the cooling following an accident, it takes about 24 seconds before the fuel could begin to melt and about 82 seconds before the cladding could begin to melt. The ACS system is designed to operate within a few seconds following any type of accident, and provides the cooling. In the event of loss of offsite power, however, starting of the emergency diesel generators and start of the ACS system could take as long as 85 seconds, providing potentially inadequate protection of the fuel. Although more detailed analysis is expected to show that cladding failure will not occur, this area requires further study. 9.2.5 Waste Heat Utilization

9.2.5.1 Direct Converter and Beam Dump.

Heat Availability. The basic principles and operation of the direct energy converter proposed for use in the MHR plant are described in Chapter 8 of Ref. 13. Most of the trapped, injected neutral beam is scattered into the loss cone (defined by the magnetic field) and lost before it can react. Only a small fraction of the injected current is consumed in fusion reactions and it is estimated that as much as 98 to 99 of the 


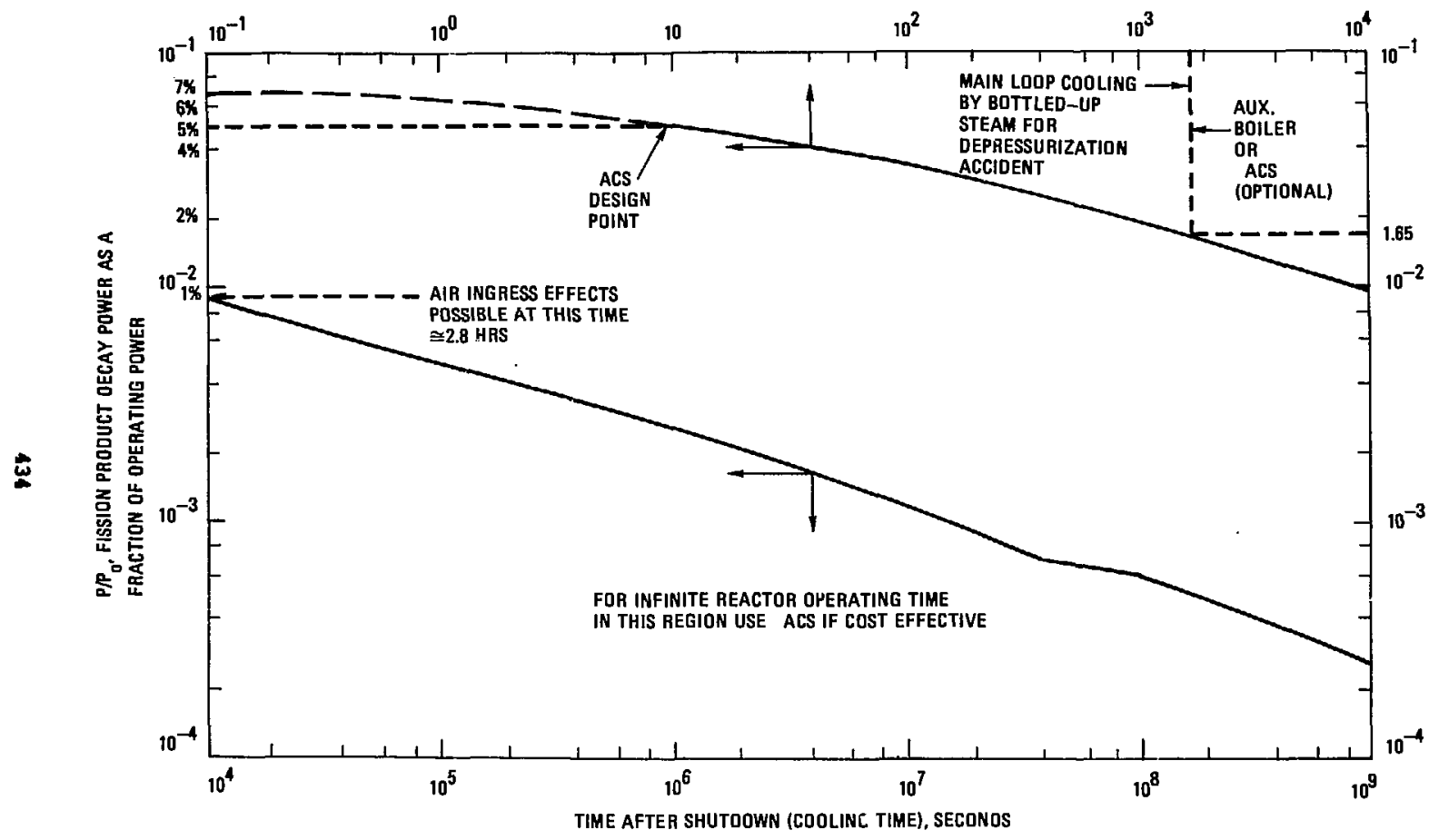

Fig. 9-14. Auxiliary cooling system operating ranges. 
injected current, plus the a-particle current, is scattered into the relocity-space loss cone and escapes through the magnetic mirrors. This loss current has to be disposed of, and the best practical means for recovering the lost energy are offered by a combination of a direct energy converter and a beam dump unit.

The net extractable thermal energy from the direct converter and the beam dump is estimated at $415 \mathrm{MWt}$ by LLL during the present MHR study, and helium will be circulated to transfer this energy. It is indicated that the heat extraction from the energy converter and the beam dump can be performed at the same operating temperatures as the fission blanket assembly, helium inlet at $282^{\circ} \mathrm{C}\left(540^{\circ} \mathrm{F}\right)$ and outlet at $540^{\circ} \mathrm{C}\left(1004^{\circ} \mathrm{F}\right)$. Thus, the available thermal energy from the direct converter and the beam dump, together with the fission blanket thermal energy, will be input to the power conversion system.

Design Bases. The positioning of the neutral beam injectors with respect to the Yin-Yang magnets as show in Fig. 8-1 requires the direct converter to be located at the bottom of the plasma chamber for recovering the unused energy. The configuration of the magnetic flux lines from the plasma lead to the direct energy converter with no obstructions. Only a small amount of leakage cocurs in the upward direction and a beam dump unit is provided at the top as shown in Fig. 8-1 for recovering the thermal energy.

Both the direct converter (DC) and the beam dump operate in an environment which is unlikely to cause a concern from the safety standpoint due to the accidental release of radioactive substances. Only mild traces of tritium are expected in the $D C$ and beam dump unit cooling systems. Therefore, the power conversion system components associated with the DC and bean dunp unit are not expected to need to neet the safety criteria established for the components associated with the fission blanket assembly.

The DC unit fans out from the plasma chamber and occupies a sizeable space. In the present design it is located under the PCRV as shown in Fig. 8-1. A reinforced concrete structure surrounds the DC, acting as a pressure vessel, and the structure will be designed to withstand the design pressure, thermal, and mechanical stresses. It is proposed to have a separate power conversion loop (PCL) for the DC and the bean dump unit, isolated from the PCI associated with the fission blanket assembly. Approximately 415 wt power is estimated to be available from the DC-bean 
dump units, ar:3 the DC-PCL, which includes a separate steam generator and steam driven circulator, will operate at the same conditions as the PCL of the fission blanket assembly. It should be noted that performancewise, both PCL's are identical. The steam pipes emerging from all steam generator units will be manifolded together, and a comon pipe will carry the superheated steam to the power turbines. The helium loop schematic is shown in Fig. 9-11.

By having separate PCL's for the DC-beam dump units and the fission blanket assembly, the helium flows circulating in the respective loops do not come in contact with each other. Thus, during a worst-case depressurization accident occurring either in the $D C$ or in the beam dump unit, no significant release of radioactivity is expected. In addition, no auxiliary or emergency cooling provision is needed during the depressurization accident in the DC or beam dump unit, because on signal from a depressurization event the plasma power will be instantaneously terminated and no decay heat exists in the affected unit.

9.2.5.2 Injector waste Heat Utilization.

Waste Heat Availability. Neutral beam injectors continuously inject beams of deuterium and tritium atoms at high ve'scities into the plasma chamber while the MHR is in operation. Injection of beams at higher velocities consume enormous amounts of power; for the selected design parameters in the present study, approximately 1147 me power is required as input for the injectors. Preliminary studies by LLL indicated that the efficiency of the injectors is about 55\%: and thus a waste heat equivalent of $519 \mathrm{wt}$ is generated and has to be rejected to sink in the plant. Studies performed by LLI further indicated that only 758 of the injector waste heat (389 $\mathrm{MH}$ ) can be extracted at a possible maximum temperature of $227^{\circ} \mathrm{C}\left(440^{\circ} \mathrm{F}\right)$. Such a low-temperature heat source is recomended for use in the feedwater heating system, thereby decreasing the amount of steam normally bled from the power turbines for feedwater heating.

Injector Waste Heat System Conceptual study and Operation. From the standpoint of the operation of the neutral beam injecors, an inert fluid which is electrically nonconductive is desired to circulate and extract the waste heat from the injector system. Helium is a good candidate gas for such an operation and is selected as the primary coolant for the waste heat system. Heat from the helium gas will be transferred to water (secondary coolant) through a waste heat exchanger, and finally the hot water will be 
cascaded with the steam cycle feed heat train. Helium exiting the heat exchanger is then circulated through the primary loop by an electrically driven circulator. It is possible for some traces of tritium to get entrained in the helium stream and hence the waste heat exchanger and circulator units will be located in the PCRV to limit any accidental release of radioactive substances.

A conceptual arrangement of the waste heat system is show in Fig. 9-15. The hot water, after leaving the heat exchanger, enters the high-pressure feedwater heater and is cascaded through the low-pressure feed heaters and finally flows down to the main condenser (see Fig. 9-12). Low-temperature water is withdrawn from the condenser and is circulated to the waste heat exchanger by a separate water pump. By cascading both turbine bleed steam and injector waste heat cooling water in parallel, the waste heat can be effectively used while retaining an independent control parameter (the bleed steam) to control the feedwater conditions.

Selection of Waste Heat Systen Parameters. The LIL stuaies have indicated that the waste heat from the injector system can be extracted at a possible maximum temperature of $227^{\circ} \mathrm{C}\left(440^{\circ} \mathrm{F}\right)$. A search for the effective use of the injector waste heat at this temperature leads to the feedwater heating system. From the steam cycle performance standpoint, it will be advantageous and efficient if the amount of steam bled from the high-pressure power turbine to heat the feedwater could be reduced; the state of the steam entering the low-pressure turbines would then have a higher enthalpy and temperature and thereby increase the output power of the turbines. Earlier steam cycle analyses have shown that the last-stage high-pressure heater (HP heater NO. 3, see Fig. 9-12) operates at a condensing temperature of approximately $160^{\circ} \mathrm{C}\left(320^{\circ} \mathrm{F}\right)$, and it is preferred that the water in the waste heat exchanger be raised to this temperature at a pressure of $0.86 \mathrm{MPa}$ (125 psia) and saturation temperature of $174^{\circ} \mathrm{C}\left(345^{\circ} \mathrm{F}\right)$ for cascading with the feed-heat system. The condensate exits the feed-heat system at a temperature of $52^{\circ} \mathrm{C}\left(125^{\circ} \mathrm{F}\right)$, and a separate feed pump circulates the condensate back through the waste heat exchanger, completing the secondary coolant loop.

The water-side pressure drop in the heat exchanger is about 30 psia. The operating water pressure in the heat exchanger was selected to be such that at any spatial point in the unit the water temperature is well below its saturation temperature, eliminating the possibility for a two-phase flow 


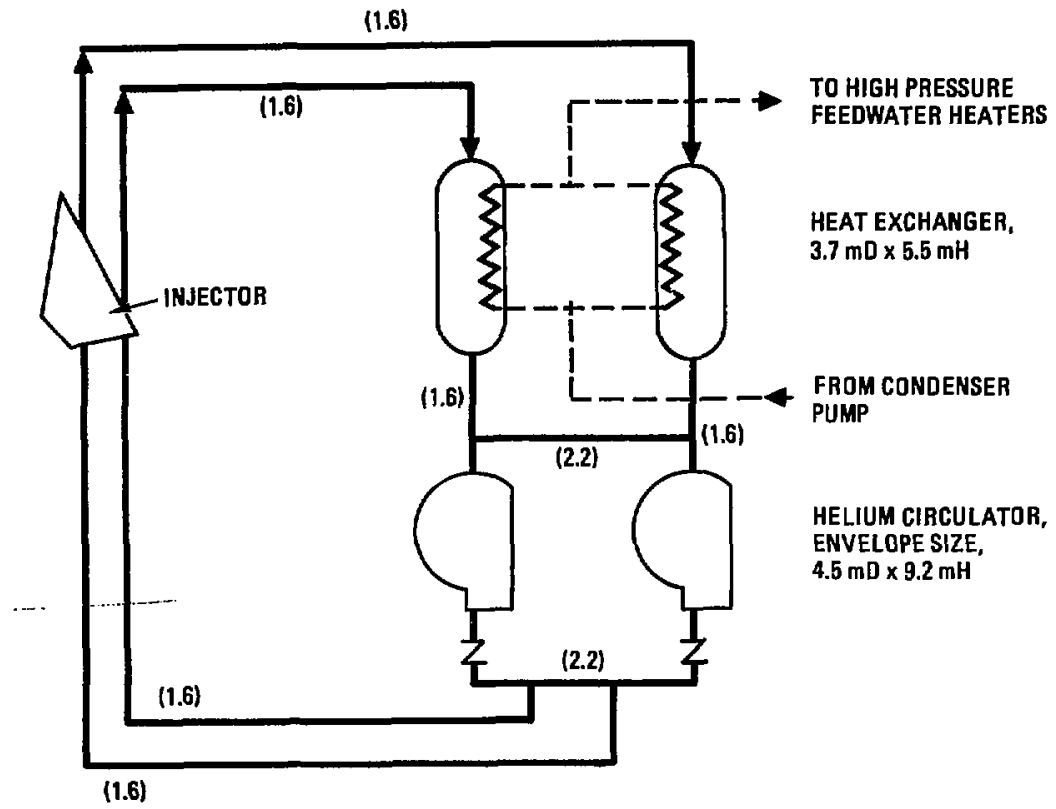

* duct Size in Meters ().

Fig. 9-15. Injector waste heat 1oop. 
condition to exist. The average helium operating pressure is about 100 psia, which is less than the water-side pressure. The reason for selecting a lower helium operating pressure is safety-related; if a leak occurs in the heat exchanger, water will ingress into the helium side, thus preventing possible release of radioactive substances entrained in the helium. The pressure drop in the helium lop is estimated at $2.5 \mathrm{psi}$. Table 9-12 shows the major design parameters for the injector waste heat system.

Waste Heat Bxchanger Design Bases. The function of the waste heat exchanger is to transfer the waste heat from the neutral beam injectors to the secondary coolant (water), which in turn rejects 1 t to the main steam feed heat system. Helium enters at a temperature of $227^{\circ} \mathrm{C}\left(440^{\circ} \mathrm{F}\right.$ ) and gives up its heat to produce hot water at $160^{\circ} \mathrm{C}\left(320^{\circ} \mathrm{F}\right)$.

The waste heat exchangers will be designed for continuous operation. Each neutral beam injector will be equipped with two waste heat exchangers, and the plant has four (4) in total. Each exchanger has an envelope size of

Table 9-12. Injector waste heat system principal design data.

Power tata

Total injector waste heat power, Mwt

Net available waste heat power, MWt

Helium circulating pumping power, MWt
519.0

389.0

11.2

Primary coolant (helium side) data

Average pressure, Pa (psia)

$6.89 \times 10^{5}(100)$

$\triangle \mathrm{P} / \mathrm{P}$,

2.5

Total flow rate, $\mathrm{kg} / \mathrm{s}(1 \mathrm{bm} / \mathrm{h})$

Inlet temperature at waste heat exchanger, ${ }^{\circ} \mathrm{C}\left({ }^{\circ} \mathrm{F}\right)$

$562.0\left(4.46 \times 10^{6}\right)$

Outlet temperature at waste heat exchanger, ${ }^{{ }} \mathbf{C},\left({ }^{{ }_{F}}\right)$

227 (440)

94 (200)

Secondary coolant (water)

Pressure at heat exchanger inlet, Pa (psia)

Pressure at heat exchanger outlet, Pa (psia)

Net pressure drop in waste heat exchanger, $\mathrm{Pa}$ (psi)

Net flow rate, $\mathrm{kg} / \mathrm{s}(\mathrm{lb} / \mathrm{h})$

Tenperature at inlet, ${ }^{\circ} \mathrm{C}\left(\mathrm{O}_{\mathrm{F}}\right)$

Temperature at outlet, ${ }^{\circ} \mathrm{C}\left({ }^{\circ} \mathrm{F}\right)$
$1.07 \times 10^{6}$

$8.6 \times 10^{5}$

$2.07 \times 10^{5}$

$830.0\left(6.59 \times 10^{6}\right)$

52 (125)

160 (320) 
about $3.66 \mathrm{~m}(12 \mathrm{ft})$ in diameter and $5.5 \mathrm{~m}(18 \mathrm{ft})$ in helght. The heat exchanger is an axial flow type and the tube bundle is helically wound. The water flow on the tube side is upward; and the helium flows downward across the tube bundle. The major design parameters are summarized in Table 9-13. Helium coolant carries the waste heat from the neutral beam injectors via ducts to the waste heat exchanger. As the gas flows downward across the tube bundle, it transfers its heat to the water in counter-flow and the cooled gas then passes to the waste heat circulator inlet. Gas leaving the circulator flows through the ducts and re-enters the injector system, completing the waste heat system primary circuit.

Table 9-13. Design data for the injector waste heat-exchanger.

General

Helium frontal flow area, $\mathrm{m}^{2}\left(\mathrm{ft}^{2}\right)$

Heat duty, Mu (Btu/h)

Logarithmic mean temperature difference, ${ }^{\circ} \mathrm{C}\left({ }_{\mathrm{F}}\right)$

Overall heat transfer coefficient, $w / m^{2} \cdot R$

$$
\text { (Btu/h-ft }{ }^{2}-o_{F} \text { ) }
$$

Heat transfer surface area, $\mathrm{m}^{2}\left(\mathrm{ft}^{2}\right)$

Hellum side

Flow rate, $\mathrm{kg} / \mathrm{s}(\mathrm{lb} / \mathrm{h})$

Inlet temperature; ${ }^{\circ} \mathrm{C}\left({ }_{F}\right)$

outlet temperature, ${ }^{\circ} \mathrm{C}\left({ }^{{ }}\right)$

Average pressure, $\mathrm{Pa}$ (psia)

Pressure drop, Pa (psi)

Water side

Flow rate, $\mathrm{kg} / \mathrm{s}(\mathrm{lb} / \mathrm{h})$

Inlet temperature, ${ }^{{ }_{C}}\left({ }_{F}\right)$

Outlet temperature, ${ }^{\circ} \mathrm{C}\left({ }_{F}\right)$

Average pressure, MPa (psia)

Pressure drop, MPa (psi)
$3.8(95.0)$

$95.0\left(324 \times 10^{6}\right)$

$37.6(99.65)$

799.0 (140.7)

2143 (23067)

$140.5\left(1.116 \times 10^{6}\right)$

227 (440)

104.5 (220)

0.6894 (100)

$8976(1.302)$

$207.0\left(1.647 \times 10^{6}\right)$

51.7 (125)

160 (320)

0.862 (125)

0.206 (30)

Tube size, $\min ($ in)

$19.05(0.75)$

Iongitudinal tube pitch, mu (in.)

$25.4(1.000)$

Transverse tube pitch, mm (in.)

$47.63(1.875)$

Tube bundle height, $m$ (ft)

$4.88(16.00)$ 
Low-temperature water exiting from the steam cycle feed heat train enters through the lead-in tubes connecting to the bottom of the waste heat exchanger.

The waste heat exchanger is of mono-tube construction, and the tube bundle has $0.02 \mathrm{~m}(0.75 \mathrm{in.})$ diameter tubes. Tube plugging to isolate defective tubes is done at the tube sheets, which are readily accessible. Injector Waste Heat System Circulator Design. The waste heat circulators are part of the waste heat system and provide helium flow for the injector waste-heat removal. Two circulators will be provided for each injector waste heat system so that in the event one should fail, the other can operate without interrupting the continuous operation of the injectors. Both the circulator and the water pump will be designed vith the potential for higher capacity when needed, resulting in a satisfactory operation of the injectors. The circulator will be an axial flow type provided with an electrical drive. During the normal operation, each waste heat circulator will provide a helium flow rate proportionate to 50 of the extracted waste heat from the injector.

The heliun-side design conditions, required for the normal operation, are for a $0.69 \mathrm{MPa}(100 \mathrm{psia})$ pressure and injector system inlet and outlet temperatures of $93^{\circ} \mathrm{C}\left(200^{\circ} \mathrm{F}\right)$ and $227^{\circ} \mathrm{C}\left(440^{\circ} \mathrm{F}\right)$, respectively. These conditions require the following circulator characteristics for each waste heat circulator.

Mass flowrate of helium

Circulator outlet pressure

Pressure rise through the circulator

Circulator inlet temperature

$$
\begin{aligned}
& 141 \mathrm{~kg} / \mathrm{s}\left(1.12 \times 10^{6} \mathrm{lb} / \mathrm{h}\right) \\
& 0.706 \mathrm{MPa}(102.5 \mathrm{psia}) \\
& 0.017 \mathrm{MPa}(2.5 \mathrm{psia}) \\
& 93.4^{\circ} \mathrm{C}\left(200^{\circ} \mathrm{F}\right)
\end{aligned}
$$

Each circulator operates independently of the other, and thus failure of one cannot cause failure in the other. Electric power for the motors will be drawn from the normal power sources. Table 9-14 sumnarizes the design paraneters of the waste heat circulator.

Injector Waste Heat System Ducting. The ducts connecting the circulators with the injectors and the heat exchangers are designed to be within practical sizes as shown in Fig. 9-15. The ducting connection also provide operability if either a heat exchanger or a circulator should have a failure. However, if a rupture occurs in the heat exchanger unit, it is imperative that the plant be shut down and appropriate remedial action be initiated. 
Table 9-14. Waste heat heliu circulator preliminary design data.

Type

Drive

Fluid

Speed, rpm

Inlet temperature, ${ }^{\circ} \mathrm{C}\left({ }^{\circ} \mathrm{F}\right)$

Inlet pressure, Pa (psia)

Outlet pressure, Ra (psia)

Mass flow, $\mathrm{kg} / \mathrm{s}$ (lb/s)

Efficiency,

Power, sis (hp)
Axial

Electric motor

Helium

2000

$93(200)$

$6.9 \times 10^{5}(100)$

$7.1 \times 10^{5}(102.5)$

140.5 (310) per circulator

82

$3.73(5000)$ 
1. K. R. Schultz, et al., Conceptual Design of the Blanket and Power Convergion System for a Mirror Hybrid Fusion-Fission Reactor, General Atomic Co., Rept. GA-Al4021 (Jul. 1976).

2. R. Leggett, et al., "Achieving High Exposure in Metallic Uranium Fuel Elements," Nuclear Appl. Tech. 9, 673 (Nov. 1970).

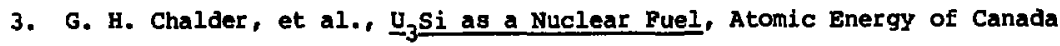
Limited, Rept. AECL-2874 (Hay 1967).

4. M. A. Feraday, et al., The Irradiation Behavior of $\mathrm{U}_{3}$ Si Elements to High Burnup, Atomic Energy of Canada Limited, Rept. AECL-4058 (Mar. 1974).

5. M. A. Feraday, et al., Irradiation Behavior of a Corrosion Resistant U-Si-Al Alloy, Atomic Energy of Canada Limited, Rept. AECL-5028 (Mar. 1975).

6. R. B. Matthews and M. L. Swanson, "Swelling of Uranium Silicide Fuel During Postirradiation Heating," Nucl. Tech. 26, 3, (Jul. 1975), 243.

7. P. C. Souvers, J. Phys. Chem. of Solids 30 (1969), pp. 2649-2556.

8. H. Fenech, "Heat Transfer Test in a Wire-Wrapped Bundle," report in preparation.

9. M. D. Carelli and D. R. Spencer, CRBRP Assemblies Hot Channel Factors Preliminary Analysis, WARD D-0050 (1974).

10. I. E. Idel'chik, Handbook of Hydraulic Resistance, AEC-TR-6630, (1966).

11. E. H. Novendstern, "Pressure Drop Prediction for Fue: Assemblieg, with Wire Wrap Spacer System," ANS Winter Annual Meeting (Miami Beach, Fla., Oct. 17-21, 1971).

12. Progress on the Conceptual Design of a Mirror Hybrid Fusion-Fission Reactor, Lawrence Livermore Laboratory, Rept. UCRL-51797 (June 25, 1975). 


\section{PLANT LAYOUT}

\subsection{General Considerations}

There are four predominant features of any large standard mirror reactor that strongly influence overall plant design. They are:

- the mass of the mirror magnet, with its attendant problems of fabrication, transport to site, and Installation.

- the force restraint structure, in this case a prestressed concrete reactor vessel (PCRV), which is very massive and dictates the order in which major plant components are constructed.

- the end tanks, which occupy a large volume and strongly influence the method of maintenance and blanket replacement.

- the secondary containment structure, which must be built to enclose the PCRV and its servicing crane.

The preferred direction for the plasma-fan axis is vertical. This permits the magnets to move vertically into and out of their pockets in the PCRV. It aj.so permits crane access to steam generators and helium circulators. Fabrication of a PCRV will be much simpler if the outside surface is circular and accessible from all directions. This implies a vertical, right-circular-cylindrical geometry for the PCRV.

The presence of a large end tank above the PCRV would inhibit servicing the thermal equipment and make blanket changing much more difficult. By making the top airror a little stronger than the bottom one, about 90\% of the leakage ions go out the bottom fan. Instead of a direct converter above the PCRV, a much simpler and smaller energy "durp" can be used - one that need not be totally removed for essential blanket change-out or servicing. This means a larger end tank underneath the plasma chamber, but size in that region has at least one advantage. If the bottom mirror coil fails, it must be lowered into the end tank before being translated horizontally toward the fabrication building. An end tank adequate in size to provide an "escape route" for one mirror coil is needed.

In 1976, a mirror hybrid was designed that required one mirror coil to be moved to gain access to the blanket for routine replacement. This was a difficult, time-consuming task, and penalized the design. We present in this design a method of blanket replacement that does not require mirror 
magnets to be moved. A smaller crane is required and a remote servicing machine enters the top of the spherical blanket through the top end tank. Small modules are removed and replaced instead of the large "orange-slice" segments of the 1976 design. (Appendix A also discusses a design that requires coil removal, but not by the hydraulic flotation method.)

A very large containment shell is planned to cover the entire nuclear system. Its diameter is $84 \mathrm{~m}$ and its height to the spring-line is $62.5 \mathrm{~m}$. The overall height is $74 \mathrm{~m}$. Construction of a shell of such a diameter has not been previously accomplished, but it should not present any unique problems. The roof is challenging, but a reasonable construction method is presented.

Because of the large excavation required for the bottom end tank, it was decided that a circular underground structure be built about $80 \mathrm{~m}$ in diameter and $42 \mathrm{~m}$ below the lower surface of the PCRV, which can be assumed to be located at-grade. The direct convertor is elliptical in cross section. The portion of the underground cylinder outside this ellipse is available for electrical-power conversion equipment associated with the neutral beam sources and the direct convertor. The cryogenics system, control room, tritium-processing equipment and thermal-unit service room will occupy the four corners of a square building surrounding the aforementioned underground cylinder. We plan that the turbine-generator building will be above ground and of conventional design. Due to the undergrounding of much of the auxiliary equipment the plant site will have only the following component visible: (see Fig. 10-1).

a. the containment building over the nuclear island.

b. the magnet-fabrication building on a filled (or natural) mesa adjacent to the containment building.

c. the turbine hall and switchyard.

d. the administration and laboratory building.

e. the cooling tower (s).

\subsection{Magnet Considerations}

The mass of one-half of the coil-set with its coil case is about 3000 tonnes. The conductor probably cannot be wound in place due to the coils' 


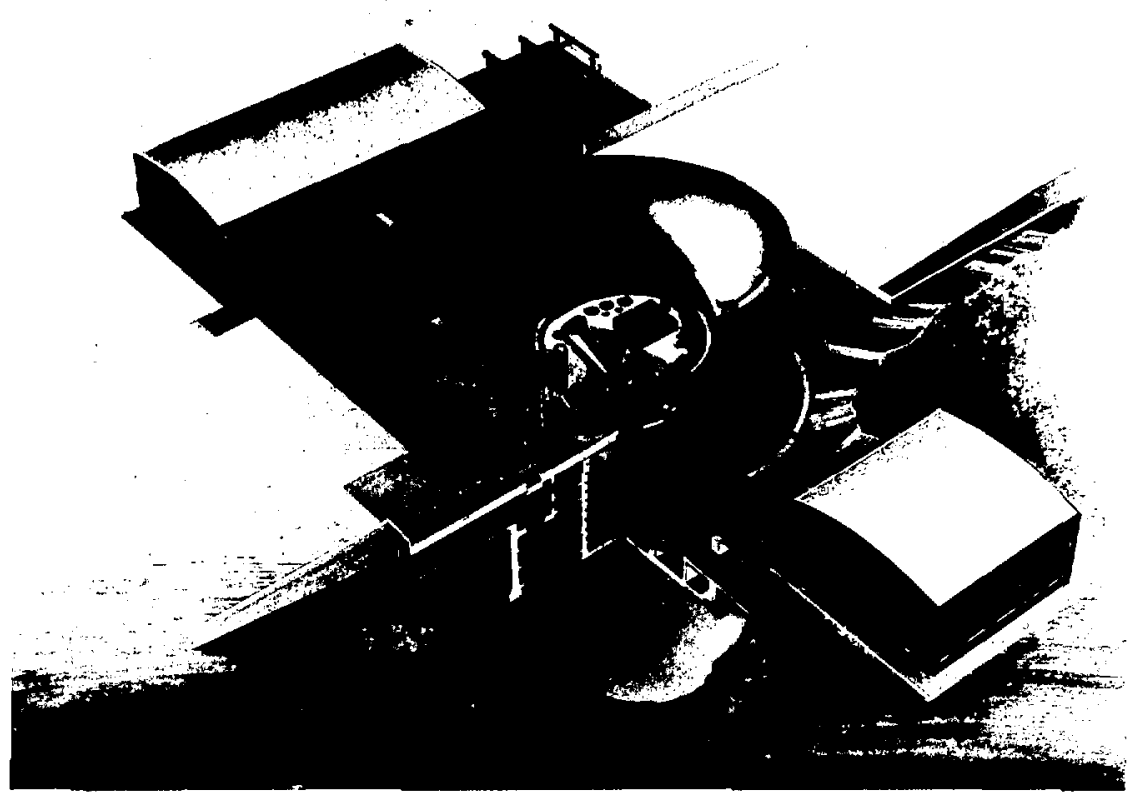

Fig. 10-1. Fusion-fission hybrid reactor - above-ground layout. 
unique geometry. It still will be necessary to renove each magnet as a unit for repair in the unlikely event of the magnet failure, so it seems prudent to plan for installation and emergency removal of a complete coil asseably.

We plan to include a magnet-fabrication building as part of the power plant, and locate that building in the best possible position for transport of the assembled coils to the reactor (or back for repair). The floor level of this factory should be flugh with the top of the PCRV and as close to the PCRV as construction of the secondary containment shell will permit.

For this purpose, it might be desirable to select a site that includes a natural hillside. Or, since a very large excavation is necessary for the botton end tank, it might be economical to build a mesa using the excavated material, relocated a short distance by a dragline.

Horizontal movenent of the magnet assembly can be on compressed air pads. Ten pads, each $5 \mathrm{~m}$ in diameter, can float the magnet with relative ease on two parallel smooth tracks from the magnet assembly floor to a position directly above its final position. Then a group of about 30 200-ton building jacks, operating on steel masts, can lower the magnet into place.

In order to install the bottom magnet easily it will be positioned prior to construction of the PCRV. The top magnet will be installed after the PCRV and the spherical blanket are finished. If the top coil must return for repair, the building-jack system run in reverse will lift it to the top of the PCRV. From there it can be air-floated back to the fabrication building. It will be necessary to cut a repairable hole in the containment shell. Failure of the lower magnet is not so easily rectified. The end tank must first be emptied and building-jack shafts installed, to perait the magnet to be lowered into the end tank, where repair must take place, working from a temporary floor or scaffold. Should rewinding be necessary, the coil would have to be translated into the end tank service room. Building-jacks would again be employed to move it up through the vacuun-pump service area and back up to mesa-top level outside the containment building. Provisions can be made in designing that portion of the bullding to minimize the expense of "clearing a path" for the botton magnet. Fortunately, the probability is very swall that major rebuilding of a magnet would ever be necessary. 


\subsection{PCRV Considerations}

The prestressed concrete reactor vessel is discussed in Sec. 8.1. A polar gantry crane, resting on a track in the top of the PCRV, serves to change-out thermal loop components and supports the blanket-module changing tool during part of its operational cycle. Figure 10-2 shows the sequence of events for removing or adding a module.

Since one magnet will be in final position before the PCRV is built, great care must be taken to protect that magnet from accidents related to construction of its environment. Metal shrouds, which may form a permanent part of the PCRV, should be used as protective fenders.

\subsection{End Tank Considerations}

The flux fan from the Yin-Yang magnet pair has an elliptical cross section. The aspect ratio of the ellipse is high (order of $10: 1$ ) and this implies a wide, thin, nearly flat-sided vacuum vessel to surround the end tank hardware. Huge vaci!nm loads on a flat self-supporting side wall demand massive structure, add great cost, and consume valuable space. Instead, we propose to use a steel menbrane anchored to the reinforced concrete wall of the building (which follows the elliptical contour of the flux-bundle). The circular arc bottom of the end tank will be similarly anchored. One large port at the bottom end nearest the direct convertor service bay provides entrance or egress for components being changed on a routine or emergency basis. A track inside the vacuum system transports components from any position in the direct convertor arc to that port.

Use of the end tank as a magnet repair region was discussed in Sec. 10.2 .

The top flux fan deposits about 108 of the escaping ions on a cooled target plate. The center of that energy dump is removable for insertion of the blanket-change tool.

Vacuum cryopumping panels will be located primarily in the bottom end tank. A bank of diffusion pumps, on both sides of the tank, pump on individual cryopump cavities which can be periodically closed, allowed to warm up, and degassed by the diffusion pumps. Some pumping will be similarly done in the top end tank. 

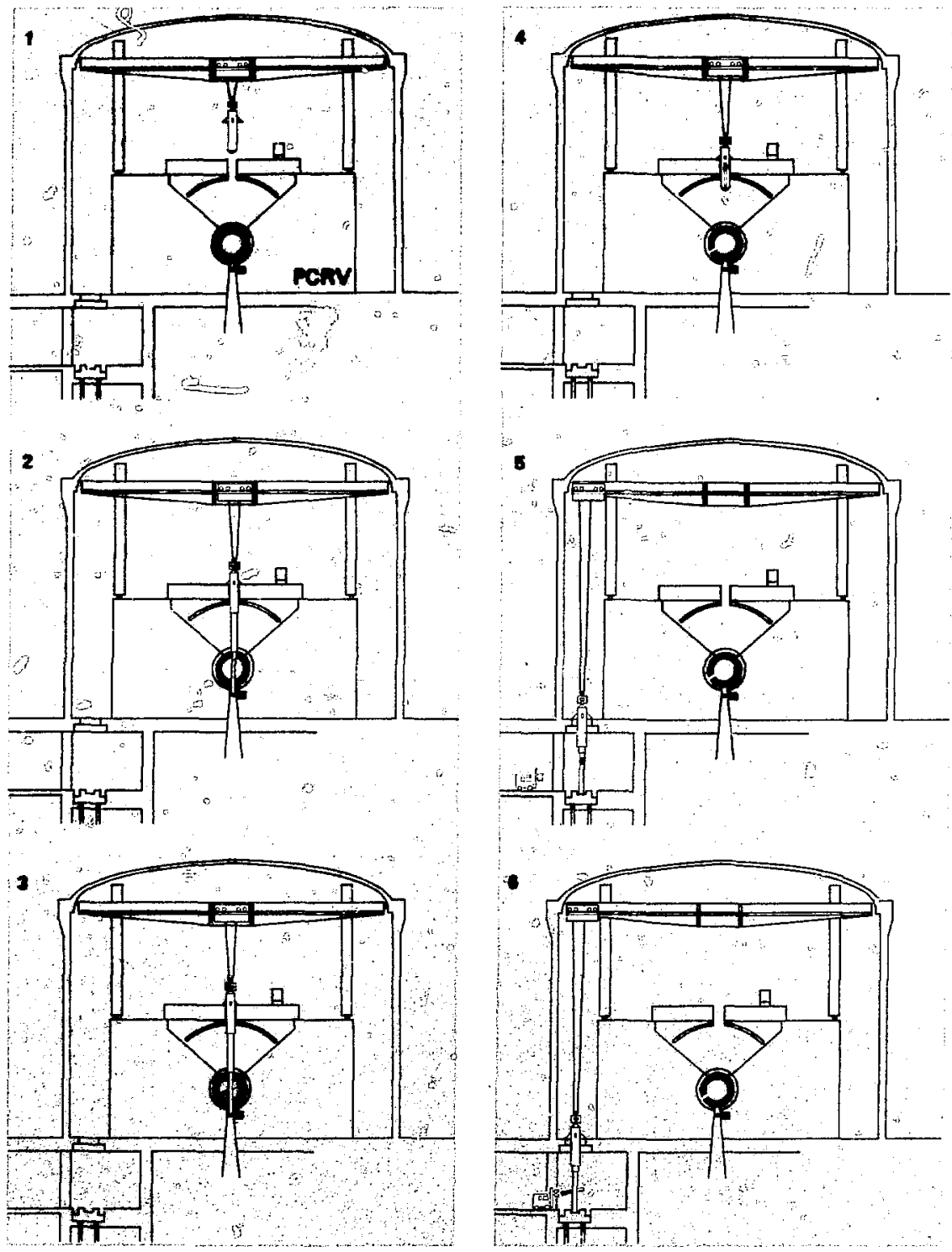

Fig. 10-2. Blanket service sequence. 


\subsection{Containment Consideration}

If a blanket module should fail structurally, the first line of defense is the vacuum system wall. This includes the very large end tank. Circulation of primary coolant under quickly reduced pressure will continue. The failed module will release a small fraction of the total coolant flow and the vacuum system pressure will approach one atmosphers. If several modules fail, and the pressure inside the vacuum system exceeds 1 atmosphere over ambient, safety burst dlaphragms will vent helium into the space inside the secondary containment shell. The volume of free space inside the containment shell is $250,000 \mathrm{~m}^{3}$. This volume is sufficient to limit the pressure rise to less than 2 atmospheres if all steam and helium were catastrophically vented from all cooling and power conversion loops. The underground structure and its relationship to the PCRV eliminates the need for any equipment openings in the containment shell. The construction method called slip-forming can be used to erect the cylindrical side wall. A fabrication sequence relating the PCRV, the 3000-tonne magnets, and the containment building is suggested below:

1. Complete the underground portion of the reactor building.

2. Install ti:e lower mirror coil.

3. Construct the PCRV.

4. Slip form the containment wall to the same elevation as the top of the PCRV.

5. Instail the upper mirror coil in the PCRV cavity.

6. Install the polar gantry crane on top of the PCRV.

7. Slip form the remainder of the circular wall to the spring line.

8. Use the gantry crane as a movable work platform to construct forms for a domed reinforced-concrete roof.

9. Pour the roof using several 100-m mast cranes. 


\section{POWER CONVERSION LOOP CONTROL SYSTEM}

\subsection{Introduction}

This chapter describes MHR plant control systems. These systems ensure that the plant can be safely operated and, in the event of an abnormal or accident condition, can be shut down in an orderly manner. The control systems consist of automatically and manually initiated systems for ensuring public health and safety under accident conditions and regulating systems used for normal plant operation. Two distinct systems are involved: (1) the plant protection system (PPS); and (2) the plant control system (PCS).

The PPS prevents an unacceptable release of radioactivity, which would constitute a hazard to the health and safety of the public. The PPS initiates action to protect the fission product barriers and limit the release of radioactivity if failures occur in the barriers. Plant availability and equipment protection are outside the scope of the PPS, and are primarily in the scope of the PCS.

The PCS is designed to regulate the plant in all normal modes of operation. It also handles conditions imposed on the system during loop trip, reactor trip, or electrical load rejection.

The MHR has certain characteristic features that affect the regulating system required. These features are:

1. Circulator turbines located in the superheated steam lines. Superheated steam flows from each steam generator through the circulator turbine and then is returned to the steam generator to be resuperheated before flowing with steam from the other loops to the main steam turbine. Steam density at the exit of the sirculator turbine varies inversely as the square root of circulator power, a characteristic used in controlling the circulator throttle valve.

2. The $\log$ mean temperature difference between the steam and helium in counter-crossflow - is relatively small. Thus, the primary and secondary systems are closely coupled in performance. 
3. To avoid large changes in the reactor outlet and steam temperatures, the feedwater and helium flow rate must be adjusted in direct proportion to the reactor power.

4. The helium loops associated with the end tanks and direct converter are separate from those that cool the blanket and can be operated essentially independently. This is especially useful during reactor startup.

\subsection{Plant Control System (PCS)}

The plant control system consists of the on-load plant control system, the operational residual-heat-removal (RHR) control system, and the startup control systera. The on-load plant control system automatically regulates and controls plant electrical output in accordance with load demand throughout the normal range of plant operation. The operational RHR system controls the secondary coolant system during the transition from normal operztion to long-term decay-heat-removal operation. The startup system performs the control necessary to bring the plant to the normal operating condition.

\subsubsection{On-Load Plant Control System}

The on-load plant control system provides automatic regulation and control of the electric power produced and delivered by the plant in accordance with load demand.

The on-load plant control system operates to fulfill the following conditions:

- Maintain the correct blanket outlet gas temperature (TBO) for the reactor power.

- Maintain an approximately constant steam temperature upstream of the main turbine throttle valve over the entire normal load range.

- Maintain the proper first-stage main turbine steam pressure (PKr) required by the load demand.

- Maintain an approximately constant circulator turbine exhaust density over the entire normal load range by controlling the circulator turbine outlet pressure in each loop.

- Maintain the proper feedwater flow rate to each steam generator as required by the load demand. 
- Maintain the correct feedwater temperature at the exit of the high-pressure heater by controlling bleed stream from the power turbines in conjunction with injector waste-heat-exchanger performance.

As a consequence of the fourth control condition given above, the ratio of helium-to-steam flow is approximately constant over the operating load range. Since steam flow is proportional to load demand, it follows that the heliun temperature $r$ ise through the blanket is approximately

constant, independent of load. The helfum temperature level will, however, vary slightly as necessary to establish the differences required to transfer the heat from the blanket to the helium and from the helium to the steam generators.

The on-load plant control syst- receives information on system behavior from instruments that measure a set of process variables. Then, by comparison with the proper set points of these quantities (which are functions of the actual load deriand), the control system generates the feedback signals for regulation.

The proposed control system, illustrated in Fig. 11-1, consists of controllers whose functions and input signals are described in the following paragraphs.

Blanket Outlet Temperature Controller (CI in Fig. 11-1). The neutron flux reaching the blankets is measured by the flux detectors $F$ and compared with the flux-level set point determined by the gas outiet temperature TBO. Controller $\mathrm{Cl}$ then varies the neutral-beam injection demand. This will control the flux level such that TBO will have the value correct for the plant load demand.

Feedwater Flow Controller (C2 in Fig. 11-1). The feedwater controller regulates feedwater flow so as to maintain steam pressure at the superheater outlet, PS, constant over the load range.

Main Turbine Throttle Valve Controlles (C3 in Fig. 11-1). The first-stage turbine pressure PMT is measured and compared to the pressure required by the load demand. If it is not correct, $c 3$ causes appropriate throttle valve action.

Circulator Turbine Flow Controller (C4 in Fig. 11-1). Each circulator is controlled by a controller whose function is to set the circulator turbine throttle valve so as to give the appropriate flow of 


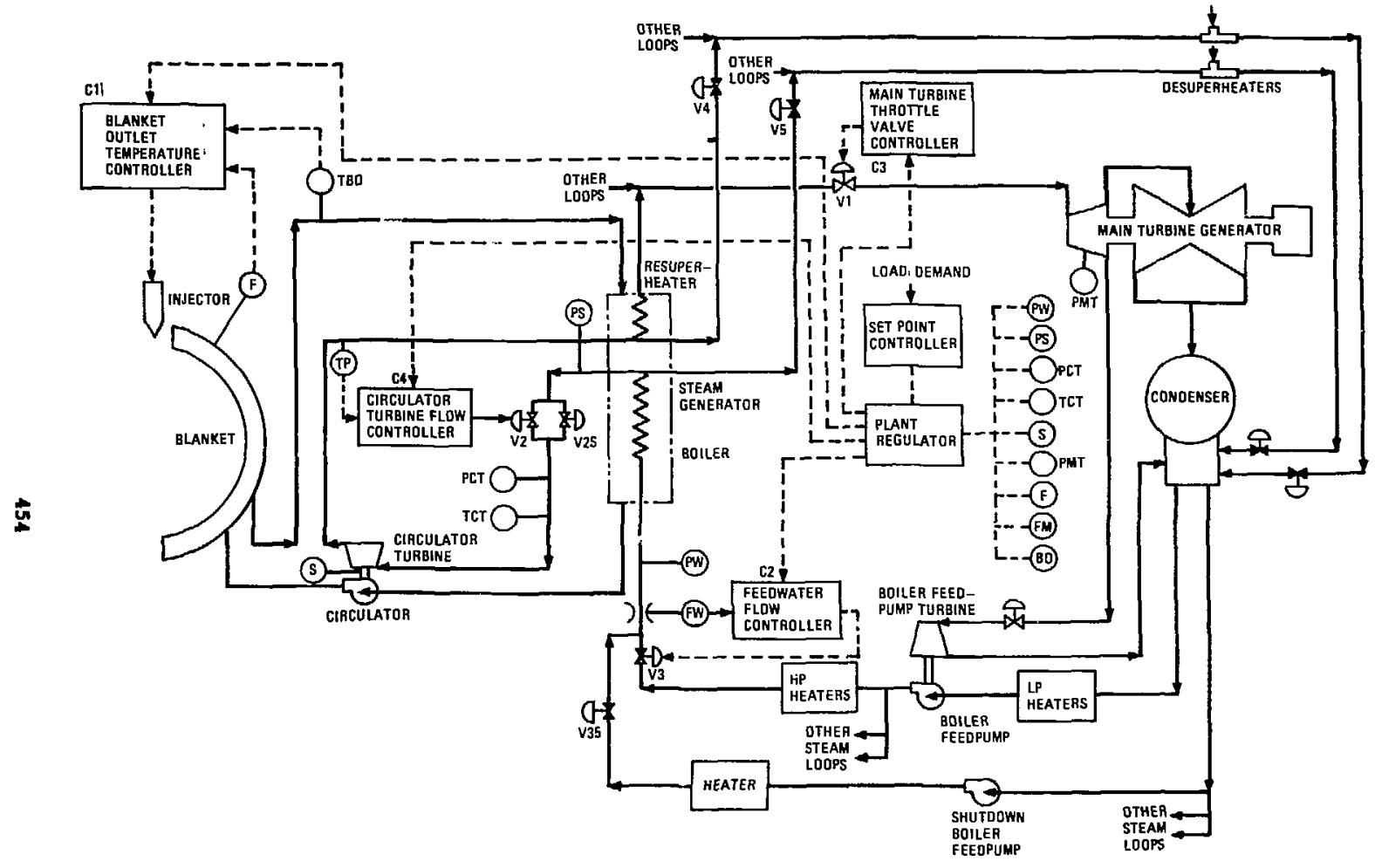

Fig. 11-1. On-load plant control system. 
helium through that loop. Analysis shows that heliun flow is proportional to steam flow if the circulator turbine exhaust density is held constant. Therefore, $\mathrm{C4}$ acts to maintain circulator exhaust density constant by controlling the turbine flow to a set point appropriate to the load demand.

Since stean generator pressure PS is maintained constant by $\mathrm{C2}$, the exhaust pressure of the circulator turbine would tend to increase with decreasing plant load if $\mathrm{C4}$ did not act to close the circulator turbine throttle valve appropriately. The actions of $\mathrm{C} 4$ and of $\mathrm{C} 3$ with decreasing load are to close the respective throttles so as to maintain PT and PMr at the proper values for the load demand.

An essential consequence of this method of control is that helium temperature rise across the reactor remains approximately constant over the load range.

The following example shows how the control system behaves. Suppose a load-demand signal is given to the main throttle controller c3 to reduce the load. It will close the main throttle valve and compare PHP to the required part-load set-point pressure. With the main turbine throttle valve partially closed, pressure at PT will increase but the circulator-turbine throttle-controller C4 will sense this and close down on the turbine throttle valve, thus increasing pressure PS. The feedwater controller C2, sensing a higher PS, will close down on the feelwater control valve, tending to increase the pump head. However, because of pump characteristics, the flow will decrease, which is actually what is reguired by the main turbine for the desired part load. With the smaller flow, the pressures PS, PWr, and PT will stabilize at the values required for the new load conditions. As the stean flow through the sirculator turbine decreases and the new values of PS and PT are established, the circulator speed decreases to establish the new helium flow required. The helium temperature rise will remais unchanged, but the blanket helium-outlet-temperature (TBO) set point will already have been decreased by a load-demand signal, thus lowering the heliun temperature level. Temperatures through the steam generator will tend to remain approximately unchanged.

\subsubsection{Operational Residual-Hent-Removal Coutrol System}

11.2.2.1 Plant shutdown Control system. The primary functional requirement of the plant shutdown control syster is to automatically provide adequate blanket cooling during the transition from on-load plant control to 
decay heat removal control following a reactor trip. The system operates in all normal and emergency shutdown situations, including coolant-system depressurization events. The control system will be designed so that the thermal transients experienced by the plant are kept to a minimum.

For reactor shutdown generated by a reactor trip signal, the following actions are initiated:

1. The neutral-beam injector power is removed, causing the plasma conditions to be less than necessary to sustain fusion.

2. Startup of the auxiliary steam supply gystem is initiated in preparation for transition to the decay-heat-removal control mode.

3. The main turbines are tripped.

4. Simultaneously with the main turbine trip, the resuperheater bypass circuits are activated and controlled by signals from the plant shutdown control system.

5. The circulator turbine large control valve (V2) is closed as the neutron flux decreases.

6. The circulator turbine small control valve (initially completely open) is controlled by signals from the plant shutdown control system.

7. The shutdown boiler feedpumps are activated approximately 1 min after reactor trip. This time is not critical because the water/steam inventory in each steam generator is ample to drive the circulator turbine for a much longer period.

8. Auxiliary cooling system operation is activated as a backup to coling by the main cooling loops.

9. The emergency diesel generator start-up sequence is initiated to provide a backup power supply.

During the power reduction from the normal power level to the decay-heat-removal-level without reactor trip, the plant shutdown control system performs actions 4 and 6 .

The plant shutdown control system, which is shown in Fig. 11-2, consists of two control loops for each reactor main coolant loop. The first control loop adjusts the circulator-turbine steam flow by varying the area 


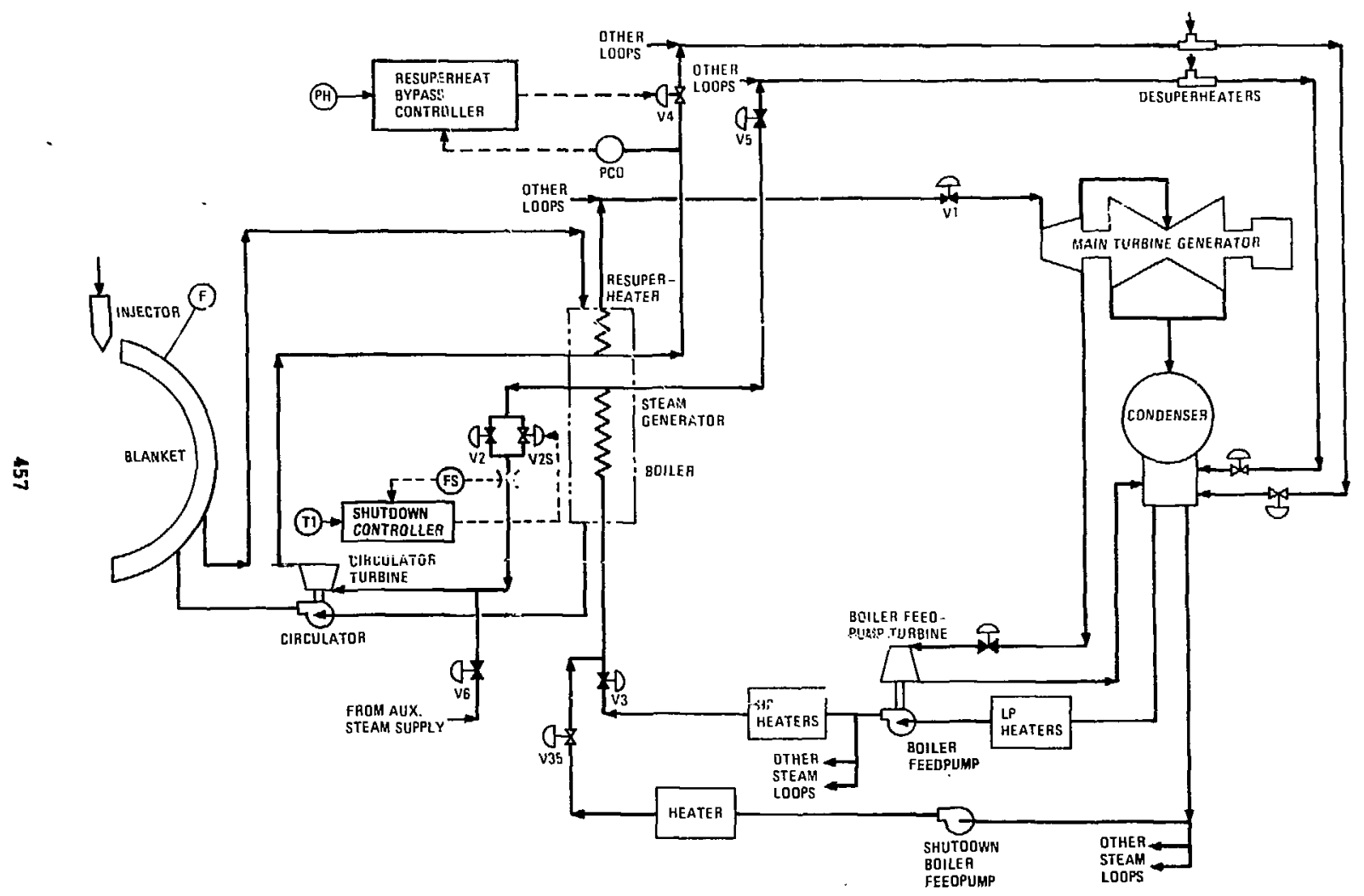

Fig. 11-2. Shutdown plant control system. 
of the circulator-turbine small control valve (V2S). The second control loop maintains the proper ratio between the circulator turbine exhaust steam pressure $(P C O)$ and the reactor inlet coolant pressure (PH) by adjusting the area of the resuperheater bypass circuit control valve (V4) until the ratio of pressures $\mathrm{PCO}$ and $\mathrm{PH}$ is at its set point. The latter control action adjusts the circulator power, and therefore the helium flow, in accordance with the reactor coolant pressure during a reactor coolant depressurization accident. This mode of control continues until orderly transition to the decay-heat-removal control system can be made.

For normal reactor shutdown, a programmed load reduction to a preset level is initiated using the on-load plant control system. Startup of the auxiliary steam supply system is initiated at the onset of the programed load reduction. Reactor power is held at reduced level until the auxiliary steam supply system is available. Plant load is then reduced to less than 5 and the turbine is tripped. The resuperheater bypass circuits are activated during this time to control circulator turbine exhaust steam pressure. Resuperheater bypass steam is used to drive the feedpump turbines. Secondary system steam and water flows are reduced consistent with reduced blanket power. Once the power has been reduced to a sufficiently low level, the shutdown boiler feedpumps are activated and orderly transition to the decay-heat-removal control system commences.

11.2.2.2 Decay-Heat-Removal Control System. The primary functional requirement of the decay-heat-removal control system is to provide adequate cooling for an indefinite period of time following transition from the plant shutdown control mode.

As the reactor shutdown proceeds with time, the quantity and quality of the nuclear-produced steam will become inadequate to drive the helium circulator turbines. The auxiliary boilers will have been started when reactor trip was initiated and will be automatically brought to operating conditions during the initial shutdown phase. Approximately 30 min after reactor 1. "y, the decay-heat-removal control system, shown in Fig. 11-3, is initiated by:

- Activating the steam-generator alternate discharge circuit in each coolant loop by opening valve v5. 


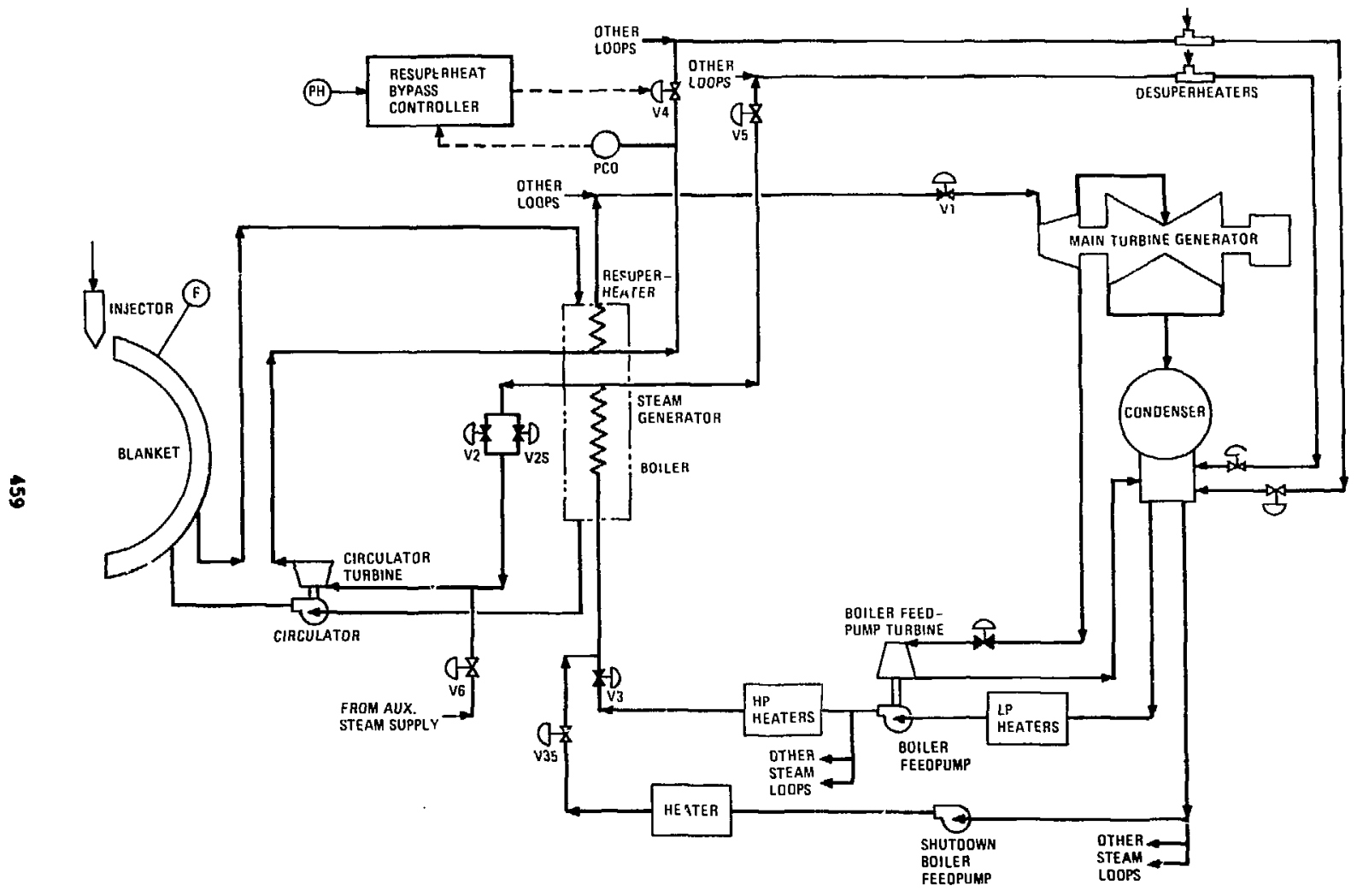

Fig. 11-3. Decay-heat removal control 3ystem. 
- Providing steam from the auxiliary steam supply system to drive the helium circulator turbines of each coolant loop by opening valve v6 and closing v2S.

This mode of operation, with each steam generator supplied by a separate shutdown boiler feedpump and each blanket quadrant supplied by a separate auxiliary boiler, can continue indefinitely to maintain heliu circulation and remove decay heat.

\subsubsection{Start-up Confrol System}

Three startup conditions are possible. These are initial startup of the plant, restart of the main steam generators and circulators from shutdown operation with the main loops, and restart of the plant from shutdow operation with the auxiliary cooling system (ACS).

For plant initiol startup, offsite power is used to cool down and energize the Yin-Yang magnets, pump down the vacuum chamber and condition the plasma system for operation. The auxiliary boilers are then utilized to provide the initial steam. Water is circulated through the steam generators using the shutdown boiler feedpunps with the blanket coolant system pressurized. The feedwater cleanup phase is then initiated with hot water until steady-state temperatures are achieved and the ion concentration is acceptable. At this point the resuperheaters and circulator turbines are drained and the bypass lines around them are opened. The shutdown boiler feedpumps are restarted, thus permitting feedwater to flow through the stean generator to the desuperheater and main condenser. Steam frsh the auxiliary boilers is fed to the circulator turbines but bypasses the resuperheater as in the shutdown mode of operation. Steam passes through the circulator turbines, starting coolant circulation, and then initially through the desuperheater and subsequently the resuperheater to the main condenser. The blankets are thus warmed up by the primary coolant transfer of heat from the steam generators. During this period, the conditioning of the fusion process is completed and the overall plant becomes ready for powered operation. With blanket coolant flow now established, the neutral-beam injector current is initiated using deuterivm only. The beam current is 
increased gradually and the direct converter and the end tank thermal power conversion loop are started. Initiation of power generation with the end tank thermal conversion loops follows the same procedure descrihed below for the blanket cooling loops. The neutral beam injectors require substantial power, and electricity generated by the direct converter and end tank loops allows offsite or auxiliary power supply requirements to be reduced. To initiate blanket power operation, tritium is jradually introduced to the injectors, which allows fusion power to begin being generated. The tritiur injection rate is gradually increased to produce reactor power and supply heat to the blanket loop steam generators. By regulating the bypass valves and the steam flows to the circulator turbines, the desired superheated steam conditions at the outlet of the steam generator are obtained. At this point, the steam supply for the circulator turbines is gradually transferred from the auxiliary boilers to the main stean generators. When the transfer is complete, steam flo:i is limited to about 10 of normal power by the capacity of the shutdown boiler feedpumps.

To increase the steam flow rate further requires operation of the steam-driven main-boiler feedpumps. This is accomplished by taking stean from the flash tank to drive the main boiler feedpump turbines. The main turbine gens fator can then be started and synchronized and a suall load applied. The turbine load is then increased to a minimum level. Once the main turbine is operating, extraction steam can be used to drive the main boiler feedpumps and the shutdown boiler feedpumps can be stopped. In addition, the ion injectors are switched from partial offsite or auxiliary power to ali on the main generator. The plant is new self-sustained and can be brought to full load.

Restart from decay-heat-removal operation with the main $100 \mathrm{ps}$ is quite simple, since the plasma systom is still fully conditioned for operation and the auxiliary boilers and main circulators are zlready in operation. In the decay-hea:-removal mode of operation, the resuperheater is bypassed and the stean senerator acts only as a heat sink for the system. To restart from this condition, the injectors are energized using off-site or auxiliary power to produce power in the blanket, the resuperheaters are put back into 
operation, and the auxillary boiler steam flow and bypass valves are regulated to give the desired superheated steam conditions at the steam generator outlets. This steam can then be directed gradually to the main circulator turbines and the auxiliary boiler steam decreased until the circulator bypass lines are closed and the plant is operating on its own steam. The plant is then brought to higher power in an identical procedure as described above for the initial startup.

Restart from sutdown operation with the auxiliary core cooling syster. is very much like the initial startup except that plasma system conditioning and the feedwater cleanup phases will be omitted unless specifically required due to the nature of the shutdown. Feedwater will be supplied by the shutdown boiler feedpumps to the steam generators and bypassed around the circulator turbines to the main condenser, as described earlier. Auxiliary boiler steam will be fed to the circulatcr turbines and the circulators will be brought up to the speed required to open the main-loop isolation valves. The auxiliary loops will then be shut down. The procedure for switching from auxiliary steam to main steam and obtaining higher power will be identical to that described above.

\subsubsection{Supplementary Comtrol Functions}

In addition to handling the normal control functions, the PCs handles conditions imposed on the system during loop trip and electrical load ejection. Since there are two generators and only one is required to keep the plant itself in operation, the pCs monitors the generator output voltage and will keep the plant in operation as long as one generator is producing power.

\subsection{Plant Protection System (PPS)}

The protection system automatically initiates appropriate action to protect the health and safety of the public during and after plant transients that wight cause a release of radioactivity from the blankets. various critical plant parameters are monf tored throughout their operating range by the protection system. Should any of these go beyond the safety limit specified by the technical specifications for the plant, the 
protection system automatically actuates raactor trip. Certain of these critical parameters are also used to initiate the plant's engineered safety features.

The reactor plant protection system includes all electrical and mechanical devices and circuitry that actuate reactor trip, and, in the event of a serious reactor accident, actuate the engineered safety features. 11.3.1 Reactor Trip System

If the protection system receives a signal that a monitored paranete. has exceeded its safety limit, the system actuates a reactor trip.

The basic reactor operating philosophy is to define an allowable range of power and to maintain the coolant flow proportional to the reactor power throughout the operating region. The allowable power range and power-to-flow ratio define the primary tripping functions. Adaitional tripping functions are provided as backup for specified abnormal conditions.

11.3.1.1 Protective Actions. Rapid reactor shutdown is provided by shutting down the neutral beam injectors. Interrupting the power to the injectors promptly causes the plasma conaitions to be less than necessary to sustain fusion. Thus, the fusion process rapidly ceases and, after the delayed neutron precursors in the blanket decay, the heat produced in the blanket is solely caused by the decay-heat. The shutdown of the plant following a reactor trip is discussed in section 11.2.2.

Flant parameters that cause reactor trip are listed in Table $11-1$ and are described below.

Manual Reactor Trip. Manual reactor trip is provided to give the operator a means to rapidly shut down the reactor.

High Neutron FIux. High neutron flux indicates excessive heat generation, requiring a reactor trip to prevent excessive blanket temperatures.

Bigh Power-to-Flow Ratio. A high ratio of neutron flux to helium flow rate through the blankets indicates an excessive reactor power level for the anount of cooling provided and requires a reactor trip to prevent excessive blanket temperatures.

High Reactor-Coolant Moisture. A signal of high blanket-coolant moisture, due to the leakage of a significant anount of water into the blanket coolant, causes the reactor to trip. Blanket coolant moisture monitors also provide siçarals for loop isolation and stean-generator dump. 
Table 11-1. Reactor-trip parameters.

\begin{tabular}{|c|c|c|}
\hline Sensed variable & Indication & Type of ingut \\
\hline Manual & - & Hand switches \\
\hline Neutron flux level & High & Linear power nuclear channels \\
\hline Power-to-flow ratio & High & $\begin{array}{l}\text { Linear power nuclear channels } \\
\text { and flow measurement }\end{array}$ \\
\hline Blanket coolant moisture & High & Woisture monitor \\
\hline $\begin{array}{l}\text { Blanket coolant delayed } \\
\text { neutron activity }\end{array}$ & High & Delayed neutron monitors \\
\hline Blanket coolant pressure & High & Pressure transmitters \\
\hline Blanket coolant pressure & Low & Pressure transmitters \\
\hline Containment pressure & High & Pressure transmitters \\
\hline Main-steam-line pressure & Low & Pressure transmitters \\
\hline Main feedwater pressure & Low & Pressure transmitters \\
\hline Plant electrical system & Power loss & Undervoltage relays \\
\hline Main loop trouble & - & Shutdown logic \\
\hline
\end{tabular}

High Delayed-Neutron Activity. A signal of high delayed-neutron activity in the primary coolant is an indication of significant fuel danage and causes the reactor to trip.

High Blanket-Coolant Pressure. High reactor-coolant pressure indicates significant water leaking into the blanket coolant system. The high blanket-coolant pressure protection constitutes a diverse backup for the high-moisture trip.

Iow Blanket-Coolant Pressure. Low blanket-coolant pressure is the primary indication of significant helium leakage from the system. A reactor trip is required when the rate of heat generation in the blankets cannot be accommodated by the reduced cooling capacity resulting from lower pressure. The safety linit may be programed with plant load to reduce the response time when the plant is at high power.

Bigh Containment Pressure. High containment pressure indicates reactor-coolant leakage or steam-line rupture within the containmant trip. The high-containment-pressure reactor trip acts as a diverse backup to the low-blanket-coolant-pressure trip. 
Low Main Steam Pressure. Low main-line steam pressure is an indication of main steam-line rupture (outside the containment). Depending on the system design, shutdown of the reactor could be appropriate in such a situation. The safety limit is below the main steam-line pressure level during normal operation and anticipated transients.

Low Main-Feedwater Pressure Low main-feedwater pressure indicates loss of both main feedpumps or a feedwater pipe rupture. In either situation, the appropriate action is reactor trip.

Blectrical-System Power Loss. Plant electrical-system power loss requires that the reactor be shut down because of the energy imbalance between the blanket and the generator.

Main-Loop Trouble. A reactor trip is required to rapidly reduce power upon receipt of a main-loop trouble signal. Blanket cooling with the renaining main loop will continue.

\subsubsection{Engineered Safeguards Actuation}

The engineered safeguards systems are actuated by the engineered safeguards actuation channels. Actuation of the engineered safety function for energency cooling with the auxiliary loops is provided only when concurrent signals of loop trouble and low reactor-coolant pressure are received by the protection system. Fast transfer to auxiliary loops is then performed automatically.

Automatic containment isolation is provided and the conteinment cleanup system initiated when either of the following signals is received by the protection system: (1) high containment pressure, or (2) high containment radioactivity. 


\section{SAFETY}

\subsection{Safety Design}

\subsubsection{Blanket Safety Evaluation}

Two aspects of the blanket safety during accident conditions were evaluated. They are the fuel heat-up rate in the event of loss of coolant flow and the forces on the fuel generated by rapid depressurization of the blanket.

12.1.1.1 Blanket Rod Heat-up Rate. Time required to cause fuel. melting with loss of cooling following reactor shutdown is an important parameter for safety analysis. In the MrR blanket, the melting point of the fuel $\left(\mathrm{U}_{3} \mathrm{Si}\right)$ is $930^{\circ} \mathrm{C}$. This is much smaller than the melting point of the clad. Heat-up rate of the fuel for complete loss of cooling (adiabatic heat-up rate) can be obtained by the following equation:

$$
\frac{d \theta}{d t}=q^{\prime \prime \prime}(t) / \rho c_{P},
$$

where

$$
\begin{aligned}
& \theta=\text { temperature of fuel, }{ }^{\circ} \mathrm{c} \\
& t=t i m e, s \\
& \dot{q}^{\prime \prime \prime}(t)=\text { decay heat, } w / \mathrm{cm}^{3} \\
& \rho c_{p}=\text { volumetric specific heat of the fuel, } w \cdot s / \mathrm{cm}^{3} \cdot{ }^{\circ} \mathrm{C} \text {. }
\end{aligned}
$$

For the MHR blanket analysis, it will be assumed that the standard fission-product decay curve ${ }^{l}$ represents the power after shutdown. Figure 12-1 shows this relation. Figure 12-1 represents the heat-up rate as a function of time for $\mathrm{U}_{3} \mathrm{Si}$ fuel at the peak power portion of the blanket rod. Increase in temperature of the fuel after time $t$, is given by:

$$
\Delta \theta=\int_{0}^{t} \frac{d \theta}{d \tau} d \tau
$$




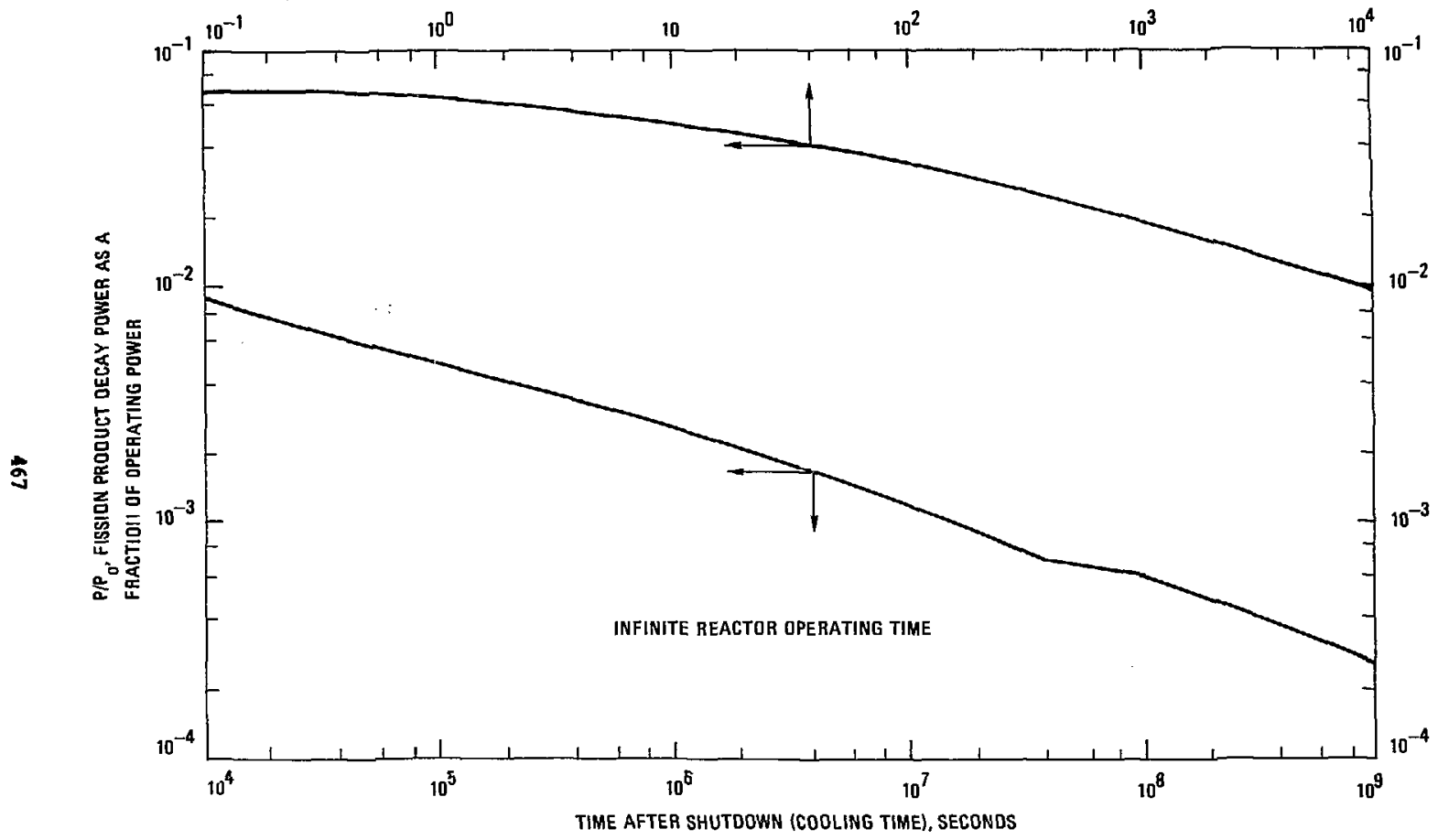

Fig. 12.1. Standard fission-product decay-heat curve. 
This relation is also plotted in Fig. 12-2 for $\dot{q}$ '' during normal operation of $=500 \mathrm{w} / \mathrm{cm}^{3}, c_{p}=0.05 \mathrm{w} \cdot \mathrm{h} / \mathrm{kg} \cdot{ }^{\circ} \mathrm{C}$ and $\rho=15.5 \mathrm{~g} / \mathrm{cm}^{3}$.

The nominal peak temperature of the MHR blanket fuel will be $663^{\circ} \mathrm{C}$ during normal operation. Hence, isc time avallable before fuel reaches the melting temperature following $108 s$ of cooling with reactor shutdown is about 24 seconds. The time available 48 hours after shutdown (refueling conditions) is 360 seconds as shown on Fig. 12-3. The time available before fuel damage occurs in the case of loss of cooling at full power, assuming adiabatic heat-up, is only 1.5 seconds as shown on Fig. 12-4.

These heat-up time estimates are based on the assumption of totally adiabatic heating and are quite conservative. In fact, coolant flow cosst down, natural convection within the modules, and conduction of heat to the water-cooled module support structure are expected to extend the time available before fuel damage occurs even in the event of complete loss of coolant flow with depressurization. Further, the auxiliary cooling system has been designed to assure maintenance of adequate cooling even in the event of loss of the main circulators with helium depressurization. Nevertheless, the blanket cooling requirements will impose stringent demands upon the response tine characteristics and reliability of the auxiliary cooling system. Further, assurance of adequate cooling during module replacement will be required, which implies the need for reliable and redundant cooling capability to be built into the fuel handling equipment. Although it is expected that these concerns can be adequately addressed, more detailed work in this area is needed.

12.1.1.2 Forces During Depressurization. A depressurization accident caused by first-wall rupture can result in large pressure differences across the blanket rod bundle. In order to reduce the resulting force, venturis have been provided in the exit manifold of the blanket. These venturis will limit the velocity of flow through the blanket and the resulting forces on the blanket during depressurization caused by first wall rupture. Since "faulted condition" design limits were used, damage to the fuel support grid is expected but a coolable geometry will be maintained.

\subsubsection{Plant Sufety Amalysis}

\subsubsection{Introduction}

Objective and scope of Safety Analysis. The objective of this Bection is to analyze the preliminary design of the 4400-Wwt Mirror Hybrid Reactor so that the adequacy of the proposed design to control expected 


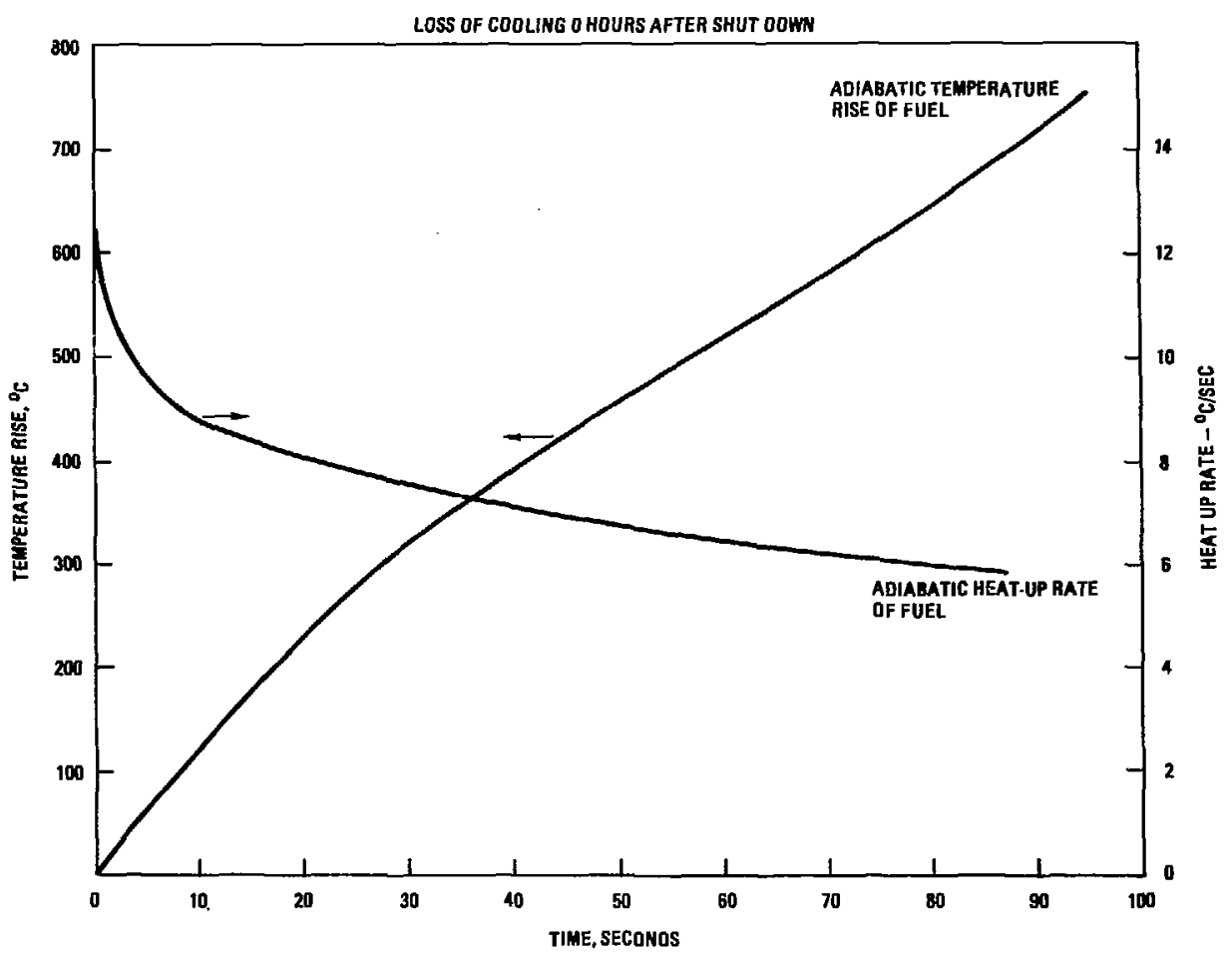

Pig. 12-2. Inss of cooling 0 hours after shutorom. 


$$
4
$$




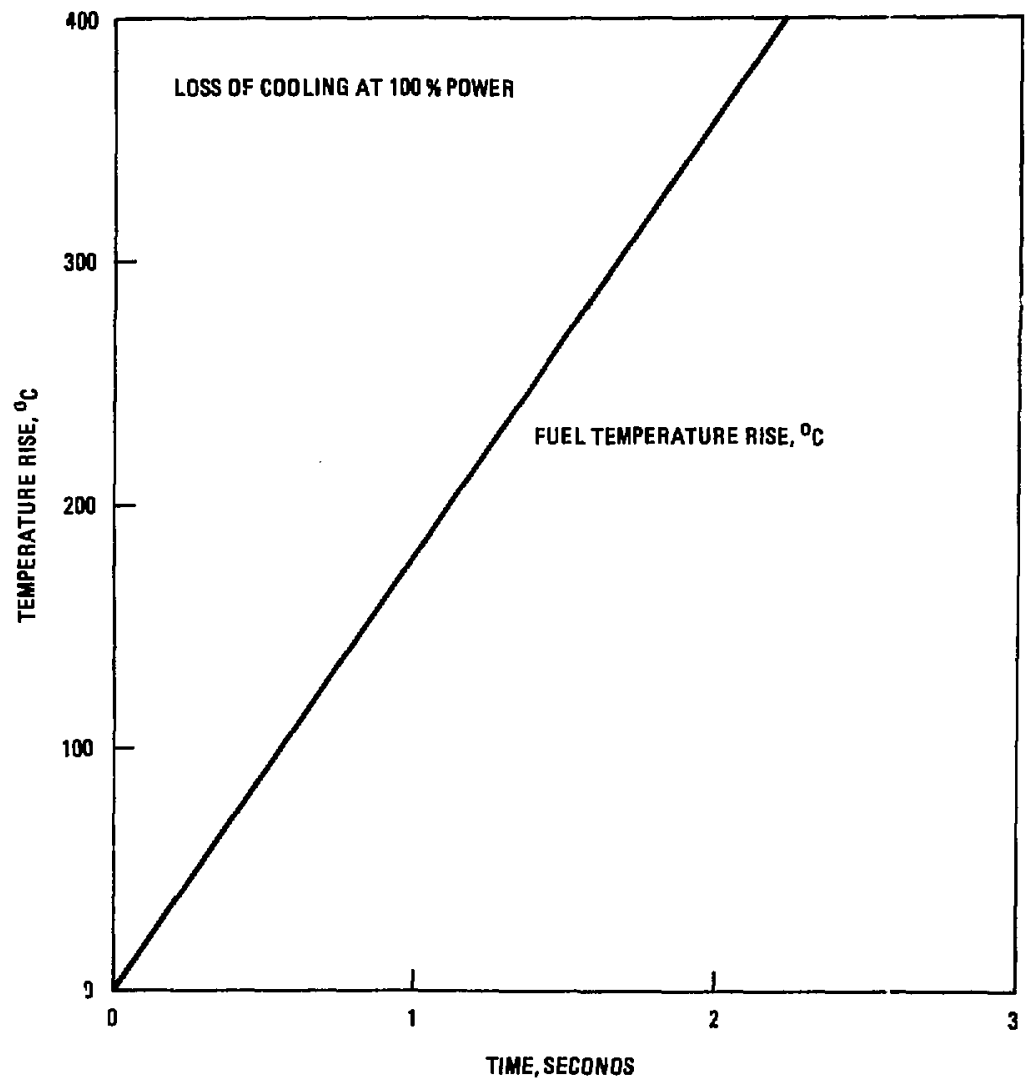

Fig. 12-4. Loss of cooling at 100 power. 
transients and prevent or mitigate accidents can be evaluated. Safety analyses of those systems which do not directly involve the blanket and power conversion system are not within the scope of this study.

This safety analysis is focused on the more severe expected plant transients and on the design-basis accident. An important objective of the safety analysis is the choice of a postulated accident for use in establishing the design bases for the engineered safeti features emergency cooling and containment atnosphere cleanup - and for the containment system. After examination of potential accidents, the design-basis accident chosen is the postulated gross failure of the helium ducting which causes rapid depressurization of the blanket coolant into the containment building. The design-basis accident is similar to that being used for the Gas-Cooled Fast Breede: Reactor (GCFR) because the blanket coolant systems and the fast-fission fuel, which have a predominant influence on safety, are essentially similar. 2,3

In the analysis, potential equipment failures and combinations of failures are considered that have a significant probability of occurrence. Systems and components with adequate redundancy are assumed to maintain their functional capabilities. As an important example, adequate reliability of the plant protection system and the neutral beam injector systems to perform reactor shutdown when required is assumed throughout this section.

Outline of Safety Analysis. In line with the objectives stated above, the following subjects are treated in this safety analysis section. Plant shutdown cooling using forced convection of helium in normal and emergency conditions other than the design-basis accident is treated in sec. 12.2.2.2.

Potential reactivity accidents are considered and seismically induced reactivity insertions are discussed in Sec. 12.1.2.3.

The development of local flow blockage accidents and their potential propagation are treated in Sec. 12.1.2.4.

Secondary coolant leakage into the reactor coolant system, due to steam generator failures, or into the containment building due to a pipe cupture, is analyzed in Sec. 12.1.2.5. 
Preliminary safety criteria have been proposed fnr fuel handling techniques. Fuel handling accidents are no: of primary importance in this evaluation of plant safety and, therefore, are only briefly discussed in Sec. 12.1 .2 .6 .

Blanket cool.ant leakage is discussed in Sec. 12.1.2.7, and the potential failure that would result in a most rapid primaiy-system depressurization is discussed.

PCRV failure is discussed in Sec. 12.1.2.8.

In Sec. 12.1.2.9, the design-basis depressurization accident (DBA) is defized and the primary-system response, containment building response, and radiological effects are evaluated.

Finally, in Sec. 12.1.2.10, conclusions and recomnendations for future efforts are given.

\subsubsection{2 plant Shutdown Cooling}

General. Assured shutdown cooling using forced circulation of helium in all normal and emergency situations is a fundamental safety requirement cf the plant. Therefore, much attention has been given to means for providing this function with sufficient redundancy and reliability. The shutdown-cooling capabilities of the design presented in this report are discussed here.

Shutdown cooling in the MrR consists of two phases: an initial period of power reduction, either in a programmed manner or rapidly through a reactor trip, followed by a long-term decay-heat-removal phase.

Normal shutdown of the MHR plant is described in $\mathrm{Ch}$. 11; reactor trip by the plant protection system is described below. Reactor trip results in rapid reactor power reduction to the blanket decay-heat level through the trip of the neutral beam injectors together with a trip of the turbine generator. The associated temperature transients are potentially the most severe that a plant would be expected to experience during normal operations.

During the decay-heat-remoral phase, the shutdown cooling of the blanket can be accomplished with either the main cooling loops or the auxiliary cooling loops. The parts of both cooling-loop systems necessary to perform emergency cooling in the MHR plant are designed to provide this function during transients and postulated accidents, including design basis earthquakes, the loss of offsite power, and sing.le equipment failures. 
Plant Operations and Performance after Reactor Trip from Full Load. The various signals that can initiate reactor trip are given in Table 11-1. upon receipt of a trip signal, the plant protection system initiates reactor trip. Power to all the neutral beam injectors is interrupted, causing the plasma to rapidly fall below fusion-producing conditions. Concurrently, a trip signal is sent to the turbine.

Closure of the turbine stop valve causes the control system to change from the normal turbine control mode to the shutdown control mode. Turbine trip causes the opening of the bypass valves around the resuperheaters and the opening of the loop feedwater discharge lines to the desuperheater. It aiso causes the programmed closure of th: Alrculator turbine throttle valves. Loss of extraction steam will result in tripping of the main boiler feedpumps shortly after turbine trip unless a separate desuperheater path from the steam generators to the feedpumps is provided.

The reactor trip signal also initiates the startup sequence of the auxiliary boilers anci associated equipment, the auxiliary circulators and associated equipment, and the emergency diesel generators.

The plant design includes many features that contribute to the reliability of cooling after reactor trip. Each main circulator continues to use steam directly from its associated steam generators. Also stean from any steam generator in any quadrant can be routed to the circulator $z$ in any other quadrant to insure forced convection cooling of the blanket. Separate electrically driven shutdown boiler feedpumps are started to supply feedwater to each steam generator. Each shutdown boiler feedpump is supplied from an irdependent shutdown feedwater header. The normal shutdown feedwater supply from the deaerator is backed up by an emergency water supply. Puim-NPSH-tanks connected in each shutdown feedwater header ensure the NPSH (net positive suction head) of the boiler feedwater during a changeover from normal to emergency water supply. The shutdown boiler feedpungs are assumed ready to supply feedwater shortly after reactor trip. However, the water inventory in the steam generators is ample to cover a considerable delay in pump startup or an interruption, such as that required to change from normal to emergency porer supply to the pumps.

To ensure an independent, unincerzupted supply to the main circulator bearings, the bearing fluid pump for each circulator may be iriven by a steam turbine using steam produced in its associated steam generators. The 
drive steam would be extracted at the outlet of the steam generators before the circulator turbine throttle valve so that it would undergo essentially no transient during a reactor trip.

The system is designed to operate without a significant transient in case of loss of nonse ismic equipment, e.g., due to an earthg sake. In case of loss of all nonseismic equipment, the steam from the circulator turbine would exhaust to the atmosphere sutside the containment building rather than to the condenser, and feedwater wuld be supplied from the emergency water supply.

The control actions required in each loop upon reactor trip have a backup wherever possible and will be made as independent of the other loops as possible, so that the most severe single equipment malfunction or failure could result in loss of only one loop.

During shutdown transients, the feedwater flow to the steam generators and the steam flow through the circulator turbines are controlled. This may be done as follows:

1. The feedwater flow is reduced to zero as the extraction steam supply for the main boiler feedpumps decreases. It remains at zero until 60 seconds and then is set constant at $\sim 2$ of full load flow for the remainder of the initial period, i.e., for more than 30 minutes. The feedwater flow should not be critical because of the large steam generator inventory; the flow could remain at zero for considerably longer than 1 minute and the set Vi-ue could depart substantially from 28 without important consequences.

2. The circulator-turbine steam flow rate is governed by two valves in parallel located upstream of the turbine. The large control valves are used for plant control during normal operations, whereas the smaller valves, which operate wide open euring normal operations, are used to control the steam flow to the circulator turbine after the reactor has been shut down. When the reastor is tripped, the large valves are closed rapidly (when a reactor trip signal and a confirmatory indication from the neutron-flux detectors that the reactor power has been reduced are received), and then the shutdown-control system manipulates the small valves to achieve the desired plant transient behavior.

Initially, after a reactor trip, the helium flow falls rapidly as a consequence of the rapid reduction in power to the circulator turbines with, of course, a rapid drop in circulator speed. 
The blanket cutlet helium temperature will probably owing down and up during the first 2 minutes over a small range but should not significantly exceed the initial full load value. The maximum cladding temperature will closely follow the blanket outlet temperature and will also probably remain well within acceptable limits. Gas inlet temperature to the steam generators is expected to vary less than blanket outlet temperature because of the gas volume in the ducting and the heat capacities of walls and other parts.

Thus, these estimates which are based on the GCFR system design indicate that this mode of shutdown cooling can maintain metal termeratures and their rates of change within acceptable limits. There is ample margin in steam conditions and supply to provide time for transfer to the decayheat-removal cooling mode.

Decay-heat Removal with Main Cooling Loops. Approximately one-half hour after reactor trip, the decay-heat-removal phase will be initiated. If the main cooling loops are used, steam from the auxiliary boilers will be introduced into each main circulator supply line ahead of the circulator turbine throttle. Then the resuperheater outlet isolation valves will be closed and the steam from the steam generators will be routed through a bypass line to a desuperheater and then to the main condenser.

Procedures for initiation of decay-heat removal with the main cooling loops are the same following any reactor trip. The procedures for an unplanned shutdown are described in Cl. 11. However, if the plant operator knows of the plant shutdown in advance and chooses to use the main loops in the decay-heat-removal phase, he or she could bring the auxiliary boilers up to the desired steam condition prior to plant shutdown.

Independent water supplies to the auxiliary boilers and to the main steam generators (which in this mode are the heat-removal exchangers) are provided. These supplies come normally from the condensate system. In case all nonseismic equipment is assumed to be inoperative, operation continues without feedwater supply, and circulator steam is exhausted to the atmosphere outside the containment until cooling is transferred to the auxiliary loops. If further analysis shows it to be necessary, a seismic class 1 feedwater supply system could be provided so that open-ended main-100p system operation could continue for an extended period. 
The auxiliary boilers and associated piping and equipment are independent, so that the loss of a piece of equipment will affect only one auxiliary boiler system. Each auxiliary boiler system is expected to be capable of providing for 1008 decay-heat removal. (The capacity of the a.xiliary boilers is actually based on plant startup requirements.)

Decay-heat Removal with Auxiliary Loops. Decay-heat removal can be provided by the auxiliary loops instead of the main loops. Normally, both loops will be used. However, the operator will not normally initiate the automatic switchover sequence that will make the transfer before 15 minutes after shutdown, at which time the decay heat being generated in the core can be removed using only one auxiliary loop. After the auxiliary blanketcooling system equipment startup sequence is completed, cooling is initiated with the auxiliary loops. The auxiliary circulators overpressure the main loops to force open the auxiliary-loop isolation valves and to close the main-loop isolation valves. These procedures are essentially the same, though the rate of transfer is different, as those when these loops are used for emergency blanket cooling.

12.1.2.3 Reactivity Insertion Accidents. Although the blankets in the MHR are designed to always be subcritical, there maybe events which could cause increases in reactivity. These potential causes must be considered: overheating in the blankets with associated fuel melting and the possibility of major changes in fuel geometry during a partial melt, earthquake or depressurization accident.

Fuel melting is the most critical of the reactivity insertion mechanisms. Any accident which leads to fuel melting - earthquakes, depressurization accidents, loss of forced cooling, flow blockage, etc. should be carefully analyzed. In the GCFR analysis, the Nuclear Regulatory Commission analyzed fuel melt accidents which resulted in reactivity insertion of $>\$ 100.00 / \mathrm{s}$ (Kef. 4). If such events are possible in the MHR, then the assumption that the blanket is always subcritical becomes open to question. A rapid increase in local multiplication could lead to potential safety problems even if the system remained subcritical.

Due to the low burnup and resulting low plutonium content of the blanket, it is expected that analysis will show criticality to be impossible under any circumstances. If this is the case, it is expected that reactivity insertion accidents will not pose any significant hazard to the 
MHR. Further, because the MHR blanket normally operates with $k_{\text {eff }} \approx 0.3$, even a large reactivity insertion should not result in any significant power increase.

Review of Reactivity Insertion Mechanism. Reactivity insertion accidents are characterized by reactor power increases caused by incidents that result in inadvertent augmentation of the blanket multiplication factor. Potential reactivity effects include: externally induced blanket element movements, changes of coolant density, introduction of steam into the blanket, thermal bowing effects, forced displacement of fuel elements, and fuel meiting.

Blanket movements induced by external forces represent a potential mechaniam for changing reactivity, particularly in a fast reactor. In general, such forces will excite vibratory fuel element motions. The effect of this motion could not be analyzed here.

Coolant-flow-induced fuei element vibrations are, at worst, expected to cause a small reactivity noise that is not a safety problem. Coolant pressure forces on the fuel support plate also will cause a small reactivity effect. Earthquakes are the most severe external forces considered in the blanket design, and seismically induced blanket vibratory motions could potentially cause a reactivity effect. Parameters that affect this phenomenon include the quality of the soil as well as the stiffness and the damping factor of the construction.

The only mechanism that can cause a sizable reduction of the coolant density is a depressurization of the reactor coolant system. Def.essurization from normal working pressure to atmospherif pzessure coula increase the core reactivity. This insertion is controlled by the rate of depressurization.

The introduction of steam into the core, which may result from a failure within a steam generator, influences the neutron energy spectrum of the core. The reactivity effect depends on the steam concentration and the state of the planket. Preliminary estimates indicate that at all times in the blanket life the effect of steam addition is negative and small and does not represent a safety problem. Other safety aspects of steam inleakage are discussed in Sec. 12.1.2.5. 
Thermal bowing of blanket elements due to temperature gradients ocross them will have reactivity effect that is expected to be very small. However, this is strongly dependent on the mechanical design of the blanket and the radial rod orientation should provide no mechanism for rod bowing.

\subsubsection{Local Flow-Blockage Accidents}

Characteristics of Local Flow-Blockage Accidents. Local flow-blockage accidents initially involve only a small portion of a blanket. Potential causes for such accidents are localized blockage within a module or flow reduction in an entire module or module assembly. Depending on the failure mode and time sequence and the response of the protection system, serious damage either may or may not be prevented and the failure may or may not propagate.

"Whole-blanket" accidents, initiated by overall loop flow reduction or reactivity insertions, are indicated by unanbiguous signals that are readily detectable and can be used for initiating protective actions; local flow-blockage accidents, in which only a restricted area of a module is involved, are more difficult to detect with sufficient assurance to make a decision for reactor shutdown. However, when the signal indicating damage is weak, a fast protective action is presumably not required, whereas a large signal from sizable damage means that fast protective action is desirable.

The objective in detecting a local blockage incident is to limit propagation of the damage. The sensitivity specifications for the protection systems can be defined with regard to an acceptable amounc of damage. This maximum tolerable blanket damage may involve more than one fuel rod or module but it must not be so great as to involve extensive loss of blanket-cooling geometry.

The occurrence of major coolant flow blockages caused by foreign matter is highly unlikely. The numerous parallel and rather tortuous coolant flow paths leading into each module would prevent large pieces of foreign matter from completely blocking a whole module. Additionally, it would be essentially impossible to totally block the annular flow entry area into each fuel rod bundle by any single piece of debris.

Analysis of locel flow-blockage accidents is hindered by the intrinsic difficulty that an actual sequence of events is unknown. There is a large range of potential local-flow-blockage-accident sequences. For example, 
consider the consequences of a local flow blockage within a fuel element. Although the deposition of material of unknown origin on a rod spacer grid would, most probably, not completely block the coolant flow around adjacent rods, the assumption of only a partial flow redictivin is ticticult to justify. Therefore, from a safety standpoint, a complete local loss of coolant to the fuel rods involved may have to be postulated. Similar degrees of conservatism may have to be Introduced when analyzing the propagation of a local fuel-rod fallure to neighboring rods and across the wall into neighboring modules.

A concern in a fuel melting accident is the possiblity of exothermic chemical reactions that could exacerbate the accident. The use of helium coolant precludes chemical reactions from that quarter. Chemical activity between $U_{3} S i$, $L i H$, and steel was investigated. Even in the molten state no chemical reactions are expected.

Partial Blockage Accidents. Partial fuel element flow blockage could potentially be caused by material being deposited on the fuel-rod spacers or support grid, or by fuel-rod swelling and thermal distortion. The consequent local undercooling could result in cladding failure and eventually in fuel-rod damage and relocation of $f, e l$ and cladding debris.

Preliminary analysis shows a relatively large mount of coolant flow reduction can be tolerated before the cladding fails. As an example, a flow reduction in excess of $70 \%$ is required in the GCFR plant in order to raise the maximum cladding temperature to the melting point. The $\mathrm{U}_{3} \mathrm{Si}$ fuel would melt but the cladding would retain the fuel rod geometry.

Mechanisms that could potentially lead to the propagation of failure from fuel rod to fuel rod include:

1. Relocation of fuel-rod debris in adjacent cooling channels and on spacers.

2. Flow reduction through the entire module as a result of the locally increased flow resistance.

3. Flow reduction through parts of module as a result of coolant bypassing through holes in the module walls as a result of a melt-through failure of the wall.

4. Reactivity changes of the entire system caused by blanket material relocation, which may result in a power increase and therefore in an acceleration of the failure development. 
Because of the complexity of the problem, a simplified model has been used to obtain lower-limit estimates of the periods of time involved for the propagation of failures from rod to rod. This model is based on the following assumption:

After the coolant channels around a particular fuel rod have been blocked, the heat transfer between the rod and its surroundings is interrupted (no convective or radiation heat transfer). Therefore, the satup of the rod is adiabatic.

The adiabatic heatup of the fuel rod to the relocation temperature can be represented by:

$$
\frac{\Delta T}{\Delta t}=\frac{Q}{c_{p}} \text {. }
$$

where $\Delta T=$ temperature increase to reach the relocation temperature.

$\Delta t=$ time to reach melting (s),

$c_{p}=$ mean volumetric specific heat of the fuel rod,

$Q=$ average full-power volumetric heat generation in the fuel.

From Eq. (3) the time to reach melting of the $U_{3} \mathrm{si}$ at full power amounts to

$$
\Delta:=\frac{\Delta \mathrm{TC}_{\mathrm{P}}}{\mathrm{Q}}=1.5 / \mathrm{s} .
$$

Cladding melting would begin in about 4.0 seconds at full power.

Propagation of the failure to other channels is difficult to assess at this time. However, for reference, GCFR propagation times (element to element) are on the order of 10-70 seconds. Propagation times are expected to be larger in the MHR blanket due to the large amount of structural material between modules.

It should be noted that because of the loss of structural material, there is less neutron scattering in the melted region. Thus the neutron spectrum is hardened, and, depending on the change in neutron leakage, there may be a local reactivity insertion. This means the multiplication factor is large and heat generation rates increase. This in turn promotes more 
melting. Prompt detection of melting and reactor trip can limit the extent of fuel melting. During a localized fuel-melt transient it is expected that the blanket will remain far subcritical.

Detection of Local Flow-blockage Accidents. Rapid detection of local failures is desirable in order to initiate protective actions that will limit the damage and reduce the contamination of the primary circuit.

According to the present design, the following signals will indicate he cccurrence of failures:

- Blanket module coolant outlet termerature

- Blanket-coolant-activity

- Power level

Whereas the coolant outlet temperature is a signal for an alarm, the coolant activity and the power level are signals that will cause a reactor trip after having exceeded preset operation limits. In the subsequent paragraphs, the response times of these signals axe discussed with respect to the propagation time of local failures. A value of 15 seconds is used as a representative number for the minimun propagation time of a local failure to an adjacent module.

a. The coolant outlet temperature of each 12-module assembly is monitored. This temperature would increase from the beginning of the accident, and, assuming a thermocouple response time of 1 to 2 seconds unambiguous signals might be expected after 2 to 3 seconds in the case of a major flow blockage accident, and thus before propagation of damage to adjacent module. In any circumstance, the thermocouples are intended as operational system devices and will not be included as part of the safety related plant protection system.

b. In cases of cladding failures the fission gases would be transmitted by convective flow into the helium coolant. Instrumentation lines monitoring the activity will pick up increased activity or delayed neutrons. Estimates indicate that the system response time is less than 5 seconds for detection of clad melting. This would allow shutdown of the reactor before the dange could propagate to adjacent modules.

To detect the potential for fuel overheating, the power level (neutron flux level) in the blanket can be monitored. Due to the geometry of the blankets an accurate determination of local flux levels may be difficult. However, if this problem can be surmounted, the flux monitors should be able to detect an overpower problem in less than 20 seconds. 
12.1.2.5 Secondary-Coolant-System Leakage. Leakage of water or steam from the secondary-coolant system can occur within the helium 10op, outside of the helium loop but inside of the containment building, or outside of the containment building. Because of the positive pressure differential from steam to helium under all normal and accident conditions (except for the resuperheater - see Sec. 12.1.2.7), it is impossible for significant amounts of fission products to leak from the reactor coolant system into the secondary system. As such, failure of a water or steam line outside of the secondary containment is not considered to be a serious safety problem.

Discussions of secondary-coolant leakage are divided into 1) steam and water leakage into the blanket coolant system and 2) steam-line breaks within the containment building.

Leakage into the Reactor Coolant System. Steam in the reactor coolant system of a gas-cooled fast reactor with metal-clad fuel does not result in significant damage to the plant. No exothermic chemical reactions with reactor internals will occur. Any significant metal-water reaction requires temperatures much higher than that of the cladding. The circulators, steam generators, and auxiliary heat exchangers will be designed to operate satisfactorily with the higher coolant densities associated with steam-leak accidents. With contint ing steam or water addition to the blanket coolant system beyond what the helium purification system can remove, the system pressure will increase. The moisture detection system will detect the moisture in the leaking loop and the plant protection system will actuati reactor trip, isolate the loop, and dump the steam-generator inventory to the atmosphere.

If there is no operator or protective action to control the leak, the blanket-coolant-system pressure would continue to increase until the safety valves would open and a steam-helium mixture would be blown into the containment building. Possible leakage sources of water or steam into the reactor coolant system are the steam generators, the auxiliary heat exchangers and, if water bearings are used, the circulator bearing-water supply. The auxiliary heat exchanger has a lower pressure and smaller tube sizes than do the steam generators, and it has a much smaller coolant inventory. Consequently, the water or steam flows due to possible ruptures in the auxiliary heat exchang ar are less severe than those of the main steam 
generators. Potential leakage of circulator bearing water could be prevented by a water seal and two helium seals between the water hearing and the reactor cavity. ${ }^{4}$ The seal leakage would be collected in the helium-water drain and discharged into a high pressure separator tank external to the circulator. Even if both buffer-helium supplies are unavailable and the jet pump of the helium-water drain system does not function properly, it is estimated that the maximum leakage of bearing water into the blanket coolant system could be 1 imited to $1 \mathrm{~kg} / \mathrm{s}$. Since this leakage rate is considerably less than the maximum rate caused by a steam-generator failure, the leakage of circulator bearing water was not treated as a separate accident.

Steam or water leakage from the steam generator into the helium can be caused by a steam-generator-tube rupture in either the economizer, evaporator, or superheater sections, or a break in a lead-in tube or a lea -out tube. The leak rates from a steam generator failuze can be readily limited by orificing. It is a commonly used practice in both the HTGR and GCFR. The leak rate limits should be based on insuring that the helium coolant pressure relief valves do not open during a water ingress transient. Preliminary estimates indicate that ingress flows in excess of $50 \mathrm{~kg} / \mathrm{s}$ could be tolerated.

It is estimated that the largest leak of secondary coolant into the blanket coolant system is caused by a rupture of a superheater leadout tube. This is considered to be a credible occurrence and, although a double-ended offset failure is very unlikely, it is taken into account in the design of the plant. The first protective action is initiated by the plant protection system upon detection of moisture. At a certain moisture level, the plant is tripped, and the loop identified to have the leak shut down. The circulator turbine is tripped, which in turn causes the helium valve to close, the leaking steam generator valves are closed, and the water inventory is released to the atmosphere. This procedure need not be very rapid.

Loop moisture detection will be made highly reliable through the use of redundant sensors to minimize the possibility of faulty detection that could possibly lead to isolating and dumping the wrong loop. The automatic loop shutdown logic will prevent dumping more than one loop. If the loop dump 
fails to work or if the wrong loop is dumped, the reactor would still be shut down. As a backup, high blanket-coolant-system pressure will cause a programmed load reduction at an intermediate high pressure and the reactor will be tripped at a higher system pressure. Upon reactor trip, the feedwater flow will decrease rapidly, and subsequently the leak flow into the helium system would be reduced. Another signal that will cause reactor trip because of a secondary system leakage is the blanket-coolant high moisture signal.

The consequences of failure of the loop steam dump system to operate properly are not severe. As a consequence of the loop shutdown, the circulator of the leaking loop stops and the steam generator loses its heat load, since the isolation valve on the helium side closes. From then on, the steam leaking into the blanket coolant is produced by stored heat in the steam generator tubes and by flash boiling of saturated water. Analyses indicate that the amount of steam leaking into the reactor coolant system could be $>5000 \mathrm{~kg}$, which is sufficient to cause the relief valve to open to relieve the pressure. About 108 of the helium inventory could be released into the containment building. The consequences of wrong loop moisture detection are similar. The wrong loop would be shut down and dumped by the plant protection system, but a high-moisture signal to the protection system would cause a reactor trip that would stop the normal flow of feedwater to the leaking loop, thus limiting the amount of water leaking into the blanket coolant system. Enough wate:. would leak to cause the helium loop relief valve to open but only a small fraction of the reactor-coolant inventory would be released into the containment building. The analysis shows that a steam or water leak into the blanket-coolant system does not endanger the plant or any equipment. It is estimated that adequate time is available for the response of the plant protection system, with respect to the initiating event and the following transient.

In the calculations of the superheater lead-out tube leakage, a rupture directly at the tubesheet was assumed, such that the break creates an ideal nozzle without any friction. The redundancy of the moisture detection system assures a proper response of the ogerational protection system. The time necessary for the durp (25 seconds) and the linear decrease of the leak rate during dump used in the analysis are also considered conservative assumptions. Even in the case of the largest possible steam leak, a dump of 
the steam generator inventory within about 50 seconds would prevent the discharge of reactor coolant into the secondary containment. The actual delay time of the moisture detection system is expected to be between 10 and 12 seconds.

Secondary-Coolant Pipe Break within the Containment Building. A rupture of a secondary-colant pipe in the containment will cause the loss of one loop and a pressure $r$ ise in the containment atmospinere. The plant protection system will initiate an automatic loop shutdown and a programmed load reduction, but the leak will not damage any plant equipment and consequently poses no radiological danger to the environment.

Separate feedwater pipes penetrate the containment and supply tach steam generator with feedwater. The steam from the resuperheater outlet flows so the main turbine through the steam lines that penetrate the containment ind:vidually and are joined together in the turbine building.

Any major steam leak within the containment could be detected by acoustic detectors, by loop resuperheater steam pressure, or by circulator - speed signals in the affected loop. Low-main-feedwater-pressure and high-containment-pressure signals would actuate a reactor trip. A conservative assumption made in this study is that the full feedwater flow of the plant is discharged into the containment until the loop is isolated or until the boiler feedpumps are shut down. Actually, the feedwater flow would drop rapidly due to flash boiling of the feedwater (at the saturation pressure) in the line and in the high-pressure heater. The control systam reduces the feedwater flow to zero after reactor trip. Reactor trip is actuated by low-feedwater-pressure and by high-containment-pressure signals.

For the GCFR plant an analysis was based on an initial leak flow which was almost twice the total normal feedwater flow for the plant and about six times the normal flow through the ruptured pipe, which accounts also for the initial transient after the pipe rupture. ${ }^{3}$ The leak rate was decreased linearly to zero within 20 seconds, which is a very conservative assumption. A total of $25,000 \mathrm{lb}$ of feedwater with an enthalpy of $909 \mathrm{~kJ} / \mathrm{kg}$ is discharged into the containment, causing a pressure $r$ ise of about $13 \mathrm{kFd}$ and a temperature rise of $6^{\circ} \mathrm{C}$. Similar results rould be expected for the MRR .

A failure of a high-pressure steam pipe upstream of the circulator turbine will cause the largest possible steam leak into the containment. In this event, the steam-generator inventory is blown off rapidly and 
subsequently a large fraction of the plant's feedwater flow will be discharged through the steam generator and the ruptured pipe into the containment. Since the circulator will lose its power supply, it will slow down very rapidly, the helium loop isolation valve will close, and the steam generator will lose its heat load. Consequently, the feedwater will not evaporate in the steam generator but will leak into the containment at its inlet enthalpy.

At full power, the circulator curbine steam flow in the turbine nozzles would be almost choked out. This limits the largest leak of a single steam-line rupture downstream of the cirsula ur turbine in the containment. If a steam-line rupture occurs after the circulator turbine, the amount of steam dumped into the containment is smaller than if a rupture occurs upstrem of the turbine, yet the enthalpy of the steam may be higher.

In event of a steam-line break, a signal from loop acoustic sensors, a low loop-resuperheater-steam pressure signal or a circulator overspeed signal. could rapidly actuate the plant, protection system to isolate the affected loop and limit the steam leakage into the containment. High containment pressure provides an additional backup, causing reactor trip and containment isolation.

Detailed analysis of the pressure and temperature histories of the containinent after different steam-line ruptures could not be assessed here. However, preliminary analysis indicates that the pressure and temperature $r$ ises caused by any secondary-coolant leak are snieller than the design containment pressure and temperature, based on a depressirization accident.

12.1.2.6 Fuel-Handling Accidents. The design of the fuel-handling equipment ard fuel storage system will make the occurrence of a serious fuel-handling accident an unlikely event.

Continuous and uninterrupted cooling of irradiated blanket modules during fuel-handling operations is essential. For this reason, each fuel-handling machine or set of machines that handles an irradiated blanket nodule will have two independent cooling systeins operating while handling that module. (These systems can be in the form of direct air, helium, or water cooling.) When the module is transferred from one machine or set of machines to another, both independent cooling systems of the machine or set of machines receiving the module will be in operation and providing cooling 
before the operation of the cooling systems of the machine or set of machines delivering the module is terminated. At no time during the module handling cycle will cooling be impaired to the point that the handling motions could not be terminated and the module remain safety cooled.

Due to the conceptiol stage or the design for the MHR, adequate assessment of fuel handing accidents could not be made. The potential for dropping modules in the containment or during handling should be assessed in future studies. Also the potential for and consequences of cocling system failure or interruption should be determined.

12.1.2.7 Reactor-Coolant Leakage. The primary hazard associated with reactor-coolant leakage is the radiological exposure from the coolant-borne tritium and fission products that leak out with the coolant. The radiological aspects of this are discussed in Sec. 12.1.2.9.

The MHR blanket coolant system is similar to that of the GCFR in that it uses high-pressure helium. The only system extensions outside the PCRV are small-diameter sample, instrument, and purge lines. In addition to having a small diameter, these lines are provided with shutoff and backflow check valves wherever necessary to prevent significant coolant leakage in the event of a break. Consequently, the only significant areas of potential accidental blanket-coolant leakage are in the PCRV penetrations, these being closed by welded seals and held in place by redundant fastenings tied into the PCRV structure. The welded seals are backed up by flow-restricting devices. Cracks in the PCRV liner could allow leakage, but such cracks are unlikely because of the design of the liner. Further, the leakage would be much smaller and slcrer than that through a penetration failure because the flow path would be through the porous concrete.

As indicated above most blanket coolant leaks are slow. They would arise primarily from failures of small piping. The sizes of the small lines have not been fixed; however, they are expected to be on the same order as those used in the 330 wWe HTGR built for Public Service Conpany of Colorado at Fort St. Vrain. The study conducted for the Fort St. Vrain HTeR indicated that the largest credible leak would be due to a failure involving the helium-purification-system lines. In the GCFR and in the HTGR, the potential leak rates are small, i.e., $1.6 \mathrm{~kg} / \mathrm{s}$ (Refs. 5, 6, and 7). The MHR, with either a sealed or separately purged LiH tritium breeding zone, should have about the same size helium purification system and thus the same size potential leak. 
The flow rates can be reduced by orificing at the tap-off points in the main dists, by going to smaller diameter piping or by incorporating fast valving in the system to limit the leak after it occurs. In other words, there are several available methods to reduce the potential leak rates at any Jucation to acceptable levels.

It should also be noted that all the radioactivity-bearing portions of the helium purification system can be contained within the containment building. This implies there can be no direct release to the public in case of a failure anici, therefore, that some latitude in design approach is available.

The operating steam pressure of the resuperheater is less than the operating pressure of the secondary loop helium. Therefore, any resuperheater fai'ure results in fission products from the helium coolant entering the steam system. Once in the steam system, the fission products can be released to the public via the turbine gland seals or air ejectors.

This accident has a precedence in the HTGR reheater. ${ }^{7}$ Analysis has been shown that in the HTGR, a reheater failure does not cause undue risk to the public. The same should be true of a resuperheater sailure in the MHR.

A resuperheater failure can easily be detected by radioactivity monitors at the outlet lines of the resuperheater. Isolation valves could then stop the release of helium to the secondary system before significant radioactive contamination ocurs.

\subsubsection{PCRV Failure}

Incredibility of Gross PCRV Failure. The PCRV is a multicavity design, with prestressing accomplished by means of linear steel tendons in the axial direction and bands of external wire wrapping in the circuaferential direction. Since the PCRV is a highly redundant structural system with conventional steel reinforcenent as well as prestressing steel, the only failure modes that could lead to structural failure are those assoriated with multiple simultaneous independent failures. These types of faicure modes, because of the PCRV design and construction practices and the testing and monitoring provisions, have an extremely low probability of occurrence and are therefore not considered as design-basis events for the MHR.

Large amounts of prestressing force could be lost around the vessel before the remaining prestress force would not balance the reactor-coolant- 
system working pressure. For exanple, each circumferential prestressing band consists of multiple, individually anchored layers of wire winding, which are designed so that failure of a layer in each circumferential prestressing band would not cause loss of vessel integrity. The nature and amount of reserve strength available in a PCRV has been demonstrated by analysis and experimental development for the Fort St. Vrain vessel. The PCRV design and operation are discussed in Sec. 8.1.

Hot gas leaking through the liner would have little effect on the stress-strain properties of the FCRV steel members and reinforcing bars. These members are widely dispersed, and small reactor coolant helium leaks are cooled by the surrounding colder concrete and liner cooling tubes. Helium flows sufficiently large to cause general increases in concrete and steel-member temperatures could only occur in the event of a complete crack of the reactor cavity liner and adjacent concrete at above normal reactor working pressure. Such flow rates would quickly terminate due to depressurization of the reactor coolant system. Local temperature increases of steel members would be less than $50^{\circ} \mathrm{F}$, as shown for Fort st. Vrain experiments. Band-type circumferential prestressing is even further removed from the heating effects of gas leaks. In addition, the vertical tendons are contained in steel ducts and the circumferential bands are located in steel-lined troughs, which provide protection from direct gas impingenent.

Because of the design of the reactor system and the system of safety relief valves, the PCRV internal pressure is prevented from exceeding the maximum cavity pressure (MCP) during accident conditions. If a total circumferential central cavity liner crack were to ocur at MCP, the prestressed concrete and reinforcing steel in the PCRV would preclude loss of structural integrity.

Each major PCRV penetration contains a penetration closure that incorporates a flow restrictor. The flow restrictors are designed to withstand the static and dynamic forces occurring after a hypothetical penetration-closure seal failure. A complete penetration assembly is redundantly retained by the penetration liner and the penetration liner shear anchors.

Failure of PCRV Penetration Closures. Failure of the basic PCRV structure or the concrete plugs is not considered to be possible. The most 
severe postulated accident is failure of the primary holddown means for the major penetration closures and the resultant gross failure of the seal welds. The nost severe of these cases is discussed in Sec. 12.1.2.9. The PCRV is designed so that in the event of a hypothetical gross failure of the primary holddown means and welded closure seal of any large concentration closure, the closure is secured by a secondary structural means and the reactor-coolant flow through that penetration is limited to a maximum of $300 \mathrm{~cm}^{2}$, which is a current GCFR design value. This is accomplished by the inclusion of a seal-ring type of flow restrictor in each penetration closure that limits the free-flow area to less than $300 \mathrm{~cm}^{2}$.

Failure of a Hodule Pressure Shell. The complete blow-out of a blanket module would expose a depressurization flow area of about $160 \mathrm{~cm}^{2}$ for helium flow from the hot and cold helium ducts into the vacuum chamber. Although verifying design work has not been done, it is expected that the upper leakage fan beam stop and lower leakage fan dirfet converter could adequately contain the helium in the event of a module failure. Thus, primary coolant would not enter the secondary containment.

\subsubsection{Design-basis Accident}

Introduction. A preliminary consideration of the design-basis accident (DBA) for the MHR is discussed here. The DBA as defined here is initiated by a hypothetical sudden, complete failure of the largest PCRV closure seal so that the reactor coolant system is depressurized into the secondary containment.

The DBA is postulated for anaiysis of the engineered safeguards capability; however, the DBA is not considered credible because of the special design features incorporated in the PCRV closures, as noted earlier.

Sequence of Bvents of Design-basis Accident. The DBA is postulated to start with the nonmechanistic gross failure of the primary holddown toggles and seal weld of a cavity closure or of a steam-generator penetration closure. It is assumed that the reactor coolant depressurizes into the secondary containment through an opening of the size limited by the flow restrictor $\left(300 \mathrm{~cm}^{2}\right)$.

The plant protection system receives a signal of iow reactor-coolant pressure and initiates reactor trip.

Rising pressure in the containnent building caused by the leaking reactor coolant causes containment isolation and starts the containmentatwosphere cleanup system. 
In the early part of the accident (first few seconds), the control system and the plant respond in a manner similar to that in a pressurized trip. The feedwater flow from the main boiler feed pumps stops shortly after the turbine stop valve closes. Steam to drive the main circulators and circulator bearing-water pumps continues to be provided by the stecm generator of each loop. Low-capacity shutdown boiler feed pumps, each supplied with power from ar essential bus; are started 60 seconds after reactor trip, and feedwater flow to each steam generator is resumed. As the reactor-coolant pressure decays, the control system provides the required increase in circulator power to maintain adequate helium circulation.

Several seconds after the start of the accident, the reactor coolant system will have reached pressure equilibrium with the pressure in the containment building. After several more minutes, the reactor temperatures reach a quasi-steady-state decay-heat-removal condition. During this period, temperatures rise to maxima and then slowly decrease.

After the plant shutdown period, which lasts for approximately one-half hour, the decay-heat-removal phase is initiated in a manner similar to that following any reactor trip. The procedures are discussed in sec. 12.1.2.2.

During depressurization of the reactor collant system, the containment atmosphere increases in temperature and pressure. Because of the energy content of the reactor colant, the containment pressure will rise rapidly from atmospheric to a peak and then fall to the equilibrium backpressure (estimated to be within approximately 20 minutes). Concurrently, the containment temperatures increase and then decay to their initial values.

The source of activity available for release to the containment building is the blanket-coolant fission-product inventory, which is primarily due to fission products released from blanket elements that may be defective at the time of the DBA. The containment-atmosphere cleanup system will remove activity from the containment building and rapidly reduce the anount available for leakage to the environnent.

The analysis assunes that the steam generator loop in which the depressurization occurs is isolated at the beginning of the accident so that only the remaining loops are available for cooling. Autonatic loop shutdown of the loop with the closure failure is initiated. The remaining main loops provide circulation for core cooling through the shutdown cooling phase. Transfer to the decay-heat-removal phase is again similar to that following any reactor trip. 
Plant Performance Following Depressurization. The plant design includes many feitures that contribute to the overall reliability and the assurance of effective blanket cooling following a DBA. Either of the two independent main loop reactor cooling systems is capable of providing this shutdown cooling. Each reactor cooling system is designed to retain functional cooling capability during and after operating basis and safe shutdown earthquakes, the loss of off-site power, and single equipment failures.

Analysis shows that the MrR plant has the capability to safely cool the blanket after a blanket-coolant depressurization accident where no blanket cooling is performed with the loop in which the failure occurs. After this accident, the reactor can be safely cooled even after a failure that terminates the cooling capability of the remaining main steam generator locps (worst single failure). Upon receipt of a loop trouble signal caused by the total termination of cooling in one of the remaining three steam generator loops (a low-reactor-coolant-pressure signal has been previously received), blanket cooling can continue with the remaining two loops or, if required, the transfer of cooling to the auxiliary cooling loops can be started.

The design of the main cooling loops is such that the loss of electrical power does not have a major efiect on the use of these locps for shutdown cooling. The loss of off-site power might cause a delay in restoration of feedwater flow to the steam generators by the shutdown boiler feed pumps or an interruption following startup. However, analyses have show that the water inventory in the steam generators is ample to cover a considerable delay in the startup or interruption in operation of the shutdown boiler feed pumps.

The auxiliary cooling system is driven by electrical power, but it is designed to provide adequate performance with the loss of off-site power. The esergency generators and the emergency power system will be designed so that the failure of off-site power and the transfer to the emergency diesel generators will not cause critical temperatures to exceed specified limits. Both the main and the auxiliary cooling systems can provide adequate shutdown cooling after a safe shutdown earthquake. All equipnent in the auxiliary cooling system is class I and, therefore, the entire system renains available for operation after a DBN with a concurrent safe shutdown earthquake. 
The parts of the main cooling system that are not seismic class I or do not have class I capability are in the secondary coolant system. If all nonseismic equipment is lost, the normal steam system is not available to receive the stean exhausted by the circulator turbine; this steam is therefore exhausted to the atmosphere outside the containnent building. All steam piping in the containment is Class I. If the normal feedwater supply is not available because of the loss of the heat sink or because of a break in the nonseismic equipment of the feedwater system, the class I emergency water system will supply water.

The containment-atmosphere cleanup system is Class $I$. The systern contains multiple units to circulate and clean up the containment atmosphere after a DBA. Should any one unit fail to start or to perform properly, sufficient capacity is available in the other units. Each unit is powered from a separate essential power bus. Loss of off-site power at any time when this equiprent is required would delay the operation of the equipment for a short period (less than 2 minutes) to permit the transfer to emergency power. The containment-atmosphere cleanup system is an engineered safety feature and has a high priority among equipment that receives emergency power. To show margin in the design of the containment and the containmentatmosphere cleanup system, a hypothetical accident involving the release of fission products associated with a TID-14844 accident ${ }^{9}$ needs to be analyzed. Also the effects of hot helium jets on other systems in the containment need to be analyzed. These analyses could not be attumpted during this study.

Blanket-coolant-system Response. The blanket-coolant-system response during a depressurization accident dejends on the size of the leak that occurs. The response also depends on the location of the leak, because for some locations the flow associated with the leak tends to help the coolant, whereas for others the tendency is the opposite. Also, for a given size of leak area, the rate of depressurization is affected by leak location because of the temperature of the leaking gas and the resistances of the paths between the various system plenums and the leak. After depressurization is complete, the system reaches a quasi-steady-state condition in which reactor temperatures are determined by the decay-heat level, the coolant flow rate, and the reactor coolant inlet temperature. The coolant inlet temerature is directly related to the temperature of the feedwater entering the stean 
generators. Furthermore, the response of the system to accidents compounded by failure of system components is also of importance. In the present context, only the worst single failure-loss of the remaining main loop was considered.

In the depressurization accident studies, the reactor is tripped by a low-pressure signal when the helium pressure drops significantly below its normal operating value. The reactor trip signal also trips the main turbine and changes the system control from the normal operation mode to the shutdown mode. In the shutdown mode, the steam flow bypasses the resuperheater section of the steam generator and the control is of steam flow through each of the two circulator turbines and of feedwater flow to each steam generator. This control is initially identical to that following any reactor trip, which is described in Sec. 12.1.2.2. The feedwater flow in each 100 was ramped to zero as extraction steam decreases following turbine trip. It remains at zero until approximately 60 seconds and then is maintained at $2 t$ of full-load flow for the remainder of the transient. The circulator turbine steam $f l o w$ is reduced to a level compatible with the decay heat being generated.

The circulator turbine steam flow is reduced further as depressurization becomes complete, and held constant at a minimum value for about 10 minutes after which it is gradually reduced so as to maintain the reactor outlet temperature below $530^{\circ} \mathrm{C}$.

Preliminary analysis shows that the steam-generator inventory stays at a satisfactory level. During the transient, each circulator speed initially increases after the turbine trip, then falls rapidly to correspond to the blanket power after the reactor trip, and then accelerates and levels off as the coolant pressure comes to equilibrium and air enters the system. Steam conditions for the circulator turbines is estimated to remain satisfactory throughout the initial 30 mintues.

For arbitrary, but reasonable, control of circulator steam and feedwater flows, preliminary estimates show that during depressurization and approach to a quasi-steady-state decay-heat-removal condition, metal temperatures are well below damage limits.

Approximately one-half hour after the depressurization is sensed, the cooling system is transferred from the shutdown phase to the decay-heatremoval phase; the auxiliary boilers will supply steam to drive the 
circulators and the steam leaving the steam generatorg will be bypassed to the condenser. The latter phase of shutdown cooling and the decay-heatremoval phase will be very similar to that following any reactor trip, as discussed in sec. 12.1.2.2. The electrically powered auxiliary cooling loops remain in standby condition as a backup cooling system.

Containment-building Response. The results of the depressurization accident studies discussed above should be used to evaluate the pressure and temperature response within the containment building during a depressurization accident. The calculated helium temperature and leak rate into the containment would be used as input for a code such as CoNTEuPT which performs detailed mass and energy balances within the containment and accounts for energy removed from the containment atmosphere by heat transfer to internal structures (walls, floors, ducting, and equipment) 10

The pressure and temperature within the containment building during a design-basis accident could approach $200 \mathrm{kPa}$ and $150^{\circ} \mathrm{C}$. This implies the containment must be designed to accomodate these pressures and remain leaktight. Industrial experience indicated that in general the seismic and missile design requirement for the containment building automatically make it capable of withstanding this moderate peak pressure.

The large mass of the cortainment building will not be significantly affected by the transient temperatures in the containment. However, future analysis should consider the effect of these tempertures on fusion-related equipment in the containment, e.g., cryogenic flasks or superconducting magnets.

Radiological Evaluation. As has been shown in the light water reactor (LNR) industry, the mechanisms for clad-fuel interactions are not clearly understood. Because of this uncertainty in long term clad behavior, it is generally assumed for design purposes that 1 of the clad is defective. This is consistent with both LWR and GCFR practice.

Given the excellent retentive properties of $\mathrm{U}_{3} \mathrm{Si}$ fuel, the fission products which leak out from the 1 defective fuel will still result in substantial "plate-out" on the internal surfaces of the helium loop. This plateout is estimated to be $s 10$ "Ci/quadrant. During a depressurization accident, significant amounts of the plate-out will be lifted off and blown into the containment building. Also, the fission product release from the fuel will lead to a steady stace circulating radioactivity level in the 
helium coolant anounting to $10^{3} \mathrm{Ci} /$ quadrant. It is expected that the coolant tritium activity will be about the same level. It would have to be assumed that this will all be released in a depressurization accident. Because tritium is much less radiologically toxic than fission products, the tritium does not contribute significantly to the hazard potential of a depressurization accident.

The problems of having the circulating fission products routinely flow to the helium purification/tritium recovery systems and building up inventories in these systems snould be addressed in future studies. If necessary these problems can be reduced by continually venting the fuel to the rad-waste system in a manner similar to that used in GCFR's Pressure Equalization System (PES). 5

The circulating activity in the HIGR is $10^{2}$ times larger than that of the MKR. Analysis has shown that releasing these fission products to the containment building does not endanger public health. Other analysis has shown that, even if all the circulating activity in the HTGR were released directly to the atmosphere the off-site doses would be well within $10 \mathrm{CFR} 100$ guidelines. 11,12 Because the MHR circulating inventory is much less than the HTGR inventory, it can be assumed that the release of the circulating inventory alone is not a significant problem.

The effects of releasing the fission products in the fuel with defective clad or the release of plutonium due to fuel melting is of much greater significance. In Ref. 3, it was shown that the release of fission products from fuel with defective cladding during a depressurization accident resulted in a maximum off-site dose of less than 0.5 rem. A similar dose should result from an MHR depressurization accident. Under all circumstances this dose is acceptable under the regulations of $10 \mathrm{CFR} 100$.

In both the GCFR and MHR analysis, it is assumed the blanket cooling systems can keep blanket temperatures below the fuel melting point. Therefore, no plutonium is released to the containment. In the analysis of the GCFR, the NRC postulated that a portion of the core did melt during a hypothetical depressurization accident; $100 \mathrm{~kg}$ of plutonium in the form of an aerosol was released to the containment. The results of site doses were calculated to exceed $10^{3} \mathrm{rem}$. Consequently, in the MrR it is of paranount importance to Insure the adequate cooling of the blanket at all times so that blanket meltdown is not a credible event. 
Pcelininary assessment indicates that the continued cooling of the blanket can be provided with the same high reliability of the GCFR cooling system. This assures that the potential for plutonium release is adequately minimized. Given this fact, it appears that all accidents in the MHR will, at nost, have a consequence comparable to those experienced in an HTGR or GCFR for a similar accident. Therefore, the MHR can be designed is such a manner that the health and safety of the public are insured.

12.1.2.1C Safety Conclusions and Recommendations. A preliminary assessment of the MHR blanket and power conversion system indicates that an adequately safe design is technically feasible. However, considerably moze analysis than that described here will have to be performed if an optimized and readily licensable design is to be developed.

To point the direction toward a design that is optimum from a safety and economic point of view, there are several areas that should be studied:

1. The potential for and effects of reactivity insertions and fuel melting with the release of plutonium aerosols and the potential for criticality occurring during a major blanket meltdown should be evalue.ted. It is anticipated that studies will show there can never by a critlcailty accident in the wh despite large-scale fuel melting. This is based on the expectation that only low concentrations of plutonium will be present in the blanket.

2. Depressurization accident design criteria should be developed, e.g. hole size, flow limiter design/feasibility, and induced forces on reftical cormonents.

3. The effects of fission products and plate-out in the helium purification and tritium recovery system due to clad defects should be estimated. Inservice inspection and maintenance could be significantly affected. The cost/benefits of vented fuel should be considered.

4. The potential for and consequences of accidents during fuel handling should be properly assessed so that appropriate jesign changes can minimize any radiological risks.

5. During any major accident there is a possibility for the fusion-related equipment to interact with the blanket-related equipment synergistically to produce more severe accident. This potential problem should be investigated. 
6. Possible occurrence of flow maldistribution in the primary circuit following a failure or accident should be investigated. This could prevent adequate blanket cooling even after the plant shuts down. This problem is highly dependent on the blanket design and should be evaluated in depth. If found necessary, flow maldistribution could be adequately limited by suitably modifying inlet and outlet ducts to each module or by insuring that the degree of flow maldistribution generated due to a failure is limited to a small fraction of the blanket cooling flow.

7. The potential for and design requirements of using the vacuum chamber as an extension of the primary coolant boundary in the event of a module failure should be investigated. It is possible that this may reduce the safety requirements inposed upon the module design. The impact on the direct converter and beam stop must be determined, however.

\section{Acknowledgment}

The safety analysis and discussion presented here relies heavily upon the safety analysis done by $G$. Baccus of General Atomic as a part of the FY 76 Mirror Hybrid Reactor work. (K. R. Schultz et al., Conceptual Design of the Blanket and Power Conversion System for a Mirror Hybrid Fusion-Fission Reactor, General Atomic Co., Rept. GA-A14021, July 1976). 


\subsection{Safety and Environmental Effects}

\subsubsection{Introduction}

In the evaluation of safety and enviconmental effects of hybrid fusion-fission power systems, as well as in other advanced technologies, comparative assessment is necessary because absolute standards do not exist. This comparison can be made with government regulations for existing technologies, directly with other advanced energy system3, or with other technological systems with acceptable public riak.

As an example, it has been argued that because of government regulatory agencies, any system that will be permitted to be built will be safe (by currently acceptable standards). ${ }^{13}$ since existing government regulations may not apply to future technologies, comparisons with other advanced technologies or competitive technologies may be necessary. Holdren ${ }^{14}$ argues that appropriate yardsticks are necessary and that these yardsticks depend on the mission of the system under consideration. Application of this concept to the fusion-fission hybrid presented in this study requires that "in the fuel-production role, the comparison is with mining and enrichment or with pure-fission breeders". 14

Although such reasoning is attractive because it effectively focuses attention on some aspect of the fuel cycle, it may also be overly simplistic because a change in one aspect of the fuel cycle may have serious consequences in another, or may even have synergistic effects.

As proposed in this study, a fusion-fission hybrid may have the potential of supplying plutonium (Pu) for approximately five light water reactors (LWR's). The LWR fuel cycle, based on uranium ( ${ }^{235} \mathrm{U}$ ) fuel (without Pu recycle), can be thought of as having some "measure of risk," with respect to public health and safety. This measure of risk includes mining and enrichment, as well as the LwR power plant risk, such as that calculated in Ref. 15. The switch to an LWR based on the Pu fuel cycle will have a different measure of $r$ isk, because the individual $r i s k$ of each component may change, including the power plant itself. For example, fuel production in a fissile-producing hybrid may require more geographic isolation for insured public safety; yet this same consideration may lead to an increase in transportation risks via vulnerability to theft. 
Another argument for examination of the total fuel cycle is as follows. It may be that high-temperature gas-cooled reactors (HIGR's), based on the thorium-usanium ( $\mathrm{Th}-{ }^{233} \mathrm{U}$ ) fuel cycle, have a safecy and environmental advantage over LWR's. One may be willing to accept an increased $\mathbf{r}$ isk in fuel production for a fusion-fission hybrid-producing ${ }^{233} \mathrm{~J}$, if the effect on the total fuel cycle is to increase safety (or decrease the total $r$ isk of energy production).

Synerglstic effects may also be overlooked when examining only one aspect of the fuel cycle. The proposnd fusion-fission hybrid presented in this design can potentially supply 5 LFR's with fissile fuel, while a comparative liquid metal fast breeder reactor (IMFBR) may only produce fuel during its life for two or three other LMFBR's. An accident in a hybrid, which in itself does not constitute an insult to public health and safety, but effectively eliminates that plant, may also leave those five ImR's with a short fuel supply. If one accepts the notion that the absence of generating capacity is also a public health risk, ${ }^{16,17}$ then the risk due to the lack of hybrid-produced fuel may be appreciable.

Before closing this general discussion, two further points should be made. The first is that public acceptance of a new technology, with respect to perceived risk, will become increasingly difficult. The public, and hence government regulatory agencies, will require more stringent regulations, whether or not real or apparent irisks are present. 18,19 second, before an assessment of a particular energy system (total fuel cycle) can be made, its various components must be analyzed. The remainder of this section, then, deals with one component of an IWR fuel cycle: the possible production of fissile fuel in a fusion-fission hybrid device. In particular, emphasis will be placed on tritium, fission products, and actinide elements. In principle, questions relasing to first-wall aduced activity would be the same as those for a pure fusion device.

\subsubsection{Potemtial Hazards and Safety}

12.2.2.1 Introduction. The first step in addressing the potential for risk of most energy-producing systems is to determine the type and quantity of each hazardous material present in the system. Once this has been accomplished, questions regarding their potential for release can be addressed. In a pure fusion power reactor, potentially hazardous meterials have been identified as tritium, induced radioactive material (structure and 
corrosion products), and nonradioactive, but toxic, material that occurs in large quantities. 20,21 For pure fission power reactors, the potential hazards include the actinide elements, radioactive fission products, and radioactive corrosion products. 15 The relative importance of the actinides vs the fission products depends upon both the stage of the fuel cycle considered and the type of system (LFR or LMFBR). ${ }^{22}$

For a fusion-fission hybrid astem, it appears that initial consideration, at least, should be made for all of these. Such a judgment is based on several considerations. First, the hybrid design considered here is based on the deuterium-tritium-lithium fuel cycle (D-T-Li). Since tritium is limited to production in nature by cosmic ray bombardment, it must be bred in the blanket via absorption of neutrons produced by the D-T reaction. Second, these neutrons also induce radioactivity in the first wall via $(n, \gamma)$ and $(n, 2 n)$ reactions and cause fission and/or tronsmutation in the actinides (uranium, thorium and plutonium), which are also in the blanket.

The fusion-fission design presented here has several design goals. Three of these are: (1) to produce enough tritium to sustain the fusion reactions of D-T during the life of the plant; (2) to produce fissile fuel (plutoiniua) for several (about five) LWR's by neutron capture in ${ }^{238}{ }^{0}$; and (3) to produce at least enough energy (via primarily fast fission in ${ }^{238}$ ) to sustain the plant.

12.2.2.2 Tritiun. The first goal, that of producing tritiun for sustaining the D-T reaction, requires that there be a significant amount of tritium on site. Various design options exist and these in turn will determine potential tritium inventories as well as the potential for tritium release. The two main design options considered for this system (and which will face future designers) are to continually recover the tritium bred in the blanket, or to allow the tritium to build up in the blanket and only recover at long intervals when the bred-fissile fuel is recovered.

These two design options have different implications for safety. In the first option, (continual recovery), there will be less tritiun in the blanket at any given time, but it will be continuously handled. In addition, greater quantities of tritium will be found throughout the plant at all times during life. Attributable to such a design is the possibility of having minor accidents with relatively high frequency of occurrence. 
Using the typical frequency-consequence diagram, ${ }^{15}$ this type of design would tend to yield points in the low-consequence high-frequency portion of the risk curve.

The second option, that of allowing the tritium bred in the blanket to continue to build up, reduces the anount of tritium handled during normal operation and consequently can eliminate many potential high-frequency low-consequence events. In addition, the amount of tritium found throughout the plant will be less. On the other hand, the anount of tritium that could be released in a blanket or fuel handling accident increases considerably. These accidents would have a much lower frequency of occurrence, but would result in a higher consequence event. In addition, a large supply of tritium external to the blanket will also be necessary and thus must be accounted for.

If safety were the only criterion for determining which design option to follow, several considerations could be made. First, one could compare the total risk of each approach (summing or integrating over the consequence-frequency curve) and make the determination on that basis alone. Inherent limitations in this approach, and at this time, are a lack of sufficient design data to achieve accurate points, lack of knowledge of all potential accidents, and lack of acceptable risk criteria. 23 second, if blanket accidents were of concern, one could compare the tritium in either option to the potential fission-related hazard to determine whether or not it adds significantly. ${ }^{24}$ This point will be addressed in a later section.

For the design presented here, the option of retaining the bred tritium in the blanket until the fissile inventory is changed is adopted. This approach is taken for two reasons. Consistent with the goals of these preliminary reactor designs, this option, which has not been used before, will permit an examination of the potential safety, economic, design, and technological problems. Second, some gain in safety is considered to occur with hold-up of tritium, although no data and little experience exist to verify this consideration.

Using this mode of operation it is ariticipated that $10^{8} \mathrm{Ci} / \mathrm{GH}$ year will be permitted to accuraulate in the blanket. 
12.2.2.3 Fission Products and Actinides. The second and third goals, to prodice plutonium by neutron absorption in depleted uranium and to use fission to produce a net energy (electricity) gain, lead to the actinide and fission product hazards.

There are basically two fissile fuel production schemes. These are the thorium-uranium scheme:

$$
{ }_{90}^{232} \mathrm{Th}+{ }_{0}^{1} \mathrm{n}+{ }_{90}^{233} \operatorname{Th} \beta^{-}{ }_{91}^{233} \mathrm{~Pa}+{ }_{92}^{233} \mathrm{v}
$$

and the uranium-plutoniun scheme:

$$
{ }_{92}^{238} \mathrm{~J}+{ }_{0}^{1} \mathrm{n}+{ }_{92}^{239} \mathrm{U} \mathrm{B}^{-}{ }_{93}^{239} \mathrm{~Np}{ }_{94}^{-239} \mathrm{Pu}
$$

The design option chosen here is the uranium-plutonium scheme. Since the plutonium is not taken out of the blanket immediately after production, some of it will fission and some of it will be transmuted to higher isotopes via $n, \gamma$ reactions. These higher isotopes include americium (Am), of which the

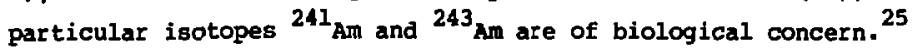

Although the major hazard with respect to the actinides is the plutoniun, some work is necessary to determine the quantities of the higher elements. For the thorium-uranium cycle, these higher actinides would only appear if the ${ }^{233} \mathrm{~J}$ were left for very long times in the blanket. From this point of view, there would appear to be less of a hazard for the Th- 0 blanket because of the lack of the higher actinide elements. However, there may be a problem with the buildup of ${ }^{232} \mathrm{U}$ which has a long decay chain of alpha emitters.

Regardless of the fuel scheme chosen, fission will take place in the blanket. Typlcal power densities considered $(250-500 \mathrm{H} / \mathrm{cc})$ are similar to power densities in Lin's. Hence, the fission product inventories in the fusion-fissiun hybria blanket will be comparable to those in pure fission devices per unit of energy produced. There is one difference, however. In the hybrid blanket, fission is caused by both 14-Mev neutrons coming from the plasina and by neutrons with energies below $2 \mathrm{MeV}$ born in fission. Approximately half the fission products are due to each source of neutron. As is well known, ${ }^{26}$ fission product yield curves are sensitive to neutron energy. In particular, this difference is accentuated for fission products pairs of nearly equal atomic weight (i.e., the two characteristic fission 
product yield bumps tend to smooth out). For many of the fission products of concern there is little change. However, ruthenium-106 is an exception. 26 This yield may be an order of magnitude larger for fast fission (14-MeV neutron-induced) than for slow fission (less than 2-MeV induced).

In summary, then, major hazard potential due to fission and/or transmutation is the result of the fission products and the actinide elements that build up. At present, detailed calculations of the guantitjes of these hazards are lacking.

12.2.2.4 Other Radioactive and Non-Radioactive Hazardous Material. Studies of pure fusion devices ${ }^{20,21}$ have indicated that the large inventories of such materials as mercury, lead, beryllium, chromium, sodium, and copper constitute potential hazards. In the absence of detailed design data, it is difficult to quantify their potential hazard. It is even more difficult to determine potential ways of making these urually solid materials mobile, and releasing them to the environment. The primary radicactive hazards other than tritium, fission products, and actinide elements are found primarily in the blanket structure and result from various neutron reactions. This potential hazard will, in all probability, be the same as that for a pure fusion device. For these reasons, only the tritium, radioactive fission products, and actinides will be considered in this context.

\subsubsection{Potential Accidents and Safety}

12.2.3.1 Introduction. Once the type, quantity, and location of material in a power plant is known, the safety or risk considerations can focus on two questions: 1) are there mechanisms for making these materials mobile? and 2) are there mechanisms for breaching containment so they can be released to the environment? For pure fusion reactors of the Tokamak type, witn lithium cooling, studies have indicated that the chemical energy released during lithium-concrete, lithium-water, and lithium-air interactions can serve as a source of energy for volatilizing radioactive structural material and the released energy from the cryogenic coolant systen can overpressurize and breach containment. ${ }^{20}$ other potential sources may be plasma-induced, magnet-induced, and site-induced (e.g., ear thquakes). 20 
The following discussion will focus on safety issues that pertain mainly to the fusion-fission hybrid blanket, although there way be sone overlap with the rest of the system. In particular, two major situations will be addressed: 1) undercooling - including loss of flow or loss or coolant (depressurization) and 2) overpower - including increase of plasna neutron source and reactivity ddditions to the blanket. Other potential accidents, such as flow blockages, fuel handling, etc.. will be briefly discussed.

12.2.3.2 Undercooling Accidents. The primary consideration in this type of accident is the removal of decay heat." Although it is possible to envision a situation where there is a loss of cooling ability (due to pump failure or depressurization) without shutdown of the plasma, such a situation is considered unlikely. Of higher probability is the potential loss of cooling ability after the plasma is quenched (e.g., loss of off-site power with failure of auxiliary power).

Preliminary estimates of the time required to reach fuel melting assuming adiabatic conditions and failure to quench the plasma are on the order of 5 to $10 \mathrm{s.}$ Without plasma heating, estimates are on the order of 100 to $200 \mathrm{s.} 27$ Typical decay heating rates are approximately 7 \% nominal power during the first 10 to $100 \mathrm{~s}$ and 1 at $10^{4} \mathrm{~s}(\sim 3 \mathrm{~h})$, and reach 0.1 at $10^{7} \mathrm{~s}$ (about $100 \mathrm{~d}$ ). Therefore, forced cooling is a necessity following plant shutdown to prevent melting.

Although there will be redundant (and perhaps eventually passive) cooling systems for decay heat removal (in this design either the main or auxillary cooling loops can be used), it is still important to understand the consequences of undercooling the blanket. In particular, the design basis accident (DBA) for this plant is a depressurization caused by gross failure of a helium duct in one blanket quadrant, ${ }^{2 \theta}$ After the depressurization is complete, the system reaches a quasi-steady state condition in which the fuel temperatures are determined by the decay-heat level, the coolant flow rate, and the inlet temperature. The ultinate course of events will be determined by the damage done to the components of the prinary heat transfer loop during the depressurization.

\footnotetext{
theating due to the radioactive decay of the fission products after shutdown.
} 
Assuming that all plant protective systems fail during an undercooling event, the decay heat will eventually cause melting to occur. As the fuel melts, some of the fission products and actinides will be released. These release fractions depend on several factors, including relative melting points, boiling points, and chemical behavior. For mixed oxide fuel, the release fractions used range from 0.005 for plutonium to 1.0 for the noble gases. Far the fuel considered here $\left(U_{3} \mathrm{Si}\right)$, these rolease fractions may be quite different.

A major question for this design is whether or not the tritium that has built up in the solid breeding material will also be released. As stated in Section 12.2.2, the design option of allowing the tritiun to build up in the blanket is chosen. Implementation of this approach has yielded a design in which the fuel zone (uranium pins) is adjacent to the lithiun (almost a continuation of the uranium pin) zone. Although the amount of heating due to the radioactive decay in the lithium pins is very small when compared to the fuel decay heat, any geometric arrangement where the molten fuel would drain or run down the lithium pin could cause a tritium release. such a tritiun relee . fraction would be close to unity.

To assess the potential risk to the public for such an event, the relevant pathways to man must be knom. This involves a detailed understanding of the containment and its behavior, as well as the biological pathways (e.g., food chains) outside the containment. For the gas-cooled blanket considered in this design, there are essentially two containment barriers. The high-pressure coolant zone constitutes one containment (inner) and the containment structure (outer) constitutes the other. Several design options exist which affect the containment boundary. A major option is the placement of the direct converter (geonetrically below the reactor) and whether or not it is inside the inner containment zone. sitting geometrically below the reactor, it would probably be the place where molten fuel would collect in the event of a melt. Its ability to hold the nelt, or even cool it, would have a profound impact on safety. If the fuel could freeze there, the release would be limited. (Similar considerations are present in the event of a dropped fuel element during refueling.) In the absence of both a final contaimment design and fission product and actinide release data from uranium sillcide, it is difficult to quantify release mechanisms. 
Atterpts are currently under way to wodel critical pathways to man for tritiun, fission products, and actinides produced in fusion-fission hybrids. ${ }^{29,30}$ Holdren has qualitatively shown that using the oxide fuel release fractions, from Ref. 15, the release weighted BAP for tritiun that has accumulated in this design option is much less than the expected plutoniu and fission products. ${ }^{24}$ To assess the impact on the health and safety of the public, the potential for release to the enviroment, the critical pathways, and total dose-comitment (total dose over the life of the element in the biosphere) should also be considered. From this type of consequence modeling, more information on the relative impact of tritium and the fission product ejements can be obtained.

12.2.3.3 Overpower Accidents, 'the two areas of concern in overpower situations are: 1) an overpower in the plasma which produces more 14-MeV source neutrons in the blanket and 2) a criticality accident in the blanket itself.

Although mirror fusion devices may not have the potential for major plasma power overshoots that may occur in a Tokamak fusion device, the ability of the blanket to withstand power changes (both transients and fluctuations) should be assessed. Any rapid overheating of the fuel in the blanket, without additional cooling, and beyond the design limits, will cause fuel failure with the potential for fission product and/or actinide release. As experienced in both LWR's and LMFBR analysis, the behavior of the fuel pins, during both steady state and transient irradiation, has a profound effect on safety. In the absence of such behavior, it is difficult to characterize the behavior of the blanket fuel during a transient.

The second consideration, perhaps of more concern, is a reactivity accident. Although the blanket is designed to be substantially subcritical at all times, there are conceivable ways in which blanket multiplication can increase. The first is that, due to a desire for higher burn-up, the blanket elements remain in the reactor for longer periods than originally designed for. In this case the plutonium will continue to build up until either the

blanket is critical or it zquilibrates. Preliminary calculations indicate

\footnotetext{
Biological hazard potential. In this work, the BHP was taken as the number of curies divided by the maximum permissible concentration in air.
} 
that the plutonium concentration equilibrates before criticality is achieved. 31 The second is that the fuel reconfigures into a more critical arrangement, or there is a reconfiguration of other materials such as water or lithium.

Studies have been made in which water (from the steam generators) is allowed to enter the fissile fuel blanket. 31 preliminary results indicate that the water wwderates the neutrons, causing a spectral shift into the Doppler region for uranium absorption. This causes a reduction in multiplication for the designed geonetry and for build-up of plutonium to about 4 at. 3 . If the fuel pin pitch is increased so that more water can enter, the neutrons can be thermalized and multiplication will increase. Here, too, there is a tradeoff between water/plutonium fraction.

The fuel can potentially reconfigure in several ways. A major reconfiguratica can occur if the fuel melts and gravity induces a flow in one portion of the blanket. Other potential sources of reconfiguration are forces due to a depressurization accident or a major magnet failure. Both could lead to large forces on the blanket modules which could cause reconfiguration.

A goal of this design is to keep the concentration of plutonium low, so that regardless of melting, reconfiguration of fuel or of other materials, the blanket will remain subcritical.

12.2.3.4 Other Considerations. There are several other considerations that should be mentioned for completeness. There are several other potential accidents which may influence a fusion-fission hybrid design. These include flow blockage accidents, fuel handling accidents, stear generator accidents, cryogenic coolant accidents, magnet disruption accidents, and energy storage accidents.

of particular concern is the possibility of a fuel handling accident, because some type of active cooling will be required during fuel handling, and because the fuel (containing fission products and actinides) will be contiguous with the tritium. Any damage to an element can potentially lead to a release of the volatile fission products and tritium. During fuel handling operations, one or more of the penetrations may be open, rendering containment less effective. 
Failure of a stean generator or a cryoganic cooling system can cause missile generation and overpressurization, which can result in containnent and/or system fallure. These, in addition to magnet failure, can cause blanket reconfiguration as discussed in section 12.2.3.3.

The major concern of a flow blockage is the possibility of propagation. Such an event, if undetected, can lead to fuel melting.

\subsubsection{Summary and Conchusions}

The major potential hazards that appear in this design are tritium, fission products, actinide elements, and induced activity in the blanket structural material. The major threat to the health and welfare of the public appears to be the release of thse materials as a result of the blanket fuel melting coupled with breaching both inner and outer containment. While other accident paths can be postulated, it is quite clear that forced (active) cooling is required at all times in the blanket to keep the fuel from melting. Of particular concern is the requirement for decay heat removal following reactor shutdown and during spent-fuel handling. Failure to do so leads to fuel melting on a relatively short tine scale.

Other potential blanket events include overpower transients due to increased plasma power, reactivity insertions aue to fuel or other material reconfiguration, and fuel handling accidents. Keeping the blanket subcritical under all conditions is a design goal. Because of the low burnup anticipated, criticality appears difficult to achieve.

Nonblanket events such as magnet failures, cryogenic coolant system failure, and gross depressurization can have potential effects on the safety of the blanket.

A good deal of information regarding the behavior of the blanket fuel during irradiation, and during mild and severe transients, is required to adequately assess the risk to the public. In particular, the behavior and release of the fission products and actinide elements up to and during fuel melting need to be known.

Lastly, a proper perspective on the risks of an IWR economy based on plutonium fuel can only be gained when the Individual, as well as the cunulative, risk of the fuel cycle is determined. 


\section{References}

1. Proposed ANS Standard Decay Energy Release Rates Following Shutdown of Uranium-Fueled Thermal Reactors, American Nuclear Society, ANs-5-1 (October 1971).

2. R. W. Moir, et al., Progress on the Conceptual Design of a Mirror Hybrid Fission-Pusion Reactor, Lawrence Livermore Laboratory, Rept. UCRL-51797 (June 25, 1975).

3. GCFR Preliminary Safety Information Document, General Atomic Co., Rtpt. GA-10298 (February 15, 1971).

4. Preapplication Safety Evaluation of the GCFR, Project 456 , Nuclear Regulatory Commission (August 1, 1974).

5. 300-MW(e) GCFR Demonstration Plant, General Atomic Co., Rept. GA-Al3045 (July 15, 1974).

6. 750-MW (e) GCFR Demonstration Plant, General Atomic Co., Rept. GA-A13459 (January 21, 1976).

7. Fulton Generating Station Preliminary Safety Analysis Report, Philadelphia Electric Company (NRC Docket 50-463/464).

8. General Atomic Standard Safety Analysis Report, February 5, 1975 (NRC Docket STN-50-535).

9. J. J. Di Nunno, et al., Calculation of Distance Factors for Power and Test Reactor Sites, U.S. Atomic Energy Commission, Rept. TID-14844 (May 23, 1962).

10. L. C. Richardion, et al., CONTEMpT A Computer Program for Predicting the Containment Pressure-Temperature Response to a Loss-of-coolant Accident, Gulf General Atomic Co., Rept. GA-8692 (May 1, 1968).

11. United States Atomic Energy Commission Rules and Regulations, Title 10, Atomic Energy, Part 100, Reactor Site Criteria (1962).

12. R. R. Deremer, et al.. Need for a Conventional Containment on HIGR's, General Atonic Co., Rept. GA-Al3465 (May 1975). 
13. K. Schultz, "Summary of Discussion Session on Fusion-Fission Reactor Safety," in Proc. US-USSR Symposium on Fusion-Fission Reactors, July 13-16, 1976, CONP-760733.

14. J. Holdren, "Sumary of Discussion Session on Fuston-Fission Reactor Safety," in Proc. US-USSR Symposium on Fusion-Fission Reactors, July 13-16, 1976, CONF-760733.

15. Reactor Safety Study: An Assessment of Accident Risks in U.S. Commercial Nuclear Power Plants, U.S. Nuclear Regulatory Commission, WASH-1100 (1976).

16. C. I. Comar, "Blological Risks of Electricity Production," Aware 63 (1965).

17. T. W. T. Burnett, "The Euman Cost of Regulatory Delays," in Proc. 22nd Annual Meeting of the American Nuclear Society, Toronto, 1976.

18. D. Okrent, "Some Thoughts on Fast. Reactor Safety," in Proc. Meeting on Fast Reactor Safety and Related Physics, Chicago, 1976.

19. C. Ashworth, "Sumary of Discussion Session on Fusion-Fission Reactor Safet:y," in Proc. of the US-USSR Symposium on Fusion-Fission Reactors, 1976, CONF-760733.

20. D. Okrent, พ. E. Kastenberg, et al., "On the Safety of Tokamak-Type, Central Station Fusion Power Reactors," Nuclear Engineering and Design 39, 215-238 (1976).

21. R. G. Clark, Safety Review of Conceptual Fusion Power P1ants, BNwL-2024 (1976).

22. L. Cave, D. Ilberg, and D. Okrent, "Designing for Safety in Fast Reactors in the Presence of Uncertainties," in Proc. International Meeting on Fast Reactor Safety and Related Physics, Chicago, 1976.

23. W. W. Kastenberg, T. E. Mckone, and D. Okrent, on Risk Assessment in the Absence of Complete Data, School of Englneering and Applied Science, University of California, Los Angeles, Rept. UCra-gar-7677 (1976).

24. J. Holdren, Lawrence Livermore Laboratory, Livermore, Calif., private communication (November 12, 1976).

25. R. Rose, et al., "Tokamak Actinide Burner Design Study," in Proc. of the US-USSR Symposium on Fusion-Pission Reactorg, 1976, CONF-760733.

26. G. R. Reepin, Physics of Nuclear Kinetics (Addison-Wesley Publishing Co., New York, 1965). 
27. R. R. Schultz, et al., Mirror Hybrid Reactor Blanket and Power Conversion System Conceptual Design," in Proc. of the US-USSR Symposium on Fusion-Fission Reactors, 1976 , CONP-750733.

28. K. R. Schultz, et al., Conceptual Design of the Blanket and Power Conversion System for a Mirror qybrid Fusion Fission Reactor, GA-A-14021, (1976).

29. T. Hakone, W. E. Kastenberg, and A. Z. Ullman, "Tritium Kealth Effects During Off Normal and Normal Releave in CF's." Mis Transactions (1977).

30. T. WcKone, in some Safety Considerations in Fusion-Fission Hybrid Reactors, Final Report to the Electric Power Research Institute (to be publiahed).

31. V. Badham, in Sone Safety Congiderations in Fusion-F1ssion Bybrid Reactors, Final Report to the Electric Power Research Institute (to be published). 


\section{APPENDIX A: ALTERNATE MECHANICAL DESIGN}

In the mirror hybrid design presented in $1976,{ }^{1}$ the blanket and shield could only be serviced by removing one of the huge mirror " $C$ " magnets (Fig. A-1). A flotation technique was employed where the mass moved was about 50,000 tonnes. One distinct advantage of that technique was the ability to vacuum-leak test, pressure test, and even coolant flow test one of the "orange section" blanket modules (Fig. A-2) prior to installation.

That concept and its advantage could not be ignored in the present 1977 work, so a refi'nement of it was developed in parallel with the "new" approach preser ed in the main body of this report (see $\mathrm{Ch}$. 8). This appendix describes a much different design approach, which has only one major drawback so far recognized - it still requires temporary relocation of one mirror magnet weighing approximately 3000 tonnes by means of a polar bridge crane. Cranes of such lifting capacity are about 50 s beyond current designs but ind'stry is willing to discuss and bid such a machine.

\section{Magnet Restraint}

A prestressed concrete reactor vessel (PCRV) is proposed as a magnet force-restraining structure and a vessel to encapsulate the primary coolant loop equipment (Figs. A-3 and A-4). Steam generators, helium circulators, and major coolant ducts would be permanently anchored in cylindrical cavities within the concrete. The probability of a major accident involving a primary coolant or steam circuit is greatly reduced by such anchoring of major conponents. Serviceability is maintained by covered ports that allow crane access to all steam generators and helium circulators by the polar bridge crane.

As in the primary design, we plan to have the PCRV at room temperature and transfer loads from the cold ( $4 \mathrm{~K}$ ) magnets to the warm structure by load-bearing insulation. Such a material as Glasrock (with better uniformity cont :ol) or an aerospace insulation developed by Lockheed Corporation (wi :h higher density) appears promising as a load-transfer

1. J. D. Lee, Ed., Mirror Hybrid Reactor Studies - July 1975 through September 1976, Lawrence Iivermore Laboratory, Rept. UCRL-50043-1 (1976). 


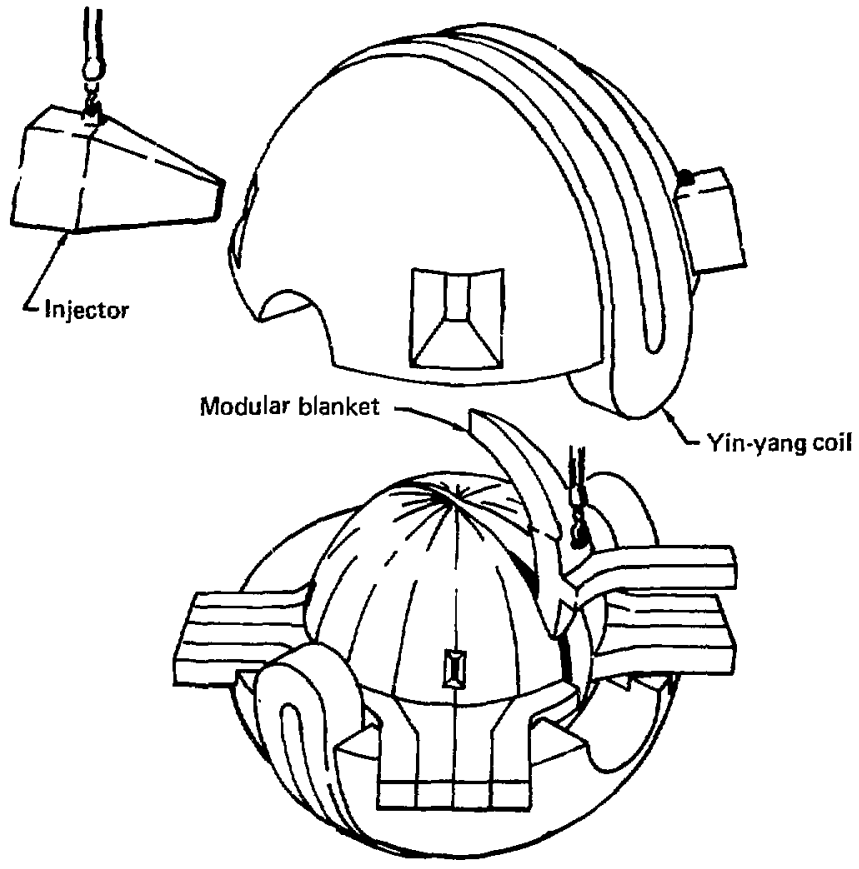

sig. A-1. Lirge blanket-module concept. 

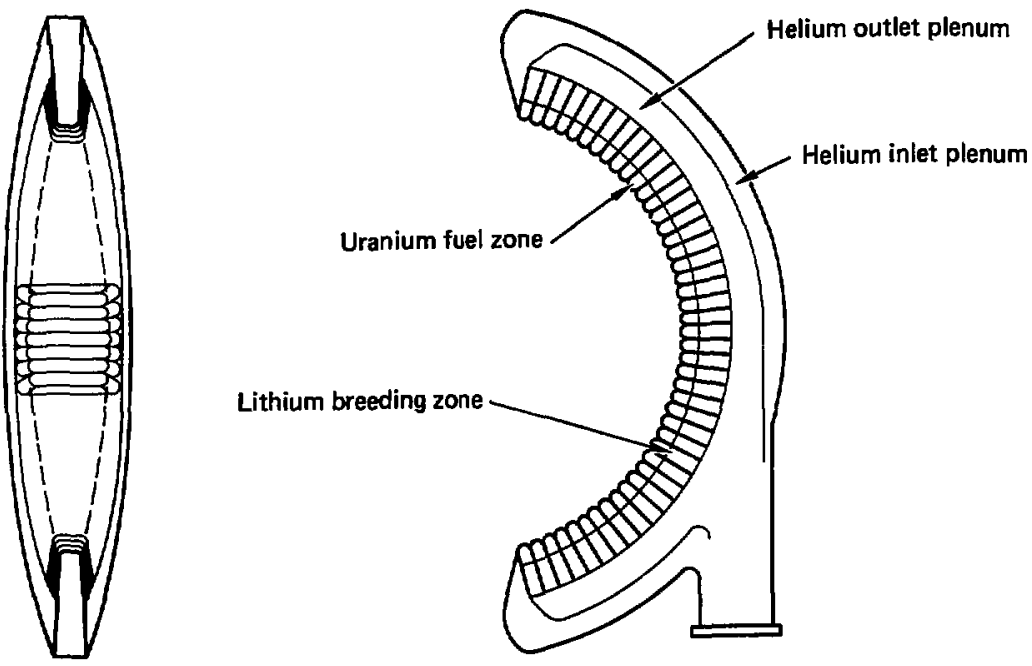

Fig. A-2. Individual blanket module. 


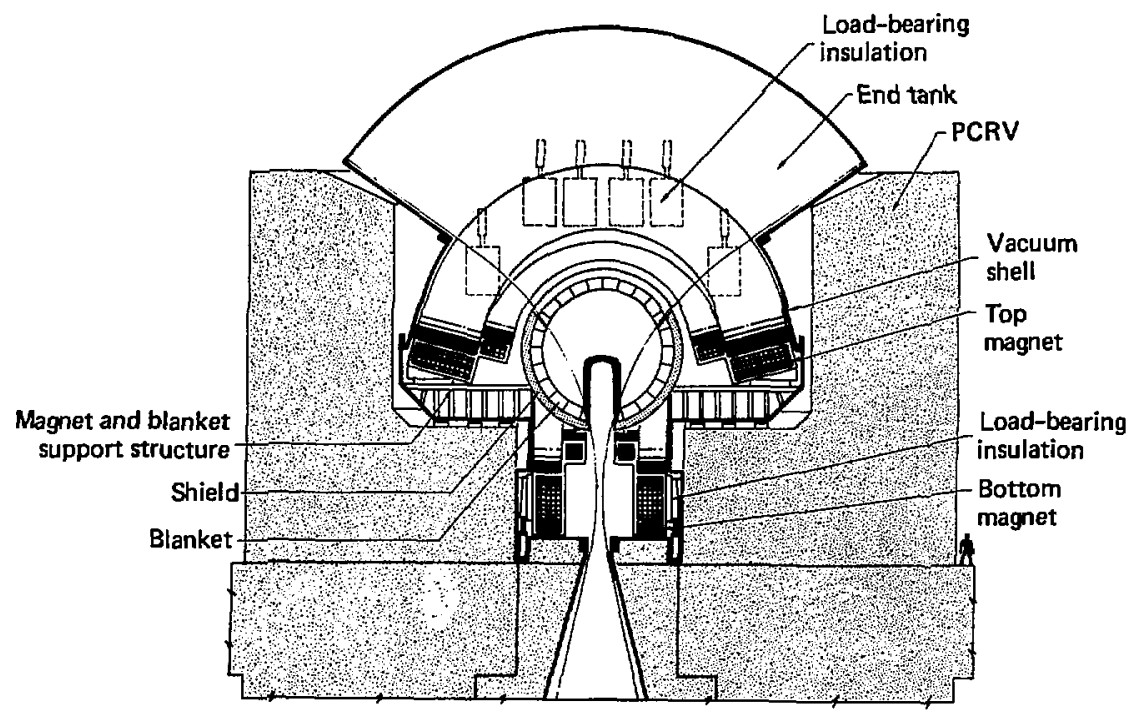

Fig. A-3. Fusion components and PCRV - elevation. 


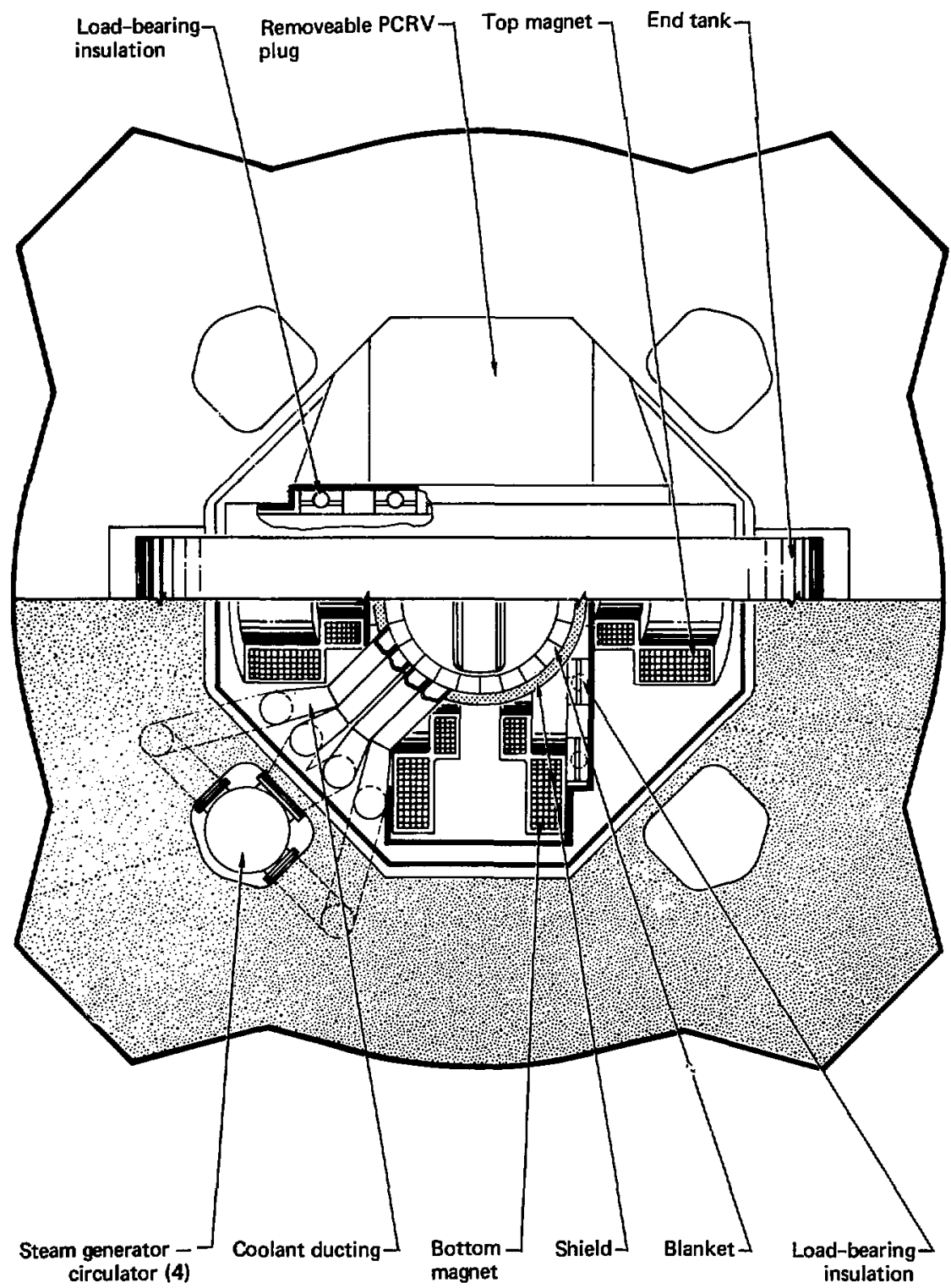

Fig. A-4. Pusion components and PCRV - plan view. 
insulator. Both are silicates, the latter employing microfibres purchased from Johns-Manville Corporation. Steps for magnet removal are the following:

1. Roll aside the top ion energy dump 7.5-m $\times 10-\mathrm{m} \times 35-\mathrm{m}$ box on rails located on the top of the PCRV.

2. Hoist the top vacuum cover (hexagon shape) and park it on a space provided that overlaps a portion of the PCRV.

3. Hoist the two identical load transfer plugs (each weighs about 1500 tonnes), and park them on the reactor building floor adjacent to the PCRV.

4. Hoist the upper " $\mathrm{C}$ " magnet and park it on fixtures provided, also overlapping the PCRV.

5. Hoist the shield dome, a hemisphere covering the top half of the blanket assembly, thus exposing the blanket "orange-section" shaped modules (Fig. A-5).

Changing the blanket involves removal of the "orange-section" moaules, transporting then to a fuel reprocessing and blanket rework shop, and replacing then with identical units prepared and tested in advance of the change-over. The biggest obstacle to quick blanket module change is the disconnect and reconnect to the large $(1.5-\mathrm{m}-\mathrm{diam})$ helium coolant ducts. We strongly believe that such duct connections need to be made or broken by a "hands-on" crew of technicans, not by a remotely operated wrench or welding and grinding machines.

To permit this, we have allowed both inlet and outlet coolant ducts to hang down in a "well" that goes down through the lower half of the PCRV and into a service cavity in the thick reinforced concrete floor supporting the PCRV (Fig. A-6). This work "cave" is $20 \mathrm{~m}$ from the center of the plasma zone and is separated by about $10 \mathrm{~m}$ of concrete from any neutron-activated materials. As the modules are hoisted for disposal, the long ducts rise up out of the "well". Lift height may be a problem and can be reduced by a simple cut-off and weld-on operation, taking place near the top level of the PCRV in a location where remotely operated tools can be much more easily operated without space restriction. Handling the modules without the long ducts attached is uncomplicated. The blanket segment design is described in Ref. 1. The only difference proposed here is that each module be $1 / 8$ instead of $1 / 16$ of a sphere. This reduces handling time and is still well within crane capacity. To maintain coolant flow symmetry in the upper and 


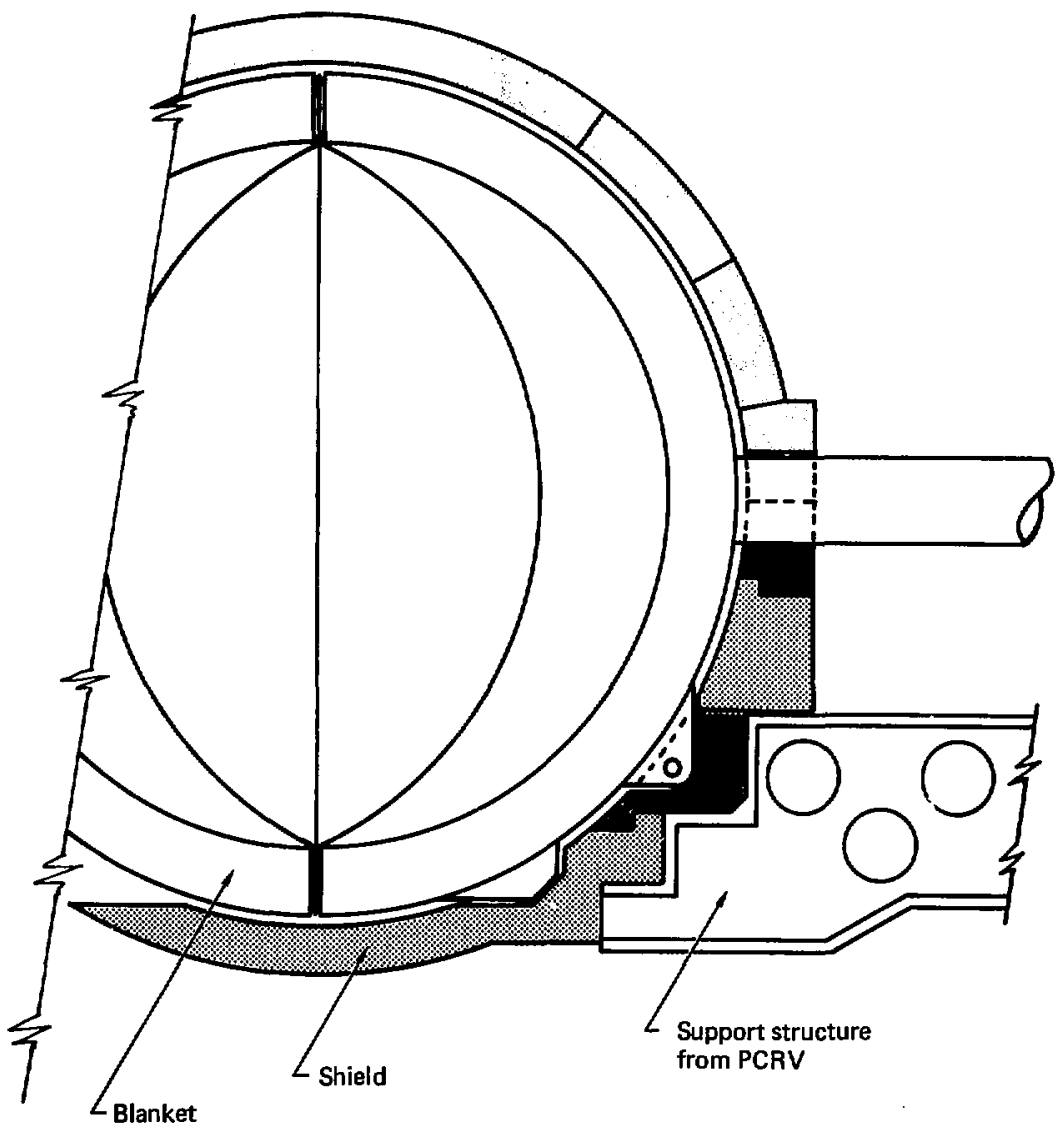

Fig. A-5. Blanket, shield, and supporting structure. 


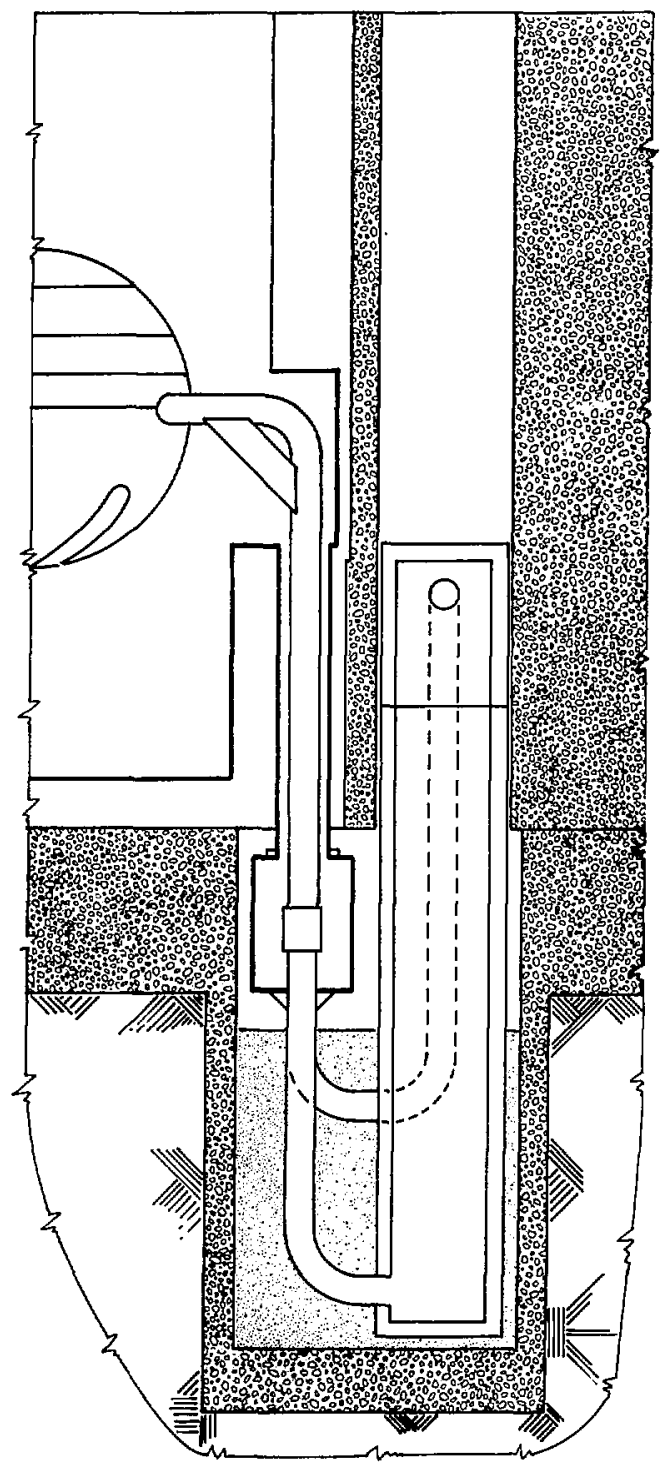

Fig. A-6. Blanket coolant piping. 
lower halves of the segaent, it is desirable to have both the inlet and exit ducts at the mid-plane (the equator of the sphere). side-by-side duct placesent at the mid-plane is possible with eight segments, but noncircular ports would be necessary if sixteen segments were used. A decision on blanket subdivision is not important at this stage of the design. The conceptual plant layout, based on this approach to the mechanical design of the reactor, is shown in Fig:- $\mathrm{A}-7$. 

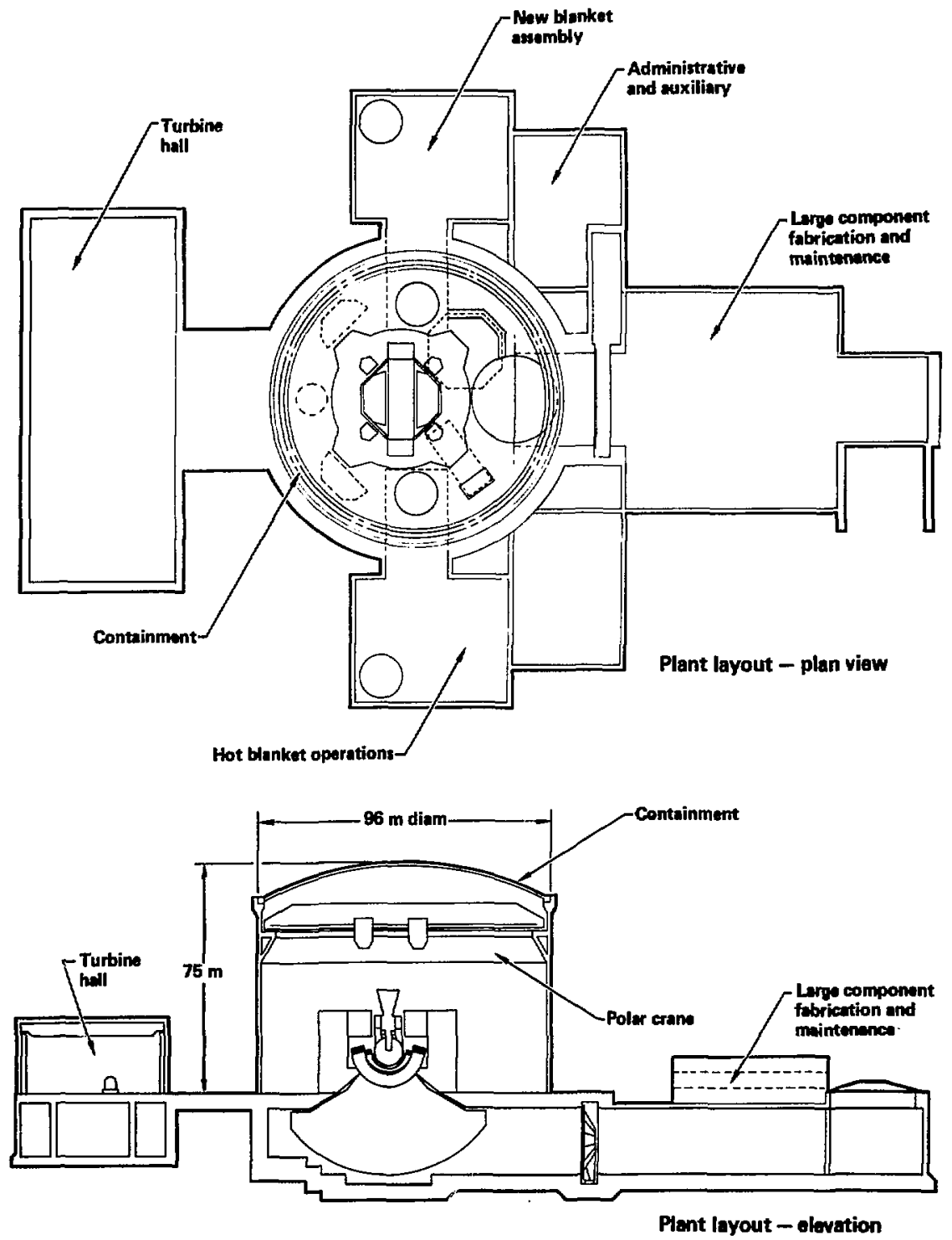

Fig. A-7. Plant layout. 


\section{APPENDIX B: EVALUATION OF HYBRID COST ACCOUNTS}

\section{B.1 Capital Costs}

The capital cost accounts that we treat are listed in Table B-1. The table is specifically tailored for the present hybrid reactor but follows the general format of Ref. 3 in Sec. 4. Accounts 21-25 are for direct capital costs; e.g., equipment, labor and materials. The sun of these accounts is the total plant direct capital costs. Accounts 91-94 are indirects, and these costs are successively compounded, starting with the total direct costs (see Table 4-3, Sec. 4), to arrive at a total plant capital cost.

Our cost estimates for the reactor components are based on the following sources: fission reactor experience (LWR and HTGR); cost estimates for HFTF; cost estimates from Bechtel Corp. for (previously published) conceptual fusion reactor designs; and some estimates were made In-house by the IrL/GA design team. Below, we do not detail the derivation of each cost, but merely quote the sources.

Several of the reactor components are based on the thermal output of the plant. Since the blanket thermal output varies with time, for costing purpnses we define the plant thermal power level as the maximum blanket power plus the thermal output from the end tanks.

In the following discussion, the notation $c(x \times x)$ is used to denote the cost of component $\mathrm{xxx}$.

211. Site Improvenent 1

$$
c(211)=\$ 1.5 / \mathrm{kwt} \text {. }
$$

\section{Reactor Building}

This cost was estimated by ILL based on the volume of material in the drawing of the reactor building in Fig. 2-1. It was assumed that the ratio of concrete voluwe to reinforcing steel was $6 \mathrm{yd}^{3} /$ ton in vertical walls and 10 $\mathrm{yd}^{3} /$ ton in horizontal slabs. Bxcavation was estimated at $\$ 2.95 / \mathrm{yd}^{3}$, fill and compaction at $\$ 3.15 / \mathrm{yd}^{3}$, concrete work at $\$ 200 / \mathrm{Yd}^{3}$, and reinforcing steel at $\$ 500 /$ ton. Thus,

$$
C(212)=\$ 69.4 \times 10^{6} .
$$


Table B-1. Capital cost accounts.

21 Structures and Facilities

211 Site improvement

212 Reactor building

213 Turbine building

215 Auxtliary butlding

218 A. Control room building

B. Diesel generator bullding

C. Administration building

D. Service bullding

22 Reactor Plant

221 Reactor equipment

.1 Magnet

.11 Hindings

.12 Coil case

.13 Insulation

.2 Injectors

.21 Sources

.22 Power supplies

.23 Accessories

.3 Direct converter

.31 Vacuum tanks

.32 Grids and collectors

.33 Electrical equipment

.34 Cooling

.4 Shielaing

.41 Coil shiela

.5 Vacuum system

.51 Cryopanels

.52 External vacuum equipment

.6 Blanket Structure

.7 Reactor Vessel

.71 PCRY

.72 Liners and insulation 
Table B-1. (continued)

222 Kain Heat Transfer Loop

.1 Stean Generators

.2 Circulatora

.3 He Purification equipment

223 Auxiliary Cooling

.1 Refrigeration

.11 Iiquia helium

.12 Liquid nitrogen

.2 Blanket Auxiliary Cooling

.3 Injectors

.4 Coil shield

224 Radioactive Waste Treatment and Disposal

225 Fuel Handling and storage

.1 Refueling equiprent

.2 Tritium reprocessing and storage

.3 Tritium clean-up

227 Instrumentation and Control

23 Turbine plant Equiprent

24 Electrical Plant Equipment

25 Miacellaneous Plant Equipment

251 Transport and lifting

252 Air, Water, and steam Service Equipment

91 Indirect Field Costs

92 Professional Services

93 Other Costs

94 Interest During Construction 
213. Turbine Building ${ }^{2,3}$

$$
C(213)=\$ 5 / k F t
$$

This cost is based on an LWR and is increased approximately 50 to account for the (zssumed) more-complex fusion reactor.

214. Auxiliary Buildings 2

For the hybrid reactor, this structure will house such components as the blanket auxiliary cooling equipment, helium coolant make-up equipment and refrigeration equipment:

$$
\text { C(215) }=\$ 4 / \mathrm{kWt} \text {. }
$$

218A. Control Room Buikding 1,2

$$
C(218 \mathrm{~A})=\$ 1.5 / \mathrm{kHt}
$$

218B. Diesel Generator Building 2

$$
C(218 B)=\$ 0.4 / k W t
$$

218C. Administration Building ${ }^{2}$

$$
c(218 \mathrm{C})=\$ 0.6 / \mathrm{kHt} .
$$

218D. Service Building 2

$$
C(218 D)=\$ 0.7 / \mathrm{kWt}
$$

\subsection{Magnet}

The magret windings cost estimate includes conductor at a cost of $\$ 0.31$ $\exp \left(0.19 B_{c}\right) \mathrm{kA}-m(\operatorname{Ref} 4)$, where $B_{c}$ is the maximum conductor field in Teslas), and winding labor, and installation at $\$ 2.5$ per $k A-m$ (Ref. 5). Thizs,

$$
C(221.11)=\left(2.5+0.31 e^{0.19 \mathrm{~B}} \mathrm{c}\right) \$ / \mathrm{kA}-\mathrm{m} \text {. }
$$


The magnet coil case is stainless steel and its costs are scaled from a previous design ${ }^{6}$ based on dimensions and maximum conductor field,

$$
C(221.12)=\$ 500 \times 10^{6} \times\left(\frac{{ }^{B}}{20}\right) \times f_{g}
$$

where $f_{g}$ is a geometric factor, related primarily to magnet surface area. Magnet insulation is the load-bearing insulation that transmits the magnet forces to the PCRV. This material was estimated to cost $\$ 25 \mathrm{CO} / \mathrm{m}^{3}$ (for 5000 psi compressive strength), and a 0.5-m-thick layer over the outside of the magnet surfaces was assumed. Thus,

$$
c(221.13)=\$ 2.5 \times 10^{3} \times t_{i} \times A_{s}
$$

where $t_{i}$ is the insulation thickness $(m)$ and $A_{s}$ the surface area $\left(m^{2}\right)$.

\subsection{Injectors}

The cost of the source scales as the ion current extracted from the plasma source. The extracted current, $I_{E}$, is related to the neutral current,

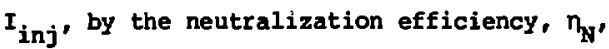

$$
I_{E}=I_{\text {inj }} / n_{N}
$$

Using a fit to existing sources and sources under development,

$$
\eta_{y}=0.7 e^{-0.006 w_{i n j}}
$$

where $\boldsymbol{w}_{\text {inj }}$ is the injection energy in kiloelectron volts. Then, using a cost of $\$ 75,000$ per $80-A$ (extracted) source, 5 and including $25 \%$ spares,

$$
c(221.21)=\$ 75 \times 10^{3} \times 1.25\left(\frac{I_{E}}{80}\right)
$$

We assune the power for the injectors is pulled directly off the line to facilitate reactor start-up. The injector power supply cost estinate includes filament and discharge power supplies, the accel-decel supplies, and the substation. Scaling from Ref. 7, 


$$
C(221.22)=\$ 80 / \mathrm{kWe} \text {, }
$$

where the total cost is based on the input power to the injectors.

The injector accessories cost estimate includes isolation valves, neutralizers (iron, ducting, and plumbing), and mounting and alignent fixtures ${ }^{5}$

$$
C(221.23)=\$ 27.5 \times 10^{3}\left(\frac{I_{E}}{80}\right) .
$$

\subsection{End Tanks}

The thin-walled lining of the vacuum tanks is assumed to be 1-can-thick stainless steel at an installed cost of $\$ 13.2 / \mathrm{kg}$, or $1.06 \times 10^{5} \$ / \mathrm{m}^{3}$ (Ref. 8). Thus,

$$
C(221.31)=\$ 1.06 \times 10^{3} \times A_{w},
$$

where $A_{W}$ is the wall area of the tanks.

The direct-converter collector structure, including coolant headers, for a single-stage unit is costed at $\$ 4,000 / \mathrm{m}^{2}$ of frontal area, ${ }^{8}$

$$
C(221.32)=\$ 4 \times 10^{3} \times A_{C} \text {, }
$$

where $A_{c}$ is the collector frontal area.

The power conditioning equipment is estimated at ${ }^{7}$

$$
C(221.33)=\$ 45 / \mathrm{kWe} \text {, }
$$

where the total cost is based on electrical output of the direct converter. The walls in the end tanks are water-cooled to remove heat radiated from the collectors and heat deposition from energetic particles. We base this cost on $\$ 2,000 / \mathrm{m}^{2}$ of wall area, 2

$$
C(221.34)=\$ 2 \times 10^{3} \times \mathrm{A} .
$$




\subsection{Shielding}

The magnet shielding is assuned to be a mixture of iron, lead and carbon at a cost of $\$ 14,000 / \mathrm{m}^{3}$ (Ref. 7).

$$
C(221.41)=\$ 14 \times 10^{3} \times\left(4 \pi R_{S B}^{2}\right) \Omega_{B L} t_{S H}
$$

where $R_{S H}$ is the radius of the (spherical) shield, $\Omega_{B L}$ the fractional blanket coverage, and $t_{s B}$ the shield thickness.

\subsection{Vacmum System}

The vacuum system equipment includes cryopanels and the external vacuum syster. The cost of the cryopanels, including the IN shield, wHe panel, chevrons, pluabing, Dewar, and Inspection is estimated to cost $\$ 6,300 / \mathrm{m}^{2}$ (Ref. 8).

$$
C(221.51)=\$ 6.3 \times 10^{3} \times A_{C p}
$$

where $A_{C p}$ is the total cryopanel area $\left(m^{2}\right)$.

The capacity of the external vacuun system is assuned to be proportional to the cryopanel capacity, and its cost is scaled from WFIF, 5

$$
C(221.52)=\$ 5 \times 10^{5} \times \frac{A \mathrm{CP}}{325}
$$

221.6 Bianket Structure 9

The cost of the blanket structure which is permanently fixed to the PCRV was estimated to be

$$
C(221.6)=\$ 1.47 \times 10^{6}
$$

\subsection{Reuctor Veasel9}

The reactor vessel consists of the PCRV (account 221.71) and insulation and liners for the PCRV (account 221.72). These costs were estinated to be

$$
\begin{aligned}
& c(221.71)=\$ 36.6 \times 10^{6}, \\
& c(221.72)=\$ 28.3 \times 10^{6} .
\end{aligned}
$$




\section{Main Heat Transfer Loop 9}

The steam generators, helium circtiators and helium purification system are similar to components developed by GA for gas-cooled reactors. Cost estimates for these three components are,

$$
\begin{aligned}
& c(222.1)=\$ 16 / \mathrm{kWt}, \\
& C(222.2)=\$ 2.6 / \mathrm{kWt}, \\
& c(222.3)=\$ 0.7 / \mathrm{kWt} .
\end{aligned}
$$

\section{Auxiliary Cooling}

The costs of the LHe and IN refrigeration systems are based on the input electrical power to the refrigerators, 10

$$
\begin{aligned}
& c(223.11)=\$ 4 \times 10^{4} \times\left[\begin{array}{ll}
P_{e} & L e^{(k W)}
\end{array}\right]^{0.7}, \\
& C(223.12)=\$ 4 \times 10^{4} \times\left[\begin{array}{ll}
P_{e} & \left.L^{(k W)}\right]^{0.7}
\end{array}\right.
\end{aligned}
$$

where $\mathrm{P}_{\mathrm{e}}$ is the input electrical power in $\mathrm{kW}$. In the above equations, the cost estimates have been increased threefold from Ref. 10 to account for transfer lines, valves, Dewars, storage, purification and installation.

The blanket auxiliary coolant loop cost estimate was developed by GA, 9

$$
C(223.13)=\$ 4.7 / k \text { Ht }
$$

where this total cost is based on the peak blanket thermal output.

The injector cooling circuit was estimated by LLL at

$$
C(223.14)=\$ 25 / \mathrm{kwt} \text {, }
$$

where the total cost is based on the heat deposited in the injectors and this estimate reflects a high-temperature coolant circuit to permit thermal conversion of the injector heat.

A simple low-temperature cooling water circuit was assuned for the coll shield cooling, estimated by IrJ at 


$$
c(223.4)=\$ 5 / k \text { it } \text {, }
$$

where total cost is based on heat deposition in the shield.

224. Radionctive Waste Treatment and Disposal 1,3

$$
C(224)=\$ 2.5 / k \text { it }
$$

\section{Fuel Hadling and Storage}

The cost of the blanket refueling equipment was estimated by $\mathrm{GA}^{9}$ to be

$$
C(225.1)=\$ 18.5 \times 10^{6}
$$

The tritiun reprocessing and storage equipment includes facilities for purification, isotopic separation, recovery and storage. This cost was estimated by ILI ${ }^{11}$ at

$$
C(225.2)=\$ 4.8 \times 10^{6}
$$

The bulk of the cost of the tritium clean-up system is for air-handling equipment for the containment building. This cost was estimated by Lr. ${ }^{11}$ at

$$
C(225.3)=\$ 3.6 \times 10^{6}
$$

227. Instrumentation and Control 9

$$
C(227)=\$ 1.6 / \mathrm{knt} \text {. }
$$

\section{Turbine Plant Equipment}

The thermal conversion system is a steam cycle. From Refs, 3 and 6, we obtain

$$
c(23)=\$ 35 / \mathrm{kWt} \text {. }
$$

This cost includes equipment, materials and installation for the turbinegenerators, mechanical equipment, condensors, circulating water system, cooling towers and plping; it does not include the steas generators. 


\section{Electrical Plant Equipment}

We compared the costs for the equipment for a conceptual fusion reactor 1 to similar equipment for an LWR. ${ }^{2,3}$ There appears to be a strong dependence on volume of the buildings and cost per unit volume. We therefore defined the following factor,

$$
V F=\frac{C(21) / k w t}{\$ 22 / k w t}
$$

to account for the relative volume and relative cost per unit volume as compared to that for an LWR. In this equation, the numerator is the sum of accounts 211 through 218D per kilowatt; the denominator is the sane sum for an IHR. 3

The switchgear cost estimate is ${ }^{3}$

$$
C(241)=V F \times \$ 0.6 / k w t,
$$

which is almost entirely for station service. The station service equipment includes station service transformers, low-voltage substation, and auxiliary power sources (batteries, diesel generator). Because the cost of this equipment should be independent of building volume, we use LwR costs ${ }^{2}$ :

$$
C(242)=\$ 2.5 / \mathrm{kNt}
$$

The switchboards equipment includes the main and auxiliary control panels and their instruments. These also should be independent of building volume and we use ${ }^{2}$

$$
C(243)=\$ 0.5 / \mathrm{kHt} .
$$

The protective equipment costs are estimated at ${ }^{1,2}$

$$
c(244)=\$ 0.2 / k n t
$$

The electrical structures and wiring containers cost estimate includes underground ducts, cable trays, and conduit ${ }^{1-3}$

$$
C(245)=\$ 3.5 / k N t .
$$


The power and control wiring estimate covers the generator circuits, station service power wiring, control and instrument wiring, and containment penetrations, at a cost of ${ }^{1-3}$

$$
C(246)=V R \times \$ 5 / k N t .
$$

\section{Miscellaneous Phant Equipment}

The transport and lifting equipment (cranes and hoists) is estimated to $\cos t^{1-3}$

$$
c(251)=\$ 0.5 / \mathrm{kWt}
$$

The cost of the air, water, and steam service (auxiliary heating boilers) is estimated to be 2,3

$$
C(252)=\$ 1.5 / \mathrm{kNt} .
$$

\section{1-94 Indirect Costs}

A review of the available literature for indirect cost factors for nuclear power plants yielded the values given in Table B-2. We adopted the following set of values:

$$
\begin{array}{ll}
\text { Acct. } 91 & =0.20 \\
\text { Acct. } 92 & =0.15 \\
\text { Acct. } 93 & =0.05 \\
\text { Acct. } 94 & =0.20 \\
\text { contingency } & =0.10
\end{array}
$$

Table B-2. Indirect cost factors.

\begin{tabular}{rllllc}
\hline Reference & 91 & 92 & 93 & 94 & Contingency \\
\hline 1 & 0.23 & 0.16 & 0.08 & 0.24 & 0.30 \\
2 & 0.072 & 0.163 & 0.052 & - & - \\
3 & 0.079 & - & - & - & - \\
6 & 0.21 & 0.15 & - & - & - \\
12 & 0.246 & - & - & 0.208 & - \\
\hline
\end{tabular}




\section{B.2 Fuel Cycle Costs}

The individual fuel cycle components and their unit costs are listed in Table B-3. The tritium costs are approximately equivalent to Pu at $\$ 100 / 9$ (i.e., $239 / 3 \times \$ 100 / g$ ). Blanket costs are for replacentent of the module pressure shell each time the fuel bundle is changed. The fabrication costs assume fuel fabrication does not have to be done in a hot facility. The uranium cost is for depleted uranium from diffusion plant tailings. 
Table B-3. Fuel cycle unit conts.

\section{Component}

Initial fertile materials

$$
\text { Uraniua }
$$

LiD

Recovered fertile materials

Urantum

LiD

Initial fusile materials

$$
\begin{gathered}
\mathrm{D}_{2} \\
\mathrm{~T}_{2}
\end{gathered}
$$

Recovered fusile materials

$$
\begin{aligned}
& \mathrm{D}_{2} \\
& \mathrm{~T}_{2}
\end{aligned}
$$

Fabrication

Uranium

LiD

Reprocessing and disposal

Uraniuri

LiD

Spent fuel shipping

$$
\text { Uranium }
$$

LiD

Bl anket
Unit Cost

$\$ 9.80 / \mathrm{kg} \mathrm{HM}$

$\$ 50.00 / \mathrm{kg}$

$\$ 9.80 / \mathrm{kg} \mathrm{Ex}$

$\$ 50.00 / \mathrm{kg}$

$\$ 60.00 / \mathrm{kg}$

$\$ 8 \times 10^{6} / \mathrm{kg}$

$\$ 60.00 / \mathbf{k g}$

$\$ 8 \times 10^{6} / \mathrm{kg}$

$\$ 105 / \mathrm{kg}$ HII

$\$ 48.00 / \mathrm{kg}$

$\$ 215 / \mathrm{kg}$ EM

-

$\$ 15 / \mathbf{k g}$ EIM

$\$ 5 \times 10^{4} / \mathrm{m}^{2}$

of first wall 


\section{B.3 Blanket and Power Conversion System}

An important aspect of the technical assessment of is design concept is the economic feasibility. Cost estimates have been made for the blanket modules and for the nuclear steam supply system of the MHR. These estimates are of necessity crude due to the preliminary nature of this study. Both the fuel and NSSS, however, are generically related to the components in General Atomic's gas cooled fast reactors which provided a comparative basis for estimating the cost of the blanket and NSS components. Costs are given in July 1977 dollars on Table B-4.

\section{B.3.1 Blanket Cost Eraluation}

The entire blanket structure is replaced when the blanket fuel is reloaded. As a consequence, the total blanket cost is treated as a fuel cycle cust rather than as a capital expenditure.

The cost estimate is based on certain unit cost assumptions:

$\begin{array}{ll}\mathrm{U}_{3} \mathrm{Si} \text { material cost (depleted uranium) } & \$ 9.80 / \mathrm{kg} \\ \text { Rod hardware cost } & \$ 7 / \mathrm{rod} \\ \text { Pelletizing cost } & \$ 6 / \mathrm{rod}\end{array}$

Based on these assumptions, the estimate for the initial blanket cost is $\$ 52$ million. One-quarter of the blanket is replaced at each reload interval. The reload blanket cost, then, is $\$ 13$ million.

\section{B.3.2 System Cost Evaluation}

A very preliminary conceptual cost estimate was made for the nuclear steam supply (NSS) for the 1611 Me (gross) Mirror Hybrid Reactor. Included in the estimate is the primary heat transfer system, the auxiliary heat transfer system, the refueling equipment, the prestressed concrete reactor vessel and special instcumentation and controls associated with the primary coolant system. The cost estimate specifically excludes the Nuclear plasma Island, fuel and the NSS interfacing system that are normally included in the balance of plant (BOP).

The principal results are given in Table $B-4$ and include project engineering costs, indirect costs, contractual risks, and fees in terms of present day dollars but without interest during construction or escalation. Also, the estimte is for an "equilibrium" commercial plant; i.e., the first-of-a-kind engineering and development costs are assumed to have already 
been recovered by the sale of earlier plants, and this is a subsequent installation (for example, this might be the tenth plant).

The estimated cost is $\$ 262$ million for these systems or $\$ 163$ per $k$ He (gross) for this portion of the plant. Due to the large power requirement of the neutral beam injectors, the peak net MHR output is $737.5 \mathrm{Mfe}$. The NSsS cost, based on net electrical output, is $\$ 355 / \mathrm{kive}$. 
Table B-4. Cost summary of blanket and power convergion sytem (in July 1977 dollars).

FUEC COST (588 modules)

Fission zone

$$
\mathrm{U}_{3} \mathrm{Si} \text { - depleted, a } \$ 9.80 / \mathrm{kg}
$$

Rod costs 3862 rods/module (fabrication and hardware)

Lithium Zone

$$
\begin{array}{lr}
\text { LiH - natural, } \$ 5.00 / \mathrm{kg} & 0.60 \\
\text { Rod costs } 168 \mathrm{rods} / \text { module (fabrication and hardware) } & 1.35
\end{array}
$$

\section{Module Cost}

Fuel assembly and pressure shell - fabricated Inconel $718, \$ 30 / 1 b$

NUCLEAR STEAM SUPPLY

Reactor Vessel

PCRV

Concrete

Lirear tendons

Circumferential wire

Miscellaneous

Total PCRV

Liner and insulation

Liner (incl, all ducts and cavities)

Module support structure

Class A thermal barrier (inlet $T$ )

Class B thermal barrier (outlet $T$ )

Loading-bearing coil insulation

Total liner and insulation

Reactor vessel indirects

(1008 of direct field labor or 408 of direct total cost) 
Blanket and Power Conversion Equipment

Primary cooling loop

Steam generators (12)

70.00

Main helium circulators and valves (8)

Main circulator service system (8)

Helium purification system

Total primary cooling loop

Auxiliary cooling loop

Auxiliary heat exchangers (5)

Auxiliary circulators, controllers, and valves (5)

Auxiliary circulator service system (5)

Total auxiliary cooling loop

Refueling equipment

In-vessel machine, including cavity seal and ports

Fuel transfer equipment

Auxiliary transfer casks

Accessories

Bridge crane

Fuel handiing control station

Total refueling equipment

Instrumentation, controls and fluid systems

Primary coolant instrumentation

Nuclear instrumentation

Plant protection system

0.56

Operational plant control system

0.56

NSS data acquisition system

Fluids regulating equipment

Radioactive gas waste system

Total instrumentation, controls and fluid system

Total blanket and PCS direct cost

Blanket indirect cost, $r$ isk, G\&A, and margin

(658, 48, 38 and 108 cumulative)

Total blanket and PCS cost 
Table B-4. (continued).

Cost

Element

\$ milition

NUCLEAR STEAM SUPPLY TOTALS

DIRECT

201.20

INDIRECT

61.19

TOTAL 


\section{References}

1. UnMAK-III, A Noncircular Tokamak Power Reactor Design, Fusion Feasibility Study Group, Fusion Technology Program, Nuclear Engineering Department, University of Wisconsin, Madison, Wisconsin, Rept. UWFDM-150 (1976).

2. Pressurized Water Reactor Plant, 1000-MWe Central Station Power Plants Investment Cost Study, United Engineers and Constructors, Philadelphia, PA, WASH-1230 (1972) Vol. 1; for our present study, costs were escalated to 1977 at 8 per year.

3. H. I. Bowers, Cost-Model Modifications for the CoNCEPT-IV Computer Code, Oak Ridge National Laboratory, Rept. ORNL-TM-4891 (1975). This study presents costs in 1971 dollars; for our present study, costs were escalated to 1977 at 8\% per year.

4. R. M. Scanlon, "Superconducting Materials for Future Magnets, Cost Projections," Lawrence Livermore Laboratory, Internal Memo (Oct., 1975).

5. T. A. Kozman, MX Cost Estimate Report, Lawrence Livermore Laboratory, Internal Report (1976); also, F. H. Coensgen, Mx Major Project Proposal, Lawrence Livermore Laboratory, Rept. LLL-PROP-142 (1976).

6. An Evaluation of the Technical and Economic Feasibility of Mirror Fusion Devices, prepared by Bechtel Corporation, San Francisco, CA for Lawrence Livermore Laboratory (August 1976).

7. Standard Mirror Fusion Reactor Design Study, R. H. Moir, Ed., Lawrence Livermore Laboratory, Rept. UCID-176d4 (1978).

8. M. A. Hoffman, Electrostatic Direct Energy Converter Performance and Cost Scaling Laws, Lawrence Livermore Laboratory, Rept. UCID-17560 (1977).

9. See Sec. B.3, this appendix.

10. T. R. Strobridge, Cryogenic Refrigerators - An Updated Survey, National Bureau of Standards, Technical Note 655 (1974). This report contains cost estimates in 1968 dollars; for our present study, costs were escalated to 1977 at 88 per year.

11. See sec. 6.6 of this report.

12. Power Plant Capital Cost Current Trends and Sensitivity to Econonic Parameters, U.S. Atomic Energy Commission, WASH-1345 (1974). 